\title{
Nuclear Magnetic Resonance Studies of Quadrupolar Nuclei and Dipolar Field Effects
}

\author{
by \\ Jeffry Todd Urban \\ B.S., B.S. (Carnegie Mellon University) 1997 \\ A dissertation submitted in partial satisfaction of the \\ requirements for the degree of \\ Doctor of Philosophy \\ in \\ Chemistry \\ in the \\ GRADUATE DIVISION \\ of the \\ UNIVERSITY OF CALIFORNIA, BERKELEY \\ Committee in charge: \\ Professor Alexander Pines, Chair \\ Professor Robert A. Harris \\ Professor Dmitry Budker
}

Fall 2004 
The dissertation of Jeffry Todd Urban is approved:

\begin{tabular}{lc}
\hline Chair & Date \\
\hline & \\
\hline Date \\
\\
\hline
\end{tabular}

University of California, Berkeley

Fall 2004 
Nuclear Magnetic Resonance Studies of Quadrupolar Nuclei and Dipolar Field Effects

Copyright 2004

by

Jeffry Todd Urban 


\begin{abstract}
Nuclear Magnetic Resonance Studies of Quadrupolar Nuclei and Dipolar Field Effects

by

Jeffry Todd Urban

Doctor of Philosophy in Chemistry

University of California, Berkeley

Professor Alexander Pines, Chair
\end{abstract}

Experimental and theoretical research conducted in two areas in the field of nuclear magnetic resonance (NMR) spectroscopy is presented: (1) studies of the coherent quantummechanical control of the angular momentum dynamics of quadrupolar (spin $I>1 / 2$ ) nuclei and its application to the determination of molecular structure; and (2) applications of the long-range nuclear dipolar field to novel NMR detection methodologies.

The dissertation is organized into six chapters. The first two chapters and associated appendices are intended to be pedagogical and include an introduction to the quantum mechanical theory of pulsed NMR spectroscopy and the time dependent theory of quantum mechanics.

The third chapter describes investigations of the solid-state multiple-quantum magic angle spinning (MQMAS) NMR experiment applied to $I=5 / 2$ quadrupolar nu- 
clei. This work reports the use of rotary resonance-matched radiofrequency irradiation for sensitivity enhancement of the $I=5 / 2$ MQMAS experiment. These experiments exhibited certain selective line narrowing effects which were investigated theoretically.

The fourth chapter extends the discussion of multiple quantum spectroscopy of quadrupolar nuclei to a mostly theoretical study of the feasibility of enhancing the resolution of nitrogen-14 NMR of large biomolecules in solution via double-quantum spectroscopy.

The fifth chapter continues to extend the principles of multiple quantum NMR spectroscopy of quadrupolar nuclei to make analogies between experiments in NMR/nuclear quadrupolar resonance (NQR) and experiments in atomic/molecular optics (AMO). These analogies are made through the Hamiltonian and density operator formalism of angular momentum dynamics in the presence of electric and magnetic fields.

The sixth chapter investigates the use of the macroscopic nuclear dipolar field to encode the NMR spectrum of an analyte nucleus indirectly in the magnetization of a sensor nucleus. This technique could potentially serve as an encoding module for the recently developed NMR remote detection experiment. The feasibility of using hyperpolarized xenon-129 gas as a sensor is discussed. This work also reports the use of an optical atomic magnetometer to detect the nuclear magnetization of Xe-129 gas, which has potential applicability as a detection module for NMR remote detection experiments.

Professor Alexander Pines

Dissertation Committee Chair 


\section{Acknowledgments}

Graduate school is as much a test of endurance as it is anything else. My time here at Berkeley has taken me 5.5 years and $2.5 \times 10^{3}$ miles from home as well as $6.5 \times 10^{2}$ pages of dissertation in 11-point font closer to old age. Needless to say, the Berkeley experience hasn't always been easy, and I would like to thank some of the people who have shaped my time here and made the journey a little bit easier.

First of all I would like to thank my advisor Alexander Pines for providing me with the freedom to investigate anything I wanted and for the equipment, funding, and collection of brilliant scientists that facilitated my studies. Alex also has an infectious enthusiasm for science which helped draw me to the research group where I eventually made my home. I would also like to thank our administrative assistants Dione Carmichael and Alyse Jacobson for keeping the group running and taking care of all the everyday tasks that still managed to bewilder me. In particular I would like to thank Alyse for finding the funding to keep me here for a few months past my time; otherwise I would surely be on Telegraph Avenue now hanging out my "will solve equations for food" shingle.

I have had good the fortune to work or interact with many extremely smart and capable people during my time in the Pines group. I was lured onto my first research project here by Alexej Jerschow after an initial attempt by John Logan. I would like to thank Alexej for the experience on the MQMAS project as well as for his notes on average Hamiltonian theory, from which I finally began to understand the technique. I worked closely with John for my first several years here. He was always around when I needed to bounce ideas off of someone. He is a hard worker and a man of his word, and was a pleasure to work with. 
I was initiated into the dipolar field project by Andreas Trabesinger. Andreas is infinitely patient and a great teacher, and most of my initial understanding of that project came from him. Andreas was also the glue that held together our initially-wavering atomic magnetometry project with the Budker group in the physics department.

Josef Granwehr joined me on the dipolar field project after Andreas left. I don’t know what I would have done without Josef; whether I was pestering him with questions about complicated scientific topics or just for help with the computers, he always came through. I also enjoyed taking time off from work to watch movies with him late at night after everyone else had left. Josef has always been a great help to me and a source of humor.

My other early collaborator on the dipolar field project was Tanya Mazur. Most of that early work didn't make it into this dissertation, but I appreciated having someone else there who was at the same stage as me as we struggled to learn. Tanya always brought a lot of excitement to the group; it was a lot quieter here after she left.

I worked with Morgan Moschetti on the nitrogen-14 project; I initially recruited him for his expertise in sample preparation, but his curiosity and drive really added a lot to the project and helped to make me really think about things. In addition Morgan is a genuinely good man; he is very easygoing and was willing to ponder all sorts of topics, scientific or otherwise. He's one of the few men I've met about whom I can honestly say I wish I were more like him.

I've had a very fruitful working relationship with Dmitry Budker and his research group in the physics department ever since I took an atomic physics class from him my first year. He thinks about science all of the time and literally day or night was willing to 
answer all my little scientific questions. He also is very kind and has a very good sense of humor. I remember his advice as I was nervously awaiting commencement of my doctoral qualifying exam, which was something like, "In Russia we had a saying that your Ph.D. exam is 40 minutes of shame, but your Ph.D. is forever." Well, the exam was actually pretty painless - aside from the ten minutes when Dima and Bob Harris ganged up on me to tell me that they thought my Hamiltonian violated a fundamental law of physics - but of course there were plenty of other opportunities for me to be shamed scientifically during my time here. Dima's graduate student Derek Kimball infected me with his enthusiasm, and he was always willing to answer my questions. His other student Simon Rochester taught me things about quantum mechanics, and of course the experimental genius of his research scientist Valeriy Yashchuk aided our collaboration immensely.

Robert Harris was my quantum mechanics professor, and he was always willing to answer my esoteric questions. He is also a good person and I was glad to have known him; I especially enjoyed the occasional lunches my classmates and I had with him. Some of my most cherished bits of knowledge about quantum mechanics - density operators, the rotating frame, quantization of the radiation field - I learned first from him.

I am indebted to Jamie Walls for the first years that we spent together here. Jamie is absolutely brilliant as well as completely willing to discuss any scientific problem for any length of time. He was my first scientific mentor, and most of the good stuff I know about NMR I learned from him. I also remember a lot of late-night breaks we took from lab to walk down to the 7-Eleven and talk. Although we never worked together directly, it was a suggestion of Jamie's that was the inspiration for my work on nitrogen-14. I think it was 
his relation to me of a conversation he had with Andreas that led me to think about the role of dipolar field effects on quadrupolar nuclei.

I would like to thank Song-I Han for always being around to have "deep" conversations with and for looking out for me. She is a good person and I admire her a lot. She is actually quite wise and is one of the few people from whom I can honestly say I gained new insights about the world.

I would like to thank Rachel Martin for many distracting conversations at lab and for introducing me to kite flying. I would also like to thank Megan Spence for her sense of humor and for looking out for me, scientifically or otherwise. I'm grateful to Kimberly Pierce who always strives to be helpful, as well as being a naturally good storyteller.

Julie Seeley and I shared a lot of ups and downs during my time here, though I can say that during some of my darkest times here one of my only sources of brightness was making Julie laugh, and she's been a good friend to me these past couple of years. She is also a very careful scientist and kept me intellectually honest. Additionally, it was a comment of hers that first really made me think about the dipolar field project in terms of remote detection NMR.

Outside of the group I'd like to thank Ben Boussert for conversations about life, science, and politics, and for always being a source of advice and a moderating non-Pines lab influence. I'm grateful to Olga Fedin for her inspiration and kindness to me, particularly during the trying times of my qualifying exam. I learned a useful phrase from her: «Лучшее-враг хорошего,» which I suppose applies to writing a dissertation as much as it does to life. I'm grateful to Astghik Shahkhatuni for her humor, friendship, and support, 
especially during the trying times of writing my dissertation.

Of course I would like to thank my parents and my brothers for getting me where I am today. My father inspired my love of science (which my mother calls "brainwashing") and my mother helped inspire my love of learning by reading to me constantly. My brother Nathan has often been willing to talk about detailed technical subjects with me and to teach me when no one else would. 


\section{Contents}

List of Figures $\quad$ xi

List of Tables $\quad$ xiv

1 Introduction to the quantum theory of pulsed NMR 1

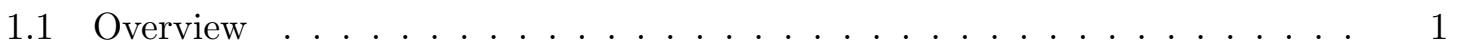

1.2 Magnetic structure of the nucleus . . . . . . . . . . . . . . 3

1.3 The Hamiltonians of NMR . . . . . . . . . . . . . . . . . . 11

1.3.1 Zeeman Hamiltonian . . . . . . . . . . . . . . . . . 13

1.3.2 Basic form of the solid state Hamiltonian . . . . . . . . . . . 17

1.3.3 High field truncation of Hamiltonians . . . . . . . . . . . . . 18

1.3.4 Chemical shielding Hamiltonian . . . . . . . . . . . . . . . . . . . 24

1.3.5 Dipolar coupling Hamiltonian . . . . . . . . . . . . . . . . . . . . 29

1.3.6 J-coupling Hamiltonian . . . . . . . . . . . . . . . . . . . . 37

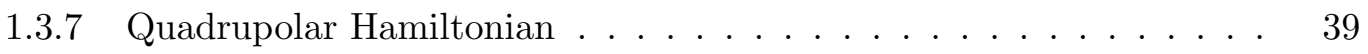

1.3.8 Radiofrequency Hamiltonian . . . . . . . . . . . . . . . 47

1.3.9 Motional averaging of Hamiltonians in fluids . . . . . . . . . . . 49

1.4 Density operator theory and propagation . . . . . . . . . . . 52

1.4.1 Introduction to the density operator . . . . . . . . . . . . 52

1.4.2 Open quantum systems and reduced density operators . . . . . . . 56

1.4.3 Ensemble-averaged density operators and thermal equilibrium . . . . 62

1.5 Relaxation of nuclear magnetization . . . . . . . . . . . 76

1.5.1 Introduction to relaxation . . . . . . . . . . . . . 76

1.5.2 Semiclassical relaxation theory . . . . . . . . . . . . . . . 79

1.5.3 Relaxation selection rules and spectral densities . . . . . . . . . . . 87

1.5.4 Dynamic frequency shifts and multiexponential relaxation under violation of extreme narrowing conditions . . . . . . . . . . . . 91

1.6 NMR excitation and spin manipulation . . . . . . . . . . . . . 96

1.7 NMR detection . . . . . . . . . . . . . . . . . . . . . . . 104

1.8 Fundamentals of two-dimensional NMR . . . . . . . . . . . . . . . . 114 
2 Time dependent theory for NMR $\quad 121$

2.1 Schrödinger time evolution . . . . . . . . . . . . . . . . . 121

2.1.1 The time evolution operator . . . . . . . . . . . . . . . . 122

2.1 .2 The density operator . . . . . . . . . . . . . . . . 129

2.2 Magnus expansion approximation for $\hat{U}\left(t ; t_{0}\right) \ldots \ldots \ldots \ldots \ldots$

2.3 Frames and representations . . . . . . . . . . . . . . . . . 144

2.3.1 Changes of frame . . . . . . . . . . . . . . . . . . . . . . . . . . . . . . 145

2.3.2 The interaction representation . . . . . . . . . . . . . 147

2.3.3 Common frame transformations in NMR . . . . . . . . . . . . . 151

2.4 Average Hamiltonian theory . . . . . . . . . . . . . . . 156

3 Mixed-coherence $I=5 / 2$ MQMAS experiments with low-amplitude RF $\begin{array}{lr}\text { pulses } & \mathbf{1 6 0}\end{array}$

3.1 Introduction . . . . . . . . . . . . . . . . . . . . 160

3.2 Removing the second-order quadrupolar broadening. The MQMAS experiment.164

3.2 .1 Conventional MQMAS . . . . . . . . . . . . . . . . . . . 167

3.2 .2 Mixed-coherence MQMAS . . . . . . . . . . . . . . . . . 173

3.3 Rotary resonance phenomena . . . . . . . . . . . . . . . 176

3.4 Rotary resonance experiments in $I=5 / 2$ systems . . . . . . . . . . . . 178

3.4 .1 Experimental procedure . . . . . . . . . . . . . . . 178

3.4.2 Sensitivity and line shape considerations in 5Q3QMAS NMR with low-amplitude pulses . . . . . . . . . . . . . . 179

3.5 Theoretical approach to rotary resonance line narrowing in $I=5 / 2$ systems 185

3.5.1 Average Hamiltonian in the quadrupolar interaction frame . . . . . 188

3.5.2 Average Hamiltonian in the quadrupolar/central transition RF interaction frame . . . . . . . . . . . . . . . 199

3.5.3 Crystallite orientation dependence of the Hamiltonian . . . . . . . . 204

3.6 Conclusions . . . . . . . . . . . . . . . . . . . 216

4 Feasibility of N-14 double quantum line narrowing in solution 222

4.1 Introduction . . . . . . . . . . . . . . . . . . . . 222

4.2 Theory of $\mathrm{N}-14$ linewidths in solution . . . . . . . . . . . . 225

4.2.1 N-14 double- and single-quantum linewidths and the comparison to

N-15 spectroscopy . . . . . . . . . . . . . . . . . 225

4.2.2 A comment on deuterium double-quantum spectroscopy . . . . . . . 233

4.3 Production of N-14 double-quantum coherence (DQC) in solution . . . . . . 236

4.3.1 DQC through the residual quadrupolar interaction in oriented media 237

4.3.2 DQC through $J$-coupling to a spin-1/2 in isotropic solution . . . . . 242

4.3.3 DQC through cross-correlation effects outside of the extreme narrowing regime . . . . . . . . . . . . . . . . . . . 244

4.4 Quadrupolar relaxation concerns for DQC creation in large- $C_{Q}$ systems . . 248

4.5 Experimental data . . . . . . . . . . . . . . . . . . 254

4.5.1 N-14 DQ-H-1 SQ correlation spectroscopy via the $J$-coupling . . . . 254

4.5.2 N-14 DQ-SQ correlation spectroscopy in oriented bicelles . . . . . . 256

4.6 Conclusions . . . . . . . . . . . . . . . . . . . . . . . 259 
5 Analogies between NMR/NQR and atomic/molecular optics 263

5.1 Introduction . . . . . . . . . . . . . . . . . . 263

5.2 Nuclear and atomic polarization . . . . . . . . . . . . . 265

5.2.1 Thermal equilibrium nuclear polarization . . . . . . . . . 268

5.2 .2 Atomic polarization . . . . . . . . . . . . . . . . 271

5.3 Atoms and nuclei with $J>1 / 2$ in electric and magnetic fields . . . . . . . 271

5.3.1 Atoms in crossed electric and magnetic fields . . . . . . . . . . . . . 272

5.3.2 Quadrupolar nuclei in low magnetic fields under radiofrequency irradiation $(\mathrm{NQR}) \ldots \ldots \ldots \ldots . \ldots \ldots . \ldots \ldots$

5.3.3 Quadrupolar nuclei in high magnetic fields under radiofrequency irradiation (quadrupolar NMR) . . . . . . . . . . . . . 276

5.4 Observables in NMR/NQR and AMO . . . . . . . . . . . . . . . 278

5.4 .1 Observables in NMR and NQR . . . . . . . . . . . . . . . 278

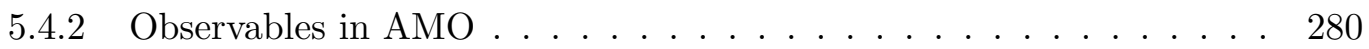

5.5 Alignment-to-orientation conversion in $J=1$ systems $\ldots \ldots$. . . . . . 282

5.5.1 Case of angular momentum $J=1$ in high-field NMR or in crossed electric and magnetic fields in atoms . . . . . . . . . . . . . . 282

5.5.2 Nuclear quadrupole resonance in $J=1$ systems: alignment-to-orientation conversion via $\mathrm{rf}$ pulses and free precession . . . . . . . . . . 285

5.6 NMR methods of manipulating polarization in quadrupolar nuclei with magnetic fields . . . . . . . . . . . . . . . . . . . . . 294

5.6.1 Efficient alignment-to-orientation conversion via adiabatic variation of the magnetic field: ARRF and ADRF . . . . . . . . . . .

5.6.2 Dynamics of the central transition in static half-integer quadrupolar nuclei in weak magnetic fields . . . . . . . . . . . . . .

5.6.3 Adiabatic coherence transfer in half-integer spins via slow modulation of the quadrupolar interaction: RIACT . . . . . . . . . . . 306

5.6.4 Coherence transfer in half-integer spins via fast resonant modulation of the magnetic field: FAM . . . . . . . . . . . . . .

5.6.5 Coherence transfer in half-integer spins via matching the quadrupolar modulation frequency to the rotating frame Larmor frequency: rotary

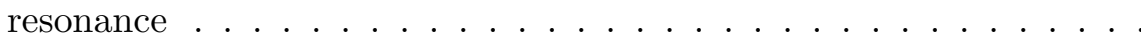

5.7 The $J=1 \rightarrow J^{\prime}=0$ transition in optics expressed as a system of two coupled spins-1/2 in NMR . . . . . . . . . . . . . . . . . . . . 319

5.7.1 The optically-pumped atomic $J=1, J^{\prime}=0$ system . . . . . . . . . 320

5.7 .2 The fictitious two-spin system . . . . . . . . . . . . . . . . 322

5.7.3 The potential Hamiltonian analogy to NMR . . . . . . . . . . . . . . 324

5.7 .4 The observables . . . . . . . . . . . . . . . . . 327

5.8 Conclusions . . . . . . . . . . . . . . . . . . . . . . . . . . . . . 329

6 Distant dipolar field effects and Xe-129 NMR "remote detection" 332

6.1 Remote detection NMR . . . . . . . . . . . . . . . . . 332

6.1 .1 Introduction to remote detection NMR . . . . . . . . . . . . . . 332

6.1.2 An example of remote detection NMR with spectroscopic encoding . 337

6.1.3 Dipolar field-encoded remote detection NMR of an analyte . . . . . 347 
6.2 The distant dipolar field . . . . . . . . . . . . . . . . . . . . 348

6.2.1 Classical calculation of DDF effects . . . . . . . . . . . . . . 352

6.2.2 Quantum-mechanical calculation of intermolecular multiple quantum coherences . . . . . . . . . . . . . . . . . . . . . 374

6.2.3 DDF effects in homonuclear systems: resonance shifts and multiple echoes .............................. 381

6.2.4 A comment on DDF effects in quadrupolar spin systems . . . . . . . 401

6.3 Indirect detection with the distant dipolar field . . . . . . . . . . . . . . 407

6.3.1 The basic indirect detection experiment . . . . . . . . . . . . 408

6.3.2 Proposed modification of the indirect detection experiment for remote detection . . . . . . . . . . . . . . . . 428

6.4 Heteronuclear DDF indirect detection experiments using Xe-129 as a sensor 438

6.4.1 Introduction to Xe-129 NMR . . . . . . . . . . . . . . . . . 439

6.4.2 Geometric considerations for dipolar field encoding: coaxial sample tube configuration . . . . . . . . . . . . . . . . . 459

6.4.3 Experimental DDF indirect spectra using the coaxial tube geometry. Results and future prospects. . . . . . . . . . . . . . 468

6.5 Calculation of the distant dipolar field for cylindrically symmetric geometries 477

6.6 Depletion and diffusion of xenon magnetization during multi-pulse batch mode experiments . . . . . . . . . . . . . . . . 488

6.6.1 Limit of extremely slow diffusion . . . . . . . . . . . . . . . 489

6.6.2 Limit of extremely fast diffusion with global equilibration of magnetization . . . . . . . . . . . . . . . 497

6.6.3 Limit of fast diffusion with local equilibration of magnetization . . . 501

6.6.4 Limit of fast diffusion with extended diffusion of magnetization . . . 507

6.7 Detection of nuclear magnetization by atomic magnetometry . . . . . . . 521

6.7.1 The Berkeley FM NMOR optical pumping magnetometer . . . . . . 522

6.7.2 Detection of hyperpolarized Xe-129 gas by atomic magnetometry . . 528

6.8 Conclusions . . . . . . . . . . . . . . . . . . . 532

$\begin{array}{ll}\text { Bibliography } & 537\end{array}$

A Spherical tensor operators and the Wigner-Eckart theorem $\quad 600$

A.1 Cartesian Tensors and Dyadics . . . . . . . . . . . . . . . . . . 601

A.2 Spherical Tensors and Tensor Operators . . . . . . . . . . . . . . . 605

A.2.1 Spherical Tensors . . . . . . . . . . . . . . . . 606

A.2.2 Spherical Tensor Operators . . . . . . . . . . . . . . 611

A.3 Wigner-Eckart Theorem . . . . . . . . . . . . . . . 615

$\begin{array}{ll}\text { B Rotations and spherical tensors } & 618\end{array}$

C Time-dependent perturbation theory via a power series expansion of the $\begin{array}{lr}\text { time development operator } & 629\end{array}$

D Dynamics of two $J$-coupled spin-1/2 nuclei $\quad 636$ 
E Simulation of the distant dipolar field for cylindrically-symmetric geometries (Matlab code) 


\section{List of Figures}

1.1 Schematic of two magnetic dipoles interacting via their magnetic fields. . .

1.2 Schematic of a non-spherical nuclear charge distribution leading to an electric quadrupole moment. . . . . . . . . . . . . . . . . . .

1.3 Schematic of an external charge distribution leading to an electric field gradient at the position of the nucleus. . . . . . . . . . . . . .

1.4 Autocorrelation spectral density functions $J^{\xi, \xi}(0), J^{\xi, \xi}\left(\omega_{0}\right)$, and $J^{\xi, \xi}\left(2 \omega_{0}\right)$ for rigid spherical molecules tumbling in isotropic solution. . . . . . . . . 91

1.5 Schematic diagram of a two dimensional NMR experiment. . . . . . . . . . 116

3.1 Pulse sequence and coherence pathway diagram for the 5Q3QMAS experiment.175

3.2 Comparison of the $2 \mathrm{D} \mathrm{Al}-27 \mathrm{NMR}$ spectra of $\mathrm{Al}(\mathrm{acac})_{3}$ in the hard pulse and low-amplitude pulse versions of the 5Q3QMAS experiment with spinning at $20 \mathrm{kHz} \ldots \ldots \ldots \ldots \ldots \ldots \ldots$

3.3 Comparison of the Fourier transforms of the first transients of the hard pulse and low-amplitude pulse versions of the 5Q3QMAS experiment on Al-27 in $\mathrm{Al}(\mathrm{acac})_{3}$ with spinning at $20 \mathrm{kHz} \ldots \ldots \ldots \ldots$. . . . . . . . .

3.4 Calculated zero-frequency quadrupolar dynamic phase Fourier coefficient $A_{0}$ as a function of crystallite angle $\beta$ for $\eta_{Q}=0 \ldots \ldots \ldots$. . . . . .

3.5 Calculated zero-frequency quadrupolar dynamic phase Fourier coefficient $B_{0}$ as a function of crystallite angle $\beta$ for $\eta_{Q}=0 \ldots \ldots \ldots . . \ldots . . .215$

3.6 Calculated zero-frequency quadrupolar dynamic phase Fourier coefficient $A_{0}$ as a function of crystallite angle $\beta$ for $\eta_{Q}=1 \ldots \ldots \ldots . \ldots . \ldots 216$

3.7 Calculated zero-frequency quadrupolar dynamic phase Fourier coefficient $B_{0}$ as a function of crystallite angle $\beta$ for $\eta_{Q}=1 \ldots \ldots$. . . . . . . . . 217

3.8 Calculated second-order average Hamiltonian coefficient $\left|\alpha^{(2)}\right|^{2}$ as a function

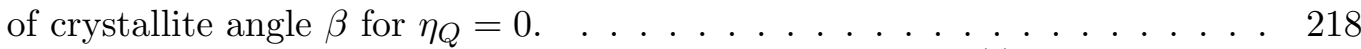

3.9 Calculated second-order average Hamiltonian coefficient $\left|\beta^{(2)}\right|^{2}$ as a function of crystallite angle $\beta$ for $\eta_{Q}=0 . \ldots \ldots \ldots \ldots$

3.10 Calculated second-order average Hamiltonian coefficient $\left|\gamma^{(2)}\right|^{2}$ as a function of crystallite angle $\beta$ for $\eta_{Q}=0 . \ldots \ldots \ldots \ldots . \ldots \ldots 220 \ldots$

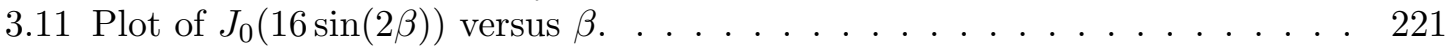


4.1 Calculated double-quantum and single-quantum N-14 quadrupolar relaxation rates as a function of rotational correlation time for a rigid spherical molecule in isotropic solution at $16.45 \mathrm{~T} . \ldots \ldots \ldots \ldots$

4.2 Calculated double-quantum N-14 quadrupolar relaxation rates for a rigid spherical molecule in isotropic solution at 16.45 $\mathrm{T}$ for two different values of $C_{Q}$ as a function of rotational correlation time, plus the calculated transverse relaxation rate of $\mathrm{N}-15$ under CSA and nitrogen-proton dipolar couplings. .

4.3 Ratio of the Lipari-Szabo spectral densities to the rigid-molecule spectral densities as a function of global correlation time with $\omega_{0} / 2 \pi=50.6 \mathrm{MHz}$ for two sets of values of the Lipari-Szabo model-free parameters. . . . . . . . .

4.4 Calculated H-1 transverse relaxation rate in the presence of a scalar-coupled $\mathrm{N}-14$ nucleus in a rigid molecule in isotropic solution at $16.45 \mathrm{~T}$ as a function of correlation time. . . . . . . . . . . . . . . . .

4.5 Calculated double-quantum and single-quantum $\mathrm{H}-2$ relaxation rates as a function of rotational correlation time for a rigid spherical molecule in isotropic solution at 16.45 T. . . . . . . . . . . . . . . . .

4.6 Pulse sequence for $I=1$ double-quantum spectroscopy in the presence of a weak quadrupolar coupling. . . . . . . . . . . . . . . .

4.7 Pulse sequence for indirect detection of $S=1$ double quantum coherence via a $J$-coupled $I=1 / 2$ partner. . . . . . . . . . . . . . .

4.8 Calculated N-14 relaxation rate and quadrupolar-CSA dynamic frequency shift evolution frequency of the $\hat{T}_{2, \pm 1}$ density operator terms as a function of rotational correlation time for a rigid spherical molecule in isotropic solution at 16.45 T. . . . . . . . . . . . . . . . . . . .

4.9 Calculated quadrupolar relaxation rate of the $\hat{I}_{ \pm} \hat{T}_{2,0}^{S}$ density operator terms in the amide N-14-H-1 $(I-S)$ system as a function of rotational correlation time for a rigid spherical molecule in isotropic solution at $16.45 \mathrm{~T}$, plus the $\mathrm{N}-\mathrm{H} J$-coupling frequency. . . . . . . . . . . . . . . . . .

$4.10 \mathrm{~N}-14$ double quantum spectrum of ${ }^{+} \mathrm{NH}_{4}(\mathrm{aq})$ in ammonium nitrate detected at $7.05 \mathrm{~T}$ on $\mathrm{H}-1$ through the $J$-coupling. . . . . . . . . . . . . 255

$4.11 \mathrm{~N}-14$ spectrum of DHPC:DMPC bicelles aligned at $B_{0}=16.43 \mathrm{~T}$ at $40{ }^{\circ} \mathrm{C} . \quad 258$

$4.12 \mathrm{~N}-14$ double-quantum-single quantum correlation spectrum in DHPC:DMPC bicelles aligned at $B_{0}=16.43 \mathrm{~T}$ at $40{ }^{\circ} \mathrm{C} \ldots \ldots \ldots$. . . . . . . 260

5.1 Calculated powder-averaged $J=1 \mathrm{NQR}$ signal as a function of rf pulse length

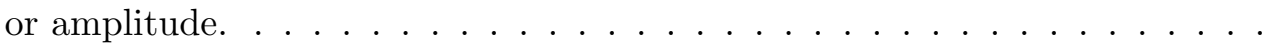

6.1 NMR remote detection experiment utilizing a two-pulse spectroscopic encoding sequence. . . . . . . . . . . . . . . . . . .

6.2 Dipolar field-induced multiple echoes in water observed after two-pulse sequences. . . . . . . . . . . . . . . . . . . . . . 389

6.3 Pulse sequence for DDF indirect detection. . . . . . . . . . . . . . . . 410

6.4 Modified DDF indirect detection pulse sequence for use as an encoding module for remote detection NMR. . . . . . . . . . . . . . . . . . . . 429 
6.5 Schematic of optical pumping of the $\mathrm{Rb}_{1}$ transition using circularly-polarized light. . . . . . . . . . . . . . . . . . . . . . . 448

6.6 Schematic of typical coaxial tube geometry used in distant dipolar field investigations. . . . . . . . . . . . . . . . . .

6.7 Plot of the intensity of the DDF-encoded H-1 spectrum of benzene as a function of magnetization modulation wavelength using a separated P-31 sensor in a coaxial tube geometry. . . . . . . . . . . . . .

6.8 Plot (pseudocolor) of a vertical cross section of the secular dipolar field generated by modulated analyte magnetization in the outer tube in a coaxial tube geometry ( 0.5 modulation wavelengths over the coil region) . . . . . .

6.9 Plot (mesh) of a vertical cross section of the secular dipolar field generated by modulated analyte magnetization in the outer tube in a coaxial tube geometry $(0.5$ modulation wavelengths over the coil region $) .. . . . .$.

6.10 Plot (pseudocolor) of a vertical cross section of the secular dipolar field generated by modulated analyte magnetization in the outer tube in a coaxial tube geometry (3 modulation wavelengths over the coil region). . . . . . . .

6.11 Plot (mesh) of a vertical cross section of the secular dipolar field generated by modulated analyte magnetization in the outer tube in a coaxial tube geometry (3 modulation wavelengths over the coil region). . . . . . . . .

$6.12 \mathrm{H}-1 \mathrm{NMR}$ spectrum of water at $7.05 \mathrm{~T}$ detected indirectly via the distant dipolar field using a separated Xe-129 sensor. . . . . . . . . . . . .

6.13 F-19 NMR spectrum of 2,2,2-trifluoroethanol at $7.05 \mathrm{~T}$ detected indirectly via the distant dipolar field using a separated P-31 sensor. . . . . . . . . .

6.14 Simulated decay of observed signal in multiple-pulse, constant tip-angle batch Xe NMR experiments in a long tube. . . . . . . . . . . . . . . . . .

6.15 Measured decay of signal in a multiple-pulse, constant tip-angle batch Xe NMR experiment in a long tube compared to a model that incorporates fast diffusion effects causing an extended equilibration of magnetization. . . . .

6.16 Measured dispersive FM NMOR resonances in the $\mathrm{Rb}-87 \mathrm{D}_{1}$ line as a function of applied bias field at a laser modulation frequency of $\Omega_{m}=200 \mathrm{~Hz}$. . . .

6.17 FM NMOR atomic magnetometer used for detecting Xe-129 nuclear magnetization. . . . . . . . . . . . . . . . .

6.18 Measurement of the nuclear magnetization of Xe-129 gas as a function of time by atomic magnetometry. . . . . . . . . . . . . . 


\section{List of Tables}




\section{Chapter 1}

\section{Introduction to the quantum}

\section{theory of pulsed NMR}

\subsection{Overview}

Pulsed nuclear magnetic resonance (NMR) spectroscopy is one of the best coherent quantum-mechanical control techniques in existence. NMR spectroscopists have the ability to use radiofrequency pulses to manipulate nuclear spins into very specific quantummechanical states in such a way that the measured spin dynamics return a wealth of information about chemical structure, molecular motion, the distribution of spin density, etc. This places NMR spectroscopy among the foremost of techniques for chemical structure determination, medical imaging, and for many other applications. This utility is not so much wrought by the spectroscopist as it is given freely by nature itself: not only are atomic nuclei quite literally "right in the middle of things" when it comes to probing molecular structure 
and dynamics, but they are weakly coupled to their environment and are subject to only a few well-understood molecular interactions. This allows much room for the spectroscopist to choreograph the spin evolution and to be well-situated to observe what the spins have to say about nature. The common motivation of the work presented in this dissertation was to gain a better understanding of nuclear spin dynamics: how to control them and how to learn from them.

In order to aid others with this understanding, I have tried to include more pedagogical information than is usual in a dissertation. After all, usually no one except for new lab members ever read dissertations. The approach I have taken is from the perspective of a theoretical, quantum-mechanical description of NMR. The Dirac notation is used exclusively; the novice reader is referred to the introductory text by Chester [1] for perhaps the best description of this formalism. For an introduction to NMR, three books will probably be the most useful. Goldman's little book [2] is perhaps the best introduction for the student who wants to learn to do quantum mechanical calculations in NMR. Levitt's book [3] is probably the most complete introductory text, and the most physical. The book by Cavanagh et al. [4] take a strong middle ground; it is not very deep, but very practical for both experimentalists and theorists. These three books will provide the student with insight, understanding, and pragmatism, respectively. Slichter's book [5] has continually pleasantly surprised me with the buried tidbits I have found from time to time. As far as understanding the fundamental interactions of NMR and their Hamiltonians goes, Abragam's treatise [6] is still the bible even after so many years. Likewise, the text by Ernst et al. [7] contains anything anyone ever wanted to know about solution-state NMR and multidimensional or 
Fourier spectroscopy. These two books are advanced; to me, Slichter has a similar style to Abragam and Cavanagh et al. have a similar style to Ernst, but their books are easier for the beginning student to learn from. The mysteries of solid-state NMR may be unlocked with Mehring's book [8], which is still a classic. The solid-state NMR book by Schmidt-Rohr and Spiess [9] is more modern and contains much useful information and clever techniques. Duer's book [10] has the most comprehensive review of modern solid-state techniques and is very accessible.

Armed with these references and hopefully with this dissertation, the student of NMR should be well-prepared for his or her initiation into the world of spin.

\subsection{Magnetic structure of the nucleus}

An atomic nucleus consists of $A$ nucleons: $Z$ protons of electric charge $+|e|$ and $A-Z$ uncharged neutrons bound together by the strong nuclear force. The simplest nucleus consists of just a single proton, whereas the most complex nuclei contain more than two hundred nucleons. The atoms themselves are characterized by an atomic number equal to $Z$, where $Z=1$ corresponds to hydrogen, $Z=7$ to nitrogen, $Z=54$ to xenon, etc. Different atomic isotopes are characterized by differing mass (nucleon) numbers $A$ for a given $Z$. The nuclear mass actually plays little role in determining the atomic electronic structure, due to the large disparity between nuclear and electron masses $\left(m_{p}=938.272 \mathrm{MeV} / c^{2} \approx m_{n}\right.$, $\left.m_{e}=0.511 \mathrm{MeV} / c^{2}\right)$. Since chemical properties are dominated by the molecular electronic structure, substitutions among isotopes of the same atomic element usually result in only minor modifications to chemical structure and reactivity. However, the difference between 
two isotopes of the same element can be enormous from the perspective of the internal structure of the nucleus, and in NMR studies the differences between isotopes can be as great as the differences between elements themselves. For example, the $99 \%$ naturally abundant carbon-12 isotope is not observable by the NMR technique, so carbon NMR spectroscopy is performed on the stable carbon-13 isotope (1\% natural abundance), often using samples that have been isotopically enriched.

The nuclear structure is in general quite complicated, and the problem involves multiple nucleons strongly interacting with each other in a potential that has no well-defined center $[11,12]$. The strength of the nuclear forces may be appreciated from a simple quantum "particle-in-a-box" model of the static nucleus, which gives an estimate for the spacing of nuclear energy levels ${ }^{1}$ on the order of $\frac{h^{2}}{8 M_{N} L^{2}}=\frac{\pi^{2}(\hbar c)^{2}}{2\left(M_{N} c^{2}\right) L^{2}} \approx \frac{(10)(200)^{2}}{(2)(20 \cdot 1000)(50)}=$ $0.2 \mathrm{MeV}$. (The mass of a light nucleus has been taken to be $M_{N} \simeq 20 m_{p}$, with nuclear dimensions of several fm translating into a cross section on the order of $L^{2} \sim 50 \mathrm{fm}^{2}$, and $\hbar c \sim 200 \mathrm{MeV} \cdot \mathrm{fm}, m_{p} c^{2} \sim 1000 \mathrm{MeV}$, and $\pi^{2} \sim 10$.) Nuclear level spacings are indeed found to be in the $\mathrm{keV}$ to $\mathrm{MeV}$ range, which is much larger than the thermal energy $k_{B} T \sim 1 / 40 \mathrm{eV}$ available at room temperature, virtually guaranteeing that only the nuclear ground state is populated under normal laboratory conditions. This fact, and the fact that conventional nuclear magnetic resonance experiments involve excitation energies far lower than those of nuclear transitions ensure that only the nuclear ground state properties are of concern in an NMR experiment (see also $\S 3.5$ of Ref. [2]). ${ }^{2}$ There turn out to be only

\footnotetext{
${ }^{1}$ The crudeness of this estimate is apparent upon consideration of the fact that the three-dimensional particle-in-a-box energy levels diverge quadratically in the quantum numbers, instead of converging to a finite value as must be the case for the discrete spectrum of a bound system that can dissociate.

${ }^{2}$ Pure radiofrequency NMR excitation energies are in the $\mu \mathrm{eV}$ range at best and optical techniques involve excitations in the $\mathrm{eV}$ range. That is not to say, however, that nuclear energies are never involved in NMR experiments. There exist techniques such as $\beta$-NMR $[13, \S 2.8]$ that involve the magnetic resonance of ground
} 
three quantities characterizing the nuclear ground state that are of relevance to the NMR spectroscopist: the total angular momentum, the magnetic dipole moment, and the electric quadrupole moment.

The Hamiltonian operator of the nucleus is considered to be symmetric under rotations and external fields are typically not large enough to break this symmetry; consequently, the nucleus has eigenstates of well-defined total angular momentum that may be labeled as $\left|\alpha ; I, m_{I}\right\rangle[14, \S 3.4]$. Here $I$ is the quantum number for the total angular momentum and $m_{I}$ is the magnetic quantum number for the component of this angular momentum along some space-fixed quantization axis; $I$ and $m_{I}$ are good quantum numbers for the system. The quantized angular momentum $I$ is a non-negative integer or half-integer, and $m_{I}$ takes on integrally spaced values from $-I$ to $+I$. The symbol $\alpha$ represents the quantum numbers for the rest of the degrees of freedom that characterize the state aside from angular momentum. From here on it will be assumed that $\left|\alpha ; I, m_{I}\right\rangle$ represents a sublevel of the nuclear ground state. The quantity $I$ is often (rather casually) referred to as the nuclear "spin". The nuclear angular momentum operator may be written as

$$
\underline{\hat{I}}=\underline{\hat{L}}+\underline{\hat{S}}=\sum_{k=1}^{A} \underline{\hat{l}}_{k}+\sum_{k=1}^{A} \underline{\hat{s}}_{k}
$$

where $\underline{\hat{L}}$ and $\underline{\hat{S}}$ are the operators for the total orbital and total intrinsic spin angular momentum of the nucleus, respectively, and $\underline{\hat{l}}_{k}$ and $\underline{\hat{s}}_{k}$ are the orbital and intrinsic spin angular momentum operators of the $k$ th nucleon. Protons and neutrons are both $s_{k}=\frac{1}{2}$ particles. Note that $L$ and $S$ are not necessarily good quantum numbers for the nucleus. ${ }^{3}$ states of nuclei undergoing radioactive decay.

${ }^{3}$ For instance, if the nuclear potential is non-central (due to the so-called tensor force $[15, \S 14.5]$ ), it does 
The measured NMR signal is proportional to the bulk ensemble nuclear magnetization of the sample, which in the simplest case is in turn proportional to the magnetic dipole moment of the ground state. ${ }^{4}$ The magnitude of this moment is defined quantummechanically as the maximum projection of the magnetic dipole moment on the quantization axis $z: \mu=\left\langle\alpha ; I, m_{I}=+I\left|\hat{\mu}_{z}\right| \alpha ; I, m_{I}=+I\right\rangle$. The magnetic dipole moment operator is

$$
\underline{\hat{\mu}}=\sum_{k=1}^{A} \underline{\hat{\mu}}_{k}^{l}+\sum_{k=1}^{A} \underline{\hat{\mu}}_{k}^{s}=\sum_{k=1}^{A} g_{l} \mu_{N} \underline{\hat{l}}_{k} / \hbar+\sum_{k=1}^{A} g_{s} \mu_{N} \underline{\hat{s}}_{k} / \hbar
$$

where $\underline{\hat{\mu}}_{k}^{l}$ and $\underline{\hat{\mu}}_{k}^{s}$ are respectively the orbital and intrinsic spin angular momentum operators for the $k$ th nucleon, $\mu_{N}=\frac{|e| \hbar}{2 m_{p}}$ is the nuclear magneton, the orbital nucleon $g$-factors are $g_{l}=+1$ for the proton and $g_{l}=0$ for the neutron, and the spin nucleon $g$-factors are $g_{s}=+5.5857$ for the proton and $g_{s}=-3.8261$ for the neutron. A semiclassical model of a structureless particle orbiting with quantized angular momentum of $\hbar$ predicts a $g$-factor of \pm 1 for particles with charge $\pm|e|$, and a $g$-factor of 0 for neutral particles (since only charged particles produce a magnetic moment upon circulation). This is consistent with the orbital $g$-factors above, but the nature of the spin $g$-factors is more complicated. The Dirac point electron has a spin $g$-factor of -2 (with small corrections from quantum electrodynamics), and the unusual numeric values for the nucleon spin $g$-factors (particularly the nonzero result for the neutron) are indicative of the internal (quark) structure of the nucleon.

The expressions in Eqs. 1.1 and 1.2 are similar, but not so similar that the relation

not commute with $\underline{\hat{L}}^{2}$ and $L$ is no longer a good quantum number. Also note that the tensor potential, though non-central, still must be rotationally invariant [16, $\S I I .5 . B]$. Another example of when $L$ is not conserved is the case of a non-spherical nuclear potential $[14, \S 5.3]$, which is mentioned below in the discussion of the nuclear electric quadrupole moment.

${ }^{4}$ It is also proportional to the nuclear polarization, since an ensemble of unpolarized nuclear moments will lead to no net magnetization of the sample. 
between the total nuclear angular momentum and the nuclear magnetic moment is clear. However, a simple relationship can be established between the matrix elements of $\underline{\hat{I}}$ and $\underline{\hat{\mu}}$ within the manifold of angular momentum substates $\left\{\left|\alpha ; I, m_{I}\right\rangle\right\}$ using a powerful theorem called the Wigner-Eckart theorem. This theorem states:

$$
\left\langle\alpha^{\prime} ; j^{\prime}, m^{\prime}\left|\hat{T}_{k, q}\right| \alpha ; j, m\right\rangle=\left\langle j^{\prime}, m^{\prime} ; k, q \mid j, m\right\rangle\left\langle\alpha^{\prime} ; j^{\prime}\left\|\hat{T}_{k}\right\| \alpha ; j\right\rangle
$$

where the operator $\hat{T}_{k, q}$ is the rank- $k$, order- $q$ component of the spherical tensor operator $\hat{T}$, the amplitude $\left\langle j^{\prime}, m^{\prime} ; k, q \mid j, m\right\rangle$ is known as a Clebsch-Gordan coefficient, and the quantity $\left\langle\alpha^{\prime} ; j^{\prime}\left\|\hat{T}_{k}\right\| \alpha ; j\right\rangle$ is known as the reduced matrix element of $\hat{T}_{k}$. The reader is referred to Appendix A for a detailed discussion of spherical tensor operators and the interpretation of the Wigner-Eckart theorem. The principal result of the theorem is that the geometrical dependence and dynamical dependence of the matrix element are separated into the ClebschGordan coefficient and the reduced matrix element, respectively. The term "geometrical" refers to the angular distribution and "dynamical" refers to the other degrees of freedom, the dependence on which must in general be found by a detailed solution of the structure. A corollary of this theorem states that any two vector operators are proportional to each other within a particular manifold of angular momentum states, where the proportionality constant is determined from the reduced matrix elements. Therefore it can be concluded that within the $\left\{\left|\alpha ; I, m_{I}\right\rangle\right\}$ manifold the two vector operators ${ }^{5} \underline{\hat{\mu}}$ and $\underline{\hat{I}}$ are proportional to

\footnotetext{
${ }^{5}$ Actually, from the perspective of certain symmetries $\hat{\mu}$ and $\underline{\hat{I}}$ are known as pseudovector operators, but the Wigner-Eckart theorem does not distinguish between vectors and pseudovectors.
} 
each other:

$$
\underline{\hat{\mu}}=\gamma \underline{\hat{I}}
$$

where $\gamma$ is a proportionality constant called the gyromagnetic ratio, which aptly enough is the ratio of the magnetic moment (which describes a magnetic property) to the angular momentum (which describes the gyroscopic motion). Since the value of the gyromagnetic ratio depends on the reduced matrix elements, it can be determined from first principles only by directly solving the nuclear structure. It also can be measured experimentally. The gyromagnetic ratio can be either positive or negative, corresponding to a nuclear magnetic moment that is either parallel or antiparallel to the angular momentum, respectively. Eq. 1.4 also implies that nuclei with ground state angular momenta $I=0$ possess no magnetic dipole moment ${ }^{6}$ and thus are NMR-inactive.

The magnetic dipole moment is just one of many possible static "multipole moments" that can characterize an electric or magnetic distribution [17]. There are in general an infinite number of possible electric and magnetic moments $E k$ and $M k$ that can be characterized by their spherical tensor rank $k[18,19,20]$ : the values $k=0,1,2,3,4$ correspond to monopole, dipole, quadrupole, octupole, and hexadecapole moments, respectively. Using this notation the magnetic dipole moment is the $M 1$ moment. It is straightforward to imagine how a collection of magnetic dipoles can lead to a detectable magnetic field. However, it is also necessary to consider how the strength or dynamics of the nuclear magnetization may depend on other nuclear multipole moments. The (spherical) components of these mo-

\footnotetext{
${ }^{6}$ This also follows from the fact that the matrix elements $\left\langle\alpha ; I=0, m_{I}\left|\hat{\mu}_{1, q}\right| \alpha ; I=0, m_{I}\right\rangle$ are proportional to the Clebsch-Gordan coefficient $\left\langle 0, m_{I} ; 1, q \mid 0, m_{I}\right\rangle$, which is zero.
} 
ments are expectation values of the form $\left\langle\alpha ; I, m_{I}=I\left|\hat{T}_{k, q}\right| \alpha ; I, m_{I}=I\right\rangle$, where $\hat{T}_{k, q}$ is an operator that characterizes the particular multipole component. The nuclear Hamiltonian to a very good approximation obeys certain symmetries such as parity (spatial inversion) and time-reversal invariance, which restrict the type of nuclear multipole moments that may be observed. Parity invariance alone results in nuclear eigenstates that are characterized by well-defined parity (i.e., the eigenstates either are invariant or acquire a sign inversion upon the spatial coordinate inversion $\underline{r} \rightarrow-\underline{r})$. A consequence of this symmetry is that all odd-rank $(k=1,3,5 \ldots)$ electric multipoles $[14, \S 3.5],[21, \S 3-3]$ and all even rank magnetic multipoles $(k=0,2,4, \ldots)$ are zero in the nuclear system. ${ }^{7}$ Small exceptions to this rule may be possible due to symmetry violations, e.g., parity violation due to the weak nuclear forces. It also should be noted that these selection rules hold for the diagonal multipole matrix elements (i.e., the static multipole moments), not for the off-diagonal matrix elements (which correspond to nuclear multipole transitions).

Upon making a survey of the magnetic multipole moments, one finds that the magnetic monopole moment $M 0$ is forbidden (indeed, a magnetic monopole has never been found in nature); the dipole moment $M 1$ has already been considered; the magnetic quadrupole moment $M 2$ is forbidden; and the octupole moment $M 3[22,23,24]$ and higher moments are small and difficult to observe. Upon considering the electric multipole moments, one finds that the electric monopole moment $E 0$ is allowed (it is proportional to the total nuclear

\footnotetext{
${ }^{7}$ The parity operator $\hat{P}$ is unitary such that $\hat{P}^{\dagger} \hat{P}=\hat{1}$, and the multipole operators transform in a definite way under the parity operation such that $\hat{P} \hat{T}_{k, q}^{(E)} \hat{P}^{\dagger}=(-1)^{k} \hat{T}_{k, q}^{(E)}$ for electric multipoles and $\hat{P} \hat{T}_{k, q}^{(M)} \hat{P}^{\dagger}=(-1)^{k+1} \hat{T}_{k, q}^{(M)}$ for magnetic multipoles. The eigenstates $\left|\alpha ; I, m_{I}\right\rangle$ (assumed to be nondegenerate except for the $m_{I}$ degeneracy) have well-defined parity such that $\hat{P}\left|\alpha ; I, m_{I}\right\rangle=( \pm 1)\left|\alpha ; I, m_{I}\right\rangle$. Therefore $\left\langle\alpha ; I, m_{I}\left|\hat{T}_{k, q}^{(E)}\right| \alpha ; I, m_{I}\right\rangle=\left\langle\alpha ; I, m_{I}\left|\hat{P}^{\dagger} \hat{P} \hat{T}_{k, q}^{(E)} \hat{P}^{\dagger} \hat{P}\right| \alpha ; I, m_{I}\right\rangle=(-1)^{k}\left\langle\alpha ; I, m_{I}\left|\hat{T}_{k, q}^{(E)}\right| \alpha ; I, m_{I}\right\rangle$ and $\left\langle\alpha ; I, m_{I}\left|\hat{T}_{k, q}^{(M)}\right| \alpha ; I, m_{I}\right\rangle=(-1)^{k+1}\left\langle\alpha ; I, m_{I}\left|\hat{T}_{k, q}^{(M)}\right| \alpha ; I, m_{I}\right\rangle$, from which the aforementioned selection rules may be deduced.
} 
charge) but causes a uniform shift in energy of the $\left\{\left|\alpha ; I, m_{I}\right\rangle\right\}$ manifold and thus may be neglected ${ }^{8}$ the electric dipole moment $E 1$ is forbidden; the electric quadrupole moment $E 2$ is allowed and will be discussed below; the octupole moment $E 3$ is forbidden; and the hexadecapole moment $E 4[24,25,26,27]$ and higher moments are small and difficult to observe.

The electric quadrupole moment of the nucleus can play a large role in the NMR studies of nuclei that have non-zero quadrupolar couplings to their local chemical environments. Non-spherical nuclei generally possess an electric quadrupole moment, corresponding to a component of the electric charge distribution that can be described by the second-rank spherical harmonics. In the field of nuclear physics it is customary to speak of the "intrinsic" quadrupole moment of the nucleus referred to a nucleus-fixed coordinate system and the "spectroscopic" quadrupole moment referred to a space-fixed coordinate system. Most nuclei are non-spherical and possess an intrinsic electric quadrupole moment. ${ }^{9}$ However, a nonspherical nucleus can undergo rotational motion (which is not apparent in the nucleus-fixed frame), and the spectroscopic quadrupole moment measured in NMR studies is actually the rotationally-averaged intrinsic quadrupole moment viewed from a space-fixed frame. This averaging can change the magnitude and even the sign of the observed quadrupole moment relative to the intrinsic quadrupole moment [14, $\S 5.2]$, and in the case of $I=0$ and $I=1 / 2$

\footnotetext{
${ }^{8} \mathrm{~A}$ uniform shift of the energy levels of a system can be shown to have no effect on their populations or on the system dynamics.

${ }^{9}$ Nuclei whose nucleon number $A$ is near one of the nuclear "magic numbers" are typically the most spherical. In addition to simple considerations of the static proton distribution, there are many internal motions that can give rise to a non-spherical charge distribution. For instance, the orbit of a single neutron (which, being uncharged, cannot directly create an electric quadrupole moment) around a spherical nuclear core can perturb the core into a quadrupolar charge distribution $[15, \S 18.1]$. One must also consider the collective motion of the nucleons in order to describe the large quadrupole moments of "permanently deformed" nuclei.[14, §5.2],[15, §18],[21, §9] (Although these nuclear potentials are treated as being non-spherical, rotational invariance is not violated because the deformation is an artifact of generating an effective potential from the mean field of the collective rotating nucleons $[21, \S 10.6]$.)
} 
nuclear states, the quadrupole moment is completely averaged to zero $[15, \S 18.2],[21, \S 3-3]$ and is not observable by NMR. This is consistent with the Wigner-Eckart theorem, which predicts that the quadrupole moment $\left\langle\alpha ; I, m_{I}=I\left|\hat{T}_{2,0}^{Q}\right| \alpha ; I, m_{I}=I\right\rangle$ is zero unless $I \geq 1$ (recall that $m_{I}$ is the component of angular momentum along a space-fixed axis). ${ }^{10}$

There is an analogous expression to Eq. 1.4 that relates the electric quadrupole moment operator components to functions of the angular momentum operator components, which will be developed in $\S 1.3 .7$. The important point to remember is that the WignerEckart theorem can be used to relate the nuclear multipole moments to the nuclear angular momentum operator, and through this formalism the nuclear dynamics due to the interaction of the multipole moments with external fields can be related to the angular momentum dynamics. ${ }^{11}$ It is for this reason that the quantum theory of angular momentum plays such a large role in the modern theory of nuclear magnetic resonance, which in a sense is the study of the interactions of atomic nuclei with external magnetic and electric fields created by the local chemical environment.

\subsection{The Hamiltonians of NMR}

The Hamiltonian operator is the quantum-mechanical operator for the energy of the system; i.e., $\langle\hat{H}\rangle$ is the expectation value of the energy. As will be seen in $\S 2$, the Hamiltonian plays a very important role in quantum mechanics: it is the generator of time

\footnotetext{
${ }^{10}$ Similar symmetry arguments can be extended to higher multipole moments, e.g., $I \geq 2$ is required to support a spectroscopic nuclear hexadecapole moment.

${ }^{11}$ For instance, it might not be immediately clear how the interaction of a nucleus with an electric field can affect the magnetic resonance. However, the coupling of the nuclear electric quadrupole moment with an external electric field gradient affects the angular momentum dynamics, which in turn guides the dynamics of the magnetic moment vector (cf. Eq. 1.4). Thus the effect of the electric quadrupole coupling ultimately may be observed in the dynamics of the nuclear magnetization vector.
} 
evolution $[28, \S 8],[29, \S 4]$. If the Hamiltonian operator for a particular system is known the quantum-mechanical time evolution of that system is completely specified. The pulsed Fourier-transform NMR technique makes direct or indirect measurements of the nuclear angular momentum dynamics using transient detection of the bulk nuclear magnetization. These dynamics are due to quantum beat effects when the angular momentum is prepared in quantum-mechanical superposition of energy eigenstates; i.e., the frequencies contained in the spectrum of the evolution are the transition frequencies between pairs of energy eigenstates. The Hamiltonians of NMR are few and well-understood and generally involve the interaction of the nuclear angular momentum with external or local magnetic or electric fields. The local fields are produced by atomic charges and currents and are characteristic of the microscopic chemical environment of the nucleus. If these fields are static they contain information about the local molecular structure; if they are rendered time dependent by molecular motion, they contain information about the molecular dynamics. ${ }^{12}$ The Hamiltonian formalism is the means by which information about molecular structure and dynamics can be extracted and interpreted from the NMR measurement of nuclear spin evolution.

The treatise of Abragam [6] provides probably the most complete description of the physical and mathematical origin of the Hamiltonians that arise in NMR studies. A more accessible but still detailed treatment is included in the text by Slichter [5]. Levitt's book [3] also contains a thorough and extremely physical description of these Hamiltonians that is accessible to novices yet useful to advanced spectroscopists. Smith et al. [30] have published a good pedagogical review of the Hamiltonians of NMR. These Hamiltonians will

\footnotetext{
${ }^{12}$ The terms "static" and "time dependent" are relative terms that mean "slow" and "fast" compared to the characteristic timescale of the nuclear spin evolution. This timescale is on the order of the reciprocal energy of the NMR interaction as measured in frequency units.
} 
be described below; the treatment will be non-relativistic and all magnetic and electric fields will be treated classically (i.e., without second quantization).

\subsubsection{Zeeman Hamiltonian}

The most fundamental NMR interaction is the Zeeman effect, which is the coupling of the nuclear magnetic moment to a magnetic field. Not only are most of the local NMR Hamiltonians interpretable as the interaction of the nuclear spin with a local magnetic field, the NMR experiment itself is conducted in a large, experimentally applied magnetic field which can be on the order of tens of Tesla in modern superconducting magnets. The fact that these experiments are conducted at high magnetic field is what puts the 'magnetic' in 'Nuclear Magnetic Resonance.' The use of high field techniques provide many advantages, as will be discussed in various parts of this dissertation: sensitivity is increased due to larger nuclear thermal polarizations and increased Larmor frequencies for inductive detection; magnetic field-induced chemical shifts are increased, leading to increased spectral resolution; the Zeeman splitting of nuclear magnetic sublevels provides a means of coherently manipulating the nuclear magnetization using resonant radiofrequency pulses; the truncation of internal spin interactions by the externally-imposed Zeeman interaction leads to simplified selection rules for NMR transitions and simplified spectra; this truncation creates an effective cylindrical symmetry of the system about the magnetic field axis which allows the experimentalist to apply coherence pathway selection techniques that result in further spectral simplification.

The quantum mechanics text by Landau and Lifshitz contains a good non-relativistic derivation of the Zeeman Hamiltonian (in atoms) from a "first principles" description uti- 
lizing the magnetic vector potential $[31, \S 112]$. A slightly different approach will be taken here. The classical energy of a magnetic dipole $\underline{\mu}$ in a magnetic field $\underline{B}$ is $[17, \S 5.7]$ :

$$
E_{Z}=-\underline{\mu} \cdot \underline{B}
$$

Eq. 1.5 indicates that the energy of the dipole is low when it is oriented parallel to the magnetic field and is high when it is oriented antiparallel to the magnetic field; the Zeeman energy is linear in the strength of the field. The promotion of the classical dipole moment vector $\underline{\mu}$ to a quantum-mechanical vector operator for the magnetic moment of the nucleus $\underline{\hat{\mu}}$ yields the correct form of the Zeeman Hamiltonian operator:

$$
\hat{H}_{Z}=-\underline{\hat{\mu}} \cdot \underline{B} .
$$

Eq. 1.6 can be rewritten in terms of the nuclear angular momentum operator $\underline{\hat{I}}$ using Eq. 1.4:

$$
\hat{H}_{Z} / \hbar=-\gamma \underline{\hat{I}} \cdot \underline{B}
$$

where $\gamma$ is the nuclear gyromagnetic ratio. For the sake of correctness Eq. 1.4 expressed the angular momentum operator in its correct units, but Eq. 1.7 and every subsequent equation in this dissertation makes all factors of $\hbar$ explicit by using dimensionless angular momentum operators. Eq. 1.7 has treated the nucleus (or rather, the nuclear ground state) as if it had no internal structure, which is a good approximation since the coupling of the nucleons to the magnetic field is very much weaker than the couplings (spin-spin or otherwise) between 
nucleons. $^{13}$

NMR studies are usually carried out in the presence of a large magnetic field which can be on the order of $\sim 10$ Tesla in a superconducting magnet. By convention this magnetic field is taken to be in the $+z$-direction,

$$
\underline{B}_{0}=B_{0} \underline{\tilde{z}}
$$

The Zeeman Hamiltonian in the presence of this field becomes

$$
\hat{H}_{Z} / \hbar=-\gamma B_{0} \hat{I}_{z}=-\omega_{0} \hat{I}_{z}
$$

where the Larmorfrequency is defined to be

$$
\omega_{0}=\gamma B_{0}
$$

Various authors define the Larmor frequency in different ways, i.e., $\omega_{0}=\gamma B_{0}, \omega_{0}=-\gamma B_{0}$, or $\omega_{0}=\left|\gamma B_{0}\right|$. In certain situations the sign of the Larmor frequency becomes very important and care should be taken to treat the signs in a consistent manner [3, 33, 34].

Nuclear Larmor frequencies are typically on the order of $\left|\omega_{0}\right| / 2 \pi \sim 10^{1}-10^{2} \mathrm{MHz}$; commercial magnets are available which produce H-1 Larmor frequencies of $900 \mathrm{MHz} .{ }^{14}$ The Zeeman interaction at these field strengths completely dominates any other interaction experiences by the nuclear spins. For this reason Eq. 1.9 is often referred to as the Zeeman

\footnotetext{
${ }^{13}$ See Refs. $[31, \S 112]$ and $[32, \S 3.3]$ for discussions of further effects of very strong magnetic fields on atomic electrons, which experience much weaker forces than the intranuclear forces.

${ }^{14} \mathrm{NMR}$ spectroscopists often refer to their magnets in terms of the H-1 Larmor frequencies they produce.
} 
Hamiltonian even if local molecular magnetic fields or other small applied fields are present; this Hamiltonian is often designated $\hat{H}_{0}$. NMR calculations are almost exclusively performed in the Zeeman eigenbasis, i.e., the states $\left\{\left|I, m_{I}\right\rangle\right\}$, where $m_{I}$ is the magnetic quantum number for the projection of angular momentum along the magnetic field axis, z. Often NMR spectroscopists use terms like " $z$-field," "z-rotation," "z-filter," etc.; here " $z$ " always refers to the direction of $\underline{B}_{0}$. Likewise, the designations "longitudinal" and "transverse" are always taken to mean "parallel to $\underline{B}_{0}$ " or "perpendicular to $\underline{B}_{0}$," respectively.

The eigenvalues of the Zeeman Hamiltonian are calculated according to $\hat{H}_{Z}|I, m\rangle=$ $E_{0}\left|I, m_{I}\right\rangle$ :

$$
E_{0} / \hbar=-m_{I} \omega_{0}
$$

The energies of the magnetic sublevels $\left\{\left|I, m_{I}\right\rangle\right\}$ (which are degenerate in zero magnetic field) split linearly in $B_{0}$. The sublevel energies are also proportional to the magnetic quantum number. When $\underline{\hat{\mu}}$ is parallel to $\underline{\underline{I}}$ (i.e, $\gamma>0$ ) the states in which $m_{I}$ (the angular momentum projection along $\left.\underline{B}_{0}\right)$ is positive have magnetic moments that are aligned with components in the direction of $\underline{B}_{0}$ and have negative energies. The $m_{I}<0$ states for $\gamma>0$ correspond to magnetic moments aligned against $\underline{B}_{0}$ and have positive energies. The ordering of the magnetic suvblevels is reversed when $\gamma<0$. These results reproduce those of the classical equation (Eq. 1.5) when $\underline{\mu}$ is replaced by one of its quantized values. Note that two levels that differ by $\left|\Delta m_{I}\right|=1$ differ in energy by $\hbar\left|\omega_{0}\right|$. The selection rule for NMR excitation and detection is $\left|\Delta m_{I}\right|=1$ (see $\S 1.6$ and $\S 1.7$ ); the Larmor frequency $\left|\omega_{0}\right|$ corresponds both to the excitation resonance frequency and to the absolute frequency of 
spin precession in the magnetic field.

\subsubsection{Basic form of the solid state Hamiltonian}

Solid state NMR is the most general case of NMR from the perspective of Hamiltonian dynamics because some of the local Hamiltonians average to zero due to molecular motion in isotropic fluids that is fast on the NMR timescale. The local Hamiltonians considered in this work are the chemical shielding, dipole-dipole coupling, electric quadrupolar coupling, and $J$-coupling (also known as the indirect or scalar coupling) Hamiltonians. These Hamiltonians are parameterized by values that depend on molecular structure; this is what makes NMR useful for structure determination. There exist some other interactions such as the spin-rotation coupling; i.e., the coupling of the nuclear spin to the magnetic field created by electric currents generated by molecular rotation. The spin-rotation interaction is generally not active in the solid state and is typically averaged away in fluids but can enter as a relaxation term, e.g., it plays a role in the nuclear spin relaxation of condensed-phase Xe-129, as is mentioned briefly in $§ 6.4 .1$.

All the common NMR Hamiltonians can be written as a contraction of a secondrank Cartesian tensor $\underline{\underline{A}}$ containing lattice degrees of freedom with a second-rank Cartesian tensor $\underline{\underline{\underline{T}}}$ containing nuclear spin operators (see Appendix A):

$$
\hat{H}_{\xi}=\underline{\underline{\hat{T}}}^{\xi}: \underline{\underline{A}}^{\xi}=\hat{T}_{j i}^{\xi} A_{i j}^{\xi}
$$

where e.g. $A_{i j}$ labels the $(i$ th, $j$ th $)$ Cartesian component of the tensor $(i, j \in\{x, y, z\})$. The transpose notation used in Appendix A is suppressed here for convenience. The symbol $\xi$ 
labels the interaction, i.e., chemical shielding, quadrupolar coupling, etc. According to Eq. A.19 this equation can be written in terms of spherical tensor components:

$$
\hat{H}_{\xi}=\sum_{k=0}^{2} \sum_{q=-k}^{k}(-1)^{q} A_{k, q}^{\xi} \hat{T}_{k,-q}^{\xi}
$$

where e.g. $A_{k, q}^{\xi}$ represents order- $q$ component of the rank- $k$ spherical tensor $A_{k}^{\xi}$. Eq. A.11 indicates that the $A_{0}^{\xi}$ spherical tensor is proportional to the trace (isotropic component) of $\underline{\underline{A}}^{\xi}$; the anisotropic part of $\underline{\underline{A}}^{\xi}$ is described by the spherical tensors $A_{1}^{\xi}$ and $A_{2}^{\xi}$, which correspond to the antisymmetric $\left(A_{j i}^{\xi}=-A_{i j}^{\xi}\right)$ and symmetric $\left(A_{j i}^{\xi}=A_{i j}^{\xi}\right)$ components of $\underline{\underline{A}}^{\xi}$, respectively. The antisymmetric component (if nonzero) usually does not make a significant contribution to the NMR spectra-i.e., in the case of the chemical shielding $[8$, $\S 2.2],[35]$ - although there are some experiments in which the effects of these terms may be observed (see Refs. [35, 36, 37] and references therein).

\subsubsection{High field truncation of Hamiltonians}

The laboratory-frame solid-state Hamiltonian in a high-field NMR experiment is

$$
\hat{H}=\hat{H}_{Z}+\sum_{\xi} \hat{H}_{\xi}
$$

The Zeeman Hamiltonian could be due to one spin or to a sum of spins. Typically in NMR experiments the magnitude of the Zeeman Hamiltonian is much larger than the Hamiltonians of any of the internal interactions, $\left\|\hat{H}_{Z}\right\| \gg\left\|\hat{H}_{\xi}\right\|$. It is then appropriate to treat the internal Hamiltonians as perturbations on the Zeeman Hamiltonian. It would be simple to 
use static perturbation theory to calculate the shifts of the Zeeman energy levels due to the local interaction. However, in pulsed FT (time domain) NMR - as opposed to continuous wave NMR - it is important to be able to calculate the dynamics and it is more useful to calculate the perturbation in terms of effective Hamiltonian operators. In this formalism the Zeeman interaction is said to "truncate" the local Hamiltonians, such that only part of them are effective at inducing spin dynamics in the presence of the dominant Zeeman interaction. The effective is called the secular Hamiltonian of the interaction. The Zeeman interaction is usually so large that first-order perturbation theory is sufficient; however, some local interactions such as the electric quadrupolar coupling are large enough that higher-order perturbation theory is necessary. In general, the truncated Hamiltonians will be of the form

$$
\hat{H}_{\xi} \approx \hat{H}_{\xi}^{(1)}+\hat{H}_{\xi}^{(2)}
$$

The perturbation treatment can be implemented in several ways. It will be assumed for now that the Zeeman Hamiltonian applies to a single spin or to a set of equivalent spins (i.e., with the same Larmor frequency). The case in which the spins have different Larmor frequencies can cause some complications, such as the truncation of the dipole-dipole coupling and $J$ coupling Hamiltonians in heteronuclear systems. The results of this type of truncation will be discussed further in the discussion of the dipole-dipole coupling in $§ 1.3 .5$; an instructive example of truncation via a static perturbation theory treatment is included in Ref. [2, $\S 5.2 .3]$.

Consider the case of a single spin $I$ where $\hat{H}_{Z} / \hbar=-\omega_{0} \hat{I}_{z}$ and $|m\rangle \equiv\left|I, m_{I}\right\rangle$ are 
the eigenstates of $\hat{H}_{Z}$, i.e., the unperturbed states. Since these states are non-degenerate in the presence of the magnetic field, the first-order truncated Hamiltonian of the local interaction will simply be diagonal: with its matrix elements being the first-order static perturbation theory corrections to the unperturbed energies:

$$
\hat{H}_{\xi}^{(1)}=\sum_{m}|m\rangle H_{m m}^{\xi}\langle m|
$$

where $H_{m m}^{\xi}=\left\langle m\left|\hat{H}_{\xi}\right| m\right\rangle=E_{m}^{(1)}$ is the first-order static perturbation theory energy correction for the unperturbed state $|m\rangle$. Note that $\left[\hat{H}_{\xi}^{(1)}, \hat{H}_{Z}\right]=0$. This is a fine matrix representation of $\hat{H}_{\xi}$, but there is a cleaner way to get an operator representation, involving a transformation into the interaction frame of the Zeeman interaction and application of average Hamiltonian theory (AHT, see $\S 2.4)$.

The expanded form of the laboratory frame Hamiltonian (Eq. 1.14) for a single spin $I$ and a single interaction may be found using Eqs. 1.9 and 1.13:

$$
\hat{H}=-\hbar \omega_{0} \hat{I}_{z}+\sum_{k=0}^{2} \sum_{q=-k}^{k}(-1)^{q} A_{2, q}^{\xi} \hat{T}_{2,-q}^{\xi} .
$$

The transformed Hamiltonian in the interaction frame of $\hat{H}_{Z}$ is given by Eqs. 2.40 and 2.41 using the unitary transformation operator $\hat{V}_{Z}(t)=e^{-i \hat{H}_{Z} t / \hbar}=e^{i \omega_{0} t \hat{I}_{z}}$ :

$$
\begin{aligned}
\hat{\tilde{H}}(t) & =e^{-i \omega_{0} t \hat{I}_{z}}\left[\sum_{k=0}^{2} \sum_{q=-k}^{k}(-1)^{q} A_{2, q} \hat{T}_{2,-q}\right] e^{+i \omega_{0} t \hat{I}_{z}} \\
& =\sum_{k=0}^{2} \sum_{q=-k}^{k}(-1)^{q} A_{2, q}^{\xi} \hat{T}_{2,-q}^{\xi} e^{i q \omega_{0} t}
\end{aligned}
$$


where Eq. B.15 has been used. This is one reason why the spherical tensor formalism is so useful: the interaction frame transformation operator $\hat{V}_{Z}(t)$ is just operator $\hat{R}_{z}\left(-\omega_{0} t\right)$ for a rotation about the $z$-axis through a time dependent angle $-\omega_{0} t$, and spherical tensors have simple transformation properties under rotations, particularly under $z$-rotations. The Zeeman Hamiltonian has been removed in Eq. 1.18 by the interaction frame transformation. Once the large Hamiltononian has been removed it is appropriate to apply time dependent perturbation theory in the interaction frame. The interaction frame of the Zeeman Hamiltonian is defined by a cycle time $\left|\omega_{0}\right|$; according to $\S 2.4$ a lowest-order average Hamiltonian can be computed as the time integral of Eq. 1.18 from 0 to $\tau=\left|\omega_{0}\right|^{-1}$,

$$
\hat{\widetilde{H}}_{\xi}^{(1)}=\int_{0}^{\tau} d t^{\prime} \hat{\widetilde{H}}\left(t^{\prime}\right)=\sum_{k=0}^{2} A_{k, 0}^{\xi} \hat{T}_{k, 0}^{\xi} .
$$

The effect of the interaction frame transformation is to go into a frame that co-rotates with the Larmor precession; in this frame the terms of the local Hamiltonian are either static or oscillate at $\omega_{0}$ or $2 \omega_{0}$. These oscillations are very fast compared to the characteristic rate at which the spins evolve under the local Hamiltonian, i.e., $\left|\omega_{0}\right| \gg\left\|\hat{H}_{\xi} / \hbar\right\|$, so these terms average to zero on the long term. The AHT treatment actually just assumes they average to zero over one Larmor period, although there are some perils to this assumption because AHT is a stroboscopic theory and the spins are not actually observed once per Larmor period. At any rate, the terms in $\hat{H}_{\xi}$ that survive are the ones that remained time independent in the interaction frame, i.e., the $\hat{T}_{k, 0}$ terms that commute with $\hat{H}_{Z}$.

The fact that the secular part of $\hat{H}_{\xi}$ is the part that commutes with $\hat{H}_{Z} \propto \hat{I}_{z}$ has a physical interpretation. As has been demonstrated, if $\left[\hat{H}_{\xi}^{(1)}, \hat{H}_{Z}\right]=0$ then $\left[\hat{H}_{\xi}^{(1)}, \hat{I}_{z}\right]=0$ 
and $\hat{H}_{\xi}^{(1)}$ is invariant under $z$-rotations. That means that $\hat{H}_{\xi}^{(1)}$ is symmetric in the spin space about the $z$-axis. The effect of the large Zeeman interaction is to impose a cylindrical symmetry about $\underline{B}_{0}$ onto the spin system. Note that in this case $\hat{H}_{\xi}^{(1)}$ is the same in both the laboratory and interaction frames. It is therefore suitable for use in rotating frame calculations. An important result of the Zeeman truncation is the introduction of a (spatial) orientational dependence of the energy eigenvalues. Eq. 1.13 is valid in any coordinate system; the zero-field local Hamiltonians are scalar operators and their eigenvalues are independent of any choice of coordinates (although in general the eigenvectors are not). Once the magnetic field truncates the interaction, however, the isotropy of space is broken and the energies of the system now depend on the molecular orientation with respect to $\underline{B}_{0}$. In a solid powder which contains a random distribution of molecular orientations there is also a distribution of resonance frequencies due to the local interactions. These anisotropic interactions lead to the so-called "powder broadening" of solid-state NMR spectra. The powder-broadened lineshapes contain useful chemical information but their widths are on the order of $\sim\left\|\hat{H}_{\xi}\right\| / \hbar$ is also on the order of the dispersion of resonances due to that interaction; therefore it will be difficult to resolve the solid-state NMR spectrum of a compound whose nuclei occupy multiple chemically- or magnetically-distinguishable sites because the resonances will overlap. Techniques such as magic angle sample spinning have been developed in order to reduce the solid-state linewidths. The powder broadening is not observed in fluids because of motional averaging of the the anisotropic Hamiltonians.

The next step is to calculate the higher order perturbation terms of the local Hamiltonian, which are sometimes necessary to consider. The higher-order perturbation treatment 
has more subtleties to it. Some of the techniques are reviewed in Refs. [38, 39, 40]; a few of the difficulties are summarized here. It is not immediately clear to what order the perturbation expansion in Eq. 1.15 can be carried out such that the terms are still secular (i.e., such that they commute with $\hat{H}_{Z}$ ) because the full $\hat{H}_{\xi}$ itself does not in general commute with $\hat{H}_{Z} \cdot{ }^{15}$ The average Hamiltonian calculation of the second-order truncated quadrupolar Hamiltonian results in certain non-secular terms as well as secular terms [38, 42]. In general one must also consider the fact that the Zeeman eigenstates must receive corrections in higher-order perturbation treatments. It is appropriate to apply a tilting matrix transformation to include these effects $[38,39,40,43]$. A standard procedure $[10, \S 4],[44]$ used to develop the second-order secular Hamiltonian $\hat{H}_{\xi}^{(2)}$ is to generate the diagonal matrix according to the usual second-order energy corrections in the static perturbation theory $[45$, $\S 5.1]$ in the manner of Eq. 1.16. The result is

$$
\hat{H}^{(2)}=\sum_{m, n \neq m} \frac{|m\rangle\left\langle m\left|\hat{H}_{\xi}\right| n\right\rangle\left\langle n\left|\hat{H}_{\xi}\right| m\right\rangle\langle m|}{\hbar \omega_{0}(m-n)} .
$$

Note that if more than one local interaction is involved, there can exist interference terms between $\hat{H}_{\xi}$ and $\hat{H}_{\xi^{\prime}}$ in the second-order secular Hamiltonian. These cross-correlation effects will not be considered here, although they are discussed further in $\S 1.5$ and $\S 4$.

Eq. 1.20 can be rewritten with the aid of Eq. 1.13:

$$
\hat{H}^{(2)}=\sum_{k, k^{\prime}=0}^{2} \sum_{q=-k, q^{\prime}=-k^{\prime}}^{k, k^{\prime}} \sum_{m, n \neq m} \frac{(-1)^{q+q^{\prime}} A_{k, q} A_{k^{\prime}, q^{\prime}}|m\rangle\left\langle m\left|\hat{T}_{k,-q}^{\xi}\right| n\right\rangle\left\langle n\left|\hat{T}_{k^{\prime},-q^{\prime}}^{\xi}\right| m\right\rangle\langle m|}{\hbar \omega_{0}(m-n)} .
$$

\footnotetext{
${ }^{15}$ Some authors use a somewhat different definition of "secular", see Ref. [41, §4.3.3].
} 
This expression can be simplified considerably upon considering the form of the Zeemanbasis matrix elements of the $\hat{T}_{k, q}$ operators. In the case of a Hamiltonian $\hat{H}_{\xi}$ that contains purely rank $k=2$ components, the second-order secular Hamiltonian can be written as [44]:

$$
\begin{aligned}
\hat{H}_{k=2}^{(2)} & =\sum_{m} \sum_{p \neq 0} \frac{A_{2,-p}^{\xi} A_{2, p}^{\xi}|m\rangle\left\langle m\left|\hat{T}_{2, p}^{\xi} \hat{T}_{2,-p}^{\xi}\right| m\right\rangle\langle m|}{\hbar \omega_{0} p} \\
& =\sum_{p>0} \frac{A_{2,-p}^{\xi} A_{2, p}^{\xi}\left[\hat{T}_{2,-p}^{\xi}, \hat{T}_{2, p}^{\xi}\right]}{\hbar \omega_{0} p} .
\end{aligned}
$$

Note that the second-order secular Hamiltonians go like $\sim \omega_{\xi}^{2} / \omega_{0}$, where $\left\|\hat{H}_{\xi}\right\| \sim \hbar \omega_{\xi}{ }^{16}$ These terms therefore become less important as the magnetic field increases, as is expected. It is important to note than in general the only NMR interaction that is large enough such that it has a non-negligible effect on the observed spectrum (i.e., it causes shifts that are larger than the linewidths) is the electric quadrupolar coupling. Second-order perturbation theory is however important in the theory of spin relaxation (see $\S 1.5)$.

\subsubsection{Chemical shielding Hamiltonian}

An excellent description of the theory of the chemical shielding in diamagnetic molecules is given in $\S 4$ of the book by Slichter [5]. See also the discussion given by Abragam $\left[6, \S\right.$ VI.II.B] and Refs. $[35,46]$. A magnetic field (call it $\underline{B}_{0}=B_{0} \underline{z}$ ) applied to an atom is capable of inducing currents of the atomic electrons. These currents are partly due to the quantum-mechanical analog of the classical diamagnetic current $[47, \S 6.1 .3]$ and partly due to mixing the atomic ground state with excited states that have orbital angular momentum.

\footnotetext{
${ }^{16}$ Another potential problem with the average Hamiltonian treatment is that the factor of $\omega_{0}^{-1}$ comes in as the reciprocal of the cycle time $t_{c}$, where $t_{c}>0$. The static perturbation theory correctly treats $\omega_{0}$ as a signed quantity.
} 
The induced currents will usually circulate in a sense such that the magnetic field they create at the center of the atom opposes the direction of $\underline{B}_{0}$. Therefore the effective magnetic field at the position of the nucleus is less than $\underline{B}_{0}$ and the nuclei are said to be "shielded" from the effects of $\underline{B}_{0}$ by the electrons. This effect is called the chemical shielding; it is the only one of the NMR interactions discussed here that disappears in the absence of an external magnetic field. Note that sometimes a "deshielding" convention is often assumed; in that case the effect is known instead as the chemical shift.

If the induced electron currents create a field $\underline{B}_{i n d}^{\sigma}(0)$ at the position of the nucleus the total Zeeman interaction can be written as

$$
\hat{H}_{Z}^{t o t}=-\underline{\hat{\mu}} \cdot\left[\underline{B}_{0}+\underline{B}_{i n d}^{\sigma}(0)\right]
$$

The induced field $\underline{B}_{\text {ind }}^{\sigma}$ is found experimentally to be linear in $B_{0}$, although its direction can vary depending on the relative orientations of the molecule and $\underline{B}_{0}$. Therefore one can write a very general expression,

$$
\underline{B}_{i n d}^{\sigma}(0)=-\underline{\sigma}_{B_{0}}
$$

where the second-rank tensor $\underline{\underline{\sigma}}$ is known as the chemical shielding tensor and provides the mapping between $\underline{B}_{0}$ and vect $B_{\text {ind }}^{\sigma}(0)$. The negative sign is introduced so that $\underline{\underline{\sigma}}$ has mostly positive components when it acts to shield the nucleus. The chemical shielding tensor is the quantity of interest in NMR because it contains information about the local chemical 
structure, i.e., how the electrons in different chemical environments respond to magnetic fields. For example, a particular type of atom located in a particular functional group usually is associated with a characteristic chemical shielding, which is used ubiquitously for molecular structural determination by NMR. NMR shielding tensors are usually on the order of $\|\underline{\underline{\sigma}}\| \sim 10^{-4}-10^{-5}$, i.e., the induced magnetic field at the nucleus is on the order of $\sim 10-100$ parts per million $(\mathrm{ppm})$ of $B_{0}$. The magnitude of the shielding depends on how easily the electron currents are induced; an atom such as hydrogen does not have a very polarizable electron cloud and its shielding ranges over about $10 \mathrm{ppm}$ in different chemical environments, whereas xenon has a chemical shielding range of up to thousands of ppm. In superconducting high field magnets the absolute value of the resonance shifts due to chemical shielding from the Larmor frequency of a bare nucleus are usually on the order of $\mathrm{kHz}$ to tens of $\mathrm{kHz}$.

The total Zeeman Hamiltonian may be written in terms of the chemical shielding as

$$
\hat{H}_{Z}^{t o t}=-\hbar \gamma \underline{\underline{\underline{I}}} \cdot(\underline{\underline{1}}-\underline{\underline{\sigma}}) \cdot \underline{B}_{0}
$$

It is customary to write this as

$$
\hat{H}_{Z}^{t o t}=\hat{H}_{Z}+\hat{H}_{\sigma}
$$

where $\hat{H}_{Z}$ is the usual Zeeman Hamiltonian due to $\underline{B}_{0}$ in the absence of chemical shielding; 
$\hat{H}_{\sigma}$ is known as the chemical shielding Hamiltonian:

$$
\hat{H}_{\sigma} / \hbar=\gamma \underline{\underline{I}} \cdot \underline{\underline{\sigma}} \cdot \underline{B}_{0}
$$

which in terms of laboratory-frame coordinates $\{x, y, z\}$ is

$$
\hat{H}_{\sigma} / \hbar=\gamma B_{0}\left(\sigma_{x z} \hat{I}_{x}+\sigma_{y z} \hat{I}_{y}+\sigma_{z z} \hat{I}_{z}\right)
$$

the secular part of which is

$$
\hat{H}_{\sigma}^{(1)} / \hbar=\sigma_{z z} \omega_{0} \hat{I}_{z},
$$

where $\omega_{0}=\gamma B_{0}$. The tensor component $\sigma_{z} z$ in the laboratory frame can be related to the principal axis system (PAS) components of $\underline{\underline{\sigma}}$ via a rotation of the coordinate system. It is also customary to make a second-rank Cartesian tensor $\underline{\underline{T}}=\underline{\underline{B_{0} \hat{I}}}$ and work in terms of a spherical tensor representation using Eq. 1.13 (see Appendix A). Then the secular approximation can be applied as described in $\S 1.3 .3$. Since $\underline{\underline{T}}$ is created from only one vector that contains spin operators, the spherical components of the rank-2 tensor $\underline{\underline{T}}$ are more conveniently written in terms of rank-1 spin operators (see Appendix A of [8]). The spatial tensors remain of $\operatorname{rank} k=0,1,2$. The $k=1$ antisymmetric components of $\underline{\underline{\sigma}}$ typically do not give a secular contribution to the spectrum (see $\S 1.3 .2$ ), but the $k=0$ isotropic and $k=2$ symmetric anisotropic components do contribute. The secular chemical 
shielding Hamiltonian may be written as $[10, \S 1.4 .1]$

$$
\hat{H}_{\sigma}^{(1)}=\left(\omega_{\sigma}^{i s o}+\omega_{\sigma}^{\text {aniso }}\right) \hat{I}_{z}
$$

where the isotropic component of the characteristic chemical shielding frequency is

$$
\omega_{\sigma}^{i s o}=\sigma_{i s o} \omega_{0}
$$

and the anisotropic shielding frequency written in terms of the polar angles $(\theta, \phi)$ that relate the $Z$-axis in the PAS of $\underline{\underline{\sigma}}$ to the laboratory frame $z$-axis is

$$
\omega_{\sigma}^{i s o}=\frac{1}{2} \Delta \sigma \omega_{0}\left[3 \cos ^{2} \theta-1+\eta_{\sigma} \sin ^{2} \theta \cos 2 \phi\right] .
$$

The isotropic chemical shielding written in terms of its PAS components $\{X, Y, Z\}$ is

$$
\sigma_{i s o}=\frac{1}{3} \operatorname{Tr}[\underline{\underline{\sigma}}]=\frac{1}{3}\left(\sigma_{X X}+\sigma_{Y Y}+\sigma_{Z Z}\right),
$$

and the chemical shielding anisotropy is referenced to $\sigma_{i s o}$ :

$$
\Delta \sigma=\sigma_{Z Z}-\sigma_{i s o}
$$

The chemical shielding asymmetry parameter is

$$
\eta_{\sigma}=\left(\sigma_{X X}-\sigma_{Y Y}\right) / \sigma_{Z Z}
$$


It vanishes when $\underline{\underline{\sigma}}$ is symmetric about some axis.

\subsubsection{Dipolar coupling Hamiltonian}

The magnetic field at a point $\underline{r}$ due to a classical magnetic dipole $\underline{\mu}_{2}$ located at the origin is $[17, \S 5.6]$ :

$$
\underline{B}_{2}(\underline{r})=\frac{\mu_{0}}{4 \pi}\left[\frac{3 \underline{\widetilde{r}}\left(\underline{\widetilde{r}} \cdot \underline{\mu}_{2}\right)-\underline{\mu}_{2}}{r^{3}}+\frac{8 \pi}{3} \underline{\mu}_{2} \delta^{3}(\underline{r})\right]
$$

where $r \equiv|\underline{r}|$ is the magnitude of $\underline{r}, \underline{\widetilde{r}} \equiv \underline{r} / r$ is a unit vector in the direction of $\underline{r}, \delta^{3}$ is the three-dimensional Dirac delta function, and $\mu_{0}$ is the magnetic permeability of free space. A diagram of two interacting magnetic dipoles is shown in Fig. ??. The classical energy of

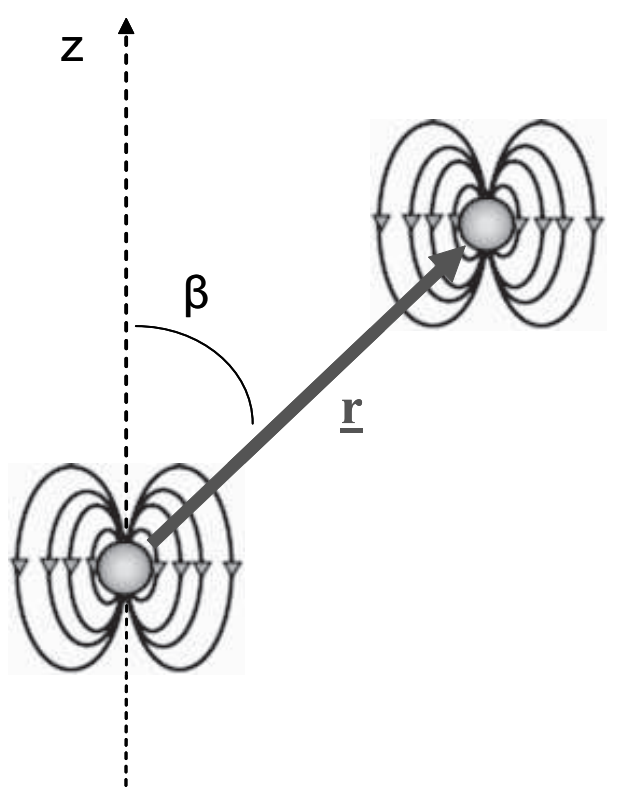

Figure 1.1: Schematic of two magnetic dipoles interacting via their magnetic fields. 
a magnetic dipole $\underline{\mu}_{1}$ in the field of $\underline{\mu}_{2}$ is:

$$
\begin{aligned}
E_{D} & =-\underline{\mu}_{1} \cdot \underline{B}_{2}(\underline{r}) \\
& =-\frac{\mu_{0}}{4 \pi}\left[\frac{3(\underline{\mu} \cdot \underline{\underline{r}})\left(\underline{\widetilde{r}} \cdot \underline{\mu}_{2}\right)-\underline{\mu}_{1} \cdot \underline{\mu}_{2}}{r^{3}}+\frac{8 \pi}{3}\left(\underline{\mu}_{1} \cdot \underline{\mu}_{2}\right) \delta^{3}(\underline{r})\right] .
\end{aligned}
$$

This expression is symmetric upon exchange of $\underline{\mu}_{1}$ and $\underline{\mu}_{2}$, and could just as well have been written as $E_{D}=-\underline{\mu}_{2} \cdot \underline{B}_{1}(\underline{r})$, the energy of $\underline{\mu}_{2}$ in the dipole field of $\underline{\mu}_{1}$. The $\delta$-function term ${ }^{17}$ becomes important if there is spatial overlap between the two dipoles, such as between a nucleus and an atomic $s$-state electron, known as the Fermi contact contribution to the hyperfine interaction. ${ }^{18}$ However, in the context of the internuclear dipole-dipole coupling, this term may be neglected because atomic nuclei do not overlap with each other to any appreciable extent under normal laboratory conditions.

Eq. 1.37 can be written in a compact Cartesian tensor notation as (see Appendix A):

$$
E_{D}=-\underline{\mu}_{1} \cdot \underline{\underline{A}}_{D} \cdot \underline{\mu}_{2}=-\underline{\underline{T}}_{D}: \underline{\underline{A}}_{D}
$$

where $\underline{\underline{A}}_{D}=\frac{\mu_{0}}{4 \pi r^{3}}\left[3 \underline{\underline{r}} / r^{2}-\underline{\underline{1}}\right]$ and $\underline{\underline{T}}_{D}=\underline{\underline{\mu_{2} \mu_{1}}}$. The $\delta$-function contact term has been neglected. The tensor $\underline{\underline{T}}_{D}$ contains the information about the orientation of the dipole vectors and the traceless tensor $\underline{\underline{A}}_{D}$ contains the information about the spatial arrangement of the dipoles relative to each other. The spherical tensor formalism is useful for determining

\footnotetext{
${ }^{17}$ This expression uses the Ampère model of a classical magnetic dipole rather than the Gilbert model to calculate the delta-function term $[47, \S 6.1 .2]$, which is consistent with hyperfine splitting measurements [48].

${ }^{18}$ The nucleus-conduction electron contact term in metals leads to a large nuclear resonance shift called the Knight shift [5, §4.7].
} 
the secular contribution to the homonuclear dipolar Hamiltonian.

A rigorous quantum-mechanical derivation of the magnetic dipole-dipole coupling Hamiltonian without the contact term yields:

$$
\begin{aligned}
\hat{H}_{D} & =-\frac{\mu_{0}}{4 \pi} \frac{1}{r^{3}}\left[3\left(\underline{\hat{\mu}}_{1} \cdot \underline{\widetilde{r}}\right)\left(\underline{\widetilde{r}} \cdot \underline{\hat{\mu}}_{2}\right)-\underline{\hat{\mu}}_{1} \cdot \underline{\hat{\mu}}_{2}\right] \\
& =\hbar \omega_{D}^{12}\left[3\left(\underline{\hat{I}}_{1} \cdot \underline{\widetilde{r}}\right)\left(\underline{\widetilde{r}} \cdot \underline{\hat{I}}_{2}\right)-\underline{\hat{I}}_{1} \cdot \underline{\hat{I}}_{2}\right],
\end{aligned}
$$

where $\omega_{D}^{12}=-\frac{\mu_{0}}{4 \pi} \gamma_{1} \gamma_{2} \hbar r^{-3}$. Eq. 1.39 has the same form as Eq. 1.37 except the classical dipole moments $\underline{\mu}_{1}$ and $\underline{\mu}_{2}$ have been promoted to quantum-mechanical operators $\underline{\hat{\mu}}_{1}=\gamma_{1} \hbar \underline{\hat{I}}_{1}$ and $\underline{\hat{\mu}}_{2}=\gamma_{2} \hbar \underline{\hat{I}}_{2}$. The angular momentum operators have been taken to be dimensionless. In the case of two nuclear magnetic moments, $\underline{r}$ is the internuclear vector and $r$ is the average internuclear distance. The dipolar coupling $\omega_{D}^{12}$ is bilinear in the gyromagnetic ratios and goes as the inverse cube of the average internuclear (interatomic) distance. Upon choosing the quantization axis $z$ to be along the internuclear vector $\underline{\mathrm{r}}$, Eq. 1.39 takes the simple form $\hat{H}_{D} / \hbar=\omega_{D}^{12}\left[3 \hat{I}_{z 1} \hat{I}_{z 2}-\underline{\hat{I}}_{1} \cdot \underline{\hat{I}}_{2}\right]$. This is easily diagonalized to give the eigenvalues $\hbar \omega_{D}^{12} / 2, \hbar \omega_{D}^{12} / 2,0$, and $-\hbar \omega_{D}^{12}$, which are independent of the direction of the internuclear vector (although interestingly, the eigenvectors are not). The single-quantum transitions are observed at the frequencies $\pm \frac{3}{2}\left|\omega_{D}^{12}\right|$, corresponding to two lines split by $3\left|\omega_{D}^{12}\right|$. This is the zero-field NMR spectrum of a pair of dipole-coupled spins.

Most NMR studies are conducted at high magnetic fields where $\left\|\hat{H}_{Z}\right\| \gg\left\|\hat{H}_{D}\right\|$. The quantization axis $z$ is normally taken to be along the direction of the external magnetic field $\underline{B}_{0}$. The chemically-shielded Zeeman Hamiltonian is $\hat{H}_{0} / \hbar=-\omega_{1}^{0} \hat{I}_{z 1}-\omega_{2}^{0} \hat{I}_{z 2}$, where $\omega_{1}^{0}=\gamma_{1} B_{0}\left(1-\sigma_{1}^{z z}\right)$ and $\omega_{2}^{0}=\gamma_{2} B_{0}\left(1-\sigma_{2}^{z z}\right)$, and the shielding tensor components are 
on the order of ppm to hundreds of ppm (see §1.3.4). The secular approximation (a firstorder perturbation theory treatment) keeps only the part of $\hat{H}_{D}$ that commutes with $\hat{H}_{0}$. There are two relevant limits: the weak coupling limit $|\Delta| \gg\left|\omega_{D}^{12}\right|$ where dipolar coupling is much less than the Larmor/chemical shift frequency difference $\Delta=\omega_{1}^{0}-\omega_{2}^{0}$, and the strong coupling limit $|\Delta| \ll\left|\omega_{D}^{12}\right|$ where the dipolar coupling is much greater than the Larmor/chemical shift frequency difference. At high magnetic fields, on the order of Tesla, virtually all heteronuclear spin pairs are in the weak coupling limit because $\Delta \approx \gamma_{1} B_{0}-\gamma_{2} B_{0}$ corresponds to a Larmor frequency difference on the order of $\mathrm{MHz}$, whereas $\left|\omega_{D}^{12}\right| / 2 \pi$ is typically on the order of $10 \mathrm{kHz}$ or less. Homonuclear spin pairs have $\gamma_{1}=\gamma_{2}=\gamma$, so $\Delta=\gamma B_{0} \sigma_{1}^{z z}-\gamma B_{0} \sigma_{2}^{z z}$ is just the chemical shift difference, which can be smaller or larger than the dipolar coupling depending on the system. From this perspective, a heteronuclear system looks like a homonuclear system with an extremely large chemical shift difference.

The secular dipolar Hamiltonian in the strong-coupling limit (also called the highfield homonuclear dipolar Hamiltonian) is:

$$
\begin{aligned}
\hat{H}_{D}^{s e c, s . c .} / \hbar & =\omega_{D}^{12}\left(\frac{3 \cos ^{2} \beta-1}{2}\right)\left[3 \hat{I}_{z 1} \hat{I}_{z 2}-\hat{I}_{1} \cdot \hat{\underline{I}}_{2}\right] \\
& =\omega_{D}^{12}\left(\frac{3 \cos ^{2} \beta-1}{2}\right)\left[2 \hat{I}_{z 1} \hat{I}_{z 2}-\frac{1}{2}\left(\hat{I}_{+1} \hat{I}_{-2}+\hat{I}_{-1} \hat{I}_{+2}\right)\right]
\end{aligned}
$$

where $\beta$ is the angle between the internuclear vector and the $z$-axis and $\hat{I}_{ \pm j}=\hat{I}_{x j} \pm i \hat{I}_{y j}$. The orientation dependent term has a second-rank Legendre polynomial dependence: $\left(3 \cos ^{2} \beta-\right.$ 1) $/ 2=P_{2}(\cos \beta)$. In the weak-coupling limit the secular dipolar Hamiltonian (also called 
the high-field heteronuclear dipolar Hamiltonian) becomes:

$$
\hat{H}_{D}^{s e c, w . c .} / \hbar=\omega_{D}^{12}\left(\frac{3 \cos ^{2} \beta-1}{2}\right)\left[2 \hat{I}_{z 1} \hat{I}_{z 2}\right]
$$

which is the same as the weak-coupling Hamiltonian except the "flip-flop" terms $\hat{I}_{ \pm 1} \hat{I}_{\mp 2}$ have been lost. These terms do not commute with $\hat{H}_{0}$ when $|\Delta| \gg\left|\omega_{D}^{12}\right|$. If the system consists of two $z$-quantized spin- $1 / 2$ nuclei in a Zeeman eigenstate, a term like $\hat{I}_{+1} \hat{I}_{-2}$ the state of spin 1 from down to up and the state of spin 2 from up to down. These two processes cost opposite amounts of energy if the spins are nearly chemically equivalent (strong coupling limit), so the net Zeeman energy of the system is conserved. However, if these two spins are strongly inequivalent due to the addition of the Zeeman field (weak coupling limit), flipping spin 1 in one direction costs a very different amount of energy than flipping spin 2 in the opposite direction, and the Zeeman energy is not conserved. Making the secular approximation in the presence of a dominant Zeeman interaction amounts to keeping only the terms in the dipolar Hamiltonian that conserve the Zeeman energy (i.e., commute with $\left.\hat{H}_{0}\right)$, which in the weak coupling limit are only the $\hat{I}_{z 1} \hat{I}_{z 2}$ terms. Note that in the strong coupling limit $\omega_{1}^{0} \approx \omega_{2}^{0}$, the Zeeman Hamiltonian is nearly proportional to the $z$-component of total angular momentum $\hat{I}_{Z}=\hat{I}_{z 1}+\hat{I}_{z 2}$, so that $\left[\hat{H}_{D}^{\text {sec,s.c. }}, \hat{H}_{0}\right] \approx\left[\hat{H}_{D}^{\text {sec,s.c. }},-\hbar \omega_{0} \hat{I}_{Z}\right]=0$ implies that the secular dipolar coupling nearly conserves this component of total angular momentum. However, the individual angular momentum components $\hat{I}_{z 1}$ and $\hat{I}_{z 2}$ are not conserved. The weak coupling limit of the secular approximation keeps only the part of the dipolar Hamiltonian that conserves the individual $z$-components of angular momentum $\hat{I}_{z 1}$ and $\hat{I}_{z 2}$; therefore, the $z$-component $\hat{I}_{Z}$ of total angular momentum is also conserved. 
There is another way of looking at the difference between the weak coupling and strong coupling secular dipolar Hamiltonians. If one goes into the interaction frame of $\hat{H}_{Z}$, this is equivalent to going into a doubly-rotating frame that goes around at frequency $\omega_{1}^{0}$ for spin 1 and frequency $\omega_{2}^{0}$ for spin 2. Any rapidly-oscillating terms in this frame are considered to time-average to zero and are therefore negligible; the remaining terms are the secular terms. From a classical perspective, the "secular" part of the dipolar magnetic field in Eq. 1.36 in the strong coupling limit is (neglecting the contact term):

$$
\underline{B}_{2}^{\text {sec,s.c. }}(\underline{r})=\frac{\mu_{0}}{4 \pi} \frac{1}{r^{3}}\left(\frac{3 \cos ^{2} \beta-1}{2}\right)\left[3 \mu_{2 z} \underline{\tilde{z}}-\underline{\mu}_{2}\right]
$$

where $\mu_{2 z}=\underline{\mu}_{2} \cdot \underline{\widetilde{z}}$ and $\beta=\cos ^{-1}(\underline{\tilde{r}} \cdot \underline{\widetilde{z}})$ is the angle between $\underline{r}$ and $\underline{\widetilde{z}}$. The secular Hamiltonian in Eq. 1.40 is recovered when the magnetic moments in the expression for the classical secular dipolar energy $-\underline{\mu}_{1} \cdot \underline{B}_{2}^{\text {sec,s.c. }}(\underline{r})$ are quantized. It is important to note that $\underline{B}_{2}^{\text {sec,s.c. }}(\underline{r})$ in no way represents the real magnetic field distribution due to $\underline{\mu}_{2}$; rather, it is the part of the dipolar field of $\underline{\mu}_{2}$ that affects the dynamics of $\underline{\mu}_{1}$ in the presence of a large external field $\underline{B}_{0}$. The effective field $\underline{B}_{2}^{\text {sec,s.c. }}$ contains transverse terms because when the nuclei are nearly chemically equivalent, a magnetic moment $\underline{\mu}_{2}$ precessing about $\underline{B}_{0}$ at the Larmor frequency $\omega_{2}^{0}$ creates a $\underline{B}_{2}^{\text {sec,s.c. }}$ field with transverse components rotating at the same frequency, which are nearly static in the frame of the magnetic moment $\underline{\mu}_{1}$ precessing about $\underline{B}_{0}$ at the frequency $\omega_{1}^{0}$. These transverse magnetic field components can cause a change in $\mu_{1 z}$ through nutation, which is consistent with the quantum-mechanical result that the individual $z$ components of angular momentum are not conserved when the spins are equivalent. If the nuclei are strongly chemically inequivalent, $\omega_{1}^{0}$ and $\omega_{2}^{0}$ are no longer nearly the same. The 
transverse components of $\underline{B}_{2}^{s e c}$ then no longer oscillate at nearly the precession frequency of $\underline{\mu}_{1}$, and thus are ineffective at changing $\underline{\mu}_{1}$. The effective dipolar field in the weak coupling limit is reached upon dropping the transverse components of $\underline{\mu}_{2}$ from the strong-coupling expression:

$$
\underline{B}_{2}^{\text {sec,w.c. }}(\underline{r})=\frac{\mu_{0}}{4 \pi} \frac{1}{r^{3}}\left(\frac{3 \cos ^{2} \beta-1}{2}\right)\left[2 \mu_{2 z}\right] \underline{\widetilde{z}},
$$

which is entirely longitudinal and conserves $\mu_{1 z}$.

The strong-coupling (homonuclear) secular dipolar Hamiltonian leads to singlequantum peaks at $\omega_{1}^{0} \pm \frac{3}{2}\left|\omega_{D}^{12}\right| P_{2}(\cos \beta)$ and $\omega_{2}^{0} \pm \frac{3}{2}\left|\omega_{D}^{12}\right| P_{2}(\cos \beta)$, which correspond to a pair of doublets of splitting $3\left|\omega_{D}^{12}\right| P_{2}(\cos \beta)$ centered on the chemical shift frequencies $\omega_{1}^{0}$ and $\omega_{2}^{0}$. The dipolar splitting depends on only one physical parameter $\left(\omega_{D}^{12}\right)$ and one angle $(\beta)$ because the dipolar spatial tensor $\underline{\underline{A}}_{D}$ is axially symmetric and traceless. The weak coupling (heteronuclear or other large chemical shift difference) secular dipolar Hamiltonian leads to doublets of splitting $2\left|\omega_{D}^{12}\right| P_{2}(\cos \beta)$ centered on $\omega_{1}^{0}$ and $\omega_{2}^{0}$. Typically only one spin or the other is observed at one time in heteronuclear systems at high field. The solid-state spectra have sharp doublets only in the case of a single crystal where a single angle $\beta$ is represented. In a polycrystalline powder the crystallite orientations are randomly distributed, causing a distribution of splitting frequencies in the bulk signal and a broadening of the dipolar doublets. The powder-broadened doublet distribution is called a Pake pattern, and its shape can be calculated analytically $[6, \S$ VII.I.A(a)], $[9, \S \mathrm{E}]$. The total secular dipolar Hamiltonian for a collection of homonuclear spins is the sum of the individual 
pairwise dipolar Hamiltonians:

$$
\hat{H}_{D}^{s e c}=\sum_{j>k} \hat{H}_{D}^{s e c ~ j k}=\hbar \sum_{j>k} \omega_{D}^{j k} P_{2}\left(\cos \beta_{j k}\right)\left[2 \hat{I}_{z j} \hat{I}_{z k}-\frac{1}{2}\left(\hat{I}_{+j} \hat{I}_{-k}+\hat{I}_{-j} \hat{I}_{+k}\right)\right]
$$

where $\omega_{D}^{j k}=-\frac{\mu_{0}}{4 \pi} \gamma^{2} \hbar r_{j k}^{-3}, \underline{r}_{j k}$ is the internuclear vector between spins $j$ and $k$, and $\beta_{j k}$ is the angle between $\underline{r}_{j k}$ and the $z$-axis. The dipolar spectrum has never been calculated analytically because $\left[\hat{H}_{D}^{i j}, \hat{H}_{D}^{j k}\right] \neq 0$ due to the flip-flop terms in the homonuclear secular dipolar Hamiltonian, and the first truly successful approximation of the dipolar linewidth in the many-body system was by Van Vleck moment expansion method [6, §IV.II],[5, §3.3]. The flip-flop terms also lead to apparently irreversible "spin-diffusion" effects in which e.g. magnetization created on one nucleus propagates throughout the dipole-coupled network.

The effects of the dipolar Hamiltonian in fluids (gases and liquids) are much different than in the solid state. The intramolecular dipole-dipole Hamiltonian averages to zero in isotropic solution under rapid molecular reorientation due to the fact that the trace (i.e., the isotropic portion) of the spatial tensor $\underline{\underline{A}}_{D}$ is zero. (Note that $\underline{\underline{A}}_{D}$ is not traceless if the contact term is included.) The intermolecular dipole-dipole Hamiltonian is also averaged to zero on a microscopic length scale due to molecular diffusion. Therefore no dipolar splittings are observed in isotropic solution, although the dipolar couplings can enter in higher-order perturbation theory as a relaxation mechanism that causes line broadening, see $\S 1.5$. The dipolar couplings also cause relaxation in solids. If the solution is not isotropic, e.g. an oriented liquid-crystalline medium, the dipolar couplings are not averaged to zero. However, fast molecular reorientation is still present, so the spectra present sharp doublets with some orientationally-averaged splitting $\omega_{D}^{12} \overline{P_{2}(\cos \beta)}$. The orientational average $\overline{P_{2}(\cos \beta)}$ is not 
zero if the averaging is anisotropic. Effective non-axially symmetric dipolar couplings in the molecule-fixed frame have been reported in some biaxial liquid crystalline phases due to motionally-averaged restricted rotations (librations) [49, 50, 51].

As is discussed in more detail in $\S 6$, the intramolecular dipolar couplings are not completely averaged away by molecular diffusion over a macroscopic length scale, since the internuclear vector $\underline{r}$ changes very little on the timescale of $1 / \omega_{D}^{12}$. Most of these dipolar couplings are quite small due to the $r^{-3}$ dependence of $\omega_{D}^{12}$, but they are not negligible due to the enormous number of them, on the order of $N^{2} / 2$ for $N \approx 10^{20}$ spins in a macroscopic sample. These effects of these distant dipoles can be described by a classical mean field approach, in which a given dipole precesses in the average magnetic field created by the rest of the dipoles. Semiclassical and other partially quantum-mechanical methods have also been developed to treat the coherent effects of long-range dipolar couplings. ${ }^{19}$

\subsubsection{J-coupling Hamiltonian}

There exists another coupling between nuclear spins called the $J$-coupling. This coupling arises from the mutual dipole-dipole coupling of two nuclear spins $I_{2}$ and $I_{2}$ to an electron. A simple model of the $J$-coupling is included in $\S 7.7$ of Ref. [32]. Since the nucleus-nucleus coupling is mediated by electrons, it is also sometimes called the indirect nuclear spin coupling. When the coupling Hamiltonian is averaged over electronic degrees

\footnotetext{
${ }^{19}$ The speed at which the oscillation of one dipole can be communicated to the other is limited by the finite speed of light, so distant dipole moments cannot feel changes in each other's orientation instantaneously. One may therefore wonder whether such time retardation effects can appreciably alter the dynamics of a pair of dipole-coupled nuclei separated by macroscopic distances. The authors of Ref. [52] have argued that such effects between a pair of oscillating dipoles actually decrease as the separation increases and are completely negligible in internuclear or any other physical systems. They did not consider collective effects in a system of many dipoles.
} 
of freedom, an interaction Hamiltonian can be written purely in terms of nuclear spin operators:

$$
\hat{H}_{J} / \hbar=\underline{\hat{I}}_{1} \cdot \underline{\underline{J}} \cdot \underline{\hat{I}}_{2} .
$$

Like the dipolar coupling tensor $\underline{\underline{A}}_{D}$, the $J$-coupling tensor $\underline{\underline{J}}$ is axially symmetric. Unlike $\underline{\underline{A}}_{D}$, however, $\underline{\underline{J}}$ is not traceless. The $J$-coupling Hamiltonian can be written as

$$
\hat{H}_{J} / \hbar=\hat{H}_{J}^{i s o}+\hat{H}_{J}^{a n i s o}
$$

where

$$
\hat{H}_{J}^{i s o} / \hbar=\omega_{J}\left(\hat{I}_{1} \cdot \hat{I}_{2}\right)
$$

is isotropic both in real space and in the combined spin space of $I_{1}$ and $I_{2}$. The $\hat{H}_{J}^{\text {aniso }}$ term is anisotropic, i.e., its value depends on the orientation of the molecule. The $J$-coupling constant is usually written in terms of frequency:

$$
J=\omega_{J} / 2 \pi
$$

The $J$-coupling constants tend to be on the order of $\mathrm{Hz}$ to hundreds of $\mathrm{Hz}$. An important property of the $J$-coupling is that it is a through-bond interaction rather than a throughspace interaction like the dipole-dipole coupling; i.e., the nuclei need to belong to two atoms that are connected through a covalently-bonded network. Therefore the $J$-couplings 
contain useful information about the connectivities between atoms. The $J$-coupling constant is often written as ${ }^{n} J_{X Y}$, where $n$ is the number of covalent bonds that separate nuclei $X$ and $Y$. The anisotropic $J$-coupling is averaged away in fluids due to molecular motion; in solids, the $J$-coupling is not usually very important because the residual powder linewidths in solids are often comparable to or larger than the $J$-couplings, even under magic angle spinning conditions. Therefore the $J$-coupling Hamiltonian in NMR is usually taken to be the secular part of $\hat{H}_{J}^{i s o}$. All of $\hat{H}_{J}^{i s o}$ is secular in the strong-coupling (homonuclear) limit when $\left|\omega_{J}\right| \gg\left|\omega_{0}^{1}-\omega_{0}^{2}\right|$ (see $\left.\S 1.3 .5\right)$. The interaction becomes truncated in the weak coupling limit $\left|\omega_{J}\right| \ll\left|\omega_{0}^{1}-\omega_{0}^{2}\right|$, which is always in effect at high field if the two spins are heteronuclear species. The secular approximation in a $J$-coupled system is described in terms of static perturbation theory in $\S 5.2 .3$ of Ref. [2]. The secular strong- and weak- $J$ coupling Hamiltonians are respectively (neglecting the anisotropic terms):

$$
\begin{aligned}
& \hat{H}_{J}^{\text {sec,s.c. }} / \hbar=\omega_{J}\left(\hat{\underline{I}}_{1} \cdot \hat{\underline{I}}_{2}\right) \\
& \hat{H}_{J}^{\text {sec,w.c. }} / \hbar=\omega_{J} \hat{I}_{z 1} \hat{I}_{z 2} .
\end{aligned}
$$

\subsubsection{Quadrupolar Hamiltonian}

The standard references for the theory of quadrupolar interactions are the monographs by Abragam [6, §VI.I], Das and Hahn [53], and Cohen and Reif [54]. Quadrupolar NMR has also been reviewed, e.g., Refs. [55], [56], and [44]. As was discussed in $§ 1.2$, spin $I>1 / 2$ nuclei in general have a non-spherical electric charge distribution and possess electric quadrupole moments that can couple to local electric field gradients. The nuclear 
charge distributions probed in NMR are averaged over the rotational motion of the nucleus and it is the rotationally-averaged "spectroscopic" quadrupole moment that is considered here. Fig. 1.2 illustrates how certain non-spherical charge distributions can lead to an electric quadrupole moment. The electric field gradients that the nucleus interacts with are typically due to external sources, e.g., the charges of nearby atoms. Note that in ionic crystals external electric field gradients can distort the electron cloud of the atom bearing the quadrupolar nucleus which in turn generates an electric field gradient of its own at the position of the nucleus; this is called the Sternheimer antishielding [6, §VI.I.B],[54, $\S 12],[57,58]$. Fig. 1.3 illustrates an external charge distribution that can create an electric field gradient at the position of the nucleus. These figures are only two-dimensional and the actual problem must be considered in three dimensions.

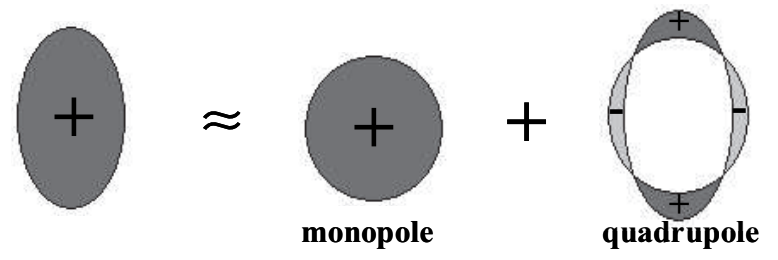

Figure 1.2: Schematic of a non-spherical nuclear charge distribution leading to an electric quadrupole moment.

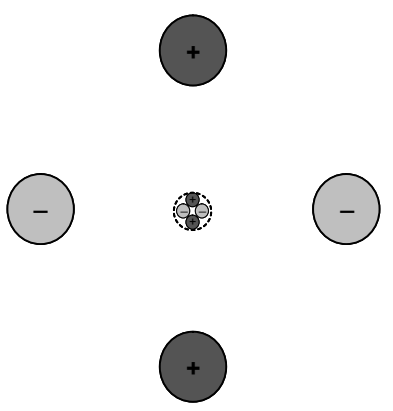

Figure 1.3: Schematic of an external charge distribution leading to an electric field gradient at the position of the nucleus. 
The classical quadrupolar energy is $[17, \S 4.2]$ :

$$
E_{Q}=-\frac{1}{6} \underline{\underline{Q}}: \underline{\underline{\nabla E}}(0)
$$

where $\underline{Q}$ is the electric quadrupole moment tensor and $\underline{\nabla E}(0)$ is the electric field gradient (EFG) tensor evaluated at the position of the nucleus. The EFG tensor can be written

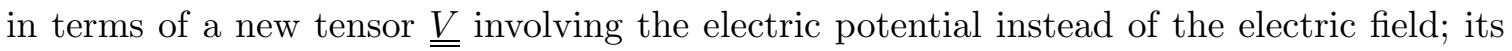
Cartesian components are

$$
V_{i j}=-[\nabla E(0)]_{i j}=\frac{\partial^{2} V}{\partial q_{i} \partial q_{j}}(0)
$$

The electric potential $V(\underline{r})$ is defined via $\underline{E}(\underline{r})=-\underline{\nabla} V(\underline{r})$ and $q_{i}, q_{j} \in\{x, y, z\}$. If the classical electric quadrupole moment tensor is promoted to a quantum operator, $\underline{\underline{Q}} \rightarrow \underline{\underline{\hat{Q}}}$, the quadrupolar Hamiltonian may be written in the form

$$
\hat{H}_{Q}=\underline{\underline{A}}^{Q}: \underline{\underline{\hat{T}}}^{Q}=\sum_{q=-2}^{2}(-1)^{q} A_{2, q}^{Q} \hat{T}_{2,-q}^{Q}
$$

where $\underline{\underline{A}}^{Q} \propto \underline{\underline{V}}$ and $\underline{\underline{\hat{T}}}^{Q} \propto \underline{\underline{\hat{Q}}}$. The neglect of the $k=1$ and $k=0$ spherical tensor terms in Eq. 1.53 as compared to Eq. 1.13 is justified as follows. The spatial tensor $\underline{\underline{A}}^{Q}$ is symmetric $\left(A_{i j}^{Q}=A_{i j}^{Q}\right)$ because of the equality of mixed partial derivatives, i.e., $\frac{\partial^{2} V}{\partial q_{i} \partial q_{j}}=\frac{\partial^{2} V}{\partial q_{j} \partial q_{i}}$ in Eq. 1.52. Therefore according to Eq. A.11 the rank-1 spherical tensor $A_{1}^{Q}$ is zero. Also note that the isotropic component of the spatial tensor is $A_{0}^{Q} \propto \operatorname{Tr}[\underline{\underline{V}}]=\frac{\partial^{2} V}{\partial x^{2}}(0)+\frac{\partial^{2} V}{\partial y^{2}}(0)+\frac{\partial^{2} V}{\partial z^{2}}(0)=$ $\nabla^{2} V(0)$. The Poisson equation of electrostatics states that $\nabla^{2} V(\underline{r})=\rho(\underline{r}) / \epsilon_{0}$, where $\rho(\underline{r})$ 
is the electric charge density at $\underline{r}$ and $\epsilon_{0}$ is the permittivity of free space. Since the charge distribution that generates the EFG typically comes from sources such as atoms that are external to the nucleus, $\rho(\underline{r})=0$, and therefore $A_{0}^{Q}=0$. Note that even if $\rho(0) \neq 0$, e.g., due to distortion of the $s$-electron distribution of the atom containing the quadrupolar nucleus, the $A_{0,0}^{Q}$ term multiples the tensor component $\hat{T}_{0,0}^{Q}$, which by the Wigner-Eckart theorem (see $\S$ A.3) is proportional to $\hat{1}$. This results in a uniform shift of the energies of the magnetic sublevels and does not affect the angular momentum dynamics.

It will be convenient to absorb the factor of $\frac{1}{6}$ into the tensors and to move any units involving the electric quadrupole moment into the spatial tensor $\underline{\underline{A}}^{Q}$ so that $\underline{\underline{\hat{T}}}^{Q}$ is dimensionless. The tensor $\underline{\underline{\hat{T}}}^{Q}$ can be written in terms of nuclear angular momentum operators via the Wigner-Eckart theorem (see §A.3). Eq. 1.53 can be written in the principal axis system (PAS) of the EFG tensor (labeled $\{X, Y, Z\}$ ) in a particularly simple form:

$$
\hat{H}_{Q}=\frac{h C_{Q}}{4\left[3 I^{2}-I(I+1)\right]}\left\{\left[3 \hat{I}_{Z}^{2}-I(I+1) \hat{1}\right]-\frac{\eta_{Q}}{2}\left(\hat{I}_{X}^{2}-\hat{I}_{Y}^{2}\right)\right\}
$$

According to a relatively standard definition the nuclear quadrupolar coupling constant is

$$
C_{Q}=e^{2} q Q / h
$$

where $e$ equals the electron charge and the principal component of the EFG tensor is

$$
e q=V_{Z Z}=\frac{\partial^{2} V}{\partial Z^{2}}(0)=-\frac{\partial E_{Z}}{\partial Z}(0)
$$


and the nuclear electric quadrupole moment is

$$
e Q=\left\langle I, m_{I}=I\left|\hat{Q}_{Z Z}\right| I, m_{I}=I\right\rangle
$$

The quadrupolar coupling constant $C_{Q}$ can be positive or negative depending on the relative signs of the nuclear quadrupole moment and the local electric field gradients, although only experimentally-determined $\left|C_{Q}\right|$ values are tabulated in the NMR literature because the sign of the quadrupolar coupling is not trivial to determine, at least by NMR methods (see pp. 261-262 of Ref. [6]. ${ }^{20}$ Typical values of $\left|C_{Q}\right|$ in solids are on the order of $\sim 100 \mathrm{kHz}$ to $\sim 1 \mathrm{MHz}$. The EFG asymmetry parameter is defined as

$$
\eta_{Q}=\left(V_{X X}-V_{Y Y}\right) / V_{Z Z}
$$

for $\left|V_{Z Z}\right| \geq\left|V_{X X}\right| \geq\left|V_{Y Y}\right|$; the case $\eta_{Q}$ corresponds to axial symmetry of the EFG about $Z$. The spherical tensors have been normalized such that

$$
\begin{aligned}
\hat{T}_{2,0} & =\left[3 \hat{I}_{Z}^{2}-I(I+1) \hat{1}\right] \\
\hat{T}_{2,+2}+\hat{T}_{2,-2} & =\sqrt{\frac{3}{2}} \hat{I}_{+}^{2}+\sqrt{\frac{3}{2}} \hat{I}_{-}^{2}=\sqrt{6}\left(\hat{I}_{X}^{2}-\hat{I}_{Y}^{2}\right) .
\end{aligned}
$$

Note that $A_{0,0}^{Q}$ and $A_{1, q}^{Q}$ are zero, and in the EFG PAS, $A_{2, \pm 1}^{Q \text { PAS }}=0$.

In NMR experiments it is more useful to express the quadrupolar Hamiltonian in laboratory frame coordinates $\{x, y, z\}$ so that the spin tensors are quantized along $z$, the magnetic field axis. The expression for $\hat{H}_{Q}$ in the laboratory frame is quite complicated

\footnotetext{
${ }^{20}$ The sign of $C_{Q}$ can be determined by other techniques, e.g., Mössbauer spectroscopy.
} 
(see Eq. 4.7 of Ref. [10]). Eq. 1.19 can be used to write the first-order secular contribution of $\hat{H}_{Q}$ as

$$
\begin{aligned}
\hat{H}_{Q}^{(1)} & =A_{2,0}^{\mathrm{LAB}} \hat{T}_{2,0}^{\mathrm{LAB}} \\
& =\hbar \omega^{1 Q}\left(C_{Q}, \eta_{Q} ; \alpha, \beta, \gamma\right)\left[3 \hat{I}_{z}^{2}-I(I+1) \hat{1}\right]
\end{aligned}
$$

where $\omega_{1 Q}\left(C_{Q}, \eta_{Q} ; \alpha, \beta, \gamma\right)$ is the characteristic first-order quadrupolar frequency is written in terms of the Euler rotation angles that relate the EFG PAS to the laboratory frame. If instead the polar angles $(\theta, \phi)$ that relate $Z$ to $z$, this frequency equals (see Eqs. 4.7, 4.11 of Ref. [10])

$$
\omega_{1 Q}=\frac{1}{2} \omega_{Q}\left[\eta_{Q} \cos 2 \phi\left(\cos ^{2} \theta-1\right)-\frac{1}{2}\left(3 \cos ^{2} \theta-1\right)\right]
$$

where

$$
\omega_{Q}=\frac{2 \pi C_{Q}}{2 I(2 I-1)}
$$

Note that $\hat{H}_{Q}^{1}$ is an odd function of $\hat{I}_{z}$. Therefore $\left\langle+|m|\left|\hat{H}_{Q}^{1}\right|+|m|\right\rangle=\left\langle-|m|\left|\hat{H}_{Q}^{1}\right|-|m|\right\rangle$ and the evolution of "symmetric" Zeeman coherences $|\mp| m|\rangle\langle \pm|m||$ is invariant to $\hat{H}_{Q}^{1}$.

Since quadrupolar couplings are so large, the effects of the second-order perturbation terms on the NMR spectrum cannot in general be neglected. The second order secular quadrupolar Hamiltonian can be written with the aid of Eq. 1.22. The products of spatial tensors, $A_{2,-p}^{Q \mathrm{LAB}} A_{2, p}^{Q \mathrm{LAB}}$, may be combined using Eq. A.17 to form higher ten- 
sors $A_{k, q}^{Q \mathrm{LAB}}$. According to the rules of angular momentum addition, $q=-p+p=0$ and $k=|2-2|, \ldots,|2+2|=0, \ldots, 4$ in integer steps. The higher tensors with odd $k$ are zero. This can be appreciated via a simple parity argument: since $P A_{l, m} P^{-1}=(-1)^{l} A_{l, m}$, where $P$ is the parity inversion operator, each of the $A_{2, p}^{Q \text { LAB }}$ terms is even under parity and therefore so is their product, which implies the product tensors have even $k$. Interestingly, the second-order secular quadrupolar Hamiltonian contains a spatially-isotropic $(k=0)$ component; this presents itself in NMR spectra in much the same way as a chemical shift. Note however that the spin operator dependence is different from that of a chemical shift, except in the case of $I+1$ where $\hat{I}_{z}^{3}=\hat{I}_{z}$. In that case the isotropic part of the second-order secular quadrupolar Hamiltonian is just proportional to the chemical shielding Hamiltonian. The two effects could however be distinguished by conducting experiments at different magnetic fields because they depend on $B_{0}$ differently.

The second-order secular quadrupolar Hamiltonian is (see Eqs. 4.8, 4.12 of Ref. $[10])$

$$
\begin{gathered}
\hat{H}_{Q}^{(2)} / \hbar=\omega_{0}^{2 Q} \hat{I}_{z}\left[I(I+1) \hat{1}-3 \hat{I}_{z}^{2}\right]+\omega_{2}^{2 Q} \hat{I}_{z}\left[8 I(I+1) \hat{1}-12 \hat{I}_{z}^{2}-3 \hat{1}\right] \\
+\omega_{4}^{2 Q}\left[18 I(I+1) \hat{1}-34 \hat{I}_{z}^{2}-5 \hat{1}\right]
\end{gathered}
$$


where the rank- $k$ second-order characteristic quadrupolar frequencies $\omega_{k}^{2 Q}$ are

$$
\begin{aligned}
& \omega_{0}^{2 Q}= \frac{1}{80 \pi^{2}} \frac{\omega_{Q}^{2}}{\omega_{0}}\left(3+\eta_{Q}^{2}\right) \\
& \omega_{2}^{2 Q}=-\frac{1}{448 \pi^{2}} \frac{\omega_{Q}^{2}}{\omega_{0}}\left[\left(\eta_{Q}-3\right)\left(3 \cos ^{2} \theta-1\right)+6 \eta_{Q} \sin ^{2} \theta \cos 2 \phi\right] \\
& \omega_{4}^{2 Q}=-\frac{1}{128 \pi^{2}} \frac{\omega_{Q}^{2}}{\omega_{0}}\left[\frac{1}{140}\left(18+\eta_{Q}^{2}\right)\left(35 \cos ^{4} \theta-30 \cos ^{2} \theta+3\right)+\right. \\
&\left.\quad+\frac{3}{7} \eta_{Q} \sin ^{2} \theta\left(7 \cos ^{2} \theta-1\right) \cos 2 \phi+\frac{1}{4} \eta_{Q}^{2} \sin ^{4} \theta \cos 4 \phi\right] .
\end{aligned}
$$

Note that $\hat{H}_{Q}^{(2)}$ is an odd function of $\hat{I}_{z} \cdot{ }^{21}$ This allows the evolution of symmetric Zeeman coherences $|\mp| m|\rangle\langle \pm|m||$ under $\hat{H}_{Q}^{(2)}$ to be "reversed" in a coherence transfer experiment by appropriately choosing the signs of the coherences. This leads to the possibility of removing parts of the $\hat{H}_{Q}^{(2)}$ evolution using a coherence transfer echo, which is a principle that the MQMAS experiment in half integer quadrupolar spins relies on (see $\S 3$ ).

Second-order cross-correlation effects can also be important when at least one of the interactions is large, such as a quadrupolar coupling. Quadrupolar-chemical shift anisotropy and quadrupolar-dipolar cross-correlation relaxation effects in solution are considered in $\S 1.5$ and $\S 4$. Cross-correlated interactions are the source of the small apparent magnetic field dependence of the quadrupolar splitting that has been observed in gaseous Xe-129 [59, 60].

It is rarely necessary to consider perturbation terms beyond second order. However, there are compounds that possess nuclear quadrupolar couplings that are large enough

\footnotetext{
${ }^{21}$ One might object that this is unphysical since $\hat{H}_{Q}$ and hence its unitary-truncated form $\hat{H}_{Q}^{(2)}$ must be invariant (even) under time reversal, but $\hat{I}$ and hence $\hat{I}_{z}$ is an odd function under time reversal $\left(T \hat{I}_{z} T^{-1} \rightarrow\right.$ $-\hat{I}_{z}$ ). However, one must also consider the time reversal properties of the $\omega_{k}^{2 Q}$ terms. Electric fields and charge densities are even under time reversal and therefore so is $\omega_{0}$; note, however that $\omega_{0}$ contains a "hidden" factor of the magnetic field $B_{0}$. Magnetic fields are odd under time reversal; therefore, $\hat{H}_{Q}^{(2)}$ as a whole is even under time reversal, as is expected.
} 
that higher-order perturbation corrections are necessary even at fairly high values of $B_{0}$. Third-order quadrupolar effects have been observed experimentally [61] and also considered theoretically $[39,40,61,44]$. Bain has made an exact calculation in Liouville space of the simultaneous effects of the Zeeman and quadrupolar interactions [62]. Grandinetti has considered the effects of large quadrupolar couplings on phase cycling [63].

\subsubsection{Radiofrequency Hamiltonian}

NMR is, as its name suggests, a resonant technique. The adjacent magnetic sublevels of a spin $I$ are split in the presence of a magnetic field $\underline{B}_{0}$ by $\hbar \omega_{0}$, where $\omega_{0}$ is the Larmor frequency. An oscillating magnetic field tuned near the resonance frequency $\omega_{0}$ can drive transitions between adjacent sublevels. This section considers this process from a Hamiltonian perspective. The magnetic field will be treated classically; the reader is referred elsewhere for quantized field descriptions of magnetic resonance [64],[65, §4],[66],[67, $\S 2]$, which are generally not necessary to describe most NMR phenomena.

An NMR probe is operated by passing an oscillating current through a coil which in turn generates an oscillating magnetic field. Typical nuclear Larmor frequencies in superconducting magnets are on the order of $10^{2} \mathrm{MHz}$, which is in the radio frequency region of the electromagnetic spectrum. NMR excitation has therefore been considered to be a radiofrequency (rf) irradiation of the nuclei. Note, however, that this technique does not subject the spins to what are commonly understood to be radio waves. Radio waves are a type of electromagnetic radiation, which is a "far field" effect, i.e., they are experienced at distances from the oscillating source that are large compared to the oscillation wavelength $[17, \S 9]$. A current oscillating at $\omega_{r}=300 \mathrm{MHz}$ corresponds to radio waves with a 
wavelength of $1 \mathrm{~m}$; however, the rf coil and the sample and the distance between the two are all typically on the order of $1 \mathrm{~cm}$. The magnetic field is experienced by the spins on a sub-wavelength scale and therefore NMR irradiation can actually be considered to be a "near field" effect [68].

Assume that the rf coil creates a homogeneous magnetic field oscillating at frequency $\omega_{r}$ with phase $\phi$ that is linearly polarized along the $x$-axis in the laboratory frame:

$$
\underline{B}_{r f}(t)=2 B_{1} \cos \left(\omega_{r} t+\phi\right) \underline{\underline{x}} .
$$

The factor of 2 is introduced for later convenience. This linearly polarized field can be decomposed into two counter-rotating circularly-polarized fields:

$$
\begin{aligned}
\underline{B}_{r f}(t) & =\underline{B}_{r f}^{+}(t)+\underline{B}_{r f}^{-}(t) \\
& =B_{1}\left[\cos \left(\omega_{r} t+\phi\right) \underline{\widetilde{x}}+\sin \left(\omega_{r} t+\phi\right) \underline{\widetilde{y}}\right]+B_{1}\left[\cos \left(\omega_{r} t+\phi\right) \underline{\widetilde{x}}-\sin \left(\omega_{r} t+\phi\right) \underline{\widetilde{y}} \cdot(1 .\right.
\end{aligned}
$$

The laboratory-frame radiofrequency Hamiltonian for a spin $I$ is just the Zeeman Hamiltonian for the spin in the presence of $\underline{B}_{r f}(t)$ :

$$
\begin{aligned}
\hat{H}_{r f}(t) / \hbar & =-\gamma \underline{\underline{I}} \cdot \underline{B}_{r f}(t)=-2 \omega_{1} \cos \left(\omega_{r} t+\phi\right) \hat{I}_{x} \\
& =-\omega_{1}\left[\cos \left(\omega_{r} t+\phi\right) \hat{I}_{x}+\sin \left(\omega_{r} t+\phi\right) \hat{I}_{y}\right]-\omega_{1}\left[\cos \left(\omega_{r} t+\phi\right) \hat{I}_{x}-\sin \left(\omega_{r} t+\phi\right) \hat{I}_{y}\right],
\end{aligned}
$$

where $\omega_{1}=\gamma B_{1}$. The effects of the rf Hamiltonian on nuclei is considered further in $\S 1.6$. 


\subsubsection{Motional averaging of Hamiltonians in fluids}

The basic solid-state NMR Hamiltonian is given by Eqs. 1.13 and 1.14:

$$
\begin{aligned}
\hat{H} & =\hat{H}_{Z}+\sum_{\xi} \hat{H}_{\xi} \\
& =\hat{H}_{Z}+\sum_{\xi} \sum_{k=0}^{2} \sum_{q=-k}^{k}(-1)^{q} A_{k, q}^{\xi} \hat{T}_{k,-q}^{\xi},
\end{aligned}
$$

where $\hat{H}_{Z}$ is the dominant Zeeman Hamiltonian and the $\hat{H}_{\xi}$ are the Hamiltonians of local interactions. There are two relevant timescales for quantum-mechanical evolution under this Hamiltonian: the Zeeman timescale $\hbar /\left\|\hat{H}_{Z}\right\| \sim \omega_{0}^{-1}$ and the set of timescales of the local interactions $\hbar /\left\|\hat{H}_{Z}\right\| \sim \omega_{\xi}^{-1}$. The timescale of Zeeman evolution (Larmor precession) in a high-field NMR experiment is very much faster than the timescale of any of the local interactions; in $§ 1.3 .3$ it was seen that this resulting in a secular averaging (truncation) in spin space in the Zeeman interaction frame. Now consider what happens when time dependence is added to the spatial part of the Hamiltonian. The once the spatial spatial tensors are referenced to some coordinate system, they can be written as (courtesy of the Wigner-Eckart theorem, see $\S$ A.3):

$$
\hat{A}_{k, q}^{\xi} \propto Y_{k, q}(\theta, \phi)
$$

where $Y_{k, q}(\theta, \phi)$ is a spherical Harmonic function. The polar angles $(\theta, \phi)$ could for example describe the orientation of an internuclear vector relative to space-fixed (laboratory frame) coordinates in the case of a dipole-dipole coupling Hamiltonian, or the orientation of the 
principal axis of a local molecular electric field gradient relative to space-fixed coordinates in the case of a quadrupolar coupling. As opposed to the situation in solids, in which the molecules are rigidly locked into one orientation, fluids are characterized by random molecular motion including tumbling and diffusion. The $\hat{A}_{k, q}^{\xi}$ are fixed to the molecular frame (neglecting internal molecular motion); i.e., the principal axis of one tensor might be along the axis of a particular chemical bond, and as the molecule rotates so does the bond. This causes a stochastic reorientation of the spatial tensor components that can be characterized by introducing a time dependence into the polar angles that parameterize them, $\theta \rightarrow \theta(t)$ and $\phi \rightarrow \phi(t)$. If the fluid is isotropic $\theta(t)$ and $\phi(t)$ will sample all possible orientations isotropically. If the timescale of molecular reorientation is much faster than any of the NMR timescales including the Zeeman timescale - e.g., molecular tumbling can be on the order of picoseconds for small molecules - it is appropriate to replace the spatial tensors with their time-averaged values. This is equivalent to replacing $A_{k, q}$ with its isotropic orientational average. The spherical harmonic functions are orientationally-averaged according to

$$
\left\langle Y_{k, q}\right\rangle_{\mathcal{O}}=\frac{1}{\int d \Omega}\left[\int d \Omega Y_{k, q}(\theta, \phi)\right]
$$

where $d \Omega=\sin \theta d \theta d \phi$ is the differential area element. Note that this can be written as

$$
\left\langle Y_{k, q}\right\rangle_{\mathcal{O}}=\frac{1}{\sqrt{4 \pi}} \int d \Omega Y_{k, q}(\theta, \phi) Y_{0,0}^{*}(\theta, \phi)
$$


where $Y_{0,0}^{*}(\theta, \phi)=Y_{0,0}(\theta, \phi)=\sqrt{\frac{1}{4 \pi}}$. The orthogonality relation for spherical harmonic functions is

$$
\int d \Omega Y_{l, m}(\theta, \phi) Y_{l^{\prime}, m^{\prime}}^{*}(\theta, \phi)=\delta_{l, l^{\prime}} \delta_{m, m^{\prime}}
$$

Therefore it can be seen that $\left\langle Y_{k, q}\right\rangle_{\mathcal{O}}=0$ unless $k=0, q=0$; i.e., unless it is an average of the isotropic component, $\left\langle A_{0,0}\right\rangle_{\mathcal{O}}=A_{0,0}=\sqrt{\frac{1}{4 \pi}}$. Therefore fast isotropic reorientation in fluids is said to average away all tensor interactions; i.e., all non-isotropic components of the local Hamiltonians are averaged to zero. This is not necessarily the case in an oriented (anisotropic) fluid medium because some orientations of the molecule are preferred over others and the isotropic orientational average must be replaced with a probability-weighted orientational average.

The only local interactions that have a spatially isotropic component are the chemical shielding and $J$-coupling interactions. Solution-state NMR spectroscopy largely consists of the measurement of chemical shifts and $J$-splittings. The quadrupolar coupling interaction is averaged away, as is the dipole-dipole coupling (at least over microscopic distances, see $\S 6)$. On the other hand the anisotropic broadening found in powdered solids is averaged away in solution so the spectral lines are very sharp.

Note that when the molecular motion is on a timescale comparable to or slower than the Zeeman timescale, it is more appropriate to apply the Zeeman secular averaging to the local interactions before the motional averaging. This can lead to small second-order frequency shifts. For example, as was seen in $§ 1.3 .7$ the second-order secular quadrupolar Hamiltonian contains an isotropic component which will not be averaged away by isotropic 
molecular reorientation. These so-called dynamic frequency shifts in solution-state NMR are considered from the perspective of the semiclassical relaxation theory in $§ 1.5 .4$.

\subsection{Density operator theory and propagation}

\subsubsection{Introduction to the density operator}

The following discussion of the density operator and its application to quantum mechanics and statistical mechanics draws heavily from the works of Blum [69], Farrar and Harriman [70], Fano [71], Giulini et al. [72], and Tolman [73, §IX].

It is well-known that if an isolated quantum system is known to be prepared in some state $|\psi\rangle$, then that state vector contains all the information necessary to specify the probabilistic results of all measurements of the internal configuration of the system. However, usually only a finite number of degrees of freedom of a system are known to the experimenter, and even the best-isolated systems exhibit some coupling to their surroundings. Therefore, sometimes only incomplete knowledge of the quantum system is available; i.e., it may not be certain that the system exists in some state $|\psi\rangle$, but there may be some statistical knowledge that the system is likely to exist in state $\left|\psi_{i}\right\rangle$ with probability $P_{i}$, with multiple $\left\{\left|\psi_{i}\right\rangle\right\}$ possible. Alternatively, in an ensemble picture one might think of a fraction $P_{i}$ of the ensemble existing purely in state $\left|\psi_{i}\right\rangle$.

It is necessary to develop a quantum-mechanical formalism that can accommodate the statistical ensemble picture. This is usually accomplished by introducing a statistical density operator. It should be clear that if the state $|\psi\rangle$ contains all the internal information of the system, then the projection operator $|\psi\rangle\langle\psi|$ contains no more and no less information 
than does $|\psi\rangle$ itself. If the system is known (by experimental control or due to natural law) to exist in some set of states $\left\{\left|\psi_{i}\right\rangle\right\}$ with probabilities $\left\{P_{i}\right\}$, then a density operator may be defined

$$
\hat{\rho}=\sum_{i} P_{i}\left|\psi_{i}\right\rangle\left\langle\psi_{i}\right|
$$

where $\hat{\rho}$ is a probability-weighted linear combination of projection operators. There is no requirement that the $\left\{\left|\psi_{i}\right\rangle\right\}$ be orthogonal or normalized; however, if they are, then the following relation is satisfied:

$$
\operatorname{Tr}[\hat{\rho}]=\sum_{i} P_{i}=1
$$

where the sum of all probabilities must equal unity. Additionally, $\hat{\rho}$ is Hermitian, such that

$$
\hat{\rho}^{\dagger}=\hat{\rho} .
$$

The general form of Eq. 1.73 specifies a mixed state, since the system is not known to be specified by any one state $\left|\psi_{i}\right\rangle$. However, if one $P_{i}$ equals unity and the rest equal zero, then $\hat{\rho}=\left|\psi_{i}\right\rangle\left\langle\psi_{i}\right|$ specifies a system in a pure state (i.e., the state $\left|\psi_{i}\right\rangle$ ). The $\left\{P_{i}\right\}$ may be interpreted in an ensemble picture as being the populations of the states $\left\{\left|\psi_{i}\right\rangle\right\}$ of the system. It can be shown that $\hat{\rho}^{2}=\hat{\rho}$ for a pure state and $\hat{\rho}^{2} \neq \hat{\rho}$ for a mixed state. It also can be shown that $\operatorname{Tr}\left[\hat{\rho}^{2}\right] \geq 0$ for any $\hat{\rho}$.

The density operator can always be expanded in some complete set of orthonormal basis states $\{|n\rangle\}$, e.g. by expanding $\left|\psi_{i}\right\rangle=\sum_{n} c_{n}^{(i)}|n\rangle$, where $c_{n}^{(i)}=\left\langle n \mid \psi_{i}\right\rangle$. In this case Eq. 


\subsection{3 becomes}

$$
\hat{\rho}=\sum_{m, n} \sum_{i} P_{i} c_{m}^{(i) *} c_{n}^{(i)}|m\rangle\langle n|,
$$

allowing the density operator can be written in terms of its matrix elements $\rho_{m n}=\langle m|\hat{\rho}| n\rangle=$ $\sum_{i} P_{i} c_{m}^{(i) *} c_{n}^{(i)}$. It is evident that the only way to get a nonzero off-diagonal matrix element $\rho_{m n}(m \neq n)$ is if more than one of the coefficients $\left\{c_{n}^{(i)}\right\}$ describing at least one of the $\left|\psi_{i}\right\rangle$ are non-zero; i.e., if $\left|\psi_{i}\right\rangle$ is in a coherent superposition of the states basis $\{|n\rangle\}$. For this reason the off-diagonal matrix elements of $\hat{\rho}$ are called quantum coherences: they indicate coherent superpositions in a given basis within the system. The diagonal elements of the density operator are $\rho_{n n}=\sum_{i} P_{i}\left|c_{n}^{(i)}\right|^{2}=\sum_{i} P_{i} P_{n}^{(i)}$, where $P_{n}^{(i)}=\left|\left\langle n \mid \psi_{i}\right\rangle\right|^{2}$ is the probability of finding the system in state $|n\rangle$ if it is prepared in the pure state $\left|\psi_{i}\right\rangle$. The $P_{i}$ are "statistical probabilities" and the $P_{n}^{(i)}$ are "quantum probabilities." It follows that $\rho_{n n}$ corresponds to the probability of finding the system in state $|n\rangle$ if it is prepared in the mixed state described by Eq. 1.73. It should be stressed that coherences are in the eye of the beholder in the sense that a system may have coherences in one set of basis states but not in another; likewise, the populations must reflect the choice of basis as well. ${ }^{22} \mathrm{~A}$ mixed state is said to be at least partially coherent if the density operator has coherences in some basis. A density operator is said to be completely incoherent if it has no coherences in any

\footnotetext{
${ }^{22}$ This may be exemplified by considering a two-state system spanned by the basis vectors $|1\rangle$ and $|2\rangle$. If the system is prepared in the pure state $|1\rangle$, then $\hat{\rho}=|1\rangle\langle 1|=\left(\begin{array}{ll}1 & 0 \\ 0 & 0\end{array}\right)$ in the basis of $|1\rangle$ and $|2\rangle$. However, in the basis of $| \pm\rangle=\frac{1}{\sqrt{2}}(|1\rangle \pm|2\rangle)$, the density operator becomes $\hat{\rho}=\frac{1}{2}(|+\rangle\langle+|+|+\rangle\langle-|+|-\rangle\langle+|+|-\rangle\langle-|)=\frac{1}{2}\left(\begin{array}{ll}1 & 1 \\ 1 & 1\end{array}\right)$. In the former case the system has populations $P_{1}=1$ and $P_{2}=0$ and no coherences; in the latter case, the system has populations $P_{ \pm}=\frac{1}{2}$ and non-zero coherences between $|+\rangle$ and $|-\rangle$. Note that this density operator is different from $\hat{\rho}=\frac{1}{2}(|+\rangle\langle+|+|-\rangle\langle-|)=\frac{1}{2}\left(\begin{array}{ll}1 & 0 \\ 0 & 1\end{array}\right)$, which corresponds to an incoherent superposition of $|+\rangle$ and $|-\rangle$; in fact, it represents the completely incoherent/mixed state because $\hat{\rho}=\frac{1}{2}\left(\begin{array}{ll}1 & 0 \\ 0 & 1\end{array}\right)$ in any basis.
} 
basis, in which case it is always diagonal with every $P_{i}$ being equal (i.e., it is proportional to the identity operator).

The density operator can be used to predict the results of experimental measurements. A measurement result corresponds to the expectation value of an observable operator, which in the case of a system in the pure state $\left|\psi_{i}\right\rangle$ is $\langle\hat{A}\rangle=\left\langle\psi_{i}|\hat{A}| \psi_{i}\right\rangle=\operatorname{Tr}\left[\left|\psi_{i}\right\rangle\left\langle\psi_{i}\right| \hat{A}\right]$. If the system exists in a mixed state, then

$$
\begin{aligned}
\langle\hat{A}\rangle & =\sum_{i} P_{i}\left\{\operatorname{Tr}\left[\left|\psi_{i}\right\rangle\left\langle\psi_{i}\right| \hat{A}\right]\right\} \\
& =\operatorname{Tr}[\hat{\rho} \hat{A}]
\end{aligned}
$$

which must be generalized to $\langle\hat{A}\rangle=\operatorname{Tr}[\hat{\rho} \hat{A}] / \operatorname{Tr}[\hat{\rho}]$ if $\hat{\rho}$ is not normalized. If the density operator and observable operator are expanded directly into some basis, then $\langle\hat{A}\rangle=$ $\sum_{m, n} \rho_{m n} A_{n m}$, where $\rho_{m n}=\langle m|\hat{\rho}| n\rangle$ and $A_{n m}=\langle n|\hat{A}| m\rangle$.

The density operator also may evolve in time under a system Hamiltonian. If the system obeys purely quantum-mechanical (unitary) time evolution, then $|\psi(t)\rangle=\hat{U}\left(t ; t_{0}\right)\left|\psi\left(t_{0}\right)\right\rangle$ in the case of a pure state, where $\hat{U}\left(t ; t_{0}\right)$ is the unitary time-development operator that propagates the state vector from time $t_{0}$ to time $t$. This may be generalized to find the time evolution of the density operator $(\S 2.1 .2)$ :

$$
\hat{\rho}(t)=\hat{U}\left(t ; t_{0}\right) \hat{\rho}\left(t_{0}\right) \hat{U}^{-1}\left(t ; t_{0}\right)
$$

The time development operator may be found by solving the Schrödinger Equation $(\S 2)$. 
The density operator satisfies von Neumann's quantum Liouville equation of motion:

$$
i \hbar \frac{\partial \hat{\rho}(t)}{\partial t}=[\hat{H}(t), \hat{\rho}(t)]
$$

where $\hat{H}(t)$ is the (possibly time-dependent) Hamiltonian of the system. The density operator does not evolve in time if it commutes with the Hamiltonian operator. The equation of motion for the expectation value of an observable is:

$$
i \hbar \frac{\partial<\hat{A}>(t)}{\partial t}=-<[\hat{H}(t), \hat{A}]>
$$

where $<\hat{A}>(t)=\operatorname{Tr}[\hat{\rho}(t) \hat{A}]$.

\subsubsection{Open quantum systems and reduced density operators}

This preceding discussion has not considered the origin of mixed states or the interaction of a quantum system with its environment, and these topics will be considered here. A quantum system that is completely isolated from any other system is considered to be closed. Consider a closed quantum system $C$ whose degrees of freedom can be divided into two parts: a subsystem of interest $S$, and the environment $E$, such that $C=S \otimes E$. The division between system and environment is somewhat arbitrary, but often a system of interest can be singled out as a particular set of degrees of freedom upon which measurements are made, e.g. NMR measurements are made within the set of angular momentum eigenstates of the nuclear ground state. Presumably, even a very large closed quantum system can be described by a pure state vector, although this hypothesis cannot really be tested 
experimentally since every system interacts with an environment (including the observer) to some degree. Suppose $C$ is prepared in some state $|\Psi\rangle$. This state can be expanded in sets of basis states within the $S$ and $E$ subspaces:

$$
\left|\Psi^{C}\right\rangle=\sum_{m \in S} \sum_{n \in E} c_{m n}\left|\psi_{m}^{S} \phi_{n}^{E}\right\rangle
$$

where $\left|\psi_{m}^{S} \phi_{n}^{E}\right\rangle \equiv\left|\psi_{m}^{S}\right\rangle \otimes\left|\phi_{n}^{E}\right\rangle, c_{m n}=\left\langle\psi_{m}^{S} \phi_{n}^{E} \mid \Psi^{C}\right\rangle$, and the $\left\{\left|\psi_{m}^{S}\right\rangle\right\}$ and $\left\{\left|\phi_{n}^{E}\right\rangle\right\}$ are complete sets of orthonormal basis vectors within the $S$ and $E$ subspaces of $C$, respectively. It should be noted that if all the expansion coefficients were uncorrelated between $S$ and $E$ such that $c_{m n}=c_{m} c_{n}$, then one could write $|\Psi\rangle=\left|\psi^{S}\right\rangle \otimes\left|\phi^{E}\right\rangle$, where $\left|\psi^{S}\right\rangle=\sum_{m} c_{m}\left|\psi_{m}^{S}\right\rangle$ and $\left|\psi^{E}\right\rangle=\sum_{n} c_{n}\left|\phi_{n}^{E}\right\rangle$. However, in general this is not the case, and the state of the system $|\Psi\rangle$ cannot be factored into a direct product of states in the $S$ and $E$ subspaces. States that cannot be factored in this manner are considered to be entangled in the product basis because quantum correlations exist between the $S$ and $E$ subspaces.

The density operator of the full system $C$ is:

$$
\hat{\rho}_{(C)}=\left|\Psi^{C}\right\rangle\left\langle\Psi^{C}\left|=\sum_{m, m^{\prime}} \sum_{n, n^{\prime}} c_{m n} c_{m^{\prime} n^{\prime}}^{*}\right| \psi_{m}^{S} \phi_{n}^{E}\right\rangle\left\langle\psi_{m^{\prime}}^{S} \phi_{n^{\prime}}^{E}\right|
$$

and in general $\hat{\rho}_{(C)}$ is correlated-i.e., $\hat{\rho}_{(C)}$ cannot be factored into a direct product of operators in the $S$ and $E$ subspaces alone - unless all of the $c_{m n}$ are uncorrelated. ${ }^{23}$ An expectation value in the combined system $C$ can be calculated in the usual way as $\left\langle\hat{A}_{(C)}\right\rangle=$ $\left\langle\Psi^{C}\left|\hat{A}_{(C)}\right| \Psi^{C}\right\rangle=\operatorname{Tr}\left[\hat{\rho}_{(C)} \hat{A}_{(C)}\right]$, where $\hat{A}_{(C)}$ is the observable that is measured. However, it

\footnotetext{
${ }^{23}$ Hereafter the parentheses will be used to denote an operator in the combined space $C$, e.g. to distinguish $\hat{A}_{(S)}=\hat{A}_{S} \otimes \hat{1}_{E}$ from $\hat{A}_{S}$.
} 
may be desirable to consider the results of a measurement just on the system $S$, with an observable of the form:

$$
\hat{A}_{(S)}=\hat{A}_{S} \otimes \hat{1}_{E}=\sum_{m, m^{\prime}} \sum_{n, n^{\prime}} A_{m m^{\prime}}^{S} \delta_{n, n^{\prime}}\left|\psi_{m}^{S} \phi_{n}^{E}\right\rangle\left\langle\psi_{m^{\prime}}^{S} \phi_{n^{\prime}}^{E}\right|
$$

where $\hat{A}_{S}=\sum_{m, m^{\prime}} A_{m m^{\prime}}^{S}\left|\psi_{m}^{S}\right\rangle\left\langle\psi_{m^{\prime}}^{S}\right|$ is in the subspace $S$ and $\hat{1}_{E}=\sum_{n}\left|\phi_{n}^{E}\right\rangle\left\langle\phi_{n}^{E}\right|$. A measurement of $\hat{A}_{(S)}$ returns information about the system $S$ while leaving the environment $E$ undisturbed. ${ }^{24}$ The expectation value of the measurement is:

$$
\begin{aligned}
\left\langle\hat{A}_{(S)}\right\rangle & =\operatorname{Tr}\left[\hat{\rho}_{(C)} \hat{A}_{(S)}\right] \\
& =\sum_{m, m^{\prime}} \sum_{n} c_{m n} c_{m^{\prime} n}^{*} A_{m^{\prime} m}^{S}
\end{aligned}
$$

where the relation $\left\langle\psi_{m}^{S} \phi_{n}^{E}\left|\hat{A}_{(S)}\right| \psi_{m^{\prime}}^{S} \phi_{n^{\prime}}^{E}\right\rangle=\left\langle\psi_{m}^{S}\left|\hat{A}_{S}\right| \psi_{m^{\prime}}^{S}\right\rangle\left\langle\phi_{n}^{E} \mid \phi_{n^{\prime}}^{E}\right\rangle=A_{m m^{\prime}}^{S} \delta_{n, n^{\prime}}$ has been used. It is convenient to introduce the reduced density operator of the system $S$ :

$$
\hat{\rho}_{S}=\operatorname{Tr}_{E}\left[\hat{\rho_{(C)}}\right]=\sum_{m, m^{\prime}} \sum_{n} c_{m n} c_{m^{\prime} n}^{*}\left|\psi_{m}^{S}\right\rangle\left\langle\psi_{m^{\prime}}^{S}\right|
$$

where $\operatorname{Tr}_{E}[\cdots]$ indicates a partial trace over the environmental states:

$$
\operatorname{Tr}_{E}\left[\hat{\rho}_{(C)}\right]=\sum_{n}\left\langle\phi_{n}^{E}\left|\hat{\rho}_{(C)}\right| \phi_{n}^{E}\right\rangle
$$

\footnotetext{
${ }^{24}$ See Ref. [72, §A3.2] for a discussion of observations of open systems that are correlated with unobserved environments.
} 
where from Eq. 1.82 it can be seen that

$$
\begin{aligned}
\left\langle\phi_{n}^{E}\left|\hat{\rho}_{(C)}\right| \phi_{n}^{E}\right\rangle & =\sum_{m, m^{\prime}} \sum_{n^{\prime}, n^{\prime \prime}} c_{m n^{\prime}} c_{m^{\prime} n^{\prime \prime}}^{*}\left|\psi_{m}^{S}\right\rangle\left\langle\phi_{n}^{E} \mid \phi_{n}^{E}\right\rangle\left\langle\psi_{m^{\prime}}^{S}\right|\left\langle\phi_{n^{\prime}}^{E} \mid \phi_{n^{\prime}}^{E}\right\rangle \\
& =\sum_{m, m^{\prime}} \sum_{n^{\prime}, n^{\prime \prime}} c_{m n^{\prime}} c_{m^{\prime} n^{\prime \prime}}^{*} \delta_{n, n^{\prime}} \delta_{n^{\prime \prime}, n}\left|\psi_{m}^{S}\right\rangle\left\langle\psi_{m^{\prime}}^{S}\right| \\
& =\sum_{m, m^{\prime}} c_{m n} c_{m^{\prime} n}^{*}\left|\psi_{m}^{S}\right\rangle\left\langle\psi_{m^{\prime}}^{S}\right| .
\end{aligned}
$$

Using these definitions the expectation value $\left\langle\hat{A}_{(S)}\right\rangle$ given in Eq. 1.84 can be calculated completely within the $S$ subspace as:

$$
\begin{aligned}
\left\langle\hat{A}_{S}\right\rangle \equiv\left\langle\hat{A}_{(S)}\right\rangle & =\operatorname{Tr}\left[\hat{\rho}_{S} \hat{A}_{S}\right] \\
& =\sum_{m, m^{\prime}} \rho_{m m^{\prime}}^{S} A_{m^{\prime} m}^{S}
\end{aligned}
$$

where $\rho_{m m^{\prime}}^{S}=\left\langle\psi_{m}^{S}\left|\hat{\rho}_{S}\right| \psi_{m^{\prime}}^{S}\right\rangle=\sum_{n} c_{m n} c_{m^{\prime} n}^{*}, A_{m m^{\prime}}^{S}=\left\langle\psi_{m}^{S}\left|\hat{A}_{S}\right| \psi_{m^{\prime}}^{S}\right\rangle$, and $\operatorname{Tr}[\cdots]=\sum_{m}\left\langle\psi_{m}^{S}|\cdots| \psi_{m}^{S}\right\rangle$ is the trace within the $S$ subspace. A reduced density operator for the environment can also be defined as $\hat{\rho}_{E}=\operatorname{Tr}_{S}\left[\hat{\rho}_{(C)}\right]$, with matrix elements $\rho_{n n^{\prime}}^{E}=$ $\left\langle\phi_{n}^{E}\left|\hat{\rho}_{E}\right| \phi_{n^{\prime}}^{E}\right\rangle=\sum_{m} c_{m n} c_{m n^{\prime}}^{*}{ }^{25}$ Note that in general the density operator in the combined space does not factor into a direct product of operators in the subspaces: i.e., $\hat{\rho}_{(C)} \neq \hat{\rho}_{S}^{\prime} \otimes \hat{\rho}_{E}^{\prime}$, where $\hat{\rho}_{S}^{\prime}=\hat{\rho}_{S} / \sqrt{\operatorname{Tr}\left[\hat{\rho}_{(C)}\right]}$ and $\hat{\rho}_{E}^{\prime}=\hat{\rho}_{E} / \sqrt{\operatorname{Tr}\left[\hat{\rho}_{(C)}\right]}$; the matrix elements are $\rho_{m, n ; m^{\prime}, n^{\prime}}^{C}=$ $c_{m n} c_{m^{\prime} n^{\prime}}^{*}$, whereas $\left(\hat{\rho}_{S}^{\prime} \otimes \hat{\rho}_{E}^{\prime}\right)_{m, n ; m^{\prime}, n^{\prime}}=\left\{\sum_{m^{\prime \prime}} \sum_{n^{\prime \prime}} c_{m n^{\prime \prime}} c_{m^{\prime} n^{\prime \prime}}^{*} c_{m^{\prime \prime} n} c_{m^{\prime \prime} n^{\prime}}^{*}\right\} /\left\{\sum_{m} \sum_{n}\left|c_{m n}\right|^{2}\right\}$. The equality $\hat{\rho}_{(C)}=\hat{\rho}_{S}^{\prime} \otimes \hat{\rho}_{E}^{\prime}$ can be proven in the special case of uncorrelated coefficients $c_{m n}=c_{m} c_{n}$ by using the normalization condition $\left\langle\Psi^{C} \mid \Psi^{C}\right\rangle=\operatorname{Tr}\left[\hat{\rho}_{(C)}\right]=\sum_{m} \sum_{n}\left|c_{m n}\right|^{2}=$ $\sum_{m^{\prime \prime}}\left|c_{m^{\prime \prime}}\right|^{2} \sum_{n^{\prime \prime}}\left|c_{n^{\prime \prime}}\right|^{2}$ to remove the sums over $m^{\prime \prime}$ and $n^{\prime \prime}$

\footnotetext{
${ }^{25}$ Incidentally, the trace in the complete space $C$ is $\operatorname{Tr}_{C}[\cdots]=\operatorname{Tr}_{E}\left\{\operatorname{Tr}_{S}[\cdots]\right\}=\operatorname{Tr}_{S}\left\{\operatorname{Tr}_{E}[\cdots]\right\}$; i.e., the partial traces can be taken in any order.
} 
The system $S$ represents an open quantum system in the sense that it is not isolated from its environment (of course, $E$ is also an open system because it is not isolated from $S$, but here it is assumed that measurements are performed on $S$ but not on $E$ ). The important point to note is that even if the combined system $C$ is described by a pure state (i.e., $\hat{\rho}_{(C)}^{2}=\hat{\rho}_{(C)}$ for $\hat{\rho}_{(C)}=\left|\Psi^{C}\right\rangle\left\langle\Psi^{C}\right|$ ), the reduced density operator of $S$ does not in general represent a pure state: $\hat{\rho}_{S}^{2} \neq \hat{\rho}_{S}$, since $\left\langle\psi_{m}^{S}\left|\hat{\rho}_{S}^{2}\right| \psi_{m^{\prime}}^{S}\right\rangle=\sum_{m^{\prime \prime}} \sum_{n, n^{\prime}} c_{m n} c_{m^{\prime \prime} n^{\prime}}^{*} c_{m^{\prime \prime} n^{\prime}} c_{m^{\prime} n^{\prime}}^{*}$, but $\left\langle\psi_{m}^{S}\left|\hat{\rho}_{S}\right| \psi_{m^{\prime}}^{S}\right\rangle=\sum_{n} c_{m n} c_{m^{\prime} n}^{*}$. An exception would be the case in which $C$ is in some unentangled pure state $\left|\Psi^{C}\right\rangle=\left|\psi^{S}\right\rangle \otimes\left|\phi^{E}\right\rangle$, in which case $\hat{\rho}_{S}=\left|\psi^{S}\right\rangle\left\langle\psi^{S}\right|$ represents a pure state in the $S$ subspace. Mixed state distributions of a subsystem can arise even from a pure state of the complete system, under the conditions that only the subsystem is observed and that there are quantum correlations between this subsystem and its surroundings. It should be noted that some information about the combined system has been lost upon tracing out the dependence of one subsystem or the other; i.e., knowing both $\hat{\rho}_{S}$ and $\hat{\rho}_{E}$ is not sufficient to specify $\hat{\rho}_{(C)}$ completely unless one of $\hat{\rho}_{S}$ or $\hat{\rho}_{E}$ represents a pure state $[72, \S \mathrm{A} 3]$, as the relation $\hat{\rho}_{(C)} \neq \hat{\rho}_{S}^{\prime} \otimes \hat{\rho}_{E}^{\prime}$ indicates.

One final topic of consideration is of the time evolution of open quantum systems. A truly closed quantum system should have a time-independent Hamiltonian operator, since the Hamiltonian is the operator for the energy of the system, which is conserved. However, energy may be exchanged between an open subsystem and its environment, leading to coupling Hamiltonians that are effectively time dependent from the perspective of the system. A classic example is the semiclassical radiation-matter theory, in which the effects of the quantized radiation field are approximated by potentially time-dependent classical 
electromagnetic fields.

Consider a time-independent Hamiltonian of the complete system $C$ of the form:

$$
\hat{H}_{(C)}=\hat{H}_{(S)}+\hat{H}_{(E)}+\hat{V}_{(S, E)}
$$

where $\hat{H}_{(S)}=\hat{H}_{S} \otimes \hat{1}_{E}$ contains only operators within the system subspace $S, \hat{H}_{(E)}=$ $\hat{1}_{S} \otimes \hat{H}_{E}$ contains only operators within $E$, and $\hat{V}_{(S, E)}$ contains operators in the combined space that couple $S$ and $E$ and is not necessarily factorable as $\hat{V}_{(S, E)}=\hat{V}_{S} \otimes \hat{V}_{E}$. Using Eqs. 1.79 and 1.85, the equation of motion for the reduced density operator of the system becomes:

$$
\begin{aligned}
i \hbar \frac{\partial \hat{\rho}_{S}(t)}{\partial t}= & \operatorname{Tr}_{E}\left\{\left[\hat{H}_{(C)}, \hat{\rho}_{(C)}(t)\right]\right\} \\
= & {\left[\hat{H}_{S}, \hat{\rho}_{S}(t)\right]+\operatorname{Tr}_{E}\left\{\left[\hat{H}_{(E)}+\hat{V}_{(S, E)}, \hat{\rho}_{(C)}(t)\right]\right\} } \\
= & {\left[\hat{H}_{S}, \hat{\rho}_{S}(t)\right]+\sum_{m, m^{\prime}} \sum_{n, n^{\prime}}\left\{H_{n n^{\prime}}^{E} \rho_{m n^{\prime} ; m^{\prime} n}^{C}(t)-\rho_{m n ; m n^{\prime}}^{C}(t) H_{n^{\prime} n}^{E}\right\}\left|\psi_{m}^{S}\right\rangle\left\langle\psi_{m^{\prime}}^{S}\right| } \\
& +\sum_{m, m^{\prime}, m^{\prime \prime}} \sum_{n, n^{\prime}}\left\{V_{m n ; m^{\prime \prime} n^{\prime}}^{S, E} \rho_{m^{\prime \prime} n^{\prime} ; m^{\prime} n}^{C}(t)-\rho_{m n ; m^{\prime \prime} n^{\prime}}^{C}(t) V_{m^{\prime \prime} n^{\prime} ; m^{\prime} n}^{S, E}\right\}\left|\psi_{m}^{S}\right\rangle\left\langle\psi_{m^{\prime}}^{S}\right|
\end{aligned}
$$

where $\hat{\rho}_{S}=\operatorname{Tr}_{E}\left[\hat{\rho}_{(C)}\right], H_{n n^{\prime}}^{E}=\left\langle\phi_{n}^{E}\left|\hat{H}_{E}\right| \phi_{n^{\prime}}^{E}\right\rangle, \rho_{m n ; m^{\prime} n^{\prime}}^{C}(t)=\left\langle\psi_{m}^{S} \phi_{n}^{E}\left|\hat{\rho}_{(C)}(t)\right| \psi_{m^{\prime}}^{S} \phi_{n^{\prime}}^{E}\right\rangle$, and $V_{m n ; m^{\prime} n^{\prime}}^{S, E}=\left\langle\psi_{m}^{S} \phi_{n}^{E}\left|\hat{V}_{(S, E)}\right| \psi_{m^{\prime}}^{S} \phi_{n^{\prime}}^{E}\right\rangle$. Note that $i \hbar \frac{\partial \hat{\rho}_{S}(t)}{\partial t} \neq\left[\hat{H}_{S}^{e f f}, \hat{\rho}_{S}(t)\right]$ in general due to the effects of correlations with and couplings to the $E$ subspace, where $\hat{H}_{S}^{e f f}$ is some effective Hamiltonian in the $S$ subspace. However, if $\hat{\rho}_{(C)}\left(t_{0}\right)=\hat{\rho}_{S}^{\prime}\left(t_{0}\right) \otimes \hat{\rho}_{E}^{\prime}\left(t_{0}\right)$ and $\hat{V}_{(E, S)}=\hat{V}_{S}^{\prime} \otimes \hat{V}_{E}^{\prime}$, then $i \hbar \frac{\partial \hat{\rho}_{S}(t)}{\partial t}=\left[\hat{H}_{S}+a \hat{V}_{S}, \hat{\rho}_{S}(t)\right]$ holds, where $a=\operatorname{Tr}\left[\hat{\rho}_{E}(t) \hat{V}_{E}\right] /\left(\operatorname{Tr}\left[\hat{\rho}_{(C)}\right] \operatorname{Tr}\left[\hat{V}_{(S, E)}\right]\right) .{ }^{26}$

\footnotetext{
${ }^{26}$ As usual, $\hat{\rho}_{S}^{\prime}=\hat{\rho}_{S} / \sqrt{\operatorname{Tr}\left[\hat{\rho}_{(C)}\right]}, \hat{\rho}_{E}^{\prime}=\hat{\rho}_{E} / \sqrt{\operatorname{Tr}\left[\hat{\rho}_{(C)}\right]}, \hat{V}_{S}^{\prime}=\hat{V}_{S} / \sqrt{\operatorname{Tr}\left[\hat{V}_{(S, E)}\right]}$, and $\hat{V}_{E}^{\prime}=\hat{V}_{E} / \sqrt{\operatorname{Tr}\left[\hat{V}_{(S, E)}\right]}$,
} 
The $\operatorname{Tr}_{E}\left\{\left[\hat{H}_{(E)}+\hat{V}_{(S, E)}, \hat{\rho}_{(C)}(t)\right]\right\}$ term contains environmental variables, and it is difficult to isolate a $\hat{\rho}_{S}(t)$ term to form a proper differential equation. This term can lead to irreversible, non-unitary dynamics within the subsystem $S$; this apparent "non-quantum mechanical" relaxation behavior arises due to the over-simplifying neglect of the full details of the interaction of the system with its environment. Eq. 1.89 can be used as the starting point of a quantum-mechanical theory of relaxation. The usual procedure includes: transforming into the interaction frame of $\hat{H}_{(S)}+\hat{H}_{(E)}$ if these Hamiltonians are much larger than the coupling term $\hat{V}_{(S, E)}$, making a perturbative approximation to the integrated reduced Liouville equation of motion in order to write Eq. 1.89 in terms of $\hat{\rho}_{(C)}\left(t_{0}\right)$ instead of $\hat{\rho}_{(C)}(t)$, and applying some thermodynamic arguments if $E$ represents a constant-temperature reservoir [6, §VIII.II.D], [69, §8.1]. A projection operator approach is described in Ref. [72, §7.3]. It is also very common to begin with a semiclassical approximation to the equation of motion of the reduced density operator, as will be discussed in $\S 1.5$.

\subsubsection{Ensemble-averaged density operators and thermal equilibrium}

Often a system can be divided into a set of identical constituent subsystems. NMR measurements are always on a macroscopic sample, and involve observations of not just one spin, but a collection of $\sim 10^{20}$ spins. A fundamental procedure encountered in statistical mechanics is the reduction of detailed information about the many-particle ensemble to only a few averaged, statistically-distributed variables that describe the macroscopic system. ${ }^{27}$ Consider an open system $S$ that is weakly coupled to an environment $E$ that has where $\hat{\rho}_{S}=\operatorname{Tr}_{E}\left[\hat{\rho}_{(C)}\right], \hat{\rho}_{E}=\operatorname{Tr}_{S}\left[\hat{\rho}_{(C)}\right], \hat{V}_{S}=\operatorname{Tr}_{E}\left[\hat{V}_{(S, E)}\right]$, and $\hat{V}_{E}=\operatorname{Tr}_{S}\left[\hat{V}_{(S, E)}\right]$. Note that $\operatorname{Tr}\left[\hat{\rho}_{(C)}(t)\right]$ must be independent of time in a closed system obeying unitary time evolution.

${ }^{27}$ The term "ensemble" in statistical mechanics can refer not only to many real identical subsystems, but also to a set of fictitious identical systems whose internal configurations vary according to thermodynamic 
many degrees of freedom, i.e, enough to form a quasi-continuum. The system $S$ could for example be a system of nuclear spins for which all the environmental degrees of freedom such as molecular vibrational and rotational degrees of freedom, electron spins, etc., have been traced out. General statistical-mechanical principles $[73,74]$ indicate that the system will eventually establish a thermal equilibrium at some temperature $T$, in which case the ensemble-averaged reduced density operator has the form

$$
\hat{\bar{\rho}}_{e q}^{S}=Q_{S}^{-1} e^{-\beta \hat{H}^{S}}
$$

where the overline indicates the ensemble averaging, $\hat{H}^{S}$ is the effective Hamiltonian operating only on system degrees of freedom, $\beta=\left(k_{B} T\right)^{-1}$, and

$$
Q_{S}=\operatorname{Tr}\left[e^{-\beta \hat{H}^{S}}\right]
$$

Note that $\hat{\bar{\rho}}_{\text {eq }}^{S}$ is diagonal in the eigenbasis of $\hat{H}^{S}$, i.e., $\left[\hat{\bar{\rho}}_{e q}^{S}, \hat{H}^{S}\right]=0$. This makes sense; the fact that the equilibrium density operator commutes with the Hamiltonian means that it does not evolve in time (see $\S 2$ ), which is expected because the ensemble-averaged state of the system at equilibrium should be static. The fact that $\hat{\bar{\rho}}_{e q}^{S}$ is diagonal in the eigenbasis of $\hat{H}^{S}$ means that there are no ensemble-averaged coherences in this basis. Note that on a microscopic level when all of the degrees of freedom are considered there may be internal dynamics, i.e., evolving coherences, etc., but from the standpoint of ensemble averaging these coherences must average away. In the rest of this work, the overline ensemble average law. 
notation will be dropped. NMR is inherently an ensemble technique due to the macroscopic number of spins involves; the ensemble averaging is implicit in all calculations. The "system" label $S$ will also be dropped; unless states otherwise the system is the collection of nuclear spins under study.

Eqs. 1.90 and Eq. 1.91 give the usual result for the thermal equilibrium population of some energy eigenstate $|m\rangle$ with energy $E_{m}$ :

$$
P\left(E_{m}\right)=\left\langle m\left|\hat{\bar{\rho}}_{e q}\right| m\right\rangle=Q^{-1} e^{-\beta E_{m}}
$$

where the canonical partition function is

$$
Q=\sum_{m} e^{-\beta E_{m}}
$$

Thermal polarization of an $I=1 / 2$ nucleus in a high magnetic field

Consider the laboratory-frame thermal equilibrium density operator of a molecule containing a single spin labeled $j$ :

$$
\hat{\rho}_{j}^{e q}=Q_{j}^{-1} e^{-\beta \hat{H}_{j}}
$$

where the canonical partition function is $Q_{j}=\operatorname{Tr}\left[e^{-\beta \hat{H}_{j}}\right]$ and $\beta=\left(k_{B} T\right)^{-1}$. In a highfield NMR experiment the Hamiltonian is dominated by the Zeeman interaction, $\hat{H}_{j} \simeq \hat{H}_{Z}^{j}$, 
where $\hat{H}_{Z}^{j}=-\hbar \omega_{0}^{j} \hat{I}_{z j}, \omega_{0}^{j}=\gamma_{j} B_{0}$, and $\underline{B}_{0}=B_{0} \underline{\widetilde{z}}$. In this case:

$$
\hat{\rho}_{j}^{e q}=\exp \left(+\beta \hbar \omega_{0}^{j} \hat{I}_{z j}\right) / \operatorname{Tr}\left[\exp \left(+\beta \hbar \omega_{0}^{j} \hat{I}_{z j}\right)\right]
$$

The partition function can be evaluated exactly by taking the trace in the eigenbasis $\left|m_{j}\right\rangle \equiv$ $\left|I_{j}, m_{I}^{j}\right\rangle$ of $\hat{I}_{z j}$ :

$$
\begin{aligned}
Q_{j} & =\operatorname{Tr}\left[\exp \left(+\beta \hbar \omega_{0}^{j} \hat{I}_{z j}\right)\right] \\
& =\sum_{m_{j}=-I_{j}}^{+I_{j}}\left\langle m_{j}\left|\exp \left(+\beta \hbar \omega_{0}^{j} \hat{I}_{z j}\right)\right| m_{j}\right\rangle=\sum_{m_{j}=-I_{j}}^{+I_{j}} \exp \left(+\beta \hbar \omega_{0}^{j}\left\langle m_{j}\left|\hat{I}_{z j}\right| m_{j}\right\rangle\right) \\
& =\sum_{m_{j}=-I_{j}}^{+I_{j}} \exp \left(+\beta \hbar \omega_{0}^{j} m_{j}\right),
\end{aligned}
$$

where the expectation values can be taken inside the exponential function only because they are eigenstates of the argument of the exponential. The partition function reduces in the case of an $I_{j}=1 / 2$ nucleus to:

$$
Q_{j}=\exp \left[+\beta \hbar \omega_{0}^{j}\left(-\frac{1}{2}\right)\right]+\exp \left[+\beta \hbar \omega_{0}^{j}\left(+\frac{1}{2}\right)\right]=2 \cosh \left(\beta \hbar \omega_{0}^{j} / 2\right)
$$


Likewise, the operator term can be simplified using the relation $\hat{I}_{z j}=\hat{1}_{j} / 4$ for a spin- $1 / 2$ nucleus:

$$
\begin{aligned}
\exp \left(+\beta \hbar \omega_{0}^{j} \hat{I}_{z j}\right)= & \sum_{n=0}^{\infty} \frac{1}{n !}\left(\beta \hbar \omega_{0}^{j}\right)^{n} \hat{I}_{z j}^{n} \\
= & {\left[\hat{1}_{j}+\frac{1}{2 !}\left(\beta \hbar \omega_{0}^{j}\right)^{2}\left(\hat{1}_{j} / 4\right)+\frac{1}{4 !}\left(\beta \hbar \omega_{0}^{j}\right)^{4}\left(\hat{1}_{j} / 16\right)+\cdots\right] } \\
& \quad+\left[\hat{I}_{z j}+\frac{1}{3 !}\left(\beta \hbar \omega_{0}^{j}\right)^{3}\left(\hat{I}_{z}^{j} / 4\right)+\frac{1}{5 !}\left(\beta \hbar \omega_{0}^{j}\right)^{5}\left(\hat{I}_{z j} / 16\right)+\cdots\right] \\
= & {\left[\hat{1}_{j}+\frac{1}{2 !}\left(\beta \hbar \omega_{0}^{j} / 2\right)^{2}+\frac{1}{4 !}\left(\beta \hbar \omega_{0}^{j} / 2\right)^{4}+\cdots\right] \hat{1}_{j} } \\
& \quad+2\left[\hat{I}_{z j}+\frac{1}{3 !}\left(\beta \hbar \omega_{0}^{j} / 2\right)^{3}+\frac{1}{5 !}\left(\beta \hbar \omega_{0}^{j} / 2\right)^{5}+\cdots\right] \hat{I}_{z j} \\
= & \cosh \left(\beta \hbar \omega_{0}^{j} / 2\right) \hat{1}_{j}+2 \sinh \left(\beta \hbar \omega_{0}^{j} / 2\right) \hat{I}_{z j} .
\end{aligned}
$$

Eq. 1.98 holds only for a spin-1/2 nucleus; higher spin nuclei do not satisfy the relation $\hat{I}_{z j}^{2}=\hat{1}_{j} / 4$, and in general the thermal equilibrium density operator includes polarization terms that are not proportional to longitudinal magnetization $\hat{I}_{z j}$. Eqs. 1.97 and 1.98 can be combined to give the high-field thermal equilibrium density operator for a spin- $1 / 2$ nucleus:

$$
\hat{\rho}_{j}^{e q}=\frac{1}{2} \hat{1}_{j}+\tanh \left(\beta \hbar \omega_{0}^{j} / 2\right) \hat{I}_{z j}
$$

This equation can be used to calculate the expectation value of the magnetic moment of spin $I_{j}$ at thermal equilibrium:

$$
\begin{aligned}
\left\langle\underline{\hat{\mu}}_{j}\right\rangle_{e q} & =\operatorname{Tr}\left[\hat{\rho}_{j}^{e q} \underline{\hat{\mu}}_{j}\right]=\operatorname{Tr}\left\{\left[\frac{1}{2} \hat{1}_{j}+\tanh \left(\beta \hbar \omega_{0}^{j} / 2\right) \hat{I}_{z j}\right]\left(\gamma_{j} \hbar \underline{\hat{I}}_{j}\right)\right\} \\
& =\frac{1}{2} \gamma_{j} \hbar \tanh \left(\beta \hbar \omega_{0}^{j} / 2\right) \underline{\widetilde{z}}
\end{aligned}
$$


where the relation $\operatorname{Tr}\left[\hat{I}_{z}^{2}\right]=\frac{1}{2}$ for an $I_{j}=1 / 2$ nucleus has been used. As expected, the nucleus is polarized parallel to the $\underline{B}_{0}$ field. Note that Eq. 1.100 can be written:

$$
\left\langle\underline{\hat{\mu}}_{j}\right\rangle_{e q}=P_{+}^{j}\left\langle\underline{\hat{\mu}}_{j}^{+}\right\rangle+P_{-}^{j}\left\langle\underline{\hat{\mu}}_{j}^{-}\right\rangle
$$

where $P_{ \pm}^{j}=Q_{j}^{-1} \exp \left[-\beta E_{ \pm}^{j}\right]$ are the fractional Boltzmann populations of the eigenstates $\left|m= \pm \frac{1}{2}\right\rangle$ with eigenenergies $E_{ \pm}^{j}= \pm|m| \hbar \omega_{0}^{j}$; the $\left\langle\underline{\hat{\mu}}_{j}^{ \pm}\right\rangle= \pm|m| \gamma_{j} \hbar \underline{\tilde{z}}$ are the average magnetic moments in the $| \pm| m|\rangle$ spin-up or spin-down states. The statistical interpretation of the density operator method is clear: the average magnetic moment $\left\langle\underline{\hat{\mu}}_{j}\right\rangle_{e q}$ is just the population/probability-weighted sum of the magnetic moments in the two states.

The equilibrium bulk magnetization density of $N$ identical $I=1 / 2$ spins is:

$$
\underline{M}_{e q}=\frac{1}{V} \sum_{j=1}^{N}\left\langle\underline{\hat{\mu}}_{j}\right\rangle_{e q}=\frac{N \gamma \hbar}{2 V} \tanh \left(\beta \hbar \omega_{0} / 2\right) \underline{\widetilde{z}},
$$

where the net thermal spin polarization of $I=1 / 2$ nuclei is:

$$
\zeta=\tanh \left(\beta \hbar \omega_{0} / 2\right)
$$

The thermal polarization goes as $\zeta \rightarrow 1$ at very low temperatures when $T \rightarrow 0$ and $\beta \rightarrow+\infty$; the polarization goes as $\zeta \rightarrow 0$ at very high temperatures when $T \rightarrow+\infty$ and $\beta \rightarrow 0$. The net polarization goes like the $\gamma$-weighted population difference $\gamma\left(P_{+}-P_{-}\right)$because the magnetic moments of the up and down states oppose each other. At infinite temperature the populations equalize, the magnetic moments randomize, and the net polarization vanishes; at zero temperature only the low-energy state is populated, all the magnetic moments are 
aligned, and the net polarization is $100 \%$. Protons at room temperature in a $700 \mathrm{MHz}$ magnet $\left(\gamma_{H 1} / 2 \pi=42.577 \mathrm{MHz} / \mathrm{T}, B_{0}=16.45 \mathrm{~T}\right)$ have a thermal polarization of $\zeta=5.6 \times 10^{-5}$. Even spins with high gyromagnetic ratios at high magnetic fields have very small thermal polarizations at room temperature; it is advantageous to work at cryogenic temperatures if the sample supports it (e.g., liquids will freeze) or, if possible, to use alternative polarization methods. The vast majority of NMR experiments are performed using thermal polarization, which is part of the reason why high-field magnets are employed. Note that in the case that some possibly non-equilibrium polarization $\zeta$ is created, the bulk magnetization density of a sample of $N$ identical spin-1/2 nuclei is just the generalization of Eq. 1.102:

$$
\underline{M}_{e q}=\frac{N \zeta \gamma \hbar}{2 V} \underline{\widetilde{z}}
$$

where $\zeta$ is on the interval $[-1,1]$.

\section{The high-temperature approximation}

It is possible to make power series expansions of the exponential functions in the single-molecule density operator Eq. 1.94:

$$
\hat{\rho}_{j}^{e q}=\left\{\operatorname{Tr}\left[\sum_{n=0}^{\infty} \frac{1}{n !}\left(-\beta \hat{H}_{j}\right)^{n}\right]\right\}^{-1}\left[\sum_{n=0}^{\infty} \frac{1}{n !}\left(-\beta \hat{H}_{j}\right)^{n}\right] .
$$


When the argument of the exponential is small such that $\beta\left\|\hat{H}_{j}\right\| \ll 1$ (i.e., $\left\|\hat{H}_{j}\right\| \ll k_{B} T$ ), it is safe to make a low-order truncation of Eq. 1.105:

$$
\begin{aligned}
\hat{\rho}_{j}^{e q} & \simeq\left\{\operatorname{Tr}\left[\hat{1}_{j}\right]\right\}^{-1}\left[\hat{1}_{j}-\beta \hat{H}_{j}\right] \\
& =\left(2 I_{j}+1\right)^{-1}\left[\hat{1}_{j}-\beta \hat{H}_{j}\right]
\end{aligned}
$$

This approximation is called the high temperature approximation; it is valid when the thermal energy is large compared to the energy level spacings in the quantum system. When the Zeeman interaction dominates (as it does in almost all high-field NMR experiments), $\hat{H}_{j} \simeq \hat{H}_{Z j}=-\hbar \omega_{0}^{j} \hat{I}_{z j}$ and:

$$
\hat{\rho}_{j}^{e q} \simeq\left(2 I_{j}+1\right)^{-1}\left[\hat{1}_{j}+\beta \hbar \omega_{0}^{j} \hat{I}_{z j}\right]
$$

The spin-1/2 equilibrium density operator in Eq. 1.99 equals Eq. 1.107 in the high temperature limit where $\tanh \left(\beta \hbar \omega_{0}^{j} / 2\right) \simeq \beta \hbar \omega_{0}^{j} / 2$. The magnitude of the temperature expansion parameter may be estimated as:

$$
\begin{aligned}
\beta\left\|\hat{H}_{j}\right\| & =\beta \sqrt{\operatorname{Tr}\left[\hat{H}_{Z j}^{\dagger} \hat{H}_{Z j}\right]}=\beta \hbar\left|\omega_{0}^{j}\right| \operatorname{Tr}\left[\hat{I}_{z j}^{2}\right] \\
& =\frac{\hbar\left|\omega_{0}^{j}\right|}{3 k_{B} T} I_{j}\left(I_{j}+1\right)\left(2 I_{j}+1\right)
\end{aligned}
$$

which equals $\frac{\hbar\left|\omega_{0}^{j}\right|}{2 k_{B} T}$ when $I_{j}=1 / 2$. The high-temperature approximation is therefore valid for a spin- $1 / 2$ nucleus when $\left|\omega_{0}^{j}\right| / 2 \pi \ll 2 k_{B} T / h$. The thermal energy is $k_{B} T / h=6.2 \times 10^{6}$ $\mathrm{MHz}$ at room temperature $(T=298 \mathrm{~K})$. Typical Larmor frequencies in a high field magnet 
are on the order of $\left|\omega_{0}^{j}\right| / 2 \pi \sim 10^{2} \mathrm{MHz}$, so the high temperature approximation is certainly adequate.

The question arises as to how the high temperature approximation should be applied in a system of many spins (perhaps even a macroscopic number of them). This problem has been considered in detail by Jeener et al. [75] and Warren et al. [76, 77]. The $N$-spin equilibrium density operator is assumed to be of the form:

$$
\hat{\rho}_{e q}^{(N)}=Q^{-1} e^{-\beta \hat{H}}
$$

where $Q=\operatorname{Tr}[\exp (-\beta \hat{H})]$. At this stage, the $N$ spins could be considered to be on the same molecule or on different molecules. Consider a case in which the spins do not interact, i.e., the Hamiltonian is a simple sum of single-spin Hamiltonians:

$$
\hat{H}=\sum_{j=1}^{N} \hat{H}_{j}
$$

This equation could also hold approximately if the couplings between spins are small compared to the single-spin terms. If Eq. 1.110 is satisfied, then the exponential function in Eq. 1.109 factors into a product of single-spin terms, and the equilibrium density operator exhibits no correlations between spins:

$$
\begin{aligned}
\hat{\rho}_{e q}^{(N)} & =\left[\prod_{j=1}^{N} \exp \left(-\beta \hat{H}_{j}\right)\right] /\left\{\operatorname{Tr}\left[\prod_{j=1}^{N} \exp \left(-\beta \hat{H}_{j}\right)\right]\right\} \\
& =\prod_{j=1}^{N} \hat{\rho}_{j}^{e q}
\end{aligned}
$$


where $\hat{\rho}_{j}^{e q}$ is defined by Eq. 1.94, and the trace of a product of single-spin terms equals the product of the single-spin traces. It is understood that $\prod_{j=1}^{N} \hat{\rho}_{j}^{e q}=\hat{\rho}_{1}^{e q} \otimes \hat{\rho}_{2}^{e q} \cdots \otimes \hat{\rho}_{N}^{e q}$. Applying the single-spin high-temperature approximation of Eq. 1.107 to Eq. 1.111 yields:

$$
\begin{aligned}
\hat{\rho}_{e q}^{(N)} & \simeq \prod_{j=1}^{N}\left\{\left[\hat{1}_{j}-\beta \hat{H}_{j}\right] / \operatorname{Tr}\left[\hat{1}_{j}\right]\right\} \\
& =(2 I+1)^{-N}\left\{\hat{1}+(-\beta) \sum_{j}^{N} \hat{H}_{j}+(-\beta)^{2} \sum_{j<k}^{N} \hat{H}_{j} \hat{H}_{k}+(-\beta)^{3} \sum_{j<k<l}^{N} \hat{H}_{j} \hat{H}_{k} \hat{H}_{l}+\ldots\right\},
\end{aligned}
$$

where it has been assumed for the sake of simplicity that all the spins have the same angular momentum $I$, and that the high-temperature condition $\beta\left\|\hat{H}_{j}\right\| \ll 1$ is satisfied for each spin $j$. The $n$ th-order sum in Eq. 1.112 contains only terms that are products of $n$ single-spin operators. If all the spins have Hamiltonians of about the same magnitude $\left\|\hat{H}_{s}\right\|$, the $n$th sum in Eq. 1.112 has a prefactor that goes like $\left[\beta\left\|\hat{H}_{s}\right\|\right]^{n}$. These prefactors are very small since $\beta\left\|\hat{H}_{s}\right\| \ll 1$, and it is tempting to apply a further truncation and keep terms only linear in $\beta$, as was done in the single-spin high temperature approximation. However, there are $\left(\begin{array}{l}N \\ n\end{array}\right)=N ! /[n !(N-n) !]$ terms in the $n$th sum. This number can be astronomical if $N \approx 10^{20}$ represents the number of molecules in a macroscopic sample, and it is not clear that the $n$th sum is actually small. Suppose, however, that $N \approx 10^{2}$ represents the number of spins in a molecule and $\beta\left\|\hat{H}_{s}\right\| \approx 10^{-4}$ for the Zeeman energy of room-temperature protons in a strong magnetic field. The quadratic and higher terms in $\beta$ are negligible in this case, since there are simply not enough terms in the sums to overcome the smallness of $\beta\left\|\hat{H}_{s}\right\|{ }^{28}$ It is generally safe to assume that the series can be truncated after the term

\footnotetext{
${ }^{28}$ For example, if $N=100$, the $n=10$ th sum has $\sim 10^{13}$ terms, but the prefactor is on the order of
} 
linear in $\beta$ when considering an intramolecular spin system, but the higher-order terms may play a role when considering a system containing a macroscopic number of spins. Note that if a high spin polarization $(|\zeta| \rightarrow 1)$ is created either at cryogenic temperatures or by non-thermal means (e.g., by spin-exchange optical pumping of a noble gas or by dynamic nuclear polarization), the high temperature approximation is not valid and the full density operator including multi-spin terms must be retained.

There is another compelling reason to keep only the terms linear in $\beta$ in Eq. 1.112: they contain multi-spin terms that do not correspond to observable magnetization, since the magnetization observable is a sum of single-spin operators. This is easily appreciated by calculating the magnetization of a generic $n$-spin density operator term $\hat{B}_{n}$ :

$$
\begin{aligned}
\underline{M}_{n} & \propto \operatorname{Tr}\left[\underline{\hat{\mu}} \hat{B}_{n}\right]=\operatorname{Tr}\left\{\sum_{j=1}^{n} \gamma_{j} \hbar \underline{\hat{I}}_{j} \prod_{k^{\prime}=1}^{n} \hat{B}_{k^{\prime}}^{n}\right\} \\
& \propto \sum_{j}^{n}\left\{\operatorname{Tr}_{j}\left[\underline{\hat{\mu}}_{j} \hat{B}_{j}\right] \prod_{k^{\prime} \neq j}^{n} \operatorname{Tr}_{k^{\prime}}\left[\hat{B}_{k^{\prime}}\right]\right\},
\end{aligned}
$$

where the factorization of partial traces has been used. ${ }^{29}$ The primed notation indicates that the index $k^{\prime}$ need not be taken sequentially from 1 to $n$, just as long as $n$ terms are taken in the product. The $k^{\prime}$ th partial trace is $\operatorname{Tr}_{k^{\prime}}\left[\hat{B}_{k^{\prime}}\right]=0$ if $\hat{B}_{n}$ is truly an $n$-spin operator, since the trace is non-zero only if $\hat{B}_{k^{\prime}}$ contains the identity operator, in which case $\hat{B}_{n}$ would contain product terms with less than $n$ spins. The only operators that contribute to observable signal are single-spin terms, which have one non-zero component of $\underline{\underline{I}}_{j}$ on $\sim\left(10^{-4}\right)^{10}=10^{-40}$

${ }^{29}$ Note that to be strictly correct, $\hat{B}_{n}$ written in the $N$-spin space also contains $N-n$ identity operators in the product; these operators contribute to the total trace only as an overall non-zero multiplicative factor. The operator $\hat{\mu}$ contains $N-n$ identity operators in the sum, but these would only contribute to a trace with the operator $\hat{1}=\prod_{k=1}^{N} \hat{1}_{k}$. 
spin $j$ and identity operator contributions from the rest of the spins. An $n$-spin term of $\hat{\rho}_{e q}^{N}$ remains an $n$-spin term under any quantum-mechanical evolution if the spins are truly non-interacting, so these terms would never be observable in an NMR experiment if spin couplings were absent. ${ }^{30}$ In that case it would be safe to truncate Eq. 1.112 to terms that are at most linear in $\beta$ :

$$
\hat{\rho}_{e q}^{(N)} \simeq Q^{-1}\left\{\hat{1}-\beta \sum_{j=1}^{N} \hat{H}_{j}\right\} .
$$

Eq. 1.114 can be obtained by expanding Eq. 1.109 as $\hat{\rho}_{e q}^{(N)} \simeq Q^{-1}[\hat{1}-\beta \hat{H}]$ if $\hat{H}$ is given by Eq. 1.110. Eq. 1.114 is sometimes called the "strong" high-temperature approximation for a many-spin system, whereas Eq. 1.112 is the "weak" high-temperature approximation. The strong high temperature approximation clearly yields a more simple equation, and is adequate when the system contains less than a macroscopic number of spins. The weak high-temperature approximation may be necessary if the system is macroscopic (i.e., bulk matter) and if there exist intermolecular spin couplings (i.e., dipolar couplings) that can convert the multi-spin terms into observable single-spin terms. Eq. 1.112 was predicated on the assumption that the spins were uncoupled, but it is still valid if the couplings contribute little to the total polarization (e.g., when the dipolar couplings are very small compared to the Zeeman interaction). The intermolecular dipolar couplings are the source of the distant dipolar field in bulk matter (see $\S 6$ ). The dipolar spin dynamics in the solid state are dominated by couplings between nearby spins (recall the dipolar coupling strength falls off as $r^{-3}$ ) which overwhelm the macroscopic contribution of distant spins in the multi-spin terms

\footnotetext{
${ }^{30}$ Note that different spins are not coupled to each other by rf fields.
} 
of Eq. 1.112. The strong high-temperature approximation therefore adequately describes solid-state systems (unless the polarization is very high $|\zeta| \rightarrow 1$ ). The intramolecular dipolar couplings between nearby spins are averaged away in an isotropic fluid due to molecular tumbling; the nearby intermolecular couplings can be neglected because they are so few (much less than a macroscopic amount) and act coherently for only short time before they are altered by diffusion. The number of distant couplings, however, is astronomical; even though each coupling acts coherently for only a short time, their sheer numbers make a nonnegligible contribution to the spin dynamics. A complete description of the system requires keeping the multi-spin terms in the weak high-temperature approximation or simply using the full $N$-spin equilibrium density operator Eq. 1.109. As will be seen in $\S 6$, distant dipolar field effects can be significant in some solution-state NMR experiments.

When the spin system consists of $N$ spins that are dominated by the Zeeman Hamiltonian $\hat{H}_{Z}=-\sum_{j=1}^{N} \hbar \omega_{0}^{j} \hat{I}_{z j}$, Eq. 1.109 becomes:

$$
\hat{\rho}_{e q}^{(N)}=Q^{-1} \exp \left[+\beta \hbar \sum_{j=1}^{N} \omega_{0}^{j} \hat{I}_{z j}\right],
$$

where $Q=\operatorname{Tr}\left\{\exp \left[+\beta \hbar \sum_{j=1}^{N} \omega_{0}^{j} \hat{I}_{z j}\right]\right\}$. The strong high-temperature approximation to the equilibrium density operator is:

$$
\hat{\rho}_{e q}^{(N)}=\{\operatorname{Tr}[\hat{1}]\}^{-1}\left\{\hat{1}+\beta \hbar \sum_{j=1}^{N} \omega_{0}^{j} \hat{I}_{z j}\right\},
$$

where $\hat{1}=\prod_{j=1}^{N} \hat{1}_{j}$. The net thermal equilibrium magnetization density is the average of the total dipole moment $\underline{\hat{\mu}}^{(N)}=\sum_{j=1}^{N} \underline{\hat{\mu}}_{j}$ per unit volume. In the high-temperature limit 
this becomes:

$$
\begin{aligned}
\underline{M}_{e q} & =\frac{1}{V}\left\langle\underline{\hat{\mu}}^{(N)}\right\rangle=\frac{1}{V} \operatorname{Tr}\left\{\underline{\hat{\mu}}^{(N)} \hat{\rho}_{e q}^{(N)}\right\} \\
& \simeq \frac{1}{V} \operatorname{Tr}\left\{\left[\sum_{j=1}^{N} \gamma_{j} \hbar \underline{\hat{I}}_{j}\right]\left[\{\operatorname{Tr}[\hat{1}]\}^{-1}\left(\hat{1}+\beta \hbar \sum_{k=1}^{N} \omega_{0}^{j} \hat{I}_{z k}\right)\right]\right\} \\
& =\frac{1}{V} \sum_{j=1}^{N}\left\{\gamma_{j} \hbar^{2} \omega_{0}^{j} \operatorname{Tr}_{j}\left[\hat{I}_{z j}^{2}\right] /\left(2 I_{j}+1\right)\right\} \underline{\widetilde{z}}
\end{aligned}
$$

where the full trace of $\hat{I}_{z j}^{2}$ has been factored according to $\operatorname{Tr}\left[\hat{I}_{z j}^{2}\right]=\operatorname{Tr}_{j}\left[\hat{I}_{z j}^{2}\right]\left\{\prod_{k \neq j}^{N} \operatorname{Tr}_{k}\left[\hat{1}_{k}\right]\right\}=$ $\operatorname{Tr}_{j}\left[\hat{I}_{z j}^{2}\right] \cdot \operatorname{Tr}[\hat{1}] /\left(2 I_{j}+1\right)$. The strong high temperature approximation is sufficient because the additional multi-spin terms in the weak approximation do not contribute to net magnetization, as has been discussed previously. The trace of $\hat{I}_{z j}^{2}$ can be evaluated in the eigenbasis $\left\{\left|m_{j}\right\rangle\right\}$ of $\hat{I}_{z j}$ as:

$$
\operatorname{Tr}\left[\hat{I}_{z}^{2}\right]=\sum_{m_{j}=-I_{j}}^{+I_{j}}\left\langle m_{j}\left|\hat{I}_{z j}^{2}\right| m_{j}\right\rangle=\sum_{m_{j}=-I_{j}}^{+I_{j}} m_{j}^{2}=\frac{1}{3} I_{j}\left(I_{j}+1\right)\left(2 I_{j}+1\right) .
$$

If all the spins belong to the same species such that $I_{j}=I, \gamma_{j}=\gamma$, and $\omega_{0}^{j}=\gamma B_{0}$ the magnetization density is:

$$
\underline{M}_{e q} \simeq \frac{N \gamma^{2} \hbar^{2} I(I+1)}{3 V k_{B} T} \underline{B}_{0}
$$

Eq. 1.119 is the Curie law for the net equilibrium spin magnetization density in the high temperature limit, and is equal to $N$ times the single-spin magnetization density. Eq. 1.119 can also be derived slightly more simply using the single-spin reduced density operator $[5$, $\S 5.4]$. Note that $\underline{M}_{e q}$ is parallel to the Zeeman field, quadratic in $\gamma$, proportional to $B_{0}$, and 
inversely proportional to temperature. This is obviously only an approximation, because $M_{e q} \rightarrow \infty$ as $B_{0} \infty$ or $T \rightarrow 0$. The thermal equilibrium magnetization density can be solved exactly for a collection of $N$ spin-1/2 nuclei using Eqs. 1.99 and 1.111:

$$
\hat{\rho}_{e q}^{(N)}=2^{-N} \prod_{j=1}^{N}\left(\hat{1}_{j}+2 \zeta_{j} \hat{I}_{z j}\right),
$$

where $\zeta_{j}=\tanh \left(\beta \hbar \omega_{0}^{j} / 2\right)$ and $\omega_{0}^{j}=\gamma_{j} B_{0}$. If all the spins are of the same species,

$$
\underline{M}_{e q}=\frac{1}{V} \operatorname{Tr}\left\{\underline{\hat{\mu}}^{(N)} \hat{\rho}_{e q}^{(N)}\right\}=\frac{N \gamma \hbar}{2 V} \tanh \left(\beta \hbar \omega_{0} / 2\right) \underline{\tilde{z}}
$$

which reproduces Eq. 1.102. Eq. 1.121 reduces to Eq. 1.119 for $I=1 / 2$ in the high temperature limit where $\tanh \left(\beta \hbar \omega_{0} / 2\right) \approx \beta \hbar \omega_{0} / 2$.

\subsection{Relaxation of nuclear magnetization}

\subsubsection{Introduction to relaxation}

It was established in the last section $(\S 5.2 .1)$ that an ensemble of nuclear spins $I$ in an external magnetic field thermally equilibrates with a nuclear magnetization aligned parallel to the external field axis (i.e., the $z$-axis). If the external Zeeman Hamiltonian dominates all of the internal spin Hamiltonians, then within the high-temperature approximation the only non-zero terms in the thermal equilibrium single-spin difference density operator will be proportional to $\hat{I}_{z}$. In other words, an ensemble of spin-1/2 nuclei in an arbitrary state of polarization left to thermally equilibrate will reach a state in which the transverse magnetization components (proportional to $\hat{I}_{x}$ and $\hat{I}_{y}$ in the density operator) 
are zero and the longitudinal magnetization component has some value corresponding to the equilibrium Curie polarization (Eq. 1.119) In $I>1 / 2$ systems, all the tensor components of the difference density operator will equilibrate to zero in the high-temperature approximation except for the $\hat{T}_{1,0} \propto \hat{I}_{z}$ component. The process of thermal equilibrationspecifically, the disappearance of transverse magnetization components and reestablishment of equilibrium longitudinal magnetization - is called relaxation of the spin system.

In order to understand how relaxation phenomena manifest themselves in NMR experiments, one must understand the nature of the relaxation processes. A completely isolated quantum system (in which energy is conserved) is characterized by a time-independent Hamiltonian $\hat{H}$ in an operator space that includes all the quantum degrees of freedom of the system. The time evolution of the system from times $t_{0}$ to $t$ is described simply by the time development operator $\hat{U}\left(t ; t_{0}\right)=\exp \left[-i \hat{H}\left(t-t_{0}\right) / \hbar\right]$. The time evolution is unitary and the dynamics are coherent. However, consider the re-equilibration that takes places when an initially unpolarized $(\hat{\rho}=0)$ spin system is suddenly placed in a magnetic field directed along the $z$-axis. The system equilibrates from $\hat{\rho}\left(t_{0}\right)=0$ to $\hat{\rho}(t) \propto \hat{I}_{z}$ as the spins polarize. The evolution of the spin system in this case is obviously not unitary, since unitary operations preserve the norm of the density operator, and the creation of longitudinal magnetization from zero magnetization does not preserve $\left\langle\left\langle\rho(t) \mid \rho\left(t_{0}\right)\right\rangle\right\rangle$. In fact, the energy of the spin system is not even conserved as the spins polarize, since an ensemble of spins aligned parallel to a magnetic field has a lower energy than a depolarized (randomly polarized) ensemble. The energy non-conservation and non-unitary time evolution suggest that something is missing from the quantum description. What have been left out are the other 
degrees of freedom that affect the time evolution besides the spin degrees of freedom. The spin system is not isolated, and it interacts with its environment, referred to as the lattice (a term that originated from solid-state studies). For example, the nuclear spins may couple to electron spins, to the vibrational, rotational, or translational degrees of freedom of the molecules in which they are contained, or even to other nuclear spins (recall that many of the degrees of freedom of the spin ensemble are lost upon going from a many-spin to a single-spin density operator). These other degrees of freedom have been averaged over in the single-spin density operator treatment, but their effects on the spin system appear in the form of random perturbations that cause incoherent, non-unitary dynamics that drive the system to equilibrium. In fact, the idea of a small system with a manageable number of degrees of freedom in thermal contact with a bath or reservoir containing an enormous number of degrees of freedom is central to the theories of equilibrium statistical mechanics and thermodynamics. ${ }^{31}$

It is possible to develop rate equations that describe the transfer of populations between the Zeeman eigenstates during relaxation processes. These equations can be used to describe longitudinal relaxation, since it is the longitudinal components of the density operator (i.e., those that commute with the Zeeman Hamiltonian) that are needed to describe the populations of the Zeeman eigenstates. These rate equations, when applied to a two-spin system are called the Solomon equations, although they can be generalized to include $N$ spins. However, in order to predict the functional forms or numerical values of the rate constants that arise in these equations, a microscopic theory of relaxation is required.

\footnotetext{
${ }^{31}$ In fact, the ideas of thermal equilibrium, temperature, and the Boltzmann law can be derived almost from this concept alone; see the notes by Feynman $[74, \S 1.1]$.
} 
Furthermore, a set of equations that describe only movements of populations (longitudinal relaxation) incompletely determine the relaxation properties of the system, since the decay of the coherences (transverse relaxation) has been neglected. The full set of relaxation equations that include all components of the density operator is called the master equation, which is often written in matrix form and also requires a microscopic relaxation theory in order to determine the matrix elements.

There are several theoretical approaches that are commonly employed to describe relaxation behavior. The following discussion will be restricted to a consideration of relaxation processes in molecules in liquid phases. The text by Abragam has an authoritative treatment of some semiclassical and quantum relaxation theories $[6, \S \mathrm{VIII}]$, and there is another seminal work by Redfield [78]. Slichter's book also contains a similar approach [5, $\S 5.11,5.12]$. The text by Cavanagh et al. has an accessible pedagogical survey of standard liquid-state relaxation theory $[4, \S 5]$, and the text by Goldman contains a concise, physical treatment of the same $[2, \S 9]$. Goldman has also presented a modern view of spin-lattice relaxation [79]. Murali and Krishnan have published a tutorial on liquid-state relaxation [80]. Luginbühl and Wüthrich wrote a review of semiclassical relaxation theory as applied to biological macromolecules [81], and Dayie et al. have published a review of the same topic [82].

\subsubsection{Semiclassical relaxation theory}

Consider the semiclassical model of nuclear spin relaxation, in which the spin system is treated quantum-mechanically and the lattice is treated classically by tracing out the environmental degrees of freedom. The classical lattice variables may become time depen- 
dent due to molecular reorientation and diffusion, yielding a laboratory-frame Hamiltonian of the form:

$$
\hat{H}(t)=\hat{H}_{0}+\hat{H}_{1}(t)
$$

where $\hat{H}_{0}$ is the deterministic Hamiltonian that leads to coherent dynamics and $\hat{H}_{1}(t)$ is the stochastic Hamiltonian that leads to incoherent relaxation. The Hamiltonian $\hat{H}_{0}$ is timeindependent and contains the Zeeman Hamiltonian, which at high magnetic fields dominates all other spin interactions; $\hat{H}_{0}$ can also include smaller time-independent Hamiltonians such as the isotropic chemical shielding and $J$-coupling interactions. The interactions included in $\hat{H}_{1}$ are assumed to be anisotropic terms rendered time dependent by random molecular motions and are typically much smaller than the Zeeman interaction; their random fluctuating nature causes $\hat{H}_{1}(t)$ to have a time average of zero. ${ }^{32}$

The most common approach to the semiclassical relaxation theory begins by treating $\hat{H}_{1}(t)$ as a time-dependent perturbation to the primary Hamiltonian $\hat{H}_{0}$, and then develops equations of motion for the density operator (the master equation) valid to second order in the perturbation theory. This is the Bloch, Wangsness, and Redfield relaxation theory $[78,83]$. The following discussion most closely parallels that of Cavanagh et al. [4] or Ernst et al. [7, §2.3.1]. Upon transforming into the interaction frame of the dominant Hamiltonian $\hat{H}_{0}$ (see $§ 1.3 .3$ ) and applying time-dependent perturbation theory, the following equation of motion for the spin density operator is reached, which is good to second

\footnotetext{
${ }^{32}$ If the molecules of interest are aligned in an oriented medium, then the residual anisotropic interactions that remain after motional averaging may also be included in $\hat{H}_{0}$, such that $\hat{H}_{1}(t)$ still time-averages to zero.
} 
order in the perturbation theory:

$$
\frac{d \hat{\widetilde{\rho}}(t)}{d t} \simeq-\frac{1}{\hbar^{2}} \int_{0}^{\infty} d \tau \overline{\left[\hat{\tilde{H}}_{1}(t),\left[\hat{\tilde{H}}_{1}(t-\tau), \hat{\widetilde{\rho}}(t)-\hat{\widetilde{\rho}}_{e q}\right]\right.}
$$

where the tilde represents an operator transformed into the interaction frame, and the overbar designates an ensemble average (it is understood that the reduced density operator is already an ensemble-averaged quantity, so the presence of the overbar is implicit). Some assumptions have been made in the derivation of this equation $[6,2]$, including: $t \gg \tau_{c}$, $\tau_{c} \ll\left[\overline{\mid \hat{H}_{1} / \hbar \|^{2}}\right]^{-1 / 2}, \overline{\hat{\widetilde{H}}_{1}(t) \hat{\tilde{\rho}}(0)}=\overline{\hat{\widetilde{H}}_{1}(t)} \overline{\hat{\widetilde{\rho}}(0)}$, and $\overline{\hat{H}_{1}(t)}=0 .{ }^{33,34}$ Here, $\tau_{c}$ is a correlation time characteristic of the stochastic Hamiltonian $\hat{H}_{1}(t)$. The equilibrium density operator $\hat{\rho}_{e q}=e^{-\beta \hat{H}_{0}} / \operatorname{Tr}\left[e^{-\beta \hat{H}_{0}}\right]$ equals $\hat{\widetilde{\rho}}_{e q}$, since $\left[\hat{\rho}_{e q}, \hat{H}_{0}\right]=0$. The equilibrium density operator does not enter naturally into the semiclassical theory (which predicts $\hat{\rho}(t \rightarrow \infty)=0=$ $\hat{\rho}_{e q}(\beta=0)$, which is the infinite temperature result), so $\hat{\rho}_{e q}$ has been added to Eq. 1.123 after the fact to ensure proper results at finite temperatures.

The stochastic Hamiltonian $\hat{H}_{1}(t)$ is usually written in terms of spherical tensors as: ${ }^{35}$

$$
\hat{H}_{1}(t)=\sum_{\xi} \hat{H}_{1}^{\xi}(t)=\sum_{\xi} \sum_{k=1}^{2} \sum_{q=-k}^{k} A_{k, q}^{\xi}(t) \hat{T}_{k, q}^{\xi},
$$

where the $A_{k, q}$ are spatial tensors, the $\hat{T}_{k, q}$ are spin tensor operators, and $\xi$ labels the

\footnotetext{
${ }^{33}$ It is also required that $\tau_{c}$ is much shorter than any of the reciprocal relaxation rates of the system.

${ }^{34}$ The condition $\overline{\hat{H}_{1}(t)}=0$ follows from the fact that $\hat{H}_{1}(t)$ has a zero time average, if the system is stationary and ergodic and thus obeys the ergodic hypothesis.

${ }^{35}$ One finds no $k=0$ components here because the $A_{0,0}$ spatial tensors are isotropic and are timeindependent under modulation by random molecular tumbling, and hence have been included in $\hat{H}_{0}$. Also, for convenience the $A_{k, q}$ have been redefined such that the sign of $q$ has been switched (using the fact that $\left.A_{k, q}^{*}=A_{k,-q}\right)$, and the factor of $(-1)^{q}$ has been absorbed.
} 
different types of anisotropic interactions (e.g., chemical shielding anisotropy, dipolar or quadrupolar coupling, etc.) ${ }^{36}$ The spherical tensor formalism is employed for the convenience of expressing the Hamiltonian and for the ease of performing calculations using the transformation properties of the spatial tensors. However, since the perturbation calculation is performed in the interaction frame of $\hat{H}_{0}$, in this case it is more convenient to expand the spin tensor operators in a set of orthogonal basis operators $\hat{K}_{k, q ; p}\left(\right.$ with $\left.\hat{K}_{k, q ; p}^{\dagger}=\hat{K}_{k,-q ; p}\right)$ that satisfy certain commutation relations with $\hat{H}_{0}$ :

$$
\hat{T}_{k, q}=\sum_{p} \hat{K}_{k, q ; p}=\sum_{p} c_{q ; p} \hat{h}_{p}
$$

where $\left[\hat{H}_{0} / \hbar, \hat{h}_{p}\right]=\omega_{p} \hat{h}_{p}$, such that $\left.\left|h_{p}\right\rangle\right\rangle$ and $\omega_{p}$ are eigenfunctions and the corresponding eigenfrequencies of the commutation superoperator of $\hat{H}_{0}$, and $c_{q ; p}^{*}=c_{-q ; p} \cdot{ }^{37}$ This commutation relation also implies:

$$
e^{+i \hat{H}_{0} t / \hbar} \hat{h}_{p} e^{-i \hat{H}_{0} t / \hbar}=e^{+i \omega_{p} t} \hat{h}_{p}
$$

Upon substituting Eq. 1.124 with Eq. 1.125 into Eq. 1.123 and using Eq. 1.126 to

\footnotetext{
${ }^{36}$ The label $\xi$ can also denote the same interaction on two different atoms, e.g. the chemical shielding anisotropies of two neighboring carbon atoms would be labeled with different $\xi$.

${ }^{37} \mathrm{An}$ example of this type of decomposition is found in the treatment of a heteronuclear two-spin system with $\hat{H}_{0} / \hbar=\omega_{0}^{I} \hat{I}_{z}+\omega_{0}^{S} \hat{S}_{z}$, where $\hat{T}_{2,0}=\frac{1}{\sqrt{6}}\left(3 \hat{I}_{z} \hat{S}_{z}-\underline{\hat{I}} \cdot \underline{\hat{S}}\right)$ is split into $\hat{K}_{2,0 ; 0}=\hat{K}_{2,0 ; 0}^{\dagger}=\frac{1}{\sqrt{6}} 2 \hat{I}_{z} \hat{S}_{z}$, $\hat{K}_{2,+0 ; 1}=-\frac{1}{\sqrt{6}} \hat{I}_{+} \hat{S}_{-}$, and $\hat{K}_{2,-0 ; 1}=\hat{K}_{2,+0 ; 1}^{\dagger}=-\frac{1}{\sqrt{6}} \hat{I}_{-} \hat{S}_{+}$, with $\omega_{p=0}=0$ and $\omega_{p=1}=\omega_{0}^{I}-\omega_{0}^{S}$. (Note that $\omega_{0}^{I}$ and $\omega_{0}^{S}$ are signed quantities.) In the case of identical homonuclear spins, $\hat{H}_{0}$ has degenerate eigenvalues, and care must be taken in defining the operator basis set within the degenerate subspace.
} 
aid in the interaction frame transformation, the following equation can be reached:

$$
\begin{aligned}
\frac{d \hat{\widetilde{\rho}}(t)}{d t}= & -\frac{1}{\hbar^{2}} \sum_{\xi, \xi^{\prime}} \sum_{k, k^{\prime}} \sum_{q, q^{\prime}} \sum_{p, p^{\prime}} e^{i\left(-\omega_{p^{\prime}}+\omega_{p}\right) t}\left[\hat{K}_{k^{\prime}, q^{\prime} ; p^{\prime}}^{\xi^{\prime}},\left[\hat{K}_{k, q ; p}^{\xi}, \hat{\widetilde{\rho}}(t)-\hat{\rho}_{e q}\right]\right] \\
& \times \int_{0}^{\infty} d \tau e^{-i \omega_{p} \tau} \overline{A_{k^{\prime}, q^{\prime}}^{\xi^{\prime}}(t) A_{k, q}^{\xi}(t-\tau)}
\end{aligned}
$$

It is usually assumed that the fluctuations of the spatial tensors $A_{k^{\prime}, q^{\prime}}(t)$ and $A_{k, q}(t)$ are uncorrelated unless $k=k^{\prime}$ and $q=-q^{\prime},{ }^{38}$ and therefore the argument of the integral in Eq. 1.127 vanishes unless this condition is fulfilled. This allows the sums over $\left(k, k^{\prime}\right)$ and $\left(q, q^{\prime}\right)$ to be reduced to sums over one variable each. Next, the secular approximation is applied, in which any terms $e^{i\left(-\omega_{p^{\prime}}+\omega_{p}\right) t}$ that oscillate rapidly compared to the relaxation timescale are considered to be ineffective in driving the time evolution of $\hat{\widetilde{\rho}}(t)$. This is physically justified because the reciprocal frequencies $\omega_{p}^{-1}$ are typically of the order of reciprocal Larmor frequencies (nanoseconds to microseconds at high field), whereas relaxation times are usually of the order of milliseconds to seconds or longer. Therefore, only the secular terms survive, i.e., the terms in Eq. 1.127 that acquire no time dependence from the interaction frame transformation. These terms have $-\omega_{p^{\prime}}+\omega_{p}=0$ (or at least $-\omega_{p^{\prime}}+\omega_{p}$ is much less than the reciprocal relaxation times), implying $p=p^{\prime}$ and removing another sum:

$$
\frac{d \hat{\widetilde{\rho}}(t)}{d t} \simeq-\frac{1}{\hbar} \sum_{\xi, \xi^{\prime}} \sum_{k, q, p}\left[\hat{K}_{k,-q ; p}^{\xi^{\prime}},\left[\hat{K}_{k, q ; p}^{\xi}, \hat{\widetilde{\rho}}(t)-\hat{\rho}_{e q}\right]\right] j_{q}^{\xi, \xi^{\prime}}\left(\omega_{p}\right)
$$

where $j_{q}^{\xi, \xi^{\prime}}(\omega)=\hbar^{-1} \int_{0}^{\infty} d \tau e^{-i \omega \tau} \overline{A_{k,-q}^{\xi^{\prime}}(t) A_{k, q}^{\xi}(t-\tau)}$ is the power spectral density function.

\footnotetext{
${ }^{38}$ Note that $A_{k, q}^{\xi^{\prime}}(t)$ and $A_{k,-q}^{\xi}(t)$ are related through $A_{k, q}^{\xi}(t)=A_{k,-q}^{\xi *}(t)$ if $\xi=\xi^{\prime}$, and that the product $A_{k, q}^{\xi^{\prime}}(t) A_{k,-q}^{\xi}(t)$ contains an isotropic component.
} 
Upon transforming back into the laboratory frame, the following relaxation master equation is obtained:

$$
\frac{d \hat{\rho}(t)}{d t}=-\frac{i}{\hbar}\left[\hat{H}_{0}, \hat{\rho}(t)\right]-\frac{1}{\hbar} \sum_{\xi, \xi^{\prime}} \sum_{k, q, p} j_{q}^{\xi, \xi^{\prime}}\left(\omega_{p}\right)\left[\hat{K}_{k,-q ; p}^{\xi^{\prime}},\left[\hat{K}_{k, q ; p}^{\xi}, \hat{\rho}(t)-\hat{\rho}_{e q}\right]\right]
$$

This equation is conveniently written in Liouville space as:

$$
\left.\left.\left.\frac{d|\rho(t)\rangle\rangle}{d t}=-\frac{i}{\hbar} \hat{\hat{H}}_{0}|\rho(t)\rangle\right\rangle-\frac{1}{\hbar} \hat{\hat{\Gamma}}(|\rho(t)\rangle\rangle-\left|\rho_{e q}\right\rangle\right\rangle\right)
$$

where $\hat{\hat{H}}_{0} \leftarrow\left[\hat{H}_{0},\right]$ is the commutation superoperator of $\hat{H}_{0}$, the relaxation superoperator is $\hat{\hat{\Gamma}} \leftarrow \sum_{\xi, \xi^{\prime}} \sum_{k, q, p} j_{q}^{\xi, \xi^{\prime}}\left(\omega_{p}\right)\left[\hat{K}_{k,-q ; p}^{\xi^{\prime}},\left[\hat{K}_{k, q ; p}^{\xi},\right]\right]$, and $\left.\left.\hat{\rho}(t) \rightarrow|\rho(t)\rangle\right\rangle, \hat{\rho}_{e q} \rightarrow\left|\rho_{e q}\right\rangle\right\rangle$. It is customary to separate $j_{q}^{\xi, \xi^{\prime}}(\omega)$ into its real and imaginary parts:

$$
\begin{aligned}
j_{q}^{\xi, \xi^{\prime}}(\omega) & =J_{q}^{\xi, \xi^{\prime}}(\omega)-i L_{q}^{\xi, \xi^{\prime}}(\omega), \\
J_{q}^{\xi, \xi^{\prime}}(\omega) & =\frac{1}{\hbar} \int_{0}^{\infty} d \tau \cos (\omega \tau) \overline{A_{k,-q}^{\xi^{\prime}}(t) A_{k, q}^{\xi}(t-\tau)}, \\
L_{q}^{\xi, \xi^{\prime}}(\omega) & =\frac{1}{\hbar} \int_{0}^{\infty} d \tau \sin (\omega \tau) \overline{A_{k,-q}^{\xi^{\prime}}(t) A_{k, q}^{\xi}(t-\tau)} .
\end{aligned}
$$

The contribution from the imaginary part of the spectral density causes small second-order frequency shifts (called dynamic shifts) and is usually neglected in the relaxation equation by including it with the deterministic $\hat{H}_{0}$ term:

$$
\left.\left.\left.\frac{d|\rho(t)\rangle\rangle}{d t}=-\frac{i}{\hbar} \hat{\hat{H}}_{0}^{\prime}|\rho(t)\rangle\right\rangle-\hat{\hat{R}}(|\rho(t)\rangle\rangle-\left|\rho_{e q}\right\rangle\right\rangle\right)
$$

where $\hat{\hat{H}}_{0}^{\prime} / \hbar=\hat{\hat{H}}_{0} / \hbar-\hat{\hat{L}}, \hat{\hat{\Gamma}} / \hbar=\hat{\hat{R}}-i \hat{\hat{L}}, \hat{\hat{R}} \leftarrow \sum_{\xi, \xi^{\prime}} \sum_{k, q, p} J_{q}^{\xi, \xi^{\prime}}\left(\omega_{p}\right)\left[\hat{K}_{k,-q ; p}^{\xi^{\prime}},\left[\hat{K}_{k, q ; p}^{\xi},\right]\right]$, 
and $\hat{\hat{L}} \leftarrow \sum_{\xi, \xi^{\prime}} \sum_{k, q, p} L_{q}^{\xi, \xi^{\prime}}\left(\omega_{p}\right)\left[\hat{K}_{k,-q ; p}^{\xi^{\prime}},\left[\hat{K}_{k, q ; p}^{\xi},\right]\right]$. That the imaginary part of $\hat{\hat{\Gamma}}$ leads to frequency shifts can be appreciated by examination of the form of the differential equation in Eq. 1.132, which indicates that imaginary terms on the right-hand-side of the equation lead to oscillatory motion of $|\rho(t)\rangle\rangle$, while real terms lead to damped motion.

If the density operator is written in terms of some set of orthonormal basis operators $\left\{\hat{B}_{s}\right\}$ such that $\hat{\rho}(t)=\sum_{s} b_{s}(t) \hat{B}_{s}$ with $b_{s}(t)=\left\langle\left\langle B_{s} \mid \rho(t)\right\rangle\right\rangle=\operatorname{Tr}\left\{\hat{B}_{s}^{\dagger} \hat{\rho}(t)\right\}$, then Eq. 1.132 written in terms of matrix elements is:

$$
\frac{d b_{r}(t)}{d t}=\sum_{s}\left\{-i \Omega_{r s}^{\prime} b_{s}(t)-R_{r s}\left(b_{s}(t)-b_{s}^{e q}\right)\right\},
$$

where $\Omega_{r s}^{\prime}=\left\langle\left\langle B_{r}\left|\hat{\hat{H}}_{0}^{\prime} / \hbar\right| B_{s}\right\rangle\right\rangle=\operatorname{Tr}\left\{\hat{B}_{r}^{\dagger}\left[\hat{H}_{0}^{\prime} / \hbar, \hat{B}_{s}\right]\right\}$ and $R_{r s}=\left\langle\left\langle B_{r}|\hat{\hat{R}}| B_{s}\right\rangle\right\rangle$. Eq. 1.133 specifies a set of coupled differential equations for the time evolution of the operator components of $\hat{\rho}$, where the existence of the component $\left.\left|B_{s}\right\rangle\right\rangle$ in the density operator is considered to affect the relaxation of the $\left.\left|B_{r}\right\rangle\right\rangle$ component if $R_{r s} \neq 0$. When $r=s$, the relaxation process is called auto-relaxation of the density operator components, whereas when $r \neq s$, the relaxation process is called cross-relaxation between the density operator components. ${ }^{39}$ An often convenient choice of basis operators is the normalized version of the set of $\left\{\hat{K}_{k, q ; p}\right\}$ operators employed in Eq. 1.125. Another possible choice is the set of operators $\hat{B}_{s}=$ $\hat{B}_{n^{\prime}, n}=\left|n^{\prime}\right\rangle\langle n|$ that represent transitions $|n\rangle \rightarrow\left|n^{\prime}\right\rangle$ between the eigenstates $\{|n\rangle\}$ of $\hat{H}_{0}$; the resulting matrix elements $R_{m^{\prime} m, n^{\prime} n}$ (specifying the so-called "Redfield relaxation matrix") give the rates of cross-relaxation between different transitions. Since $\left[\hat{H}_{0} / \hbar,\left|n^{\prime}\right\rangle\langle n|\right]=\left(\omega_{n^{\prime}}-\right.$

\footnotetext{
${ }^{39}$ Since the $\hat{I}_{x / y / z}$ and $\hat{S}_{x / y / z}$ operators can serve as a subset of the basis operators necessary for the two-spin system, relaxation induced in one spin by another is considered to be a cross-relaxation process. However, the term "cross-relaxation" properly refers to all the possible relaxation processes that occur between density operator elements, not just between two spins.
} 
$\left.\omega_{n}\right)\left|n^{\prime}\right\rangle\langle n|$, the single-transition operators $\left|n^{\prime}\right\rangle\langle n|$ can also be grouped by their coherence order $p$ indexed by the Zeeman transition frequency $\omega_{p}=\omega_{n^{\prime}}-\omega_{n} \equiv \omega_{n^{\prime} n}$, if $\hat{H}_{0}$ is dominated by the Zeeman interaction. A consequence of the secular approximation is that the density operator components $\left|m^{\prime}\right\rangle\langle m|$ and $\left|n^{\prime}\right\rangle\langle n|$ do not cross-relax with each other if $p \neq p^{\prime}$, leading to a block-diagonalization of the relaxation matrix $\hat{\hat{R}}$ into subspaces of a given $p .^{40}$ Furthermore, if none of the transitions of the system within the subspace of a given $p$ are degenerate to within about a linewidth (i.e., $\left|\Omega_{s s}^{\prime}\right| \gg R_{r s}, r=m^{\prime} m, s=n^{\prime} n, m \neq m^{\prime}, n \neq$ $n^{\prime}$ ), then the $R_{r \neq s}$ matrix elements can be neglected and none of the $r \neq s$ operators within the $p$-subspace appreciably cross-relax with each other. ${ }^{41}$ However, the matrix elements that represent populations (i.e., the $n=n^{\prime}$ elements in the $\hat{H}_{0}$ eigenbasis) do cross-relax with each other because $\Omega_{s s}=\omega_{n^{\prime} n}=0$ if $n=n^{\prime}$, and thus both the diagonal and offdiagonal matrix elements of $\left(-i \hat{\hat{H}}_{0}^{\prime} / \hbar-\hat{\hat{R}}\right)$ are of the same order of magnitude within the population subspace. Under these conditions the effective relaxation matrix $\hat{\hat{R}}$ has the so-called "Redfield-kite" structure in the single-transition basis.

Under these assumptions, the differential equations in Eq. 1.133 become completely decoupled for single-transition operators that do not commute with $\hat{H}_{0}$; i.e., there is no crossrelaxation in the transverse relaxation of individual spectral transitions, and transverse relaxation is also independent of longitudinal relaxation. The solution to Eq. 1.133 for transverse relaxation then becomes:

$$
b_{r}(t)=b(0) e^{-i \Omega_{r r}^{\prime} t} e^{-R_{r r} t}
$$

\footnotetext{
${ }^{40}$ This is true when the relaxation matrix is written in any operator basis $\left\{\hat{B}_{s}\right\}$ for which $\left[\hat{H}_{0} / \hbar, \hat{B}_{s}\right]=$ $\omega_{p} \hat{B}_{s}$.

${ }^{41}$ Note that in the single-transition basis, $\Omega_{r s}=\Omega_{m^{\prime} m, n^{\prime} n}=0$ if $r \neq s$ (i.e., if $m^{\prime} \neq n^{\prime}$ or $m \neq n$ ).
} 
The differential equations for longitudinal relaxation (involving the redistribution of populations in the $\hat{H}_{0}$ eigenbasis) remain coupled, so their solution is more complicated and cross-relaxation is possible in principle.

\subsubsection{Relaxation selection rules and spectral densities}

It is clear from Eq. 1.133 that a component $\left.\left|B_{s}\right\rangle\right\rangle$ of the density operator does not contribute to the relaxation of $\left.\left|B_{r}\right\rangle\right\rangle$ if $R_{r s}=\left\langle\left\langle B_{r}|\hat{\hat{R}}| B_{s}\right\rangle\right\rangle$ vanishes. This constitutes a "selection rule" for relaxation. The amplitude $\left\langle\left\langle B_{r}|\hat{\hat{R}}| B_{s}\right\rangle\right\rangle=$ obviously vanishes identically if $\left.\hat{\hat{R}}\left|B_{s}\right\rangle\right\rangle=0$ or $\left\langle\left\langle B_{r}\right| \hat{\hat{R}}=0\right.$. It can be seen from these relations and from the definition of $\hat{\hat{R}}$ following Eq. 1.132 that an individual term $\hat{K}_{k, q ; p}^{\xi}$ in the stochastic Hamiltonian $\hat{H}_{1}$ will contribute to the cross-relaxation of the $\hat{B}_{r}$ element of the density operator due to the $\hat{B}_{s}$ element when $\left[\hat{K}_{k, q ; p}^{\xi}, \hat{B}_{s}\right]=0$ or $\left[\hat{K}_{k,-q ; p}^{\xi^{\prime}}, \hat{B}_{r}^{\dagger}\right]=0 .{ }^{42}$ We consider the components of $\hat{H}_{1}$ that commute with $\hat{H}_{0}$, which in general will be the Hermitian $\hat{K}_{k, 0 ; 0}$ terms with $\omega_{p=0}=0$ if $\hat{H}_{0}$ is dominated by the Zeeman interaction. If $\hat{B}_{r}$ is Hermitian and also commutes with $\hat{H}_{0}$, then it can be shown that $\left[\hat{K}_{k, 0 ; 0}, \hat{B}_{r}\right]=0$ if $\hat{H}_{0}$ has a non-degenerate eigenvalue spectrum (e.g., the system does not contain chemically identical spins). ${ }^{43}$ The terms $\hat{B}_{r}$ for which $\left[\hat{B}_{r}, \hat{H}_{0}\right]$ generally represent populations in the eigenbasis of $\hat{H}_{0}$. The commuting $\hat{K}_{k, 0 ; 0}$ terms are sometimes called the secular terms in $\hat{H}_{1}$, because they remain invariant (time independent) upon a transformation into the interaction frame of $\hat{H}_{0}$ and

\footnotetext{
${ }^{42}$ Note that the Hilbert-space representation of $\left\langle\left\langle B_{r}|\hat{\hat{R}}| B_{s}\right\rangle\right\rangle$ can be manipulated using the identity $\operatorname{Tr}\{\hat{A}[\hat{B},[\hat{C}, \hat{D}]]\}=\operatorname{Tr}\{\hat{D}[\hat{C},[\hat{B}, \hat{A}]]\}$.

${ }^{43}$ This follows from the fact that if $[\hat{A}, \hat{B}]=0$ and $[\hat{A}, \hat{C}]=0$, then $[\hat{B}, \hat{C}]=0$ if all the operators are Hermitian and $\hat{A}$ has only non-degenerate eigenvalues. This result can be appreciated from the facts that: all Hermitian matrices can be diagonalized; if $\hat{A}$ has a non-degenerate spectrum, then it is diagonal in one unique eigenbasis; if $\hat{B}$ and $\hat{C}$ both commute with $\hat{A}$ then they can both be diagonalized in the eigenbasis of $\hat{A}$; and two diagonal matrices commute.
} 
thus are retained in the first-order perturbation theory secular approximation. We arrive at an important result: that the secular terms in $\hat{H}_{1}$ do not contribute to the relaxation of populations, i.e., to longitudinal relaxation. The contribution of the secular terms is also called adiabatic relaxation, and so longitudinal relaxation is adiabatic-free. Since the secular terms in $\hat{H}_{1}$ commute with $\hat{H}_{0}$, they have $\omega_{p=0}=0$ and thus adiabatic transverse relaxation processes contribute a zero-frequency $J_{0}^{\xi, \xi^{\prime}}(0)$ spectral density. The physical picture commonly associated with adiabatic relaxation is that the secular terms contribute to a randomly-fluctuating $z$-component of the local magnetic field at the nucleus, causing a random modulation of the Zeeman energy levels and hence of the Larmor frequency. This random modulation contributes to irreversible dephasing (relaxation) of the bulk transverse magnetization as different spins get out of phase with each other while precessing at different Larmor frequencies.

The non-secular terms in $\hat{H}_{1}$ are those that do not commute with $\hat{H}_{0}$ and thus become time-dependent in the interaction frame and can be neglected to first order in the perturbation theory via the secular approximation. (Note that some of these terms can be retained in the second-order secular approximation, as witnessed in Eq. 1.128.) These non-adiabatic terms can contribute to both transverse and longitudinal relaxation. ${ }^{44}$ The physical picture usually ascribed to this type of relaxation is that the terms in $\hat{H}_{1}$ that do not commute with $\hat{H}_{0}$ can perturbatively connect the eigenstates of $\hat{H}_{0}$, and thus induce random transitions between the Zeeman levels. These transitions can change populations (longitudinal relaxation), and can contribute to transverse relaxation by inducing an uncer-

\footnotetext{
${ }^{44}$ Note that the non-adiabatic terms can sometimes contribute a zero or near-zero frequency spectral density, e.g. in the two-spin $I-S$ system the $\hat{K}_{2, \pm 0 ; 1}=-\frac{1}{\sqrt{6}} \hat{I}_{ \pm} \hat{S}_{\mp}$ have $\omega_{p=1}=\omega_{0}^{I}-\omega_{0}^{S}$, which can be near zero if the spins have nearly the same Larmor frequencies. Zero-frequency spectral density contributions alone do not define an adiabatic relaxation mechanism.
} 
tainty in the energy of the $\hat{H}_{0}$ eigenstates with a corresponding natural lifetime broadening $\left[84\right.$, C. $\left.K_{I I I}\right],\left[85\right.$, C. $\left.D_{X I I I}\right] \cdot{ }^{45}$

Aside from consideration of the relaxation "selection rules", it is also apparent from the definition of $\hat{\hat{R}}$ following Eq. 1.132 that the spectral density $J_{q}^{\xi, \xi^{\prime}}(\omega)$ evaluated at the frequency $\omega_{p}$ must be non-negligible for the terms $\hat{K}_{k,-q ; p}^{\xi^{\prime}}$ and $\hat{K}_{k, q ; p}^{\xi}$ in $\hat{H}_{1}$ to induce efficient relaxation. Furthermore, the definition of $J_{q}^{\xi, \xi^{\prime}}(\omega)$ in Eq. 1.135 indicates that the spectral density vanishes if the fluctuations of $A_{k,-q}^{\xi^{\prime}}$ and $A_{k, q}^{\xi}$ are uncorrelated (i.e., $\left.\overline{A_{k,-q}^{\xi^{\prime}}(t) A_{k, q}^{\xi}(t-\tau)}=0\right)$. Spectral densities with $\xi=\xi^{\prime}$ govern auto-correlated relaxation, whereas spectral densities with $\xi \neq \xi^{\prime}$ govern cross-correlated relaxation. For example, fluctuations of the quadrupolar interaction and the chemical shielding anisotropy of a nucleus may be cross-correlated during molecular tumbling if these anisotropic interactions share a definite spatial relationship to each other in the local coordinate system (e.g., in a coordinate system fixed to a bond vector).

The analytic evaluation of the spectral densities may be simplified if certain assumptions about the molecular motion apply. The simplest case is that of autocorrelated relaxation of a rigid spherical molecule undergoing global rotational Brownian motion (i.e., with no local intramolecular motions) in isotropic solution, for which:

$$
J_{q}^{\xi, \xi}(\omega)=(-1)^{q} J^{\xi, \xi}(\omega)=(-1)^{q} \overline{\left|A_{k, q}^{\xi}\right|^{2}} \frac{\tau_{c}}{1+\omega^{2} \tau_{c}^{2}}
$$

where $\tau_{c}$ is the correlation time for global rotational motion. ${ }^{46}$ In a single spin system

\footnotetext{
${ }^{45}$ Note that the secular perturbations that commute with $\hat{H}_{0}$ may modulate the value of the energies of the $\hat{H}_{0}$ eigenstates, but they do not add an uncertainty in the determination of these energies.

${ }^{46}$ In the case of cross-correlated spectral densities with axially symmetric interaction Hamiltonians, this equation must be multiplied by a factor proportional to $P_{2}\left(\cos \theta_{\xi^{\prime}}\right)$, where $P_{2}(x)$ is the second-rank Legendre
} 
with $\hat{H}_{0} / \hbar=\omega_{0} \hat{I}_{z}$ the interaction-frame modulation frequencies are $\omega_{p}=p \omega_{0}$, where $\omega_{0}$ is the Larmor frequency of spin $I$; therefore the spectral densities $J^{\xi, \xi}\left(\omega_{p}\right)$ have the limiting behavior of $J^{\xi, \xi}\left(\omega_{p}\right) \rightarrow J^{\xi, \xi}(0)=\overline{\left|A_{k, q}^{\xi}\right|^{2}} \tau_{c}$ when $\omega_{0} \tau_{c} \ll 1$ and $J^{\xi, \xi}\left(\omega_{p}\right) \rightarrow \overline{\left|A_{k, q}^{\xi}\right|^{2}} \tau_{c} /\left(p^{2} \omega_{0}^{2}\right)$ when $\omega_{0} \tau_{c} \gg 1$. The former regime is called the extreme narrowing regime, where the motional timescale is much faster than the reciprocal Larmor frequency; for the purposes of this work the latter regime is called the slow tumbling regime, where the motional timescale is much slower than the reciprocal Larmor frequency. However, one must note that the second-order perturbative approximation implicit in Eq. 1.123 may begin to break down as $\omega_{0} \tau_{c} \geq 1 .{ }^{47}$ Some of the relevant single-spin autocorrelation spectral densities are plotted in Fig. 1.4 as a function of $\tau_{c}$. Note that $J^{\xi, \xi}\left(\omega_{0}\right)$ and $J^{\xi, \xi}\left(2 \omega_{0}\right)$ have maxima in the vicinity of $\tau_{c} \sim \omega_{0}^{-1}$, whereas $J^{\xi, \xi}(0)$ increases linearly in $\tau_{c}$.

Longitudinal and transverse terms in the density operator are usually found to decay as simple exponential functions in the extreme narrowing limit, with decay time constants of $T_{1}$ and $T_{2}$, respectively. The observed NMR linewidth is of the order of $T_{2}^{-1}$. Outside of the extreme narrowing regime, multiexponential relaxation is possible in quadrupolar spins or in groups of equivalent spins-1/2. It may be seen readily that since transverse relaxation is affected by adiabatic relaxation processes with zero-frequency $J(0)$ spectral densities, the linear dependence of $J(0)$ on $\tau_{c}$ indicates that the $T_{2}^{-1}$ transverse relaxation rate is ever-increasing as the rotational tumbling slows. Longitudinal relaxation is adiabatic-free, and the $T_{1}^{-1}$ relaxation rate has a maximum near $\tau_{c} \sim \omega_{0}^{-1}$. This is where the polynomial and $\theta_{\xi^{\prime} \xi}$ is the fixed angle between the principal axes of interaction $\xi^{\prime}$ and interaction $\xi$ in the rigid molecule. The amplitude factor must also be modified to $\overline{A_{k,-q}^{\xi^{\prime}} A_{k, q}^{\xi}}$.

${ }^{47}$ The Redfield equations may still be valid when the condition $\omega_{0} \tau_{c}<1$ is not violated too strongly, if the motional averaging of the anisotropic interactions is sufficiently rapid compared to $\left\|\hat{H}_{1}\right\|^{-1}$; see, e.g. Refs. $[86,87]$. 


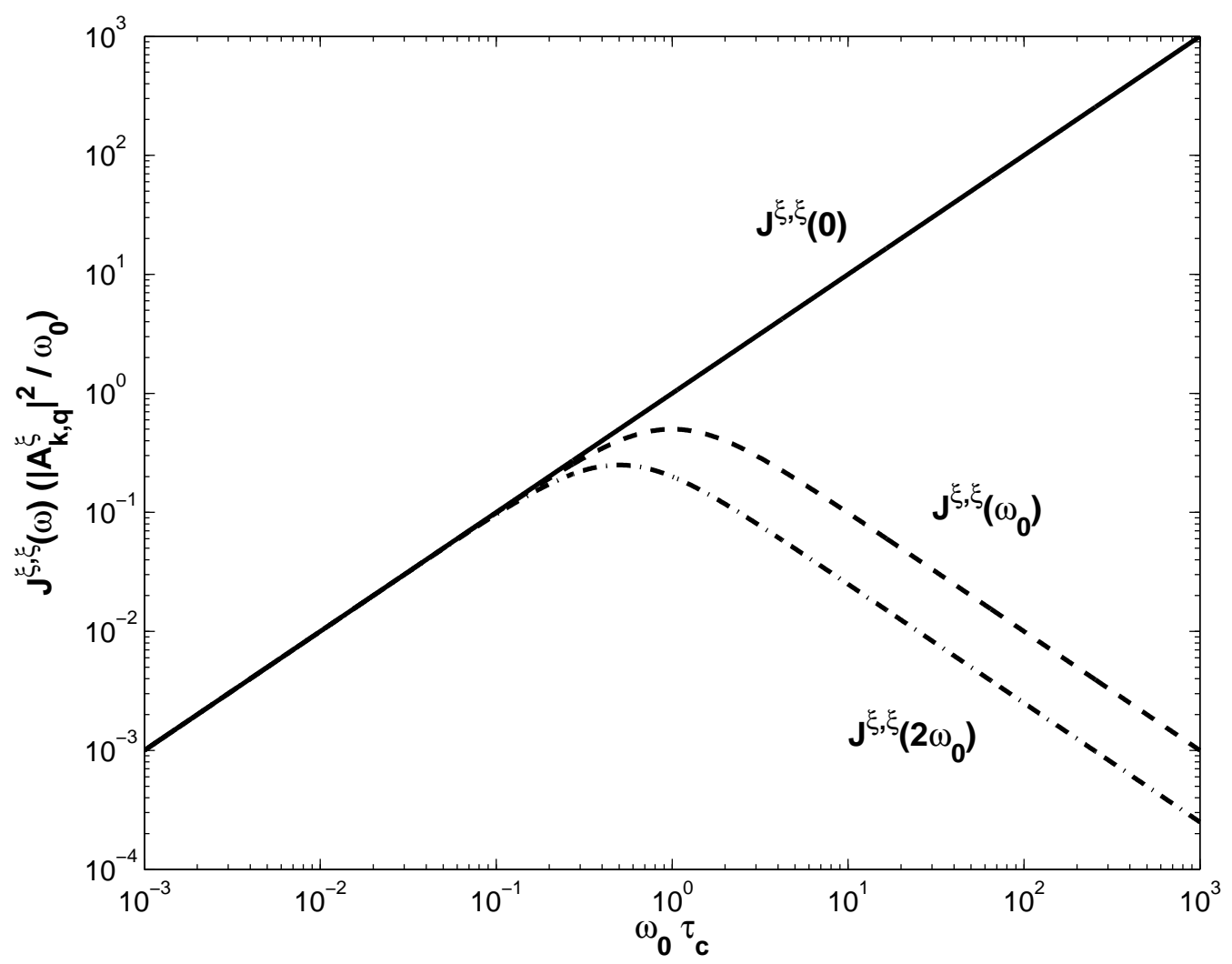

Figure 1.4: Autocorrelation spectral density functions $J^{\xi, \xi}(0), J^{\xi, \xi}\left(\omega_{0}\right)$, and $J^{\xi, \xi}\left(2 \omega_{0}\right)$ for rigid spherical molecules tumbling in isotropic solution. The spectral densities are plotted in units of $\overline{\left|A_{k, q}^{\xi}\right|^{2}} / \omega_{0}$ as a function of the dimensionless parameter $\omega_{0} \tau_{c}$.

non-adiabatic spectral densities have their maxima; the population redistribution between the Zeeman eigenstates is caused by transitions that are efficiently driven by fluctuations in the local transverse magnetic field that are resonant at the Larmor frequency.

\subsubsection{Dynamic frequency shifts and multiexponential relaxation under violation of extreme narrowing conditions}

Qualitative differences in the relaxation dynamics may begin to emerge in the "slow-tumbling" regime where $\left|\omega_{0}\right| \tau_{c} \geq 1$. This can be understood by first realizing that 
there are at least three timescales involved in the relaxation problem: the timescale of the Zeeman interaction ||$\hat{H}_{0} \|^{-1} \sim\left|\omega_{0}\right|^{-1}$, the timescale of the anisotropic interactions $\left.|| \hat{H}_{1}\right|^{-1}$ (i.e., on the order of the reciprocal of the dipolar couplings, quadrupolar coupling, etc.), and the timescale of the stochastic modulation of the anisotropic interactions $\tau_{c}$. The Redfield relaxation theory attempts to include the effects of these different timescales all at once in a second-order perturbative approximation. However, consider the first-order approximation of a system in the case when the three timescales are distinct. The proper procedure by which to approximate the dynamics of a system when multiple timescales are involved is to perform averaging with respect to the fastest timescale first, then the next fastest, etc. In the extreme narrowing regime $\left(\left|\omega_{0}\right| \tau_{c} \ll 1\right)$ the fastest timescale is the motional timescale characterized by $\tau_{c}$, and the next fastest is the Zeeman timescale $\left|\omega_{0}\right|^{-1}$. In this limit it is proper to perform motional averaging first (see $\S 1.3 .9$ ), causing all the anisotropic interactions to disappear to first order in the perturbation theory (only to reappear as relaxation terms in the higher-order treatment). A second averaging (also to first order) via the Zeeman interaction can then be applied to the motionally-averaged Hamiltonian. In the slow-tumbling regime where extreme-narrowing conditions are violated, the fastest timescale is the Zeeman timescale $\left|\omega_{0}\right|^{-1}$, and the next fastest is the motional timescale $\tau_{c}$. Under these conditions the Zeeman truncation of the full Hamiltonian should be applied first, and the motional averaging applied second.

That changing the order of the Zeeman truncation and motional averaging may lead to different dynamical results can be appreciated by a simple example due to Redfield [78]. Consider a single spin subjected to a magnetic field $\underline{B}(t)=\underline{B}_{0}+\underline{B}_{1}(t)$, where $\underline{B}_{0}$ 
is the dominant static magnetic field and $\underline{B}_{1}(t)$ is a small, randomly reorienting magnetic field of constant magnitude $B_{1}$, which could in principle be the local field due to modulated anisotropic interactions. Here $\underline{B}_{1}(t)$ is presumed to be slowly varying with time constant $\tau_{c}$, and it is assumed that $\left|\omega_{0}\right|^{-1} \ll \tau_{c} \ll\left|\omega_{1}\right|^{-1}$, where $\omega_{0}=\gamma B_{0}$ and $\omega_{1}=\gamma B_{1}$. Since $\underline{B}_{1}(t)$ is slowly varying, the variation of $\underline{B}(t)$ over some time $\Delta t \sim\left|\omega_{0}\right|^{-1}$ is small, and during this interval the spin precesses in an average field of magnitude $B^{\prime}=\sqrt{B_{0}^{2}+B_{1}^{2}} \cdot{ }^{48}$ Therefore on average the spin will precess at the Larmor frequency $\left|\omega_{0}^{\prime}\right|=\gamma B^{\prime}=\left|\omega_{0}\right| \sqrt{1+\left(\omega_{1} / \omega_{0}\right)^{2}}$ of the average magnetic field. However, if the variation of $\underline{B}_{1}(t)$ is rapid such that $\tau_{c} \ll$ $\left|\omega_{0}\right|^{-1} \ll\left|\omega_{1}\right|^{-1}$, then during the interval $\Delta t$ the stochastic field $\underline{B}_{1}(t)$ has undergone many fluctuations and averages to zero, so that the spin effectively precesses solely in the field $\underline{B}_{0}$ at frequency $\omega_{0}=\gamma B_{0}$. The difference in precession frequencies between $\left|\omega_{0}\right|$ in the extreme narrowing regime and $\left|\omega_{0}^{\prime}\right|$ in the slow tumbling regime is of the order of $\omega_{1}^{2} /\left|\omega_{0}\right|$ and corresponds to the dynamic frequency shift mentioned in $§ 1.5 .2$.

The existence of the dynamic frequency shift can also be intuited from secondorder quantum-mechanical static perturbation theory if the roles of these varied dynamical timescales are taken into account. For example, the Zeeman-truncated solid-state quadrupolar Hamiltonian (discussed in \$1.3.7) contains a second-order isotropic component, i.e., a component that does not depend on molecular orientation. If the molecular reorientation processes are very slow, say in the limit that the solid has become a glassy fluid, one expects that that motional averaging will not completely average away the quadrupolar isotropic fre-

\footnotetext{
${ }^{48}$ The average value of $\left|\underline{B}^{\prime}(t)\right|=\left|\underline{B}_{0}+\underline{B}_{1}(t)\right|$ is determined from the isotropic orientational average $\left\langle\left|\underline{B}^{\prime}\right|\right\rangle=\left\{\int d \Omega\left|\underline{B}^{\prime}\right|\right\} / \int d \Omega=\frac{1}{4 \pi} \int d \Omega \sqrt{B_{0}^{2}+B_{1}^{2}+2 B_{0} B_{1} \cos \theta} \simeq \sqrt{B_{0}^{2}+B_{1}^{2}}$ if $B_{0} \gg B_{1}$, where $\theta=\theta(t)$ is the instantaneous angle at time $t$ between $\underline{B}_{0}$ and $\underline{B}_{1}(t)$, and $d \Omega=d \phi d \theta \sin \theta$ is the differential surface element on the unit sphere.
} 
quency shift. This is the slow tumbling limit $\tau_{c} \gg\left|\omega_{0}\right|^{-1}$ in which the Zeeman truncation of the quadrupolar Hamiltonian must be performed before the motional averaging. In the opposite (extreme narrowing) limit $\tau_{c} \ll\left|\omega_{0}\right|^{-1}$, the motional averaging is the fastest timescale and must be applied first. In this case, the full quadrupolar Hamiltonian - which contains only tensor spatial components - is completely averaged away by fast isotropic molecular reorientation, and there is no quadrupolar coupling interaction remaining to which to apply the Zeeman truncation, so the quadrupolar isotropic shift disappears. It follows that in the intermediate regime, the solid-state quadrupolar isotropic shift must be gradually quenched by increasingly fast molecular motion as $\tau_{c}$ decreases [87]. The dynamic frequency shift arises in the second-order semiclassical relaxation theory from the imaginary component $\hat{\hat{L}}$ of the relaxation superoperator, with the magnitude of the shift given by the imaginary part of the spectral densities $L_{q}^{\xi^{\prime}, \xi}(\omega)$. In light of the previous discussion, it is not surprising that the second-order dynamic shift predicted from the relaxation theory asymptotically attains the value of the second-order solid-state isotropic shift in the slow-tumbling regime where $\tau_{c} \gg\left|\omega_{0}\right|^{-1}[88,89]$. Dynamic frequency shifts are not limited to autocorrelated interactions. Cross-correlated interactions (e.g., the quadrupolar-chemical shift anisotropy cross correlation) can also lead to dynamic shifts in the slow tumbling regime [90, 91], which asymptotically approach the isotropic shifts of the second-order solid-state interference terms. It should be noted that dynamic frequency shifts are usually smaller than linewidths and are therefore rarely observed, except in the slow tumbling regime for transitions for which there is no line broadening through adiabatic relaxation [92, 93].

Certain spin systems with sufficient degrees of freedom exhibit multiexponen- 
tial relaxation behavior when extreme narrowing conditions are violated. This behavior has been predicted and observed in groups of equivalent spins $[94,95,96,97,98]$ and in quadrupolar nuclei $[99,100,101]$. Again, this behavior can be intuited from physical arguments. For instance, in the slow tumbling regime where it is appropriate to perform the Zeeman truncation before the motional averaging, one can imagine that although the first-order truncated quadrupolar Hamiltonian eventually time-averages to zero (causing no frequency shift), it still dominates the relaxation dynamics. It has been noted (see \$1.3.7) that "symmetric" transitions $|I,+| m_{I}|\rangle \longleftrightarrow|I,-| m_{I}|\rangle$ in a quadrupolar spin $I$ are invariant to the effects of the quadrupolar interaction to first order in the perturbation theory. Consequently the three single-quantum transitions that correspond to observable transverse magnetization in the $I=3 / 2$ system should not relax equivalently, because the symmetric $\left|m_{I}=+\frac{1}{2}\right\rangle \longleftrightarrow\left|m_{I}=-\frac{1}{2}\right\rangle$ transition is unaffected by the first-order quadrupolar Hamiltonian and therefore should relax more slowly than the "asymmetric" $\left|m_{I}=+\frac{3}{2}\right\rangle \longleftrightarrow\left|m_{I}=+\frac{1}{2}\right\rangle$ and $\left|m_{I}=-\frac{1}{2}\right\rangle \longleftrightarrow\left|m_{I}=-\frac{3}{2}\right\rangle$ transitions. Equivalently, one can observe that there is no adiabatic contribution to quadrupolar relaxation of the symmetric transitions in the second-order semiclassical relaxation theory $[102,103]$ (some violations of this rule are discussed in Ref. [87]), and recall from the example of Fig. 1.4 that the adiabatic (zero-frequency) contribution to the relaxation rate dominates in the slow tumbling regime. The difference in relaxation rates between the symmetric and asymmetric singlequantum transitions in the slow-tumbling limit leads to biexponential transverse relaxation behavior in the $I=3 / 2$ system and a corresponding natural lineshape that is the sum of two Lorentzians of different widths. Similar arguments indicate that the two asymmetric 
single-quantum transitions in the $I=1$ system relax equivalently under the quadrupolar interaction, but in the slow tumbling regime the symmetric $\left|m_{I}=+1\right\rangle \longleftrightarrow\left|m_{I}=-1\right\rangle$ double-quantum transition relaxes more slowly than the single-quantum transitions.

A final point to consider is that the existence of dynamic frequency shifts and multiexponential relaxation processes allows for spin dynamics to occur that would otherwise be forbidden in isotropic solution. For instance, the dynamic frequency shift embodied in the superoperator $\hat{\hat{L}}$ corresponds to an effective correction to the coherent Hamiltonian $\hat{\hat{H}}_{0}$ that may be non-negligible in the slow-tumbling regime, although the time evolution under this correction may not be significantly faster than the relaxation of the system [92]. Also, multiexponential relaxation effects have already been successfully exploited to cause interconversions between density operator elements that would otherwise be forbidden in isotropic solution in the extreme narrowing regime $[97,98,100,101,104]{ }^{49}$

\subsection{NMR excitation and spin manipulation}

This section considers the actual process of magnetic resonance during rf irradiation. A simple treatment also can be found in $\S 4.4 .2$ of Ref. [41]. Consider the laboratory-

\footnotetext{
${ }^{49}$ This can be understood from a simple example. Consider an $I=3 / 2$ spin in isotropic solution pre-

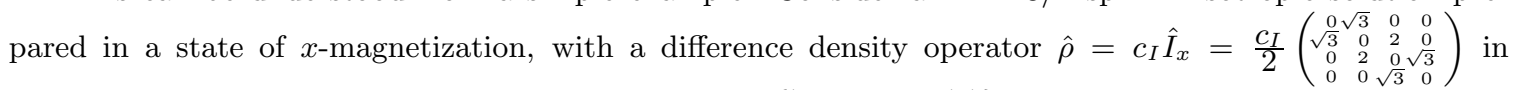
the Zeeman eigenbasis. The relaxation in the inner $\left\{\left|m_{I}= \pm 1 / 2\right\rangle\right\}$ manifold is slow because these coherences commute with the first-order quadrupolar Hamiltonian. If the fast quadrupolar relaxation dephased the other coherences to a fraction $A$ of their initial value and the central transition coherences relaxed to a fraction $B$ of their initial value $(A<B)$, then the difference density operator would be $\hat{\rho}=$ $A \frac{c_{I}}{2}\left(\begin{array}{cccc}0 & \sqrt{3} & 0 & 0 \\ \sqrt{3} & 0 & 0 & 0 \\ 0 & 0 & 0 & 0 \\ 0 & 0 & \sqrt{3} & 0\end{array}\right)+B \frac{c_{I}}{2}\left(\begin{array}{cccc}0 & 0 & 0 & 0 \\ 0 & 0 & 2 & 0 \\ 0 & 2 & 0 & 0 \\ 0 & 0 & 0 & 0\end{array}\right)=c_{I}\left[\left(\frac{3}{5} A+\frac{2}{5} B\right) \hat{I}_{x}+\sqrt{\frac{6}{5}}(A-B) \hat{T}_{3,0 ; x}\right]$, where $\hat{T}_{3,0 ; x}=-\frac{1}{\sqrt{2}}\left(\hat{T}_{3,+1}-\hat{T}_{3,-1}\right)$ and $\hat{T}_{3, \pm 1}=\mp \frac{1}{4} \sqrt{\frac{1}{15}}\left[5\left(\hat{I}_{z}^{2} \hat{I}_{ \pm}+\hat{I}_{ \pm} \hat{I}_{z}^{2}\right)-\{2 I(I+1)+1\} \hat{I}_{ \pm}\right]$. Therefore the biexponential relaxation of transverse magnetization $\hat{I}_{x}$ in the $I=3 / 2$ system also creates rank-3 single quantum coherences $\hat{T}_{3, \pm 1}$, which can be converted into double- and triple-quantum coherences $\hat{T}_{3, \pm 2}$ and $\hat{T}_{3, \pm 2}$ or octupolar order $\hat{T}_{3,0}$ via a radiofrequency pulse. If the relaxation was monoexponential $(A=B)$, only $x$-magnetization $\hat{I}_{x}$ would be present during relaxation.
} 
frame Hamiltonian of a spin- $I$ nucleus in the presence of a large magnetic field $\underline{B}_{0}=B_{0} \underline{\tilde{z}}$ and an rf field $\underline{B}_{r f}(t)=2 B_{1} \cos \left(\omega_{r} t\right)$ (see Eqs. 1.9 and 1.67:

$$
\begin{aligned}
\hat{H}(t) & =\hat{H}_{Z}+\hat{H}_{r f}(t) \\
& =-\hbar \omega_{0} \hat{I}_{z}-2 \hbar \omega_{1} \cos \left(\omega_{r} t+\phi\right) \hat{I}_{x} .
\end{aligned}
$$

Here $\omega_{1}=\gamma B_{1}$ and the Larmor frequency $\omega_{0}$ may be modified to include the chemical shielding (e.g., $\omega_{0}=\gamma\left(1-\sigma_{i s o}\right) B_{0}$ in isotropic solution), if desired. The rf carrier frequency $\omega_{r}$ is assumed to be non-negative. Note that $\hat{H}(t)$ in Eq. 1.136 does not commute with itself at different times. As discussed in $\S 2$, this implies that the time evolution under this Hamiltonian cannot be solved exactly; an approximation is necessary. When the rf irradiation is nearly resonant, $\omega_{r} \approx\left|\omega_{0}\right|$, the time evolution is best approximated in the rotating frame, i.e., the frame rotating at angular frequency $\omega_{r}$ about the $z$-axis. Eq. 1.136 can be rewritten in terms of circularly-polarized rf components (see Eq. 1.67):

$$
\begin{aligned}
\hat{H}(t)=-\hbar \omega_{0} \hat{I}_{z}-\hbar \omega_{1}\left[\cos \left(\omega_{r} t+\phi\right) \hat{I}_{x}+\sin \left(\omega_{r} t+\phi\right) \hat{I}_{y}\right] \\
-\hbar \omega_{1}\left[\cos \left(\omega_{r} t+\phi\right) \hat{I}_{x}-\sin \left(\omega_{r} t+\phi\right) \hat{I}_{y}\right] \\
=-\hbar \omega_{0} \hat{I}_{z}-\hbar \omega_{1}\left[e^{-i\left(\omega_{r} t+\phi\right) \hat{I}_{z}} \hat{I}_{x} e^{+i\left(\omega_{r} t+\phi\right) \hat{I}_{z}}\right]-\hbar \omega_{1}\left[e^{+i\left(\omega_{r} t+\phi\right) \hat{I}_{z}} \hat{I}_{x} e^{-i\left(\omega_{r} t+\phi\right) \hat{I}_{z}}\right] .
\end{aligned}
$$

The magnetic field vector of one rf component goes around nearly at the angular frequency of the Larmor precession $\underline{\omega}_{0}$; the other component rotates in the opposite sense as the spin precession. The time development operator is best approximated in the frame that rotates 
nearly with the Larmor precession, which is defined by the unitary transformation (see $\S 2.3 .3):$

$$
\hat{V}_{\text {rot }}(t)=\exp \left(+i \omega_{r} t \hat{I}_{z}\right)
$$

Note that when $\omega_{r}=\omega_{0}$, the operator $\hat{V}_{\text {rot }}(t)$ reduces to a transformation into the interaction frame of the Zeeman Hamiltonian, $\hat{V}_{Z}(t)=e^{-i \hat{H}_{Z} t / \hbar}$. The effective Hamiltonian in the rotating frame is (see $\S 2.3 .1)$ :

$$
\begin{aligned}
\hat{H}^{r o t}(t)= & \hat{V}_{\text {rot }}^{-1}(t) \hat{H}(t) \hat{V}_{\text {rot }}(t)-i \hbar \hat{V}_{\text {rot }}^{-1}(t) \frac{\partial \hat{V}_{r o t}(t)}{\partial t} \\
= & -\hbar\left(\omega_{0}-\omega_{r}\right) \hat{I}_{z}-\hbar \omega_{1}\left[e^{-i\left(2 \omega_{r} t+\phi\right) \hat{I}_{z}} \hat{I}_{x} e^{+i\left(2 \omega_{r} t+\phi\right) \hat{I}_{z}}\right]-\hbar \omega_{1}\left[e^{+i \phi \hat{I}_{z}} \hat{I}_{x} e^{-i \phi \hat{I}_{z}}\right] \\
= & -\hbar\left(\omega_{0}-\omega_{r}\right) \hat{I}_{z}-\hbar \omega_{1}\left[\hat{I}_{x} \cos \phi-\hat{I}_{y} \sin \phi\right] \\
& -\hbar \omega_{1}\left[\cos \left(2 \omega_{r} t+\phi\right) \hat{I}_{x}+\sin \left(2 \omega_{r} t+\phi\right) \hat{I}_{y}\right] .
\end{aligned}
$$

Note that this equation was derived assuming $\omega_{0} \simeq \gamma B_{0}>0$; if $\omega_{0}<0$ the other circularly rotating component of the rf magnetic field is nearly resonant with the spin precession. Also note that the Zeeman interaction term has been almost completely removed in the rotating frame, leaving only a resonance offset term $-\hbar\left(\omega_{0}-\omega_{r}\right) \hat{I}_{z}$. The laboratory frame Hamiltonian describes dynamics on two very different timescales, $\left|\omega_{0}\right|^{-1}$ and $\left|\omega_{1}\right|^{-1}$, but the rotating frame Hamiltonian describes dynamics only on the order of $\left|\omega_{1}\right|^{-1}$. It is then easy to apply time-dependent perturbation theory. The first two terms in the last line of Eq. 1.139 are time independent and the last term oscillates at $2 \omega_{r}$. First-order time-dependent perturbation theory (the secular approximation) dictates that this rapidly oscillating term 
can be time-averaged to zero and neglected when the oscillation frequency $2 \omega_{r}$ is much greater than the frequency $\left|\omega_{1}\right|$ that characterizes the dynamical evolution in the rotating frame. Therefore one half of the effectiveness of the linearly-polarized rf magnetic field is lost because it does not rotate in the same sense as the spin precession. Since $\omega_{r} \approx\left|\omega_{0}\right|$, one can say that this counter-rotating term can be neglected when the Larmor frequency of the spins due to $\underline{B}_{0}$ is much greater than than the Larmor frequency of the spins due to $\underline{B}_{1}$ (also known as the rf nutation frequency), i.e., when $\left|\omega_{0}\right| \gg\left|\omega_{1}\right|$. Since $\left|\omega_{0}\right| / 2 \pi$ is typically on the order of $10^{2} \mathrm{MHz}$ in high-field NMR experiments and $\left|\omega_{1}\right| / 2 \pi$ is at most on the order of $10^{2} \mathrm{kHz}$ in high-power solid state probes, this is usually a good approximation. The effects of the non-secular counter-rotating term can appear in a higher-order perturbation theory treatment, where they are known as Bloch-Siegert shifts of the resonance [105],[41, $\S \mathrm{H}],[41, \S 4.4 .2]$. Note that all of the rf field instead of half of it could be used to drive the spins if a crossed coil pair was employed such that a nearly-resonant circularly polarized rf field could be produced that rotated in the same sense as the spin precession. Also note that the rotating frame Hamiltonian (Eq. 1.139) would be completely time independent in that case; this is an interesting example of a system in which the time evolution cannot be solved exactly in the laboratory frame because the Hamiltonian is not homogeneous (see $\S 2.1 .1)$ but can be solved exactly in the rotating frame.

According to the arguments above, the rotating frame Hamiltonian during rf irradiation is approximately

$$
\hat{H}_{r o t} / \hbar \approx-\left(\omega_{0}-\omega_{r}\right) \hat{I}_{z}-\hbar \omega_{1}\left[\hat{I}_{x} \cos \phi-\hat{I}_{y} \sin \phi\right] .
$$


This corresponds to the Zeeman Hamiltonian of a spin $I$ in the presence of an effective static magnetic field

$$
\underline{B}^{e f f}=B_{1} \cos \phi \underline{\widetilde{x}}-B_{1} \sin \phi \underline{\widetilde{y}}+\left(B_{0}-\omega_{r} / \gamma\right) \underline{\widetilde{z}} .
$$

The phase $\phi$ of the rf field determines the direction of the transverse component of the effective field and the carrier frequency $\omega_{r}$ of the field enters such that the Larmor frequency of the effective longitudinal field is equal to the detuning from resonance $\left(\omega_{0}-\omega_{r}\right)$. Note that the transverse component of the rotating frame rf Hamiltonian is often written $\hbar \omega_{1}\left[\hat{I}_{x} \cos \phi+\right.$ $\left.\hat{I}_{y} \sin \phi\right]$ such that $\phi=0$ corresponds to a $+x$ pulse and $\phi=\pi / 2$ corresponds to a $+y$ pulse; here strict attention is paid to the sign conventions. In the absence of that attention one often finds expressions for the rotating frame rf Hamiltonian such as the following (for the $\phi=0$ case)

$$
\hat{H}_{r f} / \hbar=\delta \hat{I}_{z}+\omega_{1} \hat{I}_{x}
$$

where $\delta$ is the detuning of the $\mathrm{rf}$ carrier from resonance. This sort of expression is usually acceptable but one must remember that there are certain circumstances in which the signs of the frequencies and phases matter and should be made explicit [3, 33, 34]. Consider the action of an $x$-pulse in the rotating frame according to Eq. 1.142. If the spins are initially magnetized in the $+z$ direction - e.g., corresponding to thermal equilibrium in the high 
temperature limit $(\S 1.4 .3)$ - the difference density operator is

$$
\hat{\rho}(0) \propto \hat{I}_{z}
$$

The time evolution in the rotating frame under $+x$-irradiation is

$$
\hat{\rho}(t)=e^{-i \hat{H}_{r f} t / \hbar} \hat{\rho}(0) e^{+i \hat{H}_{r f} t / \hbar}=e^{-i \omega_{1} t \hat{I}_{x}} \hat{\rho}(0) e^{+i \omega_{1} t \hat{I}_{x}}=\hat{I}_{z} \cos \left(\omega_{1} t\right)-\hat{I}_{y} \sin \left(\omega_{1} t\right),
$$

which corresponds to pure precession (rotation) about the $+x$-field in the $y-z$ plane. Historically this motion is called "rf nutation" because of its appearance when added to the fast precession about $z$ the Larmor frequency $\omega_{0}$ in the laboratory frame. The quantity $\omega_{1}$ is called the rf nutation frequency. If the spins are irradiated for some time $\tau$ which is the length of the rf pulse, one finds that the spins are tipped through the angle

$$
\theta_{\text {nut }}=\omega_{1} \tau
$$

A pulse whose strength and duration is chosen such that $\omega_{1} \tau=\pi / 2$ is called a $90^{\circ}$ pulse; in this case the initial $z$-magnetization is rotated entirely into the transverse plane. A $90^{\circ}$ pulse maximizes the NMR signal, which is proportional to the transverse magnetization. In practice the experimentalist calibrates the $\mathrm{rf}$ pulse by varying $\tau$ at a fixed $\mathrm{rf}$ power until the signal maximum is observed [4, $§ 3.4 .1],[106]$. The presence of a resonance offset can adversely affect the ability to apply pulses because less of the magnetization is nutated into the transverse plane when the effective magnetic field in the rotating frame contains a $z$-component. When $|\delta| \gg\left|\omega_{1}\right|$ the effective field is almost entirely along the $z$-axis, which 
obviously does not do much to rotate $z$-magnetization into the transverse plane. The case when $\left|\omega_{1}\right|$ is much larger than the resonance offset or the size of any of the internal NMR Hamiltonians (measured in frequency units) is called a "hard" rf pulse and corresponds to a pure rotation of the nuclear polarization.

One may conclude from this analysis that nearly-resonant harmonic rf irradiation can be used to rotate nuclear magnetization about an axis in the transverse plane that is determined by the phase of the pulse. If the spins are initially magnetized along the $z$-axis, this corresponds to the excitation of single-quantum coherences in the Zeeman basis from populations. This can be appreciated by observing the fact that the operator $\hat{I}_{x}$ (or $\hat{I}_{y}$ ) in the rf Hamiltonian connects states $|I, m\rangle$ to states $|I, m \pm 1\rangle$. For this reason the NMR excitation selection rule is said to be $\Delta m= \pm 1$ (the reasoning for this would of course be different in a quantized field picture). The selection rule is the same for NMR detection, but for a slightly different reason (see $§ 1.7)$. An rf pulse in the absence of any other interactions can change the coherence order of the nuclear polarization, but according to arguments in Appendix B it cannot change the spherical tensor rank of the polarization. This is a consequence of the fact that the effective rf Hamiltonian (Eq. 1.140) causes a rotation of the polarization about an effective magnetic field; rotations conserve tensor rank. However, a combination of rf irradiation in the presence of certain other interactions such as an electric quadrupolar coupling can change tensor rank. These results are in contrast to the result of NMR free precession in the absence of a magnetic field; the evolution of the spins under the Zeeman-truncated secular internal Hamiltonians conserves coherence order $q$, a result that is exploited in coherence pathway selection techniques. 
As an interesting aside, it has been shown by Feynman, Vernon, and Hellwarth [107] that the quantum-mechanical dynamics of any two-level system can be interpreted geometrically as the evolution of the angular momentum of a fictitious spin- $1 / 2$ particle in a magnetic field. This is a result of the fact that a two-level Hamiltonian can be decomposed in terms of spherical tensor operators of up to rank $k=1$ (giving it the matrix form of a Zeeman Hamiltonian $\left.\propto \underline{\hat{I}} \cdot \underline{B}=\hat{I}_{x} B_{x}+\hat{I}_{y} B_{y}+\hat{I}_{z} B_{z}\right)$ and likewise for the density operator (giving it the matrix form of the components of angular momentum/magnetization $\left.\propto \underline{\hat{I}} \cdot \underline{\widetilde{\zeta}}=\zeta_{x} \hat{I}_{x}+\zeta_{y} \hat{I}_{y}+\zeta_{z} \hat{I}_{z}\right)$

A final point to note is that NMR excitation is coherent: the rf magnetic field is uniform on an atomic scale and each spin in the ensemble experiences approximately the same rf field at the same time. The spins therefore nutate in phase with each other, so in the absence of any local interactions and relaxation effects the net polarization of the ensemble is preserved under the action of the rf field. This is why ensemble-averaged coherence can exist in the sample after irradiation. If the excitation field was incoherenti.e., each spin may be excited into a coherence but the phases of the coherences are randomly distributed from spin to spin-then no ensemble-averaged coherence would persist. The fact that NMR spectroscopy is coherent makes the quantum-mechanical description of the dynamics useful. It is interesting to note that population inversions (i.e., an inversion of the magnetization) could not be achieved if the NMR excitation was incoherent (the best case in a two-level system would be to equalize the populations in the absence of spontaneous emission), whereas it is quite simple to achieve complete population inversions in NMR using $180^{\circ}$ pulses. 


\subsection{NMR detection}

Those who are versed in optical spectroscopic techniques might assume that the detection of the NMR signal takes place via the measurement of the radiative emission of the excited spins. In pulsed Fourier-transform NMR the signal is actually measured while the rf field is off, so stimulated emission is ruled out as a source of signal in this type of NMR experiment. Likewise, spontaneous emission of the nuclear spins is a completely negligible process [68]. The conventional method of NMR detection considered here involves the detection of nuclear magnetization via the current it induces in a coil. In pulsed Fourier transform NMR spectroscopy this signal is measured in the time domain. Although NMR detection can be considered at least formally in a field-quantized picture, it is best thought of simply as a near-field inductive coupling of the oscillating bulk magnetic field of the nuclear spins to the rf detection coil [68]. Typically NMR experiments use the same coil both for applying pulses and for detection, although some techniques such as NMR remote detection (see §6) separate the encoding of spins from their detection. The coil axis is transverse to $\underline{B}_{0}$; this allows both for the application of transverse rf pulses for spin excitation but also detection of the rotating transverse nuclear magnetization. The book by Fukushima and Roeder [108] is an excellent resource on NMR hardware and its role in the NMR experiment; see also $\S 3$ of the book by Cavanagh et al. [4].

Faraday's law of magnetic induction states that the electromotive force (emf) induced in a loop of wire by a time-varying magnetic field $\underline{B}(t)$ is

$$
\mathcal{E}=-\frac{d \Phi(t)}{d t}
$$


where the magnetic flux through the loop is

$$
\Phi(t)=\int \underline{B}(t) \cdot d \underline{a},
$$

where the integral is taken over the area of the loop and $d \underline{a}$ is the differential area element directed normal to the plane of the loop. Eq. 1.148 is a consequence of the Maxwell equation

$$
\underline{\nabla} \times \underline{E}=-\frac{\partial \underline{B}}{\partial t}
$$

which states that a time-varying magnetic field can create an electric field; in the case of the current loop this means that a changing magnetic field along the loop axis induces an azimuthal electric field that drives an electric current through the loop. The details of NMR coil design are outside the scope of this discussion; however, all such coils operate on this principle of Faraday induction.

As is discussed in more detail in $\S 6.2 .1$, a polarized ensemble of nuclear dipoles creates a macroscopic magnetization which in turn generates a magnetic field. This magnetization is proportional to the average nuclear angular momentum; if a component of this angular momentum is transverse to the applied field $\underline{B}_{0}$, then it rotates in the transverse plane due to Larmor precession about $\underline{B}_{0}$ and evolves in the presence of the secular contributions of whatever local molecular fields are present. The Larmor precession and spin evolution under local Hamiltonians is therefore encoded in the time-dependence of the transverse magnetic field created by the spins. The rotating magnetic field induces a timedependent flux through the coil. The NMR signal is simply due to the current induced in 
the detection coil.

NMR spectrometers typically implement what is known as quadrature detection, in which the NMR signal is measured against two reference channels: one oscillating inphase with the rf carrier and one oscillating $\pi / 2$ out-of-phase with the carrier. As was seen in $\S 1.6$, a phase shift of the carrier by $\phi$ corresponds to a rotation by $\phi$ about $z$ in the rotating frame. Therefore quadrature detection allows for the simultaneous measurement of the rotating-frame magnetization components $M_{x}$ and $M_{y}$. By convention the circularlypolarized complex transverse magnetization component $M_{+}=M_{x}+i M_{y}$ is measured; $M_{-}=M_{x}-i M_{y}$ could be measured equally well. Since the magnetization is proportional to the average of the angular momentum, $\underline{M} \propto\langle\underline{I}\rangle$, this implies that the detection operator

$$
\hat{\mathcal{O}}_{\text {q.d. }}=\hat{I}_{+}
$$

is appropriate for quadrature-detected signals. One may object that this operator is not Hermitian and that all quantum-mechanical observables must be Hermitian and possess real eigenvalue spectra. In particular, a measurement of $\hat{I}_{+}=\hat{I}_{x}+i \hat{I}_{y}$ corresponds to a simultaneous measurement of the $x$ - and $y$-components of angular momentum, which is also forbidden because $\left[\hat{I}_{x}, \hat{I}_{y}\right] \neq 0$. The resolution of this seeming paradox comes from issues explored in $\S 1.4 .2$ (see also $[2, \S 4.2]$ ); namely, the operator $\underline{\hat{I}}$ is actually a reduced singlespin operator produced by tracing over the rest of a macroscopic collection of other spins. It should never be forgotten NMR spectroscopy as it is described here is a bulk ensemble technique. Although it is not permissible to know different components of quantized angular momentum simultaneously, in the classical limit it is certainly permissible to specify different 
components of macroscopic magnetization at the same time.

Since the macroscopic magnetic field of the spins is proportional to their magnetization (see §6.2.1), the arguments cited above can be used to show that the complex current induced in the detection coil is

$$
\mathcal{I}(t) \propto \frac{\partial}{\partial t} M_{+}(t) \propto \frac{\partial}{\partial t} \operatorname{Tr}\left[\hat{I}_{+} \hat{\rho}(t)\right]
$$

The laboratory-frame circular magnetization component is of the form $M_{+}(t)=M_{0} e^{-i \omega_{0}^{\prime} t}$ for a single resonance with no relaxation, where the term $\omega_{0}^{\prime}=\left(1-\sigma_{i s o}\right) \omega_{0}$ has included the isotropic chemical shielding as an explicit reminder of the chemical information contained in the signal. In practice, $\omega_{0}^{\prime} \approx \omega_{0}$, and

$$
\mathcal{I}(t) \propto-i \omega_{0} M_{0} e^{-i \omega_{0}^{\prime} t}=-i \omega_{0} M_{+}(t)
$$

For this reason the NMR signal is often written as being proportional to the transverse magnetization itself (instead of to its time derivative).

Eq. 1.151 can be used to make some statements about the sensitivity of NMR Faraday detection. The Larmor frequency is $\omega_{0}=\gamma B_{0}$; the magnetization contains one factor of $\gamma$ because it is proportional to the magnetic moment of the nucleus and another factor of $\omega_{0}$ if the magnetization is due to thermal equilibrium spin polarization in the field $\underline{B}_{0}$ (see Eq. 1.119). Therefore under conventional conditions the magnitude of the NMR 
signal is

$$
S \propto \gamma^{3} B_{0}^{2}
$$

This demonstrates the desirability of working at high magnetic fields, particularly if low- $\gamma$ nuclei are to be detected. In practice rf coils tend to lose some of their efficiency when operated at high frequencies and the actual signal goes something like $\omega_{0}^{3 / 2}$ instead of $\omega_{0}^{2}$ [108, §VI.A.4]. Some factors affecting the sensitivity of NMR Faraday detection (including the effects of noise) are discussed in Refs. [109],[7, §4.3],[4, §3.3.3]. Note that one factor of $B_{0}$ (and one of $\gamma$ ) can be removed from Eq. 1.152 if the nuclei can be polarized by some means other than thermal equilibration at high field, e.g., spin-exchange optical pumping (see $\S 6.4 .1$ ), etc. The second factor of $B_{0}$ (and another of $\gamma$ ) can be removed if an alternative detection technique is used whose sensitivity is proportional to the magnetization of magnetic flux itself rather than to the time derivative of the flux; examples of such techniques are atomic magnetometry [110], SQUID flux magnetometry [111], and spin-exchange optical detection [112]. Therefore the use of a large magnetic field can be avoided in certain NMR experiments, although having a large field is beneficial for other reasons, such as when the chemical shift $\left(\propto B_{0}\right)$ is of interest.

The discussion thus far has glossed over an important issue, which is how the case of a nuclear magnetization density that depends on position should be treated properly. It may not be clear at first what the effect on the signal will be of a magnetization vector that varies in strength and direction over a sample, with some parts of the sample obviously being closer to the coil than others, etc. The solution is actually quite simple, because there is a 
symmetry between the case of spins generating a magnetic field that induces a current in the coil and the case of a current in the coil being used to generate a magnetic field experienced by the spins. This is called the principle of reciprocity in NMR [109],[113],[114]. If $\underline{B}_{1}(\underline{r})$ is the magnetic field generated by an $\mathrm{rf}$ coil as a function of position and $\underline{M}(\underline{r})$ is the nuclear magnetization density of the spins, the emf in the coil can be written

$$
\mathcal{E}=-\frac{\partial}{\partial t} \int_{V} d^{3} \underline{r} \underline{M}(\underline{r}) \cdot \underline{B}_{1}(\underline{r})
$$

where $V$ is the volume containing the sample. This emf is the same no matter whether it is due to the action of the spins on the coil or vice versa. NMR rf coils are usually built such that the rf field is as homogeneous as possible across the sample so that the spins are excited uniformly. Taking the rf field to be homogeneous and directed along the coil axis $x$ such that $\underline{B}_{1}(\underline{r})=B_{1} \underline{\tilde{x}}$ in $V$, one finds

$$
\mathcal{E}=-B_{1} \frac{\partial}{\partial t} \int_{V} d^{3} \underline{r} M_{x}(\underline{r})
$$

Therefore the NMR signal is proportional to the time derivative of the integral of the magnetization over the sample volume. Quadrature detection allows the simultaneous measurement of $M_{x}(\underline{r}, t)$ and $M_{y}(\underline{r}, t)$ in the rotating frame.

In practice the NMR signal is not digitized at radio frequencies but is down-mixed with the rf carrier to form an audiofrequency signal. Consider the following problem. The ensemble-averaged nuclear angular momentum component $\left\langle\hat{I}_{+}\right\rangle(t)$ in the laboratory frame 
can be written in terms of rotating frame operators using Eq. 2.38:

$$
\left\langle\hat{I}_{+}\right\rangle(t)=\operatorname{Tr}\left[\hat{\rho}^{r o t}(t) \hat{I}_{+}^{r o t}\right]
$$

The rotating frame transformation is defined according to Eq. 1.138: $\hat{\mathcal{O}}^{\text {rot }}=\hat{V}_{\text {rot }}^{-1} \hat{\mathcal{O}} \hat{V}_{\text {rot }}$, where $\hat{V}_{\text {rot }}=e^{i \omega_{r} t \hat{I}_{z}}$. The operator $\hat{I}_{+}$is a rank $k=1$, order $q=+1$ spherical tensor operator (see Appendix A) and transforms under rotations about the $z$-axis in a simple way (Eq. B.15): $\hat{I}_{+}^{\text {rot }}=\hat{V}_{\text {rot }}^{-1} \hat{\mathcal{O}} \hat{V}_{\text {rot }}=e^{-i \omega_{r} t} \hat{I}_{+}$. Therefore,

$$
\left\langle\hat{I}_{+}\right\rangle(t)=e^{-i \omega_{r} t} \operatorname{Tr}\left[\hat{\rho}^{r o t}(t) \hat{I}_{+}\right]
$$

The rf down-mixing in the in-phase and quadrature channels is achieved by electronically multiplying the sinusoidally-oscillating induced signal by the phase-shifted sinusoidal reference signals oscillating at $\omega_{r}$. This results in oscillations at the sum and the difference of the signal frequency and the carrier frequency $\omega_{r}$; the sum oscillations are near $2 \omega_{0}$ and are too fast to digitize, whereas the difference oscillations are typically in the audiofrequency range. The effect of the mixing is to multiply the quadrature-detected signal by $e^{i \omega_{r} t}$. Since $M_{+}(t) \propto\left\langle\hat{I}_{+}\right\rangle(t)$, one can use Eqs. 1.151 and 1.156 to write the digitized quadrature-detected NMR signal as

$$
S_{+}(t) \propto \operatorname{Tr}\left[\hat{\rho}^{r o t}(t) \hat{I}_{+}\right]
$$

This is an important result: the time-domain signal observed by the experimentalist is effectively a measurement of the rotating frame dynamics because the rf carrier down- 
mixing "cancels" the time dependence acquired by transforming from the rotating frame back into the laboratory frame. This is one reason why NMR spectroscopists always think in terms of the rotating frame: they are used to seeing the measured rotating frame dynamics with their own eyes. The recorded signal, called the free induction decay (FID), oscillates at the evolution frequencies of the spins under their own local Hamiltonians (i.e., chemical shielding, quadrupolar, etc.). These measured frequencies can be shifted by an arbitrary amount by changing the rf carrier frequency (i.e., changing the resonance detuning $\delta$ ), so it is usually not the absolute frequency of a resonance that matters but rather the relative frequency compared to the resonance of a reference compound or to other peaks in the spectrum. One interesting feature of NMR spectrometers is that the phase of the receiver can often be shifted-usually $\phi_{r}=0^{\circ}, 90^{\circ}, 180^{\circ}$, or $270^{\circ}$ — which corresponds to multiplying $S_{+}(t)$ by $e^{i \phi_{r}}$. This results in multiplying the observed signal by $1, i,-1$, or $-i$, which is useful for NMR coherence pathway selection by phase cycling schemes.

Note that the operator $\hat{I}_{+}$in Eq. 1.157 connects states $|I, m\rangle$ to states $|I, m+1\rangle$. In general the detection operator $\hat{I}_{+}$selects out $\hat{I}_{-}=\hat{I}_{+}^{\dagger}$ terms from the density operator, i.e., the -1-quantum coherences. The fact that NMR measures transverse magnetization, $M_{ \pm}$(or $\hat{I}_{ \pm}$in the quantum picture), is the reason why the NMR selection rule is $\Delta m=$ \pm 1 for detection (the interpretation of this selection rule will be different in a quantized field picture). As an interesting example, consider $x$-magnetization (e.g., prepared from equilibrium $z$-magnetization by a $90^{\circ}$-y pulse) that precesses with a rotating-frame difference density operator equal to

$$
\hat{\rho}(t)=\hat{I}_{x} \cos (\delta t)+\hat{I}_{y} \sin (\delta t) .
$$


Say that quadrature detection is not employed and $x$-magnetization is detected. In that case the signal is

$$
\begin{aligned}
S_{x}(t) & \propto \operatorname{Tr}\left[\hat{\rho}(t) \hat{I}_{x}\right]=\operatorname{Tr}\left[\hat{I}_{x}^{2}\right] \cos (\delta t) \\
& =\left[\sum_{m, m^{\prime}}\left|\left\langle I, m^{\prime}\left|\hat{I}_{x}\right| I, m\right\rangle\right|^{2}\right] \cos (\delta t) .
\end{aligned}
$$

The intensity of the NMR line will therefore be proportional to the incoherent sum of probabilities $\left|\left\langle I, m^{\prime}\left|\hat{I}_{x}\right| I, m\right\rangle\right|^{2}$. This is the same result as the standard Fermi Golden Rule description of transition probabilities, in this case the single-quantum transitions $|I, m\rangle \rightarrow$ $\left|I, m^{\prime}=m \pm 1\right\rangle$. The Fermi Golden Rule result is applicable to continuous-wave NMR in which the resonant transition between Zeeman eigenstates occurs in the presence of a perturbing rf term $\hat{H}_{r f} \propto \hat{I}_{x}$. This is an interesting result, but really only says that the time domain/pulsed NMR technique has the same selection rules as the continuous wave technique.

A final topic to consider is how one goes from the time domain signal $S_{+}(t)$ to the NMR spectrum. This is performed using a Fourier transform (FT) with respect to the time variable $t$. As an example, consider the time-domain NMR signal due to a spin undergoing free precession in the presence of some resonance offset Hamiltonian $\hat{H} / \hbar=\omega^{\prime} \hat{I}_{z}$ and in the absence of relaxation:

$$
S_{+}(t)=S_{0} e^{i \omega^{\prime} t}
$$


The NMR spectrum is given by the Fourier transform of the signal:

$$
\begin{aligned}
S(\omega) & =\mathcal{F}\left\{S_{+}(t)\right\}=\int_{-\infty}^{\infty} d t e^{-i \omega t} S_{+}(t) \\
& =2 \pi S_{0} \delta\left(\omega-\omega^{\prime}\right)
\end{aligned}
$$

which corresponds to a sharp line at frequency $\omega=\omega^{\prime}$. This satisfies the expectation of a Fourier transform pair: since the signal extends indefinitely in the time domain, it is infinitely sharp in the frequency domain. If $S_{+}(t)$ is modified to include the effects of transverse relaxation for $t>0$,

$$
S_{+}(t)=S_{0} e^{i \omega^{\prime} t} e^{-t / T_{2}}
$$

the spectrum is $[4, \S 3.3 .1]$ :

$$
S(\omega)=A(\omega)+i D(\omega)
$$

where the absorptive and dispersive lineshape factors are (respectively):

$$
\begin{aligned}
A(\omega) & =\frac{T_{2}^{-1}}{T_{2}^{-2}+\left(\omega^{\prime}-\omega\right)^{2}} \\
D(\omega) & =\frac{\left(\omega^{\prime}-\omega\right)}{T_{2}^{-2}+\left(\omega^{\prime}-\omega\right)^{2}} .
\end{aligned}
$$

The time domain signal decays exponentially with a characteristic time constant $T_{2}$; its spectrum has a Lorentzian profile with a full width at half maximum $2 T_{2}^{-1}$. The quantity $\operatorname{Re}[S(\omega)]$ shows a pure absorption-mode lineshape, which is desirable in terms of resolution 
because its wings decay to zero more rapidly than those of a dispersive line. It is possible for the instrumentation to introduce phase factors into the experiment such that $S^{\prime}(t)=e^{i \phi} S(t)$ (where $\phi$ is not known ahead of time); this makes the real and imaginary parts of the spectrum into mixtures of absorptive and dispersive components. The quantity $\operatorname{Re}[S(\omega)]$ can be made purely absorptive by multiplying the signal by making a "zero-order" phase correction (i.e., multiplying by $\left.e^{-i \phi}\right)[4, \S 3.3 .2 .3]$. Another interesting case is when the FID is delayed by a time $\tau$, e.g. during a spin echo experiment, such that the signal becomes $S^{\prime}(t)=S(t-\tau)$. There is a Fourier transform identity [4, $\left.\S 3.3\right]$ :

$$
\mathcal{F}\{S(t-\tau)\}=e^{-i \omega \tau} \mathcal{F}\{S(t)\}=e^{-i \omega \tau} S(\omega)
$$

which indicates that an extra time-dependent phase is acquired which can be removed by making a "first-order" phase correction.

In practice the time domain signal is sampled discretely and a discrete Fourier transform is performed. If the sampling time (called the "dwell time") is $d t$, the spectral width is $s w=1 / d t$. Frequencies in the signal of up to $\pm s w / 2$ from zero are observable; this is a result of the Nyquist sampling theorem [4, §3.2]. Higher frequencies get aliased back into the spectral window if they are not first filtered out.

\subsection{Fundamentals of two-dimensional NMR}

The basic goal of NMR is to measure the nuclear spin dynamics as a function of time in order to gain information about their environment. The two dimensional (2D) NMR technique provides a means of mapping out spin evolution indirectly in a time di- 
mension that is additional to the time dimension that corresponds to direct measurement of the spins. The two-dimensional technique was first proposed by Jeener and developed by Ernst's group [115, 116]. The authoritative reference on this topic is Ernst's text [7], but the books by Cavanagh et al. [4] and Goldman [2] also explain these techniques well and perhaps more simply. The usual goal of $2 \mathrm{D}$ NMR is to correlate the time evolution in the indirect dimension with the time evolution in the direct dimension to form a so-called NMR interferogram. Sometimes the elucidation of these correlations yields more information than does determining the time evolution in either dimension alone. Sometimes these correlations can be used to disperse the resonances of a crowded spectrum into another dimension. The use of an indirect measurement technique is also what allows multiple quantum spectroscopy experiments to be conducted, since multiple quantum coherences do not lead to bulk magnetization therefore are not directly observable by NMR techniques. The principles of multidimensional and multiple quantum NMR has been reviewed by Pines et al. [117, §2]. Two dimensional spectroscopy can even be used to correlate spin evolution under the action of two different Hamiltonians.

A diagram of a generic two dimensional NMR experiment is presented in Fig. 1.5. The preparation interval is used to perturb the spins from their equilibrium state and manipulate them into a state that will have time evolution of interest. For example, in multiple quantum spectroscopy the preparation step could consist of an rf pulse or sequence of pulses that is used to excite multiple quantum coherences from initial $z$-magnetization. The nuclei are then allowed to evolve for a time $t_{1}$, which is the indirect time parameter. Radiofrequency pulses and other manipulations of the sample can be applied during this 
time to modify the Hamiltonian, if desired. The optional mixing period is used to convert undetectable coherences into observable transverse magnetization for detection or to otherwise aid in selecting which coherences in the indirect dimension will be correlated with the detected coherences. The detection interval is used to measure the evolution of the spins as a function of the direct time, $t_{2}$. The state of the nuclei at the beginning of the detection interval depends on their evolution during $t_{1}$. This is a completely general technique; for example, coherences of spin $I$ could be prepared and transferred during the mixing period to coherences of spin $S$ for detection, without ever having to detect spin $I$ directly.

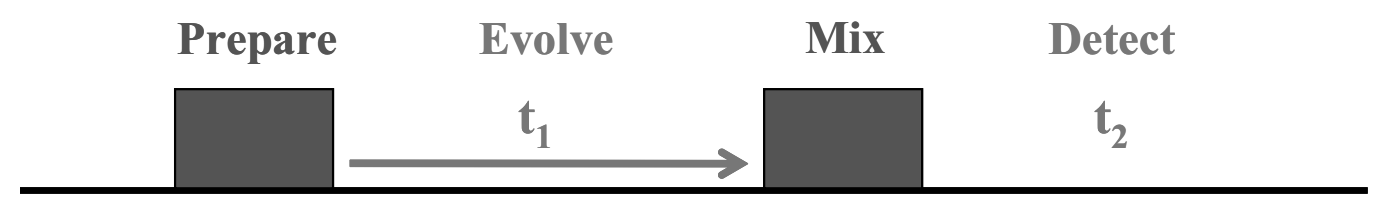

Figure 1.5: Schematic diagram of a two dimensional NMR experiment.

The key to the two dimensional technique is to perform a series of experiments while incrementing the value of $t_{1}$ in order to mimic a discretely sampled transient acquisition such as the ones that occur during the detection interval $t_{2}$ at the end of each experiment. Whereas the signal in the direct dimension $t_{2}=n d t_{2}$ may be discretely sampled in $N_{2}$ points in increments of $d t_{2}$, the time evolution in the indirect dimension is followed by performing $N_{1}$ transient experiments, each using an indirect evolution time of $t_{1}=m d t_{1}$. In this manner a two-dimensional $\left(N_{1} \times N_{2}\right)$ interferogram $S\left(t_{1}, t_{2}\right)$ can be built. Note that two-dimensional experiments are inherently much longer than onedimensional experiments; whereas a one-dimensional FID with $N_{2}$ points can be acquired in $1 s$, a $N_{1} \times N_{2}$-dimensional data set takes $N_{1}$ times as long to acquire (i.e., $\sim N_{1}$ s). The experiment will take even longer $\left(\sim p N_{1}\right)$ if a $p$-step phase cycle is employed per transient 
acquisition. The two-dimensional NMR technique is easily extended to higher dimensions but the experiment takes even longer, e.g., a three dimensional experiment would require $N_{1} \times N_{3}$ transient acquisitions to map out the time evolution in the indirect dimensions $t_{1}$ and $t_{3}$, respectively. For this reason, several approaches have been developed to perform fast multidimensional NMR spectroscopy, some of which have been reviewed in Ref. [118].

It is a useful exercise to examine mathematically how the 2D NMR signal arises. This discussion largely follows that of Ernst et al. [7, §6.2], except the effects of relaxation will be neglected. Define $\hat{V}_{p}, \hat{U}_{1}\left(t_{1}\right), \hat{V}_{m}$, and $\hat{U}_{2}\left(t_{2}\right)$ to be the time development operators for the preparation, indirect evolution, mixing, and (direct) detection intervals, respectively. All time evolution will be calculated in the rotating frame. The total time development operator for the experiment is

$$
\hat{U}\left(t_{1}, t_{2}\right)=\hat{U}_{2}\left(t_{2}\right) \hat{V}_{m} \hat{U}_{1}\left(t_{1}\right) \hat{V}_{p}
$$

Usually $\hat{V}_{p}$ and $\hat{V}_{m}$ involve rf pulses or pulse sequences and can have complicated forms. The time development operators during the evolution periods will be assumed to be of the form

$$
\begin{aligned}
& \hat{U}_{1}\left(t_{1}\right)=e^{-i \hat{H}_{1} t_{1} / \hbar}, \\
& \hat{U}_{2}\left(t_{2}\right)=e^{-i \hat{H}_{2} t_{2} / \hbar},
\end{aligned}
$$

i.e., they will be considered to be the evolution operators under the effective time-independent Hamiltonians $\hat{H}_{1}$ and $\hat{H}_{2}$, respectively. These could, for example, be secular spin Hamil- 
tonians, but also could be average Hamiltonians over the intervals $t_{1}$ or $t_{2}$ while the spins were subjected to sample spinning or to rf pulse sequences. It will be convenient to work in the eigenbases of $\hat{H}_{1}$ and $\hat{H}_{2}$ :

$$
\begin{aligned}
& \hat{H}_{1}|t\rangle=\hbar \omega_{t}^{(1)} \\
& \hat{H}_{2}|r\rangle=\hbar \omega_{r}^{(2)} .
\end{aligned}
$$

The two Hamiltonians need not share the same eigenbases, but in practice they often do, e.g., the Zeeman eigenbasis. The coherences $|t\rangle\langle u|$ and $|r\rangle\langle s|$ evolve under these Hamiltonians in the indirect and direct dimensions (respectively) as

$$
\begin{aligned}
& \hat{U}_{1}\left(t_{1}\right)|t\rangle\left\langle u\left|\hat{U}_{1}^{-1}\left(t_{1}\right)=e^{-i \hat{H}_{1} t_{1} / \hbar}\right| t\right\rangle\langle u| e^{+i \hat{H}_{1} t_{1} / \hbar}=e^{-i \omega_{t u}^{(1)} t_{1}} \\
& \hat{U}_{2}\left(t_{2}\right)|r\rangle\left\langle s\left|\hat{U}_{2}^{-1}\left(t_{2}\right)=e^{-i \hat{H}_{2} t_{2} / \hbar}\right| t\right\rangle\langle u| e^{+i \hat{H}_{2} t_{2} / \hbar}=e^{-i \omega_{r s}^{(2)} t_{2}}
\end{aligned}
$$

where the quantum beat frequencies are $\omega_{t u}^{(1)}=\omega_{t}^{(1)}-\omega_{u}^{(1)}$ and $\omega_{r s}^{(2)}=\omega_{r}^{(2)}-\omega_{s}^{(2)}$, respectively. According to Eqs. 1.157 and 1.167 the two-dimensional interferogram is

$$
\begin{aligned}
S\left(t_{1}, t_{2}\right) & \propto \operatorname{Tr}\left[\hat{\mathcal{O}}_{+} \hat{U}\left(t_{1}, t_{2}\right) \hat{\rho}(0) \hat{U}^{-1}\left(t_{1}, t_{2}\right)\right] \\
& =\operatorname{Tr}\left[\hat{\mathcal{O}}_{+} \hat{U}_{2}\left(t_{2}\right) \hat{V}_{m} \hat{U}_{1}\left(t_{1}\right) \hat{V}_{p} \hat{\rho}(0) \hat{V}_{p}^{-1} \hat{U}_{1}^{-1}\left(t_{1}\right) \hat{V}_{m}^{-1} \hat{U}_{2}^{-1}\left(t_{2}\right)\right] \\
& =\left\langle\left\langle\mathcal{O}_{+}^{\dagger}\left|\hat{\hat{U}}_{2}\left(t_{2}\right) \hat{\hat{V}}_{m} \hat{\hat{U}}_{1}\left(t_{1}\right) \hat{\hat{V}}_{p}\right| \rho(0)\right\rangle\right\rangle,
\end{aligned}
$$

where $\hat{\mathcal{O}}_{+}$is a generalized detection operator; usually it is the sum $\hat{\mathcal{O}}_{+}=\sum_{j} \hat{I}_{+}^{j}$ over the observed spins $I_{j}$. The last line of Eq. 1.175 has been written in terms of operators on the 
Liouville space, which is a convenient formalism for these types of calculation. The density operator after preparation of the system via $\hat{V}_{p}$ but before the indirect time evolution has occurred may be expanded in the eigenbasis of $\hat{H}_{1}$ :

$$
\begin{aligned}
\hat{\rho}_{p}\left(t_{1}=0\right) & =\hat{V}_{p} \hat{\rho}(0) \hat{V}_{p}^{-1}=\sum_{t, u} \rho_{p}^{t u}|t\rangle\langle u| \\
\left.\left|\rho_{p}\left(t_{1}=0\right)\right\rangle\right\rangle & \left.\left.=\hat{\hat{V}}_{p}|\rho(0)\rangle\right\rangle=\sum_{t, u} \rho_{p}^{t u}|t u\rangle\right\rangle,
\end{aligned}
$$

where $\rho_{p}^{t u}=\left\langle t\left|\hat{V}_{p} \hat{\rho}_{p}(0) \hat{V}_{p}^{-1}\right| u\right\rangle=\left\langle\left\langle t u\left|\hat{\hat{V}}_{m}\right| \rho(0)\right\rangle\right\rangle$. The matrix elements of the indirectdimension time evolution operator are (see Eq. 1.172): $\left\langle t\left|\hat{U}_{1}\left(t_{1}\right) \hat{\rho}_{p}\left(t_{1}=0\right) \hat{U}_{1}^{-1}\left(t_{1}\right)\right| u\right\rangle=$ $\left\langle\left\langle t u\left|\hat{\hat{U}}_{1}\left(t_{1}\right)\right| \rho_{p}\left(t_{1}=0\right)\right\rangle\right\rangle=e^{-i \omega_{t u}^{1} t} \rho_{p}^{t u}$. Therefore:

$$
\begin{aligned}
S\left(t_{1}, t_{2}\right) & =\operatorname{Tr}\left[\hat{\mathcal{O}}_{+} \hat{U}_{2}\left(t_{2}\right) \hat{V}_{m} \hat{U}_{1}\left(t_{1}\right) \hat{\rho}_{p}(0) \hat{U}_{1}^{-1}\left(t_{1}\right) \hat{V}_{m}^{-1} \hat{U}_{2}^{-1}\left(t_{2}\right)\right] \\
& =\sum_{t, u} \rho_{p}^{t u} e^{-i \omega_{t u}^{(1)} t_{1}} \operatorname{Tr}\left[\hat{\mathcal{O}}_{+} \hat{U}_{2}\left(t_{2}\right) \hat{V}_{m}|t\rangle\langle u| \hat{V}_{m}^{-1} \hat{U}_{2}^{-1}\left(t_{2}\right)\right] \\
& =\left\langle\left\langle\mathcal{O}_{+}^{\dagger}\left|\hat{\hat{U}}_{2}\left(t_{2}\right) \hat{\hat{V}}_{m} \hat{\hat{U}}_{1}\left(t_{1}\right)\right| \rho_{p}(0)\right\rangle\right\rangle=\sum_{t, u} \rho_{p}^{t u}\left\langle\left\langle\mathcal{O}_{+}^{\dagger}\left|\hat{\hat{U}}_{2}\left(t_{2}\right) \hat{\hat{V}}_{m}\right| t u\right\rangle\right\rangle .
\end{aligned}
$$

Now insert factors of the identity operator $\hat{1}=\sum_{r}|r\rangle\langle r|$ between $\hat{U}_{2}\left(t_{2}\right)$ and $\hat{V}_{m}$ and $\hat{1}=\sum_{s}|s\rangle\langle s|$ between $\hat{V}_{m}^{-1}$ and $\hat{U}_{2}^{-1}\left(t_{2}\right)$ (or insert $\left.\hat{1}=\sum_{r, s}|r s\rangle\right\rangle\left\langle\langle r s|\right.$ between $\hat{\hat{U}}_{2}\left(t_{2}\right)$ and $\left.\hat{\hat{V}}_{m}\right)$. The interferogram becomes:

$$
S\left(t_{1}, t_{2}\right)=\sum_{r, s} \sum_{t, u} \mathcal{O}_{+}^{s r} V_{m}^{r s, t u} \rho_{p}^{t u} e^{-i \omega_{t u}^{(1)} t_{1}} e^{-i \omega_{r s}^{(2)} t_{2}}
$$

where the matrix elements of the mixing superoperator are $V_{m}^{r s, t u}=V_{m}^{r t}\left(V_{m}^{-1}\right)^{u s}=V_{m}^{r t}\left(V_{m}^{s u}\right)^{*}$ $=\left\langle\left\langle r s\left|\hat{\hat{V}}_{m}\right| t u\right\rangle\right\rangle$ and the matrix elements of the detection operator matrix are $\mathcal{O}_{+}^{s r}=\left\langle s\left|\hat{\mathcal{O}}_{+}\right| r\right\rangle=$ 
$\operatorname{Tr}\left[\hat{\mathcal{O}}_{+}|r\rangle\langle s|\right]=\left\langle\left\langle\mathcal{O}_{+}^{\dagger} \mid r s\right\rangle\right\rangle$. Eq. 1.180 may be written as

$$
S\left(t_{1}, t_{2}\right)=\sum_{r, s} \sum_{t, u} Z^{r s, t u} e^{-i \omega_{t u}^{(1)} t_{1}} e^{-i \omega_{r s}^{(2)} t_{2}}
$$

Eq. 1.181 describes the correlation between the evolution of $\hat{H}_{1}$-eigenbasis coherences $|t\rangle\langle u|$ (or $|t u\rangle\rangle$ ) (initially prepared with amplitudes $\rho_{p}^{t u}$ ) and the evolution of $\hat{H}_{2}$-eigenbasis coherences $|r\rangle\langle s|$ (or $|r s\rangle\rangle)$. The $|t\rangle\langle u|\rightarrow| r\rangle\langle s|$ coherence transfer occurs with the complex probability amplitude $Z^{r s, t u}=\left\langle\left\langle\mathcal{O}_{+}^{\dagger} \mid r s\right\rangle\right\rangle\left\langle\left\langle r s\left|\hat{\hat{V}}_{m}\right| t u\right\rangle\right\rangle\left\langle\left\langle t u \mid \rho_{p}\left(t_{1}=0\right)\right\rangle\right\rangle=\mathcal{O}_{+}^{s r} V_{m}^{r s, t u} \rho_{p}^{t u}$. The two-dimensional Fourier transform of the complex interferogram with respect to $t_{1}$ and $t_{2}$ yields the $2 \mathrm{D}$ spectrum:

$$
\begin{aligned}
S\left(\omega_{1}, \omega_{2}\right) & =\mathcal{F}\left\{S\left(t_{1}, t_{2}\right)\right\}=\int_{-\infty}^{\infty} d t_{1} \int_{-\infty}^{\infty} d t_{2} e^{-i \omega_{1} t_{1}} e^{-i \omega_{2} t_{2}} S\left(t_{1}, t_{2}\right) \\
& =4 \pi^{2} \sum_{r, s} \sum_{t, u} Z^{r s, t u} \delta\left(\omega_{1}+\omega_{t u}^{(1)}\right) \delta\left(\omega_{2}+\omega_{r s}^{(2)}\right),
\end{aligned}
$$

which contains peaks at the frequency pairs $\left(\omega_{1}=-\omega_{t u}^{(1)}, \omega_{2}=-\omega_{r s}^{(2)}\right)$ if $Z^{r s, t u} \neq 0$. The frequencies $\omega_{t u}^{(1)}$ and $\omega_{r s}^{(2)}$ contain the information about the dynamics during $t_{1}$ and $t_{2}$ respectively, and the amplitudes $Z^{r s, t u} \neq 0$ contain the information about the nature of their correlation (including the selection rules for coherence transfer). Note that it is not necessary to mix $S\left(t_{1}, t_{2}\right)$ with the carrier frequency $\omega_{r}$ twice for detection (see $\S 1.7$ ). The dynamics during the indirect time evolution are automatically tracked in the rotating frame (i.e., only one rotating frame transformation is needed to go between Eq. 1.167 and the laboratory-frame time development operator). 


\section{Chapter 2}

\section{Time dependent theory for NMR}

\subsection{Schrödinger time evolution}

Consider a quantum-mechanical system that is described by a state vector $|\psi(t)\rangle$ and a Hamiltonian operator $\hat{H}(t)$. The time evolution of the state vector is dictated by the time-dependent Schrödinger equation:

$$
i \hbar \frac{\partial|\psi(t)\rangle}{\partial t}=\hat{H}(t)|\psi(t)\rangle
$$

This partial differential equation is first-order in the time $t$, and so its solution $|\psi(t)\rangle$ may be specified in terms of only one boundary condition: the initial condition $\left|\psi\left(t_{0}\right)\right\rangle$. (Usually we will take $t_{0}=0$.) Given an initial state of the system $\left|\psi\left(t_{0}\right)\right\rangle$, the state $|\psi(t)\rangle$ of the system at any arbitrary time $t$ may be found by solving the Schrödinger equation using the particular Hamiltonian that specifies the dynamics of the system.

The expectation value of an operator $\hat{A}$ that corresponds to an observable quantity 
may be calculated as $\langle\hat{A}\rangle(t)=\langle\psi(t)|\hat{A}| \psi(t)\rangle$. Here the state vectors $|\psi(t)\rangle$ have time dependence, whereas the operators $\hat{A}$ typically do not (excepting e.g. $\hat{H}(t))$. This is called the Schrödinger representation of time-dependent quantum mechanics.

\subsubsection{The time evolution operator}

Quantum-mechanical states may be described as vectors on a Hilbert linear vector space. When an operator acts on a vector within that space, the result is another vector within the space. One might ask what sort of operator, when acting on $\left|\psi\left(t_{0}\right)\right\rangle$, returns $|\psi(t)\rangle$. If such an operator can be found, it will contain a complete prescription for how the system's state vector evolves in time from $t_{0}$ to $t$. One might anticipate that this "time evolution operator" or "time propagator" will be a function of the Hamiltonian $\hat{H}(t)$, since it is known from the Schrödinger equation that the Hamiltonian dictates the time evolution. The propagator is defined by the expression

$$
|\psi(t)\rangle=\hat{U}\left(t ; t_{0}\right)\left|\psi\left(t_{0}\right)\right\rangle
$$

Sometimes hereafter the initial time index will be suppressed, such that $\hat{U}(t) \equiv \hat{U}\left(t ; t_{0}=0\right)$. The time propagator is unitary $\left(\hat{U}^{\dagger}=\hat{U}^{-1}\right)$, such that $\hat{U}^{\dagger} \hat{U}=\hat{U}^{-1} \hat{U}=\hat{1}$, and $\hat{U} \hat{U}^{\dagger}=$

$\hat{U} \hat{U}^{-1}=\hat{1}$. It must be a unitary operator for physical reasons: unitary operators preserve the norm of the state vectors on which they act, which is necessary to conserve probability 
within the system. That is, from Eq. 2.2 we have

$$
\begin{array}{r}
\langle\psi(t)|=(|\psi(t)\rangle)^{\dagger}=\left(\hat{U}\left(t ; t_{0}\right)\left|\psi\left(t_{0}\right)\right\rangle\right)^{\dagger}=\left\langle\psi\left(t_{0}\right)\right| \hat{U}^{\dagger}\left(t ; t_{0}\right)=\left\langle\psi\left(t_{0}\right)\right| \hat{U}^{-1}\left(t ; t_{0}\right) \\
\Longrightarrow\langle\psi(t) \mid \psi(t)\rangle=\left\langle\psi\left(t_{0}\right)\left|\hat{U}^{-1}\left(t ; t_{0}\right) \hat{U}\left(t ; t_{0}\right)\right| \psi\left(t_{0}\right)\right\rangle=\left\langle\psi\left(t_{0}\right)|\hat{1}| \psi\left(t_{0}\right)\right\rangle=\left\langle\psi\left(t_{0}\right) \mid \psi\left(t_{0}\right)\right\rangle .
\end{array}
$$

The total probability of finding the system in the state $|\psi(t)\rangle$ at time $t$ is $P(t)=|\langle\psi(t) \mid \psi(t)\rangle|^{2}$. Assuming $P\left(t_{0}\right)=\left|\left\langle\psi\left(t_{0}\right) \mid \psi\left(t_{0}\right)\right\rangle\right|^{2}$, we find from above that $P(t)=P\left(t_{0}\right)=1$, and probability is conserved in time.

Eq. 2.1 may be rewritten as:

$$
i \hbar \frac{\partial}{\partial t}\left[\hat{U}\left(t ; t_{0}\right)\left|\psi\left(t_{0}\right)\right\rangle\right]=\hat{H}(t) \hat{U}\left(t ; t_{0}\right)\left|\psi\left(t_{0}\right)\right\rangle
$$

Since $\left|\psi\left(t_{0}\right)\right\rangle$ is time-independent it may be factored out to yield:

$$
i \hbar \frac{\partial \hat{U}\left(t ; t_{0}\right)}{\partial t}=\hat{H}(t) \hat{U}\left(t ; t_{0}\right)
$$

Solving Eq. 2.3 for $\hat{U}\left(t ; t_{0}\right)$ is equivalent to solving the Schrödinger equation for $|\psi(t)\rangle$. Here the initial condition is $\hat{U}\left(t_{0} ; t_{0}\right)=\hat{1}$, since

$$
\left|\psi\left(t=t_{0}\right)\right\rangle=\hat{U}\left(t=t_{0} ; t_{0}\right)\left|\psi\left(t_{0}\right)\right\rangle \Longrightarrow\left|\psi\left(t_{0}\right)\right\rangle=\hat{1}\left|\psi\left(t_{0}\right)\right\rangle
$$


$\hat{U}\left(t ; t_{0}\right)$ in the case of a time-independent Hamiltonian

When $\hat{H}(t)=\hat{H}$ (i.e., the Hamiltonian has no explicit time dependence), Eq. 2.3 may be integrated directly to yield:

$$
\hat{U}\left(t ; t_{0}\right)=e^{-i \hat{H}\left(t-t_{0}\right) / \hbar}
$$

This is verified easily by substituting Eq. 2.4 back into Eq. 2.3 and differentiating. The initial condition $\hat{U}\left(t_{0} ; t_{0}\right)=\hat{1}$ also is satisfied: $\hat{U}\left(t_{0} ; t_{0}\right)=\exp [-i \hat{H} \cdot 0 / \hbar]=\exp (\hat{0})=\hat{1}$. Eq. 2.4 is an exact solution for $\hat{U}\left(t ; t_{0}\right)$ if $\hat{H}$ is time-independent. When the Hamiltonian is time-dependent, $\hat{U}\left(t ; t_{0}\right) \neq e^{-i \hat{H}(t)\left(t-t_{0}\right) / \hbar}$.

\section{$\hat{U}\left(t ; t_{0}\right)$ in the case of an inhomogeneous time-dependent Hamiltonian}

Portis [119] introduced the concept of "homogeneous" and "inhomogeneous" interactions into the field of magnetic resonance while attempting to describe the sources of homogeneous and inhomogeneous line broadening in electron spin resonance experiments. Following the convention of Maricq and Waugh [42], a time-dependent Hamiltonian $\hat{H}(t)$ is considered inhomogeneous if it commutes with itself at different times; i.e., if $\left[\hat{H}(t), \hat{H}\left(t^{\prime}\right)\right]=0$ for all $t, t^{\prime}$. The Hamiltonian $\hat{H}(t)$ is homogeneous if in general $\left[\hat{H}(t), \hat{H}\left(t^{\prime}\right)\right] \neq 0$. It must be noted that these definitions are not completely in accord with Portis' original conception of homogeneous and inhomogeneous interactions $[42,120]$.

Consider the case of an inhomogeneous Hamiltonian $\hat{H}(t)$. A piecewise approximation to the Hamiltonian may be created in which $\hat{H}(t)$ is divided up into $N$ regions of (possibly unequal) lengths $\Delta t_{n} \equiv t_{n}-t_{n-1}$ over which $\hat{H}(t)$ is approximately time- 
independent:

$$
\hat{H}(t) \simeq \sum_{n=1}^{N} \hat{H}_{n} t_{n}
$$

where $\hat{H}_{n}=\frac{1}{2}\left\{\hat{H}\left(t_{n}\right)+\hat{H}\left(t_{n-1}\right)\right\}$ on the interval $\left(t_{n}, t_{n-1}\right)$ and $=0$ otherwise. (We have $t_{n}=n \Delta t+t_{0}$ in the special case of equal time intervals, with $\Delta t_{n}=\left(t-t_{0}\right) / N \equiv \Delta t$.) The $\hat{H}_{n}$ should be approximately time independent if the $\Delta t_{n}$ are small enough such that $\|\hat{H}(t)\| \ll h / \Delta t_{n}$ for all $\Delta t_{n}$, where here $\|\hat{H}(t)\|$ is some measure of the highest frequency contained in $\hat{H}(t)$. The time evolution operator now follows from the expression:

$$
\begin{aligned}
& \hat{U}\left(t ; t_{0}\right)\left|\psi\left(t_{0}\right)\right\rangle=\hat{U}_{N}\left(t_{N} ; t_{N-1}\right) \hat{U}_{N-1}\left(t_{N-1} ; t_{N-2}\right) \cdots \hat{U}_{2}\left(t_{2} ; t_{1}\right) \hat{U}_{1}\left(t_{1} ; t_{0}\right)\left|\psi\left(t_{0}\right)\right\rangle \\
& \equiv\left\{\prod_{n=1}^{N} \hat{U}_{n}\left(\Delta t_{n}\right)\right\}\left|\psi\left(t_{0}\right)\right\rangle \simeq\left\{\prod_{n=1}^{N} e^{-i \hat{H}_{n} \Delta t_{n} / \hbar}\right\}\left|\psi\left(t_{0}\right)\right\rangle \\
& \Longrightarrow \quad \hat{U}\left(t ; t_{0}\right) \simeq \prod_{n=1}^{N} e^{-i \hat{H}_{n} \Delta t_{n} / \hbar}
\end{aligned}
$$

where the last approximation follows from Eqs. 2.4 and 2.5. There exists an identity for the exponential function of operators:

$$
e^{\hat{A}} e^{\hat{B}}=e^{(\hat{A}+\hat{B})}=e^{\hat{B}} e^{\hat{A}} \text { iff }[\hat{A}, \hat{B}]=0 .
$$

In other words, this standard identity for exponentials holds only if the arguments of the exponentials commute with each other (i.e., they are "c-numbers"). It does not hold if they do not commute (i.e., they are "q-numbers"). If $\hat{H}(t)$ is inhomogeneous, then all the $\hat{H}_{n}$ for different times commute with each other: $\left[\hat{H}_{n}, \hat{H}_{n^{\prime}}\right]=0$. Then Eq. 2.7 may be used to 
exponentiate the piecewise Hamiltonians in Eq. 2.6:

$$
\hat{U}\left(t ; t_{0}\right) \simeq \exp \left\{-\frac{i}{\hbar} \sum_{n=1}^{N} \hat{H}_{n} \Delta t_{n}\right\}
$$

In the continuum limit as $N \rightarrow \infty$ and $\Delta t_{n} \rightarrow 0$, the approximation becomes exact and the sum goes to an integral, and:

$$
\hat{U}\left(t ; t_{0}\right)=\exp \left\{-\frac{i}{\hbar} \int_{t_{0}}^{t} d t^{\prime} \hat{H}\left(t^{\prime}\right)\right\} \equiv e^{-i \hat{\Phi}(t)}
$$

where the matrix elements of $\hat{\Phi}(t)=\hbar^{-1} \int_{t_{0}}^{t} d t^{\prime} \hat{H}\left(t^{\prime}\right)$ are called "dynamic phases." Eq. 2.8 is an exact solution for $\hat{U}\left(t ; t_{0}\right)$ if $\hat{H}(t)$ is inhomogeneous. We note that Eq. 2.8 reduces to Eq. 2.4 in the case of a time-independent $\hat{H}(t)=\hat{H}$, which of course commutes with itself at different times. Thus we find that in the cases of time-independent and inhomogeneous time-dependent Hamiltonians, $\hat{U}\left(t ; t_{0}\right)$ may be calculated easily by exponentiating the time integral of the Hamiltonian. Eq. 2.8 does not hold in the case of a homogeneous timedependent Hamiltonian.

\section{$\hat{U}\left(t ; t_{0}\right)$ in the case of a homogeneous time-dependent Hamiltonian}

It first is worthwhile to examine the conditions under which a particular Hamiltonian $\hat{H}(t)$ is homogeneous (i.e., $\left[\hat{H}(t), \hat{H}\left(t^{\prime}\right)\right] \neq 0$ ). Consider a Hamiltonian of the general form

$$
\hat{H}(t)=a(t) \hat{A}+b(t) \hat{B}
$$


Therefore:

$$
\left[\hat{H}(t), \hat{H}\left(t^{\prime}\right)\right]=\left\{a(t) b\left(t^{\prime}\right)-b(t) a\left(t^{\prime}\right)\right\}[\hat{A}, \hat{B}]
$$

It follows that $\left[\hat{H}(t), \hat{H}\left(t^{\prime}\right)\right] \neq 0$ for all $t$ and $t^{\prime}$ if $[\hat{A}, \hat{B}] \neq 0$ and if $a(t)$ and $b(t)$ are not proportional to each other by a time-independent constant; otherwise $\hat{H}(t)$ is inhomogeneous. ${ }^{1}$ Increasingly complicated homogeneous Hamiltonians may be constructed by adding more mutually non-commuting terms in which the time coefficients are not proportional to each other. A simple example of a homogeneous Hamiltonian is the laboratory-frame Hamiltonian of a single spin that precesses due to the combination of an external magnetic field along the $z$-axis and a sinusoidally-modulated radiofrequency field along the $x$-axis: $\hat{H}(t) / \hbar=-\omega_{0} \hat{I}_{z}-2 \omega_{1} \hat{I}_{x} \cos \left(\omega_{r} t\right)$.

The expansion in Eq. 2.6 is of the form $\hat{U}\left(t ; t_{0}\right)=\prod_{n=1}^{N} \hat{U}_{n}$, where $\hat{U}_{n} \equiv \hat{U}\left(t_{n} ; t_{n-1}\right)$ $\simeq \exp \left(-i \hat{H}_{n} \Delta t / \hbar\right)$, and $t_{n}>t_{n-1}$. It follows from Eqs. 2.6 and 2.7 that the ordering of the $\hat{U}_{n}$ in the product does not matter for an inhomogeneous Hamiltonian, in which all the $\hat{H}_{n}$ commute with each other. However, if the Hamiltonian is homogeneous (such that $\left[\hat{H}_{n}, \hat{H}_{n^{\prime}}\right] \neq 0$ for $n \neq n^{\prime}$, then Eq. 2.7 does not hold. Therefore, the $N$ propagators $\hat{U}_{n}$ cannot simply be exponentiated, and their ordering in the product must be such that propagators acting earlier in time are further to the right. That is, the time-ordering of the $\hat{U}_{n}$ as written in the first line of Eq. 2.6 must be maintained.

\footnotetext{
${ }^{1}$ An interesting NMR example of a case in which the proportionality of $a(t)$ and $b(t)$ matters is the quadrupolar-driven recoupling of the dipolar interaction in homonuclear spin systems under magic-angle spinning conditions [121]. The recoupling effect relies on a non-commutivity of the high-field dipolar and quadrupolar Hamiltonians, whose spatial terms become time-dependent due to sample rotation. If the principal axes of the quadrupolar and dipolar interactions are colinear, the time-dependent spatial terms become proportional to each other, the Hamiltonian becomes inhomogeneous, and the recoupling effect vanishes.
} 
The formal solution for $\hat{U}\left(t ; t_{0}\right)$ in the case of a homogeneous Hamiltonian $\hat{H}(t)$ is written as:

$$
\hat{U}\left(t ; t_{0}\right)=T\left\{\exp \left[-\frac{i}{\hbar} \int_{t_{0}}^{t} d t^{\prime} \hat{H}\left(t^{\prime}\right)\right]\right\}
$$

where $T$ is the time-ordering operator that ensures that the operators in the expansion of $\hat{U}(t)$ are properly ordered in time. Here the time-ordering operator is defined in a somewhat different manner than that originally due to Dyson [122]:

$$
T\left\{\hat{H}(t) \hat{H}\left(t^{\prime}\right)\right\}= \begin{cases}\hat{H}(t) \hat{H}\left(t^{\prime}\right), & t \geq t^{\prime} \\ \hat{H}\left(t^{\prime}\right) \hat{H}(t), & t<t^{\prime}\end{cases}
$$

We note that $\hat{U}^{-1}\left(t ; t_{0}\right)=\hat{U}^{\dagger}\left(t ; t_{0}\right)=T_{-}\left\{\exp \left[+\frac{i}{\hbar} \int_{t_{0}}^{t} d t^{\prime} \hat{H}\left(t^{\prime}\right)\right]\right\}$, where $T_{-}$reverse timeorders the operator product, and we have used the fact that $\hat{H}^{\dagger}(t)=\hat{H}(t)$.

There are few physical cases for which Eq. 2.9 can be evaluated analytically; typically it is evaluated approximately using a technique such as average Hamiltonian theory. We observe that the time-ordering does not matter in the case of an inhomogeneous Hamiltonian, so that Eq. 2.9 reduces to Eq. 2.8.

We reiterate that once a solution for the time development operator $\hat{U}\left(t ; t_{0}\right)$ has been found, the time evolution of the system is known. Such a solution may be found numerically by a piecewise approximation to the Hamiltonian (as in Eq. 2.6), and in practice one need not proceed further. Other formalisms (e.g., frame transformations, average Hamiltonian theory, Floquet theory, etc.), some of which will be described later, are not necessary to solve the time evolution; rather, they are useful for developing insight into how 
to manipulate quantum systems through the experimental application of time-dependent perturbations.

\subsubsection{The density operator}

A detailed description of the density operator is given in $§ 1.4$. The description that follows is meant to be self-contained. Typically, the states of the quantum systems studied in NMR problems are known only statistically. A single state vector generally is no longer sufficient to describe the system completely, and it is necessary to specify the system by the density operator $\hat{\rho}(t)$ instead of a single state vector $|\psi(t)\rangle$.

We define the density operator for a system in a pure state $|\psi(t)\rangle$ as the outer product of $|\psi(t)\rangle$ with itself: $\hat{\rho}(t) \equiv|\psi(t)\rangle\langle\psi(t)|$. The generalization to a mixed statistical ensemble of discrete quantum states is:

$$
\hat{\rho}(t) \equiv \sum_{i} P_{i}\left|\psi_{i}(t)\right\rangle\left\langle\psi_{i}(t)\right|
$$

where the $P_{i}$ are the probabilities of finding the system in the state $\left|\psi_{i}(t)\right\rangle$ (i.e., the fractional populations of the states $\left.\left|\psi_{i}(t)\right\rangle\right)$. The $P_{i}$ are considered to be time independent during coherent (unitary) time evolution. Substituting Eq. 2.2 into Eq. 2.11 gives:

$$
\begin{aligned}
\hat{\rho}(t) & \equiv \sum_{i} P_{i}\left|\psi_{i}(t)\right\rangle\left\langle\psi_{i}(t)\right| \\
& =\sum_{i} P_{i} \hat{U}\left(t ; t_{0}\right)\left|\psi_{i}\left(t_{0}\right)\right\rangle\left\langle\psi_{i}\left(t_{0}\right)\right| \hat{U}^{-1}\left(t ; t_{0}\right)=\hat{U}\left(t ; t_{0}\right)\left\{\sum_{i} P_{i}\left|\psi_{i}\left(t_{0}\right)\right\rangle\left\langle\psi_{i}\left(t_{0}\right)\right|\right\} \hat{U}^{-1}\left(t ; t_{0}\right) \\
& =\hat{U}\left(t ; t_{0}\right) \hat{\rho}\left(t_{0}\right) \hat{U}^{-1}\left(t ; t_{0}\right),
\end{aligned}
$$


where $\hat{\rho}\left(t_{0}\right) \equiv \sum_{i} P_{i}\left|\psi_{i}\left(t_{0}\right)\right\rangle\left\langle\psi_{i}\left(t_{0}\right)\right|$. Eq. 2.12 is the prescription for how the density operator evolves in time, just as Eq. 2.2 is the prescription for how state vectors evolve in time.

We now consider the equation of motion for the density operator:

$$
\begin{aligned}
\frac{\partial \hat{\rho}(t)}{\partial t} & =\frac{\partial}{\partial t}\left[\sum_{i} P_{i}\left|\psi_{i}(t)\right\rangle\left\langle\psi_{i}(t)\right|\right] \\
& =\sum_{i} P_{i}\left[\frac{\partial\left|\psi_{i}(t)\right\rangle}{\partial t}\left\langle\psi_{i}(t)|+| \psi_{i}(t)\right\rangle \frac{\partial\left\langle\psi_{i}(t)\right|}{\partial t}\right] .
\end{aligned}
$$

Eq. 2.1 yields $\frac{\partial|\psi(t)\rangle}{\partial t}=\frac{1}{i \hbar} \hat{H}(t)|\psi(t)\rangle$ and $\frac{\partial\langle\psi(t)|}{\partial t}=-\frac{1}{i \hbar}\langle\psi(t)| \hat{H}^{\dagger}(t)$ (where $\hat{H}^{\dagger}(t)=\hat{H}(t)$ because the Hamiltonian is Hermitian), so that:

$$
\begin{aligned}
\frac{\partial \hat{\rho}(t)}{\partial t} & =\sum_{i} P_{i}\left[\frac{1}{i \hbar} \hat{H}(t)\left|\psi_{i}(t)\right\rangle\left\langle\psi_{i}(t)\left|-\frac{1}{i \hbar}\right| \psi_{i}(t)\right\rangle\left\langle\psi_{i}(t)\right| \hat{H}(t)\right] \\
& =\frac{1}{i \hbar}\left\{\hat{H}(t)\left[\sum_{i} P_{i}\left|\psi_{i}(t)\right\rangle\left\langle\psi_{i}(t)\right|\right]-\left[\sum_{i} P_{i}\left|\psi_{i}(t)\right\rangle\left\langle\psi_{i}(t)\right|\right] \hat{H}(t)\right\} \\
& =\frac{1}{i \hbar}\{\hat{H}(t) \hat{\rho}(t)-\hat{\rho}(t) \hat{H}(t)\}=\frac{1}{i \hbar}[\hat{H}(t), \hat{\rho}(t)] \\
\Longrightarrow i \hbar \frac{\partial \hat{\rho}(t)}{\partial t}=[\hat{H}(t), \hat{\rho}(t)] . &
\end{aligned}
$$

Eq. 2.13 is the von Neumann quantum Liouville equation of motion for the density operator. It carries the same physical content as the Schrödinger equation, but is generalized to the case of mixed statistical ensembles of quantum states. Eq. 2.13 may be solved for $\hat{\rho}(t)$ with the initial condition $\hat{\rho}\left(t_{0}\right)$. In the case of a time-independent Hamiltonian $\hat{H}(t)=\hat{H}$ the 
Liouville equation can be solved iteratively to give a power series in time:

$$
\begin{gathered}
\hat{\rho}(t)=\hat{\rho}\left(t_{0}\right)+\left(\frac{-i}{\hbar}\right)\left(t-t_{0}\right)\left[\hat{H}, \hat{\rho}\left(t_{0}\right)\right]+\frac{1}{2 !}\left(\frac{-i}{\hbar}\right)^{2}\left(t-t_{0}\right)^{2}\left[\hat{H},\left[\hat{H}, \hat{\rho}\left(t_{0}\right)\right]\right] \\
+\frac{1}{3 !}\left(\frac{-i}{\hbar}\right)^{3}\left(t-t_{0}\right)^{3}\left[\hat{H},\left[\hat{H},\left[\hat{H}, \hat{\rho}\left(t_{0}\right)\right]\right]\right]+\ldots,
\end{gathered}
$$

where the $\mathcal{O}\left(t^{n}\right)$ term involves $n$ commutators of the initial density operator with the Hamiltonian.

Finally, we consider how to use the density operator to calculate the time evolution of an observable $\hat{A}$. We make use of the completeness relation $\hat{1}=\sum_{n}|n\rangle\langle n|$ in some orthonormal basis $\{|n\rangle\}$ :

$$
\begin{aligned}
\langle\hat{A}>(t) & =\sum_{i} P_{i}\left\langle\psi_{i}(t)|\hat{A}| \psi_{i}(t)\right\rangle=\sum_{i} P_{i}\left\langle\psi_{i}(t)|\hat{A} \hat{1}| \psi_{i}(t)\right\rangle=\sum_{i} P_{i}\left\langle\psi_{i}(t)\left|\hat{A}\left(\sum_{n}|n\rangle\langle n|\right)\right| \psi_{i}(t)\right\rangle \\
& =\sum_{i} P_{i} \sum_{n}\left\langle\psi_{i}(t)|\hat{A}| n\right\rangle\left\langle n \mid \psi_{i}(t)\right\rangle=\sum_{i} P_{i} \sum_{n}\left\langle n \mid \psi_{i}(t)\right\rangle\left\langle\psi_{i}(t)|\hat{A}| n\right\rangle \\
& =\sum_{n}\left\langle n\left|\left(\sum_{i} P_{i}\left|\psi_{i}(t)\right\rangle\left\langle\psi_{i}(t)\right|\right) \hat{A}\right| n\right\rangle=\sum_{n}\langle n|\hat{\rho}(t) \hat{A}| n\rangle \\
\Longrightarrow \quad & <\hat{A}>(t)=\operatorname{Tr}\{\hat{\rho}(t) \hat{A}\}=\operatorname{Tr}\{\hat{A} \hat{\rho}(t)\}
\end{aligned}
$$

since $\operatorname{Tr}(\hat{A} \hat{B})=\operatorname{Tr}(\hat{B} \hat{A})$. The trace is invariant under a change of basis, so the choice of an arbitrary basis set $\{|n\rangle\}$ is justified.

Using Eqs. 2.13 and 2.15 it can be shown that:

$$
i \hbar \frac{\partial<\hat{A}>(t)}{\partial t}=-<[\hat{H}(t), \hat{A}]>
$$

where $\langle\cdots\rangle \equiv \operatorname{Tr}\{\hat{\rho}(t) \cdots\}$. This has a very similar form to Eq. 2.13, except for a sign 
difference.

\subsection{Magnus expansion approximation for $\hat{U}\left(t ; t_{0}\right)$}

It is easy to evaluate the propagator in the cases of time-independent and inhomogeneous time-dependent Hamiltonians, which additionally are special cases of Eq. 2.9 for a homogeneous time-dependent Hamiltonian. Therefore, the following discussion focuses strategies to approximate $\hat{U}\left(t ; t_{0}\right)$ in the case of a homogeneous Hamiltonian.

Before discussing the Magnus expansion, we first consider making a series expansion of the exponential in Eq. 2.9:

$$
\begin{aligned}
& \hat{U}\left(t ; t_{0}\right)=T\left\{\exp \left[-\frac{i}{\hbar} \int_{t_{0}}^{t} d t^{\prime} \hat{H}\left(t^{\prime}\right)\right]\right\} \\
& =T\left\{\hat{1}+\left[\left(\frac{-i}{\hbar}\right) \int_{t_{0}}^{t} d t^{\prime} \hat{H}\left(t^{\prime}\right)\right]+\frac{1}{2 !}\left[\left(\frac{-i}{\hbar}\right) \int_{t_{0}}^{t} d t^{\prime} \hat{H}\left(t^{\prime}\right)\right]^{2}+\ldots\right\} \\
& =T\left\{\hat{1}+\left[\left(\frac{-i}{\hbar}\right) \int_{t_{0}}^{t} d t^{\prime} \hat{H}\left(t^{\prime}\right)\right]+\left[\frac{1}{2 !}\left(\frac{-i}{\hbar}\right)^{2} \int_{t_{0}}^{t} d t^{\prime \prime} \int_{t_{0}}^{t} d t^{\prime} \hat{H}\left(t^{\prime \prime}\right) \hat{H}\left(t^{\prime}\right)\right]+\ldots\right\}
\end{aligned}
$$

and truncating the higher-order terms (neglecting for now consideration of the convergence of the series). This expansion (along with an interaction representation transformation, which will be discussed later) is a cornerstone of time-dependent perturbation theory, as is discussed in Appendix C. However, it is not often used in time-dependent NMR theory, and other methods of approximating the time development operator (i.e., the Magnus expansion) will be discussed below. 
Incidentally, Eq. 2.17 is equivalent to the Dyson series [122, 45]:

$$
\hat{U}\left(t ; t_{0}\right)=\hat{1}+\left[\left(\frac{-i}{\hbar}\right) \int_{t_{0}}^{t} d t^{\prime} \hat{H}\left(t^{\prime}\right)\right]+\left[\left(\frac{-i}{\hbar}\right)^{2} \int_{t_{0}}^{t} d t^{\prime \prime} \int_{t_{0}}^{t^{\prime \prime}} d t^{\prime} \hat{H}\left(t^{\prime \prime}\right) \hat{H}\left(t^{\prime}\right)\right]+\ldots
$$

where the $n^{\text {th }}$ term in the series is larger by a factor of $n$ ! than the $n^{\text {th }}$ term in Eq. 2.17, because the domain of integration is proportionally smaller. (The domain of integration also ensures proper ordering of the time variables.) This series comes easily from the iterative solution of the integral equation form of Eq. 2.3: $\hat{U}\left(t ; t_{0}\right)=\hat{1}+\left(-\frac{i}{\hbar}\right) \int_{t_{0}}^{t} d t^{\prime} \hat{H}\left(t^{\prime}\right) \hat{U}\left(t^{\prime} ; t_{0}\right)$, for $\hat{U}\left(t_{0} ; t_{0}\right)=\hat{1}$. Appendix $\mathrm{C}$ describes how the standard equations of time-dependent perturbation theory may be obtained directly from this expansion. It is important to note that in general neither Eq. 2.17 nor Eq. 2.18 is unitary if the series is truncated.

It is easy to calculate and apply the time evolution operator in the case of a timeindependent Hamiltonian $\hat{H}$ (Eq. 2.4); we therefore ask if it is possible to write an arbitrary $\hat{U}\left(t ; t_{0}\right)$ (as in Eq. 2.9) in the form:

$$
\hat{U}\left(t ; t_{0}\right)=\exp \left\{-i \hat{\bar{H}}\left(t-t_{0}\right) / \hbar\right\}
$$

where $\hat{\bar{H}}$ is an effective average time-independent "Hamiltonian" that describes the time evolution on the interval $\left[t_{0}, t\right]$. Later we will discuss when this ansatz for the propagator is appropriate. We now seek a series expansion of $\hat{\bar{H}}$ :

$$
\hat{\bar{H}}=\lambda \hat{\bar{H}}^{(1)}+\lambda^{2} \hat{\bar{H}}^{(2)}+\lambda^{3} \hat{\bar{H}}^{(3)}+\lambda^{4} \hat{\bar{H}}^{(4)}+\ldots
$$

where $\lambda$ is a parameter introduced to keep track of the order of the expansion $(\lambda=1$ 
is assumed in the end). We wish to find the expansion for $\hat{\bar{H}}$ such that all the terms are Hermitian, so a truncation of the series still will leave an approximate expression for $\hat{\bar{H}}$ that is Hermitian. Since $e^{i \hat{A}}$ is unitary if $\hat{A}$ is Hermitian, then the approximate expression for $\hat{U}\left(t ; t_{0}\right)$ that is reached by substituting a truncated Eq. 2.20 into Eq. 2.19 will be guaranteed to be unitary. It is not immediately clear that Eq. 2.20 will converge and that the $\hat{\bar{H}}^{(n)}$ decrease in size with increasing $n$; later rough expressions for the relative sizes of the $\hat{H}^{(n)}$ will be derived. Eq. 2.20 is called the "Magnus expansion" of the average Hamiltonian $\hat{H}$. (It should be noted that the more recent literature uses the convention that the Magnus series begins with a first-order term as in Eq. 2.20, while the older literature uses the convention that the series begins with a zeroth-order term.)

The discussion now closely follows Appendix B of Haeberlen [123]. (An alternative reference is the description of the Magnus expansion by Pechukas and Light [124].) Eq. 2.19 is expanded as (for simplicity we choose $t_{0}=0$ ):

$$
\begin{aligned}
\hat{U}(t) & =\exp (-i \hat{\bar{H}} t / \hbar) \\
& =\hat{1}+\left(\frac{-i t}{\hbar}\right) \hat{\bar{H}}+\frac{1}{2 !}\left(\frac{-i t}{\hbar}\right)^{2}(\hat{\bar{H}})^{2}+\frac{1}{3 !}\left(\frac{-i t}{\hbar}\right)^{3}(\hat{\bar{H}})^{3}+\ldots
\end{aligned}
$$

For simplicity the initial condition $t_{0}=0$ has been chosen. Inserting the Magnus expansion 
for $\hat{\bar{H}}$ (Eq. 2.20) into this expression yields (up to $\mathcal{O}\left(\lambda^{4}\right)$ ):

$$
\begin{aligned}
\hat{U}(t)= & \hat{1}+\left(\frac{-i t}{\hbar}\right)\left\{\lambda \hat{\bar{H}}^{(1)}+\lambda^{2} \hat{\bar{H}}^{(2)}+\lambda^{3} \hat{\bar{H}}^{(3)}+\lambda^{4} \hat{\bar{H}}^{(4)}+\ldots\right\} \\
+ & \frac{1}{2 !}\left(\frac{-i t}{\hbar}\right)^{2}\left\{\lambda^{2}\left(\hat{\bar{H}}^{(0)}\right)^{2}+\lambda^{3} \hat{\bar{H}}^{(1)} \hat{\bar{H}}^{(2)}+\lambda^{3} \hat{\bar{H}}^{(2)} \hat{\bar{H}}^{(1)}\right. \\
& \left.+\lambda^{4}\left(\hat{\bar{H}}^{(2)}\right)^{2}+\lambda^{4} \hat{\bar{H}}^{(1)} \hat{\bar{H}}^{(3)}+\lambda^{4} \hat{\bar{H}}^{(3)} \hat{\bar{H}}^{(1)}+\ldots\right\} \\
+ & \frac{1}{3 !}\left(\frac{-i t}{\hbar}\right)^{3}\left\{\lambda^{3}\left(\hat{\bar{H}}^{(1)}\right)^{3}+\lambda^{4}\left(\hat{\bar{H}}^{(1)}\right)^{2} \hat{\bar{H}}^{(2)}+\lambda^{4} \hat{\bar{H}}^{(1)} \hat{\bar{H}}^{(2)} \hat{\bar{H}}^{(1)}+\lambda^{4} \hat{\bar{H}}^{(2)}\left(\hat{\bar{H}}^{(1)}\right)^{2}+\ldots\right\} \\
+ & \frac{1}{4 !}\left(\frac{-i t}{\hbar}\right)^{4}\left\{\lambda^{4}\left(\hat{\bar{H}}^{(1)}\right)^{4}+\ldots\right\} \\
+ & \ldots,
\end{aligned}
$$

where the expressions in braces come from direct expansions of $(\hat{\bar{H}})^{n}$. The terms may be regrouped by their order of the expansion parameter $\lambda$ :

$$
\begin{aligned}
\hat{U}(t)= & \hat{1} \\
& +\lambda\left\{\left(\frac{-i t}{\hbar}\right) \hat{\bar{H}}^{(1)}\right\} \\
& +\lambda^{2}\left\{\left(\frac{-i t}{\hbar}\right) \hat{\bar{H}}^{(2)}+\frac{1}{2 !}\left(-\frac{i t}{\hbar}\right)^{2}\left(\hat{\bar{H}}^{(1)}\right)^{2}\right\} \\
& +\lambda^{3}\left\{\left(\frac{-i t}{\hbar}\right) \hat{\bar{H}}^{(3)}+\frac{1}{2 !}\left(-\frac{i t}{\hbar}\right)^{2}\left[\hat{\bar{H}}^{(1)} \hat{\bar{H}}^{(2)}+\hat{\bar{H}}^{(2)} \hat{\bar{H}}^{(1)}\right]+\frac{1}{3 !}\left(-\frac{i t}{\hbar}\right)^{3}\left(\hat{\bar{H}}^{(1)}\right)^{3}\right\} \\
& +\lambda^{4}\left\{\left(\frac{-i t}{\hbar}\right) \hat{\bar{H}}^{(4)}+\frac{1}{2 !}\left(-\frac{i t}{\hbar}\right)^{2} \hat{\bar{H}}^{(1)} \hat{\bar{H}}^{(3)}+\hat{\bar{H}}^{(3)} \hat{\bar{H}}^{(1)}\right] \\
& \left.+\frac{1}{3 !}\left(-\frac{i t}{\hbar}\right)^{3}\left[\left(\hat{\bar{H}}^{(1)}\right)^{2} \hat{\bar{H}}^{(2)}+\hat{\bar{H}}^{(2)}\left(\hat{\bar{H}}^{(1)}\right)^{2}+\hat{\bar{H}}^{(1)} \hat{\bar{H}}^{(2)} \hat{\bar{H}}^{(1)}\right]+\frac{1}{4 !}\left(\frac{-i t}{\hbar}\right)^{4}\left(\hat{\bar{H}}^{(1)}\right)^{4}\right\} \\
& +\ldots
\end{aligned}
$$

The Magnus series expansion (Eq. 2.21) of the ansatz for the propagator (Eq. 2.19) now must be equated with a series expansion of the formal expression for the propagator (Eq. 2.9). 
This is done following the method of Haeberlen [123], which is lengthy but pedagogically useful. Reconsider the (properly time-ordered!) discrete approximation to $\hat{U}(t)$ in Eq. 2.6 in the case of a homogeneous Hamiltonian (i.e., $\left[\hat{H}_{n}, \hat{H}_{n^{\prime}}\right] \neq 0$ for some or all $\left.n \neq n^{\prime}\right)$. We expand:

$\exp \left(-i \lambda \hat{H}_{n} \Delta t_{n} / \hbar\right)=\hat{1}+\lambda\left(\frac{-i \Delta t_{n}}{\hbar}\right) \hat{H}_{n}+\lambda^{2} \frac{1}{2 !}\left(\frac{-i \Delta t_{n}}{\hbar}\right)^{2}\left(\hat{H}_{n}\right)^{2}+\lambda^{3} \frac{1}{3 !}\left(\frac{-i \Delta t_{n}}{\hbar}\right)^{3}\left(\hat{H}_{n}\right)^{3}+\ldots$ where again the parameter $\lambda$ has been inserted to keep track of orders of the expansion. Then the propagator from Eq. 2.6 becomes:

$$
\begin{aligned}
& \hat{U}(t)=e^{-i \hat{H}_{N} \Delta t_{N} / \hbar} e^{-i \hat{H}_{N-1} \Delta t_{N-1} / \hbar} \ldots e^{-i \hat{H}_{2} \Delta t_{2} / \hbar} e^{-i \hat{H}_{1} \Delta t_{1} / \hbar} \\
& =\left[\hat{1}+\lambda(-i / \hbar) \hat{H}_{N} \Delta t_{N}+\left(\lambda^{2} / 2 !\right)(-i / \hbar)^{2}\left(\hat{H}_{N} \Delta t_{N}\right)^{2}+\left(\lambda^{3} / 3 !\right)(-i / \hbar)^{3}\left(\hat{H}_{N} \Delta t_{N}\right)^{3}+\ldots\right] \\
& \times\left[\hat{1}+\lambda(-i / \hbar)\left(\hat{H}_{N-1} \Delta t_{N-1}\right)+\left(\lambda^{2} / 2 !\right)(-i / \hbar)^{2}\left(\hat{H}_{N-1} \Delta t_{N-1}\right)^{2}\right. \\
& \left.\quad+\left(\lambda^{3} / 3 !\right)(-i / \hbar)^{3}\left(\hat{H}_{N-1} \Delta t_{N-1}\right)^{3}+\ldots\right] \\
& \quad \vdots \quad \\
& \times\left[\hat{1}+\lambda(-i / \hbar) \hat{H}_{2} \Delta t_{2}+\left(\lambda^{2} / 2 !\right)(-i / \hbar)^{2}\left(\hat{H}_{2} \Delta t_{2}\right)^{2}+\left(\lambda^{3} / 3 !\right)(-i / \hbar)^{3}\left(\hat{H}_{2} \Delta t_{2}\right)^{3}+\ldots\right] \\
& \times\left[\hat{1}+\lambda(-i / \hbar) \hat{H}_{1} \Delta t_{1}+\left(\lambda^{2} / 2 !\right)(-i / \hbar)^{2}\left(\hat{H}_{1} \Delta t_{1}\right)^{2}+\left(\lambda^{3} / 3 !\right)(-i / \hbar)^{3}\left(\hat{H}_{1} \Delta t_{1}\right)^{3}+\ldots\right] .
\end{aligned}
$$


Expanding the product and regrouping by orders of $\lambda$ (up to $\mathcal{O}\left(\lambda^{3}\right)$ ) gives:

$$
\begin{aligned}
& \hat{U}(t)=\hat{1} \\
& +\lambda\left(\frac{-i}{\hbar}\right)\left\{\hat{H}_{1} \Delta t_{1}+\hat{H}_{2} \Delta t_{2}+\ldots+\hat{H}_{N-1} \Delta t_{N-1}+\hat{H}_{N} \Delta t_{N}\right\} \\
& +\lambda^{2} \frac{1}{2 !}\left(\frac{-i}{\hbar}\right)^{2}\left\{\left[\left(\hat{H}_{1} \Delta t_{1}\right)^{2}+\left(\hat{H}_{2} \Delta t_{2}\right)^{2}+\ldots+\left(\hat{H}_{N-1} \Delta t_{N-1}\right)^{2}+\left(\hat{H}_{N} \Delta t_{N}\right)^{2}\right]\right. \\
& +2 ! \cdot\left[\hat{H}_{2} \hat{H}_{1} \Delta t_{2} \Delta t_{1}+\hat{H}_{3} \hat{H}_{1} \Delta t_{3} \Delta t_{1}+\hat{H}_{4} \hat{H}_{1} \Delta t_{4} \Delta t_{1}+\ldots\right. \\
& \left.\left.\quad+\hat{H}_{3} \hat{H}_{2} \Delta t_{3} \Delta t_{2}+\ldots+\hat{H}_{N} \hat{H}_{N-1} \Delta t_{N} \Delta t_{N-1}\right]\right\} \\
& +\lambda^{3} \frac{1}{3 !}\left(\frac{-i}{\hbar}\right)^{3}\left\{\left(\hat{H}_{1} \Delta t_{1}\right)^{3}+\ldots+(3 ! / 2 !) \cdot\left[\hat{H}_{2}\left(\hat{H}_{1}\right)^{2} \Delta t_{2}\left(\Delta t_{1}\right)^{2}+\left(\hat{H}_{2}\right)^{2} \hat{H}_{1}\left(\Delta t_{1}\right)^{2} \Delta t_{2}+\ldots\right]\right. \\
& \left.\quad+3 ! \cdot\left[\hat{H}_{3} \hat{H}_{2} \hat{H}_{1} \Delta t_{3} \Delta t_{2} \Delta t_{1}+\ldots\right]\right\} \\
& +\ldots
\end{aligned}
$$

Factorial functions have been factored out in front of each term of $\mathcal{O}\left(\lambda^{n}\right)$ to emphasize the similarity between these terms and those in the expansion of an exponential function, as will be seen below. Note that the expressions in braces are time-ordered, since Eq. 2.6 is time-ordered. These expressions can be written more compactly by using the timeordering operator. The time-ordered propagator of Eq. 2.9, when expressed in the piecewise 
approximation of Eqs. 2.6 and 2.22, becomes:

$$
\begin{aligned}
\hat{U}(t)= & \hat{1} \\
& +\lambda\left(\frac{-i}{\hbar}\right)\left\{\hat{H}_{1} \Delta t_{1}+\hat{H}_{2} \Delta t_{2}+\ldots+\hat{H}_{N-1} \Delta t_{N-1}+\hat{H}_{N} \Delta t_{N}\right\} \\
& +\lambda^{2} \frac{1}{2 !}\left(\frac{-i}{\hbar}\right)^{2} T\left\{\left[\hat{H}_{1} \Delta t_{1}+\hat{H}_{2} \Delta t_{2}+\ldots+\hat{H}_{N-1} \Delta t_{N-1}+\hat{H}_{N} \Delta t_{N}\right]^{2}\right\} \\
& +\lambda^{3} \frac{1}{3 !}\left(\frac{-i}{\hbar}\right)^{3} T\left\{\left[\hat{H}_{1} \Delta t_{1}+\hat{H}_{2} \Delta t_{2}+\ldots+\hat{H}_{N-1} \Delta t_{N-1}+\hat{H}_{N} \Delta t_{N}\right]^{3}\right\} \\
& +\ldots \\
\Longrightarrow \hat{U}(t)= & T\left\{\hat{1}+\lambda\left(\frac{-i}{\hbar}\right)\left[\sum_{i=1}^{N} \hat{H}_{i} \Delta t_{i}\right]+\lambda^{2} \frac{1}{2 !}\left(\frac{-i}{\hbar}\right)^{2}\left[\sum_{i=1}^{N} \hat{H}_{i} \Delta t_{i}\right]^{2}\right. \\
& \left.\quad+\lambda^{3} \frac{1}{3 !}\left(\frac{-i}{\hbar}\right)^{3}\left[\sum_{i=1}^{N} \hat{H}_{i} \Delta t_{i}\right]^{3}+\ldots\right\} .
\end{aligned}
$$

In the continuum limit as $N \rightarrow \infty$, we have $\sum_{i} \hat{H}_{i} \Delta t_{i} \rightarrow \int_{0}^{t} d t^{\prime} \hat{H}\left(t^{\prime}\right)$. Thus Eq. 2.23 is the discrete approximation to the exponential expansion of the propagator in Eq. 2.17. Therefore, the second way to reach Eq. 2.23 is simply to expand directly the exponential in $\hat{U}(t)=T\left\{\exp \left[-\lambda \frac{i}{\hbar} \int_{t_{0}}^{t} d t^{\prime} \hat{H}\left(t^{\prime}\right)\right]\right\}$ in the manner of Eq. 2.17.

The propagator expansion in Eq. 2.23 may be equated with the expansion of the Magnus series propagator ansatz of Eq. 2.21 in order to find expressions for the terms in 
the Magnus series. Equating terms of like order $\lambda$ :

$$
\begin{aligned}
& \hat{1}=\hat{1} \\
& \lambda\left\{\left(\frac{-i t}{\hbar}\right) \hat{\bar{H}}^{(1)}\right\}=\lambda\left(\frac{-i}{\hbar}\right) T\left\{\sum_{i=1}^{N} \hat{H}_{i} \Delta t_{i}\right\} \\
& \lambda^{2}\left\{\left(\frac{-i t}{\hbar}\right) \hat{\bar{H}}^{(2)}+\frac{1}{2 !}\left(\frac{-i t}{\hbar}\right)^{2}\left(\hat{\bar{H}}^{(1)}\right)^{2}\right\}=\lambda^{2} \frac{1}{2 !}\left(\frac{-i}{\hbar}\right)^{2} T\left\{\left[\sum_{i=1}^{N} \hat{H}_{i} \Delta t_{i}\right]^{2}\right\} \\
& \lambda^{3}\left\{\left(\frac{-i t}{\hbar}\right) \hat{\bar{H}}^{(3)}+\frac{1}{2 !}\left(\frac{-i t}{\hbar}\right)^{2}\left[\hat{\bar{H}}^{(1)} \hat{H}^{(2)}+\hat{\bar{H}}^{(2)} \hat{H}^{(1)}\right]+\frac{1}{3 !}\left(\frac{-i t}{\hbar}\right)^{3}\left(\hat{\bar{H}}^{(1)}\right)^{3}\right\} \\
& \quad=\lambda^{3} \frac{1}{3 !}\left(\frac{-i}{\hbar}\right)^{3} T\left\{\left[\sum_{i=1}^{N} \hat{H}_{i} \Delta t_{i}\right]^{3}\right\}
\end{aligned}
$$

We find that

$$
\left.\hat{\bar{H}}^{(1)}=\frac{1}{t} T\left\{\sum_{i=1}^{N} \hat{H}_{i} \Delta t_{i}\right]\right\}=\frac{1}{t} \sum_{i=1}^{N} \hat{H}_{i} \Delta t_{i} .
$$

Upon substituting this result into the $\mathcal{O}\left(\lambda^{2}\right)$ equation in Eq. 2.24 and solving for $\hat{\bar{H}}^{(2)}$, we 
have $^{2}$ :

$$
\begin{aligned}
\hat{\bar{H}}^{(2)} & =-\frac{(i / \hbar)}{2 t}\left(T\left\{\left[\sum_{i=1}^{N} \hat{H}_{i} \Delta t_{i}\right]^{2}\right\}-\left[T\left\{\sum_{i=1}^{N} \hat{H}_{i} \Delta t_{i}\right\}\right]^{2}\right) \\
& =-\frac{(i / \hbar)}{2 t}\left(\left[T\left\{\sum_{i, j} \hat{H}_{i} \hat{H}_{j} \Delta t_{i} \Delta t_{j}\right\}\right]-\left[\sum_{i, j} \hat{H}_{i} \hat{H}_{j} \Delta t_{i} \Delta t_{j}\right]\right) \\
& =-\frac{(i / \hbar)}{2 t}\left(\left[\sum_{i}\left(\hat{H}_{i} \Delta t_{i}\right)^{2}+2 \sum_{i>j} \hat{H}_{i} \hat{H}_{j} \Delta t_{i} \Delta t_{j}\right]\right. \\
& \left.=-\frac{(i / \hbar)}{2 t}\left(\sum_{i>j}\left(\hat{H}_{i} \Delta t_{i}\right)^{2}+\sum_{i>j} \hat{H}_{j} \hat{H}_{j} \Delta t_{i} \Delta t_{j}+\sum_{i<j} \hat{H}_{i} \hat{H}_{j} \Delta t_{i} \Delta t_{j}\right]\right) \\
& =-\frac{(i / \hbar)}{2 t}\left(\sum_{i>j} \hat{H}_{i} \hat{H}_{j} \Delta t_{i} \Delta t_{j}\right) \\
& =-\frac{(i / \hbar)}{2 t}\left(\sum_{i>j}\left(\hat{H}_{i} \hat{H}_{j}-\hat{H}_{j}-\sum_{i>j} \hat{H}_{j} \hat{H}_{i} \Delta t_{j} \Delta t_{i}\right)\right. \\
\hat{\bar{H}}^{(2)} & =-\frac{(i / \hbar)}{2 t}\left(\sum_{i>j}^{N}\left[\hat{H}_{i}, \hat{H}_{j}\right] \Delta t_{i} \Delta t_{j}\right) .
\end{aligned}
$$

Similar discrete approximations to the higher $\hat{\bar{H}}^{(n)}$ may be developed by extending Eq. 2.24. It can be seen that the terms $t \hat{\bar{H}}^{(1)}$ and $\hat{t}^{(2)}$ are indeed of first and second order in the smallness parameter $\lambda$ (or alternatively, in the $\Delta t_{n}$ ), and therefore it does appear that

${ }^{2}$ As an aside, it has been noted by Haeberlen [123] that for a Hamiltonian that is piecewise constant over only two finite time intervals $\Delta t_{1}$ and $\Delta t_{2}$, the expansion of Eq. 2.6 given by Eqs. 2.25, 2.26 and their higher-order analogs may be used to derive a version of the Baker-Campbell-Hausdorff relation:

$$
\begin{aligned}
\hat{A} & \equiv \hat{H}_{2} \Delta t_{2} / \hbar, \quad \hat{B} \equiv \hat{H}_{1} \Delta t_{1} / \hbar \\
\hat{U}(t) & =\hat{U}_{2}\left(\Delta t_{2}\right) \hat{U}_{1}\left(\Delta t_{1}\right)=e^{-i \hat{H}_{2} \Delta t_{2} / \hbar} e^{-i \hat{H}_{1} \Delta t_{1} / \hbar} \equiv e^{-i \hat{A}} e^{-i \hat{B}} \\
& =\exp \left\{-\frac{i t}{\hbar}\left(\hat{\bar{H}}^{(1)}+\hat{\bar{H}}^{(2)}+\hat{\bar{H}}^{(3)}+\ldots\right)\right\} \\
& =\exp \left\{-\frac{i t}{\hbar}\left[\frac{1}{t}\left(\hat{H}_{1} \Delta t_{1}+\hat{H}_{2} \Delta t_{2}\right)-\frac{i}{2 t \hbar}\left(\hat{H}_{2} \Delta t_{2} \hat{H}_{1} \Delta t_{1}-\hat{H}_{1} \Delta t_{1} \hat{H}_{2} \Delta t_{2}\right)+\hat{\bar{H}}^{(3)}+\ldots\right]\right\} \\
\Longrightarrow e^{-i \hat{A}} e^{-i \hat{B}} & =\exp \left\{(-i)(\hat{A}+\hat{B})+\frac{(-i)^{2}}{2}([\hat{A}, \hat{B}])+\frac{(-i)^{3}}{12}([\hat{A},[\hat{A}, \hat{B}]+[[\hat{A}, \hat{B}], \hat{B}])+\ldots\}\right.
\end{aligned}
$$


successive terms in the Magnus series should decrease in size. This of course depends on the length of time $t$ over which the series is calculated. More details on the convergence of the Magnus series will be given later.

We now take the continuum limits of Eqs. 2.25 and 2.26, and also give the result for the third-order term:

$$
\begin{aligned}
\hat{\bar{H}}^{(1)} & =\frac{1}{t} \int_{0}^{t} d t^{\prime} \hat{H}\left(t^{\prime}\right) \\
\hat{\bar{H}}^{(2)} & =-\frac{(i / \hbar)}{2 t} \int_{0}^{t} d t^{\prime \prime} \int_{0}^{t^{\prime \prime}} d t^{\prime}\left[\hat{H}\left(t^{\prime \prime}\right), \hat{H}\left(t^{\prime}\right)\right] \\
\hat{\bar{H}}^{(3)} & =-\frac{\left(1 / \hbar^{2}\right)}{6 t} \int_{0}^{t} d t^{\prime \prime \prime} \int_{0}^{t^{\prime \prime \prime}} d t^{\prime \prime} \int_{0}^{t^{\prime \prime}} d t^{\prime}\left\{\left[\hat{H}\left(t^{\prime \prime \prime}\right),\left[\hat{H}\left(t^{\prime \prime}\right), \hat{H}\left(t^{\prime}\right)\right]\right]+\left[\left[\hat{H}\left(t^{\prime \prime \prime}\right), \hat{H}\left(t^{\prime \prime}\right)\right], \hat{H}\left(t^{\prime}\right)\right]\right\}
\end{aligned}
$$

where the domain of integration of the multiple integrals ensures that $0 \leq t^{\prime} \leq t^{\prime \prime} \leq t^{\prime \prime \prime} \leq$ $\cdots \leq t$ for each value of the integrand, so that proper time-ordering of the Hamiltonian operators is enforced. Note that Eq. 2.27 is just the time average of the Hamiltonian $\hat{H}\left(t^{\prime}\right)$ on the interval $[0, t]$; Eqs. 2.28, 2.29 are higher-order corrections to the first-order average Hamiltonian. Furthermore, if $\hat{H}(t)$ is inhomogeneous, then all the commutators in the $\hat{\bar{H}}^{(n)}$ are zero, and $\hat{H}=\hat{\bar{H}}^{(1)}$. We observe that $\hat{\bar{H}}^{(1)} t / \hbar$ equals the dynamic phase operator $\hat{\Phi}(t)$ in Eq. 2.8, so that Eq. 2.19 reduces to Eq. 2.8 in the case of an inhomogeneous Hamiltonian.

The expressions Eqs. 2.27-2.29 for the Magnus series (upon setting $\lambda=1$ in Eq. 2.20) are the key results used in average Hamiltonian theory to calculate effective propagators over a given time interval. It is seldom necessary in most practical applications to extend the series to the third-order term or beyond. 


\section{Validity of the Magnus expansion}

It is pertinent to ask under which conditions is a Magnus expansion of the propagator valid. We consider the time evolution of a quantum system from $t=0$ to some time $t=t_{c}$. A necessary condition for the Magnus expansion treatment to be acceptable is that the Magnus series (Eq. 2.20) must converge on the interval $\left[0, t_{c}\right]$. We examine some of the conditions of convergence.

Ignoring for the moment the time dependence of the Hamiltonian, we estimate $\|\hat{H}(t)\| \sim \hbar \omega_{H}$. We define $\omega_{c} \equiv 2 \pi / t_{c}$. It follows from Eq. 2.27 that $\hat{\bar{H}}^{(1)}=\frac{1}{t_{c}} \int_{0}^{t_{c}} d t^{\prime} \hat{H}\left(t^{\prime}\right) \sim$ $\frac{1}{t_{c}}\left(\hbar \omega_{H}\right) t_{c}=\hbar \omega_{H}$, where one power of $t_{c}$ has come in from the integration from $t=0$ to $t_{c}$. We estimate $\left[\hat{H}\left(t^{\prime \prime}\right), \hat{H}\left(t^{\prime}\right)\right] \sim\left(\hbar \omega_{H}\right)^{2}$, so that $\hat{\bar{H}}^{(2)} \sim \frac{\hbar^{-1}}{t_{c}}\left(\hbar \omega_{H}\right)^{2}\left(t_{c}\right)\left(t_{c}\right)=\hbar\left(\omega_{H}\right)^{2}\left(t_{c}\right)$. Continuing this reasoning:

$$
\|\hat{\bar{H}}\|=\left\|\hat{\bar{H}}^{(1)}+\hat{\bar{H}}^{(2)}+\hat{\bar{H}}^{(3)}+\ldots\right\| \sim \hbar \omega_{H}\left[1+\left(\omega_{H} t_{c}\right)+\left(\omega_{H} t_{c}\right)^{2}+\ldots\right]
$$

This crude treatment suggests that successive terms of the Magnus series decrease by a factor of $\omega_{H} t_{c}=2 \pi\left(\omega_{H} / \omega_{c}\right)$. The approximate series given above certainly diverges if $\omega_{H} t_{c} \geq 1$, so that $t_{c}<\omega_{H}^{-1}$ is a rough criterion for the convergence of the Magnus series. In the context of average Hamiltonian theory (to be discussed later), $t_{c}$ is chosen to be the "cycle time": i.e., the period of the time evolution if the system is periodic. Therefore, in this case our convergence criterion on the interval $\left[0, t_{c}\right]$ may be interpreted as indicating that the cycle time $t_{c}$ should be greater than the reciprocal "size" of the Hamiltonian $\omega_{H}^{-1}$ (as measured 
in reciprocal angular frequency units). Maricq [125] notes that a better condition for the convergence of the Magnus series up to some time $t_{c}$ is: $\frac{1}{2 \pi} \int_{0}^{t_{c}} d t^{\prime}\left|\omega_{i}\left(t^{\prime}\right)-\omega_{j}\left(t^{\prime}\right)\right|<1$ for all pairs of time-dependent instantaneous eigenvalues $\hbar \omega_{i}(t)$ of $\hat{H}(t)$.

We now ask how many terms in the Magnus series are necessary to describe accurately the time evolution of the system. Of course, this depends on the accuracy needed for a particular calculation. We might estimate that the system evolves under $\hat{\bar{H}}^{(n)}$ at frequencies on the order of $\omega_{n} \sim\left\|\hat{\bar{H}}^{(n)}\right\| / \hbar$. (Here we have used the supremum norm definition of the magnitude of an operator, in which $\|\hat{A}\|$ is equal to the largest eigenvalue of $\hat{A}$.) Therefore, we estimate that $\hat{\bar{H}}^{(n)}$ starts to contribute significantly to the time evolution when the system oscillates one or more times under $\hat{\bar{H}}^{(n)}$ : i.e., when $h /\left\|\hat{\bar{H}}^{(n)}\right\| \leq t_{c}$. In practice, it may be best just to calculate explicitly the first few $\hat{\bar{H}}^{(n)}$ and see how large they are; after all, certain $\hat{\bar{H}}^{(n)}$ may even vanish for a particular choice of $\hat{H}(t)$ and $t_{c}$.

As a final note, we consider the conditions under which Eq. 2.19 for the average Hamiltonian expression for the propagator is valid. Maricq [126] has observed that in the case of a non-commuting (homogeneous) $\hat{H}(t)$, the expectation that there is a simple exponential solution for the propagator is too rigid, and therefore there may be cases in which the average Hamiltonian solution $\hat{\bar{H}}(t)$ does not exist. If we specialize to the case of a periodic Hamiltonian $\left(\hat{H}\left(t+n t_{c}\right)=\hat{H}(t)\right.$, where $n$ is an integer $)$, then the Floquet theorem $[126,127]$ states that the propagator for even a homogeneous Hamiltonian may be written 
of the form

$$
\begin{aligned}
\hat{U}(t) & =\hat{F}(t) \exp (-i \hat{\bar{H}} t / \hbar) \\
\Longrightarrow \hat{U}\left(t_{c}\right) & =\hat{F}\left(t_{c}\right) \exp \left(-i \hat{\bar{H}} t_{c} / \hbar\right) \\
& =\exp \left(-i \hat{\bar{H}} t_{c} / \hbar\right),
\end{aligned}
$$

where $\hat{F}(t)$ is periodic with period $t_{c}$ (subject to the initial condition $\hat{U}(0)=\hat{1} \Longrightarrow \hat{F}(0)=$ $\hat{F}\left(t_{c}\right)=\hat{1}$ ), and $\hat{\bar{H}}$ is Hermitian. Eq. 2.31 is equivalent to Eq. 2.19 when the system is observed only at $t=0$ and $t=t_{c}$, but Eq. 2.30 is valid for all times. The generalization of Eq. 2.31 to the case of "stroboscopic observation"-i.e., when the system is observed only at $t=n t_{c}$ ( $n$ integral) - forms the foundation of average Hamiltonian theory in NMR.

\subsection{Frames and representations}

We begin with a very brief review of our development of the formalism of timedependent quantum mechanics. A state vector $|\psi(t)\rangle$ obeying the Schrödinger Equation (Eq. 2.1) $i \hbar \frac{\partial}{\partial t}|\psi(t)\rangle=\hat{H}(t)|\psi(t)\rangle$ evolves as $|\psi(t)\rangle=\hat{U}\left(t ; t_{0}\right)\left|\psi\left(t_{0}\right)\right\rangle$ (Eq. 2.2), where in general $\hat{U}\left(t ; t_{0}\right)=T\left\{\exp \left[-\frac{i}{\hbar} \int_{t_{0}}^{t} d t^{\prime} \hat{H}\left(t^{\prime}\right)\right]\right\}$ (Eq. 2.9). The density operator of the system evolves as $\hat{\rho}(t)=\hat{U}\left(t ; t_{0}\right) \hat{\rho}(0) \hat{U}^{-1}\left(t ; t_{0}\right)$ (Eq. 2.12). The expectation value of an observable $\hat{A}$ evolves as $<\hat{A}>(t)=\operatorname{Tr}\{\hat{\rho}(t) \hat{A}\}($ Eq. 2.15). 


\subsubsection{Changes of frame}

We now consider the equation of motion for a state vector subjected to a unitary

rotation in the Hilbert space $\hat{U}_{R} \cdot{ }^{3}$ This transformation is sometimes called a change of frame. The rotation may be in general time-dependent, such that $\hat{U}_{R}=\hat{U}_{R}(t)$, but here we suppress the time $t$ in the notation. We define:

$$
\hat{U}_{R}\left|\phi_{R}(t)\right\rangle \equiv|\psi(t)\rangle
$$

where $|\psi(t)\rangle$ obeys Eq. 2.1, and $\left|\phi_{R}(t)\right\rangle \equiv \hat{U}_{R}^{-1}|\psi(t)\rangle .{ }^{4}$ The equation of motion for $\left|\phi_{R}(t)\right\rangle$ directly follows from Eqs. 2.1 and 2.32:

$$
\begin{aligned}
& i \hbar \frac{\partial|\psi(t)\rangle}{\partial t}=\hat{H}(t)|\psi(t)\rangle \\
\Longrightarrow & i \hbar \frac{\partial}{\partial t}\left(\hat{U}_{R}\left|\phi_{R}(t)\right\rangle\right)=\hat{H}(t)\left(\hat{U}_{R}\left|\phi_{R}(t)\right\rangle\right) \\
\Longrightarrow & i \hbar\left(\frac{\partial \hat{U}_{R}}{\partial t}\left|\phi_{R}(t)\right\rangle+\hat{U}_{R} \frac{\partial\left|\phi_{R}(t)\right\rangle}{\partial t}\right)=\hat{H}(t) \hat{U}_{R}\left|\phi_{R}(t)\right\rangle \\
\Longrightarrow & i \hbar\left(\hat{U}_{R}^{-1} \frac{\partial \hat{U}_{R}}{\partial t}\left|\phi_{R}(t)\right\rangle+\hat{U}_{R}^{-1} \hat{U}_{R} \frac{\partial\left|\phi_{R}(t)\right\rangle}{\partial t}\right)=\hat{U}_{R}^{-1} \hat{H}(t) \hat{U}_{R}\left|\phi_{R}(t)\right\rangle
\end{aligned}
$$

Now, $\hat{U}_{R}^{-1} \hat{U}_{R}=\hat{1}$. Upon rearranging:

$$
\begin{array}{r}
i \hbar \frac{\partial\left|\phi_{R}(t)\right\rangle}{\partial t}=\hat{\widetilde{H}}(t)\left|\phi_{R}(t)\right\rangle ; \\
\hat{\widetilde{H}}(t) \equiv \hat{U}_{R}^{-1} \hat{H}(t) \hat{U}_{R}-i \hbar \hat{U}_{R}^{-1} \frac{\partial \hat{U}_{R}}{\partial t}
\end{array}
$$

\footnotetext{
${ }^{3} \hat{U}_{R}$ is not to be confused with the time development operator.

${ }^{4}$ We just as well could have chosen the convention $\left|\phi_{R}(t)\right\rangle \equiv \hat{U}_{R}|\psi(t)\rangle$; in that case $\hat{U}_{R}$ and $\hat{U}_{R}^{-1}$ would have to be transposed in the following equations.
} 
We see that the rotated state $\left|\phi_{R}(t)\right\rangle$ obeys a Schrödinger-type equation with an effective Hamiltonian $\hat{\widetilde{H}}(t)$ as defined in Eq. 2.35. This effective Hamiltonian is not just $\hat{H}(t)$ rotated by $\hat{U}_{R}$, except in the case of a time-independent $\hat{U}_{R}$ (i.e., one for which $\left.\frac{\partial \hat{U}_{R}}{\partial t}=0\right)$. Since the rotated states obey an effective Schrödinger equation, their time evolution already has been solved:

$$
\begin{aligned}
\left|\phi_{R}(t)\right\rangle & =\hat{\widetilde{U}}(t)\left|\phi_{R}\left(t_{0}\right)\right\rangle \\
\Longrightarrow \hat{\rho}_{R}(t) & =\hat{\widetilde{U}}(t) \hat{\rho}_{R}\left(t_{0}\right) \hat{\widetilde{U}}^{-1}(t) ; \\
\hat{\widetilde{U}}\left(t ; t_{0}\right) & =T\left\{\exp \left[-\frac{i}{\hbar} \int_{t_{0}}^{t} d t^{\prime} \hat{\widetilde{H}}\left(t^{\prime}\right)\right]\right\} .
\end{aligned}
$$

It follows from Eq. 2.32 that $\hat{\rho}(t)=\hat{U}_{R} \hat{\rho}_{R}(t) \hat{U}_{R}^{-1}$. This implies that $\hat{\rho}\left(t_{0}\right)=\hat{\rho}_{R}\left(t_{0}\right)$ if $\hat{U}_{R}\left(t=t_{0} ; t_{0}\right)=\hat{1}$ (as is often chosen to be the case), but this equation does not hold for a time-independent $\hat{U}_{R} \neq \hat{1}$. It should be stressed that the propagator $\hat{\tilde{U}}(t)$ in the transformed frame is not simply $\hat{U}(t)$ rotated by $\hat{U}_{R}$; rather, it is an effective propagator in the new frame calculated from a new "effective" Hamiltonian.

Upon using Eq. 2.15 the expectation value of an observable can be calculated in the transformed frame:

$$
\begin{aligned}
<\hat{A}>(t) & =\operatorname{Tr}\left\{\left(\hat{U}_{R} \hat{\rho}_{R}(t) \hat{U}_{R}^{-1}\right) \hat{A}\right\} \\
& =\operatorname{Tr}\left\{\hat{\rho}_{R}(t) \hat{A}_{R}\right\}
\end{aligned}
$$

where $\hat{\rho}_{R}(t)=\hat{U}_{R}^{-1} \hat{\rho}(t) \hat{U}_{R}$, and $\hat{A}_{R}=\hat{U}_{R}^{-1} \hat{A} \hat{U}_{R}$.

What is the advantage of performing a frame transformation on the system? The 
time evolution of the system in the transformed frame is governed by the effective Hamiltonian $\hat{\widetilde{H}}(t)$ of Eq. 2.35. Sometimes the time evolution under the effective Hamiltonian may be solved more simply than under the Hamiltonian $\hat{H}(t)$. In fact, for some $\hat{H}(t)$, the effective Hamiltonian may be rendered time-independent through a suitable choice of frame. This is the case for the homogeneous Hamiltonian in the problem of Rabi oscillations: $\hat{H}(t) / \hbar=-\omega_{0} \hat{I}_{z}-\omega_{1}\left[\hat{I}_{x} \cos \left(\omega_{r} t\right)-\hat{I}_{y} \sin \left(\omega_{r} t\right)\right]$, where $\hat{U}_{R}=\exp \left(+i \omega_{r} t \hat{I}_{z}\right)$. There is another justification for frame transformations that is more prevalent in NMR theory. We note that in Eq. 2.35 the $\hat{U}_{R}^{-1} \hat{H}(t) \hat{U}_{R}$ term has the same magnitude as $\hat{H}(t)$, since $\hat{U}_{R}$ is a unitary rotation of $\hat{H}(t)$ (and therefore preserves its norm). (Here we have used the trace norm definition of the magnitude of an operator: $\|\hat{A}\|=\sqrt{\sum_{i, j}\left|A_{i j}\right|^{2}} / \sqrt{\sum_{i, j}\left|1_{i j}\right|^{2}}=\sqrt{\left[\operatorname{Tr}\left(\hat{A}^{\dagger} \hat{A}\right)\right] / D}$, where $\hat{1}$ is the identity operator and $D$ is the dimensionality of the Hilbert space.) However, with a proper choice of $\hat{U}_{R}$, the $\left(-i \hbar \hat{U}_{R}^{-1} \frac{\partial \hat{U}_{R}}{\partial t}\right)$ term may be subtractive, and thus may be used to shrink the magnitude of $\hat{\widetilde{H}}(t)$. This is very useful if $\hat{\widetilde{U}}(t)$ is to be approximated by a Magnus expansion, because when $\hat{H}(t)$ is smaller the Magnus series may converge when it otherwise might not (or at least converge much more quickly). Therefore a low-order truncation of the series may be better justified.

\subsubsection{The interaction representation}

Consider a particular unitary transformation that takes a system described by the Schrödinger equations of motion (Eqs. 2.1, 2.3, 2.12, etc.) into a new frame called the interaction representation. The transformation that will be examined first is slightly different from the one found in textbook descriptions of the interaction representation, although it is consistent with the usage in the NMR literature. 
We begin with a time-dependent, possibly homogeneous Hamiltonian that may be divided into the sum of two pieces:

$$
\hat{H}(t)=\hat{H}_{0}(t)+\hat{H}_{1}(t)
$$

We apply the frame transformation

$$
\hat{U}_{I}(t)=T\left\{\exp \left[-\frac{i}{\hbar} \int_{t_{0}}^{t} d t^{\prime} \hat{H}_{0}\left(t^{\prime}\right)\right]\right\}
$$

The operator $\hat{U}_{I}(t)$ has the same form as a time development operator for a Hamiltonian $\hat{H}_{0}(t)$. Since Eq. 2.9 is the formal solution to Eq. 2.3, it follows that $\frac{\partial \hat{U}_{I}(t)}{\partial t}=\frac{1}{i \hbar} \hat{H}_{0}(t) \hat{U}_{I}(t)$. Substituting into Eq. 2.35 for $\hat{\widetilde{H}}(t)^{5}$ :

$$
\begin{aligned}
\hat{\widetilde{H}}(t) & =\hat{U}_{I}^{-1}(t) \hat{H}(t) \hat{U}_{I}(t)-i \hbar \hat{U}_{I}^{-1}(t) \frac{\partial \hat{U}_{I}(t)}{\partial t} \\
& =\hat{U}_{I}^{-1}(t)\left[\hat{H}_{0}(t)+\hat{H}_{1}(t)\right] \hat{U}_{I}(t)-i \hbar \hat{U}_{I}^{-1}(t)\left[\frac{1}{i \hbar} \hat{H}_{0}(t) \hat{U}_{I}(t)\right] \\
& =\hat{U}_{I}^{-1}(t) \hat{H}_{0}(t) \hat{U}_{I}(t)+\hat{U}_{I}^{-1}(t) \hat{H}_{1}(t) \hat{U}_{I}(t)-\hat{U}_{I}^{-1}(t) \hat{H}_{0}(t) \hat{U}_{I}(t) \\
\Longrightarrow \hat{\widetilde{H}}(t) & =\hat{U}_{I}^{-1}(t) \hat{H}_{1}(t) \hat{U}_{I}(t) \equiv \hat{\widetilde{H}}_{I}(t) .
\end{aligned}
$$

Haeberlen and Waugh [128] have demonstrated that the Schrödinger representation time development operator $\hat{U}\left(t ; t_{0}\right)$ (Eq. 2.9) may be written in terms of the interaction

\footnotetext{
${ }^{5}$ One occasionally finds that in the NMR literature, interaction representation transformations along the lines of Eq. 2.41 are misstated as $\hat{\widetilde{H}}(t)=\hat{U}_{I}^{-1}(t) \hat{H}(t) \hat{U}_{I}(t)$ instead of $\hat{U}_{I}^{-1}(t) \hat{H}_{1}(t) \hat{U}_{I}(t)$, although the end result of the transformation typically is correct.
} 
representation time development operator $\hat{\widetilde{U}}\left(t ; t_{0}\right)=T\left\{\exp \left[-\frac{i}{\hbar} \int_{t_{0}}^{t} d t^{\prime} \hat{\widetilde{H}}\left(t^{\prime}\right)\right]\right\}$ (Eq. 2.37) $)^{6}$ :

$$
\hat{U}\left(t ; t_{0}\right)=\hat{U}_{I}(t) \hat{\tilde{U}}\left(t ; t_{0}\right) .
$$

This result may also be obtained using an operator calculus due to Feynman [129]. When $\hat{H}_{0}(t)$ is time-independent, then $\hat{H}(t)=\hat{H}_{0}+\hat{H}_{1}(t)$, and $\hat{U}_{I}(t)=\exp \left(-i \hat{H}_{0} t / \hbar\right)$. This is the "textbook" [45] Hamiltonian for the interaction representation, also called the interaction picture or Dirac picture of time-dependent quantum mechanics. There is a difference between the interaction representation and the previously discussed Schrödinger representation. State vectors (and hence density operators $\hat{\rho}(t)$ ) in the Schrödinger representation have time dependence, whereas operators corresponding to observables $(\hat{A})$ do not. The state vectors (and density operators $\left.\hat{\rho}_{I}(t) \equiv \hat{U}_{I}^{-1}(t) \hat{\rho}(t) \hat{U}_{I}(t)\right)$ and observable operators $\left(\hat{A}_{I}(t) \equiv \hat{U}_{I}^{-1}(t) \hat{A} \hat{U}_{I}(t)\right)$ both have time dependence in the interaction representation. In the interaction picture, the time evolution of $\hat{\rho}_{I}(t)$ is determined only by the effective Hamiltonian $\hat{\widetilde{H}}_{I}(t)$, and the time evolution of $\hat{A}_{I}(t)$ is determined by only $\hat{H}_{0}$.

There is another representation that is commonly used in quantum mechanics

${ }^{6}$ The proof is as follows. It utilizes Eqs. 2.3, 2.9, and 2.39-2.42:

$$
\begin{aligned}
i \hbar \frac{\partial \hat{U}\left(t ; t_{0}\right)}{\partial t} & =i \hbar \frac{\partial}{\partial t}\left[\hat{U}_{I}(t) \hat{\tilde{U}}\left(t ; t_{0}\right)\right]=i \hbar\left[\frac{\partial \hat{U}_{I}(t)}{\partial t} \hat{\tilde{U}}\left(t ; t_{0}\right)+\hat{U}_{I}(t) \frac{\partial \hat{\tilde{U}}\left(t ; t_{0}\right)}{\partial t}\right] \\
& =i \hbar\left[\left\{\frac{1}{i \hbar} \hat{H}_{0}(t) \hat{U}_{I}(t)\right\} \hat{\tilde{U}}\left(t ; t_{0}\right)+\hat{U}_{I}(t)\left\{\frac{1}{i \hbar} \hat{\tilde{H}}(t) \hat{\tilde{U}}\left(t ; t_{0}\right)\right\}\right] \\
& =\hat{H}_{0}(t) \hat{U}_{I}(t) \hat{\tilde{U}}\left(t ; t_{0}\right)+\hat{U}_{I}(t)\left[\hat{U}_{I}^{-1}(t) \hat{H}_{1}(t) \hat{U}_{I}(t)\right] \hat{\tilde{U}}\left(t ; t_{0}\right) \\
& =\hat{H}_{0}(t) \hat{U}_{I}(t) \hat{\tilde{U}}\left(t ; t_{0}\right)+\hat{1} \hat{H}_{1}(t) \hat{U}_{I}(t) \hat{\tilde{U}}\left(t ; t_{0}\right) \\
& =\left[\hat{H}_{0}(t)+\hat{H}_{1}(t)\right] \hat{U}_{I}(t) \hat{\tilde{U}}\left(t ; t_{0}\right) \\
\Longrightarrow i \hbar \frac{\partial \hat{U}\left(t ; t_{0}\right)}{\partial t} & =\hat{H}(t) \hat{U}\left(t ; t_{0}\right) .
\end{aligned}
$$

The last line is the correct differential equation for $\hat{U}\left(t ; t_{0}\right)$ (Eq. 2.3), so Eq. 2.42 must be a correct form for the time development operator. 
called the Heisenberg representation. This representation is defined by the transformation $\hat{U}_{H}=T\left\{\exp \left[-\frac{i}{\hbar} \int_{t_{0}}^{t} d t^{\prime} \hat{H}\left(t^{\prime}\right)\right]\right\}$, which reduces to $\hat{U}_{H}=\exp \left[-i \hat{H}\left(t-t_{0}\right) / \hbar\right]$ in the case of a time-independent $\hat{H}(t)=\hat{H}$. Under this transformation the Heisenberg picture state vectors/density operators are time-independent, whereas the operators $\hat{A}_{H}(t) \equiv \hat{U}_{H}^{-1}(t) \hat{A} \hat{U}_{H}(t)$ that correspond to observables carry the full time dependence of the system.

The interaction representation transformation will the be most useful in the case of a homogeneous Hamiltonian $\hat{H}(t)$ (i.e., one for which the propagator cannot be calculated simply in the Schrödinger representation), when additionally $\left\|\hat{H}_{0}(t)\right\| \gg\left\|\hat{H}_{1}(t)\right\|$. In this case the Schrödinger representation Hamiltonian has a magnitude $\|\hat{H}(t)\| \sim\left\|\hat{H}_{0}(t)\right\|$, whereas the interaction representation effective Hamiltonian has a magnitude $\|\hat{\widetilde{H}}(t)\|=$ $\left\|\hat{\widetilde{H}}_{1}(t)\right\|=\left\|\hat{H}_{1}(t)\right\|$. (The trace norm definition of the magnitude of an operator has been used, which is invariant under unitary transformations.) Therefore, the interaction representation effective Hamiltonian is much smaller in magnitude than the Schrödinger representation Hamiltonian, and the propagator may be better approximated by a low-order truncation of the Magnus series. It usually will be a good strategy to go into the interaction representation of the largest piece of a homogeneous Hamiltonian, whenever the magnitude of that interaction is much larger than any of the other interactions in the Hamiltonian. Incidentally, another requirement for the utility of an interaction frame transformation is that $\hat{U}_{I}(t)$ must be able to be evaluated analytically (i.e., $\hat{H}_{0}(t)$ is inhomogeneous). It obviously complicates matters to transform into a frame for which the transformation operator must be approximated. 


\subsubsection{Common frame transformations in NMR}

\section{Rotating frame}

The most common frame transformation encountered in magnetic resonance theory is the rotating frame transformation. It is suitably applied to the Hamiltonian of magnetic moments (labeled $I)$ in the presence of a large static magnetic field along the $z$-axis $\left(\hat{H}_{0} / \hbar=\right.$ $\left.-\omega_{0} \hat{I}_{z}\right)$, under the influence of either a rotating $\left(\hat{H}_{1}(t) / \hbar=-\omega_{1}\left[\hat{I}_{x} \cos \left(\omega_{r} t\right)-\hat{I}_{y} \sin \left(\omega_{r} t\right)\right]\right)$ or oscillating $\left(\hat{H}_{1}(t) / \hbar=-2 \omega_{1} \hat{I}_{x} \cos \left(\omega_{r} t\right)\right)$ transverse radiofrequency $(\mathrm{rf})$ field. These expressions define the laboratory frame Hamiltonian $\hat{H}(t)=\hat{H}_{0}+\hat{H}_{1}(t)$. The rotating frame transformation for this Hamiltonian is $\hat{U}_{R}(t)=\exp \left(+i \omega_{r} t \hat{I}_{z}\right)$. It is called a rotating frame transformation because $\exp \left(-i \alpha \hat{I}_{z}\right)$ is the operator for rotations of the system by an angle $\alpha$ about the $z$-axis, so that here $\hat{U}_{R}(t)$ rotates the system at a constant angular velocity $-\omega_{r}$ by the time-dependent angle $\alpha(t)=-\omega_{r} t$. Spins tilted off the $z$-axis precess under $\hat{H}_{0}$ at the velocity $-\omega_{0}$, and when at resonance $\left(\omega_{r}=\omega_{0}\right)$, the frame rotates about the $z$-axis at the same rate as the spin precession. The rotating frame transformation effectively shrinks the Zeeman interaction $\hat{H}_{0} / \hbar$ from $-\omega_{0} \hat{I}_{z}$ to $-\left(\omega_{0}-\omega_{r}\right) \hat{I}_{z}$; it renders $\hat{\widetilde{H}}(t)$ time-independent in the case of the rotating rf field, and allows the use of the Magnus expansion to calculate a simple effective propagator in the case of the oscillating rf field.

\section{Tilted frame}

A tilted frame transformation is defined by the time-independent rotation $\hat{U}_{T}=$ $\exp (-i \alpha \underline{\hat{I}} \cdot \underline{\widetilde{n}})$, where $\underline{\hat{I}}=\hat{I}_{x} \underline{\widetilde{i}}+\hat{I}_{y} \underline{\tilde{j}}+\hat{I}_{z} \underline{\widetilde{k}}$. (Here $\underline{\widetilde{n}}, \underline{\tilde{i}}, \underline{\tilde{j}}, \underline{\widetilde{k}}$ are unit vectors in real space, not quantum mechanical operators on a Hilbert space.) This transformation rotates ("tilts") 
the system by an angle $\alpha$ about the axis defined by $\underline{\widetilde{\widetilde{n}}}$. An example of a tilted frame transformation is one that is applied commonly to rotating frame effective Hamiltonians of the form $\hat{H}=-\Delta \omega \hat{I}_{z}-\omega_{1} \hat{I}_{x}$. Such a Hamiltonian describes spins under the influence of an effective magnetic field in the $z$-direction with a magnitude proportional to $\Delta \omega$ and an effective magnetic field in the $x$-direction with a magnitude proportional to $\omega_{1}$. These effective fields may be summed as vectors to give an effective field $\underline{\hat{B}}_{e}$ of magnitude proportional to $\omega_{e}=\sqrt{(\Delta \omega)^{2}+\omega_{1}^{2}}$ in the $\underline{\widetilde{n}}$ direction, where $\underline{\widetilde{n}}$ is in the $x-z$ plane and makes an angle $\alpha$ with the $z$-axis $\left(\alpha \equiv \tan ^{-1}\left(\omega_{1} / \Delta \omega\right)\right)$. The operation $\hat{U}_{T}=\exp \left(-i \alpha \hat{I}_{y}\right)$ then rotates $\underline{B}_{e}$ into the direction of the $z$-axis, so that $\hat{\widetilde{H}}=\hat{U}_{T}^{-1} \hat{H} \hat{U}_{T}=-\omega_{e} \hat{I}_{z}$.

If one wishes to apply both the rotating and tilted frame transformations at once (the so-called "tilted rotating frame" transformation) [130], this may be accomplished by using $\hat{U}_{R T}(t)=\hat{U}_{R}(t) \hat{U}_{T}=\exp \left(+i \omega_{r} t \hat{I}_{z}\right) \exp \left(-i \alpha \hat{I}_{y}\right)$. This sort of formalism may be used to describe Lee-Goldburg decoupling of the homonuclear dipolar interaction [128, 131]. Waugh et al. [132] have used a similar transformation to simplify the dipole-coupled Hamiltonian in studies of heteronuclear two-spin (double resonance) cross-relaxation. The operators $\hat{U}_{R}(t)$ and $\hat{U}_{T}$ do not commute with each other (since $\left[\hat{I}_{z}, \hat{I}_{x}\right] \neq 0$ ), so their ordering in the product is important $\left(i . e ., \hat{U}_{R}(t) \hat{U}_{T} \neq \hat{U}_{T} \hat{U}_{R}(t)\right)$. Here $\hat{\widetilde{H}}(t)=\hat{U}_{R T}^{-1}(t) \hat{H}(t) \hat{U}_{R T}(t)=$ $\hat{U}_{T}^{-1} \hat{U}_{R}^{-1}(t) \hat{H}(t) \hat{U}_{R}(t) \hat{U}_{T}$, so that first the rotating frame transformation is applied to the Hamiltonian, and next the tilted frame transformation is applied. ${ }^{7}$ One might also imagine "rotating tilted frame" transformations, in which a tilting transformation is applied to some Hamiltonian before a rotating transformation.

\footnotetext{
${ }^{7}$ Waugh's equations are different because he chooses the convention $\hat{\widetilde{H}}(t)=\hat{U}_{T R}(t) \hat{H}(t) \hat{U}_{T R}^{-1}(t)$, with $\hat{U}_{T R}=\hat{U}_{T} \hat{U}_{R}(t)$.
} 


\section{Toggling frame}

The toggling frame is used to calculate average Hamiltonians for pulse sequences $[7,123]$. We consider an effective Hamiltonian in the rotating frame $\hat{H}(t)=\hat{H}_{0}+\hat{H}_{1}(t)$. Here $\hat{H}_{0}$ is the time-independent rotating frame effective Hamiltonian for the spin system in the absence of the rf field (i.e., it is the rotating frame internal Hamiltonian). The $\hat{H}_{1}(t)$ term is the time-dependent rotating frame effective Hamiltonian for a series of rf pulses in the plane transverse to the external magnetic field (which lies along the $z$-axis). The pulses may be considered to be negligibly short in the limit that the magnitude of the rf Hamiltonian far exceeds that of the internal Hamiltonian of the spins, so that the spins nutate under the rf field much more quickly than they evolve due to interactions with their local environments. This is the limit of "delta-function pulses." 8 For example, $\hat{H}=\hbar \omega_{1} \hat{I}_{x}$ is taken to be the rotating frame rf Hamiltonian for a pulse that nutates the spins about the $x$-axis, where $\omega_{1} t_{p}=\alpha$ for spins that are rotated through an angle $\alpha$ in a time $t_{p}$. When the limit of delta-function pulses $\left(t_{p} \rightarrow 0\right)$ is taken, the Hamiltonian for an " $\alpha_{x}$ " pulse applied at time $t=t_{i}$ is $\hat{H}_{i}(t)=\hbar \alpha \hat{I}_{x} \delta\left(t-t_{i}\right)$. Therefore $\hat{H}_{1}(t)=\hbar \sum_{i=1}^{p} \alpha_{i} \hat{I}_{\phi_{i}} \delta\left(t-t_{i}\right)$, where e.g., $\phi_{i}=0$ for an $x$-pulse and $\phi_{i}=\pi / 2$ for a $y$-pulse. The Hamiltonian $\hat{H}(t)$ then appears to consist of a sequence of $\mathrm{rf}$ pulses spaced at intervals $t=t_{i}$ (during which evolution under $\hat{H}_{0}$ is neglected), separated by "windows" of free precession of the spins.

We now go into the interaction representation of the Hamiltonian $\hat{H}_{1}(t)$ of the rf pulses, where as usual the interaction transformation looks like the time propagator for the interaction $\hat{H}_{1}(t): \hat{U}_{I}(t) \equiv \hat{U}_{1}(t)=T\left\{\exp \left[-\frac{i}{\hbar} \int_{t_{0}}^{t} d t^{\prime} \hat{H}_{1}\left(t^{\prime}\right)\right]\right\}$. This is the "toggling

\footnotetext{
${ }^{8}$ Any rf pulse that is strong/short enough so that internal evolution/relaxation can be ignored during the pulse is usually referred to as a "hard" pulse, but it is often computationally convenient to assume that these pulses also have negligible duration (the "delta-function pulse" limit).
} 
frame" transformation of the rotating frame Hamiltonian, as will be seen below. It is especially appropriate to go into the interaction representation of the Hamiltonian of the pulses, because we are in the limit that the magnitude of the rf Hamiltonian is much larger than that of the internal spin Hamiltonian. ${ }^{9}$

The propagator for a single pulse $P_{i}\left(\alpha_{i \phi_{i}}\right)$ is

$$
\begin{aligned}
\hat{U}_{P_{i}}\left(t ; t_{0}\right) & =T\left\{\exp \left[-\frac{i}{\hbar} \int_{t_{0}}^{t} d t^{\prime} \hat{H}_{i}\left(t^{\prime}\right)\right]\right\} \\
& =T\left\{\exp \left[-\frac{i}{\hbar} \int_{t_{0}}^{t} d t^{\prime} \hbar \alpha_{i} \hat{I}_{\phi_{i}} \delta\left(t^{\prime}-t_{i}\right)\right]\right\} \\
& =T\left\{\exp \left[-i \alpha_{i} \hat{I}_{\phi_{i}} \int_{t_{0}}^{t} d t^{\prime} \delta\left(t^{\prime}-t_{i}\right)\right]\right\} \\
\Longrightarrow \hat{U}_{P_{i}}\left(t \geq t_{i} ; t_{0}\right) & =\exp \left(-i \alpha_{i} \hat{I}_{\phi_{i}}\right) \\
\hat{U}_{P_{i}}\left(t<t_{i} ; t_{0}\right) & =\hat{1}
\end{aligned}
$$

since $\int_{t_{0}}^{t} d t^{\prime} \delta\left(t^{\prime}-t_{i}\right)=1$, if $t_{0} \leq t_{i} \leq t$ (and $=0$ otherwise). The toggling frame transformation operator $\hat{U}_{1}(t)$ is found most easily by discretizing the propagator for $\hat{H}_{1}(t)$ in the manner of Eq. 2.6. Note that $\hat{U}_{n}\left(\Delta t_{n}\right)=\hat{1}$ unless the interval $\Delta t_{n}$ contains a pulse $P_{i}\left(\alpha_{i \phi_{i}}\right)$, in which case $\hat{U}_{n}\left(\Delta t_{n}\right)=\exp \left(-i \alpha_{i} \hat{I}_{\phi_{i}}\right)$. Therefore

$$
\hat{U}_{1}(t)=T\left\{\prod_{i=1}^{N} \hat{U}_{P_{i}}\left(t_{i}\right)\right\}=e^{-i \alpha_{N} \hat{I}_{\phi_{N}}} \cdots e^{-i \alpha_{2} \hat{I}_{\phi_{2}}} e^{-i \alpha_{1} \hat{I}_{\phi_{1}}} \quad\left(t_{i} \leq t<t_{N}\right)
$$

It can be seen that $\hat{U}_{1}(t)$ is a product of the first $\mathrm{N}$ pulse propagators $\hat{U}_{P_{i}}\left(t_{i}\right)$ up until time $t$; pulse propagators for pulses that occur after time $t$ are excluded. It follows (in analogy

\footnotetext{
${ }^{9}$ Note that in the prior discussion of the interaction representation a transformation is made into the frame of $\hat{H}_{0}(t)$ (Eq. 2.40), but this tends to be practical in the case when $\hat{H}_{0}(t)$, not $\hat{H}_{1}(t)$ is the larger interaction.
} 
to Eq. 2.41) that $\hat{\widetilde{H}}(t)=\hat{U}_{1}^{-1}(t) \hat{H}_{0} \hat{U}_{1}(t)$, where $\hat{U}_{1}^{-1}(t)=T_{-}\left\{\exp \left[+\frac{i}{\hbar} \int_{0}^{t} d t^{\prime} \hat{H}_{1}\left(t^{\prime}\right)\right]\right\}$. Therefore, for $p$ pulses in the sequence:

$$
\begin{aligned}
& \hat{\widetilde{H}}\left(t_{0} \leq t<t_{1}\right) \equiv \hat{\widetilde{H}}_{0}=\hat{1} \\
& \hat{\widetilde{H}}\left(t_{1} \leq t<t_{2}\right) \equiv \hat{\widetilde{H}}_{1}=e^{+i \alpha_{1} \hat{I}_{\phi_{1}}} \hat{H}_{0} e^{-i \alpha_{1} \hat{I}_{\phi_{1}}} \\
& \hat{\widetilde{H}}\left(t_{2} \leq t<t_{3}\right) \equiv \hat{\widetilde{H}}_{2}=e^{+i \alpha_{1} \hat{I}_{\phi_{1}}} e^{+i \alpha_{2} \hat{I}_{\phi_{2}}} \hat{H}_{0} e^{-i \alpha_{2} \hat{I}_{\phi_{2}}} e^{-i \alpha_{1} \hat{I}_{\phi_{1}}} \\
& \quad \vdots \\
& \hat{\widetilde{H}}\left(t \geq t_{p}\right) \equiv \hat{\widetilde{H}}_{p}=e^{+i \alpha_{1} \hat{I}_{\phi_{1}}} e^{+i \alpha_{2} \hat{I}_{\phi_{2}} \cdots e^{+i \alpha_{p} \hat{I}_{\phi_{p}}} \hat{H}_{0} e^{-i \alpha_{p} \hat{I}_{\phi_{p}}} \cdots e^{-i \alpha_{2} \hat{I}_{\phi_{2}}} e^{-i \alpha_{1} \hat{I}_{\phi_{1}}}}
\end{aligned}
$$

Note that the pulse propagators are applied to $\hat{H}_{0}$ in "reverse order," with $\hat{U}_{P_{p}}$ acting on $\hat{H}_{0}$ first in the expression for $\hat{\widetilde{H}}_{p}$, and $\hat{U}_{P_{1}}$ acting on $\hat{H}_{0}$ last.

We finally see why $\hat{U}_{1}(t)$ is called the toggling frame transformation. As $t$ exceeds each $t_{i}$ and pulse $P_{i}\left(\alpha_{i \phi_{i}}\right)$ is applied, the toggling frame Hamiltonian $\hat{\widetilde{H}}(t)$ is transformed by the application of the pulse propagator $\hat{U}_{P_{i}}$ to $\hat{H}_{0}$. When $\hat{U}_{P_{i}}=\exp \left(-i \alpha_{i} \hat{I}_{\phi_{i}}\right)$ in the case of delta-function pulses, these transformations look like rotations by an angle $\alpha_{i}$ about the axis defined by $\widetilde{\phi}_{i}$. Therefore each pulse effectively "toggles" the rotating frame Hamiltonian into a rotated set of coordinates. When the pulses are not infinitesimally short delta-function pulses, the "toggling" is continuous over the duration of the pulse, and the finite lengths of the pulses must be included in the expression for $\hat{U}_{1}(t)$. 


\subsection{Average Hamiltonian theory}

The techniques of average Hamiltonian theory (AHT) have become entrenched in the field of solid-state NMR largely due to the work of Waugh's group [123, 128, 8]. The "essential" references for this subject are Haeberlen and Waugh's classic treatment [128] and Haeberlen's excellent and thorough monograph [123]. The basic idea of AHT is to find approximately the time evolution of the system over a short time $t_{c}$ using a Magnus expansion approach (usually after first transforming the Hamiltonian into an appropriate interaction representation), and then extend the evolution to long times $n t_{c}$ for periodic interaction frame effective Hamiltonians $\hat{\widetilde{H}}\left(t+n t_{c}\right)=\hat{\widetilde{H}}(t)$.

We consider the interaction representation transformations specified by Eqs. 2.392.41, where in practice $\left\|\hat{H}_{0}(t)\right\| \gg\left\|\hat{H}_{1}(t)\right\|$ for $\hat{H}(t)=\hat{H}_{0}(t)+\hat{H}_{1}(t) .{ }^{10}$ For simplicity we take $t_{0}=0$. The interaction representation transformation then will be the time development operator for $\hat{H}_{0}(t)$ :

$$
\hat{U}_{I}(t)=T\left\{\exp \left[-\frac{i}{\hbar} \int_{0}^{t} d t^{\prime} \hat{H}_{0}\left(t^{\prime}\right)\right]\right\}
$$

We assume that $\hat{H}(t)$ is periodic, so that $\hat{H}_{0}(t)$ may be periodic with period $t_{h}$ and $\hat{H}_{1}(t)$ is time-independent, or vice-versa, or both $\hat{H}_{0}(t)$ and $\hat{H}_{1}(t)$ are periodic with period $t_{h}$, or both are time-independent:

$$
\hat{H}\left(t+N t_{h}\right)=\hat{H}(t)
$$

\footnotetext{
${ }^{10}$ Often AHT is applied in the toggling frame of a series of rf pulses rather than in the interaction frame of the dominant Hamiltonian [123, 128].
} 
where $N$ is an integer. We consider the special case where additionally $\hat{U}_{I}(t)$ is periodic with period $t_{c}$ :

$$
\hat{U}_{I}\left(t+N t_{c}\right)=\hat{U}_{I}(t)
$$

where $t_{c}$ is an integer multiple of $t_{h} \cdot{ }^{11}$ Haeberlen and Waugh $[123,128]$ call a $\hat{U}_{I}(t)$ operator with this property "cyclic," and note that in some cases $\hat{U}_{I}(t)$ may be cyclic over a time $t_{s}$ that is an integer submultiple of the period $t_{c} \cdot{ }^{12}$ It immediately follows from Eq. 2.46 with $t=0$ and the initial condition $\hat{U}_{I}(t=0)=\hat{1}$ that:

$$
\hat{U}_{I}\left(N t_{c}\right)=\hat{1}
$$

In other words, $\hat{U}_{I}$ has no effect on the time evolution for times $t=N t_{c}$.

Next one must consider the time evolution in the interaction frame. Eq. 2.42 states that the time development operator is $\hat{U}\left(t ; t_{0}\right)=\hat{U}_{I}(t) \hat{\widetilde{U}}(t ; 0)$, where $\hat{\widetilde{U}}(t ; 0)=$ $T\left\{\exp \left[-\frac{i}{\hbar} \int_{0}^{t} d t^{\prime} \hat{\widetilde{H}}\left(t^{\prime}\right)\right]\right\}$ is the time development operator in the interaction frame, and $\hat{\widetilde{H}}(t)=\hat{U}_{I}^{-1}(t) \hat{H}_{1}(t) \hat{U}_{I}(t)$. The interaction frame effective Hamiltonian $\hat{\widetilde{H}}(t)$ may have explicit time dependence due to the time dependence of $\hat{H}_{1}(t)$, plus additional time dependence due to the time-dependent interaction frame transformation $\hat{U}_{I}(t)$. If $\hat{H}_{1}(t)$ is

\footnotetext{
${ }^{11}$ The relation between $t_{h}$ and $t_{c}$ can be demonstrated for the contrived example of a periodic Hamiltonian of the form $\hat{H}_{0}(t)=\sum_{n \neq 0} \hat{H}_{0}^{(n)} \exp \left(i n \omega_{h} t\right)$ with $\left[\hat{H}_{0}^{(n)}, \hat{H}_{0}^{\left(n^{\prime}\right)}\right]=0$ for all $n, n^{\prime}$, where $\omega_{h}=2 \pi / t_{h}$. In this case $\hat{U}_{I}(t)=\Pi_{n \neq 0} \exp \left\{-\hat{H}_{0}^{(n)}\left[\exp \left(i n \omega_{h} t\right)-1\right] /\left(n \hbar \omega_{h}\right)\right\}$, which is periodic with some period $t_{c}=m t_{h}$, where $m$ is an integer. If $\hat{H}_{0}$ is time-independent (i.e., it has no well-defined period), then $\hat{U}_{I}(t)=e^{-i \hat{H}_{0} t / \hbar}$ still may be periodic if the eigenfrequencies $\hat{H}_{0} / \hbar$ are all rational multiples of some base frequency.

${ }^{12}$ In general the periodicity of the Hamiltonian does not guarantee the periodicity of $\hat{U}_{I}(t)$, as Haeberlen notes [123] for the case of a toggling frame transformation for a pulse sequence made up of equal, periodicallyspaced pulses with flip angles equal to an irrational fraction of $2 \pi$. In that case the (rf) Hamiltonian is periodic, but the system is not cyclic because no amount of pulses will rotate the system through exactly the angle $2 \pi$.
} 
periodic with period $t_{h}$ and $\hat{U}_{I}(t)$ is cyclic with period $t_{c}=m t_{h}$, it is reasonable to expect that $\hat{\widetilde{H}}(t)$ also is periodic with period $t_{h}$. For simplicity's sake, we take $t_{c}=t_{h}$, so that

$$
\hat{\widetilde{H}}\left(t+N t_{c}\right)=\hat{\widetilde{H}}(t)
$$

This relation certainly holds if $\hat{H}_{1}$ is time-independent and $\hat{U}_{I}(t)$ is cyclic with period $t_{c}$. From Eq. 2.48 it follows that

$$
\hat{\tilde{U}}\left(N t_{c}\right)=\left[\hat{\tilde{U}}\left(t_{c}\right)\right]^{N}
$$

because all the $\hat{\tilde{U}}\left(t_{c}\right)$ time development operators over the $N$ intervals are equal. It follows from Eqs. 2.49, 2.47, and 2.42 that the time development operator in the untransformed frame is:

$$
\hat{U}\left(N t_{c}\right)=\left[\hat{\tilde{U}}\left(t_{c}\right)\right]^{N}
$$

If the interaction frame effective Hamiltonian $\hat{\widetilde{H}}(t)$ is homogeneous the time evolution must be approximated, e.g. by the Magnus expansion (Eq. 2.19):

$$
\hat{\tilde{U}}\left(N t_{c}\right) \simeq \exp \left[-i \hat{\bar{H}}\left(N t_{c}\right) / \hbar\right]
$$

where the "average Hamiltonian" $\hat{H}$ has been calculated on the interval $\left[0, t_{c}\right]$ and truncated at an appropriate level of the Magnus expansion; i.e., the lowest order approximation from Eq. 2.27 is $\hat{\bar{H}} \simeq \hat{\bar{H}}^{(1)}=\frac{1}{t_{c}} \int_{0}^{t_{c}} d t^{\prime} \hat{\widetilde{H}}\left(t^{\prime}\right)$. Note that in this example, the Magnus expansion 
is being used to approximate the interaction frame time evolution, using the appropriate effective Hamiltonian. Eq. 2.50 indicates that

$$
\hat{U}\left(N t_{c}\right) \simeq\left[\exp \left(-i \hat{\bar{H}} t_{c} / \hbar\right)\right]^{N}
$$

Eq. 2.52 is the fundamental result of average Hamiltonian theory. It states that if a system has a cycle time $t_{c}$ and an average Hamiltonian for the system calculated via the Magnus expansion converges on the interval $\left[0, t_{c}\right]$, then the calculation of the time evolution can be extended to long times $t=N t_{c}$ without worries about convergence. (Recall that $\|\hat{\widetilde{H}}(t)\|$ $t \leq 1$ is a rough criterion for convergence of the Magnus series on the interval $[0, t]$, so there is a limit to how far the calculation of an average Hamiltonian can be extended in time.) However, it is crucial to note that AHT is a stroboscopic theory, i.e., it is only valid when the system is observed stroboscopically at discrete times $t_{N}=N t_{c}$. Refs. [38, 39, 40] mention some of the perils that await if calculations are performed without heed of the stroboscopic condition. ${ }^{13}$ Ref. $[41, \S 4.3]$ discusses the potential problem with AHT if the Hamiltonian that dictates the time evolution (i.e., either $\hat{H}(t)$ or $\hat{\widetilde{H}}(t)$ if an interaction representation transformation is made) contains a time-independent piece.

\footnotetext{
${ }^{13}$ Ref. $[133, \S 1.3 .1]$ notes that the stroboscopic observations are not necessary if the observable commutes with $\hat{U}_{I}(t)$ :

$$
\begin{aligned}
<\hat{A}>(t) & =\operatorname{Tr}[\hat{A} \rho \hat{(t)}]=\operatorname{Tr}\left[\hat{A} \hat{U}(t) \rho \hat{(0)} \hat{U}^{\dagger}(t)\right]=\operatorname{Tr}\left[\hat{A}\left\{\hat{U}_{I}(t) \hat{\tilde{U}}(t) \rho \hat{\rho}(0) \hat{\tilde{U}}^{\dagger}(t) \hat{U}^{\dagger}(t)\right\}\right] \\
& =\operatorname{Tr}\left[\left\{\hat{U}^{\dagger}(t) \hat{A} \hat{U}_{I}(t)\right\} \hat{\widetilde{U}}(t) \rho(0) \hat{\tilde{U}}^{\dagger}(t)\right]=\operatorname{Tr}\left[\hat{A}\left\{\hat{\widetilde{U}}(t) \rho \hat{(0)} \hat{\tilde{U}}^{\dagger}(t)\right\}\right],
\end{aligned}
$$

where operators have been permuted through the trace and $\left[\hat{U}_{I}(t), \hat{A}\right]=0$ guarantees that $\hat{A}$ is invariant to transformation by $\hat{U}_{I}(t)$. Thus the condition of Eq. 2.47 is not necessary. However, the cyclic nature of the system is still used via Eq. 2.49 to extend the results of the Magnus expansion to long times.
} 


\section{Chapter 3}

\section{Mixed-coherence $I=5 / 2$ MQMAS}

\section{experiments with low-amplitude}

\section{RF pulses}

\subsection{Introduction}

Most elements in the periodic table have nuclei with non-zero ground state magnetic moments have an angular momentum quantum number greater than $1 / 2$ and therefore possess an electric quadrupole moment. In particular, most of the NMR-active nuclei observed in materials studies (e.g., most metal atoms) are half-integer quadrupolar spins. The study of the structure of materials requires the ability to perform solid state experiments. Solid-state NMR is a useful technique for any type of sample that is not soluble or has solid-state properties that are interesting, or when extra structural information that cannot 
be obtained in the liquid state is desired.

The most challenging type of sample in high-field solid-state NMR studies is the polycrystalline powder or amorphous solid, which contains a random distribution of local molecular orientations. The application of a large magnetic field to the sample is desirable to increase chemical shift resolution of the NMR spectra and enhance sensitivity through an increased thermal polarization of the sample and higher Larmor frequency of the inductively-detected nuclear magnetization. However, the application of a magnetic field breaks the isotropy of space and makes the nuclear resonance frequencies dependent on the molecular orientation with respect to the magnetic field. This is manifested in the orientation-dependent (anisotropic) Zeeman-truncated internal nuclear Hamiltonians, such as the magnetic dipole-dipole, chemical shift anisotropy (CSA), and electric quadrupolar interactions, which are motionally averaged away in liquids but retain valuable structural information in the solid state. The trouble is that the orientation-dependent powder pattern that contains the structural information is roughly as broad as the interaction that causes it. Typical couplings between nuclear electric quadrupole moments to molecular electric field gradients (EFGs) range from hundreds of $\mathrm{kHz}$ to tens of $\mathrm{MHz}$. When this is compared to a range of chemical shifts that may be only tens of $\mathrm{kHz}$ for a particular nucleus, it is clear that the quadrupolar broadening in a polycrystalline powder completely precludes the acquisition of chemical shift-resolved NMR spectra. Furthermore, the spreading of the resonance over a broad spectral region presents further difficulties for the already sensitivitychallenged NMR experiment, since the powder spectrum can be broadened almost into the noise baseline. 
The most common method of reducing the anisotropic broadening in solids is high-speed magic-angle sample spinning (MAS). This technique is capable of averaging away interactions with second-rank spatial spherical tensor terms via spinning the sample about an axis that makes the "magic angle" $\theta_{m}=\tan ^{-1}(\sqrt{2})$ with the external magnetic field. Second-rank interactions include the dipole-dipole, CSA, and quadrupolar interactions truncated to first order in the perturbation theory by the Zeeman interaction. MAS techniques actually break the powder spectrum into a pattern of "spinning sidebands" spaced at integer multiples of the spinning frequency; these sidebands can be removed by discrete rotor-synchronized acquisition of the time domain signal, at the expense of limiting the spectral width to twice the spinning frequency. The spectral resolution obtained by MAS techniques is impressive, but the question of whether "high resolution" has been achieved is a relative one, i.e., whether the linewidths are smaller than the typical spacings between NMR lines.

Unfortunately, quadrupolar interactions are so large that the second-order perturbation theory contribution cannot be ignored when trying to resolve small chemical shifts. The second-order contribution goes as $\sim \omega_{Q}^{2} / \omega_{0}$ (where $\omega_{Q}$ is the quadrupolar coupling frequency and $\omega_{0}$ is the Larmor frequency), and can be of the order of hundreds of $\mathrm{Hz}$ to hundreds of $\mathrm{kHz}$ in a typical high-field magnet, depending on the size of the quadrupolar coupling, spin quantum number, and magnetic field strength. The use of high magnetic field strengths can ameliorate, but not remove the second-order broadening. The second-order contribution contains both rank-0 isotropic and rank-2 and rank-4 anisotropic spatial terms (see §1.3.7). The rank-0 term adds an isotropic frequency shift of the NMR resonances that 
adds to the usual isotropic chemical shift. The rank-2 term can be removed by MAS, along with the first-order quadrupolar, dipole-dipole, and CSA broadening. However, the rank-4 term is not averaged away by MAS, but only scaled down by a factor of $P_{4}\left(\cos \theta_{m}\right)$, where $P_{4}(x)$ is the fourth-rank Legendre polynomial. This residual quadrupolar broadening even under MAS conditions was formerly a major obstacle to the acquisition of high resolution NMR spectra of quadrupolar nuclei until experiments were designed to overcome it. The most widely-employed technique for half-integer quadrupolar nuclei is the two-dimensional multiple-quantum magic-angle spinning (MQMAS) experiment, which employs a combination of MAS and multiple-quantum coherence evolutions, as will be described later.

The large quadrupolar coupling presents problems not only for spectral resolution. Even high-power solid-state NMR probes are only capable of producing radiofrequency magnetic fields that cause nutations on the order of $10^{5} \mathrm{~Hz}$. Therefore the rf field strength is often much smaller than the quadrupolar coupling, which dominates the time evolution. It then becomes difficult to control the dynamics of the nuclear polarization precisely using rf fields, and the loss of sensitivity due to inefficient multiple quantum coherence conversion pulses in the MQMAS experiment is a major problem that has stimulated the development of many new pulse techniques (see $§ 5.6 .2$ ). Most of these techniques use rf pulses that are as strong as possible, and some incorporate adiabatic coherence transfers due to sample rotation. The work described in this chapter is an investigation of the effects of very lowamplitude rf pulses in $I=5 / 2$ MQMAS experiments. These pulses can not only enhance the sensitivity of the MQMAS experiment, but a new line-narrowing mechanism has also been observed in certain systems. 


\subsection{Removing the second-order quadrupolar broadening. The MQMAS experiment.}

Under fast magic-angle spinning conditions the dipole-dipole and CSA interactions are assumed to be averaged away. However, since even high-speed MAS probes cannot spin faster than a large quadrupolar coupling (i.e., MHz), if the acquisition is not rotorsynchronized the signal intensity is spread out over a series of spinning sidebands that span the powder spectrum. The Fourier-transform NMR experiment records the time evolution of single-quantum coherences in the Zeeman basis that correspond to transverse magnetization. Nuclei with half-integer spins possess symmetric single-quantum coherences $\mid I, m_{I}=$ $\left.\pm \frac{1}{2}\right\rangle\left\langle I, m_{I}=\mp \frac{1}{2}\right|$ of coherence order $p= \pm 1$ which correspond to the "central transition" of the spectrum. The first-order quadrupolar Hamiltonian $\hat{H}_{Q}^{(1)} \propto\left[3 \hat{I}_{z}^{2}-I(I+1) \hat{1}\right]$ is an even function of $\hat{I}_{z}$ and does not shift the transition frequency of any symmetric transition between states of $+\left|m_{I}\right|$ and $-\left|m_{I}\right|$, since $\left\langle I,+\left|m_{I}\right|\left|\hat{H}_{Q}^{(1)}\right| I,+\left|m_{I}\right|\right\rangle-\left\langle I,-\left|m_{I}\right|\left|\hat{H}_{Q}^{(1)}\right| I,-\left|m_{I}\right|\right\rangle=$ 0 and therefore does not cause any time evolution of a symmetric coherence. Consequently, the satellite transitions corresponding to non-symmetric single quantum coherences are broadened by the first-order quadrupolar Hamiltonian, whereas the central transition is not. Most quadrupolar NMR experiments therefore focus on the central transition of the spectrum, which is subject to quadrupolar broadening only under the second order Hamiltonian $\hat{H}_{Q}^{(2)}$. As will be seen, this broadening is not completely removed by MAS.

During sample spinning the evolution frequency of a symmetric p-quantum coherence $\left|I, m_{I}=+p / 2\right\rangle\left\langle I, m_{I}=-p / 2\right|$ under a time-averaged single-crystallite rotating frame 


\subsection{REMOVING THE SECOND-ORDER QUADRUPOLAR}

Hamiltonian that includes chemical shift and second-order quadrupolar effects is

$$
\begin{aligned}
\omega_{p}^{(2)} & =\left\langle I,+\frac{p}{2}\left|\left[\hat{H}_{c s}+\hat{\bar{H}}_{Q}^{(2)}\right] / \hbar\right| I,+\frac{p}{2}\right\rangle-\left\langle I,-\frac{p}{2}\left|\left[\hat{H}_{c s}+\hat{\bar{H}}_{Q}^{(2)}\right] / \hbar\right| I,-\frac{p}{2}\right\rangle \\
& =\left(\delta+\omega_{0}^{c s}\right) p+\left[\omega_{0}^{2 Q} C_{0}^{I}(p) P_{0}(\cos \theta)+\omega_{2}^{2 Q} C_{2}^{I}(p) P_{2}(\cos \theta)+\omega_{4}^{2 Q} C_{4}^{I}(p) P_{4}(\cos \theta)\right],
\end{aligned}
$$

where $\delta$ is the resonance offset frequency, $\omega_{0}^{c s}$ is the isotropic chemical shift frequency, the $\omega_{k}^{2 Q}$ are rank- $k$ spatial tensor contributions to the second-order quadrupolar frequency shift, the $C_{k}^{I}(p)$ are coefficients that depend on the spin degrees of freedom of the system (including the spin quantum number $I$ and the coherence order $p$ ), the $P_{k}(x)$ are the rank- $k$ Legendre polynomials, and $\theta$ is the angle between the rotor (spinning) axis and the Zeeman magnetic field. ${ }^{1}$ The $\omega_{k}^{2 Q}$ arise from the spatially dependent terms of the Hamiltonian; the rank-0 term $\omega_{0}^{2 Q}$ is isotropic and does not depend on crystallite orientation, whereas the $\omega_{2}^{2 Q}$ and $\omega_{4}^{2 Q}$ terms are anisotropic and depend on crystallite orientation. It is the anisotropic terms that lead to the second-order quadrupolar broadening, and it is these terms which must somehow be removed in order to narrow the central transition linewidth.

One strategy to eliminate both the second- and fourth-rank quadrupolar terms is to rotate the sample about two axes $\theta_{1}$ and $\theta_{2}$ simultaneously such that $P_{2}\left(\cos \theta_{1}\right)=0$ and $P_{4}\left(\cos \theta_{2}\right)=0$ (the condition $P_{2}\left(\cos \theta_{2}\right)=0$ and $P_{4}\left(\cos \theta_{1}\right)=0$ also works). The first angle $\theta_{1}$ is measured relative to the magnetic field axis, and the second angle $\theta_{2}$ is measured relative to the first spinning axis. The first angle is the inverse cosine of the zero of the

\footnotetext{
${ }^{1}$ The rank-0 polynomial $P_{0}(x)$ always equals 1 regardless of its argument, so this term is actually independent of the angle of the spinning axis. It is included here for the sake of consistency, since sample spinning generates a $P_{k}(\cos \theta)$ dependence from rank- $k$ spatial tensor terms. Strictly speaking, the isotropic chemical shift term should also have a $P_{0}(\cos \theta)$ "dependence."
} 


\subsection{REMOVING THE SECOND-ORDER QUADRUPOLAR BROADENING. THE MQMAS EXPERIMENT.}

second-rank Legendre polynomial, which is the magic angle $\theta_{1}=\theta_{m}=\tan ^{-1}(\sqrt{2}) \simeq 54.74^{\circ}$. The second is the inverse cosine of the zero of the fourth-rank Legendre polynomial, $\theta_{2} \simeq$ $30.56^{\circ}$ or $\theta_{2} \simeq 70.12^{\circ}$. This technique is called double rotation (DOR) [134]; it not only averages away the second- and fourth-rank second-order quadrupolar broadening, but also other second-rank anisotropic terms such as the dipole-dipole coupling and CSA. Another technique that was developed to remove the second-order broadening is dynamic angle spinning (DAS) [135, 136, 137]. The DAS principle involves switching the spinning axis between two angles $\theta_{1}$ and $\theta_{2}$ (called a DAS angle pair) during the indirect dimension delay in a two-dimensional experiment such that the anisotropic quadrupolar (and also CSA and dipole-dipole) evolution is refocused in a type of generalized spin echo. The echo is induced entirely by spatial manipulation of the sample.

The DAS and DOR techniques both require the use of special NMR probes. In particular, DOR involves a "rotor inside a rotor" design, in which the speed of the outer rotor is limited for mechanical reasons. This can make spectral interpretation difficult due to the presence of many closely-spaced spinning sidebands. However, DOR has the advantage of being a one dimensional experiment, so it is fast. The DAS experiment is two dimensional and takes longer, but the 2D anisotropic-isotropic correlation spectra can be useful. However, the DAS technique requires the magnetization to be stored along the Zeeman field axis for $>10 \mathrm{~ms}$ while the rotor switches positions in order to avoid dephasing, and significant magnetization could be lost during the storage time if longitudinal relaxation is fast (as is often the case for quadrupolar nuclei).

Both the DAS and DOR techniques use manipulations of two spatial degrees of 


\subsection{REMOVING THE SECOND-ORDER QUADRUPOLAR BROADENING. THE MQMAS EXPERIMENT.}

freedom to remove the two independent anisotropic second-order quadrupolar terms simultaneously. Frydman and coworkers have introduced an experiment called multiple-quantum magic-angle spinning (MQMAS) that employs a spin manipulation to refocus the fourthrank second-order quadrupolar broadening in combination with spinning solely at the magic angle to remove all second-rank anisotropic broadening. Unlike DAS and DOR which use only $|p|=1$ single-quantum coherences, MQMAS also uses $|p|>1$ multiple-quantum coherences. This technique has the advantage that it can be implemented in a standard solid-state MAS probe. Other characteristics of the experiment are described below.

\subsubsection{Conventional MQMAS}

This section describes the original MQMAS experiment due to Frydman et al. that uses a multiple-quantum evolution time in conjunction with a single-quantum evolution time to refocus the fourth-rank second order quadrupolar broadening [138, 139]. Some of the developments in this field have been reviewed by Goldbourt and Madhu [140]. Like DAS, MQMAS is a two-dimensional experiment that correlates the quadrupolar-broadened (anisotropic) MAS spectrum in the direct dimension with the narrow isotropic spectrum in the indirect dimension.

The MQMAS experiment is performed entirely at the magic angle, so consider Eq. 3.1 when evaluated at $\theta=\theta_{m}$ :

$$
\omega_{p}^{(2)}=\left(\delta+\omega_{0}^{c s}\right) p+\left[\omega_{0}^{2 Q} C_{0}^{I}(p)+\omega_{4}^{2 Q} C_{4}^{I}(p) P_{4}\left(\cos \theta_{m}\right)\right] .
$$

The magic angle spinning has removed the second-rank quadrupolar broadening $\omega_{2}^{2 Q}$ since 


\subsection{REMOVING THE SECOND-ORDER QUADRUPOLAR BROADENING. THE MQMAS EXPERIMENT.}

$P_{2}\left(\cos \theta_{m}\right)=0$. This equation holds for any symmetric $p$-quantum coherence. Imagine performing a two dimensional NMR experiment in which a symmetric $p_{1}$-quantum coherence is created and evolves during the indirect dimension time interval $t_{1}$ before it is converted to symmetric $p_{2}$-quantum coherence during the direct dimension time interval $t_{2}$. The only symmetric coherence that is directly observable by quadrature detection is the $p_{2}=-1$ central transition coherence, and phase cycling can be used to select the proper $0 \rightarrow p_{1} \rightarrow-1$ coherence pathway. The NMR signal is proportional to:

$$
S\left(t_{1}, t_{2}\right) \propto \exp \left[-i \omega_{p_{1}}^{(2)} t_{1}\right] \exp \left[-i \omega_{p_{2}}^{(2)} t_{2}\right]
$$

The phase acquired by anisotropic quadrupolar evolution in the indirect dimension is $\phi_{1}^{2 Q}=\omega_{4}^{2 Q} C_{4}^{I}\left(p_{1}\right) P_{4}\left(\cos \theta_{m}\right) t_{1}$, whereas the phase acquired by anisotropic quadrupolar evolution in the direct dimension is $\phi_{2}^{2 Q}=\omega_{4}^{2 Q} C_{4}^{I}\left(p_{2}\right) P_{4}\left(\cos \theta_{m}\right) t_{2}$, such that $\phi_{2}^{2 Q} / \phi_{1}^{2 Q}=$ $\left[C_{4}^{I}\left(p_{1}\right) t_{1}\right] /\left[C_{4}^{I}\left(p_{2}\right) t_{2}\right]$. In other words, these two phases are proportional to each other by a factor that does not contain any orientation dependence. They only depend on the spin quantum number, coherence order, and evolution time. The coherence order of the system and evolution time are under experimental control. Therefore the use of symmetric coherences not only rids the spectrum of any first order quadrupolar broadening, but also can be used to refocus the rank- 4 quadrupolar broadening when $\phi_{1}+\phi_{2}=0$ by choosing $t_{2}=k t_{1}$, where $k=-C_{4}^{I}\left(p_{1}\right) / C_{4}^{I}\left(p_{2}\right) \cdot{ }^{2}$ The constant $k$ must be positive, which means $C_{4}^{I}\left(p_{1}\right)$ and $C_{4}^{I}\left(p_{2}\right)$ must have opposite signs; another way of saying this is that the coherences $p_{1}$ and $p_{2}$ must evolve in opposite directions under the fourth-rank quadrupolar terms in order for

\footnotetext{
${ }^{2}$ The use of the symbol $k$ is common in the literature and should not be confused with the spherical tensor rank $k$ used elsewhere in this treatment.
} 


\subsection{REMOVING THE SECOND-ORDER QUADRUPOLAR}

this evolution to be refocused. At the point of the refocusing the signal is:

$$
S\left(t_{1}, t_{2}=k t_{1}\right) \propto \exp \left[-i\left(\widetilde{\omega}_{0}^{c s}+\widetilde{\omega}_{0}^{2 Q}\right) t_{1}\right]
$$

where the scaled isotropic chemical shift/resonance offset and scaled isotropic second-order quadrupolar shift are $\widetilde{\omega}_{0}^{c s}=\left(\delta+\omega_{0}^{c s}\right)\left(p_{1}+k p_{2}\right)$ and $\widetilde{\omega}_{0}^{2 Q}=\omega_{0}^{2 Q}\left(C_{0}^{I}\left(p_{1}\right)+k C_{0}^{I}\left(p_{2}\right)\right)$. This amounts to a spin echo of the fourth-rank quadrupolar broadening that forms at a time $t_{2}=k t_{1}$. The $C_{k}^{I}(p)$ coefficients are given by Eq. 1.63:

$$
\begin{aligned}
& C_{0}^{I}(p)=p\left[I(I+1)-\frac{3}{4} p^{2}\right] \\
& C_{2}^{I}(p)=p\left[8 I(I+1)-4 p^{2}-3\right] \\
& C_{4}^{I}(p)=p\left[18 I(I+1)-\frac{17}{2} p^{2}-5\right] .
\end{aligned}
$$

These coefficients are odd functions of the coherence order $p$, reflecting the fact that the second-order quadrupolar Hamiltonian $\hat{H}_{Q}^{(2)}$ is an odd function of the spin operator $\hat{I}_{z}$ (see §1.3.7). Therefore it is always possible to ensure that the constant $k$ is positive by choosing the appropriate sign of the coherence order $p_{1}$ (the coherence order $p_{2}=-1$ is fixed). ${ }^{3}$

Consider a spin $I=5 / 2$ nucleus. Aside from the $p= \pm 1$ symmetric central transition single-quantum coherences $\left|\frac{5}{2}, \pm \frac{1}{2}\right\rangle\left\langle\frac{5}{2}, \mp \frac{1}{2}\right|$, this system also supports symmetric $p= \pm 3$ triple-quantum coherences $\left|\frac{5}{2}, \pm \frac{3}{2}\right\rangle\left\langle\frac{5}{2}, \mp \frac{3}{2}\right|$ and symmetric $p= \pm 5$ quintuple-quantum coherences $\left|\frac{5}{2}, \pm \frac{5}{2}\right\rangle\left\langle\frac{5}{2}, \mp \frac{5}{2}\right|$. The conventional MQMAS experiment that uses $p_{1}=-5$ and

\footnotetext{
${ }^{3}$ Note that both the second- and fourth-rank second-order quadrupolar anisotropies can be refocused simultaneously by a simple $\pi$-pulse induced spin echo, which selects the $p_{1}=+1$ to $p_{2}=-1$ coherence pathway. Unfortunately, this type of spin echo inverts all coherences $p$ and thus also refocuses the isotropic chemical shift and isotropic quadrupolar shift, so the information about the sample of interest is lost, and all resonances would occur at zero frequency in the indirect dimension.
} 


\subsection{REMOVING THE SECOND-ORDER QUADRUPOLAR BROADENING. THE MQMAS EXPERIMENT.}

$p_{2}=-1$ quantum coherences to form the MQMAS echo is known as the 5Q1QMAS or simply 5QMAS experiment. In this case $k=-C_{4}^{I}(-5) / C_{4}^{I}(-1)=25 / 12 \simeq 2.083$. Another possibility is the 3QMAS experiment with $p_{1}=+3$ and $p_{2}=-1$, with $k=19 / 12 \simeq 1.583$. The choice of 5QMAS versus 3QMAS experiment depends mainly on two factors: which experiment is more sensitive, and which offers higher spectral resolution in the indirect dimension. In general, the creation of high coherence orders $p$ is less efficient than the creation of low coherence orders, so one expects the 5QMAS experiment to be less sensitive. However, in the ratio of the scaled chemical shift in the MQMAS experiment to the chemical shift that one would observe under evolution as only -1-quantum coherence is $\kappa_{c s}=\left(p_{1} \omega_{0}^{c s}+k p_{2} \omega_{0}^{c s}\right) /\left(p_{2} \omega_{0}^{c s}+k p_{2} \omega_{0}^{c s}\right)=-\left(p_{1} 1-k\right) /(1+k)$. The 5QMAS experiment therefore gives a chemical shift resolution enhancement of $\kappa_{c s}=2.297$ in the indirect dimension over the chemical shift that is observed in the direct dimension, whereas the 3QMAS experiment only offers a relative chemical shift resolution scaling of $\kappa_{c s}=-0.548$. In other words, the 5QMAS experiment more than doubles the effective chemical shift resolution, whereas the 3QMAS experiment cuts the chemical shift resolution nearly in half. Note that the observed peak in the indirect dimension is at the sum of the isotropic chemical and quadrupolar shift frequencies, where the isotropic quadrupolar shift scaling factor is $\kappa_{2 Q}=\left[C_{0}^{I}\left(p_{1}\right)+k C_{0}^{I}(-1)\right] /\left[C_{0}^{I}(-1)+k C_{0}^{I}(-1)\right]=\left[C_{0}^{I}\left(p_{1}\right) / C_{0}^{I}(-1)+k\right] /(1+k)$. Therefore any resolution enhancement or decrease would actually be the sum of two factors that can possibly compete with each other depending on the signs of the $\kappa$ factors. Also, any "resolution enhancement" is predicated on the fact that the linewidth in the indirect dimension does not increase by the same amount (or more) as the isotropic shift scaling. In principle, 


\subsection{REMOVING THE SECOND-ORDER QUADRUPOLAR BROADENING. THE MQMAS EXPERIMENT.}

the use of the highest symmetric multiple quantum coherence available to the system (i.e., $\left|p_{1}\right|=2 I$ ) offers the best chemical shift scaling factor. However, Ref. [141] (and references therein) discusses some of the subtleties of achieving actual resolution enhancement in such experiments.

Since the MQMAS echo forms at a time $t_{2}=k t_{1}$ in the direct dimension, the echo moves further and further into the acquisition window as $t_{1}$ is incremented. The hallmark of any coherence transfer echo experiment in which the echo travels in the acquisition window in proportion to $t_{1}$ is a "shearing" effect in the two-dimensional Fourier-transformed spectrum $[7, \S 6.6 .1]$, where the peaks do not fall along the diagonal in the $2 \mathrm{D}$ spectrum but rather along a line of slope $k$ (where the indirect $F 1$ dimension is the vertical axis and the direct $F 2$ dimension is the horizontal axis). In this case the projection of the 2D Fouriertransformed data set along the indirect frequency dimension $F 1$ does not yield the desired isotropic spectrum. This can be corrected by applying a mathematical shearing transformation to the data. It also can be corrected experimentally by ensuring that the echo does not travel in the acquisition window from transient to transient. Brown et al. [142] have introduced the "split- $t_{1}$ " MQMAS experiment, in which some of the single-quantum evolution is included in the indirect dimension so that the fourth-rank quadrupolar broadening is refocused entirely during the $t_{1}$ interval. Therefore the peak of the echo forms at the beginning of $t_{2}$ for every transient, and there is no shearing effect after Fourier transformation. Unfortunately, a time domain data set collected in this manner does not have a pure absorptive-phase lineshape after the double Fourier transform. This problem can be overcome by the States method, in which a $t_{1}$-amplitude-modulated signal is collected by 


\subsection{REMOVING THE SECOND-ORDER QUADRUPOLAR BROADENING. THE MQMAS EXPERIMENT.}

retaining both the echo $\left(+p_{1}\right)$ and anti-echo $\left(-p_{1}\right)$ coherence pathways to form a hypercomplex data set [143]. It is also possible to apply pulses such that the top of the echo forms at a time $\tau$ into the acquisition window instead of at the beginning. If $\tau$ is long enough, the entire spin echo can be moved into the acquisition window. The acquisition of the whole echo allows pure absorption-mode lineshapes after the 2D Fourier transformation even if a single $0 \rightarrow p_{1} \rightarrow-1$ coherence pathway is collected $[144,145]$. This "shifted echo" method is advantageous when combined with the split- $t_{1}$ technique $[146,147]$.

The sensitivity of the MQMAS experiment hinges on the efficiency of the pulses that create multiple-quantum coherence $p_{1}$ from longitudinal magnetization and convert this MQC to single-quantum coherence $p_{2}=-1$ for detection. It is difficult to manipulate the nuclear polarization with rf pulses that are weak compared to the quadrupolar interaction. The simplest MQMAS experiment uses a single "hard" (high-amplitude) rf pulse for multiple-quantum excitation, and another hard pulse for conversion $[139,144,148] .{ }^{4}$ The single-pulse multiple quantum excitation technique is based on old ideas by Vega and Pines for double quantum excitation in $I=1$ systems $[149,150]$ that were extended by Vega and Naor to experiments on half-integer spin systems [151]; single-pulse excitation represents an improvement over the two-pulse excitation sequence in the original MQMAS experiment [138], which is more appropriate when the rf strength is stronger than the quadrupolar interaction. Some other techniques for inducing coherence transfer in half-integer quadrupolar nuclei are discussed in $\S 5.6 .2$.

Finally, it should be mentioned that most methods of creating or converting

\footnotetext{
${ }^{4}$ Conventional NMR terminology dictates that a "hard" pulse is one for which the rf amplitude dominates any other internal interaction. Since this is almost never the case when a large quadrupolar coupling is present, the term "hard" pulse is sometimes used in the quadrupolar NMR community to mean an rf pulse that is as strong as possible, as opposed to a "soft" (low amplitude) selective or adiabatic pulse.
} 


\subsection{REMOVING THE SECOND-ORDER QUADRUPOLAR BROADENING. THE MQMAS EXPERIMENT.}

multiple-quantum coherence also form undesired coherences that can lead to spectral artifacts, so these unwanted coherences need to be suppressed. Certainly, a density operator that contains $\mathrm{a}+p_{1}$ coherence without a $-p_{1}$ coherence is non-Hermitian, so some manner of coherence pathway selection must be applied to suppress the $-p_{1}$ multiple-quantum coherence pathway in the final signal. This can be accomplished by standard Bodenhausentype phase cycling [152],[7, §6.3],[4, $\S 4.3]$, and Levitt's cogwheel phase cycling procedure has also been applied to MQMAS experiment [153, 154], as have magic-angle pulsed field gradient coherence pathway selection methods [155].

\subsubsection{Mixed-coherence MQMAS}

Jerschow has observed [156] that the split- $t_{1}$ MQMAS experiment can be modified such that the anisotropic quadrupolar broadening is refocused completely during $t_{1}$ using two symmetric multiple quantum coherences (MQCs) instead of one symmetric MQC and one symmetric single-quantum coherence, and that better isotropic shift scaling factors can be obtained this way. This is the mixed-multiple quantum MAS (MMQMAS) experiment. It is applicable to nuclei with $I>3 / 2$ that are capable of supporting at least two symmetric multiple-quantum coherences $p_{1}$ and $p_{2}$, and in a sense the conventional split- $t_{1}$ MQMAS experiment is a special case of MMQMAS with $\left|p_{2}\right|=1$. The highest isotropic shift scaling factors (and hence the best theoretical resolution enhancement) are obtained when the two highest symmetric MQCs available to the system are used, e.g. $p_{1}=+5$ and $p_{2}=+3$ in the $I=5 / 2$ 5Q3QMAS experiment, or $p_{1}=+7$ and $p_{2}=+5$ in the $I=7 / 2$ 7Q5QMAS experiment. Jerschow et al. have also adopted the shifted echo approach for pure absorptivephase lineshapes. 


\subsection{REMOVING THE SECOND-ORDER QUADRUPOLAR BROADENING. THE MQMAS EXPERIMENT.}

The basic $I=5 / 2$ 5Q3QMAS pulse sequence and coherence pathway diagram is shown in Fig. 3.1. The $+5 \mathrm{QC}$ is excited from longitudinal magnetization with a single pulse and allowed to evolve for a time $a t_{1}$ before another pulse converts it to $+3 \mathrm{QC}$, which evolves for a time $b t_{1}$, where $a+b=1$. In terms of the previous notation one finds $k=b / a$, where $k=-C_{4}^{I}(+5) / C_{4}^{I}(+3)=25 / 19$, such that $a=19 / 44$ and $b=25 / 44$. At the end of the $t_{1}$ interval the fourth-rank, second-order quadrupolar broadening is refocused. The $+3 \mathrm{QC}$ could then be transferred directly to $-1 \mathrm{QC}$ for detection during $t_{2}$, but in the shifted echo approach it is converted to $+1 \mathrm{QC}$ instead and allowed to evolve for a time $\tau$, during which the coherence dephases. Then a central-transition selective $\pi$-pulse inverts the $+1 \mathrm{QC}$ to $-1 \mathrm{QC}$ for quadrature detection in the interval $t_{2}$, and the coherence rephases at a time $t_{2}=\tau$ to form a spin echo. ${ }^{5}$ The delay $\tau$ is chosen to be long enough so that the entire echo forms in the acquisition window. The proper coherence pathway is selected by phase cycling, e.g. a 640-step phase cycle [156] calculated using the CCCP program [157], ${ }^{6}$ or a shorter 201-step cogwheel phase cycle [154]. Any such phase cycling procedure also lets through non-symmetric coherences, which in principle could contribute to the final signal, but these coherences are assumed to be dephased during $t_{1}$ (or $t_{2}$ in case of the single-quantum coherences) by evolution under the large, anisotropic first-order quadrupolar coupling.

Although the mixed-MQMAS experiments potentially can yield higher-resolution isotropic spectra in the indirect dimension than conventional MQMAS experiments, they suffer from sensitivity problems since they involve an extra coherence conversion pulse,

\footnotetext{
${ }^{5}$ Conversion pulses that cause a small change in coherence order $\Delta p$ are usually more efficient, so if $+3 Q \mathrm{C}$ is converted to $+1 \mathrm{QC}$ with $\Delta p=-2$ and then efficiently to $-1 \mathrm{QC}$ with a selective pulse, better signal may be obtained than if $+3 \mathrm{QC}$ was converted to $-1 \mathrm{QC}$ directly with $\Delta p=-4$.

${ }^{6}$ This phase cycle may actually let through some other coherence pathways that were determined to have small amplitudes.
} 


\subsection{REMOVING THE SECOND-ORDER QUADRUPOLAR

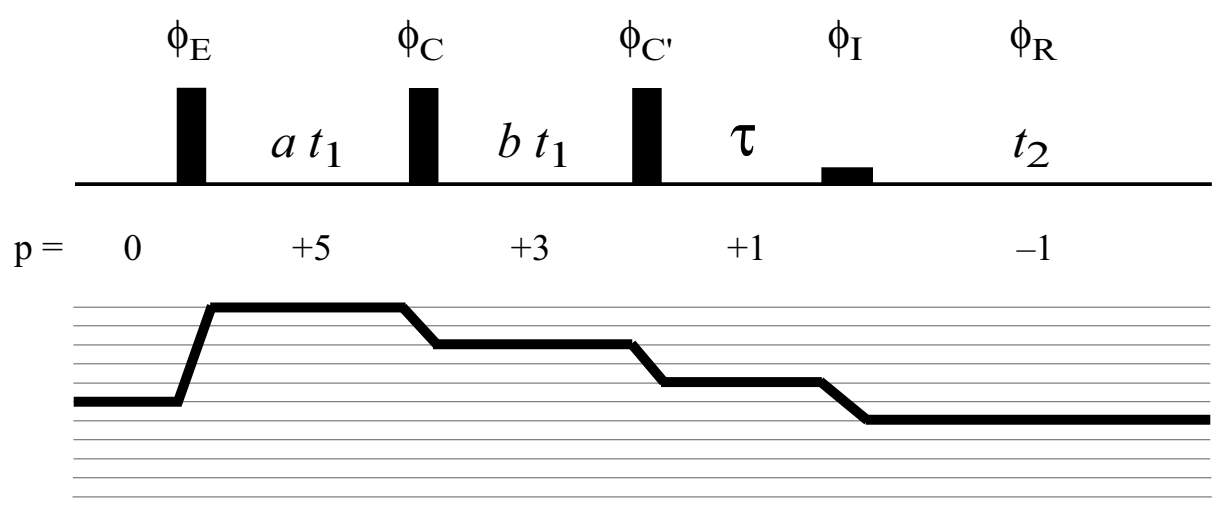

Figure 3.1: Pulse sequence and coherence pathway diagram for the 5Q3QMAS experiment. The fourth-rank second-order quadrupolar broadening is refocused after split evolution in the indirect dimension during the intervals $a t_{1}$ and $b t_{1}$ as +5 -quantum and +3 -quantum coherence, respectively, where $a=19 / 44$ and $b=25 / 44$. The multiple-quantum coherence is then converted to single-quantum coherence for detection, where a selective central transition inversion pulse causes a spin echo at a time $\tau$ in the direct dimension $t_{2}$. The phases of the multiple-quantum excitation, first and second conversion, and soft inversion rf pulses and the receiver phase are $\phi_{E}, \phi_{C}, \phi_{C^{\prime}}, \phi_{I}$, and $\phi_{R}$, respectively.

since at the end of $t_{1}$ the multiple-quantum coherence is not directly observable and must be converted to single-quantum coherence for detection. Therefore the MMQMAS experiments are even more in need of efficient multiple-quantum excitation and conversion pulses than are the conventional MQMAS experiments.

One other interesting property of the MMQMAS experiments is that can they extend the generality of MQMAS to integer spins. Conventional MQMAS cannot be applied to integer spins because it involves a symmetric single-quantum (central transition) coherence, which integer spins do not possess. However, the integer spins with $I>1$ all have at least two symmetric multiple quantum coherences that can be used to refocus the second-order quadrupolar broadening, for instance the $p_{1}=+6$ coherence $|3,+3\rangle\langle 3,-3|$ and the $p_{2}=+4$ coherence $|3,+2\rangle\langle 3,-2|$. The extension to integer spins is of limited utility; other than the $I=3$ B-10 nucleus that exists in $20 \%$ natural abundance, there are no common nuclides of 
$I>1$ with significant natural abundance. The MMQMAS experiment is not applicable to $I=1$ nuclei since they possess only one symmetric coherence, the unique double-quantum coherence. The second-order quadrupolar broadening is difficult to remove in $I=1$ nuclei using spin manipulations, because the spin operator $\hat{I}_{z}^{3}$ is then proportional to $\hat{I}_{z}$, so that $\hat{H}_{Q}^{(2)} \propto \hat{I}_{z}$ (see $\left.\S 1.3 .7\right)$. In this case the second-order quadrupolar Hamiltonian shares the same spin operator dependence as the chemical shift Hamiltonian, so any attempt to refocus the quadrupolar broadening using rf pulses also refocuses the chemical shift.

\subsection{Rotary resonance phenomena}

There has been much work dedicated toward improving the sensitivity of the MQMAS experiment, most of which has focused on increasing the efficiencies of multiplequantum coherence conversion and excitation pulses under sample spinning conditions. Some of these techniques are reviewed in $§ 5.6 .2$. Most are applicable in the limit where the rotationally-induced time dependent quadrupolar coupling can induce adiabatic coherence transfers in the presence of an rf field. An adiabaticity parameter $\alpha=\left|\omega_{1}^{2} /\left(\omega_{Q} \omega_{R}\right)\right|$ has been defined by A. Vega, where $\omega_{1}=\gamma B_{1}$ is the rotating-frame rf field strength in frequency units, $\omega_{Q}$ is the quadrupolar coupling frequency, and $\omega_{R}$ is the sample spinning frequency [158]. The limit $\alpha \gg 1$ characterizes the adiabatic passage regime and $\alpha \ll 1$ characterizes the sudden passage regime. The adiabatic transfers are effective when $\alpha$ is well into the adiabatic regime, i.e., when the rf power is high and the sample spinning is slow, and the quadrupolar coupling is not too large.

Some studies of quadrupolar spin dynamics in the presence of radiofrequency ir- 
radiation have been performed in the sudden passage regime, i.e., under fast spinning and low rf power conditions $[158,159,160]$. Several workers noted that unusual spin dynamics are observed at resonant conditions when the rf strength $\omega_{1}$ is appropriately matched to the spinning speed $\omega_{R}[159,160]$, as is discussed further in $\S 5.6 .5$. The "rotary resonance" condition for a nucleus with half-integer angular momentum $I$ is:

$$
\omega_{1}=\frac{4 N}{2 I+1} \omega_{R}
$$

where $N$ is an integer. This condition reduces to $\omega_{1}=N \omega_{R}$ for $I=3 / 2$ and $\omega_{1}=\frac{2}{3} N \omega_{R}$ for $I=5 / 2$. The origin of these resonances is explored in $§ 3.5 .2$. These resonant effects remained largely a curiosity until Vosegaard et al. discovered that certain coherence transfers could be induced under fast spinning and low rf power conditions when the rf amplitude was matched to one of these resonances, and other coherence transfers were possible when the rf amplitude was set in between the resonances. These effects formed the basis of the first so-called "FASTER" (FAst Spinning gives Transfer Enhancement at Rotary resonance) MQMAS experiment in $I=3 / 2$ nuclei [161]. Unlike the adiabatic techniques, FASTER MQMAS operates in the sudden passage regime and is easily applicable to nuclei with relatively large quadrupolar coupling constants of several $\mathrm{MHz}$ (although there is eventually a limit to how large the quadrupolar coupling can be). Walls [162] and Gan and Grandinetti [163] have developed successful Floquet theories of these effects in $I=3 / 2$ systems, and Walls extended the theory to include $I=5 / 2$ nuclei [164]. The work presented in this chapter represents the first experimental investigation of rotary resonance effects in the sudden passage regime in $I=5 / 2$ nuclei $[164,165,166]$. It also represents the first extension 
of the FASTER technique to the mixed-MQMAS experiment, which is applicable only in $I>3 / 2$ systems.

\subsection{Rotary resonance experiments in $I=5 / 2$ systems}

Although detailed investigations of the efficiencies of various multiple-quantum excitation and conversion pulses as functions of pulse amplitude and length were conducted in $I=5 / 2$ 3QMAS, 5QMAS, and 5Q3QMAS experiments $[164,165,166]$, these results will not be presented here. Rather, the results of these investigations will be used to show how the sensitivity of the 5Q3QMAS experiment can be increased using low-amplitude pulses matched at or away from rotary resonance conditions. Also, a new line narrowing effect in the anisotropic dimension of the 5Q3QMAS experiment will be discussed.

\subsubsection{Experimental procedure}

The low-amplitude pulse experiments were performed on the $I=5 / 2 \mathrm{Al}-27$ nucleus in a sample of aluminum acetylacetonate (Aldrich, 99\%), $\mathrm{Al}(\mathrm{acac})_{3}$, where acac $=\left[\mathrm{H}_{3} \mathrm{C}\right.$ (CO)-CH- $\left.(\mathrm{CO})-\mathrm{CH}_{3}\right]^{-}$. This compound is reported to have one unique Al-27 site with a quadrupolar coupling constant of $C_{Q}=3.0 \mathrm{MHz}$ and an electric field gradient asymmetry parameter of $\eta_{Q}=0.15$ [167]. The $\mathrm{Al}(\mathrm{acac})_{3}$ sample was ground with a mortar and pestle to ensure homogeneity before being packed into a $3.2 \mathrm{~mm}$ outer diameter zirconia rotor. ${ }^{7}$ All experiments were performed on a Chemagnetics/Varian CMX Infinity 500 spectrometer with a $3.2 \mathrm{~mm}$ Chemagnetics solid-state magic-angle spinning probe operated at $\omega_{R} / 2 \pi=$ $20 \mathrm{kHz}$. The magnetic field strength was 11.7 Tesla, corresponding to an Al-27 Larmor

\footnotetext{
${ }^{7}$ It is important to choose a rotor material that gives no Al-27 background signal.
} 
frequency of $130.3 \mathrm{MHz}$. The rf pulse amplitudes were calibrated against a sample of $1 \mathrm{M}$ $\mathrm{AlCl}_{3}(\mathrm{aq})$ solution. ${ }^{8}$

The 5Q3QMAS pulse sequence was implemented as illustrated in Fig. 3.1. The rf carrier frequency was set to the center of the central transition anisotropic powder pattern. A 640-step phase cycle was used to select the proper coherence pathway [165]. The dwell time was $40 \mu \mathrm{s}$ in the indirect dimension $t_{1}$ and $50 \mu \mathrm{s}$ (rotor-synchronized) in the direct dimension $t_{2}$, where there were 128 and 256 data points collected in $t_{1}$ and $t_{2}$, respectively. The soft inversion pulse was experimentally optimized to be $12.5 \mu \mathrm{s}$ long with an rf amplitude of $\omega_{1} / 2 \pi=3.7 \mathrm{kHz}$. The echo delay was $\tau=5 \mathrm{~ms}$. Two types of multiple-quantum excitation and conversion pulse were implemented. The "hard" pulse (HP) version used an $\omega_{1} / 2 \pi=150 \mathrm{kHz}$ amplitude pulse experimentally optimized to be $0.47 \mu \mathrm{s}$ long. The low-amplitude pulse (LAP) conditions were chosen from the optimal values found in experimental investigations of low-amplitude pulses near rotary resonance conditions $[165,166]$. For example, a $190 \mu \mathrm{s}, 19 \mathrm{kHz}$ rf pulse was used for $+5 \mathrm{QC}$ excitation from longitudinal magnetization, and a $95 \mu \mathrm{s}, 6.5 \mathrm{kHz}$ rf pulse was used for $+5 \mathrm{QC}$ to $+3 \mathrm{QC}$ conversion.

\subsubsection{Sensitivity and line shape considerations in 5Q3QMAS NMR with low-amplitude pulses}

Theoretical investigations of low-amplitude rf pulses in $I=5 / 2$ systems under fast spinning conditions have indicated the following results, which were confirmed by experiment [164]. At rotary resonance conditions $\omega_{1}=\frac{2}{3} N \omega_{R}$ symmetric triple-quantum coher-

\footnotetext{
${ }^{8}$ It is important to calibrate the rf pulses against a solution state sample in which the quadrupolar coupling is motionally averaged away, in order to avoid quadrupolar-induced truncations of the rf field and central transition-selective excitation $(\S 5.6 .2)$.
} 
ence is strongly mixed with symmetric single-quantum (central transition) coherence, inducing efficient triple-quantum to single-quantum conversions. The conversion of quintuplequantum coherence to single-quantum coherence is also efficient at rotary resonance conditions, mainly due to some transfer of quintuple to triple quantum coherence which is then efficiently transferred to single-quantum coherence. Multiple quantum excitation from longitudinal magnetization is minimal at rotary resonance conditions.

In between adjacent rotary resonance conditions $\left(\frac{2}{3}(N-1) \omega_{R}<\omega_{1}<\frac{2}{3} N \omega_{R}\right)$ multiple-quantum excitations are efficient, although there are efficiency minima roughly at the halfway points between rotary resonances due to powder averaging effects. Quintuplequantum to triple-quantum conversion is efficient at roughly the halfway points between rotary resonances; these coherences are also mixed at rotary resonance conditions but the transfer is suppressed by the subsequent strong triple-quantum to single-quantum conversion. In between rotary resonance conditions the triple-quantum to single-quantum conversion efficiency is minimal.

The rotary resonance conditions in an $I=5 / 2$ system spinning at $\omega_{R} / 2 \pi=20$ $\mathrm{kHz}$ are $\omega_{1} / 2 \pi=N \times 13.33 \mathrm{kHz}$. The $\omega_{1} / 2 \pi=19 \mathrm{kHz}$ experimental optimum amplitude for the $+5 \mathrm{QC}$ excitation pulse used in the 5Q3QMAS experiments is somewhat less than halfway between the $N=1$ and $N=2$ rotary resonance conditions. The $\omega_{1} / 2 \pi=6.5$ $\mathrm{kHz}$ experimental optimum amplitude for the $+5 \mathrm{QC}$ to $+3 \mathrm{QC}$ conversion pulse is close to halfway between the "zeroth" resonance condition and the $N=1$ resonance condition. These results are consistent with the theoretical results outlined above. It should be noted that an uncertainty in the low-power rf calibration was suspected after comparison of the 
experimental data with numerical simulations [166].

Using values consistent with Vega's notation of $\omega_{Q} / 2 \pi=3 C_{Q} /[2 I(2 I-1)]=$ $450 \mathrm{kHz}, \omega_{R} / 2 \pi=20 \mathrm{kHz}$ and $\omega_{1} / 2 \pi=20 \mathrm{kHz}$, the adiabaticity parameter is $\alpha=$ $\left|\omega_{1}^{2} /\left(\omega_{Q} \omega_{R}\right)\right|=0.044$. This is less than Vega's empirically-determined cutoff (in $I=3 / 2$ systems) of $\alpha \simeq 0.4$ for crossover between the sudden-passage and adiabatic regimes [158], placing the system well inside the sudden-passage regime where rotary resonance effects are prominent. The system is even farther inside the sudden passage regime when weaker rf pulses are used. The $\omega_{1} / 2 \pi=150 \mathrm{kHz}$ "hard" pulses are definitely outside of the suddenpassage regime.

There are three important rf pulses in the 5Q3QMAS experiment that need to be optimized: the $+5 \mathrm{QC}$ excitation pulse, the $+5 \mathrm{QC}$ to $+3 \mathrm{QC}$ conversion pulse, and the $+3 \mathrm{QC}$ to $+1 \mathrm{QC}$ conversion pulse. A series of 5Q3QMAS experiments were performed using all possible combinations of hard pulses and optimized low-amplitude pulses. The standard against which these experiments were compared was the version of the experiment that used three hard pulses. The version that yielded the best sensitivity was the combination of $\mathrm{LAP}+5 \mathrm{QC}$ excitation, $\mathrm{LAP}+5 \mathrm{QC}$ to $+3 \mathrm{QC}$ conversion, and $\mathrm{HP}+3 \mathrm{QC}$ to $+1 \mathrm{QC}$ conversion. Fig. 3.2 shows a comparison of the 2D MQMAS spectrum for this experiment with that of the all-hard pulse experiment. The signal-to-noise ratio of the peak in the isotropic (indirect) dimension is a factor of two higher in the LAP-LAP-HP experiment than in the HP-HP-HP experiment, demonstrating that significant sensitivity increases in the MMQMAS experiment can be achieved using FASTER MQMAS-type techniques.

Closer inspection of Fig. 3.2 shows that there is a considerable narrowing of the 

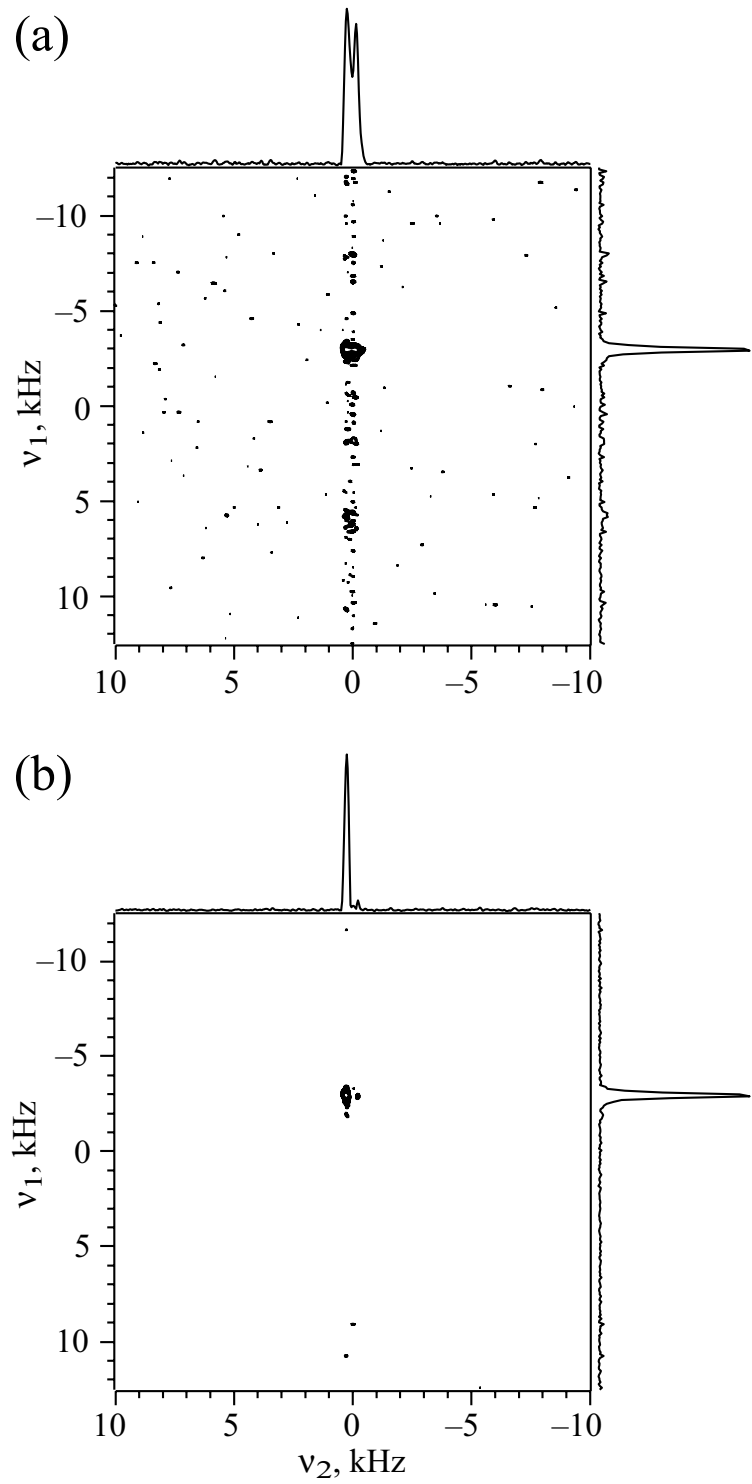

Figure 3.2: Comparison of the 2D Al-27 NMR spectra of $\mathrm{Al}(\mathrm{acac})_{3}$ in the (a) hard pulse and (b) low-amplitude pulse versions of the 5Q3QMAS experiment with spinning at $20 \mathrm{kHz}$. The hard-pulse version used three $\omega_{1} / 2 \pi=150 \mathrm{kHz}$ pulses for the multiple quantum excitations and conversions. The low-amplitude version used optimized low-amplitude pulses for $+5 \mathrm{QC}$ excitation and $+5 \mathrm{QC}$ to $+3 \mathrm{QC}$ conversion, and a $150 \mathrm{kHz}$ hard pulse for $+3 \mathrm{QC}$ to $+1 \mathrm{QC}$ conversion. The direct (anisotropic) dimension is along the horizontal axis and the indirect (isotropic) dimension is along the vertical axis. The isotropic projection has twice the signalto-noise ratio in the low-amplitude pulse experiment as in the all-hard pulse experiment. 
anisotropic spectrum in the direct dimension of the LAP version of the 5Q3QMAS experiment versus the all-HP version, which shows the normal MAS spectrum in the anisotropic dimension. In order to see this better, a one-dimensional data set was generated from the LAP-LAP-HP and HP-HP-HP experiments by taking the first transient in $t_{1}$ in which the delays between the three multiple-quantum pulses are negligible. The Fourier-transformed spectra are shown in Fig. 3.3. The narrowing of the LAP-LAP-HP anisotropic spectrum does not appear to be due to the creation of an isotropic line as occurs in the indirect dimension of an MQMAS experiment. Rather, it seems to be due to the suppression of a certain frequency range in the normal MAS powder pattern. This suggests that the HPHP-HP combination uniformly affects all of the crystallites in the powder, whereas the LAP-LAP-HP experiment selects out only certain crystallite orientations that correspond to a particular region of the second-order quadrupolar-broadened spectrum. This line narrowing effect was not observed in the conventional 5QMAS and 3QMAS experiments, and Vosegaard et al. did not report a similar effect in their $I=3 / 2$ experiments [161]. ${ }^{9}$ Simulations indicate that the orientation selection occurs in two bands of crystallite Euler angles $\beta=35^{\circ}$ to $62^{\circ}$ and $\beta=118^{\circ}$ to $145^{\circ}[165,166]$. The simulations also indicate that the orientation selection occurs primarily due to the low-amplitude $+5 \mathrm{QC}$ excitation and $+5 \mathrm{QC}$ to $+3 \mathrm{QC}$ conversion pulses, especially due to the excitation pulse. ${ }^{10}$ The orientation selectivity seems breaks down for nuclei with large quadrupolar asymmetry parameters $\eta_{Q}$ $\left(\mathrm{Al}(\mathrm{acac})_{3}\right.$ has a small $\left.\eta_{Q}=0.15\right)$. The origin of the orientation-selective line narrowing effects is explored in the next section.

\footnotetext{
${ }^{9}$ Note however that the line narrowing effect is sensitive to resonance offset effects [166] and therefore might not be observed under the wrong experimental conditions.

${ }^{10}$ These simulations were of single-pulse excitations and transfers; the net effect of a series of pulses over
} 

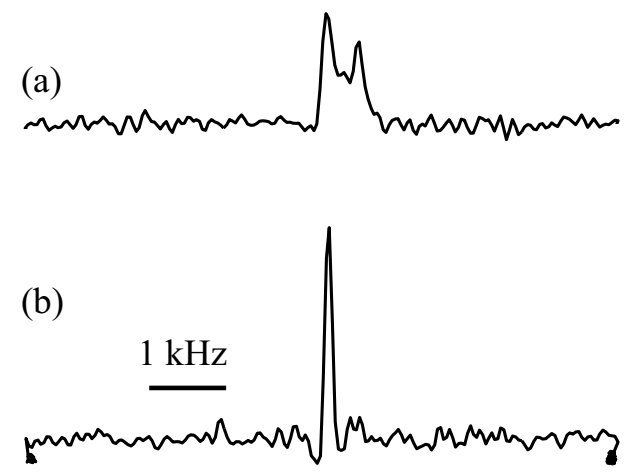

Figure 3.3: Comparison of the Fourier transforms of the first transients of the (a) hard pulse and (b) low-amplitude pulse versions of the 5Q3QMAS experiment on $\mathrm{Al}-27$ in $\mathrm{Al}(\mathrm{acac})_{3}$ with spinning at $20 \mathrm{kHz}$. The anisotropic spectrum in the low-amplitude pulse version is considerably narrowed over the spectrum in the all-hard pulse version, which exhibits the normal quadrupolar-broadened MAS lineshape.

This line narrowing effect suggests a possible new experiment that could be of some utility. As was done shown above, a one-dimensional NMR experiment can be designed such that the $I=5 / 2$ system goes through the $0 \rightarrow+5 \rightarrow+3 \rightarrow+1 \rightarrow-1$ coherence pathway. If the LAP-LAP-HP pulse scheme is implemented, the 1D anisotropic NMR spectrum should be considerably narrowed for quadrupolar sites for which $\eta_{Q} \simeq 0$. If a sample has several such sites whose quadrupolar-broadened resonances overlap in the MAS spectrum, this line narrowing could allow these sites to be resolved without having to resort to a long twodimensional MQMAS experiment. Unfortunately, the quadrupolar parameters could not be extracted from this spectrum without having a model of the narrowed lineshape. The narrowed line also does not correspond to the isotropic line that is observed in the indirect dimension of an MQMAS experiment, so the isotropic shifts also cannot be determined directly from this one-dimensional experiment. However, one could imagine situations in which it would be useful to screen a number of samples rapidly based on the qualitative the course of the experiment may be different. 
distribution of resonances in their NMR spectrum, so quantitative spectral information would be unnecessary. It should be noted that some signal intensity may be lost in the multiple-pulse experiment relative to a standard single-pulse 1D MAS experiment. Also, some form of coherence pathway selection still needs to be employed, requiring the averaging of 640 transients using standard phase cycling (corresponding to a $\sim 5$ minute experiment if the recycle delay is $0.5 \mathrm{~s}$ ), 201 transients using cogwheel phase cycling, or potentially just one transient using pulsed field gradient selection. However, signal averaging can help make up for losses of signal due to inefficiencies in the multiple-quantum pulses. One further disadvantage of the 1D low-amplitude pulse experiment is that the observed signal intensity is sensitive to resonance offset effects $[165,166]$, so this technique would not be quantitative in the sense that the intensities of different resonances would not be in proportion to the concentration of that nucleus in the sample.

\subsection{Theoretical approach to rotary resonance line narrowing in $I=5 / 2$ systems}

An understanding of the rotary resonance and line narrowing effects described above can come only from a proper theory of the $I=5 / 2$ spin dynamics in the sudden passage regime. A complete Floquet theory of $I=5 / 2$ spin dynamics under low-power $\mathrm{rf}$ irradiation and magic-angle spinning conditions has been developed by Walls $[133,164]$. The Floquet theoretical approach involves transforming the time-dependent Hamiltonian into an infinite-dimensional time-independent space and applying static perturbation theory. This method is quite generally applicable, and can explain the spin dynamics at and in between 


\subsection{THEORETICAL APPROACH TO ROTARY RESONANCE LINE}

NARROWING IN $I=5 / 2$ SYSTEMS

rotary resonance conditions as a continuous function of time. However, the expressions that result from the perturbation theory can be quite complicated, and it is somewhat difficult to see how crystallite orientation enters into the expressions.

The favorite tool that NMR spectroscopists use to evaluate dynamics under periodic Hamiltonians (as is the case of the quadrupolar Hamiltonian under magic angle spinning) is average Hamiltonian theory (AHT), which is a Magnus expansion-based approach (see $\S 2$ ). The AHT approach has several major drawbacks when applied to rotary resonance problems. First of all, AHT is a stroboscopic theory, so it will be suitable to describe the time evolution only at times equal to integer multiples of the rotor period $\tau_{R}$. Second, as will be seen, AHT will be able to provide insight as to how the rotary resonances arise, but will be unable to explain the spin dynamics when the rf amplitude is not matched to a resonance condition. Most disturbingly, when AHT is expressed in the interaction frame of the quadrupolar coupling (which is the dominant interaction), the $n^{\text {th }}$-order term in the Magnus series goes as $\left(\omega_{1} / \omega_{R}\right)^{n}$, so the series may not even converge (or at least will converge slowly) at low rf powers where the rf amplitude is comparable to or even smaller than the spinning speed. However, the AHT results are rather simple to compute and to express, so it would be worthwhile to see if AHT-type expressions can capture the essential features of rotary resonance phenomena in $I=5 / 2$ systems (the approach to $I=3 / 2$ systems is outlined in $\S 5.6 .5)$.

The basic rotating-frame Hamiltonian that will be considered here includes the MAS-modulated first-order quadrupolar coupling plus a constant resonant radiofrequency 
radiation term:

$$
\hat{H}_{Q, r f}(t)=\hat{H}_{Q}^{(1)}(t)+\hat{H}_{r f}
$$

Second-order quadrupolar effects and chemical shifts or other resonance offset effects will be neglected during the pulse, although they do play a role. It is convenient to write the Hamiltonian in a in terms of fictitious Cartesian spin- $1 / 2$ single-transition operators $\hat{I}_{x}^{j-k}=\frac{1}{2}(|j\rangle\langle k|+| k\rangle\langle j|), \hat{I}_{y}^{j-k}=-\frac{i}{2}(|j\rangle\langle k|-| k\rangle\langle j|)$, and $\hat{I}_{z}^{j-k}=\frac{1}{2}(|j\rangle\langle j|-| k\rangle\langle k|)$, where $|j\rangle,|k\rangle \in\left\{\left|I, m_{I}\right\rangle\right\}[168,169]$. These operators have many useful properties, including the fact that any two fictitious spin- $1 / 2$ operators that do not share any indices commute with each other. The $I=5 / 2$ magnetic sublevels will be labeled $|1\rangle \equiv\left|I=\frac{5}{2}, m_{I}=+\frac{5}{2}\right\rangle$, $|2\rangle \equiv\left|I=\frac{5}{2}, m_{I}=+\frac{3}{2}\right\rangle,|3\rangle \equiv\left|I=\frac{5}{2}, m_{I}=+\frac{1}{2}\right\rangle,|4\rangle \equiv\left|I=\frac{3}{2}, m_{I}=-\frac{1}{2}\right\rangle,|5\rangle \equiv \mid I=$ $\left.\frac{5}{2}, m_{I}=-\frac{3}{2}\right\rangle$, and $|6\rangle \equiv\left|I=\frac{5}{2}, m_{I}=-\frac{5}{2}\right\rangle$. As is usual, the quantization axis is parallel to the Zeeman field. Using the fictitious spin-1/2 operators, the rotating-frame quadrupolar and rf Hamiltonians become:

$$
\begin{aligned}
\hat{H}_{Q}^{(1)}(t) / \hbar & =\omega_{Q}(t)\left[3 \hat{I}_{z}^{2}-I(I+1) \hat{1}\right] \\
& =\omega_{Q}(t)\left[20 \hat{I}_{z}^{1-2}+16 \hat{I}_{z}^{2-3}-16 \hat{I}_{z}^{4-5}-20 \hat{I}_{z}^{5-6}\right] \\
\hat{H}_{r f} / \hbar & =\omega_{1} \hat{I}_{x}=\left(\hat{H}_{r f}^{C T}+\hat{H}_{r f}^{i S T}+\hat{H}_{r f}^{o S T}\right) / \hbar \\
& \left.=3 \omega_{1} \hat{I}_{x}^{3-4}+2 \sqrt{2} \omega_{1}\left(\hat{I}_{x}^{2-3}+\hat{I}_{x}^{4-5}\right)+\sqrt{5} \omega_{1}\left(\hat{I}_{x}^{1-2}+\hat{I}_{x}^{5-6}\right)\right],
\end{aligned}
$$

where the rf Hamiltonian has been divided into terms that, if isolated from each other, would directly drive the central, inner satellite, or outer satellite single-quantum transitions. Here, 
the rf interaction is assumed to be of constant phase $\phi=0$ and amplitude $\omega_{1}=-\gamma B_{1} / 2$, where $B_{1}$ is the rf magnetic field amplitude in the laboratory frame. The quadrupolar Hamiltonian is that of a single crystallite in the polycrystalline powder; powder-averaged results can be obtained by averaging over random crystallite orientations. The time-dependent quadrupolar frequency $\omega_{Q}(t)$ depends on the quadrupolar parameters $C_{Q}$ and $\eta_{Q}$ and on the Euler angles $\alpha, \beta$, and $\gamma$ that relate the crystallite electric field gradient principal axis system (EFG PAS) to the laboratory frame coordinate system. Under magic-angle spinning conditions, $\omega_{Q}(t)$ is harmonically modulated at the frequencies $\omega_{R}$ and $2 \omega_{R}$, and has a zero time average over one rotor period $\tau_{R}=2 \pi / \omega_{R}$. The functional form of $\omega_{Q}(t)$ will be considered in more detail in $§ 3.5 .3$.

\subsubsection{Average Hamiltonian in the quadrupolar interaction frame}

In most systems the quadrupolar coupling interaction is much larger than the rf interaction, since the quadrupolar coupling could on the order of $\mathrm{MHz}$ and $\left|\omega_{1}\right|$ can be up to $200 \mathrm{kHz}$ or so using solid-state spectrometers. Therefore, it makes sense to perform any calculations in the interaction frame of the quadrupolar interaction, which is dominant at least for the majority of crystallite orientations. The quadrupolar interaction frame is defined by the transformation:

$$
\begin{aligned}
\hat{V}_{Q}(t) & =T\left\{\exp \left[-\frac{i}{\hbar} \int_{0}^{t} d t^{\prime} \hat{H}_{Q}^{(1)}\left(t^{\prime}\right)\right]\right\} \\
& =\exp \left[-i \Phi_{Q}(t)\left(20 \hat{I}_{z}^{1-2}+16 \hat{I}_{z}^{2-3}-16 \hat{I}_{z}^{4-5}-20 \hat{I}_{z}^{5-6}\right)\right]
\end{aligned}
$$


where the quadrupolar dynamic phase is $\Phi_{Q}(t)=\int_{0}^{t} d t^{\prime} \omega_{Q}\left(t^{\prime}\right)$. The commutation and rotation properties of the fictitious spin- $1 / 2$ operators may be used to convert Eq. 3.7 into the quadrupolar interaction frame:

$$
\begin{aligned}
\hat{\widetilde{H}}_{Q, r f}^{Q}(t) / \hbar= & \hat{V}_{Q}^{-1}(t)\left[\hat{H}_{r f} / \hbar\right] \hat{V}_{Q}(t) \\
= & 3 \omega_{1} \hat{I}_{x}^{3-4}+2 \sqrt{2} \omega_{1}\left\{\left(\hat{I}_{x}^{2-3}+\hat{I}_{x}^{4-5}\right) \cos \left[6 \Phi_{Q}(t)\right]-\left(\hat{I}_{y}^{2-3}-\hat{I}_{y}^{4-5}\right) \sin \left[6 \Phi_{Q}(t)\right]\right\} \\
& \quad+\sqrt{5} \omega_{1}\left\{\left(\hat{I}_{x}^{1-2}+\hat{I}_{x}^{5-6}\right) \cos \left[12 \Phi_{Q}(t)\right]-\left(\hat{I}_{y}^{1-2}-\hat{I}_{y}^{5-6}\right) \sin \left[12 \Phi_{Q}(t)\right]\right\}(3.11)
\end{aligned}
$$

Since the quadrupolar frequency is periodic such that $\omega_{Q}\left(t+N \tau_{R}\right)=\omega_{Q}(t)$ (where $N$ is an integer), the dynamic phase $\Phi_{Q}(t)$ and the trigonometric functions of $\Phi_{Q}(t)$ also share the same periodicity. In fact, the interaction frame Hamiltonian remains periodic with period $\tau_{R}$. The periodicity of the trigonometric functions allows for the following Fourier series expansions of the complex exponential functions:

$$
\begin{aligned}
\exp \left[+i 6 \Phi_{Q}(t)\right] & =\sum_{n=-\infty}^{+\infty} A_{n} e^{i n \omega_{R} t} \\
\exp \left[-i 6 \Phi_{Q}(t)\right] & =\sum_{n=-\infty}^{+\infty} A_{n}^{*} e^{-i n \omega_{R} t}=\sum_{n=-\infty}^{+\infty} A_{-n}^{*} e^{i n \omega_{R} t} \\
\exp \left[+i 12 \Phi_{Q}(t)\right] & =\sum_{n=-\infty}^{+\infty} B_{n} e^{i n \omega_{R} t} \\
\exp \left[-i 12 \Phi_{Q}(t)\right] & =\sum_{n=-\infty}^{+\infty} B_{n}^{*} e^{-i n \omega_{R} t}=\sum_{n=-\infty}^{+\infty} B_{-n}^{*} e^{i n \omega_{R} t}
\end{aligned}
$$

where the $A_{n}$ and $B_{n}$ are complex coefficients that bear the dependence on the quadrupolar parameters and crystallite orientation. Using these definitions it is possible to rewrite Eq. 
3.11 in the form:

$$
\hat{\widetilde{H}}_{Q, r f}^{Q}(t)=\sum_{n=-\infty}^{+\infty} \hat{\tilde{H}}_{n}^{Q} e^{i n \omega_{R} t}
$$

where the operators $\hat{\widetilde{H}}_{n}^{Q}$ are non-Hermitian such that $\hat{\widetilde{H}}_{n}^{Q \dagger}=\hat{\widetilde{H}}_{-n}^{Q}$. The $n=0$ term is $\hat{\widetilde{H}}_{0}^{Q} / \hbar=3 \omega_{1} \hat{I}_{x}^{3-4}+2 \sqrt{2} \omega_{1} A_{0}\left(\hat{I}_{x}^{2-3}+\hat{I}_{x}^{4-5}\right)+\sqrt{5} \omega_{1} B_{0}\left(\hat{I}_{x}^{1-2}+\hat{I}_{x}^{5-6}\right)$. For $n \neq 0$, these operators are defined as:

$$
\begin{aligned}
\hat{\tilde{H}}_{n}^{Q} / \hbar= & \sqrt{2} \omega_{1}\left[A_{n}\left(\hat{I}_{+}^{2-3}+\hat{I}_{-}^{4-5}\right)+A_{-n}^{*}\left(\hat{I}_{-}^{2-3}+\hat{I}_{+}^{4-5}\right)\right] \\
& +\frac{\sqrt{5}}{2} \omega_{1}\left[B_{n}\left(\hat{I}_{+}^{1-2}+\hat{I}_{-}^{5-6}\right)+B_{-n}^{*}\left(\hat{I}_{-}^{1-2}+\hat{I}_{+}^{5-6}\right)\right], \\
= & \sqrt{2} \omega_{1}\left[A_{n}(|T+\rangle\langle C+|+| T-\rangle\langle C-|)+A_{-n}^{*}(|C+\rangle\langle T+|+| C-\rangle\langle T-|)\right] \\
& +\frac{\sqrt{5}}{2} \omega_{1}\left[B_{n}(|Q+\rangle\langle T+|+| Q-\rangle\langle T-|)+B_{-n}^{*}(|T+\rangle\langle Q+|+| T-\rangle\langle Q-|)\right],
\end{aligned}
$$

where the sine and cosine trigonometric functions have been written in terms of the complex exponential functions, and $\hat{I}_{ \pm}^{j-k}=\hat{I}_{x}^{j-k} \pm \hat{I}_{y}^{j-k}$. The Hamiltonian operator components also have been written in terms of the eigenstates of the first-order quadrupolar Hamiltonian that also represent the symmetric single-, triple-, and quintuple-quantum coherences in the Zeeman basis [158]: $|C \pm\rangle=\frac{1}{\sqrt{2}}\left(\left|\frac{5}{2},+\frac{1}{2}\right\rangle \pm\left|\frac{5}{2},-\frac{1}{2}\right\rangle\right),|T \pm\rangle=\frac{1}{\sqrt{2}}\left(\left|\frac{5}{2},+\frac{3}{2}\right\rangle \pm\left|\frac{5}{2},-\frac{3}{2}\right\rangle\right)$, and $|Q \pm\rangle=\frac{1}{\sqrt{2}}\left(\left|\frac{5}{2},+\frac{5}{2}\right\rangle \pm\left|\frac{5}{2},-\frac{5}{2}\right\rangle\right)$. This basis is convenient when considering transfers between the symmetric coherences. Interestingly, the $\hat{\widetilde{H}}_{n}^{Q}$ terms with $n \neq 0$ have the same matrix representation in the Zeeman basis and in the quadrupolar eigenbasis. This property holds for any operator $\hat{M}$ that not only block-diagonalizes into separate $+\left|m_{I}\right|$ 
and $-\left|m_{I}\right|$ subspaces such that $\left\langle I,+\left|m_{I}\right||\hat{M}| I,-\left|m_{I}^{\prime}\right|\right\rangle=0$, but also has the symmetry $\left\langle I,+\left|m_{I}\right||\hat{M}| I,+\left|m_{I}^{\prime}\right|\right\rangle=\left\langle I,-\left|m_{I}\right||\hat{M}| I,-\left|m_{I}^{\prime}\right|\right\rangle$.

The average Hamiltonian theory approach outlined in $\S 2.4$ results in an approximate time-dependent Hamiltonian $\hat{\widetilde{\widetilde{H}}}_{Q, r f}^{Q}=\hat{\widetilde{\widetilde{H}}}_{Q, r f}^{Q(1)}+\hat{\widetilde{\widetilde{H}}}_{Q, r f}^{Q(2)}+\ldots$, where the lowest-order term is just the time average of $\hat{\widetilde{H}}_{Q, r f}^{Q}(t)$ over one period $\tau_{R}$. This is equivalent to making the secular approximation in the quadrupolar interaction frame. The lowest-order term is therefore $\hat{\widetilde{H}}_{Q, r f}^{Q(1)}=\frac{1}{\tau_{R}} \int_{0}^{\tau_{R}} d t \hat{\widetilde{H}}_{Q, r f}^{Q}(t)=\hat{\widetilde{H}}_{0}^{Q}$, which in the Zeeman basis equals:

$$
\begin{aligned}
\hat{\widetilde{H}}_{Q, r f}^{Q(1)} / \hbar & =3 \omega_{1} \hat{I}_{x}^{3-4}+2 \sqrt{2} \omega_{1} A_{0}\left(\hat{I}_{x}^{2-3}+\hat{I}_{x}^{4-5}\right)+\sqrt{5} \omega_{1} B_{0}\left(\hat{I}_{x}^{1-2}+\hat{I}_{x}^{5-6}\right) \\
& =\left(\hat{H}_{r f}^{C T}+A_{0} \hat{H}_{r f}^{i S T}+B_{0} \hat{H}_{r f}^{o S T}\right) / \hbar
\end{aligned}
$$

where it can be seen that this average Hamiltonian looks like the rf Hamiltonian with the inner and outer satellite transition terms scaled down by factors of $A_{0}$ and $B_{0}$, respectively. ${ }^{11}$ It should be noted that if the sample is static such that $\omega_{Q}(t)=\omega_{Q}$, then the average Hamiltonian in the quadrupolar interaction frame over the time $\tau_{Q}=2 \pi / \omega_{Q}$ equals $\hat{H}_{r f}^{C T} / \hbar=3 \omega_{1} \hat{I}_{x}^{3-4}$, which causes a selective nutation of the central transition magnetization at the frequency $3 \omega_{1}$. This $\left(I+\frac{1}{2}\right) \omega_{1}$ selective nutation behavior of the central transition is due to the truncation of the rf Hamiltonian by the quadrupolar interaction, as is described in more detail in $\S 5.6 .2$. Therefore it may be concluded that the modulation of the quadrupolar coupling due to sample rotation partially recouples the part of the rf Hamiltonian that drives the satellite transitions, which is rendered ineffective in the presence of a strong static quadrupolar coupling.

\footnotetext{
${ }^{11}$ Here and below the symbol $B_{0}$ will refer to the Fourier coefficient, not the Zeeman magnetic field strength.
} 


\subsection{THEORETICAL APPROACH TO ROTARY RESONANCE LINE} NARROWING IN $I=5 / 2$ SYSTEMS

These points warrant further consideration. In the absence of a quadrupolar coupling the only Hamiltonian that remains is the rf Hamiltonian (which is the Hamiltonian in Eq. 3.16 with $A_{0}=B_{0}=1$ ). This Hamiltonian causes a pure rotation of the nuclear polarization about the $x$-axis, and so is incapable of intermixing density operator terms of different spherical tensor rank, as discussed in Appendix B. Therefore an rf pulse in the absence of any other interaction cannot create multiple quantum coherence from magnetization, or convert between symmetric multiple quantum coherences. On the other hand, a weak rf pulse in the presence of a static strong quadrupolar interaction only causes rotations within the central transition subspace $\left\{\left|\frac{5}{2}, \pm \frac{1}{2}\right\rangle\right\}$ to lowest order in the perturbation theory, so is ineffective at converting magnetization into multiple quantum coherences, especially those with high coherence orders. However, in the case of a weak rf pulse in the presence of a strong modulated quadrupolar coupling, the Hamiltonian in Eq. 3.16 contains partially recoupled satellite transition terms and is no longer proportional to $\hat{I}_{x}$ and is not limited to pure rotations of the spin system. Thus a weak rf pulse during sample spinning is able to induce more complicated dynamics than a simple rotation of either the total magnetization. This phenomenon is exploited not only in low-amplitude pulse experiments, but also to perform adiabatic coherence transfers in high-rf power RIACT and FAM experiments. The first-order average Hamiltonian Eq. 3.16 written in the symmetric coherence basis is:

$$
\begin{aligned}
\hat{\widetilde{\widetilde{H}}}_{Q, r f}^{Q(1)} / \hbar= & \frac{3}{2} \omega_{1}[|C+\rangle\langle C+|-| C-\rangle\langle C-|] \\
& +\sqrt{2} \omega_{1} A_{0}[(|T+\rangle\langle C+|+| C+\rangle\langle T+|)+(|T-\rangle\langle C-|+| C-\rangle\langle T-|)] \\
& +\frac{\sqrt{5}}{2} \omega_{1} B_{0}[(|Q+\rangle\langle T+|+| T+\rangle\langle Q+|)+(|Q-\rangle\langle T-|+| T-\rangle\langle Q-|)],
\end{aligned}
$$


where, as expected, it can be seen that the inner satellite transition terms couple the triplequantum $|T \pm\rangle$ states to the $|C \pm\rangle$ states and the outer satellite transition terms couple the quintuple-quantum $|Q \pm\rangle$ states to the triple-quantum $|T \pm\rangle$ states.

It is of particular interest to have a theory that can explain the excitation of +5 quantum coherence from $z$-magnetization or the conversion of $+5 \mathrm{QC}$ to symmetric $+3 \mathrm{QC}$ during low-amplitude pulses, since these seem to be the orientation-selective transfers in the 5Q3Q MMQMAS experiment, as was discussed in $§ 3.4 .2$. Therefore, what needs to be calculated is the development of +5 -quantum coherence $\hat{Q}_{+}=\left|\frac{5}{2},+\frac{5}{2}\right\rangle\left\langle\frac{5}{2},-\frac{5}{2}\right|$ from an initial density operator $\hat{\rho}(0) \propto \hat{I}_{z}$, as well as the symmetric +3 -quantum coherence $\hat{T}_{+}^{s}=$ $\left|\frac{5}{2},+\frac{3}{2}\right\rangle\left\langle\frac{5}{2},-\frac{3}{2}\right|$ from $\hat{\rho}(0) \propto \hat{Q}_{+}$. The time development operator in the rotating frame is:

$$
\hat{U}(t)=\hat{V}_{Q}(t) \hat{\widetilde{U}}^{Q}(t)
$$

where to first order in the AHT perturbation theory,

$$
\hat{\widetilde{U}}^{Q}(t) \simeq \exp \left[-i \hat{\widetilde{H}}_{Q, r f}^{Q(1)} t / \hbar\right]
$$

The time development of some normalized basis operator term $\hat{B}$ in the density operator is 
given by:

$$
\begin{aligned}
\hat{B}(t) & =\langle\langle B \mid \rho(t)\rangle\rangle=\operatorname{Tr}\left[\hat{B}^{\dagger} \hat{\rho}(t)\right]=\operatorname{Tr}\left[\hat{B}^{\dagger} \hat{U}(t) \hat{\rho}(0) \hat{U}^{-1}(t)\right] \\
& =\operatorname{Tr}\left[\hat{B}^{\dagger} \hat{V}_{Q}(t) \hat{\tilde{U}}^{Q}(t) \hat{\rho}(0) \hat{\tilde{U}}^{Q-1}(t) \hat{V}_{Q}^{-1}(t)\right] \\
& =\operatorname{Tr}\left[\hat{V}_{Q}^{-1}(t) \hat{B}^{\dagger} \hat{V}_{Q}(t) \hat{\tilde{U}}^{Q}(t) \hat{\rho}(0) \hat{\tilde{U}}^{Q-1}(t)\right] \\
& =\operatorname{Tr}\left[\hat{\tilde{U}}^{Q-1}(t) \hat{V}_{Q}^{-1}(t) \hat{B}^{\dagger} \hat{V}_{Q}(t) \hat{\tilde{U}}^{Q}(t) \hat{\rho}(0)\right],
\end{aligned}
$$

where the trace permutation property $\operatorname{Tr}[\hat{A} \hat{B}]=\operatorname{Tr}[\hat{B} \hat{A}]$ has been used. In a proper stroboscopic application of AHT the time dependence us evaluated only at integer multiples of the period $\tau_{R}=N \tau_{R}$, in which case the quadrupolar interaction frame transformation operator is $\hat{V}_{Q}\left(N \tau_{R}\right)=\hat{1}$ and may be neglected. However, $\left[\hat{V}_{Q}(t), \hat{Q}_{+}\right]=0$ and $\left[\hat{V}_{Q}(t), \hat{T}_{+}^{s}\right]=0$ since the symmetric transition operators commute with the first-order quadrupolar Hamiltonian, so $\hat{V}_{Q}(t)$ can be neglected at any time, and $\hat{B}(t)=\operatorname{Tr}\left[\hat{B}^{\dagger} \hat{\widetilde{U}}^{Q}(t) \hat{\rho}(0) \hat{\widetilde{U}}^{Q-1}(t)\right]$ for $\hat{B}=\hat{Q}_{+}$ or $\hat{B}=\hat{T}_{+}^{s}$. Rather than attempt an exact solution of $\hat{\rho}(t)$ using the average Hamiltonian approximation, the approach used here will be to approximate the time evolution in a power series in time according to Eq. 2.14, and then examine the leading short-time effects.

Starting from $\hat{\rho}(0) \propto \hat{I}_{z}$, the first-order average Hamiltonian in Eq. 3.16 can create the $+5 \mathrm{QC}$ operator $\hat{Q}_{+}$only in $\mathcal{O}\left(t^{7}\right)$. Therefore, this level of theory is only marginally sufficient to describe $+5 \mathrm{QC}$ excitation from longitudinal magnetization. However, starting from +5 -quantum coherence $\hat{\rho}(0) \propto \hat{Q}_{+}$, symmetric $+3 \mathrm{QC}$ is created in $\mathcal{O}\left(t^{2}\right)$ with $\hat{T}_{+}^{s}(t) \sim$ $B_{0}^{2} \omega_{1}^{2} t^{2}$, where the Fourier coefficient $B_{0}$ carries the crystallite orientation dependence that would be necessary to explain any orientation-selective pulse effects. 


\subsection{THEORETICAL APPROACH TO ROTARY RESONANCE LINE} NARROWING IN $I=5 / 2$ SYSTEMS

By way of comparison, the +1-quantum symmetric central transition coherence $\hat{C}_{+}^{s}=\left|\frac{5}{2},+\frac{1}{2}\right\rangle\left\langle\frac{5}{2},-\frac{1}{2}\right|$ is excited from $\hat{\rho}(0) \propto \hat{I}_{z}$ in $\mathcal{O}\left(t^{1}\right)$ as $\sim \omega_{1} t$, and the first crystallite orientation dependence occurs in $\mathcal{O}\left(t^{3}\right)$ as $\sim\left(A_{0}^{2}-8 / 9\right) \omega_{1}^{3} t^{3}$. Symmetric +1 QC is converted from $+5 \mathrm{QC}$ with $\hat{\rho}(0) \propto \hat{Q}_{+}$in $\mathcal{O}\left(t^{4}\right)$ as $\sim A_{0}^{2} B_{0}^{2} \omega_{1}^{4} t^{4}$, and is converted from $\hat{\rho}(0) \propto \hat{T}_{+}^{s}$ in $\mathcal{O}\left(t^{2}\right)$ as $\sim A_{0}^{2} \omega_{1}^{2} t^{2}{ }^{12}$ These coherence transfers have not been observed to be orientation selective.

It seems likely that a higher order of average Hamiltonian theory is necessary to describe the desired coherence transfers; certainly it is necessary in the case of the quintuple-quantum excitation from longitudinal magnetization. It has already been noted that when a periodic Hamiltonian $\hat{H}(t+\tau)=\hat{H}(t)$ is expanded in a Fourier series $\hat{H}(t)=$ $\sum_{n=-\infty}^{+\infty} \hat{H}_{n} e^{i n \omega t}$ as in Eq. 3.13, the first-order AHT term over the period $\tau=2 \pi / \omega$ is $\hat{\bar{H}}^{(1)}=\hat{H}_{0}$. The second-order AHT term can be found by directly integration of Eq. 2.28 over the period $\tau$; it is:

$$
\begin{aligned}
\hat{\bar{H}}^{(2)} & =-\frac{1}{2 \hbar} \sum_{n \neq 0} \frac{1}{n \omega}\left\{\left[\hat{H}_{-n}, \hat{H}_{n}\right]-2\left[H_{-n}, \hat{H}_{0}\right]\right\} \\
& =-\frac{1}{\hbar} \sum_{n>0}^{+\infty} \frac{1}{n \omega}\left\{\left[\hat{H}_{-n}, \hat{H}_{n}\right]-\left[\left(\hat{H}_{-n}-\hat{H}_{n}\right), \hat{H}_{0}\right]\right\} \\
& =\hat{\bar{H}}_{\text {sec }}^{(2)}+\hat{\bar{H}}_{\text {nonsec }}^{(2)},
\end{aligned}
$$

where the second-order average Hamiltonian has been divided into its secular and nonsecular contributions. Mehring has objected that the inclusion of the non-secular terms can lead to

\footnotetext{
${ }^{12}$ The operator $\hat{C}_{+}^{s}$ represents $+1 \mathrm{QC}$; the $-1 \mathrm{QC}$ operator $\hat{C}_{-}^{s}=\left|\frac{5}{2},-\frac{1}{2}\right\rangle\left\langle\frac{5}{2},+\frac{1}{2}\right|$ is created from $+5 \mathrm{QC}$ in $\mathcal{O}\left(t^{6}\right)$ and from $+3 \mathrm{QC}$ in $\mathcal{O}\left(t^{4}\right)$, which suggests that the $+5 \rightarrow+1 \mathrm{QC}$ and $+3 \rightarrow+1 \mathrm{QC}$ conversions followed by a quantitative selective inversion of the single-quantum coherence are more efficient than direct $+5 \rightarrow-1 \mathrm{QC}$ and $+3 \rightarrow-1 \mathrm{QC}$ conversions.
} 


\subsection{THEORETICAL APPROACH TO ROTARY RESONANCE LINE} NARROWING IN $I=5 / 2$ SYSTEMS

improper results, and has instead proposed the "secular averaging approach" [8, Appendix G], $[41, \S 4.3 .3, \S 15.3] .{ }^{13}$ The first-order AHT and SAA Hamiltonians coincide, but the second-order SAA Hamiltonian has the same form as a second-order AHT term calculated with the time-independent portion $\hat{H}_{0}=\hat{\bar{H}}^{(1)}$ of the Hamiltonian previously subtracted out. The second-order secularly-averaged Hamiltonian is:

$$
\hat{\bar{H}}^{(2)}=-\frac{1}{2 \hbar} \sum_{n \neq 0} \frac{1}{n \omega}\left[\hat{H}_{-n}, \hat{H}_{n}\right]=-\frac{1}{\hbar} \sum_{n>0}^{+\infty} \frac{1}{n \omega}\left[\hat{H}_{-n}, \hat{H}_{n}\right]
$$

which lacks the non-secular contribution of Eq. 3.21. The SAA also is not limited to only stroboscopic observation.

The second-order SAA term for the rf Hamiltonian in the quadrupolar interaction frame is:

$$
\begin{aligned}
& \hat{\widetilde{H}}_{Q, r f}^{Q(2)} / \hbar= \frac{\omega_{1}^{2}}{\omega_{R}}\left\{2 \alpha^{(2)}\left(\hat{I}_{z}^{1-2}-\hat{I}_{z}^{5-6}\right)+2 \beta^{(2)}\left(\hat{I}_{z}^{2-3}-\hat{I}_{z}^{4-5}\right)\right. \\
&\left.\quad-\left[\gamma^{(2)}\left(\hat{I}_{+}^{1-3}+\hat{I}_{-}^{4-6}\right)+\gamma^{(2) *}\left(\hat{I}_{-}^{1-3}+\hat{I}_{+}^{4-6}\right)\right]\right\} \\
&=\frac{\omega_{1}^{2}}{\omega_{R}}\left\{\alpha^{(2)}[(|Q+\rangle\langle Q+|+| Q-\rangle\langle Q-|)-(|T+\rangle\langle T+|+| T-\rangle\langle T-|)]\right. \\
& \quad+\beta^{(2)}[(|T+\rangle\langle T+|+| T-\rangle\langle T-|)-(|C+\rangle\langle C+|+| C-\rangle\langle C-|)] \\
&\left.\quad-\left[\gamma^{(2)}(|Q+\rangle\langle C+|+| Q-\rangle\langle C-|)+\gamma^{(2) *}(|C+\rangle\langle Q+|+| C-\rangle\langle Q-|)\right]\right\},
\end{aligned}
$$

\footnotetext{
${ }^{13}$ Ref. [8] correctly states the AHT result in Eq. 3.21 when the summation index runs over positive and negative values, but Ref. [41] incorrectly states the result when the summation index is positive.
} 
where the Hamiltonian coefficients are given by:

$$
\begin{aligned}
& \alpha^{(2)}=\Theta\left(b_{+}, b_{-}\right)=\frac{5}{4} \sum_{n \neq 0} \frac{\left|B_{n}\right|^{2}}{n}=\frac{5}{4} \sum_{n>0}^{+\infty} \frac{1}{n}\left(\left|B_{n}\right|^{2}-\left|B_{-n}\right|^{2}\right) \\
& \beta^{(2)}=\Theta\left(a_{+}, a_{-}\right)=2 \sum_{n \neq 0} \frac{\left|A_{n}\right|^{2}}{n}=2 \sum_{n>0}^{+\infty} \frac{1}{n}\left(\left|A_{n}\right|^{2}-\left|A_{-n}\right|^{2}\right) \\
& \gamma^{(2)}=\Theta\left(a_{+}, b_{+}\right)=\sqrt{\frac{5}{8}} \sum_{n \neq 0} \frac{1}{n}\left(A_{n} B_{-n}-B_{n} A_{-n}\right)=\sqrt{\frac{5}{2}} \sum_{n>0}^{+\infty} \frac{1}{n}\left(A_{n} B_{-n}-B_{n} A_{-n}\right),
\end{aligned}
$$

where e.g., $\Theta\left(a_{+}, a_{-}\right) \equiv-\frac{i}{2 \tau_{R}} \int_{0}^{\tau_{R}} d t^{\prime} \int_{0}^{t} d t\left[a_{+}\left(t^{\prime}\right) a_{-}(t)-a_{-}\left(t^{\prime}\right) a_{+}(t)\right]$, and the time-dependent functions are written in terms of the quadrupolar dynamic phases as $a_{ \pm}(t)=\left(\exp \left[ \pm i 6 \Phi_{Q}(t)\right]-\right.$ $\left.A_{0}\right)$ and $b_{ \pm}(t)=\left(\exp \left[ \pm i 12 \Phi_{Q}(t)\right]-B_{0}\right) \cdot{ }^{14}$ The Hamiltonian coefficients can be evaluated either by a (probably numerical) two-dimensional time integral or by summing the infinite series of Fourier coefficients. One method may be preferable over another depending on how many crystallite orientations are used in the powder averaging and how quickly the series of Fourier coefficients converges.

It can be seen that the $+\left|m_{I}\right|$ manifold is decoupled from the $-\left|m_{I}\right|$ manifold in the SAA second-order Hamiltonian, since the $\hat{\widetilde{H}}_{n \neq 0}^{Q}$ Fourier terms are decoupled this way. Additionally, the second-order Hamiltonian has the same matrix representation in both the Zeeman and quadrupolar eigenbases. The second-order Hamiltonian introduces new terms that can directly couple the quintuple-quantum states $|Q \pm\rangle$ to the single-quantum states $|C \pm\rangle$. As expected, the ratio $\left\|\hat{\widetilde{H}}_{Q, r f}^{Q(2)}\right\| /\left\|\hat{\widetilde{H}}_{Q, r f}^{Q(1)}\right\|$ is on the order of $\left|\omega_{1}\right| / \omega_{R}$, which

\footnotetext{
${ }^{14}$ The second-order AHT approach does not require the subtraction of the zero-frequency $A_{0}$ and $B_{0}$ terms when evaluating the double time integral, but the non-secular contribution does contain additional terms that couple the $|T \pm\rangle$ states to the $|C \pm\rangle$ states.
} 
means that the second-order term actually may not be smaller than the first-order term for low rf-amplitude pulses.

The spin dynamics can now be calculated under the averaged Hamiltonian $\hat{\widetilde{H}}_{Q, r f}^{Q} \simeq$ $\hat{\widetilde{H}}_{Q, r f}^{Q(1)}+\hat{\widetilde{H}}_{Q, r f}^{Q(2)}$. Using Eqs. 3.17 and 3.24 with Eq. 3.20 yields the result that $+5 \mathrm{QC}$ excitation of $\hat{Q}_{+}$from $\hat{\rho}(0) \propto \hat{I}_{z}$ now occurs in $\mathcal{O}\left(t^{3}\right)$ as $\sim\left|\gamma^{(2)}\right|^{2}\left(\omega_{1}^{5} / \omega_{R}^{2}\right) t^{3}$. The $+5 \mathrm{QC}$ to symmetric $+3 \mathrm{QC}$ conversion again occurs in $\mathcal{O}\left(t^{2}\right)$ as $\sim B_{0}^{2} \omega_{1}^{2} t^{2}$, but also in $\mathcal{O}\left(t^{3}\right)$ as $A_{0} B_{0}\left(\gamma^{(2)}-\gamma^{(2) *}\right)$. The symmetric $+1 \mathrm{QC}$ excitation from longitudinal magnetization again shows up in $\mathcal{O}\left(t^{1}\right)$ as $\sim \omega_{1} t$, and in $\mathcal{O}\left(t^{3}\right)$ as before, but now including a term $\sim\left(\omega_{1}^{5} / \omega_{R}^{2}\right)\left|\gamma^{(2)}\right|^{2}$. The +5 QC to symmetric $+1 \mathrm{QC}$ conversion now occurs in $\mathcal{O}\left(t^{2}\right)$ as $\sim\left(\omega_{1}^{4} / \omega_{R}^{2}\right)\left|\gamma^{(2)}\right|^{2}$. The symmetric $+3 \mathrm{QC}$ to symmetric $+1 \mathrm{QC}$ conversion again shows up in $\mathcal{O}\left(t^{2}\right)$ as $\sim A_{0}^{2} \omega_{1}^{2} t^{2}$, and also in $\mathcal{O}\left(t^{3}\right)$ as $\sim\left|\gamma^{(2)}\right|^{2}\left(\omega_{1}^{5} / \omega_{R}^{2}\right) t^{3}$.

It is clear that this approach even to second order does not yield results that can differentiate between the orientation-selective pulses and the non-orientation-selective pulses, plus there are serious flaws with the convergence of the perturbative approach at low rf powers. Some insight may potentially be gained as to why certain crystallite orientations are selected over others by examining the orientation-dependent Fourier coefficients, and this will be done in §3.5.3. A further major objection to the average Hamiltonian (or secular averaging) theory in the quadrupolar interaction frame is that it does not even seem to explain why rotary resonances occur. This problem will be addressed in the next section. 


\subsection{THEORETICAL APPROACH TO ROTARY RESONANCE LINE}

\subsubsection{Average Hamiltonian in the quadrupolar/central transition RF in- teraction frame}

Walls has noted that since the first-order quadrupolar Hamiltonian commutes with the central transition term of the radiofrequency Hamiltonian, the transformation into a joint quadrupolar-central transition rf frame is simple to achieve. As will be seen, this transformation induces an additional $\omega_{1}$-dependent time modulation to the Hamiltonian that can interfere with the rotation-induced modulation, causing the rotary resonance effects. The quadrupolar-CT rf transformation operator is:

$$
\begin{aligned}
\hat{V}_{Q, C T}(t) & =T\left\{\exp \left[-\frac{i}{\hbar} \int_{0}^{t} d t^{\prime}\left(\hat{H}_{Q}^{(1)}\left(t^{\prime}\right)+\hat{H}_{r f}^{C T}\right)\right]\right\} \\
& =\exp \left\{-i\left[\Phi_{Q}(t)\left(20 \hat{I}_{z}^{1-2}+16 \hat{I}_{z}^{2-3}-16 \hat{I}_{z}^{4-5}-20 \hat{I}_{z}^{5-6}\right)+3 \omega_{1} t \hat{I}_{x}^{3-4}\right]\right\}
\end{aligned}
$$

where the quadrupolar dynamic phase is again $\Phi_{Q}(t)=\int_{0}^{t} d t^{\prime} \omega_{Q}\left(t^{\prime}\right)$. The quadrupolar-rf Hamiltonian in the new quadrupolar-CT rf interaction frame is [164]:

$$
\begin{aligned}
\hat{\widetilde{H}}_{Q, r f}^{Q, C T}(t) / \hbar= & \hat{V}_{Q, C T}^{-1}(t)\left[\left(\hat{H}_{r f}^{i S T}+\hat{H}_{r f}^{o S T}\right) / \hbar\right] \hat{V}_{Q, C T}(t)=e^{+i 3 \omega_{1} t \hat{I}_{x}^{3-4}}\left[\hat{\widetilde{H}}_{Q, r f}^{Q}(t) / \hbar\right] e^{-i 3 \omega_{1} t \hat{I}_{x}^{3-4}} \\
= & 2 \sqrt{2} \omega_{1}\left\{\left[\left(\hat{I}_{x}^{2-3}+\hat{I}_{x}^{4-5}\right) \cos \left(\frac{3}{2} \omega_{1} t\right)+\left(\hat{I}_{y}^{2-4}-\hat{I}_{y}^{3-5}\right) \sin \left(\frac{3}{2} \omega_{1} t\right)\right] \cos \left[6 \Phi_{Q}(t)\right]\right. \\
& \left.-\left[\left(\hat{I}_{y}^{2-3}-\hat{I}_{y}^{4-5}\right) \cos \left(\frac{3}{2} \omega_{1} t\right)-\left(\hat{I}_{x}^{3-5}+\hat{I}_{x}^{2-4}\right) \sin \left(\frac{3}{2} \omega_{1} t\right)\right] \sin \left[6 \Phi_{Q}(t)\right]\right\} \\
& +\sqrt{5} \omega_{1}\left\{\left(\hat{I}_{x}^{1-2}+\hat{I}_{x}^{5-6}\right) \cos \left[12 \Phi_{Q}(t)\right]-\left(\hat{I}_{y}^{1-2}-\hat{I}_{y}^{5-6}\right) \sin \left[12 \Phi_{Q}(t)\right]\right\}, \quad(3.27)
\end{aligned}
$$

where the central transition $\mathrm{rf}$ transformation causes an additional $\omega_{1}$-dependent modulation of the inner satellite transition rf terms. This Hamiltonian may be written in the following 
form:

$$
\hat{\widetilde{H}}_{Q, r f}^{Q, C T}(t)=\sum_{n=-\infty}^{+\infty} \sum_{m=0, \pm 1} \hat{\widetilde{H}}_{n, m}^{Q, C T} e^{i n \omega_{R} t} e^{i m\left(3 \omega_{1} / 2\right) t}
$$

where the the Fourier expansions in Eq. 3.12 can be used to express the Hamiltonian coefficients as:

$$
\begin{aligned}
& \hat{\widetilde{H}}_{n, 0}^{Q, C T} / \hbar=\frac{\sqrt{5}}{2} \omega_{1}\left[B_{n}(|Q+\rangle\langle T+|+| Q-\rangle\langle T-|)+B_{-n}^{*}(|T+\rangle\langle Q+|+| T-\rangle\langle Q-|)\right](3.2 \\
& \hat{\widetilde{H}}_{n, \pm 1}^{Q, C T} / \hbar=\sqrt{2} \omega_{1}\left[A_{n}|T \mp\rangle\left\langle C \mp\left|+A_{-n}^{*}\right| C \pm\right\rangle\langle T \pm|\right]
\end{aligned}
$$

Eq. 3.28 indicates a bimodal time dependence of the quadrupolar-CT rf interaction frame Hamiltonian, i.e., there is evolution at the two fundamental frequencies $\omega_{R}$ and $3 \omega_{1} / 2$. In comparison, the time dependence of the quadrupolar interaction frame Hamiltonian in Eq. 3.13 is unimodal with the fundamental frequency $\omega_{R}$. Time evolution at two fundamental frequencies will in general cause destructive interferences in the Hamiltonian of Eq. 3.28, since only the $\hat{\widetilde{H}}_{0,0}^{Q, C T}$ term is time-independent. However, when the rotary resonance condition $\omega_{1}=\frac{2}{3} N \omega_{R}$ is satisfied, the Hamiltonian again has only one fundamental frequency $\omega_{R}$, and different time-independent pieces for different resonance conditions $N$. The resonance conditions appear because of the dual modulation at $\omega_{R}$ due to sample spinning and at $\frac{2}{3} \omega_{1}$ due to the interaction frame transformation. In general, rotary resonance phenomena in a nucleus of half-integer spin $I$ can be explained by going into a quadrupolarCT rf interaction frame, which causes modulation of the Hamiltonian at the frequency $\omega_{m}=\left\langle I, m_{I}= \pm \frac{1}{2}\left|\hat{H}_{r f}\right| I, m_{I}=\mp \frac{1}{2}\right\rangle=\frac{1}{2}\left(I+\frac{1}{2}\right) \omega_{1}$ for $\hat{H}_{r f}=\omega_{1} \hat{I}_{x}$. Resonances occur 
when $\omega_{m}=N \omega_{R}$, i.e., when $\omega_{1}=\frac{4 N}{2 I+1} \omega_{R}$, at which point the interaction frame Hamiltonian contains an additional time-independent piece that dominates the spin dynamics in a lowest-order perturbative treatment.

At the resonance condition $\omega_{1}=\frac{2}{3} N \omega_{R}$, Eq. 3.28 can be rewritten without loss of generality as:

$$
\hat{\widetilde{H}}_{Q, r f}^{Q, C T}(t)=\sum_{k=-\infty}^{+\infty} \hat{\widetilde{H}}_{k}^{Q, C T} e^{i k \omega_{R} t},
$$

where

$$
\hat{\widetilde{H}}_{k}^{Q, C T}=\hat{\widetilde{H}}_{k, 0}^{Q, C T}+\hat{\widetilde{H}}_{k-N,+1}^{Q, C T}+\hat{\widetilde{H}}_{k+N,-1}^{Q, C T}
$$

The first-order AHT or SAA approximation to the effective interaction frame Hamiltonian is:

$$
\begin{aligned}
& \hat{\widetilde{\widetilde{H}}}_{Q, r f}^{Q, C T(1)} / \hbar=\left(\hat{\tilde{H}}_{0,0}^{Q, C T}+\hat{\tilde{\widetilde{H}}}_{-N,+1}^{Q, C T}+\hat{\tilde{H}}_{+N,-1}^{Q, C T}\right) / \hbar \\
& =\sqrt{2} \omega_{1}\left[\left(A_{+N}|T+\rangle\left\langle C+\left|+A_{+N}^{*}\right| C+\right\rangle\langle T+|\right)+\left(A_{-N}|T-\rangle\left\langle C-\left|+A_{-N}^{*}\right| C-\right\rangle\langle T-|\right)\right] \\
& \quad+\frac{\sqrt{5}}{2} \omega_{1} B_{0}[(|Q+\rangle\langle T+|+| T+\rangle\langle Q+|)+(|Q-\rangle\langle T-|+| T-\rangle\langle Q-|)] .
\end{aligned}
$$

Eq. 3.33 has the same form as the first-order Hamiltonian in Eq. 3.17 in the quadrupolar interaction frame but without the central transition rf term, plus the symmetry between the $+\left|m_{I}\right|$ and $-\left|m_{I}\right|$ manifolds is broken by the inner satellite transition terms through differ- 
ences in the coefficients $A_{+N}$ versus $A_{-N}$. The condition $N=0$ recovers the quadrupolar interaction frame average Hamiltonian for $n \neq 0$, since in the $\omega_{1}=\frac{2}{3} N \omega_{R}=0$ limit the transformations $\hat{V}_{Q}(t)$ and $\hat{V}_{Q, C T}(t)$ are identical.

The second-order SAA Hamiltonian is:

$$
\begin{aligned}
\hat{\widetilde{H}}_{Q, C T}^{(2)} / \hbar= & \frac{\omega_{1}^{2}}{\omega_{R}}\left\{\alpha^{(2)}[(|Q+\rangle\langle Q+|+| Q-\rangle\langle Q-|)-(|T+\rangle\langle T+|+| T-\rangle\langle T-|)]\right. \\
& +\left[\left(\beta_{+N}^{(2)}|T+\rangle\left\langle T+\left|+\beta_{-N}^{(2)}\right| T-\right\rangle\langle T-|\right)-\left(\beta_{+N}^{(2)}|C+\rangle\left\langle C+\left|+\beta_{-N}^{(2)}\right| C-\right\rangle\langle C-|\right)\right] \\
& \left.-\left[\left(\gamma_{+N}^{(2)}|Q+\rangle\left\langle C+\left|+\gamma_{-N}^{(2)}\right| Q-\right\rangle\langle C-|\right)+\left(\gamma_{+N}^{(2) *}|C+\rangle\left\langle Q+\left|+\gamma_{-N}^{(2) *}\right| C-\right\rangle\langle Q-|\right)\right]\right\},
\end{aligned}
$$

where

$$
\begin{aligned}
& \alpha^{(2)}=\Theta\left(b_{+}, b_{-}\right)=\frac{5}{4} \sum_{k>0}^{+\infty} \frac{1}{k}\left(\left|B_{k}\right|^{2}-\left|B_{-k}\right|^{2}\right) \\
& \beta_{ \pm N}^{(2)}=\Theta\left(a_{+}^{ \pm N}, a_{-}^{ \pm N}\right)=2 \sum_{k>0}^{+\infty} \frac{1}{k}\left(\left|A_{k \pm N}\right|^{2}-\left|A_{-k \pm N}\right|^{2}\right) \\
& \gamma_{ \pm N}^{(2)}=\Theta\left(a_{+}^{ \pm N}, b_{+}\right)=\sqrt{\frac{5}{2} \sum_{k>0}^{+\infty} \frac{1}{k}\left(A_{k \pm N} B_{-k}-B_{k} A_{-k \pm N}\right),}
\end{aligned}
$$

where e.g., $\Theta\left(a_{+}^{+N}, a_{-}^{+N}\right) \equiv-\frac{i}{2 \tau_{R}} \int_{0}^{\tau_{R}} d t^{\prime} \int_{0}^{t} d t\left[a_{+}^{+N}\left(t^{\prime}\right) a_{-}^{+N}(t)-a_{-}^{+N}\left(t^{\prime}\right) a_{+}^{+N}(t)\right]$, with $a_{ \pm}^{+N}(t)=$ $\left(e^{-i N \omega_{R} t} \exp \left[ \pm i 6 \Phi_{Q}(t)\right]-A_{+N}\right), a_{ \pm}^{-N}(t)=\left(e^{+i N \omega_{R} t} \exp \left[ \pm i 6 \Phi_{Q}(t)\right]-A_{-N}\right)$, and $b_{ \pm}(t)=$ $\left(\exp \left[ \pm i 12 \Phi_{Q}(t)\right]-B_{0}\right)$. Again, Eq. 3.34 reduces to the quadrupolar interaction frame result Eq. 3.24 in the $N=0$ limit where $A_{k \pm N}=A_{k}$. Both the first- and second-order average Hamiltonians in the quadrupolar-CT rf interaction frame are decoupled into $+\left|m_{I}\right|$ and 
$-\left|m_{I}\right|$ manifolds, but the symmetry between these two manifolds is broken in the $N \neq 0$ limit at which rotary resonance conditions are satisfied. However, since the forms of the average Hamiltonians in both interaction frames are the same within each $+\left|m_{I}\right|$ or $-\left|m_{I}\right|$ subspace apart from some $N$-dependence in the coefficients, it is not expected that the use of the quadrupolar-CT rf interaction frame will provide much more insight into the spin dynamics beyond making the rotary resonance conditions clear. For instance, the calculations in both frames indicate that to first order the $|Q \pm\rangle$ states are coupled to the $|T \pm\rangle$ states and the $|T \pm\rangle$ states are coupled to the $|C \pm\rangle$ states, whereas to second order there is an additional coupling between the $|Q \pm\rangle$ and $|C \pm\rangle$ states. This has been predicted by the Floquet theory [164], which also suggests that the dominant $+5 \mathrm{QC}$ to $+1 \mathrm{QC}$ transfer occurs indirectly by the mutual coupling of these states to the triple-quantum states in the first-order approximation, rather than from the direct coupling of the quintuple- and single-quantum coherences in the second-order approximation.

It should also be noted that since the AHT or SAA approach in the quadrupolarCT rf interaction frame is only valid exactly at rotary resonance conditions when the problem becomes unimodal, this calculations in this frame cannot be used to explain what happens when $\omega_{1}$ is not matched to a rotary resonance condition. Since it is the low-amplitude $+5 \mathrm{QC}$ excitation and $+5 \mathrm{QC} \rightarrow+3 \mathrm{QC}$ conversion pulses that are known to be orientation selective, and these coherence transfers have been observed to have efficiency minima at rotary resonance conditions $(\S 3.4 .2)$, it is expected that calculations in the quadrupolarcentral transition rf interaction frame will not be able to explain the orientation-selective effects. 


\subsection{THEORETICAL APPROACH TO ROTARY RESONANCE LINE}

\subsubsection{Crystallite orientation dependence of the Hamiltonian}

The discussion thus far has focused on the effective form of the Hamiltonian under sample rotation and low amplitude rf irradiation, which is necessary to explain the differences between coherence transfer mechanisms, e.g., $+5 \mathrm{QC}$ excitation versus $+5 \mathrm{QC}$ to $+3 \mathrm{QC}$ transfer versus $+5 \mathrm{QC}$ to $+1 \mathrm{QC}$ transfer. However, little has been said so far about how the actual orientation dependence of these Hamiltonian terms can manifest itself. Particularly, at this point it is not yet understood why the pulses select only a particular band of crystallite orientations, or why the effects seem to disappear at high values of the local electric field gradient asymmetry parameter $\eta_{Q}$.

The qualitative account of how crystallite orientation affects the spin dynamics is as follows. The breaking of the spatial isotropy of the nuclear system by a large magnetic field means that in different crystallites the nuclei will feel different effective portions of the local electric field gradient, depending on how these gradients are oriented with respect to the magnetic field. In turn, this causes nuclei in different crystallites to experience different electric quadrupolar couplings, which are modulated as sample rotation causes these crystallites to change their orientation with respect to the magnetic field. During a radiofrequency pulse the nuclear spins evolve under a combination of the quadrupolar coupling and the rf field. The quadrupolar coupling dominates the rf interaction and renders certain portions of the rf magnetic field ineffective at inducing spin dynamics, as can be seen by transforming into the interaction frame of the quadrupolar Hamiltonian. Since the quadrupolar coupling is orientation- and time-dependent, it imposes an orientation dependence on the effective portion of the rf field, and this time-dependent orientation effect causes different 


\subsection{THEORETICAL APPROACH TO ROTARY RESONANCE LINE} NARROWING IN $I=5 / 2$ SYSTEMS

spin dynamics in different nuclei. These effects are quantitatively manifested in the different dependences of the central and inner and outer satellite transition terms of the radiofrequency Hamiltonian on the crystallite orientation-dependent quadrupolar dynamic phase. Since the spin dynamics vary from crystallite to crystallite, a distribution of nuclear polarizations is created across the sample during the pulse. Ultimately, these individual crystallite polarizations can cause constructive or destructive interference in the NMR lineshape. The details of the orientation dependence of the spin dynamics and the orientational average of the resulting nuclear polarizations are what will explain the orientation-dependent pulse effects.

The time dependent single-crystallite quadrupolar coupling during magic angle sample spinning is ${ }^{15}$ :

$$
\begin{aligned}
\omega_{Q}(t) & \equiv \omega_{Q}(\alpha, \beta, \gamma ; t) \\
& =\omega_{Q}\left[C_{1} \cos \left(\omega_{R} t+\gamma\right)+C_{2} \cos \left(2 \omega_{R} t+2 \gamma\right)\right]+S_{1} \sin \left(\omega_{R} t+\gamma\right)+S_{2} \sin \left(2 \omega_{R} t+2 \gamma\right),
\end{aligned}
$$

\footnotetext{
${ }^{15}$ The normalization is such that $A_{2,0}=\frac{1}{2}, A_{2, \pm 2}=\eta /(2 \sqrt{6})$ in units of $\omega_{Q}$.
} 
where

$$
\begin{aligned}
\omega_{Q} & =2 \pi C_{Q} /[2 I(2 I-1)] \\
C_{1} & =-\frac{3}{8} \sin \left(2 \theta_{m}\right) \sin (2 \beta)\left[1-\frac{\eta_{Q}}{3} \cos (2 \alpha)\right] \\
C_{2} & =\frac{3}{8} \sin ^{2}\left(\theta_{m}\right)\left\{\sin ^{2}(\beta)+\frac{\eta_{Q}}{3}\left[\cos ^{2}(\beta)+1\right] \cos (2 \alpha)\right\} \\
S_{1} & =-\frac{\eta_{Q}}{4} \sin \left(2 \theta_{m}\right) \sin (\beta) \sin (2 \alpha) \\
S_{2} & =-\frac{\eta_{Q}}{4} \sin ^{2}\left(\theta_{m}\right) \cos (\beta) \sin (2 \alpha)
\end{aligned}
$$

where $\alpha, \beta$, and $\gamma$ are the Euler angles that relate the crystallite-fixed coordinate system (assumed to coincide with the principal axis system of the local electric field gradient tensor in a single-site system) to the rotor-fixed coordinate system. ${ }^{16}$ The quadrupolar coupling constant is $C_{Q}=e^{2} q Q / h$, the EFG asymmetry parameter is $\eta_{Q}$, the rotation frequency is $\omega_{R}$, and the magic angle is $\theta_{m}=\tan ^{-1}(\sqrt{2})$. Eq. 3.36 indicates that at the magic angle the time-dependent quadrupolar coupling is periodic with period $\tau_{R}=2 \pi / \omega_{R}$ and has no time-independent component, such that its time average over the interval $\left[0, \tau_{R}\right]$ is zero.

There are at least two interesting points to note about the orientation dependence of the time-dependent quadrupolar coupling $\omega_{Q}(t)$. First, the crystallite angle $\gamma$ does not affect the amplitudes of the sinusoidal modulations, only their phases. The amplitudes depend only on the angles $\alpha$ and $\beta$. When $\eta_{Q}=0$, the local electric field gradients are cylindrically symmetric about the EFG principal axis and only the angle $\beta$ between the EFG principal axis and the rotor axis uniquely specifies the quadrupolar coupling strength.

\footnotetext{
${ }^{16}$ The symbols for these crystallite angles should not be confused with the second-order average Hamiltonian coefficients $\alpha^{(2)}, \beta^{(2)}$ and $\gamma^{(2)}$ used in previous sections.
} 


\subsection{THEORETICAL APPROACH TO ROTARY RESONANCE LINE}

NARROWING IN $I=5 / 2$ SYSTEMS

The result is that the amplitude coefficients $C_{1}, C_{2}, S_{1}$, and $S_{2}$ lose their $\alpha$-dependence, and in fact the sine-modulation amplitudes $S_{1}$ and $S_{2}$ vanish entirely.

The $\alpha$-dependence of the quadrupolar coupling amplitudes suggests a potential mechanism of the breakdown of orientation selectivity in systems with highly asymmetric electric field gradients $\eta_{Q} \rightarrow 1$. Consider a system in which the nuclei in all the different crystallites are uniformly polarized, e.g., every nucleus is initially magnetized along the $z$-axis. If the nuclei experience a distribution of quadrupolar couplings from crystallite to crystallite, they will evolve at different rates under the quadrupolar and rf interactions and dephase. However, if the nuclei all experience approximately the same quadrupolar coupling from crystallite to crystallite, then they will evolve in phase with each other. Assuming for the sake of argument that the system evolves into an observable coherence, the bulk signal is the sum of the signals of the individual crystallites. Every crystallite orientation makes a slightly different frequency contribution to the observed lineshape, and crystallites with nearly the same orientation contributing to nearly the same frequency component. It is possible that there is a range of crystallite angles $\beta$ over which the quadrupolar coupling varies slowly, such that the nuclei in these crystallites will evolve in phase and the spectral lines of nearby frequencies will add together constructively. Conversely, dephasing among crystallites will occur for ranges of angles for which there is a large distribution of quadrupolar couplings, and neighboring spectral lines will add destructively and cause a suppression of the NMR signal in these parts of the lineshape distribution. This may be the source of the orientation-selective effects that have been observed near $\eta_{Q}=0$. However, even if there are regions of the angle $\beta$ for which the quadrupolar coupling is relatively uniform, this uni- 


\subsection{THEORETICAL APPROACH TO ROTARY RESONANCE LINE} NARROWING IN $I=5 / 2$ SYSTEMS

formity might be destroyed as different crystallites with the same angle $\beta$ exhibit different quadrupolar couplings due to the $\alpha$-dependence. Therefore there could be no selection of preferred regions of crystallite orientations in the NMR spectrum.

The preceding argument is qualitative at best, and gives no explanation why one type of coherence transfer would exhibit orientation selection and another would not. Such an explanation would require an understanding of the terms in the Hamiltonian that drive these coherences and their orientation dependence. The average Hamiltonian-type approaches of $\S 3.5 .1$ and $§ 3.5 .2$ are insufficient to explain these effects. However, it is possible to examine the problem more quantitatively to see how some of the Hamiltonian coefficients that appeared in these treatments depend on the crystallite angles. Therefore, it may be possible to gain more insight as to whether particular regions of crystallite orientations have relatively constant quadrupolar couplings, why certain orientations are preferred, and whether a dependence on $\eta_{Q}$ and the crystallite angle $\alpha$ does indeed destroy the uniformity of coupling strengths.

The time-dependent quadrupolar coupling enters into the time development operator through the dynamic phase $\Phi_{Q}(t)$. An explicit expression for $\Phi_{Q}(t)$ can be found by direct integration of Eq. 3.36:

$$
\begin{aligned}
\Phi_{Q}(t)= & \int_{0}^{t} d t^{\prime} \omega_{Q}\left(t^{\prime}\right) \\
= & \frac{\omega_{Q}}{\omega_{R}}\left\{\left[S_{1} \cos (\gamma)+\frac{S_{2}}{2} \cos (2 \gamma)-C_{1} \sin (\gamma)-\frac{C_{2}}{2} \sin (2 \gamma)\right]\right. \\
& \left.\quad+C_{1} \sin \left(\omega_{R} t+\gamma\right)+\frac{C_{2}}{2} \sin \left(2 \omega_{R} t+2 \gamma\right)-S_{1} \cos \left(\omega_{R} t+\gamma\right)-\frac{S_{2}}{2} \cos \left(2 \omega_{R} t+2 \gamma\right)\right\} .
\end{aligned}
$$


The time development operator can be written such that it involves complex exponentials of the dynamic phase:

$$
\begin{aligned}
\exp \left[i \lambda \Phi_{Q}(t)\right]= & C_{\Phi} \exp \left[i \frac{\lambda \omega_{Q} C_{1}}{\omega_{R}} \sin \left(\omega_{R} t+\gamma\right)\right] \exp \left[i \frac{\lambda \omega_{Q} C_{2}}{2 \omega_{R}} \sin \left(2 \omega_{R} t+2 \gamma\right)\right] \\
& \times \exp \left[i \frac{-\lambda \omega_{Q} S_{1}}{\omega_{R}} \cos \left(\omega_{R} t+\gamma\right)\right] \exp \left[i \frac{-\lambda \omega_{Q} S_{2}}{2 \omega_{R}} \cos \left(2 \omega_{R} t+2 \gamma\right)\right]
\end{aligned}
$$

where $C_{\Phi}=\exp \left\{-i \frac{\lambda \omega_{Q}}{\omega_{R}}\left[C_{1} \sin (\gamma)+\frac{1}{2} C_{2} \sin (2 \gamma)-S_{1} \cos (\gamma)-\frac{1}{2} S_{2} \cos (2 \gamma)\right]\right\}$. Eq. 3.12 indicates that $\lambda= \pm 6$ for inner satellite transition $\mathrm{rf}$ terms and $\lambda= \pm 12$ for outer satellite transition rf terms. Eq. 3.39 can be expanded in a Fourier series using the Jacobi-Anger relations $[170, \S 2 \cdot 22]$ :

$$
\begin{aligned}
e^{i z \cos \theta} & =\sum_{n=-\infty}^{+\infty} i^{n} J_{n}(z) e^{i n \theta} \\
e^{i z \sin \theta} & =\sum_{n=-\infty}^{+\infty} J_{n}(z) e^{i n \theta}
\end{aligned}
$$

where $J_{n}(z)$ is the Bessel function of the first kind of order $n$. The result is:

$$
\begin{aligned}
\exp \left[i \lambda \Phi_{Q}(t)\right]= & C_{\Phi} \sum_{p, q, r, s=-\infty}^{+\infty}\left\{J_{p}\left(\frac{\lambda \omega_{Q} C_{1}}{\omega_{R}}\right) e^{i p\left(\omega_{R} t+\gamma\right)} \cdot J_{q}\left(\frac{\lambda \omega_{Q} C_{2}}{2 \omega_{R}}\right) e^{i q\left(2 \omega_{R} t+2 \gamma\right)}\right. \\
& \left.\times i^{r} J_{r}\left(\frac{-\lambda \omega_{Q} S_{1}}{\omega_{R}}\right) e^{i r\left(\omega_{R} t+\gamma\right)} \cdot i^{s} J_{s}\left(\frac{-\lambda \omega_{Q} S_{2}}{2 \omega_{R}}\right) e^{i s\left(2 \omega_{R} t+2 \gamma\right)}\right\} \\
= & C_{\Phi} \sum_{p, q, r, s=-\infty}^{+\infty} i^{(r+s)} e^{i[(p+2 q+r+2 s) \gamma]} J_{p}\left(\frac{\lambda \omega_{Q} C_{1}}{\omega_{R}}\right) J_{q}\left(\frac{\lambda \omega_{Q} C_{2}}{2 \omega_{R}}\right) \\
& \times J_{r}\left(\frac{-\lambda \omega_{Q} S_{1}}{\omega_{R}}\right) J_{s}\left(\frac{-\lambda \omega_{Q} S_{2}}{2 \omega_{R}}\right) e^{i\left[(p+2 q+r+2 s) \omega_{R} t\right]}
\end{aligned}
$$


This expression may be equated with a generic unimodal Fourier expansion:

$$
\exp \left[i \lambda \Phi_{Q}(t)\right]=\sum_{n=-\infty}^{+\infty} \Lambda_{n} e^{i n \omega_{R} t}
$$

where comparison with Eq. 3.12 indicates that the Fourier coefficients are $\Lambda_{n}=A_{n}$ for $\lambda=6$ and $\Lambda_{n}=B_{n}$ for $\lambda=12$. These coefficients become:

$$
\Lambda_{n}=C_{\Phi}^{\prime} \sum_{q, r, s=-\infty}^{+\infty} i^{(r+s)} J_{n-2 q-r-2 s}\left(\frac{\lambda \omega_{Q} C_{1}}{\omega_{R}}\right) J_{q}\left(\frac{\lambda \omega_{Q} C_{2}}{2 \omega_{R}}\right) J_{r}\left(\frac{-\lambda \omega_{Q} S_{1}}{\omega_{R}}\right) J_{s}\left(\frac{-\lambda \omega_{Q} S_{2}}{2 \omega_{R}}\right)
$$

where $n=p+2 q+r+2 s$ and $C_{\Phi}^{\prime}=e^{i n \gamma} C_{\Phi}$. This expression involves infinite sums over three indices. One of the sums can be removed upon applying the the Graf generalization of Neumann's Bessel function addition theorem $[170, \S 11 \cdot 3]$ :

$$
e^{i \nu \psi} J_{\nu}(\chi)=\sum_{m=-\infty}^{+\infty} J_{\nu+m}(Z) J_{m}(z) e^{i m \phi}
$$

where $\chi=\sqrt{Z^{2}+z^{2}-2 Z z \cos \phi}$ and $\psi$ is defined by $\chi \cos \psi=Z-z \cos \phi$ and $\chi \sin \psi=$ $z \sin \phi$. In the limit of real arguments $Z=x$ and $z=y$ and taking $\phi=\pi / 2$, one finds $\chi=$ $\sqrt{x^{2}+y^{2}}$ and $\psi=\tan ^{-1}(y / x)$. This result along with the relations $J_{m}(z)=(-1)^{m} J_{-m}(x)$ for integer $m$ and $(-1)^{m}=e^{-i m \pi}$ plus $\sum_{m=-\infty}^{+\infty} a_{m}=\sum_{m=-\infty}^{+\infty} a_{-m}$ and $i^{m}=e^{i m \pi / 2}$ can be used to write a special case of the addition theorem:

$$
\sum_{m=-\infty}^{+\infty} i^{m} J_{\nu-m}(x) J_{m}(y)=e^{i \nu \tan ^{-1}(y / x)} J_{\nu}\left(\sqrt{x^{2}+y^{2}}\right)=\left(\frac{\zeta}{|\zeta|}\right)^{\nu} J_{\nu}(|\zeta|)
$$


where $\zeta=x+i y$. The sum over the index $r$ in Eq. 3.44 can be removed by applying Eq. 3.46 with $m=r, \nu=n-2 q-2 s, x=\lambda \omega_{Q} C_{1} / \omega_{R}$, and $y=-\lambda \omega_{Q} S_{1} / \omega_{R}$ :

$$
\Lambda_{n}=C_{\Phi}^{\prime} \sum_{q, s=-\infty}^{+\infty} i^{s} e^{-i(n-2 q-2 s) \tan ^{-1}\left(\frac{S_{1}}{C_{1}}\right)} J_{n-2 q-2 s}\left(\left|\frac{\lambda \omega_{Q}}{\omega_{R}}\right| \sqrt{C_{1}^{2}+S_{1}^{2}}\right) J_{q}\left(\frac{\lambda \omega_{Q} C_{2}}{2 \omega_{R}}\right) J_{s}\left(\frac{-\lambda \omega_{Q} S_{2}}{2 \omega_{R}}\right) .
$$

This expression allows the Fourier coefficients $A_{n}$ and $B_{n}$ in Eq. 3.12 to be calculated as double infinite sums over a product of three Bessel functions weighted by an orientationdependent phase factor. However, when a large number of crystallite orientations must be considered, the double infinite sum could be difficult to evaluate even if truncated. Also, not much insight can be gained as to the orientation dependence of these coefficients, except that the dependence enters through the Bessel functions; this dependence will be discussed later.

A somewhat simpler expression can be developed in the case of axially symmetric field gradients where $\eta_{Q}=0$. In this case the coefficients of the time dependent quadrupolar coupling in Eq. 3.37 become $C_{1}^{0}=-\frac{3}{8} \sin \left(2 \theta_{m}\right) \sin (2 \beta), C_{2}^{0}=\frac{3}{8} \sin ^{2}\left(\theta_{m}\right) \sin ^{2}(\beta), S_{1}^{0}=0$, and $S_{2}^{0}=0$. A procedure similar to the one outlined above yields:

$$
\Lambda_{n}^{0}=e^{-i \lambda \frac{\omega_{Q}}{\omega_{R}}\left[C_{1}^{0} \sin (\gamma)+\frac{1}{2} C_{2}^{0} \sin (2 \gamma)\right]} e^{i n \gamma} \sum_{m=-\infty}^{+\infty} J_{n-2 m}\left(\frac{\lambda \omega_{Q} C_{1}^{0}}{\omega_{R}}\right) J_{m}\left(\frac{\lambda \omega_{Q} C_{2}^{0}}{2 \omega_{R}}\right)
$$

which involves only a single infinite sum with no orientation-dependent phase factors within 
the sum. The $n=0$ special case can be written as:

$$
\Lambda_{0}^{0}=e^{-i \lambda \frac{\omega_{Q}}{\omega_{R}}\left[C_{1}^{0} \sin (\gamma)+\frac{1}{2} C_{2}^{0} \sin (2 \gamma)\right]}\left[J_{0}\left(\frac{\lambda \omega_{Q} C_{1}^{0}}{\omega_{R}}\right) J_{0}\left(\frac{\lambda \omega_{Q} C_{2}^{0}}{2 \omega_{R}}\right)+2 \sum_{m>0}^{+\infty} J_{4 m}\left(\frac{\lambda \omega_{Q} C_{1}^{0}}{\omega_{R}}\right) J_{2 m}\left(\frac{\lambda \omega_{Q} C_{2}^{0}}{2 \omega_{R}}\right)\right]
$$

where the relation $J_{m}(z)=(-1)^{m} J_{-m}(z)$ has been used. This equation gives the $\eta_{Q}=0$ limit of the $A_{0}$ and $B_{0}$ Fourier coefficients that show up in a first-order average Hamiltonian treatment in the interaction frame.

Eq. 3.47 can be used to make plots of the orientation dependence of the Hamiltonian coefficients. First, some comments should be made as to the role of the crystallite angle $\gamma$. As is apparent from Eq. 3.36, only the phase of the time-dependent quadrupolar coupling depends on $\gamma$, such that the quadrupolar coupling of a crystallite with angle $\gamma$ arrives at the same point in its oscillation at a time $\gamma / \omega_{R}$ later than a crystallite with $\gamma=0$. This leads to the so-called "carousel symmetry" of a rotating isotropic powder [171, 172]. The $\gamma$ distribution typically contributes to the intensity of the spinning sidebands in MAS experiments on powders but otherwise does not affect the powder lineshapes. However, in these treatments it is usually assumed that the initial density operator does not depend on crystallite orientation and the isotropy of the powder is not broken by the application of an rf field. Once the first low-amplitude rf pulse is applied to the system these conditions can break down, since the "soft" pulse will affect each crystallite differently according to its orientation. The problem of $\gamma$-averaging is subtle and involves cancellation effects among 


\subsection{THEORETICAL APPROACH TO ROTARY RESONANCE LINE}

NARROWING IN $I=5 / 2$ SYSTEMS

crystallites [171]. For the sake of simplicity, it will be assumed that the $\gamma$-dependence does not dominate the orientation-selective effects. If it did, the argument that the $\beta$-dependence leads to the orientation selectivity would fail. It will be seen that the $\beta$-dependence of the Hamiltonian coefficients does indeed match the observed orientation-selective effects, as has been reproduced in simulations $[165,166]$. Therefore $\gamma$ will be set to zero in the orientation plots that follow.

A truncated summation of Eq. 3.47 was used to calculate the zero-frequency Fourier coefficients $A_{0}$ (Fig. 3.4) and $B_{0}$ (Fig. 3.5) of Eq. 3.12 as a function of the crystallite angle $\beta$ in the limit $\eta_{Q}=0$ with $C_{Q}=3.0 \mathrm{MHz}$ and $\omega_{R} / 2 \pi=20 \mathrm{kHz}$. The calculation was performed using 320 random crystallite angle pairs $(\alpha, \beta)$ generated by the REPULSION sampling method [173]; the $\eta_{Q}=0$ condition makes the coefficients dependent only on the

angle $\beta$. Both the coefficients (particularly $A_{0}$ ) exhibit regions of relative uniformity in the approximate ranges of $\beta=35^{\circ}$ to $62^{\circ}$ and $\beta=118^{\circ}$ to $145^{\circ}$. This is consistent with the numerical simulation predictions of which crystallite angles are selected via the low-amplitude pulses $[165,166]$. The same calculation was performed for $\eta=1$ for the coefficients $A_{0}$ (Fig. 3.6) and $B_{0}$ (Fig. 3.7). The $\eta_{Q} \neq 0$ condition introduces a dependence on the crystallite angle $\alpha$, so that for a given $\beta$ the coefficients can take on several values depending on the value of $\alpha$. As can be seen, this destroys any uniformity of the Fourier coefficients as a function of crystallite orientation. As a consequence, it is expected that the selection of any set of nearby crystallite orientations by the rf pulse will be suppressed.

The (truncated) summations in Eq. 3.25 can be used to calculate the dependence of some of the second-order Hamiltonian coefficients on crystallite orientation. Figs. 3.8, 


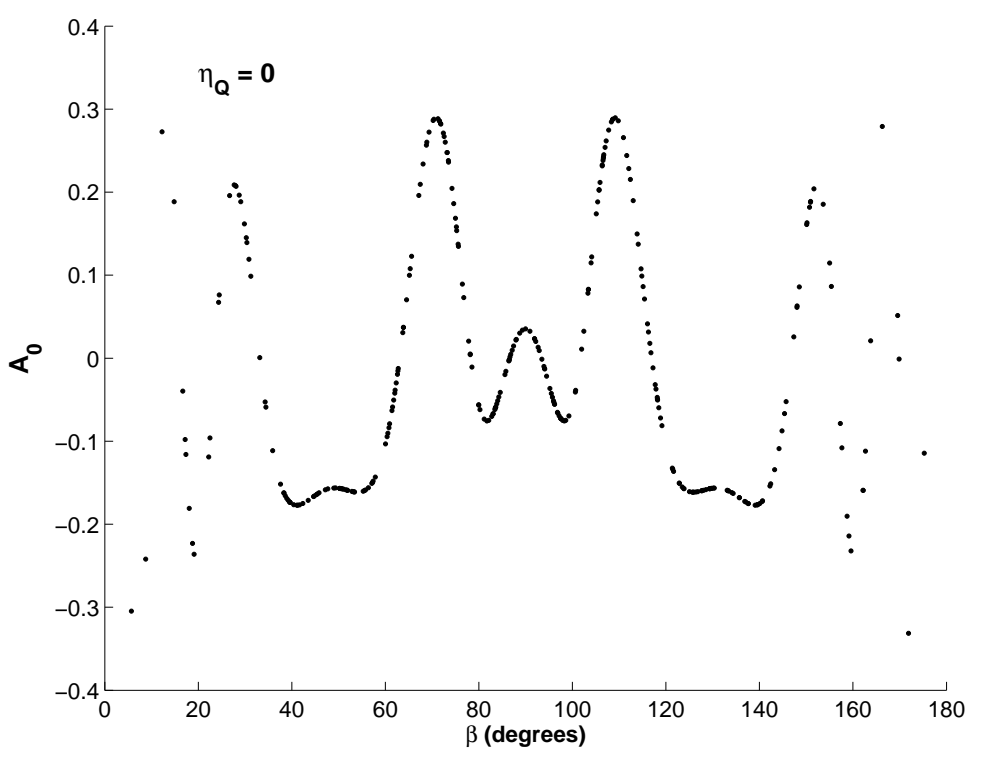

Figure 3.4: Calculated zero-frequency quadrupolar dynamic phase Fourier coefficient $A_{0}$ as a function of crystallite angle $\beta$ for $\eta_{Q}=0$. The quadrupolar coupling constant was $C_{Q}=3.0 \mathrm{MHz}$ and the spinning frequency was $\omega_{R} / 2 \pi=20 \mathrm{kHz}$. The double sums in Eq. 3.47 were taken from -20 to +20 . The calculation used 320 random crystallite angle pairs $(\alpha, \beta)$ generated by REPULSION sampling; no $\gamma$-dependence was considered.

3.9 , and 3.10 plot the absolute-squared coefficients $\left|\alpha^{(2)}\right|^{2},\left|\beta^{(2)}\right|^{2},\left|\gamma^{(2)}\right|^{2}$, respectively, as functions of the crystallite angle $\beta$ for $\eta_{Q}=0$. The other calculation parameters are the same as before. It can be seen that the $\left|\alpha^{(2)}\right|^{2}$ and $\left|\beta^{(2)}\right|^{2}$ functions do not seem to have the same regions of relative uniformity as do the coefficients $A_{0}$ and $B_{0}$ that occur in the first-order perturbation theory. The function $\left|\gamma^{(2)}\right|^{2}$ exhibits some of the same uniformity, although its value is small in these regions. The parameter $\left|\gamma^{(2)}\right|^{2}$ enters into some of the short-time dynamics during certain excitations and conversions of multiple quantum coherences, as was noted in $\S 3.5 .1$ and $§ 3.5 .2$. However, the perturbation theory employed in those sections is suspect.

Ultimately, the orientation dependence of the effective Hamiltonian in the average Hamiltonian model arises from the properties of the Bessel functions. Bands of unifor- 


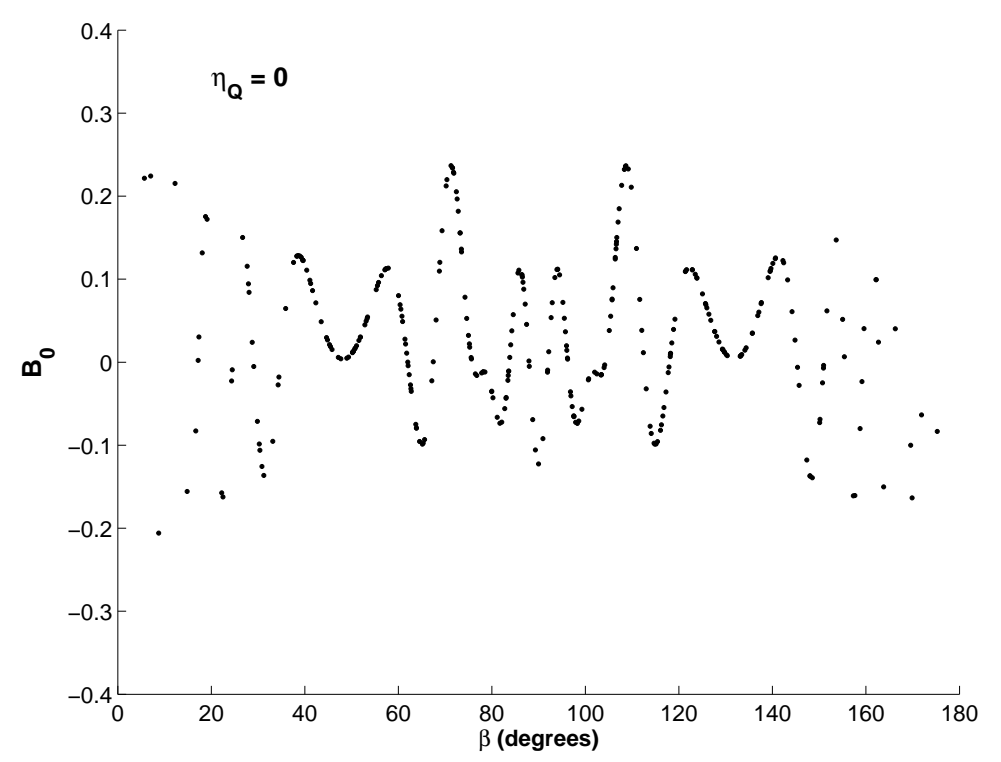

Figure 3.5: Calculated zero-frequency quadrupolar dynamic phase Fourier coefficient $B_{0}$ as a function of crystallite angle $\beta$ for $\eta_{Q}=0$. The quadrupolar coupling constant was $C_{Q}=3.0 \mathrm{MHz}$ and the spinning frequency was $\omega_{R} / 2 \pi=20 \mathrm{kHz}$. The double sums in Eq. 3.47 were taken from -20 to +20 . The calculation used 320 random crystallite angle pairs $(\alpha, \beta)$ generated by REPULSION sampling; no $\gamma$-dependence was considered.

mity can be seen in some of the individual Bessel functions, as demonstrated by Fig. 3.11, which plots the function $J_{0}(16 \sin (2 \beta))$ as a function of $\beta$, which approximates the function $J_{0}\left(6 \frac{\omega_{Q}}{\omega_{R}} C_{1}^{0}\right)$ under the conditions of the calculations above. As can be seen, dense bands of crystallite orientations over which the Bessel function varies slowly are observed from $\beta=35^{\circ}$ to $62^{\circ}$ and $\beta=118^{\circ}$ to $145^{\circ}$. There is some fortuity involved in the ratio $\omega_{Q} / \omega_{R}$ that is employed in these experiments: similar prominent bands in the plot of $J_{0}(x)$ are not observed for values of the Bessel function argument that are much (e.g. ten times) larger than $16 \sin (2 \beta)$, and the bands become less prominent as the Bessel function becomes smoother for arguments that are much (e.g. ten to one hundred times) smaller. It is unclear whether there exist more profound sources than the properties of Bessel functions ${ }^{17}$ for the

\footnotetext{
${ }^{17}$ Or rather, more profound than the properties of the functions that are approximated by Bessel functions.
} 


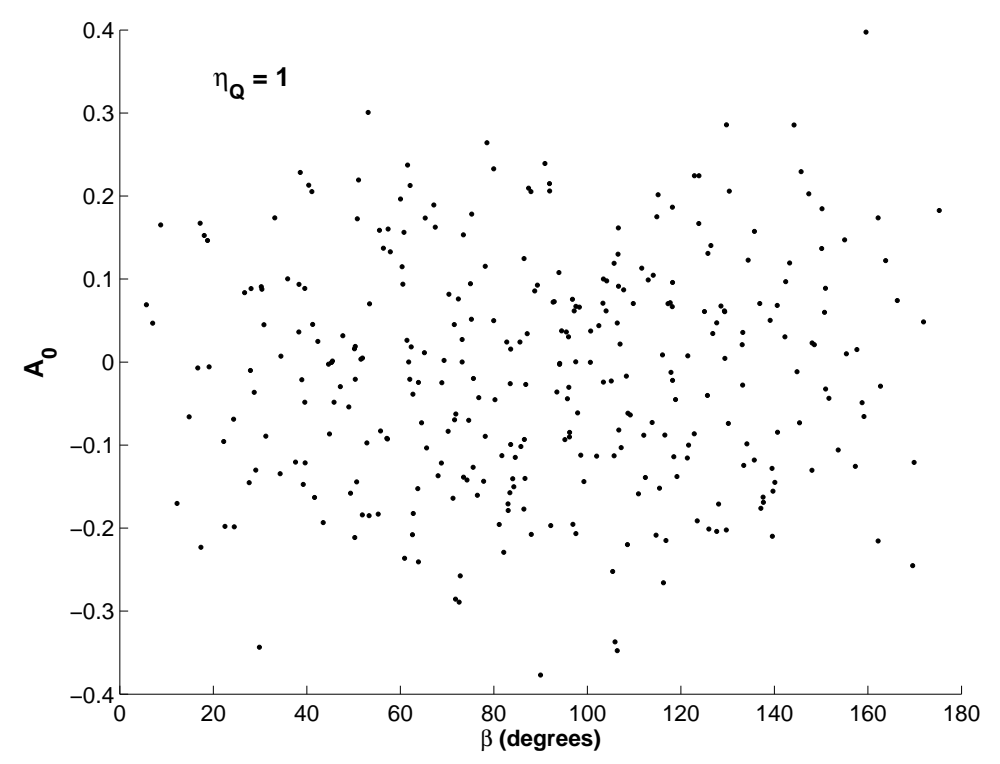

Figure 3.6: Calculated zero-frequency quadrupolar dynamic phase Fourier coefficient $A_{0}$ as a function of crystallite angle $\beta$ for $\eta_{Q}=1$. The quadrupolar coupling constant was $C_{Q}=3.0 \mathrm{MHz}$ and the spinning frequency was $\omega_{R} / 2 \pi=20 \mathrm{kHz}$. The double sums in Eq. 3.47 were taken from -20 to +20 . The calculation used 320 random crystallite angle pairs $(\alpha, \beta)$ generated by REPULSION sampling; no $\gamma$-dependence was considered.

selection of certain crystallite orientations by low-amplitude rf pulses in the quadrupolar NMR of rotating solids.

\subsection{Conclusions}

In this work, the use of low-amplitude rf multiple-quantum excitation and conversion pulses in fast-spinning $I=5 / 2$ quintuple-quantum/triple-quantum mixed-multiple quantum magic angle spinning studies was investigated both experimentally and theoretically. The low-amplitude pulses were optimized at or away from rotary resonance conditions depending on the desired coherence transfer. The experiments demonstrated that the incorporation of low amplitude pulses into the 5Q3QMAS experiment can result in a factor 


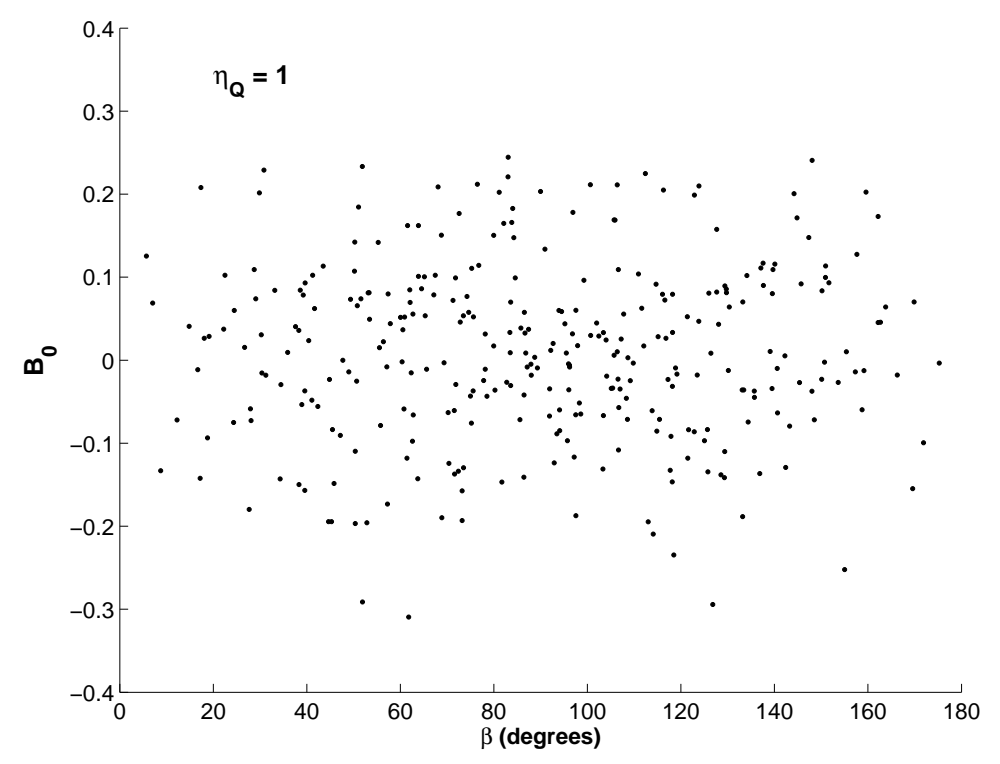

Figure 3.7: Calculated zero-frequency quadrupolar dynamic phase Fourier coefficient $B_{0}$ as a function of crystallite angle $\beta$ for $\eta_{Q}=1$. The quadrupolar coupling constant was $C_{Q}=3.0 \mathrm{MHz}$ and the spinning frequency was $\omega_{R} / 2 \pi=20 \mathrm{kHz}$. The double sums in Eq. 3.47 were taken from -20 to +20 . The calculation used 320 random crystallite angle pairs $(\alpha, \beta)$ generated by REPULSION sampling; no $\gamma$-dependence was considered.

of two gain in signal-to-noise over the all-hard pulse experiment in the $\mathrm{Al}(\mathrm{acac})_{3}$ system with moderate $C_{Q}=3.0 \mathrm{MHz}$ and low $\eta_{Q}=0.15$.

The low-amplitude pulse version of the 5Q3QMAS experiment also exhibits unusually narrow lineshapes in the anisotropic dimension for low- $\eta_{Q}$ systems. This phenomenon has suggested a non-quantitative one-dimensional low-amplitude pulse experiment for screening systems with multiple overlapping low- $\eta_{Q}$ sites. The origin of the linenarrowing effect was investigated theoretically using a second-order average Hamiltonian theory/secular averaging approach. This approach was unable to explain why certain types of low-amplitude pulses lead to line narrowing effects and other types of pulses do not. Moderate success was achieved at explaining the line narrowing effect as being the result of orientation-selective pulses. The effect seems to be due to the in-phase excitation of 


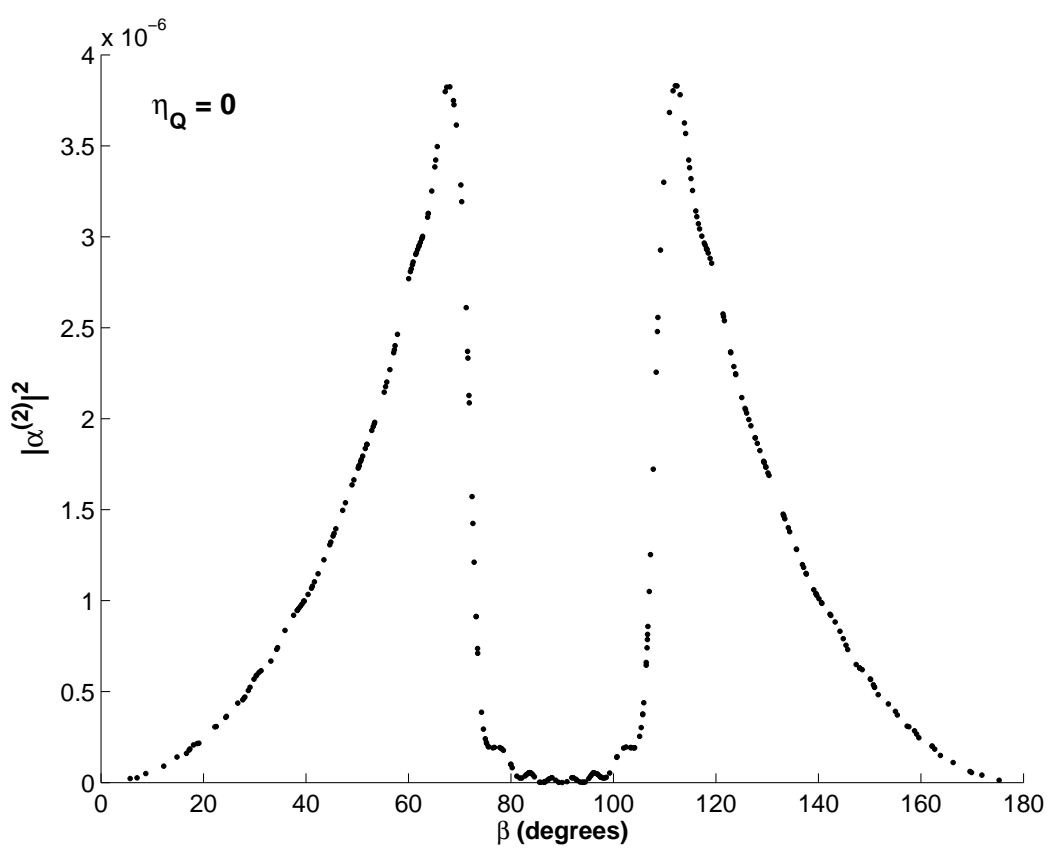

Figure 3.8: Calculated second-order average Hamiltonian coefficient $\left|\alpha^{(2)}\right|^{2}$ as a function of crystallite angle $\beta$ for $\eta_{Q}=0$. The quadrupolar coupling constant was $C_{Q}=3.0 \mathrm{MHz}$ and the spinning frequency was $\omega_{R} / 2 \pi=20 \mathrm{kHz}$. The double sums in Eq. 3.47 were taken from -20 to +20 , and the coefficient sum in Eq. 3.25 was taken from $n=-200$ to 200 . The calculation used 320 random crystallite angle pairs $(\alpha, \beta)$ generated by REPULSION sampling; no $\gamma$-dependence was considered.

a certain set of crystallite orientations by a radiofrequency field that is truncated by the orientation- and time-dependent quadrupolar Hamiltonian under MAS conditions. Other crystallite orientations do not contribute to the anisotropic spectrum because they are excited out of phase with each other, leading to the mutual cancellation of neighboring spectral components. The line-narrowing effect disappears in sites with highly-asymmetric electric field gradients $\left(\right.$ high $\left.-\eta_{Q}\right)$, probably due to an additional orientational dependence of the quadrupolar Hamiltonian that destroys the uniform excitation of certain crystallite orientations. 


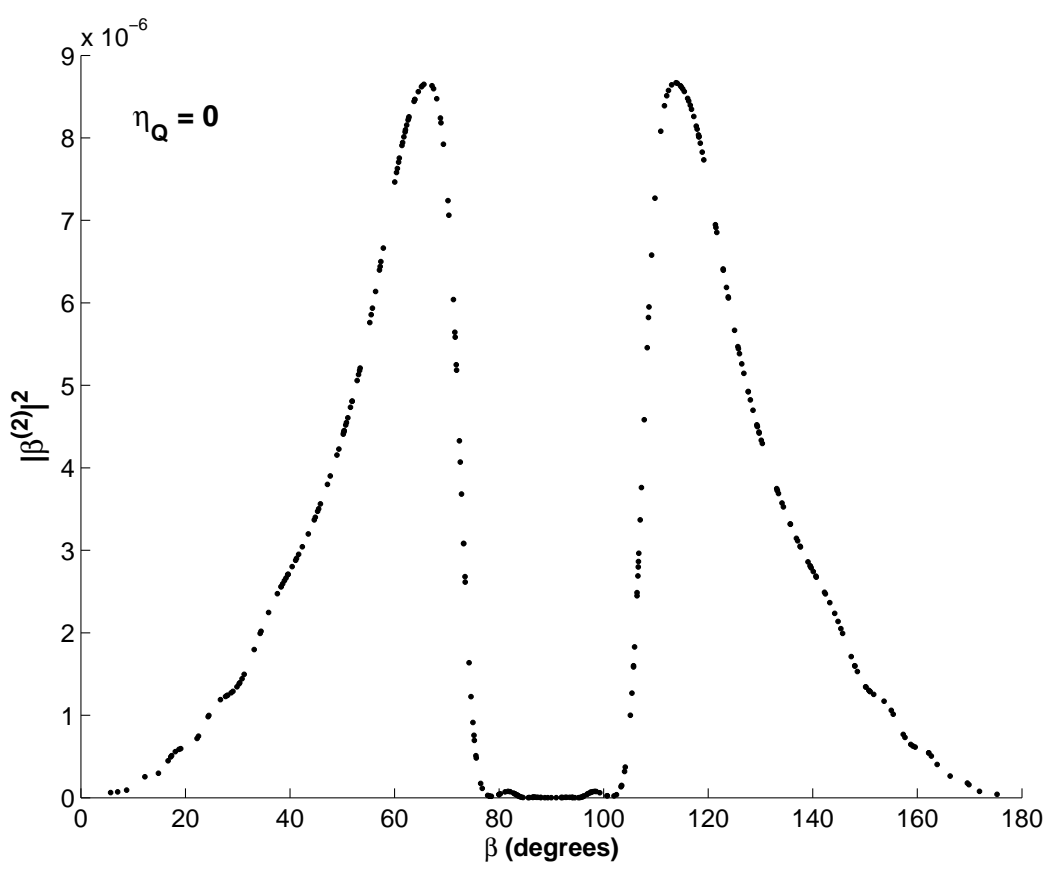

Figure 3.9: Calculated second-order average Hamiltonian coefficient $\left|\beta^{(2)}\right|^{2}$ as a function of crystallite angle $\beta$ for $\eta_{Q}=0$. The quadrupolar coupling constant was $C_{Q}=3.0 \mathrm{MHz}$ and the spinning frequency was $\omega_{R} / 2 \pi=20 \mathrm{kHz}$. The double sums in Eq. 3.47 were taken from -20 to +20 , and the coefficient sum in Eq. 3.25 was taken from $n=-200$ to 200 . The calculation used 320 random crystallite angle pairs $(\alpha, \beta)$ generated by REPULSION sampling; no $\gamma$-dependence was considered. 


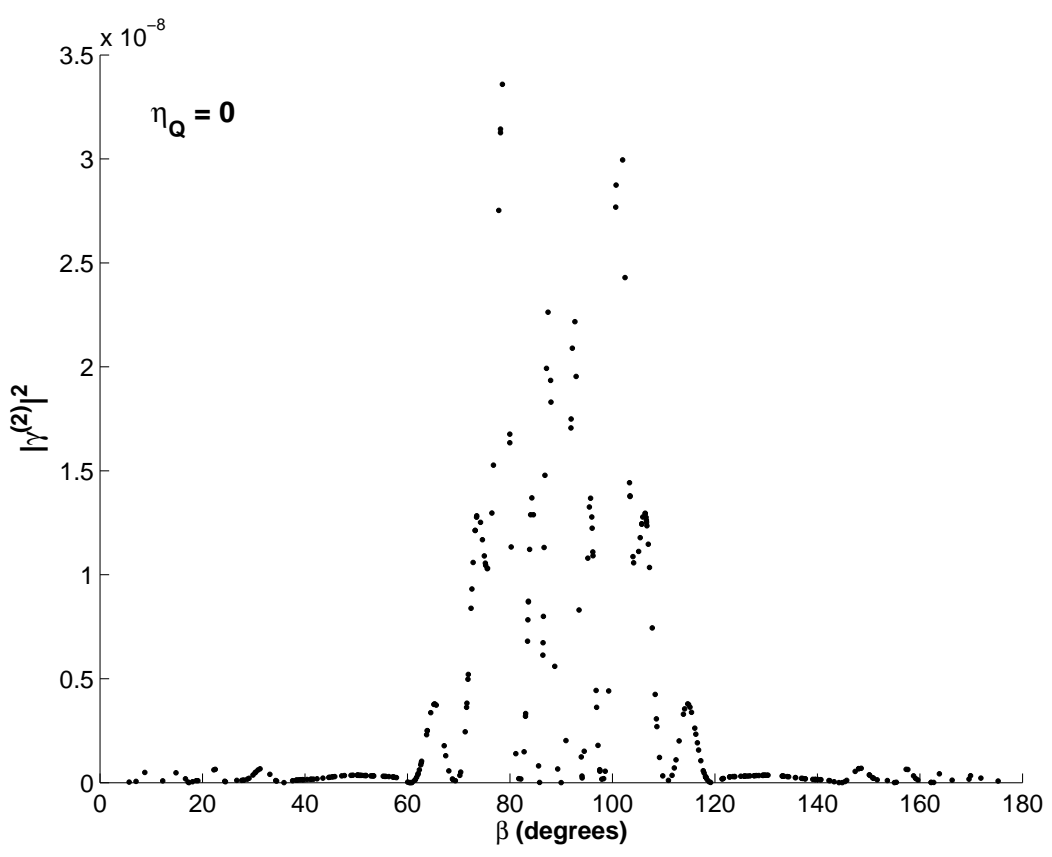

Figure 3.10: Calculated second-order average Hamiltonian coefficient $\left|\gamma^{(2)}\right|^{2}$ as a function of crystallite angle $\beta$ for $\eta_{Q}=0$. The quadrupolar coupling constant was $C_{Q}=3.0 \mathrm{MHz}$ and the spinning frequency was $\omega_{R} / 2 \pi=20 \mathrm{kHz}$. The double sums in Eq. 3.47 were taken from -20 to +20 , and the coefficient sum in Eq. 3.25 was taken from $n=-200$ to 200 . The calculation used 320 random crystallite angle pairs $(\alpha, \beta)$ generated by REPULSION sampling; no $\gamma$-dependence was considered. 


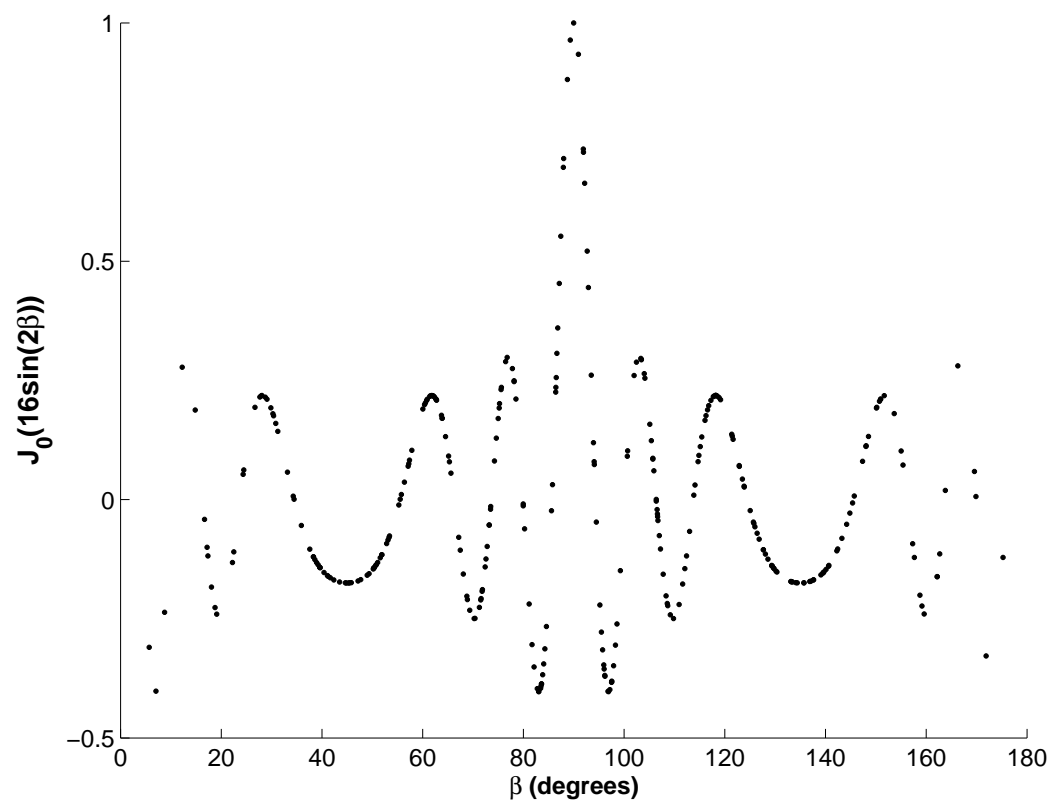

Figure 3.11: Plot of $J_{0}(16 \sin (2 \beta))$ versus $\beta$. 


\section{Chapter 4}

\section{Feasibility of N-14 double quantum}

\section{line narrowing in solution}

\subsection{Introduction}

Nitrogen is an extremely prevalent heteroatom in organic molecules and plays an important role in the structure of biomolecules, where it is present in all nucleic acids and amino acids. Proteins are sequences of amino acids joined by peptide bonds, and since every peptide bond contains a nitrogen-bearing amide functional group, nitrogen atoms trace out the entire protein backbone. NMR experiments that correlate nitrogen chemical shifts with those of neighboring atoms are widely employed to assist in the elucidation of protein structures.

The NMR-active N-14 isotope exists in $99.63 \%$ natural abundance, making it the obvious choice for NMR spectroscopic studies. However, N-14 is a spin $S=1$ nucleus, 
and therefore possesses an electric quadrupole moment. Nitrogen-14 quadrupolar coupling constants can be large, ranging from $\geq 1 \mathrm{MHz}$ in protonated amine groups in amino acids [174] to as much as $3-4 \mathrm{MHz}$ in amide groups in peptide bonds $[175,176]$. Molecules in solution are subject to rapid tumbling and other internal reorientational motions that cause a stochastic modulation of the large quadrupolar coupling, inducing efficient (rapid) relaxation of the N-14 nuclear magnetization. Since the linewidth of a particular resonance is inversely proportional to the transverse relaxation time, the fast quadrupolar relaxation leads to very broad spectral lines. The large linewidths are problematic because of the corresponding decrease in spectral resolution, and also because the area of the line is spread out over a large frequency range, leading to a small maximum intensity and low signal-tonoise ratio. The sensitivity problem is exacerbated if the nitrogen resonance is detected directly, since N-14 has a relatively low gyromagnetic ratio of $\gamma / 2 \pi=3.078 \mathrm{MHz} / \mathrm{T}$ (compared to $42.577 \mathrm{MHz} / \mathrm{T}$ for $\mathrm{H}-1)$. Furthermore, even if nitrogen coherence is transferred (i.e., through a $J$-coupling) to protons for more sensitive detection, rapid quadrupolar relaxation may attenuate the coherence during the transfer step. For all these reasons, the non-quadrupolar, spin-1/2 N-15 isotope $(\gamma / 2 \pi=-4.317 \mathrm{MHz} / \mathrm{T})$ is almost exclusively used in protein NMR studies involving nitrogen. However, the N-15 isotope exists in only $0.37 \%$ natural abundance, so the proteins typically are first artifically labeled (often uniformly) with N-15. Isotopic N-15 labeling can be a tedious and costly, but necessary step when performing nitrogen NMR studies of proteins.

Line broadening of all types is particularly problematic for large biomolecules such as proteins, which are often massive enough so that their global rotational motion is in the 
slow tumbling regime and is dominated by adiabatic relaxation mechanisms (see $§ 1.5$ ). The rotational correlation time of a spherical globular protein can be estimated from Stokes' law $[4, \S 1.4]$ :

$$
\tau_{c}=\frac{4 \pi \eta_{w} r_{H}^{3}}{3 k_{B} T}
$$

where $\eta_{w}$ is the viscosity of the solvent (i.e., water), $r_{H}$ is the effective hydrodynamic radius of the protein ( $\propto M^{1 / 3}$ excluding the protein hydration shell, where $M$ is the protein mass), $k_{B}$ is the Boltzmann constant, and $T$ is the temperature. This model predicts a value near the experimental value of $\tau_{c}=4.1 \mathrm{~ns}$ for the small $(8.6 \mathrm{kDa})$ protein ubiquitin in water at 25 ${ }^{\circ} \mathrm{C}$ [177], which yields a value of $\omega_{0} \tau_{c}=1.3$ using a Larmor frequency of $\omega_{0} / 2 \pi=50.6 \mathrm{MHz}$ for $\mathrm{N}-14$ at $16.45 \mathrm{~T}$. This value is just within the slow tumbling regime where $\omega_{0} \tau_{c}>1$. It can be seen from Eq. 4.1 that large $(>100 \mathrm{kDa})$ proteins have rotational correlation times long enough to be well into the slow tumbling regime with $\omega_{0} \tau_{c}>10$ for N-14 at such high magnetic field strengths.

The idea of performing nitrogen NMR in proteins using naturally-abundant N-14 is attractive, but to do so effectively would require a method that reduces the quadrupolarbroadened linewidths dramatically. Furthermore, any such experiment that is to be applicable to large biomolecules must be valid in the slow-tumbling motional regime. As was mentioned in $\S 1.5 .4$, the double-quantum coherence in $S=1$ systems is largely invariant to the adiabatic quadrupolar broadening mechanism that dominates the relaxation of the single-quantum coherences in the slow tumbling regime. Consequently, the doublequantum coherence relaxes more slowly and has sharper lines in this regime than do the 
single-quantum coherences. Therefore, it is worth investigating whether double-quantum spectroscopy could make N-14 protein NMR experiments viable. ${ }^{1}$

\subsection{Theory of $\mathrm{N}-14$ linewidths in solution}

\subsubsection{N-14 double- and single-quantum linewidths and the comparison to N-15 spectroscopy}

The $S=1 \mathrm{~N}-14$ spin system is characterized by nine independent density operator elements: three corresponding to populations, four corresponding to single-quantum coherences (two +1-quantum and two -1-quantum), and two corresponding to +2 - and -2-quantum coherence. If the quantization axis is along $z$, then a $p$-quantum coherence ${ }^{2}$ evolves under the Hamiltonian $\hat{H} / \hbar=\omega \hat{I}_{z}$ at the frequency $p \omega$. If one considers two resonances with equal linewidths $\Gamma$ (in frequency units) separated by a frequency difference $\Delta \omega$, then an effective resolution factor can be defined as $R=\Delta \omega / \Gamma$. Since the frequency separation of a $|p|$-quantum coherence is $|p|$ times that of a single quantum coherence, one finds that if the linewidths of two pairs of coherences are equal such that $\Gamma_{|p|}=\Gamma_{\left|p^{\prime}\right|}$, then $R_{|p|} / R_{\left|p^{\prime}\right|}=|p| /\left|p^{\prime}\right| .^{3}$ Thus, if the N-14 double quantum coherence relaxed at the same rate as the single quantum coherences, then the double-quantum spectrum would have two

\footnotetext{
${ }^{1} \mathrm{~A}$ similar approach has been used to reduce amide proton linewidths by creating multiple-quantum coherences in the two-spin N-15-H-1 J-coupled system [178].

${ }^{2}$ The density operator may be written in terms of single-transition basis operators $\left|S, m_{S}^{\prime}\right\rangle\left\langle S, m_{S}\right|$, where the coherence order is $p=m_{S}^{\prime}-m_{S}$. It is often more convenient to use a spherical tensor operator basis $\left\{\hat{T}_{k, p}\right\}$, where the double-quantum coherences are represented by the $\hat{T}_{2, \pm 2}$ elements, and observable transverse magnetization corresponds to the $\hat{T}_{1, \pm 1}$ elements. Populations (the diagonal elements of the density operator) are not coherences but are considered to have $p=0$.

${ }^{3}$ This assumes that the observed linewidths are actually the natural linewidths of the system, which is typically the case for quadrupolar-broadened systems in a modern high-field NMR magnet. If the observed linewidths are dominated by dephasing due to magnetic field inhomogeneity, then these linewidths also scale with $|p|$, such that there is no inherent change in resolution based solely upon changing coherence order.
} 
times higher resolution than the single-quantum spectrum.

Of course, the N-14 double- and single-quantum coherences actually relax at different rates. In the case of relaxation due to an axially symmetric $\left(\eta_{Q}=0\right) S=1$ quadrupolar interaction modulated by rotational diffusion of a rigid spherical molecule in isotropic solution, the transverse magnetization (the $\hat{T}_{1, \pm 1}$ single-quantum coherences in the density operator) relaxes according to $[6,4,103]$ :

$$
\Gamma_{S Q}^{Q}=1 / T_{2}^{Q}=\frac{3}{80}\left(2 \pi C_{Q}\right)^{2}\left[3 \tau_{c}+\frac{5 \tau_{c}}{1+\omega_{0}^{2} \tau_{c}^{2}}+\frac{2 \tau_{c}}{1+4 \omega_{0}^{2} \tau_{c}^{2}}\right]
$$

where $C_{Q}=e^{2} q Q / h$ is the quadrupolar coupling constant, $\tau_{c}$ is the global rotational correlation time of the molecule, and $\omega_{0}$ is the Larmor frequency. The three terms in brackets correspond to $J(0), J\left(\omega_{0}\right)$, and $J\left(2 \omega_{0}\right)$ reduced spectral densities, respectively. ${ }^{4}$ Under the same conditions, the relaxation rate of the $S=1 \hat{T}_{2, \pm 2}$ double quantum coherences (DQCs) is $[103]$ :

$$
\Gamma_{D Q}^{Q}=\frac{3}{80}\left(2 \pi C_{Q}\right)^{2}\left[\frac{2 \tau_{c}}{1+\omega_{0}^{2} \tau_{c}^{2}}+\frac{4 \tau_{c}}{1+4 \omega_{0}^{2} \tau_{c}^{2}}\right]
$$

The first and second term in brackets correspond to $J\left(\omega_{0}\right)$ and $J\left(2 \omega_{0}\right)$ reduced spectral densities, respectively, and there is no zero-frequency (adiabatic) relaxation component. Eqs. 4.2 and 4.3 are plotted in Fig. 4.1 as a function of $\tau_{c}$ for N-14 with $C_{Q}=-3.2 \mathrm{MHz}$ [175] in a $16.45 \mathrm{~T}$ magnetic field $\left(\omega_{0}^{N 14} / 2 \pi=50.6 \mathrm{MHz}\right)$. As can be seen, the difference between the transverse relaxation rate and the adiabatic-free double-quantum relaxation

\footnotetext{
${ }^{4}$ More complicated expressions for the spectral densities in the cases of non-axially symmetric quadrupolar couplings or non-spherical molecules may be found in Ref. [103].
} 
rate is quite significant in the slow tumbling $\left(\tau_{c}>\left|\omega_{0}^{-1}\right|\right)$ regime, particularly for large molecules with $\tau_{c}$ in the tens of nanoseconds range. Unfortunately, since the linewidths are of the order of $\Gamma^{Q}$ (assuming that the quadrupolar relaxation dominates all other relaxation mechanisms), the absolute magnitudes of these relaxation rates would seem to prohibit high-resolution NMR experiments, for which linewidths in the low tens of $\mathrm{Hz}$ or better are desirable.

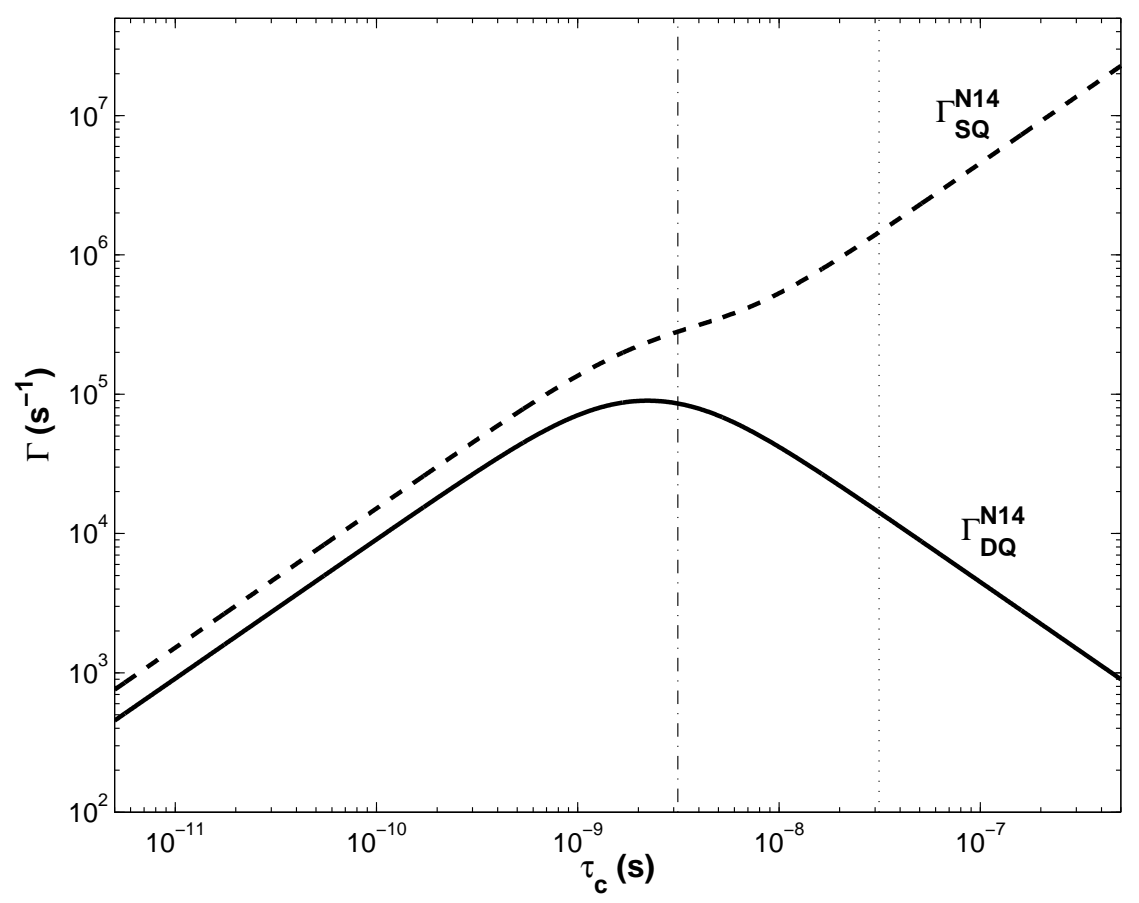

Figure 4.1: Calculated double-quantum and single-quantum N-14 quadrupolar relaxation rates as a function of rotational correlation time $\tau_{c}$ for a rigid spherical molecule in isotropic solution at $16.45 \mathrm{~T}$. The quadrupolar parameters are $C_{Q}=-3.2 \mathrm{MHz}$ and $\eta_{Q}=0$, and the Larmor frequency is $\omega_{0} / 2 \pi=50.6 \mathrm{MHz}$. The vertical dash-dot line marks the onset of the slow tumbling regime at $\tau_{c}=\left|\omega_{0}\right|^{-1}$, and the vertical dotted line marks $\tau_{c}=10\left|\omega_{0}\right|^{-1}$. Here $\tau_{c}$ ranges from 5 ps to $500 \mathrm{~ns}$, but the perturbative relaxation theory should be considered suspect when $\tau_{c}$ is beyond about 100 ns.

As a demonstration of the size of quadrupolar coupling for which N-14 doublequantum spectroscopy may be advantageous over conventional N-15 spectroscopy, Fig. 4.2 
plots the double-quantum quadrupolar relaxation rate for N-14 (Eq. 4.3) at $16.45 \mathrm{~T}$ scaled down by a factor of two (to take into account the increased frequency spread between doublequantum lines vs. single-quantum lines) for $C_{Q}=-3.2 \mathrm{MHz}$ and $C_{Q}=100 \mathrm{kHz}$, along with the transverse relaxation rate of an N-15 nucleus relaxed by dipolar coupling to a nearby (amide) proton at $1.02 \AA$ and a typical amide $[179,180]$ nitrogen chemical shift anisotropy (CSA) of $-170 \mathrm{ppm}$. The dipolar and CSA relaxation rates were calculated according to Ref. [4, §5.4]. Dipolar-CSA cross-correlated relaxation [91, 181] has been ignored. As can be seen, double-quantum spectroscopy of N-14 is not competitive with conventional $\mathrm{N}-15$ spectroscopy even well into the slow tumbling limit in the case of typical $C_{Q}=$ $-3.2 \mathrm{MHz}$ amide N-14 quadrupolar couplings. However, in the case of slowly-tumbling molecules with $\mathrm{N}-14$ sites for which $C_{Q}$ is up to several hundred $\mathrm{kHz}$, double quantum spectroscopy may be practical both relative to conventional N-15 spectroscopy and in terms of absolute quadrupolar linewidth. It should be mentioned that the N-14 relaxation rates in Fig. 4.2 do not include dipolar and CSA effects, which will begin to dominate quadrupolar relaxation for small $C_{Q}$ sites in the same way as do the dipolar and CSA relaxation effects in $\mathrm{N}-15 .^{5}$ However, this is not necessarily a liability because the ability to decrease the effects of quadrupolar relaxation to a point where N-14 linewidths are comparable to N-15 linewidths ${ }^{6}$ would be sufficient to justify the technique, since isotopic N-15 labeling would

\footnotetext{
${ }^{5}$ It would be interesting to investigate whether a technique analogous to the TROSY experiment of Pervushin et al. [182] could reduce the CSA and dipolar contributions to N-14 double-quantum relaxation.

${ }^{6}$ The dipolar and CSA relaxation effects are of comparable magnitude for the N-14 double quantum coherence and the N-15 single-quantum coherence (N-14 even has slightly smaller dipolar couplings and CSAs than N-15 due to its lower gyromagnetic ratio). There also exist quadrupolar-dipolar and quadrupolar-CSA cross-correlated relaxation effects that can be quite significant, particularly for large quadrupolar couplings. However, like autocorrelated quadrupolar relaxation, these mechanisms make no adiabatic contribution to the relaxation of double-quantum coherence (refer to the relaxation selection rules in $\S 1.5 .3$ ), so their contribution would be small in the slow tumbling regime, and is certainly smaller than the autocorrelated quadrupolar relaxation contribution.
} 


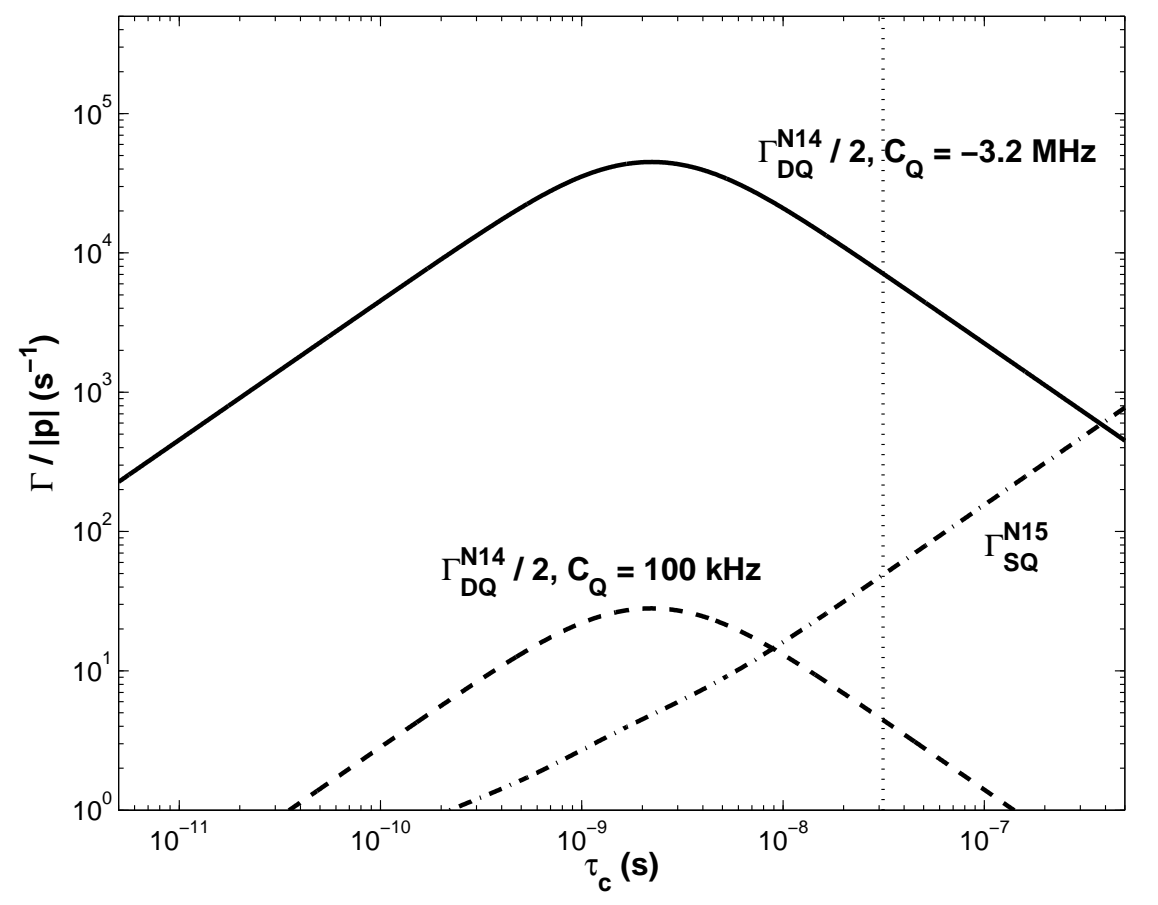

Figure 4.2: Calculated double-quantum N-14 quadrupolar relaxation rates for a rigid spherical molecule in isotropic solution for two different values of $C_{Q}$ as a function of rotational correlation time $\tau_{c}$, plus the calculated transverse relaxation rate of $\mathrm{N}-15$ under CSA and nitrogen-proton dipolar coupling. The relaxation rates were scaled by a factor of $1 /|p|$ (where $p$ is the coherence order) in order to make a fair comparison of the resolution of double-quantum spectra to that of single-quantum spectra. The magnetic field was taken to be $16.45 \mathrm{~T}$, corresponding to $\omega_{0}^{N 14} / 2 \pi=50.6 \mathrm{MHz}$ and $\omega_{0}^{N 15} / 2 \pi=-71.0 \mathrm{MHz}$. The N-14 quadrupolar parameters are $C_{Q}=-3.2 \mathrm{MHz}$ or $C_{Q}=100 \mathrm{kHz}$, with $\eta_{Q}=0$ in both cases. The N-15-H-1 average bond length was taken to be $r_{N H}=1.02 \AA$, and the N-15 CSA was taken to be $\Delta \sigma=-170 \mathrm{ppm}$ with $\eta_{C S A}=0$. Dipolar-CSA cross-correlations were neglected. The dotted vertical line marks $\tau_{c}=10\left|\omega_{0}^{N 14}\right|^{-1}$.

be unnecessary.

Modifications of the rigid-molecule model that include the effects of local internal molecular motions alter the spectral densities $J(\omega)$ from the simple form given in Eq. 1.135 and plotted in Fig. 1.4, and hence the calculated relaxation rates will differ from those plotted in Figs. 4.1 and 4.2 when internal motions are considered. A widely-used, simple (two-parameter) model-free approach to the calculation of the spectral densities was 
introduced by Lipari and Szabo $[183,184] .^{7}$ The ratio of the Lipari-Szabo spectral density $J_{L-S}(\omega)$ to the rigid-molecule spectral density $J_{\text {rigid }}(\omega)$ of Eq. 1.135 is given by:

$$
\frac{J_{L-S}(\omega)}{J_{\text {rigid }}(\omega)}=\mathcal{S}^{2}+\left(1-\mathcal{S}^{2}\right)\left(\frac{\tau}{\tau_{c}}\right)\left(\frac{1+\omega^{2} \tau_{c}^{2}}{1+\omega^{2} \tau^{2}}\right)
$$

where $\mathcal{S}$ is a generalized order parameter characterizing the amplitude of the internal motion $\left(0 \leq \mathcal{S}^{2} \leq 1, \mathcal{S}^{2}=1\right.$ corresponds to rigid motion) and $\tau^{-1}=\tau_{c}^{-1}+\tau_{e}^{-1}$, where $\tau_{c}$ is the global rotational correlation time and $\tau_{e}$ is an effective correlation time characterizing the internal motion. It can be seen that $\tau \simeq \tau_{e}$ in the limit $\tau_{e} \ll \tau_{c}$ where the internal motion is much faster than the global rotation. The effect of internal motion on the spectral density functions in the slow tumbling regime is not straightforward, as illustrated in Fig. 4.3 for the adiabatic $J(0)$ and non-adiabatic $J\left(2 \omega_{0}\right)$ spectral densities as functions of $\tau_{c}$ with fixed $\omega_{0} / 2 \pi=50.6 \mathrm{MHz}$, for $\mathcal{S}^{2}=0.5$ with $\tau_{e}=250 \mathrm{ps}$ and for $\mathcal{S}^{2}=0.05$ with $\tau_{e}=25 \mathrm{ps}$ The inclusion of internal motion effects may effectively increase or decrease the calculated relaxation rates depending on the value of the motional parameters.

One final point that has not yet been addressed is the effect of fast N-14 quadrupolar relaxation on the rate of relaxation of nearby coupled nuclei, i.e., H-1 and C-13. It is important to consider the linewidths of these nuclei in order to assess how the presence of N-14 will affect their spectral resolution. Protein NMR experiments involving nitrogen typically correlate the nitrogen resonances with $\mathrm{C}-13$ and/or $\mathrm{H}-1$ resonances, often with a transfer of nitrogen coherence to protons for sensitive detection. The N-14 quadrupolar relaxation can have deleterious effects on the structure of the $J$-split multiplet of a

\footnotetext{
${ }^{7}$ Relaxation in the amide N-H system in proteins has been found to be better described by an approach that includes parameters characterizing both fast and slow internal motional timescales, see Ref. [185].
} 


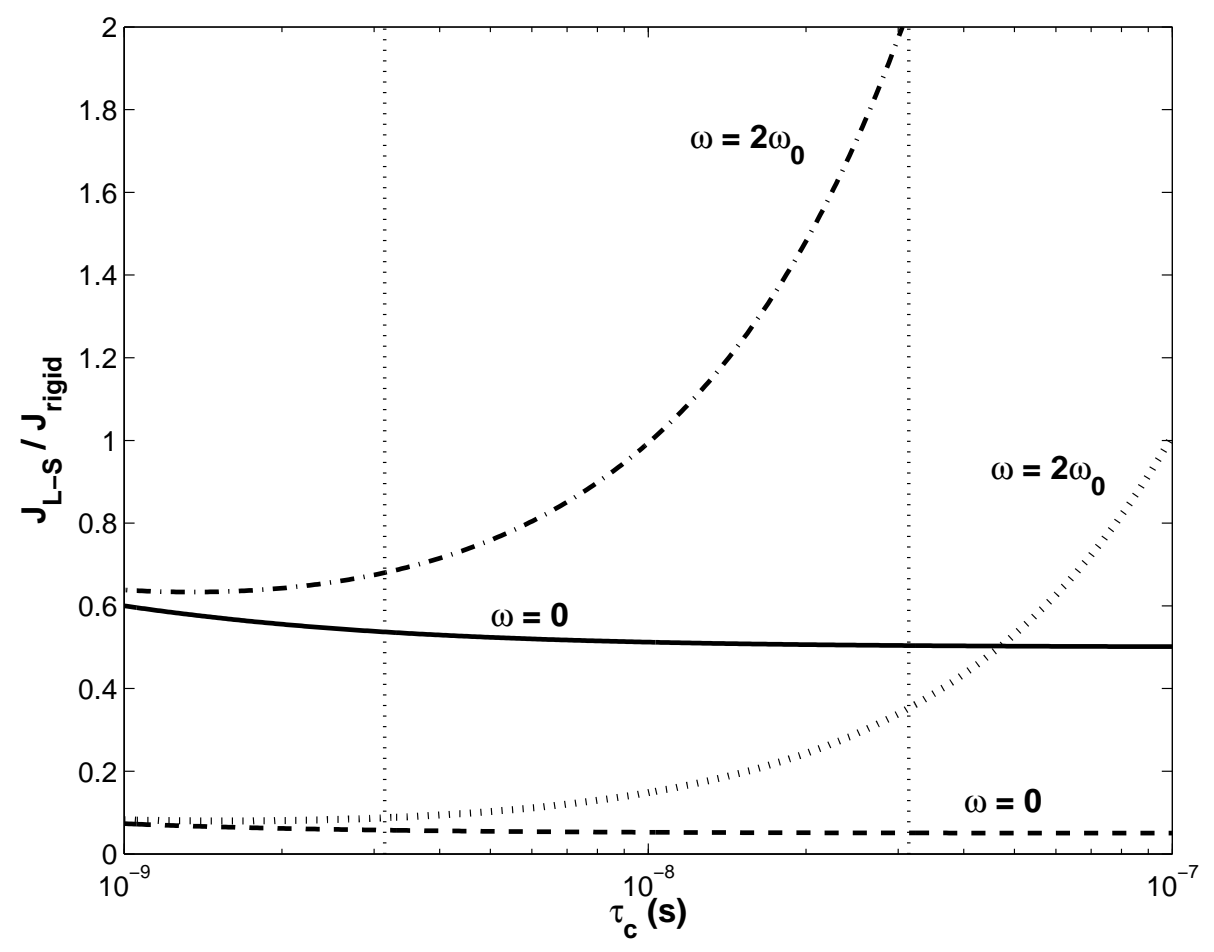

Figure 4.3: Ratio of the Lipari-Szabo spectral densities to the rigid-molecule spectral densities as a function of global correlation time with $\omega_{0} / 2 \pi=50.6 \mathrm{MHz}$ for two sets of values of the Lipari-Szabo model-free parameters. The ratios $J_{L-S}(0) / J_{\text {rigid }}(0)$ (solid line) and $J_{L-S}\left(2 \omega_{0}\right) / J_{\text {rigid }}\left(2 \omega_{0}\right)$ (dash-dot line) are plotted for $\mathcal{S}^{2}=0.5$ and $\tau_{e}=250 \mathrm{ps}$, and for $\mathcal{S}^{2}=0.05$ and $\tau_{e}=25 \mathrm{ps}$ (dashed and dotted lines, respectively). The two vertical dotted lines mark $\tau_{c}=\left|\omega_{0}\right|^{-1}$ (left) and $\tau_{c}=10\left|\omega_{0}\right|^{-1}$ (right).

coupled spin-1/2 nucleus $[186,187,188]$ and on the spin- $1 / 2$ linewidths (to the extent of collapse of the multiplet structure). In the solution state the relaxation effects are typically transmitted through the $J$-coupling, and are often referred to as "scalar relaxation of the second kind" $[6, \S$ VIII.II.F(b)(3)],[4, §5.4.5]. Other effects can contribute in the slow tumbling regime, particularly quadrupolar-dipolar cross-correlation [189] $]^{8}$, [190]. Fig. 4.4 plots the transverse relaxation rate of a proton $J$-coupled to an $\mathrm{N}-14$ nucleus in a rigid spherical molecule in isotropic solution (in the limit that the quadrupolar interaction dom-

\footnotetext{
${ }^{8}$ Ref. [189] contains some errors: on the first page, one of the spin operators should be corrected from $T_{Q}^{-1}=+\mathbf{I}_{-}\left(2 \mathbf{I}_{\mathbf{z}}+1\right) /(I(2 I-1))$ to $T_{Q}^{-1}=+\left(2 \mathbf{I}_{\mathbf{z}}+1\right) \mathbf{I}_{-} /(I(2 I-1))$, and the $\omega \tau_{2}^{2} /\left(1+\omega^{2} \tau_{2}^{2}\right)$ factor in Eq. 13 should be corrected to $\tau_{2} /\left(1+\omega^{2} \tau_{2}^{2}\right)$.
} 
inates all other interactions in the system) using Eqs. 19-21 of Ref. [189]. The proton was

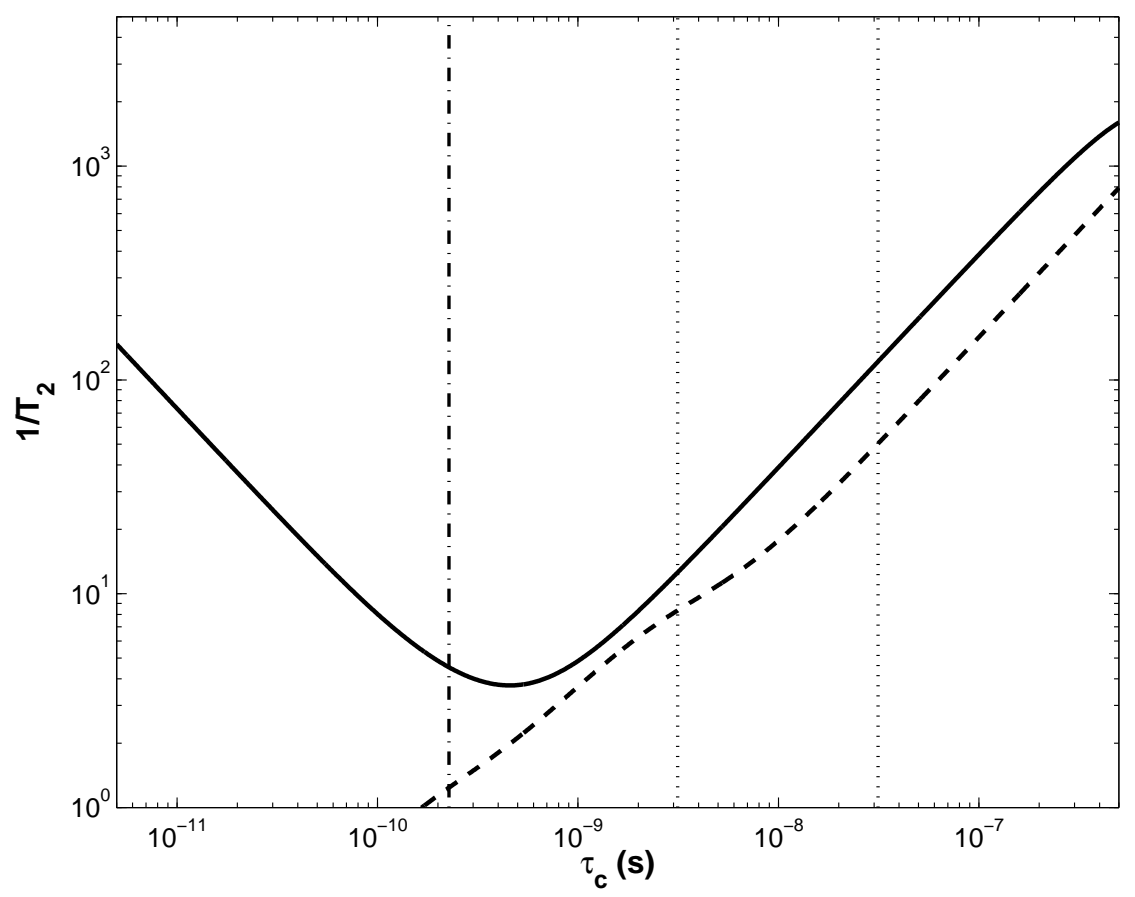

Figure 4.4: Calculated H-1 transverse relaxation rate in the presence of a scalar-coupled $\mathrm{N}-14$ nucleus in a rigid molecule in isotropic solution at $16.45 \mathrm{~T}$ as a function of correlation time. The dashed line corresponds to relaxation under $\mathrm{H}-1$ dipolar and chemical shift anisotropy interactions, and the solid line additionally includes the N-14 quadrupolar effects. The relevant Larmor frequencies are $\omega_{0}^{H 1} / 2 \pi=700 . \mathrm{MHz}$ and $\omega_{0}^{N 14} / 2 \pi=50.6 \mathrm{MHz}$. The dipolar coupling was calculated using $r_{N H}=1.02 \AA$ and the H-1 chemical shift anisotropy was $\Delta \sigma=10 \mathrm{ppm}$ with $\eta_{C S A}=0$. The N-14 quadrupolar parameters are $C_{Q}=-3.2 \mathrm{MHz}$ and $\eta_{Q}=0$. The nitrogen-proton $J$-coupling was taken to be $\left|J_{N 14-H 1}\right|=\left|\frac{\gamma_{N 14}}{\gamma_{N 15}} J_{N 15-H 1}\right|=$ $0.71 \cdot 91 \mathrm{~Hz}=65 \mathrm{~Hz}$ (the value of $J_{N 15-H 1}$ was taken from Refs. [4, 191]). Cross-correlation effects were included, and the nitrogen-proton dipolar axis was taken to be parallel to the N14 quadrupolar and H-1 CSA principal axes. The vertical dash-dot line marks $\tau_{c}=\left|\omega_{0}^{H 1}\right|^{-1}$, and the vertical dotted lines mark $\tau_{c}=\left|\omega_{0}^{N 14}\right|^{-1}$ (left) and $\tau_{c}=10\left|\omega_{0}^{N 14}\right|^{-1}$ (right).

considered to relax under dipolar and chemical shift anisotropy-induced relaxation as well as quadrupolar-induced relaxation from the N-14 nucleus, and cross-correlation effects between all three interactions were included for the special case of coincident principal axis systems. The quadrupolar and dipolar parameters were those of an amide N-14-H-1 pair, and the proton CSA was taken to be $\Delta \sigma=10 \mathrm{ppm}$ [192]. The scalar coupling was taken 
to be $\left|J_{N 14-H 1}\right|=65 \mathrm{~Hz}$. As is apparent from Fig. 4.4, the rapid relaxation of the N-14 nucleus can increase the $\mathrm{H}-1$ transverse relaxation rate quite significantly. It is tempting to try to ameliorate this effect by applying an N-14 spin decoupling pulse sequence during the H-1 evolution time, as has been done for the quadrupolar nucleus H-2 $(S=1)$ coupled to C-13 $(I=1 / 2)$ [193]. However, the N-14 decoupling strength is required to be much greater than the longitudinal relaxation rate of the quadrupolar nucleus, which in the case a rigid molecule in isotropic solution is given by $[6,4,103]^{9}$ :

$$
1 / T_{1}^{Q}=\frac{3}{80}\left(2 \pi C_{Q}\right)^{2}\left[\frac{2 \tau_{c}}{1+\omega_{0}^{2} \tau_{c}^{2}}+\frac{8 \tau_{c}}{1+4 \omega_{0}^{2} \tau_{c}^{2}}\right]
$$

for $S=1$ and $\eta_{Q}=0$. Eq. 4.5 yields a value of $1 / T_{1}^{N 14}=19 \mathrm{kHz}$ for pure quadrupolar relaxation of N-14 with $C_{Q}=-3.2 \mathrm{MHz}$ and $\eta_{Q}=0$ at $16.45 \mathrm{~T}$ with $\tau_{c}=10\left|\omega_{0}^{N 14}\right|^{-1}=31$ ns. It would be difficult to apply N-14 decoupling at a high enough power to remove the H-1 scalar relaxation effectively, even in a high-power solid-state NMR probe.

\subsubsection{A comment on deuterium double-quantum spectroscopy}

Nitrogen-14 is not the only $S=1$ nucleus available for spectroscopic studies; often NMR experiments are carried out in systems isotopically labeled with deuterium (H-2, 0.015\% natural abundance). Deuterium labeling is favored because of the decreased effect of dipolar relaxation to nearby heteronuclei; the dipolar relaxation rate goes as the square of the size of the dipolar coupling, which in turn is bilinear in the gyromagnetic ratios of the

\footnotetext{
${ }^{9}$ Table 5.7 of Ref. [4] contains an error: the $R_{1}=3 d_{00}\left\{J\left(\omega_{I}\right)+2 J\left(2 \omega_{I}\right)\right\}$ expression for longitudinal quadrupolar relaxation should be corrected to $\operatorname{read} R_{1}=3 d_{00}\left\{J\left(\omega_{I}\right)+4 J\left(2 \omega_{I}\right)\right\}$.
} 
two coupled spins. ${ }^{10}$ The gyromagnetic ratio of $\mathrm{H}-2(\gamma / 2 \pi=6.536 \mathrm{MHz} / \mathrm{T})$ is 6.5 times less than that of H-1. Scalar relaxation of a coupled $I=1 / 2$ nucleus via the $S$-spin quadrupolar coupling is much less pronounced for systems containing deuterium compared to those containing $\mathrm{N}-14$, because the deuterium quadrupolar coupling is much smaller $\left(C_{Q} \sim 200\right.$ $220 \mathrm{kHz}$ for protein backbone amide deuterons [195] and $C_{Q} \sim 170 \mathrm{kHz}$ in perdeuterated saturated hydrocarbons [196]). The scalar relaxation rate of a spin-1/2 nucleus coupled to a quadrupolar nucleus depends largely on the relaxation rates of the quadrupolar nucleus, which in turn are quadratic in the quadrupolar coupling constant (Eqs. 4.2, 4.5).

In addition to deuteration for the purpose of reducing the linewidths of coupled heteronuclei, it may be of interest to perform spectroscopy on the deuterium itself. In this case, it is possible to revisit the problem of double-quantum line narrowing in $S=1$ systems in the case of a smaller quadrupolar coupling than in amide nitrogens. The N-14 quadrupolar double-quantum relaxation rate for a small value of $C_{Q}$ was already compared to the N-15 single-quantum relaxation rate in Fig. 4.2. However, it is useful to consider the comparison of double- and single-quantum relaxation rates in a single $S=1$ system with small $C_{Q}$, as was plotted in Fig. 4.1 for a large- $C_{Q}$ system. The double-quantum and single-quantum quadrupolar plus dipole-dipole relaxation rates of a deuterium nucleus dipole-coupled to a nearby C-13 nucleus at $16.45 \mathrm{~T}$ are plotted in Fig. 4.5. The rates have not been scaled by a factor of $1 /|p|$ to account for increased double-quantum chemical shift

\footnotetext{
${ }^{10}$ However, the gyromagnetic ratio enters additionally into the spectral density functions through their Larmor frequency dependence, primarily outside of the extreme narrowing limit. It should also be noted that the dipolar relaxation rate also has a dependence on the spin quantum numbers $S$ and $I$; for instance, the longitudinal dipolar relaxation of a proton coupled to an $S=1 \mathrm{~N}-14$ nucleus is actually faster than that of a proton coupled to an $S=1 / 2 \mathrm{~N}-15$ nucleus in the extreme narrowing limit, despite N-14 having a smaller gyromagnetic ratio [194]. The authors of Ref. [194] note that scalar longitudinal relaxation of the proton via the N-14 quadrupolar coupling is negligible in the extreme narrowing limit, as predicted by theory $[6]$.
} 


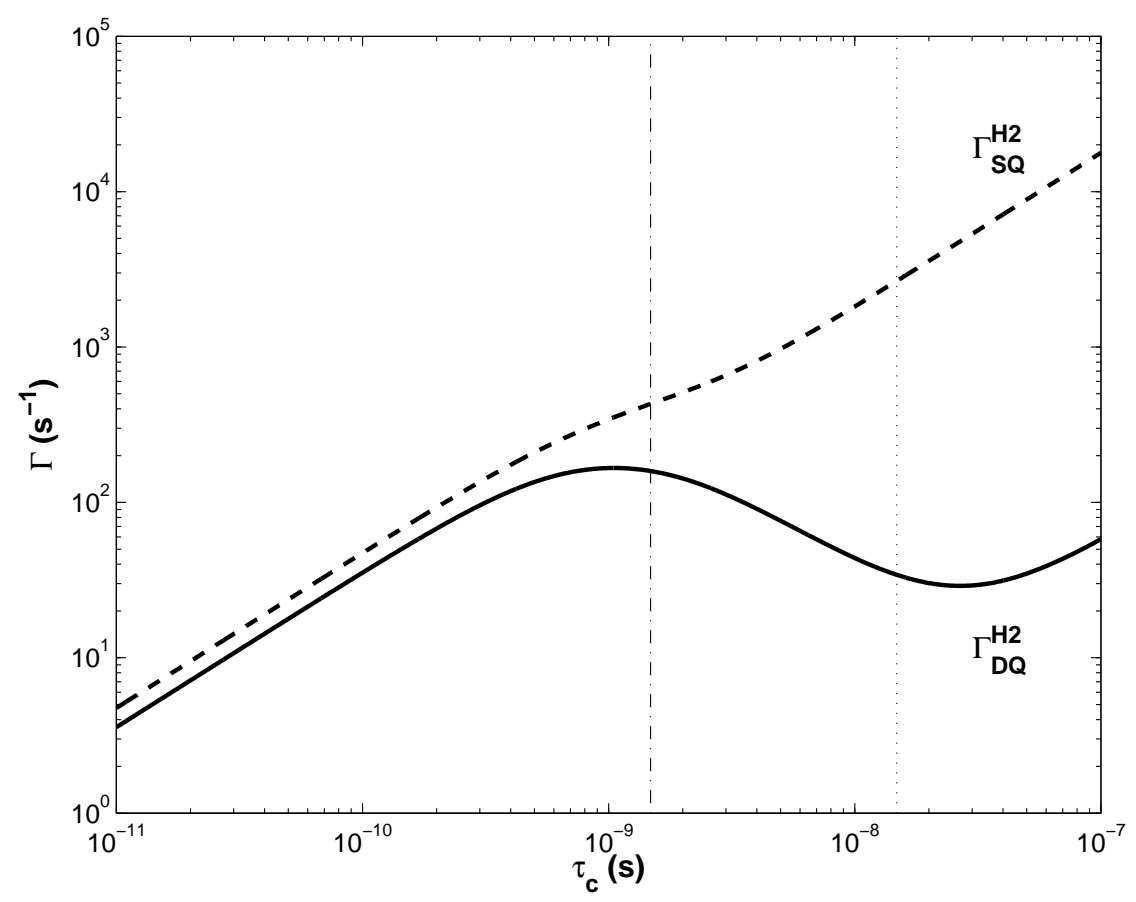

Figure 4.5: Calculated double-quantum and single-quantum H-2 relaxation rates as a function of rotational correlation time $\tau_{c}$ for a rigid spherical molecule in isotropic solution at $16.45 \mathrm{~T}$. The relaxation mechanisms include the $\mathrm{H}-2$ quadrupolar interaction and dipolecoupling to a nearby $\mathrm{C}-13$ nucleus $\left(r_{D C}=1.09 \AA\right)$, with no cross-correlation effects. The quadrupolar parameters are $C_{Q}=200 \mathrm{kHz}$ and $\eta_{Q}=0$, and the relevant Larmor frequencies are $\omega_{0}^{H 2} / 2 \pi=107.5 \mathrm{MHz}$ and $\omega_{0}^{C 13} / 2 \pi=176.2 \mathrm{MHz}$. The vertical dash-dot line marks $\tau_{c}=\left|\omega_{0}^{H 2}\right|^{-1}$, and the vertical dotted line marks $\tau_{c}=10\left|\omega_{0}^{H 2}\right|^{-1}$. Here $\tau_{c}$ ranges from $10 \mathrm{ps}$ to $100 \mathrm{~ns}$.

scaling. Deuterium CSA relaxation was not included (although it is not negligible), and the H-2-C-13 J-coupling has been neglected. The effect of quadrupolar-dipolar cross-correlation on the relaxation of the deuterium coherences was considered to be negligible. The H-2 quadrupolar parameters were taken to be $C_{Q}=200 \mathrm{kHz}$ with $\eta_{Q}=0$. The deuteriumcarbon average bond length was taken to be $r_{D C}=1.09 \AA$. As usual, the relaxation rates were calculated under the assumption of a rigid spherical molecule in isotropic solution, using Eqs. 4.2 and 4.3 for the quadrupolar relaxation. The H-2 double- and single-quantum dipole-dipole relaxation rates were calculated according to Ref. [197]. 


\subsection{PRODUCTION OF N-14 DOUBLE-QUANTUM COHERENCE (DQC) IN SOLUTION

As can be seen from a comparison of Fig. 4.5 to Fig. 4.1, the possibility of a resolution enhancement via double-quantum deuterium NMR spectroscopy in the slow tumbling regime is much more attractive in terms of the absolute linewidth values, as well as retaining the usual double-quantum versus single-quantum line narrowing feature. (The factor of two increase in the double-quantum chemical shift scaling should also be considered when examining Figs. 4.1 and 4.5.) The effects of dipole-dipole relaxation on the deuterium single-quantum relaxation rate are negligible compared to the quadrupolar relaxation effects, but in the case of the double-quantum coherence, far into the slow-tumbling regime the adiabatic dipole-dipole contribution begins to dominate the adiabatic-free quadrupolar contribution.

\subsection{Production of $\mathrm{N}-14$ double-quantum coherence (DQC) in solution}

There are really two questions in the problem of N-14 double-quantum spectroscopy: not only "is it useful?", but also "can it be done?" The first question was addressed in $\S 4.2$; the second question is not trivial, for we have already seen that fast relaxation due to an extremely large quadrupolar coupling can present formidable challenges. This section explores a few potential methods by which $S=1$ double quantum coherence may be created in solution, some of which have been explored experimentally in this work or elsewhere, some of which have not. 


\subsection{PRODUCTION OF N-14 DOUBLE-QUANTUM COHERENCE (DQC) IN SOLUTION

\subsubsection{DQC through the residual quadrupolar interaction in oriented me- dia}

Perhaps the most obvious way to create N-14 double-quantum coherence in solution is to perform the experiments on samples in anisotropic (oriented) media in which the quadrupolar Hamiltonian is not motionally averaged to zero. The prototypical example is that of a liquid crystalline medium which forms an ordered mesophase at the proper concentration and temperature. In such a medium there is a preferred (space-fixed) direction for molecular orientation, which is called the director axis. There are many cases in which the liquid crystal is oriented in an external magnetic field; in such cases, the director is usually parallel or perpendicular to the magnetic field. Not only do the liquid crystal molecules have a preferred orientation, but solute molecules dissolved in the liquid crystalline solvent will also tend to have a preferred orientation. From the perspective of NMR, anisotropic liquid crystalline systems resemble conventional fluids in the sense that fast molecular diffusion and tumbling motionally average the NMR interactions, but they resemble solids in the sense that the anisotropic orientational averaging they impose does not remove the tensor terms of the solid state Hamiltonians. Rather, when the weighted orientational average is performed, the solid state Hamiltonians tend to be motionally averaged to some smaller, non-zero value that depends on the degree of ordering of the system and the projection of the interaction onto the director axis. However, unlike in a powdered solid where the interaction strengths depend strongly on molecular orientation, all of the molecules in a liquid crystal tend to have the same interaction strengths, with some small distribution due to fluctuations in orientation about the director. 


\subsection{PRODUCTION OF N-14 DOUBLE-QUANTUM COHERENCE (DQC) IN SOLUTION

Consider an $S>1 / 2$ nucleus in an anisotropic medium. In general, this nucleus will experience a motionally averaged first-order quadrupolar Hamiltonian

$$
\hat{H}_{Q}^{\prime} / \hbar=\frac{1}{3} \omega_{Q}^{\prime}\left[3 \hat{S}_{z}^{2}-S(S+1) \hat{1}\right]
$$

where the prime indicates a motionally-averaged quantity and the averaged quadrupolar frequency $\omega_{Q}^{\prime}$ depends on the spin quantum number $S$, the $C_{Q}$ and $\eta_{Q}$ quadrupolar parameters, and the degree of orientation of the molecule. For an $S=1$ nucleus, the $\hat{S}_{x}$ term of the density operator evolves under this Hamiltonian as [198 $]^{11}$ :

$$
\hat{S}_{x}(t)=e^{-i \hat{H}_{Q}^{\prime} t / \hbar} \hat{S}_{x} e^{+i \hat{H}_{Q}^{\prime} t / \hbar}=\hat{S}_{x} \cos \omega_{Q}^{\prime} t+i\left(\hat{T}_{2,+1}+\hat{T}_{2,-1}\right) \sin \omega_{Q}^{\prime} t,
$$

where $\hat{T}_{2, \pm 1}=\mp \frac{1}{2}\left(\hat{S}_{z} \hat{S}_{ \pm}+\hat{S}_{ \pm} \hat{S}_{z}\right)$. The quadrature-detected NMR signal is proportional to $\operatorname{Tr}\left[\hat{S}_{x}(t) \hat{S}_{+}\right] \propto \cos \omega_{Q}^{\prime} t$, which corresponds to a doublet at $\omega= \pm \omega_{Q}^{\prime}$ centered on $\omega=0$ and split by $2 \omega_{Q}^{\prime}$. If a resonance offset is added to the Hamiltonian in Eq. 4.6, the doublet is centered on the offset frequency.

It has long been known that the $90_{\phi}^{\circ}-\tau-90_{\phi}^{\circ}$ two-pulse sequence can create double-quantum coherence from equilibrium magnetization in $S=1$ systems with weak quadrupolar couplings $[198,199,200] .{ }^{12}$ The conversion of magnetization to double-quantum coherence is complete with the proper choice of the delay $\tau$. This can be seen as follows. The initial thermal equilibrium difference density operator for spin $S$ is $\hat{\rho}(0) \propto \hat{S}_{z}$, and

\footnotetext{
${ }^{11}$ In Eq. 3 of Ref. [198], $\mathbf{I}_{y}=\frac{1}{\sqrt{2}}\left[\mathbf{T}_{1}^{1}+\mathbf{T}_{-1}^{1}\right]$ should be corrected to $\mathbf{I}_{y}=\frac{i}{\sqrt{2}}\left[\mathbf{T}_{1}^{1}+\mathbf{T}_{-1}^{1}\right]$.

${ }^{12}$ By "weak" it is meant that the quadrupolar coupling is small enough that quadrupolar evolution can be neglected during a "hard" radiofrequency pulse; the motionally-averaged quadrupolar frequency $\omega_{Q}^{\prime}$ in liquid crystalline media will almost always be small enough to satisfy this condition, even in low-power liquid-state NMR probes.
} 


\subsection{PRODUCTION OF N-14 DOUBLE-QUANTUM COHERENCE (DQC) IN SOLUTION

a hard $90_{y}^{\circ}$ pulse (where the $y$-direction corresponds to an rf phase of $\phi=\pi / 2$ ) converts $\hat{S}_{z}$ to $\hat{S}_{x}$. According to Eq. 4.7, the density operator at time $\tau$ is then $\hat{\rho}(\tau) \propto$ $\hat{S}_{x} \cos \omega_{Q}^{\prime} \tau+i\left(\hat{T}_{2,+1}+\hat{T}_{2,-1}\right) \sin \omega_{Q}^{\prime} \tau$, such that $\hat{\rho}\left(\tau=\pi /\left(2 \omega_{Q}^{\prime}\right)\right) \propto i\left(\hat{T}_{2,+1}+\hat{T}_{2,-1}\right)$. A second $90_{y}^{\circ}$ pulse converts this to $-i\left(\hat{T}_{2,+2}-\hat{T}_{2,-2}\right)$ [198], which in the spherical tensor operator formalism corresponds to pure double-quantum coherence, where $\hat{T}_{2, \pm 2}=\frac{1}{2} \hat{S}_{ \pm}^{2}$.

Interestingly, the double-quantum coherence terms $\hat{T}_{2, \pm 2}$ in the density operator do not evolve under the first-order quadrupolar Hamiltonian in Eq. 4.6. This means that the double quantum spectrum exhibits no quadrupolar splitting. This can be an advantage when performing $S=1$ NMR spectroscopy in oriented media because the quadrupolar-split doublets of different sites may overlap in a conventional NMR spectrum, but all these doublets are collapsed in the double-quantum spectrum so that only chemical shift information is retained.

The problem of DQC excitation becomes more complicated in the presence of a resonance offset $\delta$. In such a case the full spin- $S$ Hamiltonian is

$$
\hat{H}^{\prime} / \hbar=\delta \hat{S}_{z}+\frac{1}{3} \omega_{Q}^{\prime}\left[3 \hat{S}_{z}^{2}-S(S+1) \hat{1}\right]
$$

where the fact that the resonance offset Hamiltonian commutes with the quadrupolar Hamiltonian conveniently allows the time evolution under these two Hamiltonians to be calculated separately (sequentially). Starting from equilibrium magnetization $\hat{\rho}(0) \propto \hat{S}_{z}$, the density 


\subsection{PRODUCTION OF N-14 DOUBLE-QUANTUM COHERENCE (DQC) IN SOLUTION

operator after a $90_{y}^{\circ}-\tau-90_{y}^{\circ}$ excitation sequence is:

$$
\begin{aligned}
\hat{\rho}(\tau) \propto- & {\left[\cos \delta \tau \hat{S}_{z}-\sin \delta \tau \hat{S}_{y}\right] \cos \omega_{Q}^{\prime} \tau } \\
& \quad-\left[i \cos \delta \tau\left(\hat{T}_{2,+2}-\hat{T}_{2,-2}\right)+\sin \delta \tau\left(\hat{T}_{2,+1}-\hat{T}_{2,-1}\right)\right] \sin \omega_{Q}^{\prime} \tau
\end{aligned}
$$

In the special case that $\tau=\pi /\left(2 \omega_{Q}^{\prime}\right)$ and $\delta=0$, Eq. 4.9 reduces to $\hat{\rho}(\tau) \propto-i\left(\hat{T}_{2,+2}-\hat{T}_{2,-2}\right)$, as expected. If $\delta \neq 0$, not only is the DQC attenuated by a factor of $\cos \delta \tau$, but the presence other density operator terms can cause spectral artifacts if they end up as observable signal at the end of a pulse sequence, if proper coherence pathway selection is not employed. The resonance offset effects on DQC excitation can be removed if $\delta \tau=2 \pi n$, where $n$ is an integer. Alternatively, one can choose $\tau=\pi /\left(2 \omega_{Q}^{\prime}\right)$ and $\delta=n \pi / \tau$; however, in general both of these conditions cannot be satisfied for two different resonances (i.e., two quadrupolar sites) at the same time. A better approach may to be modify the excitation sequence to $90_{\phi}^{\circ}-\tau / 2-180^{\circ}-\tau / 2-90_{\phi}^{\circ}$, where the rf phase of the $180^{\circ}$ pulse is arbitrary. This spin echo sequence refocuses evolution under a resonance offset, but retains evolution under the first-order quadrupolar Hamiltonian. Employing this excitation sequence gives a result equivalent to setting $\delta=0$ in Eq. 4.9 .

Since the observable in NMR is transverse magnetization (by convention $\hat{S}_{+}$for quadrature-detected spectra), double quantum coherence is not directly observable because $\operatorname{Tr}\left[\hat{T}_{2, \pm 2} \hat{S}_{+}\right]=0$. However, DQC can be observed indirectly in a two-dimensional NMR experiment, after it is converted to observable (single-quantum) transverse magnetization. This conversion can be accomplished by the reverse process of the double-quantum excitation described above: DQC can be converted to (unobservable) $\hat{T}_{2, \pm 1}$ single-quantum 


\subsection{PRODUCTION OF N-14 DOUBLE-QUANTUM COHERENCE (DQC) IN SOLUTION

coherence with a $90^{\circ}$ pulse, which then evolves into observable $\hat{T}_{1, \pm 1} \propto \hat{S}_{ \pm}$single-quantum coherence (transverse magnetization) during evolution under $\hat{H}_{Q}^{\prime}$. Perhaps the simplest double-quantum 2D pulse sequence is (see Fig. 4.6):

$$
\left(90_{\phi_{1}}^{\circ}-\tau-90_{\phi_{1}}^{\circ}\right)-t_{1}-\left(90_{\phi_{2}}^{\circ}\right)-t_{2}-\operatorname{detect}\left(\phi_{\mathrm{r}}\right)
$$

where $\phi_{1}$ and $\phi_{2}$ are radiofrequency pulse phases and $\phi_{r}$ is the receiver phase. The $90_{\phi_{1}}^{\circ}-\tau-90_{\phi_{1}}^{\circ}$ DQC excitation sequence can be replaced by $90_{\phi_{1}}^{\circ}-\tau / 2-180^{\circ}-\tau / 2-90_{\phi_{1}}^{\circ}$ in order to make it robust against resonance offset effects. The optimal choice of $\tau$ for DQC creation is $\tau=\pi /\left(2 \omega_{Q}^{\prime}\right)$ can be experimentally parameterized as $\tau=1 /(2 \Delta)$, where $2 \pi \Delta=2 \omega_{Q}^{\prime}$ is the doublet splitting in the directly-detected spectrum due to the residual quadrupolar interaction. The DQC evolves during the indirect dimension time interval $t_{1}$, where it is converted by the final $90^{\circ}$ pulse into a form that evolves into transverse magnetization that is measured as a function of the direct dimension time interval $t_{2}$. Fourier transformation with respect to both time dimensions yields the double-quantum spectrum (a singlet at frequency $2 \delta$ ) in the indirect dimension projection and the single-quantum (conventional) spectrum (a doublet at $\delta \pm \omega_{Q}^{\prime}$ ) in the direct dimension projection.

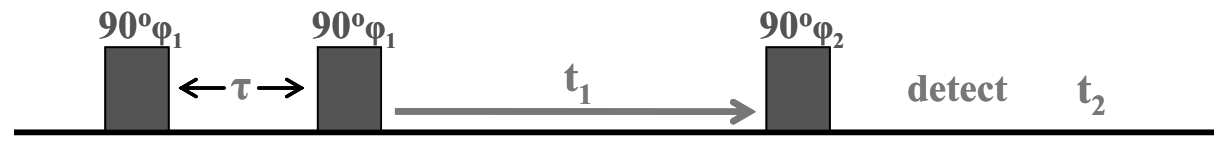

Figure 4.6: Pulse sequence for $I=1$ double-quantum spectroscopy in the presence of a weak quadrupolar coupling. A $180^{\circ}$ pulse can be inserted in the middle of the two-pulse excitation sequence to make the excitation robust against resonance offset effects.

Some experimental results on N-14 double-quantum spectroscopy of oriented phospholipid bicelles in aqueous solution at $16.45 \mathrm{~T}$ are presented in $§ 4.5 .2$. 


\subsection{PRODUCTION OF N-14 DOUBLE-QUANTUM COHERENCE (DQC) IN SOLUTION

\subsubsection{DQC through $J$-coupling to a spin-1/2 in isotropic solution}

The discussion in $§ 4.3 .1$ is applicable to oriented systems in which a residual quadrupolar interaction exists. However, it is also useful to have a way to create double quantum coherence in isotropic solution. A method of creating $S=1$ DQC in isotropic solution through the $J$-coupling to a neighboring spin- $1 / 2$ nucleus ${ }^{13}$ was invented more than twenty years ago by Yen and Weitekamp [202], with an obvious potential application being the creation of N-14 DQC in proteins through $J$-coupling to amide protons. Their pulse sequence is shown in Fig. 4.7; this 2D experiment correlates the $S=1$ double-quantum spectrum in the indirect dimension with the $I=1 / 2$ directly-detected spectrum. The basic elements of this sequence are familiar: aside from the choice of pulse phases, the excitation sequence resembles the INEPT heteronuclear polarization transfer sequence [203], and the sequence as a whole resembles the HSQC heteronuclear correlation experiment [204] used in protein NMR spectroscopy. However, the effect of this pulse sequence is very different in the $S=1, I=1 / 2 J$-coupled system. ${ }^{14}$ As is shown in Ref. [202], under the $J$-coupled heteronuclear Hamiltonian

$$
\hat{H} / \hbar=\delta_{I} \hat{I}_{z}+\delta_{S} \hat{S}_{z}+J^{\prime} \hat{I}_{z} \hat{S}_{z},
$$

\footnotetext{
${ }^{13}$ Ref. [201] describes a method capable of creating single-spin DQC in a weakly-coupled pair of spin-1 nuclei in isotropic solution.

${ }^{14}$ Ref. [205] describes a heteronuclear pulse sequence for use in anisotropic media that is structurally similar to Yen and Weitekamp's, but it includes a selective $S$-spin pulse for double-quantum excitation through the residual quadrupolar interaction, and uses a different choice of pulse phases and delays.
} 


\subsection{PRODUCTION OF N-14 DOUBLE-QUANTUM COHERENCE (DQC) IN SOLUTION

the $\left(90_{x}^{\circ}\right)_{I}-\tau / 2-\left(180_{x}^{\circ}\right)_{I},\left(180_{x}^{\circ}\right)_{S}-\tau / 2-\left(90_{x}^{\circ}\right)_{I},\left(90_{x}^{\circ}\right)_{S}$ excitation sequence creates the density operator term

$$
\hat{\rho}_{I}(\tau)=\left\{\hat{I}_{z}\left[1+\hat{S}_{y}^{2}\left(\cos J^{\prime} \tau-1\right)\right]+\hat{I}_{x} \hat{S}_{y} \sin J^{\prime} \tau\right\}
$$

from equilibrium $I$-spin magnetization $\left(\hat{\rho}_{I}(0)=c_{I} \hat{I}_{z}\right)$. The $180^{\circ}$ pulses in the center of the evolution interval $\tau$ refocus any effects due to chemical shift evolution while retaining evolution under the due to heteronuclear $J$-coupling. The $\hat{I}_{z} \hat{S}_{y}^{2}=-\frac{1}{4} \hat{I}_{z}\left[\left(\hat{S}_{+}^{2}+\hat{S}_{-}^{2}\right)-\left(\hat{S}_{+} \hat{S}_{-}+\right.\right.$ $\left.\left.\hat{S}_{-} \hat{S}_{+}\right)\right]$term contains spin- $S$ double- and zero-quantum coherences. The $-\frac{1}{4} \hat{I}_{z}\left(\hat{S}_{+} \hat{S}_{-}+\right.$ $\left.\hat{S}_{-} \hat{S}_{+}\right)$zero-quantum term is unwanted, as is the $\hat{I}_{z} z$-magnetization term and the $\hat{I}_{x} \hat{S}_{y}$ heteronuclear double- and zero-quantum term; the effects of these terms can be removed from the final spectrum by proper coherence pathway selection. Furthermore, the $\hat{I}_{x} \hat{S}_{y}$ term can be nulled by choosing $\tau=\pi / J^{\prime}=1 /(2 J)$ (where $J^{\prime}=2 \pi J$ ), which is also the condition that maximizes the $\hat{I}_{z} \hat{S}_{y}^{2}$ term that contains the $S=1$ DQC.

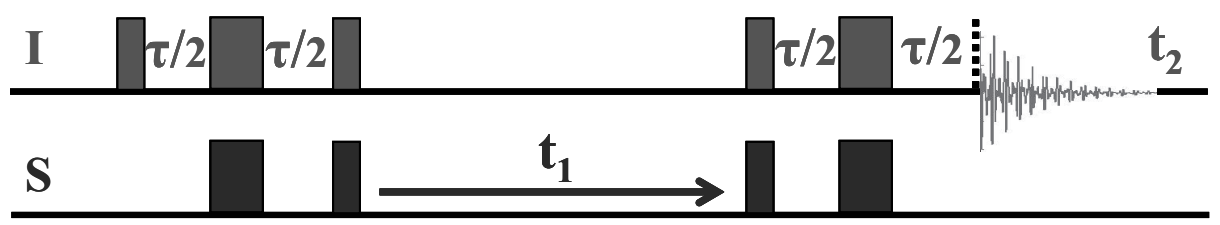

Figure 4.7: Pulse sequence for indirect detection of $S=1$ double quantum coherence via a $J$-coupled $I=1 / 2$ partner. The thin blocks represent $90^{\circ}$ pulses and the thick blocks represent $180^{\circ}$ pulses.

The spectra of systems in which the linewidths are not greater than the $J$-coupling exhibit $J$-split multiplet structures. In a system of $n$ equivalent spins $I=1 / 2$ coupled to an $S=1$ nucleus, the $I=1 / 2$ directly-detected (single-quantum) spectrum consists of a $1: 1: 1$ intensity-ratio triplet with splitting $J$. The multiplet structure of the $S=1$ 


\subsection{PRODUCTION OF N-14 DOUBLE-QUANTUM COHERENCE (DQC) IN SOLUTION}

double-quantum spectrum varies according to the number $n$ of coupled spin partners. It is important to note that the DQ multiplet structure differs in the Yen and Weitekamp heteronuclear experiment from that which would be observed if DQC was created directly by, say, a residual quadrupolar interaction; this is because the DQC in the heteronuclear experiment has the form $\hat{I}_{z} \hat{S}_{ \pm}^{2}$, whereas in the single $S=1$ system the DQC has the form $\hat{S}_{ \pm}^{2}$, and these operators have different matrix elements. In the Yen and Weitekamp experiment, the $\hat{I}_{z} \hat{S}_{ \pm}^{2}$-type DQ spectrum in the $S-I$ system corresponds to a $1: 1$ intensity-ratio doublet of splitting $2 J$; in the $S I_{2}$ system the DQ spectrum corresponds to a 1:0:1 triplet of splitting $2 J$; and in the $S I_{4}$ system the DQ spectrum corresponds to a $1: 1: 0: 1: 1$ intensity-ratio quintet of splitting $2 J$ [202]. In these three systems, the $S=1$ directly-detected spectrum corresponds to a $1: 1,1: 2: 1$, or $1: 4: 6: 4: 1$ intensity-ratio multiplet, respectively, of splitting $J$.

The result of a nitrogen-proton DQ-SQ correlation experiment at $7.05 \mathrm{~T}$ is presented in $\S 4.5 .1$ in the ammonium ion $\left({ }^{+} \mathrm{NH}_{4}\right)$ system, which is in the extreme narrowing limit.

\subsubsection{DQC through cross-correlation effects outside of the extreme nar- rowing regime}

As was discussed in $§ 1.5 .4$, outside of the extreme narrowing regime it is sometimes possible to observe dynamic frequency shifts (DFSs) and multiexponential relaxation behavior in certain spin systems. The dynamic frequency shifts are discussed first. These shifts can be thought of as arising from coherent evolution due to incomplete motional averaging of the cross (interference) terms in the second-order (Zeeman-truncated) solid- 


\subsection{PRODUCTION OF N-14 DOUBLE-QUANTUM COHERENCE (DQC) IN SOLUTION

state Hamiltonian. The largest of these effects involve correlation with the quadrupolar interaction, assuming the quadrupolar coupling is large; Wi et al. have considered in some detail the cases of quadrupolar-CSA cross-correlation $[37,206]$ and quadrupolar-dipolar cross-correlation $[207,208]$ in the solid state (particularly under magic-angle spinning conditions). The motionally averaged solution-state Hamiltonians may be deduced from the expressions for the dynamic frequency shifts in Refs. [88] and [89], ${ }^{15}$ and compared to the expressions of Wi et al. The dynamic shift Hamiltonians in isotropic solution are given for the cases of quadrupolar-quadrupolar autocorrelation on spin $S$, quadrupolar-CSA crosscorrelation on spin $S$, and quadrupolar-dipolar cross-correlation in the $I-S$ heteronuclear system, where the quadrupolar coupling is on spin $S$ and the dipolar coupling is between spins $S$ and $I$ :

$$
\begin{aligned}
& \overline{\hat{H}}_{Q_{S}, Q_{S}}^{(2)} / \hbar= \frac{3}{20} \frac{\omega_{Q_{S}}^{2}}{\omega_{0}^{S}}\left\{L_{Q_{S}, Q_{S}}\left(\omega_{0}^{S}\right)\left[(4 S(S+1)-1) \hat{S}_{z}-8 \hat{S}_{z}^{3}\right]\right. \\
&\left.-L_{Q_{S}, Q_{S}}\left(2 \omega_{0}^{S}\right)\left[(2 S(S+1)-1) \hat{S}_{z}-2 \hat{S}_{z}^{3}\right]\right\} \\
& \hat{\hat{H}}_{Q_{S}, C S A_{S}}^{(2)} / \hbar=-\frac{1}{5} \frac{\omega_{Q_{S}} \omega_{C S A_{S}}}{\omega_{0}^{S}} L_{Q_{S}, C S A_{S}}\left(\omega_{0}^{S}\right)\left[3 \hat{S}_{z}^{2}-S(S+1) \hat{1}_{S}\right] \\
& \overline{\hat{H}}_{Q_{S}, D_{I S}}^{(2)} / \hbar=-\frac{3}{5} \frac{\omega_{Q_{S}} \omega_{D_{I S}}}{\omega_{0}^{S}} L_{Q_{S}, D_{I S}}\left(\omega_{0}^{S}\right)\left[3 \hat{S}_{z}^{2}-S(S+1) \hat{1}_{S}\right] \hat{I}_{z}
\end{aligned}
$$

where $\omega_{Q_{S}}=\left(2 \pi C_{Q_{S}}\right) /[2 S(2 S-1)], \omega_{C S A_{S}}=\Delta \sigma_{S} \omega_{0}^{S}, \omega_{D_{I S}}=-\frac{\mu_{0}}{4 \pi} \frac{\gamma_{I} \gamma_{S} \hbar}{r_{I S}^{3}}$, and $\omega_{0}^{S}=\gamma_{S} B_{0}$. Axial symmetry has been assumed for the quadrupolar and CSA interactions $\left(\eta_{Q_{S}}=0\right.$, $\left.\eta_{C S A_{S}}=0\right)$. The dimensionless function $L_{\xi, \xi^{\prime}}(\omega)$ is related to the imaginary part of the spectral density and reaches an asymptotic value as the rotational correlation time $\tau_{c} \rightarrow \infty$,

\footnotetext{
${ }^{15}$ Refs. [88] and [89] incorrectly omit factors of $\omega^{2} \tau^{2}$ from the numerators of their definitions of $L(\omega)$, the imaginary part of the spectral density function.
} 


\subsection{PRODUCTION OF N-14 DOUBLE-QUANTUM COHERENCE (DQC) IN SOLUTION

which recovers the orientationally-averaged solid-state Hamiltonian. In the case of a rigid, spherical molecule, $L_{\xi, \xi^{\prime}}(\omega)=P_{2}\left(\cos \theta_{\xi, \xi^{\prime}}\right)\left[\omega^{2} \tau_{c}^{2} /\left(1+\omega^{2} \tau_{c}^{2}\right)\right]$, where $P_{2}(\cos \theta)=\frac{1}{2}\left(3 \cos ^{2} \theta-\right.$ 1 ) is the second-rank Legendre polynomial, and $\theta_{\xi, \xi^{\prime}}$ is the angle between the principal axes of interactions $\xi$ and $\xi^{\prime}\left(\theta_{\xi, \xi}=0\right.$ for autocorrelated interactions). In the case of an amide N-14 at $B_{0}=16.45 \mathrm{~T}$ with $C_{Q}=-3.2 \mathrm{MHz}, r_{N H}=1.02 \AA$, and $\Delta \sigma_{N 14}=$ $-170 \mathrm{ppm}$, the relevant parameters are $\omega_{Q_{N}}=2 \pi \times-1.6 \mathrm{MHz}, \omega_{C S A_{N}}=2 \pi \times-8.61$ $\mathrm{kHz}, \omega_{D_{N H}}=2 \pi \times-8.18 \mathrm{kHz}$, and $\omega_{0}^{N}=2 \pi \times 50.6 \mathrm{MHz}$. Thus in this type of system the quadrupolar autocorrelated dynamic frequency shift can approach the $10 \mathrm{kHz}$ range, whereas the quadrupolar-CSA and quadrupolar-dipolar cross-correlated dynamic frequency shifts are at most in the tens to hundreds of $\mathrm{Hz}$ range. It is interesting to note that the asymptotic values of the quadrupolar autocorrelated and quadrupolar-dipolar crosscorrelated DFSs decrease like $1 / B_{0}$ with increasing magnetic field strength, whereas the quadrupolar-CSA cross-correlated DFS is independent of $B_{0}$ (due to the fact that $\omega_{C S A} \propto$ $\left.B_{0}\right)$.

The next question is whether any of these dynamic frequency shift Hamiltonians can be used to create double-quantum coherence in the $S=1$ system. It is well-known that rotation due to a hard radiofrequency pulse can change only the coherence order of a density operator term, not its tensor rank, and the creation of $|q|>1$ multiple-quantum coherence $\hat{T}_{k, q}$ on spin $S$ necessarily requires converting rank $k=1$ magnetization to some higher rank tensor term. The usual strategy in NMR for creating multiple-quantum coher-

ence in the spin- $S$ system is to create transverse magnetization $\hat{S}_{ \pm} \propto \hat{T}_{1, \pm 1}$ from equilibrium magnetization via a hard rf pulse, which then evolves during free precession under an appro- 


\subsection{PRODUCTION OF N-14 DOUBLE-QUANTUM COHERENCE (DQC) IN SOLUTION

priate Zeeman-truncated Hamiltonian into $\hat{T}_{k, \pm 1}$ terms (conserving the coherence order), which then may be converted to $\hat{T}_{k, q}$ terms via a second pulse. ${ }^{16}$ It should be considered whether the Hamiltonians in Eqs. 4.13, 4.14, and 4.15 are capable of creating higher-rank spin- $S$ tensor terms under free precession. In general, all of them can be used to create spin- $S$ multiple-quantum coherence given the appropriate pulse sequence; in fact, the quadrupolar-CSA DFS Hamiltonian has the same spin-operator dependence as the firstorder quadrupolar Hamiltonian used in $\S 4.3 .1$. However, in the special case of $S=1$, the autocorrelated quadrupolar DFS Hamiltonian in Eq. 4.13 is proportional to $\hat{S}_{z}$, since $\hat{S}_{z}^{3}=\hat{S}_{z}$ for $S=1$. Evolution under such a Hamiltonian corresponds to a $z$-rotation of spin- $S$, which is not capable of changing the tensor rank of the density operator terms; in fact, the system evolves under this Hamiltonian in the same way as it would under a chemical shift. ${ }^{17}$ It is unfortunate that the autocorrelated quadrupolar DFS Hamiltonian cannot be used in $S=1$ systems, because it is relatively large and in general the larger the Hamiltonian the better, in order to be able to create multiple-quantum coherence faster than the relaxation processes can take effect. The remaining candidates appear to be the quadrupolar-CSA and quadrupolar-dipolar DFS Hamiltonians, preferably well into the slow tumbling regime where the DFSs are large.

The other possibility for DQC creation in the slow tumbling regime is to utilize the quadrupolar-dominated multiexponential relaxation phenomena to create higher-rank

\footnotetext{
${ }^{16}$ As was seen in in $\S 4.3 .2$, in a two-spin $I-S$ system it is possible to create $S$-spin multiple quantum coherence starting with $I$ magnetization instead, but a Hamiltonian is still necessary which can create higher-rank spin- $S$ tensor terms in the density operator upon free precession.

${ }^{17}$ The fact that both the second-order quadrupolar Hamiltonian and the chemical shift Hamiltonian are proportional to $\hat{S}_{z}$ is what makes using spin manipulations (i.e., rf pulses) to help to remove the anisotropic second-order quadrupolar broadening in solids without removing the chemical shift seems to be an impossible task. However, it is possible to implement DAS- and DOR-type spatial manipulations.
} 


\subsection{QUADRUPOLAR RELAXATION CONCERNS FOR DQC CREATION IN LARGE- $C_{Q}$ SYSTEMS

density operator terms. However, magnetization in $S=1$ systems is known to relax monoexponentially even outside of the extreme narrowing regime [103], so the possibility of DQC creation through this type of mechanism is precluded, at least for an isolated $S=1$ system.

\subsection{Quadrupolar relaxation concerns for DQC creation in large- $C_{Q}$ systems}

The discussion of $\S 4.2$ made it clear that the major obstacle to the successful implementation of double-quantum line narrowing in amide N-14 systems is the absolute size of the relaxation rates when the quadrupolar coupling is very large. The fast quadrupolar relaxation is also the major obstacle to creating $\mathrm{N}-14$ double quantum coherence, as will be discussed below for the cases of DQC creation through the dynamic frequency shift Hamiltonians, $J$-coupling to neighboring nuclei, or residual quadrupolar interaction in anisotropic solution.

The effects of relaxation on DQC creation through the cross-correlated dynamic frequency shift terms of $\S 4.3 .3$ will be considered first, in particular the example of the quadrupolar-CSA cross-correlation. The motionally-averaged Hamiltonian that dictates the cross-correlated dynamics is given in Eq. 4.14. This Hamiltonian has the same spin-operator dependence as the first-order quadrupolar Hamiltonian in Eq. 4.6; therefore, DQC can be created via the excitation pulse sequence in $\S 4.3 .1$. As can be seen from Eq. 4.7 evolution

of transverse magnetization under this type of Hamiltonian creates $\hat{T}_{2, \pm 1}$ density operator terms that can be converted into DQC via rf pulses. A comparison of the Hamiltonians in 


\subsection{QUADRUPOLAR RELAXATION CONCERNS FOR DQC CREATION IN LARGE- $C_{Q}$ SYSTEMS

Eqs. 4.6 and 4.14 indicates that the $\hat{T}_{2, \pm 1}$ density operator terms will develop sinusoidally under the quadrupolar-CSA DFS Hamiltonian with a frequency

$$
\omega_{Q, C S A}^{(2)}=-\frac{3}{5} \omega_{Q} \omega_{C S A} P_{2}\left(\cos \theta_{Q, C S A}\right) \frac{\omega_{0} \tau_{c}^{2}}{1+\omega_{0}^{2} \tau_{c}^{2}},
$$

where the usual conditions of a rigid spherical molecule in isotropic solution with axiallysymmetric quadrupolar and CSA interactions have been assumed. By comparison, the relaxation rate of the $\hat{T}_{2, \pm}$ density operator terms under the same conditions is given by Eqs. 10, 35, and 63-65 of Ref. [103]:

$$
\begin{aligned}
\Gamma_{2, \pm 1}= & \Gamma_{2, \pm 1}^{Q}+\Gamma_{2, \pm 1}^{C S A}+\Gamma_{2, \pm 1}^{Q, C S A} \\
\simeq & \frac{3}{80}\left(2 \pi C_{Q}\right)^{2}\left[3 J(0)+J\left(\omega_{0}\right)+2 J\left(2 \omega_{0}\right)\right]+\frac{1}{30}\left(\Delta \sigma \omega_{0}\right)^{2}\left[\frac{8}{3} J(0)+10 J\left(\omega_{0}\right)\right] \\
& \quad+\frac{1}{5}\left(2 \pi C_{Q}\right)\left(\Delta \sigma \omega_{0}\right) P_{2}\left(\cos \theta_{Q, C S A}\right) J(0)
\end{aligned}
$$

where $J(\omega)=\tau_{c} /\left(1+\omega^{2} \tau_{c}^{2}\right)$ is related to the real part of the spectral density function, and the quadrupolar-CSA cross-correlated relaxation term $\Gamma_{2, \pm 1}^{Q, C S A}$ has been approximated by its adiabatic component (which is expected to dominate in the slow tumbling regime). Obviously, if the rate $\Gamma_{2, \pm 1}$ in Eq. 4.17 that the $\hat{T}_{2, \pm 1}$ density operator terms exponentially relax to zero is greater than the frequency $\left|\omega_{Q, C S A}^{(2)}\right|$ in Eq. 4.16 at which these terms develop under the cross-correlated Hamiltonian, then trying to create DQC via this mechanism is a losing battle. These two quantities are plotted in Fig. 4.8 for $C_{Q}=-3.2 \mathrm{MHz}, \eta_{Q}=0$, $\Delta \sigma=-170 \mathrm{ppm}, \eta_{C S A}=0, \theta_{Q, C S A}=0$, and $\omega_{0} / 2 \pi=50.6 \mathrm{MHz}$. The relaxation in this case is dominated completely by the $\Gamma_{2, \pm 1}^{Q}$ quadrupolar term, and as can be seen, the 


\subsection{QUADRUPOLAR RELAXATION CONCERNS FOR DQC CREATION

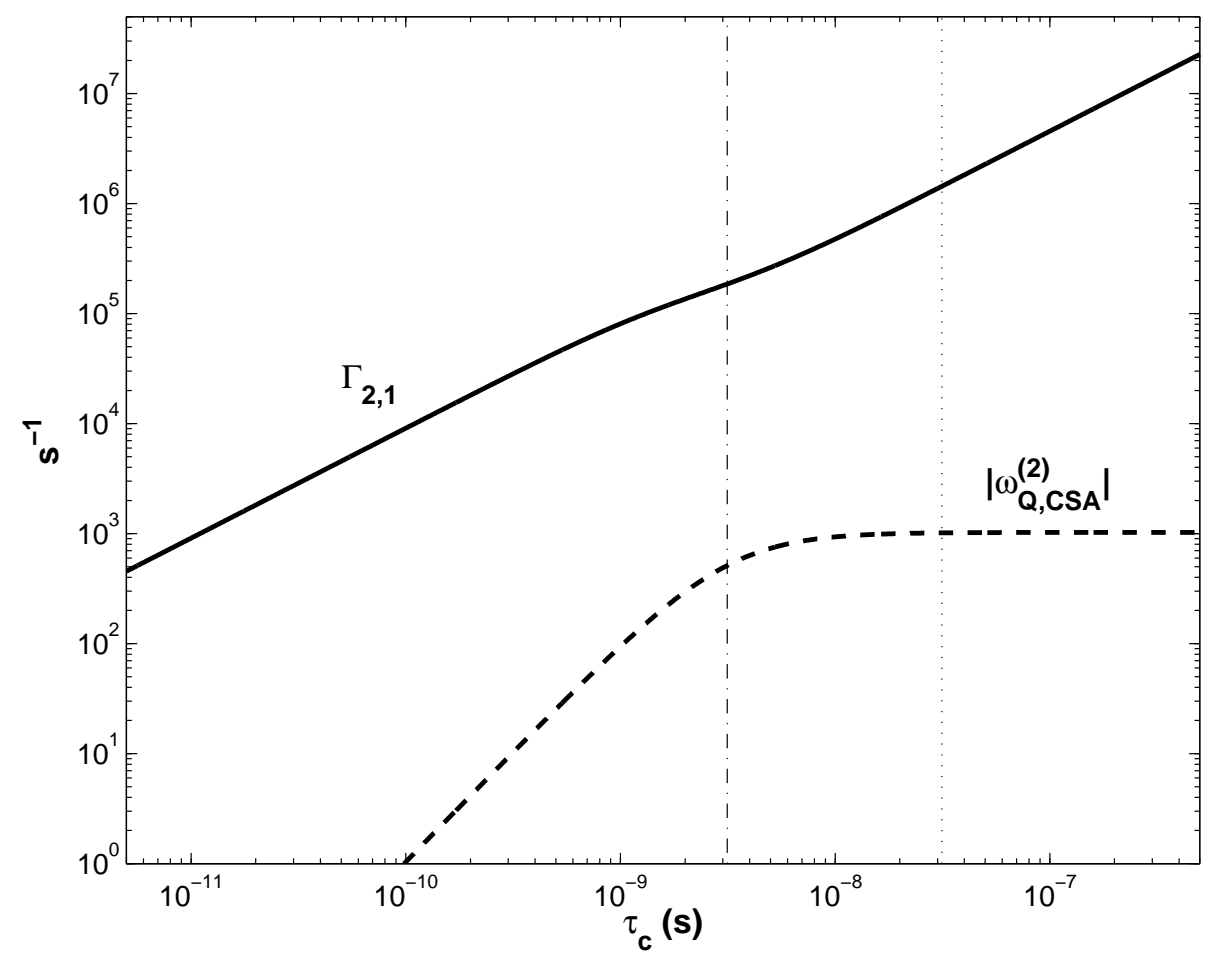

Figure 4.8: Calculated N-14 relaxation rate and quadrupolar-CSA dynamic frequency shift evolution frequency of the $\hat{T}_{2, \pm 1}$ density operator terms as a function of rotational correlation time for a rigid spherical molecule in isotropic solution at $16.45 \mathrm{~T}$. The quadrupolar parameters are $C_{Q}=-3.2 \mathrm{MHz}$ and $\eta_{Q}=0$, and the CSA parameters are $\Delta \sigma=-170 \mathrm{ppm}$ and $\eta_{C S A}=0$, with a Larmor frequency of $\omega_{0} / 2 \pi=50.6 \mathrm{MHz}$. The quadrupolar and CSA interactions are assumed to be coaxial. The vertical dash-dot line marks $\tau_{c}=\left|\omega_{0}\right|^{-1}$, and the vertical dotted line marks $\tau_{c}=10\left|\omega_{0}\right|^{-1}$.

quadrupolar relaxation overwhelms the dynamic frequency shift for all values of $\tau_{c}$, with the smallest difference between the relaxation rate and the DFS occurring near $\omega_{0} \tau_{c} \sim 1$ [6, §X.III.B]. It is perhaps not unexpected that the quadrupolar relaxation dominates the DFS, particularly in the slow tumbling regime where the relaxation is governed almost solely by the adiabatic quadrupolar term which goes as $C_{Q}^{2} \tau_{c}$, whereas the DFS goes as $C_{Q}$ scaled down by a factor of $\omega_{C S A} / \omega_{0}$. There is a simple way to determine experimentally for a given $\tau_{c}$ whether the quadrupolar-dominated relaxation of the $\hat{T}_{2,1}$ terms is faster than their creation through the quadrupolar-CSA DFS. The $R_{2, \pm 1}$ quadrupolar relaxation 


\subsection{QUADRUPOLAR RELAXATION CONCERNS FOR DQC CREATION IN LARGE- $C_{Q}$ SYSTEMS

rate coincides with the $R_{1, \pm 1}=1 / T_{2}$ transverse relaxation rate in the slow tumbling limit (and is less than $1 / T_{2}$ in the extreme narrowing limit) [103], and $1 / T_{2}$ is of the order of the linewidth in the directly-detected spectrum. The DFS-induced splitting of the directlydetected spectrum as given by the Hamiltonian in Eq. 4.14 is $\omega_{Q, C S A}^{(2)} / \pi$. Therefore, by equating the $\Gamma_{2, \pm 1}$ relaxation rate with $1 / T_{2}$ with the observed linewidth, one can estimate that $\left|\omega_{Q, C S A}^{(2)}\right|$ is comparable to or greater than the $\Gamma_{2, \pm 1}$ relaxation rate if the doublet splitting is large enough to be observed (i.e., if the splitting is at least of the order of the linewidth). Similar arguments hold for assessing the size of the quadrupolar-dipolar cross-correlated DFS in two-spin systems.

Next to be considered is the influence of spin relaxation on the creation of $S=1$ DQC via $J$-coupling to an $I=1 / 2$ neighbor, as was discussed in $\S 4.3 .2$. A $-90^{\circ}$ rotation of the $I-S$ system about the $x$-axis after the DQC excitation described in Eq. 4.12 shows that following the $\left(90_{x}^{\circ}\right)_{I}-\tau / 2-\left(180_{x}^{\circ}\right)_{I},\left(180_{x}^{\circ}\right)_{S}-\tau / 2$ portion of the DQC excitation sequence the spin- $I$ density operator is $\hat{\rho}_{I}\left(\tau_{-}\right)=\left\{\hat{I}_{y}\left[1+\hat{S}_{z}^{2}\left(\cos J^{\prime} \tau-1\right)\right]-\hat{I}_{x} \hat{S}_{z} \sin J^{\prime} \tau\right\}$. The density operator term $\hat{I}_{y} \hat{S}_{z}^{2}$ equals $\frac{2}{3} \hat{I}_{y}-\frac{i}{6}\left(\hat{I}_{+} \hat{T}_{2,0}^{S}-\hat{I}_{-} \hat{T}_{2,0}^{S}\right)$, where $\hat{T}_{2,0}^{S}=3 \hat{S}_{z}^{2}-S(S+1) \hat{1}_{S}$. It is the $\hat{I}_{ \pm} \hat{T}_{2,0}^{S}$ terms that are converted into spin-S DQC via the final pair of heteronuclear $90_{x}^{\circ}$ pulses. Ref. [189] estimates the relaxation rate of the $\hat{I}_{ \pm} \hat{T}_{2,0}^{S}$ terms to be:

$$
\Gamma_{I_{z} T_{2,0}^{S}} \simeq 12 \cdot \frac{3}{160}\left(2 \pi C_{Q}\right)^{2} \frac{\tau_{c}}{1+\omega_{0}^{S} 2 \tau_{c}^{2}}
$$

in the limit that quadrupolar interaction is much greater than the CSA, dipolar, and $J$ coupling interactions. Following the argument made about the creation of DQC through DFS evolution, the relaxation rate of the $\hat{I}_{ \pm} \hat{T}_{2,0}^{S}$ density operator terms given by Eq. 4.18 


\subsection{QUADRUPOLAR RELAXATION CONCERNS FOR DQC CREATION IN LARGE- $C_{Q}$ SYSTEMS

at $16.45 \mathrm{~T}$ is plotted in Fig. 4.9 for an amide $\mathrm{N}-14-\mathrm{H}-1$ pair along with the frequency $J^{\prime}=2 \pi J$ that characterizes the development of these terms. Eq. 4.18 contains no adiabatic

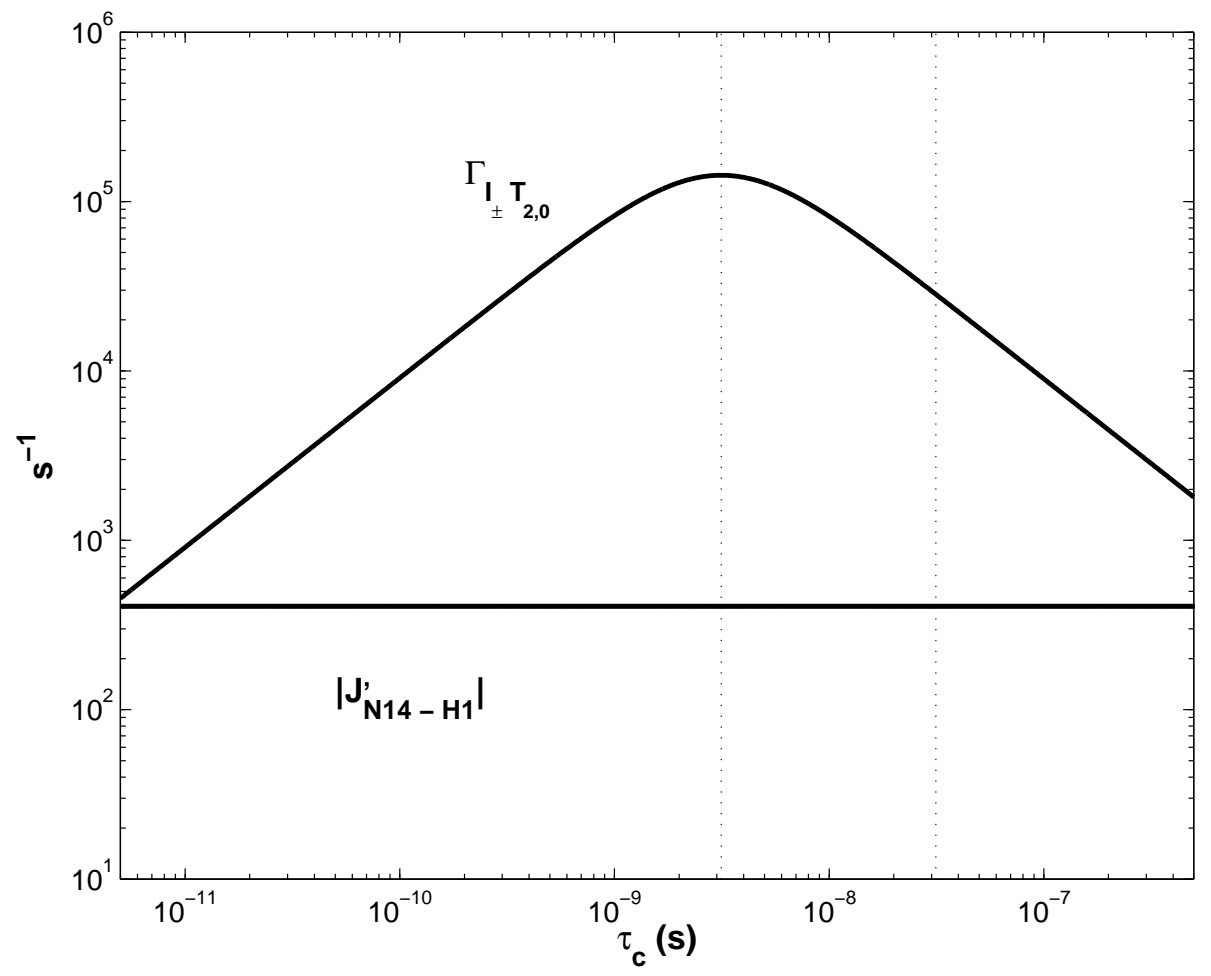

Figure 4.9: Calculated quadrupolar relaxation rate of the $\hat{I}_{ \pm} \hat{T}_{2,0}^{S}$ density operator terms in the amide N-14-H-1 $(I-S)$ system as a function of rotational correlation time for a rigid spherical molecule in isotropic solution at $16.45 \mathrm{~T}$, plus the $\mathrm{N}-\mathrm{H} J$-coupling frequency. The quadrupolar parameters are $C_{Q}=-3.2 \mathrm{MHz}$ and $\eta_{Q}=0$, with Larmor frequency of $\omega_{0} / 2 \pi=50.6 \mathrm{MHz}$. The N-14-H-1 $J$-coupling frequency is $\left|J_{N 14-H 1}^{\prime}\right|=2 \pi\left|J_{N 14-H 1}\right|=$ $2 \pi \times 65 \mathrm{~Hz}$. The vertical dotted lines mark $\tau_{c}=\left|\omega_{0}^{N 14}\right|^{-1}$ (left) and $\tau_{c}=10\left|\omega_{0}^{N 14}\right|^{-1}$ (right).

contribution in this approximation, so the relaxation rate decreases away from the $\omega_{0}^{N 14} \tau_{c} \sim$ 1 region, but with an amide $\mathrm{N}-14 C_{Q}$ of more than $3 \mathrm{MHz}$, the difficulty of using the nitrogen-proton $J$-coupling to create N-14 DQC for any reasonable value of $\tau_{c}$ is apparent.

Finally, the case of DQC excitation in anisotropic media is considered. According to Eq. 4.6, the Hamiltonian used for DQC creation in an oriented system is just the orientationally-averaged first-order quadrupolar Hamiltonian, which has the form as the 


\subsection{QUADRUPOLAR RELAXATION CONCERNS FOR DQC CREATION IN LARGE- $C_{Q}$ SYSTEMS

quadrupolar-CSA dynamic shift Hamiltonian in Eq. 4.14. Therefore, the effect of relaxation on DQC excitation is much the same as in the dynamic frequency shift case. ${ }^{18}$ However, the quadrupolar Hamiltonian in an anisotropic system can be quite large, depending on the degree of alignment of the medium. The relevant quantities to be compared are the quadrupolar evolution frequency $\omega_{Q}^{\prime}$ in Eq. 4.6 with the $\hat{T}_{2, \pm 1}$ relaxation rate in Eq. 4.17, assuming only quadrupolar and CSA relaxation. The quantity $\omega_{Q}^{\prime}$ can be determined from the $S=1$ doublet splitting $\Delta=\omega_{Q}^{\prime} / \pi$. If the quadrupolar linewidths are small enough that the doublet is resolved, it is likely that DQC excitation is possible. However, it should be pointed out that in protein NMR spectroscopy the concentration of the protein is so low $(\sim 1 \mathrm{mM})$ and the gyromagnetic ratio of $\mathrm{N}-14$ is so low that any practical experiment would still require the $\mathrm{N}-14 \mathrm{DQC}$ that develops in the indirect dimension of a $2 \mathrm{D}$ experiment to be transferred to $\mathrm{H}-1$ nuclei in the direct dimension for more sensitive detection [7, §8.5.1]. If that transfer is to be accomplished through the $J$-coupling, the same types of problems arise as were discussed previously: e.g. the $\hat{I}_{ \pm} \hat{T}_{2,0}^{S}$ density operator terms may relax before the transfer to spin- $I$ transverse magnetization is complete. It is possible, however, that the residual dipolar couplings in an aligned medium may be somewhat larger than the $J$-couplings, so the transfer may be able to proceed slightly faster.

\footnotetext{
${ }^{18}$ Note that the relaxation rates in an oriented medium differ somewhat from the rates in isotropic solution.
} 


\subsection{Experimental data}

\subsubsection{N-14 DQ-H-1 SQ correlation spectroscopy via the J-coupling}

N-14 DQ NMR experiments were performed on Yen and Weitekamp's [202] test sample in order to test the pulse sequence in Fig. 4.7. The sample was $8 \mathrm{M}$ aqueous ammonium nitrate $\left(\mathrm{NH}_{4} \mathrm{NO}_{3}(\mathrm{aq})\right)$ acidified to $\mathrm{pH} \sim 1$ with concentrated hydrochloric acid to ensure that the ${ }^{+} \mathrm{NH}_{4}$ remained in its protonated form. There are two resonances in the $\mathrm{N}-14$ spectrum, one due to ${ }^{+} \mathrm{NH}_{4}$ and the other due to $\mathrm{NO}_{3}^{-}$; only the ${ }^{+} \mathrm{NH}_{4}$ resonance was studied here because the N-14-H-1 $J$-coupling was used. The experiments were performed at 7.05 Tesla on a Varian Unity Inova spectrometer using a Varian 5-mm H-X broadband solution-state NMR probe. The $\mathrm{H}-1$ frequency was $299.78 \mathrm{MHz}$ and the N-14 frequency was 21.65 MHz. The pulse sequence in Fig. 4.7 was modified to include pulsed field gradient selection of the N-14 double quantum coherence. The experimental spectrum is shown in Fig. 4.10. As expected, the N-14 DQ spectrum showed a 1:1:0:1:1 intensity ratio $J$-split quintet centered at twice the frequency of the directly-detected ${ }^{+} \mathrm{NH}_{4} \mathrm{~N}-14$ resonance. The $J$-splitting was $\sim 100 \mathrm{~Hz}$. The $\mathrm{H}-1$ directly-detected spectrum showed a 1:1:1 intensity ratio $J$-split triplet. The N-14 line was on the order of $\mathrm{Hz}$; the tetrahedral symmetry of the ${ }^{+} \mathrm{NH}_{4}$ ion results in a small electric field gradient at the position of the nitrogen nucleus and hence in a small quadrupolar coupling. The quadrupolar relaxation is therefore slow. This system is expected to be well within the extreme narrowing regime: no line narrowing in the double quantum was observed, and none was expected.

The N-14 DQ-H1 SQ correlation experiment was tried using other samples to see how well the method could be extended to systems with faster quadrupolar relaxation 


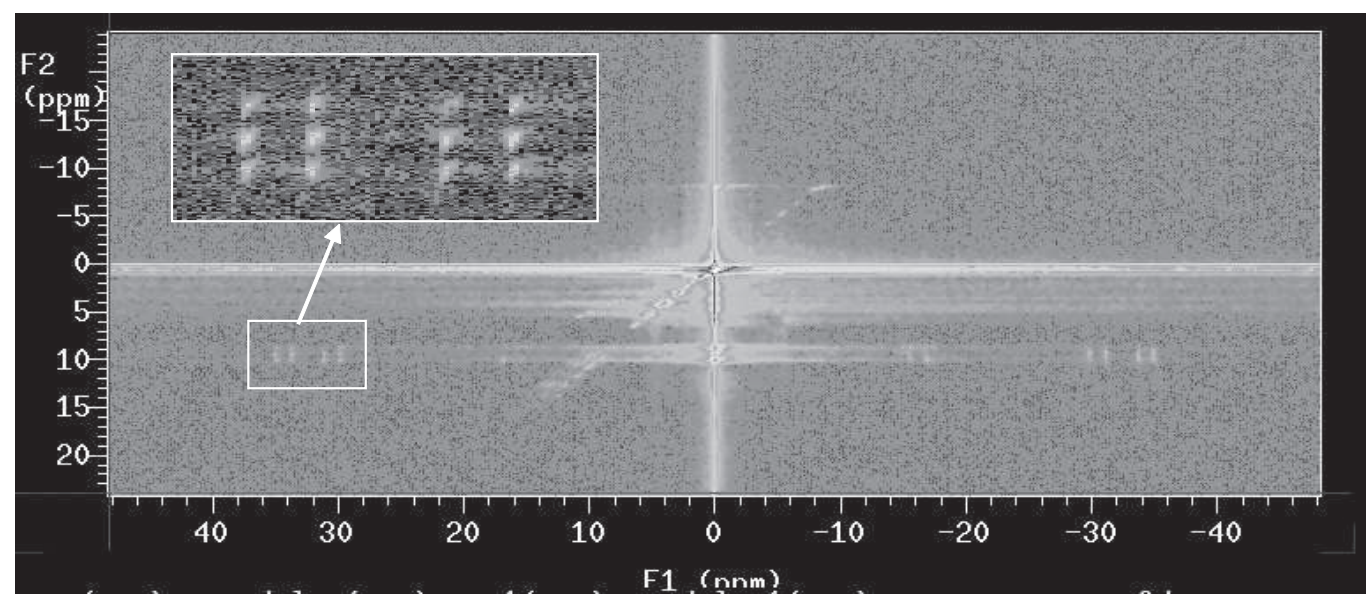

Figure 4.10: N-14 double quantum spectrum of ${ }^{+} \mathrm{NH}_{4}(\mathrm{aq})$ in ammonium nitrate detected at $7.05 \mathrm{~T}$ on $\mathrm{H}-1$ through the $J$-coupling. The vertical axis is the direct ( $\mathrm{H}-1$ single quantum) dimension and the horizontal axis is the indirect (N-14 double quantum) dimension. The ppm scales on both axes are mislabeled; see below for the correct spectral widths in both dimensions. The pulse sequence in Fig. 4.7 was used along with pulsed field gradient coherence pathway selection. A $z$-gradient pulse of strength $G=0.42 \mathrm{G} / \mathrm{cm}$ was applied for $\tau_{G 1}=6.922 \mathrm{~ms}$ at the end of the $t_{1}$ interval just before the simultaneous $90^{\circ}$ pulses; a $z$-gradient pulse of strength $G=0.42 \mathrm{G} / \mathrm{cm}$ was applied for $\tau_{G 2}=1 \mathrm{~ms}$ just after the simultaneous $90^{\circ}$ pulses. The use of equally-strong gradient pulses with durations in the ratio $\tau_{G 1} / \tau_{G 2}=6.922=\gamma_{H 1} /\left(2 \gamma_{N 14}\right)$ ensured selection of the N-14 DQC in the indirect dimension. A total of 8000 points were taken in the direct dimension with a spectral window of $4000 \mathrm{~Hz}$ and an acquisition time of $1 \mathrm{~s}$. A total of 2048 points were taken in the indirect dimension with a spectral window of $8000 \mathrm{~Hz} ; 8$ acquisitions were taken per indirect point. The $\mathrm{H}-190^{\circ}$ pulse was $55 \mu$ s long and the N-14 $90^{\circ}$ pulse was $35 \mu$ s long. A recycle delay of $1 \mathrm{~s}$ was used along with a $4 \mathrm{~ms}, 22 \mathrm{G} / \mathrm{cm}$ spoiler $z$-gradient pulse before the recycle delay to dephase any remaining proton transverse magnetization before the start of the next experiment.

rates. The only successful attempt was in neat formamide $\left(\mathrm{HCONH}_{2}\right)$, which exhibited a weak double quantum signal. The N-14 linewidth in this system was $\sim 250 \mathrm{~Hz}$ and no $J$-structure was resolved, so it is expected that this system is near the limit of practicality for this experiment. No line narrowing in the double quantum dimension was observed. Formamide reorients in the extreme narrowing regime. The DQ-SQ correlation experiment was also attempted in methyl formamide and in piperidine; no N-14 double quantum signal 
was observed in these experiments.

\subsubsection{N-14 DQ-SQ correlation spectroscopy in oriented bicelles}

Nitrogen-14 double-quantum spectroscopy was performed in a system of magneticallyoriented phosphocholine lipid bicelles. Bicelles, short for "bilayered micelles", are thought to be discoidal bilayers that self-assemble in aqueous solution from mixtures of short- and longchain polar lipids at the proper temperature and concentration. The polar (hydrophilic) head groups of the lipids orient toward the outside of the disc and the hydrophobic chains are directed inward. Phospholipid bicelles have been well-characterized by NMR [209, 210, 211]. A magnetic field such as the one in an NMR magnet can be used to align the bicelles into an oriented liquid crystalline phase. Under normal conditions the discs orient themselves so that the normal vectors of their faces are perpendicular to the magnetic field.

The bicelles used in this work were a $25 \%(\mathrm{w} / \mathrm{v})$ solution of dimyristoylphosphatidylcholine (DMPC) and dihexanoylphosphatidylcholine (DHPC) lipids in $\mathrm{D}_{2} \mathrm{O}$ in a concentration ratio of $q=[\mathrm{DMPC}]:[\mathrm{DHPC}]=2.75$. The general structure of these lipids is:

$$
\left[\mathrm{R}-(\mathrm{C}=\mathrm{O})-\mathrm{O}-\mathrm{CH}_{2}\right]-[\mathrm{CH}-\mathrm{O}-(\mathrm{C}=\mathrm{O})-\mathrm{R}] \mathrm{CH}_{2}-\left[\mathrm{O}-\left(\mathrm{PO}_{2}^{-}\right)-\mathrm{O}\right]-(\mathrm{CH} 2)_{2}-\mathrm{N}^{+}-\left(\mathrm{CH}_{3}\right)_{3}
$$

The group $-\mathrm{R}$ is the long alkyl chain $-\left(\mathrm{CH}_{2}\right)_{12}-\mathrm{CH}_{3}$ in the case of DMPC and is the short alkyl chain $-\left(\mathrm{CH}_{2}\right)_{4}-\mathrm{CH}_{3}$ in the case of DHPC. The long-chain lipids form the faces of the bicelles and the short-chain lipids cap the edges. The size of the bicelle can be adjusted by varying the concentration ratio of the two lipids.

The polar heads of the lipids are phosphocholine groups, which in turn contain an ethyltrimethylammonium group. The nitrogen atom in this group is situated in a fairly sym- 
metric environment where the electric field gradients are relatively small; consequently the quadrupolar coupling is not very large. The N-14 quadrupolar coupling constant has been estimated in an NMR relaxation study to be $\approx 100 \mathrm{kHz}$ in similar $n$-alkylmethylammonium lipids [212]. This is of the same order of magnitude (perhaps somewhat smaller) than typical quadrupolar coupling constants of H-2 nuclei; since deuterium double quantum spectroscopy has been performed long ago, DQ spectroscopy in the N-14 bicellar system should have similar success. Note that the bicelles are large, $\approx 40 \mathrm{~nm}$ in diameter and $\approx 4 \mathrm{~nm}$ thick, so they should tumble with a global rotational correlation time that is outside of the extreme narrowing regime. However, the bilayers are fluid and internal motion about the lipid axis is also possible.

The DHPC:DMPC bicelles used in this study were reconstituted from a previous mixture that had been frozen for storage. The mixture was a $25 \%(\mathrm{w} / \mathrm{v})$ 2.75:1 DMPC:DHPC solution in $\mathrm{D}_{2} \mathrm{O}$. The reconstitution procedure is as follows. The sample was mixed in a vortexer for 1 minute and then cooled in a liquid nitrogen/isopropanol bath at $-4{ }^{\circ} \mathrm{C}$ for $2-3$ minutes. It was then heated in a water bath at $50{ }^{\circ} \mathrm{C}$ for 10 minutes, followed by centrifugation for 5 minutes. This procedure was repeated three or more times.

The NMR experiments were conducted at $16.43 \mathrm{~T}$ on a Bruker Avance-700 spectrometer using a Bruker 5-mm H-X broadband solution-state NMR probe. The N-14 Larmor frequency was $50.577 \mathrm{MHz}$. Fig. 4.11 shows the N-14 spectrum of DHPC:DMPC bicelles aligned in a magnetic field of $16.43 \mathrm{~T}$ at $40{ }^{\circ} \mathrm{C}$. The quadrupolar splitting of the $\mathrm{D}_{2} \mathrm{O} \mathrm{H}-2$ nuclei was measured to be $29 \mathrm{~Hz}$; this served as an external measure of the alignment of the medium. The N-14 spectrum exhibits two quadrupolar-split doublets centered on ap- 
proximately the same frequency. The inner doublet was split by $\Delta_{i}=1.625 \mathrm{kHz}$ and the outer doublet was split by $\Delta_{o} \sim 5.3 \mathrm{kHz}$. The full-width at half maximum of the components of the inner doublet was $80 \mathrm{~Hz}$. The inner doublet was chosen for further study via

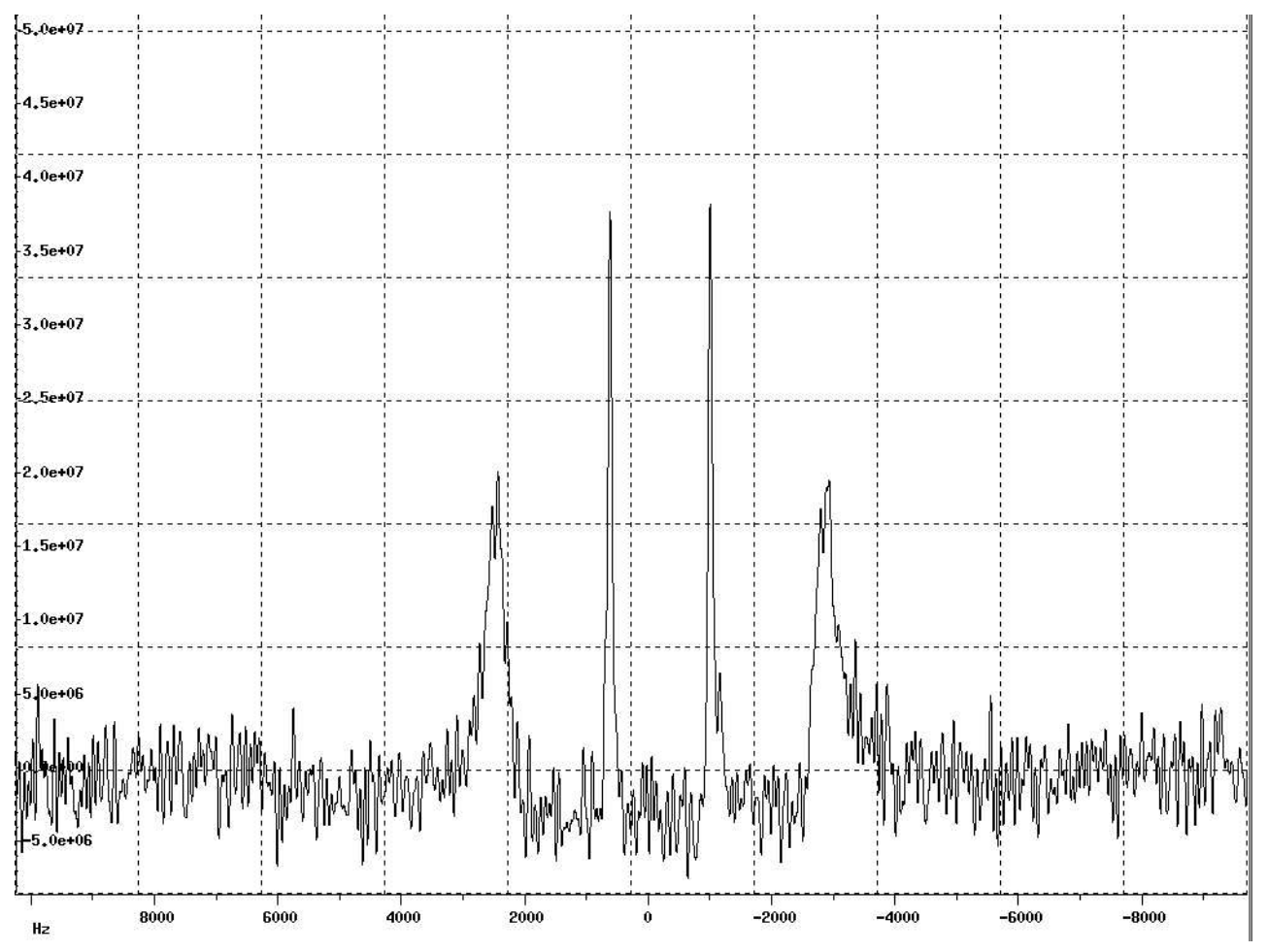

Figure 4.11: N-14 spectrum of DHPC:DMPC bicelles aligned at $B_{0}=16.43 \mathrm{~T}$ at $40{ }^{\circ} \mathrm{C}$. The spectrum was the sum of 1024 scans. The spectral window was $20 \mathrm{kHz}$, the acquisition time was $15 \mathrm{~ms}$, and the recycle delay was $350 \mathrm{~ms}$.

double-quantum spectroscopy using the pulse sequence in Fig. 4.6. The excitation time $\tau$ was set to $1 /\left(2 \Delta_{i}\right)=307 \mu \mathrm{s}$. Coincidentally, this choice of $\tau$ almost nulls the DQ excitation of the outer doublet (see Eq. 4.9). No $180^{\circ}$ refocusing pulse was used in the center of the excitation window; a $180^{\circ}$ pulse at the rf power that was used would be $80 \mu$ s long, which is a significant fraction of $\tau$. The $\mathrm{N}-14$ double-quantum-single quantum correlation spectrum is shown in Fig. 4.12. Frequency discrimination was not employed in the indirect dimension so the spectrum is mirrored across $\omega_{1}=0$. The experiment also generated phase-twist 
lineshapes rather than pure-absorptive mode lineshapes. As is expected the observed -2 $\mathrm{kHz}$ resonance offset in the double-quantum (indirect) dimension is twice the $-1 \mathrm{kHz}$ offset in the single-quantum (direct) dimension. Only a single resonance is observed in the double quantum dimension because the DQC is not split by the quadrupolar coupling. No line narrowing was observed in the double quantum dimension. A DQ-SQ correlation of the outer doublet is not observed. This may be the first $I=1$ double quantum spectrum taken in oriented bicelles.

\subsection{Conclusions}

The work reported here investigated the feasibility of obtaining higher-resolution solution-state N-14 NMR spectra in large biomolecules via the use of double-quantum spectroscopy. The ability to perform N-14 spectroscopy would obviate the need for isotopic labeling with the spin-1/2 N-15 nucleus. The spin $S=1 \mathrm{~N}-14$ nucleus typically exhibits extremely large linewidths due to the fast relaxation induced by its large quadrupolar coupling ( $C_{Q} \sim 3 \mathrm{MHz}$ for amide nitrogens). This broadening is even worse in slowly tumbling molecules whose rotational correlation time is outside of the extreme narrowing limit. However, in this limit the linewidth of the $I=1$ double quantum coherence (DQC) decreases (although not indefinitely) as the rotational correlation time increases. The double-quantum spectrum also exhibits a chemical shift resolution twice that of the normal single-quantum if the linewidths are held constant. For these reasons the idea of N-14 double quantum spectroscopy of biomolecules is appealing. The DQC is not directly observable so it is necessary to conduct a multidimensional NMR experiment to measure it indirectly. This 


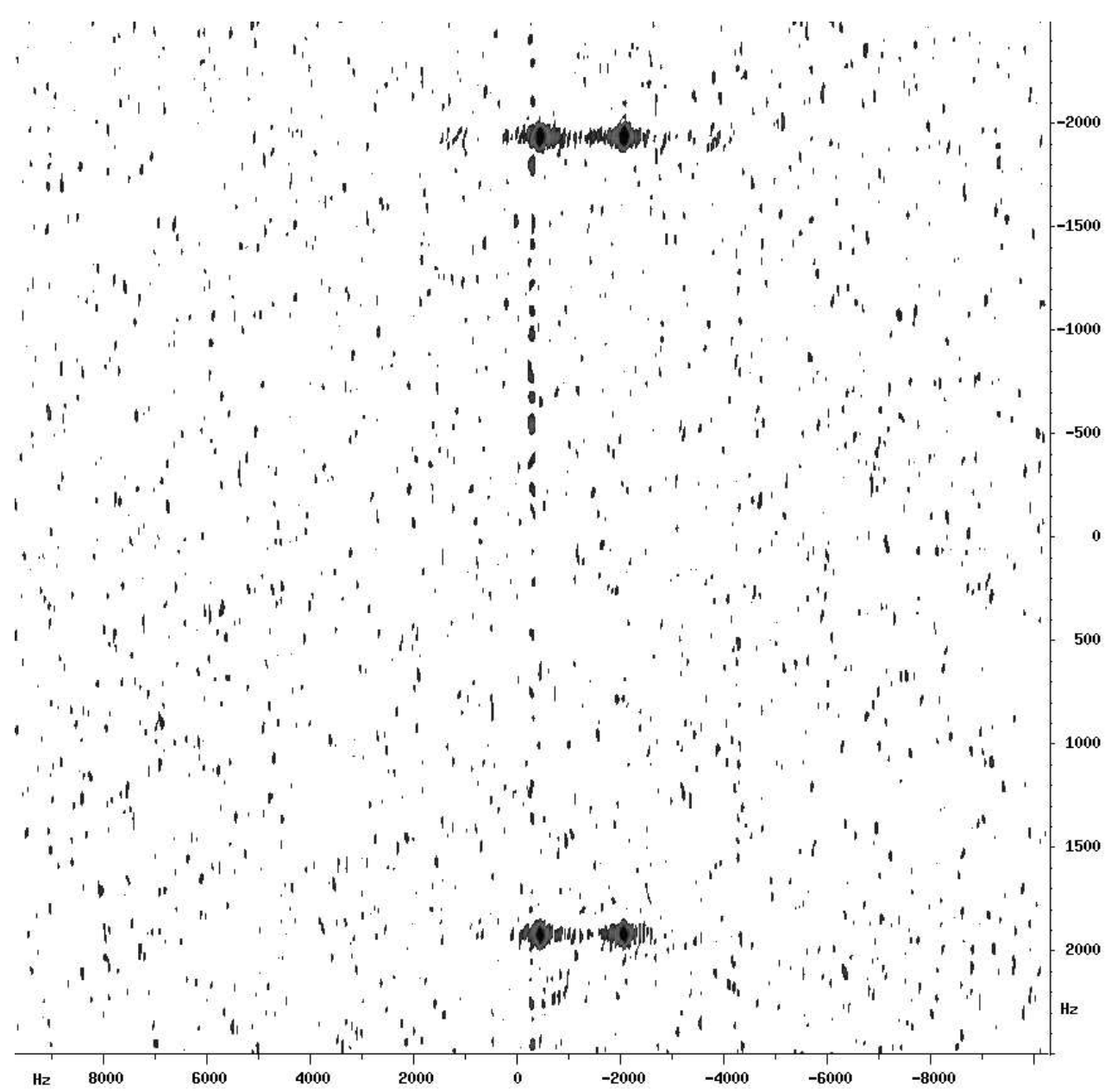

Figure 4.12: N-14 double-quantum-single quantum correlation spectrum in DHPC:DMPC bicelles aligned at $B_{0}=16.43 \mathrm{~T}$ at $40{ }^{\circ} \mathrm{C}$. The vertical axis is the indirect (DQ) dimension and the horizontal axis is the direct (SQ) dimension. The spectrum was displayed in absolute-value mode. The pulse sequence in Fig. 4.6 was used with $\tau=307 \mu$ s and a $90^{\circ}$ time of $40 \mu \mathrm{s}$. The spectral window was $20 \mathrm{kHz}$ in the direct dimension and $5 \mathrm{kHz}$ in the indirect dimension. A total of 128 points were taken in the indirect dimension with 1024 scans per indirect point. An 8-step phase cycle was employed to select the DQ spectrum while being robust toward artifacts that may occur due to residual magnetization from the previous transient acquisition [213]. A recycle delay of $300 \mathrm{~ms}$ was used.

would be a disadvantage compared to normal one-dimensional spectroscopy, but most NMR experiments in biomolecules already use multidimensional spectroscopy to establish spin correlations.

Theoretical calculations were performed to estimate the size of the double quantum 
line narrowing in the slow tumbling regime for $S=1$ nuclei and also to estimate the relevant relaxation rates. While the line narrowing effect can be substantial for very slowly tumbling molecules in a relative sense, in terms of absolute linewidth it is probably impossible to achieve the degree of spectral resolution that is enjoyed in N-15 spectroscopy if nitrogen sites with large N-14 quadrupolar couplings are considered. Furthermore, it seems unlikely in a system with a large quadrupolar coupling that the double quantum coherence can be created with any efficiency due to the competition of fast quadrupolar relaxation. Three potential methods of creating DQC in the solution state were considered: DQC creation via a reintroduced, motionally-averaged quadrupolar interaction in oriented media; DQC creation via the $J$-coupling to a spin $I=1 / 2$ partner; and DQC creation through coherent second-order dynamic shift evolution in the slow tumbling dynamical regime. It was shown that the rate of DQC production by the dynamic shift method will never compete favorably with the quadrupolar relaxation. The $J$-coupling method is feasible only when the quadrupolar relaxation rates do not exceed the value of the $J$-coupling, which will usually not be the case due to the large size of N-14 quadrupolar couplings and the relatively small size of $J$-couplings. The DQ spectroscopy in oriented media is more promising, particularly if the molecular alignment is strong enough that a large motionally-averaged quadrupolar interaction is re-introduced. It should also be noted that some of these techniques may become useful in other $S=1$ systems in the slow tumbling regime with smaller quadrupolar couplings, e.g., deuterium-labeled proteins.

Some N-14 DQ spectroscopy experiments were performed using the well-established $J$-coupling and reintroduced quadrupolar coupling methods. Proton-detected DQ spec- 
troscopy of $\mathrm{N}-14$ in the ammonium ion in the extreme narrowing limit was successfully performed but similar results were not achieved in systems with larger quadrupolar relaxation rates. Double quantum line narrowing is not expected in the extreme narrowing limit and was not observed. Nitrogen-14 DQ spectroscopy was also successfully performed in magnetically-aligned phospholipid bicelles. No DQ line narrowing was observed. This may have been the first instance of double quantum NMR spectroscopy in aligned bicelles. 


\section{Chapter 5}

\section{Analogies between NMR/NQR}

\section{and atomic/molecular optics}

\subsection{Introduction}

The great utility and versatility of nuclear resonance experiments stems from the ability of the experimenter to control the nuclear angular momentum dynamics through the application of static and radiofrequency magnetic fields. Many of these experiments can be performed in continuous wave (cw) mode, although pulsed Fourier transform techniques allow the full power of NMR quantum control to be exploited. New pulse sequences are usually designed with the following thoughts in mind: what types of quantum coherences can be created in the system? What effective Hamiltonians would be necessary in order to extract certain desired physical information from the spin system? How can the experimentalist generate the necessary effective Hamiltonians? The NMR spectroscopists have 
developed some of the most sophisticated techniques of quantum control largely due to several fortuitous conditions. First of all, the spin systems are simple, usually involving only a few discrete states that are relatively weakly coupled to their environment, which results in slow relaxation of the nuclear polarization. The experimenter often has the ability to apply "hard" rf pulses to the system during which the dynamics under any internal Hamiltonians can be neglected, allowing for the precise manipulation of the nuclear angular momenta. Furthermore, experiments conducted at high magnetic fields allow for the possibility of applying coherence pathway selection techniques that can simplify the observed spectra.

It is often the case in an atomic or molecular system ${ }^{1}$ that angular momentum is a good (or approximately good) quantum number. This angular momentum could be the total electronic angular momentum, or the total electronic plus nuclear angular momentum if a strong hyperfine coupling is present. If one or two of these angular momentum states are isolated, the situation in atomic and molecular optics (AMO) is quite analogous to NMR.

The field of AMO is quite broad, and only a small subset of those studies will be considered here. It is not the goal of this work to explore some of the analogies between ultrafast (coherent) laser spectroscopy and magnetic resonance, or mixed optical/magnetic resonance techniques. Rather, the discussion will be limited to experiments that involve the simple dynamics of quantized atomic or molecular angular momenta in (usually static) electric and magnetic fields, which are not regularly considered from the perspective of quantum control in the density operator formalism. There have been decades of work in this field and the AMO physicists have strong insights into these systems that may be

\footnotetext{
${ }^{1}$ It may be assumed for the purposes of this chapter that the atomic/molecular systems are in the gas phase.
} 
applied to the design or understanding of NMR experiments.

The goal of this chapter is simple: to make a mathematical analogy between several basic NMR and AMO systems, particularly systems with more than two levels. This analogy will be grounded in a density operator and Hamiltonian formalism for the angular momenta and will involve a comparison of the methods of polarization of nuclear and atomic systems, their respective dynamics in the presence of external or local electric and magnetic fields, and the system observables. The emphasis here is not on the proposal of new experiments but rather on a systematic exploration of the similarities between NMR and AMO angular momentum dynamics and experiments. However, it is hoped that the analogies between NMR and AMO will provide not only additional insight into these varied fields of study but also stimulate new avenues of thought in experimental design.

The language used in optical and NMR spectroscopies somewhat differs, and the NMR spectroscopist may find the optical spectroscopy books by the following authors to be both penetrable and enlightening: Budker et al. [32], Auzinsh and Ferber [214], Suter [215], Alexandrov et al. [216], Macomber [217], Sobelman [218], and Allen and Eberly [219].

\subsection{Nuclear and atomic polarization}

The first comparison to be made is between methods of polarization of nuclear and atomic ensembles. Here, polarization refers to the deviation of the state of the system from the state in which the projection of angular momentum along any direction is the same; in a single-spin system this state is the completely depolarized state. We consider

an ensemble of angular momenta described by the quantum number $J$, which is assumed 
to be a good quantum number for the system. For example, $J$ could describe a particular electronic or hyperfine state in an atom, or the angular momentum of a nuclear spin. (Typically $F$ is used to denote hyperfine states and $I$ is used to represent nuclear angular momenta, but the symbol $J$ is used everywhere in this chapter to emphasize the generality of angular momentum dynamics across systems.) This ensemble is completely specified quantum mechanically by the density operator in the manifold of the $2 J+1$ magnetic sublevels $\{|J, m=+J\rangle, \ldots,|J, m=-J\rangle\}$, where $m$ is the quantum number for the projection of the angular momentum along the quantization axis. The density operator can be decomposed in a basis of spherical tensor operators of rank $k$ and order $q$ (see Appendix A):

$$
\hat{\rho}(t)=\sum_{k=0}^{2 J+1} \sum_{q=-k}^{+k} \rho_{k, q}(t) \hat{T}_{k, q}
$$

where where the operators $\hat{T}_{k, q}$ are assumed to be orthonormal, that is, $\left\langle\left\langle T_{k^{\prime}, q^{\prime}} \mid T_{k, q}\right\rangle\right\rangle=$ $\operatorname{Tr}\left[\hat{T}_{k^{\prime}, q^{\prime}}^{\dagger} \hat{T}_{k, q}\right]=\delta_{k, k^{\prime}} \delta_{q, q^{\prime}}$. The coefficients $\rho_{k, q}(t)=\left\langle\left\langle T_{k, q} \mid \rho(t)\right\rangle\right\rangle=\operatorname{Tr}\left[\hat{T}_{k, q}^{\dagger} \hat{\rho}(t)\right]$ are called the Fano statistical tensors [71] or polarization moments of the system [214]. It is sometimes also convenient to choose a tensor basis in which symmetric and antisymmetric linear combinations of the $\hat{T}_{k, \pm q}(q>0)$ tensors are taken in order to form normalized Hermitian basis operators $[220]$ :

$$
\hat{T}_{k, q ; \phi}=\frac{1}{\sqrt{2}}\left[\cos (q \phi)\left(\hat{T}_{k,-q}+(-1)^{q} \hat{T}_{k,+q}\right)+i \sin (q \phi)\left(\hat{T}_{k,-q}-(-1)^{q} \hat{T}_{k,+q}\right)\right],
$$

where $\phi$ is the usual polar angle measured from the $x$-axis, with the quantization axis taken to be in the $+z$ direction. 
If the ensemble is completely unpolarized, the density operator is just equal to the identity operator, $\hat{\rho}=\hat{1} \propto \hat{T}_{0,0}$. However, if the system is polarized so that the probability of finding the total angular momentum in a particular direction is not isotropic, then non-zero polarization moments exist with rank $k>0$. It is useful to group together these polarization moments by rank: by one convention, the odd-rank moments correspond to orientation and the even-rank moments correspond to alignment. ${ }^{2}$ If the density matrix in the $\{|J, m\rangle\}$ basis is diagonal (i.e., it contains only terms of the form $\hat{T}_{k, 0}$ ), then alignment corresponds to the case in which the populations of the $|J, \pm m\rangle$ states are equal (excluding the case of complete depolarization), and orientation corresponds to cases in which the populations of the $|J, \pm m\rangle$ states differ [216]. In the terminology of NMR, in which the quantization axis is chosen to be along the external magnetic field, rank $k=1$ orientation is called magnetization ( $q=0$ for longitudinal magnetization, $q= \pm 1$ for transverse magnetization), and $k=2, q=0$ alignment is called quadrupolar order. ${ }^{3}$

Several methods exist by which angular momentum polarization may be visualized. The Wigner-Eckart theorem dictates that the maximum rank of polarization that can be produced in a system of angular momentum $J$ is $k_{\max }=2 J$. A two-level $J=1 / 2$ system thus can be described completely by the identity operator and the three rank $k=1$ components of the density operator. Linear combinations of these three components can be associated with the components of a vector in a three-dimensional space, enabling the dynamics of the system to be interpreted geometrically as evolution of the polarization

\footnotetext{
${ }^{2}$ Another convention specifically designates $k=1$ polarization to be orientation (for which it is possible to define a preferred direction for the polarization) and $k=2$ polarization to be alignment (for which only a preferred axis can be defined).

${ }^{3}$ Strictly speaking, the spin magnetization is proportional to the polarization by the number of spins and the average nuclear magnetic moment, but "magnetization" and "polarization" will be used somewhat interchangeably here.
} 
vector on the so-called Bloch sphere. The magnetization (rank 1 orientation) of the system is proportional to this vector, as follows from the Wigner-Eckart theorem. However, for systems with $J>1 / 2$, the density matrix has too many degrees of freedom to be described by a simple three-dimensional vector, and different methods of visualization must be employed. One useful method utilizes plots of a three dimensional surface whose radius $r$ from the origin equals the probability of finding the maximum projection of angular momentum $m=J$ along the radial direction [221]:

$$
r(\theta, \phi ; t)=\left\langle J, m=J\left|\hat{R}^{-1}(\phi, \theta, 0) \hat{\rho}(t) \hat{R}(\phi, \theta, 0)\right| J, m=J\right\rangle
$$

where $\hat{R}(\phi, \theta, 0)$ is the Euler angle-parameterized rotation operator that rotates the quantization axis into the radial direction, where $\theta$ and $\phi$ are the polar angles of the radial vector. This approach is similar to one that has been employed to describe molecular polarization $[214,222]$, and methods also have been developed to visualize the different tensor elements of nuclear polarization in terms of the corresponding spherical harmonics [223].

\subsubsection{Thermal equilibrium nuclear polarization}

The overwhelmingly common method of polarization in NMR experiments is the thermal equilibration of the spin system in the presence of a dominant magnetic field. The density operator for a system at thermal equilibrium with its environment is given by $\hat{\rho}_{e q}=Q^{-1} e^{-\beta \hat{H}}$, where $Q=\operatorname{Tr}\left[e^{-\beta \hat{H}}\right]$ is the canonical partition function and $\beta=\left(k_{B} T\right)^{-1}$ (see $§ 1.4 .3)$. At high magnetic fields the Zeeman interaction typically dominates all other spin interactions, so $\hat{H} \simeq \hat{H}_{Z}=-\hbar \omega_{0} \hat{J}_{z}$, where $\omega_{0}=\gamma B_{0}$ is the Larmor frequency of the 
spin $J$ with gyromagnetic ratio $\gamma$ in an external field $B_{0}$. The quantization axis $z$ is taken to be along the magnetic field direction. Typically $\hbar\left|\omega_{0}\right| \ll k_{B} T$ at temperatures above $\sim 10^{-3} \mathrm{~K}$ if $B_{0}$ is on the order of Tesla, so in the high-temperature approximation, the high-field equilibrium density operator becomes

$$
\hat{\rho}_{e q}^{H T} \simeq e^{-\beta \hat{H}_{Z}} / \operatorname{Tr}\left[e^{-\beta \hat{H}_{Z}}\right] \simeq\left(\hat{1}+\frac{\hbar \omega_{0}}{k_{B} T} \hat{J}_{z}\right) / \operatorname{Tr}[\hat{1}]
$$

which corresponds to rank-1 polarization in the $z$-direction, otherwise known as orientation, longitudinal magnetization, or Zeeman order (because the first non-trivial term is proportional to the Zeeman Hamiltonian).

When the externally-applied magnetic field is very small, it is then possible that the internal Hamiltonians may be the dominant interactions, which is often the case in the solid state where large internal interactions are not motionally averaged away. Usually if the nucleus possesses an electric quadrupole moment $(J>1 / 2)$, the quadrupolar interaction dominates at low magnetic fields $\left(\left\|\hat{H}_{Q}\right\| \gg\left\|\hat{H}_{Z}\right\|\right)$ and $\hat{H} \simeq \hat{H}_{Q}$. Under these conditions it is convenient to pick the quantization axis $z$ not to be along the direction of the magnetic field (if any is present) but to be along the principal axis of the local nuclear electric field gradient tensor. In this case $\hat{H}_{Q}=\frac{h C_{Q}}{4 I(2 I-1)}\left[\left(3 \hat{J}_{z}^{2}-\underline{\hat{J}} \cdot \underline{\hat{J}}\right)+\eta_{Q}\left(\hat{J}_{x}^{2}-\hat{J}_{y}^{2}\right)\right]$, where $C_{Q}$ is the quadrupolar coupling constant and $\eta_{Q}$ is the electric field gradient asymmetry parameter (see §1.3.7). Hereafter it will be assumed that the quadrupolar coupling is axially symmetric $\left(\eta_{Q}=0\right)$. The high temperature approximation applies when $h C_{Q} \ll k_{B} T$ and the equilibrium density operator of a quadrupolar nucleus at low magnetic fields can be 
approximated as:

$$
\hat{\rho}_{e q}^{L F, Q} \simeq e^{-\beta \hat{H}_{Q}} / \operatorname{Tr}\left[e^{-\beta \hat{H}_{Q}}\right] \simeq\left\{\hat{1}-\frac{\hbar \omega_{Q}}{k_{B} T} \frac{1}{\sqrt{6}}\left[3 \hat{J}_{z}^{2}-J(J+1) \hat{1}\right]\right\} / \operatorname{Tr}[\hat{1}]
$$

where in this case, $\omega_{Q}=2 \pi \sqrt{6} C_{Q} /[4 I(2 I-1)]$ and $\eta_{Q}=0$. The polarized part of $\hat{\rho}_{e q}^{L F, Q}$ corresponds to $\hat{T}_{2,0}$ alignment, also known as quadrupolar order (because the first non-trivial term looks like the quadrupolar Hamiltonian $\hat{H}_{Q}$, or because the polarization moment is of rank 2). This is the type of initial polarization found in low-field nuclear quadrupole resonance (NQR) experiments in the solid state. Quadrupolar order (alignment) does not correspond to magnetization (orientation), so the alignment must be converted to orientation in order to be detected as magnetization.

It should be noted that the density operators above were written with a single nucleus in mind. However, in the case of a polycrystalline powder, individual crystallites (and hence local electric field gradients) are oriented randomly. Therefore the direction of the quadrupolar alignment axis will also be randomly distributed, and a macroscopic collection of quadrupolar nuclei in a small magnetic field will have no net initial polarization at thermal equilibrium. This is not the case at high magnetic fields if the Zeeman interaction dominates all the local (orientation-dependent) interactions; even in a powder, nuclei in each crystallite will be magnetized (oriented) equally along the external magnetic field axis.

A final point to mention is that some techniques exist in which nuclei are polarized in a non-equilibrium state, such as spin-exchange optical pumping or dynamic nuclear polarization. Most of these techniques are used to create enhanced magnetization (orientation), and they will not be discussed here. 


\subsubsection{Atomic polarization}

There are many ways of polarizing atoms. The most classic example is perhaps

the Stern-Gerlach experiment (see $\S[45, \S 1.1]$ ), in which an initially unpolarized beam of electrically-neutral $J=1 / 2$ atoms was separated into two beams of opposite spin polarization via passage through an inhomogeneous magnetic field. A more versatile method is to use light-atom interactions, i.e., the dynamic preparation of atomic polarization via optical pumping techniques [224]. A photon is a spin-1 particle and therefore can support polarization of up to rank $\kappa=2$; therefore, a single photon interaction can transfer polarization of rank $\kappa=0,1,2$ to an atom. For example, circular light polarization can be used to define a direction in space (i.e., with or against the light propagation axis depending on the sense of the circular polarization) and therefore can prepare atoms in a state of polarization for which a direction is defined, i.e., rank-1 orientation. Linearly polarized light only defines a preferred axis in space (i.e., the light polarization axis) and therefore can prepare atoms in a state of polarization in which only a preferred axis but not a direction is defined, i.e., rank-2 alignment. This is only a simplified picture of the light-atom dynamics. Note that higher-rank polarization moments can be created via multiple photon-atom interactions. This is generally considered to be in the realm of nonlinear optics.

\subsection{Atoms and nuclei with $J>1 / 2$ in electric and magnetic fields}

The dynamical evolution of a quantum system is defined by its Hamiltonian, and the analogy between atomic systems and quadrupolar nuclei becomes clear through compar- 
ison of their respective Hamiltonians. It will be shown that the magnetic Zeeman interaction has the same form in the nuclear and atomic cases, but that the quadratic Stark interaction in atoms has the same form as the nuclear electric quadrupolar Hamiltonian in NQR (for axially symmetric quadrupole couplings) and as the high-magnetic field NMR quadrupolar Hamiltonian to first order in the perturbation theory in the rotating frame. More specifically, the NMR rotating frame dynamics of a quadrupolar nucleus during a resonant rf pulse are analogous to those of an atomic state in crossed static electric and magnetic fields. The dynamics during an NQR pulse for a nucleus with an axially-symmetric quadrupolar coupling are analogous to those of an atom in an oscillating magnetic field that makes some random angle with respect to a static electric field. Due to the experimental inability to apply rf pulses that are stronger than the nuclear quadrupolar interaction, the analogies will be complete only when the atomic Stark splittings are much larger than the Zeeman splittings.

\subsubsection{Atoms in crossed electric and magnetic fields}

An atom in a state of total angular momentum $J$, when subjected to a uniform electric field $\underline{E}$ along the $z$-axis and a uniform magnetic field $\underline{B}$ along the $x$-axis, has the following Hamiltonian [31] due to the quadratic Stark and (linear) Zeeman effects:

$$
\begin{aligned}
\hat{H} & =\hat{H}_{E}+\hat{H}_{B} \\
& =-\frac{1}{2} E^{2}\left[\alpha_{0} \hat{1}+\frac{2}{3} \alpha_{2} E^{2}\left(3 \hat{J}_{z}^{2}-\underline{\hat{J}} \cdot \underline{\hat{J}}\right)\right]-g_{J} \mu_{B} B \hat{J}_{x}
\end{aligned}
$$


where the angular momentum operators have been taken to be dimensionless, $\alpha_{0}$ and $\alpha_{2}$ are the isotropic (rank-0) and anisotropic (rank-2) components of the electronic polarizability tensor, $g_{J}$ is the $g$-factor of the state, and $\mu_{B}$ is the Bohr magneton. As is expected, the Stark Hamiltonian is symmetric about the electric field axis, and the Zeeman Hamiltonian is symmetric about the magnetic field axis. The quadratic Zeeman effect is assumed to be negligible. It is permissible to drop the term in the Stark Hamiltonian proportional to the identity operator, as this term causes only a constant shift of all the magnetic sublevels and does not affect the dynamics. ${ }^{4}$ The remaining effect of the electric field is due to the anisotropic polarizability of the atom, which is quadratic in the field strength. This effect vanishes when $J=1 / 2$ and causes a splitting of the magnetic sublevels when $J>1 / 2$. The usual Zeeman interaction is linear in the magnetic field strength. Upon dropping the isotropic polarizability term the Hamiltonian becomes:

$$
\hat{H}=-\hbar \omega_{E} \hat{T}_{2,0}-\hbar \omega_{B} \hat{J}_{x}
$$

where $\omega_{E} \propto \alpha_{2} E^{2},{ }^{5}$ and the Larmor frequency is $\omega_{B}=g_{J} \mu_{B} B$. The quantization axis has been taken to be along the electric field axis $z$. It will be assumed that all atoms in the sample feel identical fields and therefore undergo the same dynamics.

\footnotetext{
${ }^{4}$ Of course, the Stark shift of a level $J$ relative to another level $J^{\prime}$ can be extremely important when considering transitions between $J$ and $J^{\prime}$.

${ }^{5}$ Some definitions of the Stark Hamiltonian take a $J$-dependent normalization factor outside of $\alpha_{2}$, or include one in the definition of $\hat{T}_{2,0}$.
} 


\subsubsection{Quadrupolar nuclei in low magnetic fields under radiofrequency irradiation (NQR)}

Typical NQR experiments are performed on either crystalline or polycrystalline solids that contain quadrupolar nuclei of angular momentum $J$ that are coupled to local electric field gradients (EFGs) due to the atomic electrons and other neighboring charges. The quadrupolar Hamiltonian is (see $\S 1.3 .7)$ :

$$
\hat{H}_{Q}=\frac{2 \pi \hbar C_{Q}}{4 J(2 J-1)}\left[\left(3 \hat{J}_{z}^{2}-\underline{\hat{J}} \cdot \underline{\hat{J}}\right)+\eta_{Q}\left(\hat{J}_{x}^{2}-\hat{J}_{y}^{2}\right)\right]
$$

Here $C_{Q}=e q e Q$ is the quadrupolar coupling constant, where $e q$ is the largest component of the EFG tensor in its principal axis system (PAS) and $e Q$ is the electric quadrupole moment of the nucleus. $C_{Q}$ can be positive or negative. The asymmetry parameter of the electric field gradient is denoted $\eta_{Q}$, and the $z$-axis is taken to be along the principal axis of the EFG tensor. The nuclear quadrupole moment vanishes for $J=1 / 2$, so this discussion applies to nuclei with $J>1 / 2 . \hat{H}_{Q}$ can be written in the form

$$
\hat{H}_{Q}=\sum_{q=-2}^{+2}(-1)^{q} A_{2, q} \hat{T}_{2,-q}
$$

where the spatial tensors $A_{2, q}$ are decompositions of the EFG tensor in a spherical basis, and the spin tensors $\hat{T}_{2, q}$ are defined as usual.

In NQR studies on a single crystal, all the nuclear EFG tensors have the same orientation in the laboratory frame, which can be can be experimentally controlled by orienting the crystal. However, most NQR studies are performed on polycrystalline powders, 
which consist of many randomly oriented crystallites that possess different orientations of their EFG tensor with respect to laboratory frame coordinates. NQR is often performed at (or near) zero magnetic field, but a sizable magnetic field can be present during any required radiofrequency (rf) irradiation, for example, during an $\mathrm{rf}$ excitation of the system. If the rf magnetic field is linearly polarized along some axis in the laboratory frame, the rf field will appear to be along a different, random direction in the nuclear EFG principal axis system (PAS) due to the random orientation of the crystallite. Since the quadrupolar coupling usually dominates the rf interaction or any other interaction in the solid, it is convenient to perform calculations in the EFG PAS frame. It will be assumed that the nuclear site has an axially symmetric EFG tensor $\left(\eta_{Q}=0\right)$, so that the quadrupolar Hamiltonian of a single crystallite in the presence of linearly polarized rf irradiation $\underline{B}_{1}(t)=\underline{B}_{1} \cos \omega_{r} t$ becomes:

$$
\begin{aligned}
\hat{H}(t) & =\hat{H}_{Q}+\hat{H}_{r f}(t) \\
& =\hbar \omega_{Q} \hat{T}_{2,0}-\hbar \omega_{1}\left(a_{x} \hat{J}_{x}+a_{y} \hat{J}_{y}+a_{z} \hat{J}_{z}\right) \cos \omega_{r} t
\end{aligned}
$$

where $\omega_{1}=\gamma B_{1}, a_{x, y, z}=B_{1 x, y, z} / B_{1}$ are coefficients that give the components of $\underline{B}_{1}$ in the EFG PAS, $\gamma$ is the nuclear gyromagnetic ratio, and $\omega_{Q} \propto C_{Q}$. Due to the fact that the coefficients $a_{x, y, z}$ differ for each crystallite orientation in the powder, the nuclear dynamics within each crystallite will be different. Therefore, the total NQR signal must be averaged over crystallite orientation. Note that this Hamiltonian would have the same form as Eq. 5.7 if the magnetic field were time-independent and happened to be oriented along the $x$-direction in the EFG PAS. 


\subsubsection{Quadrupolar nuclei in high magnetic fields under radiofrequency irradiation (quadrupolar NMR)}

This section considers a high field NMR experiment in which an rf pulse resonant with the Larmor frequency and polarized along the laboratory-frame $x$-axis is applied to a quadrupolar nucleus in a solid powder in the presence of a strong magnetic field $\underline{B}_{0}$ in the $+z$-direction. The laboratory frame Hamiltonian for a single crystallite is:

$$
\begin{aligned}
\hat{H}_{L A B}(t) & =\hat{H}_{Z}+\hat{H}_{Q, L A B}+\hat{H}_{r f}(t) \\
& =-\hbar \omega_{0} \hat{J}_{z}+\hat{R}(\alpha, \beta, \gamma) \hat{H}_{Q} \hat{R}^{-1}(\alpha, \beta, \gamma)-2 \hbar \omega_{1} \hat{J}_{x} \cos \left(\omega_{0} t\right)
\end{aligned}
$$

where $\omega_{0}=\gamma B_{0}$ and $2 \omega_{1}=\gamma B_{1}$ (the convention for including the factor of 2 in the rf amplitude will be apparent shortly). $\hat{H}_{Q}$ is the quadrupolar Hamiltonian (Eqs. 5.8, 5.9) and $\hat{R}(\alpha, \beta, \gamma)=R_{A}(\alpha, \beta, \gamma) \hat{R}_{T}(\alpha, \beta, \gamma)$ is the rotation operator acting upon the spatial and spin tensors in Eq. 5.9 that transforms the quadrupolar EFG PAS into the laboratory frame axes according to the crystallite Euler angles $(\alpha, \beta, \gamma)$.

At high magnetic fields $\left(\left|\omega_{0}\right| \gg\left|\omega_{1}\right|\right)$ it is customary to transform into a rotating frame corresponding to the interaction representation defined by the dominant $\hat{H}_{Z}$ term. The effective Hamiltonian in the rotating frame is found by retaining only the time independent part of the transformed Hamiltonian (this is called the rotating frame or secular 
approximation):

$$
\begin{aligned}
\hat{H}_{r o t} & \simeq \hat{H}_{Q}^{(1)}+\hat{H}_{r f}^{r o t} \\
& =\hbar \omega_{Q} \hat{T}_{2,0}-\hbar \omega_{1} \hat{J}_{x}
\end{aligned}
$$

where the quantization axis has been taken to be along the magnetic field axis $z$. The quadrupolar frequency $\omega_{Q}=\omega_{Q}\left(\alpha, \beta, \gamma ; C_{Q}, \eta\right)$ depends on the crystallite orientation (see §1.3.7). This truncation of $\hat{H}_{Q}$ is equivalent to applying static perturbation theory to first order in $\hat{H}_{Q}$ with respect to the dominant Hamiltonian $\hat{H}_{Z}$. The first-order result is generally sufficient to describe the response of nuclear spin systems to rf pulses. The first-order high field-truncated quadrupolar Hamiltonian is proportional to $\hat{T}_{2,0}$ even when $\eta_{Q} \neq 0$, as opposed to the full zero-field (NQR) quadrupolar Hamiltonian. The effective size of the rf field in the rotating frame is $\hbar \omega_{1}$, which is a factor of 2 smaller than that in the laboratory frame (Eq. 5.11) due to the fact that the rotating frame approximation selects only the circular component of the rf radiation that goes around in the same direction as the spin Larmor precession about $\underline{B}_{0}$.

As in NQR, nuclei in crystallites with different orientations will evolve differently, and the total signal must be averaged over all crystallite orientations. However, there may be an even closer analogy between quadrupolar NMR and the atomic system in crossed $\underline{E}$ and $\underline{B}$ fields described by Eq. 5.7 than is possible in the example of NMR of a random powder. Consider the example of solution-phase NMR of molecules in liquid crystalline or other oriented media. As can be seen from Eq. 5.9, $\hat{H}_{Q}$ contains no isotropic $\left(A_{0,0}\right)$ component, and in solution rapid isotropic molecular tumbling on a timescale faster than $\left|\omega_{0}\right|^{-1}$ com- 
pletely averages away the anisotropic quadrupolar Hamiltonian. However, in an oriented medium the molecular tumbling is not isotropic, and a component of the quadrupolar coupling is retained, scaled down by a geometric factor due to anisotropic motional averaging of the EFG tensor. In this case the characteristic quadrupolar frequency $\omega_{Q}$ is orientationally averaged and is generally the same for every nucleus in the sample, which obviates the need for powder averaging and makes the nuclear evolution in the rotating frame under the quadrupole coupling and rf field equivalent to the atomic evolution under crossed $\underline{E}$ and $\underline{B}$ fields according to Eq. 5.7. Furthermore, the scaling of the quadrupolar coupling makes it possible to apply rf fields that are stronger than the quadrupolar coupling $\left(\left|\omega_{1}\right|>\left|\omega_{Q}\right|\right)$, which is usually not possible in solid-state NMR, but by contrast it is possible in atomic systems to cause Larmor splittings that are larger than the Stark splittings $\left(\left|\omega_{B}\right|>\left|\omega_{E}\right|\right)$.

\subsection{Observables in NMR/NQR and AMO}

\subsubsection{Observables in NMR and NQR}

Both NMR and NQR experiments measure the evolution of the bulk nuclear magnetization. The magnetization is proportional to the net magnetic moment, which in turn is proportional to the average nuclear angular momentum. In conventional NMR and NQR detection schemes, oscillating nuclear magnetization creates a time-dependent magnetic flux through a detection coil that induces a measurable current in the coil. A summary of conventional NMR detection is given in $§ 1.7$. Assuming the coil axis is along the $x$-direction 
in the laboratory frame, the detection operator is:

$$
\hat{\mathcal{O}}_{N M R / N Q R} \propto \hat{J}_{x} \propto-\frac{1}{\sqrt{2}}\left(\hat{T}_{1,+1}-\hat{T}_{1,-1}\right)
$$

In practice the detected signal is down-mixed with the rf carrier frequency, and the mixing is often performed in quadrature (virtually always in the case of high-field NMR). In high-field NMR experiments the rf coil axis is perpendicular to the dominant magnetic field along $z$. Therefore only magnetization transverse to the Zeeman field is detected, and the quadrature mixing/detection selects only one circularly-polarized component of transverse magnetization. This corresponds to a detection observable of $\hat{J}_{+} \propto-\hat{T}_{1,+1}$ in the rotating frame. The situation in NQR is more complicated because the natural coordinate system (the local EFG PAS) does not possess a fixed geometry with respect to the coil axis. It is also convenient to perform NQR calculations in the interaction frame of the dominant quadrupolar Hamiltonian, which does not correspond to a simple rotating frame transformation. A comparison between NQR and NMR detection is made in Ref. [225].

The preceding discussion refers only to observables in NMR/NQR experiments that are directly detectable by Faraday induction in a coil. It does not consider overtone effects or the detection of magnetization by magnetometric techniques. However, it should be noted that in many cases every density operator component can be addressed by density matrix tomographic techniques. Multidimensional NMR is an example of a technique that can be used to record the evolution of quantities that are not directly observable; the normally unobservable coherences are created and allowed to evolve in the indirect dimension or dimensions of the experiment before being converted to observable transverse 
magnetization for direct detection.

\subsubsection{Observables in AMO}

Unlike the case in NMR, a variety of polarization moments can be measured in different optics experiments, including those of high spherical tensor rank (e.g. Ref. [226]). The most straightforward way of determining the atomic or molecular polarization is by measuring the properties of light that is passed through the optical medium. Following the review by Budker et al. [110], the oscillating light field can be characterized by an electric field amplitude $\mathcal{E}_{0}$, a frequency $\omega$ (with corresponding vacuum wave number $k=\omega / c$, where $c$ is the speed of light), phase $\phi$, a polarization angle $\varphi$, and a polarization ellipticity $\epsilon$. It is convenient to define a vector for the polarization of the optical medium $\underline{P}=\operatorname{Tr}[\hat{\rho} \underline{\hat{d}}]$ that is equal to the projection of the density operator $\hat{\rho}$ onto the electric dipole operator $\underline{\hat{d}}$. This is just another way of representing the components of the density operator. The polarization of the medium has four independent components that can be measured by light, defined by:

$$
\begin{aligned}
& \underline{P}=\frac{1}{2}\left[\left(P_{1}-i P_{2}\right) e^{i(\omega t-k z)}+\left(P_{1}+i P_{2}\right) e^{-i(\omega t-k z)}\right] \underline{\widetilde{x}} \\
&+ \frac{1}{2}\left[\left(P_{3}-i P_{4}\right) e^{i(\omega t-k z)}+\left(P_{3}+i P_{4}\right) e^{-i(\omega t-k z)}\right] \underline{\widetilde{y}},
\end{aligned}
$$

where the $z$-axis is along the direction of light propagation. Alternatively, the four Stokes parameters could be used to characterize the system. Since only these four polarization components are detected, one might wonder how all the different possible multipole polarizations $\rho_{\kappa, q}$ of the system can be measured. The presence of various multipole polarizations can be inferred by examining the dynamics of the polarization components [224]. The same 
principle is true in NMR; although only transverse magnetization is detected, the dynamics of this magnetization can be manipulated to express much information about the quantum state of the system.

If the system is optically thin (i.e., one in which absorption is negligible and $\varphi$ and $\epsilon$ are small), the change in polarization rotation, absorption, light phase, and polarization ellipticity per unit length can be written:

$$
\begin{aligned}
\frac{d \varphi}{d z} & =-\frac{2 \pi \omega}{\mathcal{E}_{0} c} \sec 2 \epsilon\left[\cos \varphi\left(P_{1} \sin \epsilon+P_{4} \cos \epsilon\right)+\sin \varphi\left(-P_{2} \cos \epsilon+P_{3} \sin \epsilon\right)\right] \\
\frac{d \mathcal{E}_{0}}{d z} & =-\frac{2 \pi \omega}{c}\left[\sin \varphi\left(-P_{1} \sin \epsilon+P_{4} \cos \epsilon\right)+\cos \varphi\left(P_{2} \cos \epsilon+P_{3} \sin \epsilon\right)\right] \\
\frac{d \phi}{d z} & =-\frac{2 \pi \omega}{\mathcal{E}_{0} c} \sec 2 \epsilon\left[\cos \varphi\left(P_{1} \cos \epsilon+P_{4} \sin \epsilon\right)+\sin \varphi\left(-P_{2} \sin \epsilon+P_{3} \cos \epsilon\right)\right] \\
\frac{d \epsilon}{d z} & =\frac{2 \pi \omega}{\mathcal{E}_{0} c}\left[\sin \varphi\left(P_{1} \cos \epsilon+P_{4} \sin \epsilon\right)+\cos \varphi\left(P_{2} \sin \epsilon-P_{3} \cos \epsilon\right)\right]
\end{aligned}
$$

The presence of different polarization components leads to different optical effects. For instance, consider a Faraday rotation experiment where the linear polarization of light is rotated by an angle $\varphi$ by an optical medium in the presence of a magnetic field parallel to the light propagation. The ellipticity $\epsilon$ is zero for linearly polarized light and if the rotation is small such that $\varphi \ll 1$, Eq. 5.15 indicates that the rotation is $\Delta \varphi \propto-P_{4}$. The "observable operator" for optical rotation is written for the $J=0, J^{\prime}=1$ system in $§ 5.7 .4$. These methods are general and a similar approach has been used in the same system to write the observable operator for the fluorescence polarization degree, as measured in Hanle effect experiments where a magnetic field is applied perpendicular to the direction of light propagation $[227, \S 2]$. 


\subsection{Alignment-to-orientation conversion in $J=1$ systems}

The analogies between $J>1 / 2 \mathrm{NMR} / \mathrm{NQR}$ and AMO systems can be better illustrated by considering a few examples of how these systems evolve under different experimental conditions. Some analytic equations for the time evolution of the density operator will be presented in spherical tensor notation for the case of continuous rf irradiation of $J=1$ nuclei in high-field NMR, where the nucleus is polarized either in a state of longitudinal magnetization or quadrupolar order. This case is analogous to that of a $J=1$ atomic state subjected to perpendicular static electric and magnetic fields or of a $J=1$ nucleus subjected to a magnetic field that is static in the laboratory frame and applied perpendicular to the EFG PAS. Next, the production of observable signal from quadrupolar order in the $J=1$ nuclear quadrupole resonance experiment will be explained as an alignment-to-orientation conversion process, which is a phenomenon that is well-known in atomic physics studies. The spherical tensor operator formalism will be used to develop an analytic expression for the powder-averaged $J=1 \mathrm{NQR}$ signal.

\subsubsection{Case of angular momentum $J=1$ in high-field NMR or in crossed electric and magnetic fields in atoms}

Consider the Hamiltonian in Eq. 5.12, which describes the NMR rotating frame dynamics of a single quadrupolar nucleus during rf irradiation. The case of atoms in perpendicular electric and magnetic fields can be obtained by substituting $\omega_{Q} \rightarrow-\omega_{E}, \omega_{1} \rightarrow \omega_{B}$ (Eq. 5.7). Eq. 5.12 can be diagonalized exactly for the three-level $J=1$ system to obtain 
the following eigenvalues and eigenvectors:

$$
\begin{aligned}
\hbar \omega_{1 Q}^{0} & =\frac{\hbar \omega_{Q}}{\sqrt{6}} \\
\hbar \omega_{1 Q}^{ \pm} & =\frac{\hbar}{2}\left[-\frac{\omega_{Q}}{\sqrt{6}} \pm \sqrt{\frac{3}{2} \omega_{Q}^{2}+4 \omega_{1}^{2}}\right] \\
\left|\psi_{0}\right\rangle & =-\frac{1}{\sqrt{2}}[|1,+1\rangle-|1,-1\rangle] \\
\left|\psi_{ \pm}\right\rangle & =\sqrt{n_{ \pm}}\left[|1,+1\rangle-\alpha_{ \pm}|1,0\rangle+|1,-1\rangle\right]
\end{aligned}
$$

where $\alpha_{ \pm}=\sqrt{2}\left(\omega_{1 Q}^{ \pm}-\omega_{1 Q}^{0}\right) / \omega_{1}$ and $n_{ \pm}=\left(2+\alpha_{ \pm}^{2}\right)^{-1}$. All spherical tensor operators in this discussion will be assumed to be normalized, e.g. $\hat{T}_{2,0}=\sqrt{\frac{1}{6}}\left[3 \hat{J}_{z}^{2}-J(J+1) \hat{1}\right]$.

First consider a system prepared in a state of longitudinal magnetization, oriented along the $+z$ direction. So, $\hat{\rho}(0)=\hat{J}_{z}$, neglecting proportionality constants and the term containing the identity operator. The results of the Hamiltonian diagonalization (Eqs. 5.16, 5.17) can be used to calculate the dynamics in the $\{|J, m\rangle\}$ basis, leading to:

$$
\begin{aligned}
\hat{\rho}(t)= & e^{-i t\left(\omega_{Q} \hat{T}_{2,0}-\omega_{1} \hat{J}_{x}\right)} \hat{J}_{z} e^{+i t\left(\omega_{Q} \hat{T}_{2,0}-\omega_{1} \hat{J}_{x}\right)} \\
= & 2\left\{n_{+} \cos \left[\left(\omega_{1 Q}^{+}-\omega_{1 Q}^{0}\right) t\right]+n_{-} \cos \left[\left(\omega_{1 Q}^{-}-\omega_{1 Q}^{0}\right) t\right]\right\} \hat{J}_{z} \\
& +2\left\{\alpha_{+} n_{+} \sin \left[\left(\omega_{1 Q}^{+}-\omega_{1 Q}^{0}\right) t\right]+\alpha_{-} n_{-} \sin \left[\left(\omega_{1 Q}^{-}-\omega_{1 Q}^{0}\right) t\right]\right\} \hat{J}_{y} \\
& -2\left\{\alpha_{+} n_{+} \cos \left[\left(\omega_{1 Q}^{+}-\omega_{1 Q}^{0}\right) t\right]+\alpha_{-} n_{-} \cos \left[\left(\omega_{1 Q}^{-}-\omega_{1 Q}^{0}\right) t\right]\right\} \hat{T}_{2,1 ; 0^{\circ}} \\
& +2 \sqrt{2}\left\{n_{+} \sin \left[\left(\omega_{1 Q}^{+}-\omega_{1 Q}^{0}\right) t\right]+n_{-} \sin \left[\left(\omega_{1 Q}^{-}-\omega_{1 Q}^{0}\right) t\right]\right\} \hat{T}_{2,2 ; 45^{\circ}}
\end{aligned}
$$

where the normalized Hermitian spherical tensor operator combinations are defined accord- 
ing to Eq. 5.2. This exact diagonalization/spherical tensor operator approach has also been applied to the problem of $J=3 / 2$ nuclei under $\mathrm{rf}$ irradiation $[228,229]$. As can be seen from Eq. 5.18, initial longitudinal magnetization oscillates into transverse magnetization $\hat{J}_{y}$ and into rank-2 single- and double-quantum coherences $\hat{T}_{2,1 ; 0^{\circ}}$ and $\hat{T}_{2,2 ; 45^{\circ}}$ at the quantum beat frequencies $\omega_{1 Q}^{ \pm}-\omega_{1 Q}^{0}$. Note that conversions of polarization moment rank would be forbidden in the absence of the quadrupolar (or Stark) splitting because the interaction with the magnetic field conserves rank (see $\S \mathrm{B}$ ), a phenomenon that is well-known in AMO studies of the Hanle effect [227]. On the other hand, longitudinal magnetization would not evolve under the first-order quadrupolar Hamiltonian $\hat{H}_{Q}^{(1)} \propto \hat{T}_{2,0}$ alone. Only the combination of electric quadrupolar and magnetic interactions can convert longitudinal magnetization to rank-2 polarization.

Similar results can be obtained in the case of a system initially aligned into a state of quadrupolar order, where the difference density operator is $\hat{\rho}(0)=\hat{T}_{2,0}$ (neglecting proportionality constants). In this case the time evolution can be solved simply by resumming the series in Eq. 2.14 after employing the relevant commutation relationships between the spherical tensor operators [198]:

$$
\begin{aligned}
\hat{\rho}(t)= & e^{-i t\left(\omega_{Q} \hat{T}_{2,0}-\omega_{1} \hat{J}_{x}\right)} \hat{T}_{2,0} e^{+i t\left(\omega_{Q} \hat{T}_{2,0}-\omega_{1} \hat{J}_{x}\right)} \\
= & \left\{1-\frac{3 \omega_{1}^{2}}{\omega_{n}^{2}}\left[1-\cos \left(\omega_{n} t\right)\right]\right\} \hat{T}_{2,0}+\frac{\sqrt{3} \omega_{1}}{\omega_{n}} \sin \left(\omega_{n} t\right) \hat{T}_{2,1 ; 90^{\circ}} \\
& \quad-\frac{\sqrt{3} \omega_{1}^{2}}{\omega_{n}^{2}}\left[1-\cos \left(\omega_{n} t\right)\right] \hat{T}_{2,2 ; 0^{\circ}}-\frac{3 \omega_{Q} \omega_{1}}{2 \omega_{n}^{2}}\left[1-\cos \left(\omega_{n} t\right)\right] \hat{J}_{x},
\end{aligned}
$$

where $\omega_{n}=\omega_{1 Q}^{+}-\omega_{1 Q}^{-}=\sqrt{\frac{3}{2} \omega_{Q}^{2}+4 \omega_{1}^{2}}$. The mutual influence of the quadrupolar Hamiltonian and the orthogonal magnetic field partially converts initial alignment into orientation 
along the magnetic field axis $x$.

\subsubsection{Nuclear quadrupole resonance in $J=1$ systems: alignment-to- orientation conversion via rf pulses and free precession}

The origin of the signal in the NQR experiment also can be understood as an alignment-to-orientation conversion (AOC) process. AOC phenomena are well known among atomic physicists in the context of atomic dynamics in electric and magnetic fields [230, $231,222,232,233,234]$. Consider the case of a $J=1$ quadrupolar nucleus in a single crystallite at zero magnetic field, assuming zero EFG asymmetry $\left(\eta_{Q}=0\right)$. The axially

symmetric quadrupolar Hamiltonian $\hat{H}_{Q}=\omega_{Q} \hat{T}_{2,0}$ has the same form as the high-field NMR quadrupolar Hamiltonian (however, with a different definition of $\omega_{Q}$ ), and from Eq. 5.16 with $\omega_{1}=0$ the eigenvalues are determined to be $\hbar \omega_{Q} / \sqrt{6}, \hbar \omega_{Q} / \sqrt{6}$, and $-2 \hbar \omega_{Q} / \sqrt{6}$. The double degeneracy means there is only one unique single-quantum transition frequency, at $3\left|\omega_{Q}\right| / \sqrt{6}$.

The evolution during a linearly-polarized radiofrequency pulse is given by Eq. 5.10, where the quantization axis $z$ is along the crystallite EFG PAS. The typical case in NQR is $\left|\omega_{Q}\right| \gg\left|\omega_{1}\right|$, so it is appropriate to go into the interaction frame of the quadrupolar Hamiltonian, defined by $\hat{V}_{Q}(t)=\exp \left(-i \omega_{Q} t \hat{T}_{2,0}\right)$ (see $\left.\S 2.3 .2\right)$. The effective Hamiltonian in 
the interaction frame is:

$$
\begin{aligned}
\hat{\widetilde{H}}(t) / \hbar= & \hat{V}_{Q}^{-1}(t)\left[-\omega_{1}\left(a_{x} \hat{J}_{x}+a_{y} \hat{J}_{y}+a_{z} \hat{J}_{z}\right) \cos \omega_{r} t\right] \hat{V}_{Q}(t) \\
= & -\omega_{1} \cos \left(\omega_{r} t\right)\left[\left(a_{x} \hat{J}_{x}+a_{y} \hat{J}_{y}\right) \cos \left(\frac{3 \omega_{Q} t}{\sqrt{6}}\right)\right. \\
& \left.\quad-\sqrt{2}\left(a_{x} \hat{T}_{2,1 ; 90^{\circ}}-a_{y} \hat{T}_{2,1 ; 0^{\circ}}\right) \sin \left(\frac{3 \omega_{Q} t}{\sqrt{6}}\right)+a_{z} \hat{J}_{z}\right] .
\end{aligned}
$$

If the rf frequency is set on-resonance to the transition frequency $\omega_{r}=3\left|\omega_{Q}\right| / \sqrt{6}$, then Eq. 5.20 contains a time-independent piece:

$$
\hat{\widetilde{\widetilde{H}}} / \hbar=-\frac{1}{2} \omega_{1}\left(a_{x} \hat{J}_{x}+a_{y} \hat{J}_{y}\right)
$$

where this approximate effective Hamiltonian holds for $\left|\omega_{Q}\right| \gg\left|\omega_{1}\right|$. This result corresponds to the lowest-order Magnus expansion (average Hamiltonian) approximation (see §2.4). Eq. 5.21 demonstrates that the resonant NQR pulse in the quadrupolar interaction frame looks like the transverse component of a static $\underline{B}_{1}$ field scaled down by $1 / 2$. Upon transforming back out of the interaction frame, the time propagator during the pulse under this approximate Hamiltonian is given by (see $\S 2.4)$ :

$$
\hat{U}_{\text {pulse }}(t) \simeq \exp \left[-i \omega_{Q} t \hat{T}_{2,0}\right] \exp \left[+i \omega_{1} t\left(a_{x} \hat{J}_{x}+a_{y} \hat{J}_{y}\right) / 2\right]
$$

where the rf pulse is assumed to be turned on at time $t_{0}=0$. In this approximation, the time evolution factors into an apparent precession about an effective field $\underline{B}_{1}^{\text {eff }}=$ $\frac{1}{2} B_{1}^{e f f}\left(a_{x} \underline{\widetilde{x}}+a_{y} \underline{\widetilde{y}}\right)$, followed by evolution under the quadrupolar Hamiltonian, which can be 
added to any period of quadrupolar free precession after the pulse. ${ }^{6}$ Note that this result is really only valid when the system is observed stroboscopically with the quadrupolar quantum beat frequency. The effective field strength is $B_{1}^{e f f}=\frac{1}{2} B_{1} \sqrt{a_{x}^{2}+a_{y}^{2}}=B_{1}^{\perp} / 2$, where $B_{1}^{\perp}$ is the transverse component of $\underline{B}_{1}$. The cycle time of $\hat{V}_{Q}(t)$ is $t_{c}=2 \pi \sqrt{6} / \omega_{Q}$, so an average Hamiltonian treatment may be used to extend the time evolution to pulses of length $t=n t_{c}$, where $n$ is a positive integer and $\hat{V}_{Q}\left(n t_{c}\right)=\hat{1}$. This approach to the treatment of NQR pulses (and also the following approach to the calculation of the NQR signal) may be compared to the approaches of Refs. [225, 235, 236, 237, 238, 239].

Two questions must be answered in order to understand the creation of NQR signal: how is the initial quadrupolar order converted to observable magnetization, and how does net polarization arise in a powder sample from an initial random distribution of polarized nuclei? These questions can be answered by an examination of Eqs. 5.21 and 5.22. As was mentioned in $§ 5.2 .1$, unlike the high-field NMR case in which each nucleus is oriented along the external magnetic field axis, at zero field each nucleus is aligned along its local EFG principal axis; this results in no net alignment (or any other polarization) in a random powder. Eq. 5.21 demonstrates that the strong quadrupolar interaction truncates a weak resonant $\mathrm{rf}$ magnetic field, such that half of the transverse component of the rf field and all of the longitudinal component in the EFG PAS is ineffective at rotating the nuclear polarization. ${ }^{7}$ The effective magnetic field rotates the polarization by an angle proportional to $B_{1}^{\perp}$, the magnitude of which depends on the angle between the magnetic

\footnotetext{
${ }^{6}$ The magnetic resonance term "free precession" is used here to mean any period of evolution in the absence of a driving rf field, even though the dynamics under the quadrupolar Hamiltonian are more complicated than simple precession.

${ }^{7}$ However, this is not the same as the NMR case in which the effective loss of one half the linearlypolarized rf field corresponds to the selection of one circularly-polarized rf component. NQR excitation and detection is linearly polarized, unlike NMR which has circularly-polarized excitation and detection [235].
} 
field and the EFG principal axis. If the nuclear polarization was rotated by the same angle in every crystallite, the initial random polarization distribution would be rotated into another random distribution. However, the fact that the nuclear polarization in each crystallite is rotated by a different orientation-dependent angle during the pulse results in a partial ordering of the random ensemble and a net nuclear alignment [240].

The rotation of the alignment serves another purpose other than producing a net polarization in the sample. Every nucleus whose EFG principal axis is not parallel to the rf magnetic field experiences a rotation of its alignment away from that equilibrium axis. This rotation prepares each nucleus in a state of polarization that no longer commutes with the quadrupolar Hamiltonian, so the polarization evolves due to the quadrupolar interaction even after the rf field has been turned off. Put another way, the pulse causes a rotation that converts initial $\hat{T}_{2,0}$ order into some linear combination of $\hat{T}_{2, q}$ polarization. In particular, the $\hat{T}_{2, \pm 1}$ polarization will evolve into $\hat{T}_{1, \pm 1}$ orientation, while the $\hat{T}_{2,0}$ and $\hat{T}_{2, \pm 2}$ terms do not evolve under the quadrupolar Hamiltonian $\hat{H}_{Q}=\hbar \omega_{Q} \hat{T}_{2,0}$. The conversion of alignment into orientation corresponds to the creation of magnetization from quadrupolar order; the net oscillating magnetization of the sample is what creates the detected NQR signal. The oscillations occur at the quantum beat frequency $3\left|\omega_{Q}\right| / \sqrt{6}$, which corresponds to the energy difference between the $|J=1, m= \pm 1\rangle$ states and the $|J=1, m=0\rangle$ state. Therefore, a Fourier transform of this signal gives a line at the frequency of the quadrupolar transition. The NQR alignment-to-orientation conversion process can be visualized easily via the method described at the end of $\S 5.2[240]$.

It is instructive to calculate the NQR signal in a random powder using the methods 
described above. The signal due to a single crystallite can be calculated either in the laboratory frame or in the EFG PAS:

$$
\begin{aligned}
\hat{S}(t) & =\operatorname{Tr}\left[\hat{\rho}^{L A B}(t) \hat{\mathcal{O}}^{L A B}\right] \\
& =\operatorname{Tr}\left[\hat{\rho}^{P A S}(t) \hat{\mathcal{O}}^{P A S}\right]=\operatorname{Tr}\left[\hat{U}^{P A S}(t) \hat{\rho}^{P A S}(0) \hat{U}^{P A S}(t) \hat{\mathcal{O}}^{P A S}\right],
\end{aligned}
$$

where $\hat{\mathcal{O}}$ is the observable, and $\hat{U}(t)$ is the time development operator. Since the initial density operator and the dynamics under an rf pulse are already known in the EFG PAS, it is convenient to evaluate Eq. 5.23 in this frame. There is no natural direction in the laboratory frame except along the rf magnetic field direction, so the $z$-axis will be defined to be along $\hat{B}_{1}$. The $z$-axis is the rf coil axis, and the same coil is assumed to be used for both excitation and detection. In this case, the observable in the laboratory frame $\hat{\mathcal{O}}^{L A B}$ is proportional to $\hat{J}_{z}$, which in the EFG PAS is already known to be

$$
\hat{\mathcal{O}}^{P A S} \propto a_{x} \hat{J}_{x}+a_{y} \hat{J}_{y}+a_{z} \hat{J}_{z}
$$

It will be convenient to define two spherical polar angles, $\alpha=\tan ^{-1}\left(a_{y} / a_{x}\right)$ and $\beta=$ $\cos ^{-1}\left(a_{z}\right)$, which relate the EFG principal axis to the laboratory $z$-axis (i.e., the direction of the $\underline{B}_{1}$ field), where $a_{x}=\sin \beta \cos \alpha, a_{y}=\sin \beta \sin \alpha$, and $a_{z}=\cos \beta$. Here $\beta$ is the angle between the laboratory and EFG PAS $z$-axes (i.e., the angle between $\underline{B}_{1}$ and the EFG principal axis). 
The initial difference density operator is:

$$
\hat{\rho}^{P A S}(0) \propto \hat{T}_{2,0}
$$

corresponding to thermal equilibrium quadrupolar order for an axially-symmetric EFG.

The dynamics will be calculated under the action of an $\mathrm{rf}$ pulse of duration $\tau$ followed by evolution for time $t$ under the quadrupolar Hamiltonian. Eq. 5.22 can be used to approximate the time development operator for the rf pulse. The total time development operator is:

$$
\begin{aligned}
\hat{U}^{P A S}(t) & =e^{-i \hat{H}_{Q}^{P A S} t / \hbar} \hat{U}_{\text {pulse }}(\tau) \\
& \simeq \exp \left[-i \omega_{Q} t \hat{T}_{2,0}\right]\left\{\exp \left[-i \omega_{Q} \tau \hat{T}_{2,0}\right] \exp \left[+i \omega_{1} \tau\left(a_{x} \hat{J}_{x}+a_{y} \hat{J}_{y}\right) / 2\right]\right\} \\
& =\exp \left[-i \omega_{Q}(t+\tau) \hat{T}_{2,0}\right] \exp \left[+i \omega_{1} \tau\left(a_{x} \hat{J}_{x}+a_{y} \hat{J}_{y}\right) / 2\right] \\
& =\hat{U}_{Q} \hat{U}_{r}
\end{aligned}
$$

where $\hat{U}_{r}=\exp \left[+i \omega_{1} \tau\left(a_{x} \hat{J}_{x}+a_{y} \hat{J}_{y}\right) / 2\right]=\exp \left[+i \omega_{1} \tau\left(\sin \beta \cos \alpha \hat{J}_{x}+\sin \beta \sin \alpha \hat{J}_{y}\right) / 2\right]$ corresponds to a rotation about the static effective magnetic field $\underline{B}_{1}^{e f f}$, and $\hat{U}_{Q}=\exp \left[-i \omega_{Q}(t+\right.$ $\left.\tau) \hat{T}_{2,0}\right]$ corresponds to evolution under the quadrupolar Hamiltonian. One can write $\hat{U}_{r}=$ $\exp \left[-i \alpha \hat{J}_{z}\right] \exp \left[+i\left(\omega_{1} \tau \sin \beta / 2\right) \hat{J}_{x}\right] \exp \left[+i \alpha \hat{J}_{z}\right]$ using the identity $\hat{U} f(\hat{A}) \hat{U}^{-1}=f\left(\hat{U} \hat{A} \hat{U}^{-1}\right)$, 
and the application of the spherical tensor operator transformations of Ref. [198] yield: ${ }^{8}$

$$
\begin{aligned}
\hat{\rho}_{r}= & \hat{U}_{r}\left[\hat{\rho}^{P A S}(0)\right] \hat{U}_{r}^{-1} \\
= & \frac{1}{4}\left[1+3 \cos \left(\omega_{1} \tau \sin \beta\right)\right] \hat{T}_{2,0}+\frac{\sqrt{3}}{2} \sin \left(\omega_{1} \tau \sin \beta\right)\left[\hat{T}_{2,1 ; 90^{\circ}} \cos \alpha-\hat{T}_{2,1 ; 0^{\circ}} \sin \alpha\right] \\
& -\frac{\sqrt{3}}{4}\left[1-\cos \left(\omega_{1} \tau \sin \beta\right)\right]\left[\hat{T}_{2,2 ; 0^{\circ}} \cos 2 \alpha+\hat{T}_{\left.2,2 ; 45^{\circ} \sin 2 \alpha\right]}\right.
\end{aligned}
$$

where the transformation under the first $z$-rotation is simple because it commutes with $\hat{\rho}^{P A S}(0)=\hat{T}_{2,0} ;$ and

$$
\begin{aligned}
\hat{\rho}^{P A S}(t)= & \hat{U}_{Q} \hat{\rho}_{r} \hat{U}_{Q}^{-1} \\
= & \frac{1}{4}\left[1+3 \cos \left(\omega_{1} \tau \sin \beta\right)\right] \hat{T}_{2,0} \\
& +\frac{\sqrt{3}}{2} \sin \left(\omega_{1} \tau \sin \beta\right)\left\{\cos \alpha\left[\cos \left(\frac{3 \omega_{Q}}{\sqrt{6}}(t+\tau)\right) \hat{T}_{2,1 ; 90^{\circ}}-\frac{1}{\sqrt{2}} \sin \left(\frac{3 \omega_{Q}}{\sqrt{6}}(t+\tau)\right) \hat{J}_{x}\right]\right. \\
& \left.\quad-\sin \alpha\left[\cos \left(\frac{3 \omega_{Q}}{\sqrt{6}}(t+\tau)\right) \hat{T}_{2,1 ; 0^{\circ}}+\frac{1}{\sqrt{2}} \sin \left(\frac{3 \omega_{Q}}{\sqrt{6}}(t+\tau)\right) \hat{J}_{y}\right]\right\} \\
& -\frac{\sqrt{3}}{4}\left[1-\cos \left(\omega_{1} \tau \sin \beta\right)\right]\left[\hat{T}_{2,2 ; 0^{\circ}} \cos 2 \alpha+\hat{T}_{\left.2,2 ; 45^{\circ} \sin 2 \alpha\right],}\right.
\end{aligned}
$$

where only the $\hat{T}_{2, \pm 1}$ operators are affected by $\hat{U}_{Q}$ since $\left[\hat{T}_{2,0}, \hat{T}_{2,0}\right]=0$ and $\left[\hat{T}_{2,0}, \hat{T}_{2, \pm 2}\right]=0.9$ The appearance of $\hat{J}_{x}$ and $\hat{J}_{y}$ orientation is due to the conversion of the rotated alignment in Eq. 5.27 during evolution under the quadrupolar Hamiltonian. This magnetization has a projection on the laboratory-frame $z$-axis (the coil detection axis), which may be calculated

\footnotetext{
${ }^{8}$ Also note that the identity $\exp \left[-i \phi \hat{J}_{z}\right] \hat{T}_{k, q} \exp \left[+i \phi \hat{J}_{z}\right]=\exp [-i q \phi] \hat{T}_{k, q}$ leads to the relation $\exp \left[-i \phi \hat{J}_{z}\right]\left[\hat{T}_{k, q} \pm \hat{T}_{k,-q}\right] \exp \left[+i \phi \hat{J}_{z}\right]=\cos (q \phi)\left[\hat{T}_{k, q} \pm \hat{T}_{k,-q}\right]-i \sin (q \phi)\left[\hat{T}_{k, q} \mp \hat{T}_{k,-q}\right]$.

${ }^{9}$ Recall that the entire double-quantum line-narrowing premise of Chapter 4 relies on the fact that $J=1$ double-quantum coherences $\hat{T}_{2, \pm 2}$ commute with the first order quadrupolar Hamiltonian $\propto \hat{T}_{2,0}$.
} 
using Eqs. 5.23 and 5.24 to yield the detected signal of a single crystallite:

$$
\begin{aligned}
S(t) & \propto \operatorname{Tr}\left[\hat{\rho}^{P A S}(t) \hat{\mathcal{O}}^{P A S}\right] \\
& =\operatorname{Tr}\left[\hat{\rho}^{P A S}(t)\left(\sin \beta \cos \alpha \hat{J}_{x}+\sin \beta \sin \alpha \hat{J}_{y}+\cos \beta \hat{J}_{z}\right)\right] \\
& =-\sqrt{\frac{3}{2}} \sin \left[\omega_{1} \tau \sin (\beta)\right] \sin (\beta) \sin \left[\frac{3 \omega_{Q}}{\sqrt{6}}(t+\tau)\right]
\end{aligned}
$$

which is independent of the crystallite angle $\alpha$. The net signal can be obtained by averaging over crystallite orientations:

$$
\begin{aligned}
\langle S(t)\rangle & =\frac{1}{\int d \Omega}\left[\int d \Omega S(t)\right] \\
& =-\frac{1}{4 \pi} \int_{0}^{2 \pi} d \alpha \int_{0}^{\pi} d \beta \sin (\beta)\left\{\sqrt{\frac{3}{2}} \sin \left[\omega_{1} \tau \sin (\beta)\right] \sin (\beta) \sin \left[\frac{3 \omega_{Q}}{\sqrt{6}}(t+\tau)\right]\right\} \\
& =-\frac{1}{2} \sqrt{\frac{3}{2}} \sin \left[\frac{3 \omega_{Q}}{\sqrt{6}}(t+\tau)\right] \int_{0}^{\pi} d \beta \sin ^{2}(\beta) \sin \left[\omega_{1} \tau \sin (\beta)\right] \\
& =-\frac{\pi}{2} \sqrt{\frac{3}{2}}\left[\mathbf{H}_{0}\left(\omega_{1} \tau\right)-\frac{1}{\omega_{1} \tau} \mathbf{H}_{1}\left(\omega_{1} \tau\right)\right] \sin \left[\frac{3 \omega_{Q}}{\sqrt{6}}(t+\tau)\right]
\end{aligned}
$$

where $\mathbf{H}_{n}(x)$ is the Struve function of order $n[241, \S 12]$. The integral over $\beta$ was evaluated using Mathematica ${ }^{\circledR}[242]$. As expected, the bulk NQR signal $\langle S(t)\rangle$ oscillates sinusoidally at the sole (for $\eta_{Q}=0$ ) NQR transition frequency $\Delta \omega=3 \omega_{Q} / \sqrt{6}$. The sine modulation of the NQR signal is $90^{\circ}$ out of phase with the cosine-modulated excitation field. The pulse width/rf amplitude can be varied to optimize the NQR signal. The amplitude $\langle S\rangle_{0}=\frac{\pi}{2} \sqrt{\frac{3}{2}}\left[\mathbf{H}_{0}(x)-\mathbf{H}_{1}(x) / x\right]$ is plotted as a function of $x=\omega_{1} \tau$ in Fig. 5.1. The result corresponds to damped oscillation, compared to sinusoidal oscillation as a function of $\omega_{1} \tau \sin (\beta)$ in the case of a single crystallite. This behavior is qualitatively consistent 
with that of a calculation done by Lee [225], but the numerical values differ. For instance, Eq. 5.30 predicts a signal maximum at the tip angle $\omega_{1} \tau=1.7729=101.6^{\circ}$ and a signal inversion at $\omega_{1} \tau=5.2172=298.9^{\circ}$, whereas Ref. [225] predicts $119^{\circ}$ and $339^{\circ}$, respectively. The source of the discrepancy is unclear; the details of the numerical calculation in Ref. [225] were not given. Note that the results that have been obtained here only hold for $J=1$ nuclei with $\eta_{Q}=0$ and $\left|\omega_{Q}\right| \gg\left|\omega_{1}\right|$.

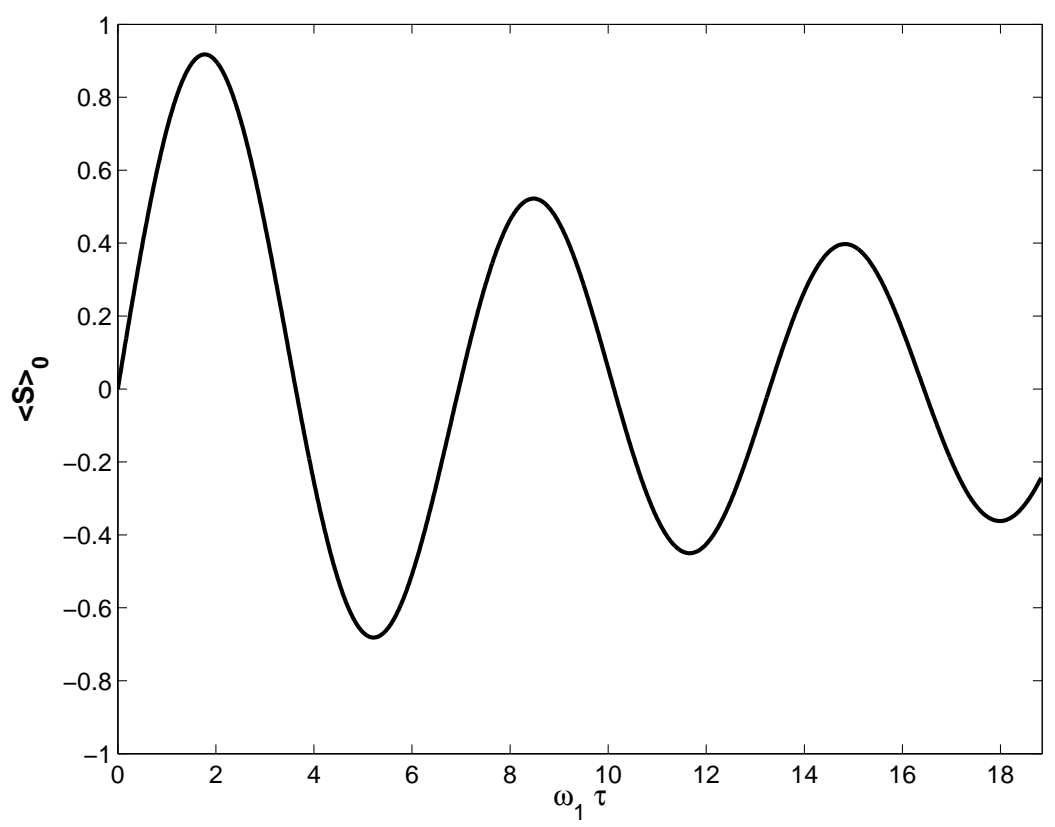

Figure 5.1: Plot of the function $\langle S\rangle_{0}=\frac{\pi}{2} \sqrt{\frac{3}{2}}\left[\mathbf{H}_{0}\left(\omega_{1} \tau\right)-\mathbf{H}_{1}\left(\omega_{1} \tau\right) /\left(\omega_{1} \tau\right)\right]$ versus $\omega_{1} \tau$, corresponding to the calculated powder-averaged $J=1 \mathrm{NQR}$ signal as a function of rf pulse length or amplitude. 


\subsection{NMR methods of manipulating polarization in quadrupo- lar nuclei with magnetic fields}

The high-field NMR of quadrupolar nuclei poses an interesting problem: how can the nuclear polarization be manipulated efficiently when the experimenter is usually only able to apply magnetic fields with strengths much smaller than the quadrupolar interaction? ${ }^{10}$ As has already been mentioned, the high-field NMR case corresponds to the atomic case in crossed electric and magnetic fields. Therefore, some of the techniques of high-field quadrupolar NMR may be useful to atomic physicists working with crossed electric and magnetic field geometries. The NMR techniques are probably most applicable to atomic systems for which the Stark splitting exceeds the Zeeman splitting by no more than one or possibly two orders of magnitude; this is because the NMR methods become inefficient when the quadrupolar coupling exceeds the rf field strength by about that amount. Most of these types of NMR techniques have been developed for systems of half-integer angular momentum, since the vast majority of quadrupolar nuclei have half-integer spin in their ground state.

The variables most typically under the NMR experimentalist's control are the amplitude, frequency, and phase of the radiofrequency radiation. The following discussion will neglect the possibilities of phase and frequency modulation and will only consider the amplitude modulation of the rf field. The size of the quadrupolar coupling is a physical property of the material and is generally not under experimental control, since it is not currently experimentally possible to apply electric field gradients to nuclei that exceed the

\footnotetext{
${ }^{10}$ The case of adiabatic demagnetization and remagnetization treated in the next section is one example where the magnetic field splittings must at some point exceed the quadrupolar splitting.
} 
local field gradients on the atomic scale. However, it is possible to modulate local electric field gradients by using an oscillating electric field to induce motion on the atomic level [243]; this technique has been used to excite quadrupolar transitions [244, 245, 246] and even to induce adiabatic demagnetization and remagnetization [247, 248], but these effects will not be considered here. ${ }^{11}$ It is much more common to induce a harmonic modulation of the quadrupolar coupling through sample spinning, usually about an axis that is at the magic angle with respect to the polarization field.

The general form of the rotating frame Hamiltonian in the case of a modulated quadrupolar coupling and rf pulse of varying amplitude when the second-order quadrupolar and resonance offset terms can be neglected is:

$$
\hat{H}(t) / \hbar=\omega_{Q}(t) \hat{T}_{2,0}-\omega_{1}(t) \hat{J}_{x}
$$

The magnetic field can be varied in a laboratory frame AMO experiment in exactly the same way as in a rotating frame NMR experiment, but it remains to be seen how similar NMR sample spinning is to the modulation of the Stark field in atomic and molecular systems.

\section{The dynamic Stark effect and quadrupolar couplings in rotating solids}

The theory of the quadratic Stark effect in a constant electric field has been long known and, for instance, has been applied to explain the angular momentum dynamics in hyperfine-coupled systems $[249,250]$. This theory, which has been extended to the case of oscillating electric fields in what is known as the AC or dynamic Stark effect, and was first

\footnotetext{
${ }^{11}$ It is also possible to achieve a similar effect via a ultrasonic (acoustic) excitation, see [6, §IX.IV.D] and references therein.
} 
applied to describe the so-called light shifts due to the oscillating optical field in hyperfinecoupled systems, particularly in the case of optical pumping experiments $[251,252,253$, $254,255,256]$. The theory of light shifts in hyperfine-coupled systems is interesting in its own right, as the effective Hamiltonian bears many resemblances to those of NMR systems [254]. However, the situation to be considered here simply corresponds to the Hamiltonian in Eq. 5.6 for the case of a time dependent electric field $\underline{E}(t)$, which, in the case of an optical field, corresponds to the light shift induced by virtual transitions. Electric dipole transitions between angular momentum manifolds will not be considered explicitly in this section (e.g., coherent excitation of the optical transition will be neglected). Rather, only the dynamics of the manifold of a particular angular momentum $J$ will be examined. Therefore, the scalar polarizability term in the Stark Hamiltonian again will not be considered in the following discussion.

The Stark splitting depends on the square of the absolute value of the electric field $|\underline{E}(t)|^{2}$. In the case of a constant-magnitude linearly-polarized optical field $\underline{E}(t)=$ $E_{0} \cos \left(\omega_{0} t\right) \underline{\widetilde{z}}$ (where $\omega_{0}$ is the laser frequency), $|\underline{E}(t)|^{2}=\left|E_{0}\right|^{2} \cos ^{2}\left(\omega_{0} t\right)$. However, if the laser frequency is much larger than the characteristic field-induced dynamical frequencies of the system (i.e., $\left.\left|\omega_{0}\right| \gg\left|\omega_{E}\right|,\left|\omega_{B}\right|\right)$ and does not coincide with a resonance of the optical medium, it is acceptable to replace the $\cos ^{2}\left(\omega_{0} t\right)$ term with $1 / 2$, its time-averaged value [257]. Therefore, the effective electric field again looks time independent. Note that the situation is much more complicated if there is a modulation of $\underline{E}(t)$ that is fast enough such that its frequency is comparable to an atomic linewidth or even to a transition frequency $[258, \S 10]$. These effects will not be considered here, but it is important to remember the 
complexity that arises near a resonance. For example, one can effectively modify the tensor polarizability $\alpha_{2}$ (see Eq. 5.6) to be a function of the resonance detuning of the laser. The sign of the quadratic Stark coupling can be inverted by sweeping the laser frequency through the resonance by changing the sign of the effective polarizability, even though $|\underline{E}(0)|^{2}$ itself does not change sign.

Consider the case where the optical field contains a slow, amplitude-modulated component at a frequency $\omega_{R}$, perhaps as a result of a binary (on-off) modulation by an optical chopper. If the modulation is approximated as sinusoidal, then $E(t)=\frac{1}{2} E_{0}[1+$ $\left.\cos \left(\omega_{R} t\right)\right] \cos \left(\omega_{0} t\right)$, in which case $|\underline{E}(t)|^{2} \simeq \frac{1}{4}\left|E_{0}\right|^{2}\left[3+4 \cos \left(\omega_{R} t\right)+\cos \left(2 \omega_{R} t\right)\right]$, where the relation $\overline{\cos ^{2}\left(\omega_{0} t\right)} \simeq \frac{1}{2}$ has been used. Therefore, for simple sinusoidal amplitude modulations, it would be reasonable to write the time-dependent tensor Stark splitting frequency as:

$$
\omega_{E}(t)=\omega_{E} \sum_{n=-2}^{2} c_{n}^{E} e^{i n \omega_{R} t},
$$

where $\omega_{E}=-\frac{1}{2} \alpha_{2} \overline{\left|\underline{E}_{0}\right|^{2}}$. This Fourier expansion can be extended to include higher harmonics of $\omega_{R}$ if necessary. Since $\omega_{E}(t) \propto|\underline{E}(t)|^{2}, \omega_{E}(t)$ itself does not undergo any sign inversions during its oscillation.

This result can be compared to the modulation of the first-order quadrupolar Hamiltonian under sample spinning conditions at high magnetic fields. A rotation of a typical crystallite in a solid sample at frequency $\omega_{R}$ induces a modulation of the quadrupolar 
coupling via a modulation of the components of the rank-2 electric field gradient tensor:

$$
\omega_{Q}(t)=\omega_{Q} \sum_{n=-2}^{2} c_{n}^{Q} e^{i n \omega_{R} t},
$$

where the coefficients $c_{n}^{Q}$ depend on the crystallite orientation. However, at the magic angle $\omega_{Q}(t)$ contains no zero-frequency component, and the quadrupolar Hamiltonian is modulated only at $\omega_{R}$ and $2 \omega_{R}$. Therefore the time average of $\omega_{Q}(t)$ is zero over one rotor period $\tau_{R}=2 \pi / \omega_{R}$, ensuring that $\omega_{Q}(t)$ undergoes at least two sign inversions over the course of a rotor cycle. The timing of the zero-crossings depends on crystallite orientation (i.e., the inversions are not synchronized among nuclei in different crystallites), and the actual number of sign inversions can be two or four $[42,259]$. As will be seen later, this inversion of the quadrupolar splitting via magic-angle sample spinning can be exploited in NMR experiments in ways important ways that can be emulated in AMO systems only by sweeping the laser frequency through a resonance.

\subsubsection{Efficient alignment-to-orientation conversion via adiabatic variation of the magnetic field: ARRF and ADRF}

It has long been known that in the solid state, a collection of nuclear spins that is prepared in a state of Zeeman order (longitudinal magnetization, orientation) at thermal equilibrium in the presence of a dominant magnetic field $\underline{B}_{0}$ can be demagnetized by decreasing $\underline{B}_{0}$ adiabatically (in the thermodynamic sense) to zero such that the polarization is converted into local order $[5, \S 6.4]$. In the case of $J>1 / 2$ nuclei the quadrupolar interaction dominates, and the Zeeman order is transformed into quadrupolar order along the 


\subsection{NMR METHODS OF MANIPULATING POLARIZATION IN QUADRUPOLAR NUCLEI WITH MAGNETIC FIELDS}

local EFG principal axis as was discussed previously in the case of zero-field NQR. This adiabatic demagnetization is an example of thermodynamic orientation-to-alignment conversion in the laboratory frame, and it can be reversed by adiabatically converting alignment back into orientation in a process called adiabatic (re)magnetization. However, in modernday NMR experiments involving superconducting magnets, it is generally not possible to decrease and increase the Zeeman field to zero at will, much less adiabatically. Methods that involve removing the sample from the field adiabatically or re-inserting it in a similar manner would require sample manipulations that are experimentally difficult, to say the least.

Not surprisingly, NMR spectroscopists have figured out how to apply rf magnetic fields to perform these conversions quantum-mechanically while the $\underline{B}_{0}$ field remains constant. The resulting techniques are called adiabatic demagnetization in the rotating frame $(\mathrm{ADRF})$ and adiabatic remagnetization in the rotating frame (ARRF). The ADRF technique was initially used to convert Zeeman order into dipolar order [260], where "dipolar order" refers to polarization that commutes with the dipole-dipole coupling Hamiltonian. ${ }^{12}$ However, these methods also can be used to convert Zeeman order into quadrupolar order and back again, and the theory of these conversions is well understood [264]. The quadrupolar order is created along the spin quantization axis parallel to $\underline{B}_{0}$.

The ADRF technique is applied after the preparation of magnetization (orientation) that is transverse to the laboratory-frame quantization axis $z$, e.g. $\hat{\rho}(0) \propto \hat{J}_{x} \cdot{ }^{13}$

\footnotetext{
${ }^{12}$ Adiabatic techniques have also been used to create " $J$-order" in $J$-coupled systems in liquids [261, 262, $263]$.

${ }^{13}$ This could be accomplished in an NMR experiment by applying a "hard" $90^{\circ} \mathrm{rf}$ pulse along the $y$-axis in the rotating frame to initial equilibrium $z$-magnetization; i.e., $\left|\omega_{1}\right| \gg\left|\omega_{Q}\right|$ and $\omega_{1} \tau_{p}=\pi / 2$, where $\tau_{p}$ is the pulse duration.
} 
An initially large rf magnetic field is applied along the orientation axis to "spin lock" the magnetization along the $x$-axis in the rotating frame, such that $\hat{H}_{r f}^{r o t}(0) \simeq-\hbar \omega_{1}(0) \hat{J}_{x}$, with $\left|\omega_{1}(0)\right| \gg\left|\omega_{Q}\right|$. The quadrupolar splitting $\omega_{Q}$ is constant during this experiment, and the necessity of applying such a strong rf field (stronger than the quadrupolar interaction) would make this technique inefficient in systems with large quadrupolar couplings but might find an ideal application in studying molecules in solution-state oriented media in which the quadrupolar coupling is motionally averaged to a smaller value. In the limit $\left|\omega_{1}(0)\right| \gg\left|\omega_{Q}\right|$ the rotating-frame quantization axis is along $x$, and ADRF is induced by adiabatically decreasing the rf field until $\left|\omega_{1}\left(\tau_{p}\right)\right| \ll\left|\omega_{Q}\right|$ and the rotating frame Hamiltonian is $\hat{H}_{r f}^{r o t}\left(\tau_{p}\right) \simeq \hbar \omega_{Q} \hat{T}_{2,0}$, rotating the rotating-frame quantization axis to the $z$-direction and creating quadrupolar order (alignment) $\hat{\rho}\left(\tau_{p}\right) \propto \hat{T}_{2,0}$. Ref. [264] shows how the adiabatic pulse shapes $\omega_{1}(t)$ can be designed for $J=1$ and $J=3 / 2$ systems after a transformation into a rotating-frame basis where the angular momentum is quantized along the $x$-axis. This reduces the dynamics to that of an effective spin-1/2 (in the case of $J=1$ ) or two isolated spin-1/2 angular momenta (in the case of $J=3 / 2$ ). ARRF can be accomplished with an amplitude variation $\omega_{1}(t)$ that is time-reversed from the ADRF case.

The authors of Ref. [264] note that in $J=1$ and $J=3 / 2$ systems ADRF creates $\hat{T}_{2,0}$ alignment from $\hat{J}_{x}$ orientation in a process that saturates the unitary bound, i.e., it creates the maximal amount of $\hat{T}_{2,0}$ allowed by a unitary (e.g., quantum-mechanical) evolution. It also creates $\hat{T}_{2, \pm 2}$ double-quantum coherence in $J=1$ systems and rank-3 single- and triple-quantum coherences $\hat{T}_{3, \pm 1}$ and $\hat{T}_{3, \pm 3}$ in $J=3 / 2$ systems, while leaving some residual transverse magnetization in both cases. The ARRF procedure also effects 
alignment-to-orientation conversion with a saturation of the unitary limit in both systems.

There exist other pulse sequences that also can convert between orientation and alignment; in fact, §5.5.1 demonstrated how a single weak rf pulse can cause these interconversions in $J=1$ systems, although the efficiency was less than complete. Another widely-used method is the Jeener-Broekaert $(\pi / 2)_{x}-\tau-(\pi / 4)_{y}$ two-pulse sequence (origi-

nally designed to convert Zeeman order into dipolar order) [265] and modifications thereof $[266,267]$, which creates maximal alignment, $\hat{T}_{2,0}$, in $J=1$ systems if $\tau=\pi /(2 \Delta)$, where $\Delta$ is the quadrupolar splitting frequency. In NMR experiments this condition on the pulse delay $\tau$ may not be achievable if multiple nuclei with different quadrupolar couplings are present (Ref. [267] attempts to remedy this), whereas the adiabatic methods are robust to this problem as long as $\left|\omega_{1}(0)\right| \gg\left|\omega_{Q}\right|$ and $\left|\omega_{1}\left(\tau_{p}\right)\right| \ll\left|\omega_{Q}\right|$ for all values of $\omega_{Q}$. Also, the Jeener-Broekaert method is not completely efficient for AOC in $J=3 / 2$ systems [268, 269]. A limitation of both the adiabatic and Jeener-Broekaert methods is the need for rf pulses that are much stronger than the quadrupolar interaction.

\subsubsection{Dynamics of the central transition in static half-integer quadrupolar nuclei in weak magnetic fields}

The nuclei with half-integer angular momentum are of particular interest in NMR because they comprise nearly all of the quadrupolar nuclei (the $J=1$ nuclei H-2, Li-6, and N-14 and the $J=3$ nucleus B-10 are exceptions) and are amenable to the MQMAS technique (see $\S 3$ ) for producing high-resolution spectra in the solid state. The directly observable nuclear transitions are single-quantum, i.e. $\left|m-m^{\prime}\right|=1$, where $m$ and $m^{\prime}$ are the magnetic quantum numbers involved in the transition. As was discussed in $\S 3$, 


\subsection{NMR METHODS OF MANIPULATING POLARIZATION IN QUADRUPOLAR NUCLEI WITH MAGNETIC FIELDS}

any coherence between $|J,+| m|\rangle$ and $|J,-| m|\rangle$ (i.e., involving the so-called "symmetric" transitions) commutes with the first-order quadrupolar Hamiltonian $\hat{H}_{Q}^{(1)} \propto \hat{T}_{2,0}$, and thus experiences no first-order quadrupolar broadening. Therefore, among the single-quantum coherences the only ones that lead to narrow lines are the $\left|J, m= \pm \frac{1}{2}\right\rangle\left\langle J, m=\mp \frac{1}{2}\right|$ "central transition" coherences. The other "satellite transition" single quantum coherences are often broadened too much to be observed (or at least too much to be useful) in a solid-state experiment. Thus, the ability to manipulate the central transition coherence in half-integer spin systems is of great interest to NMR spectroscopists.

It is instructive to consider the central transition dynamics in half-integer spins under the conditions of a static quadrupolar coupling and constant rf irradiation. This is the same situation as was described in $\S 5.5 .1$ for $J=1$ spins, except here only an approximate solution will be attempted in the weak magnetic field limit $\left|\omega_{1}\right| \ll\left|\omega_{Q}\right|$, which is the most common case in the solid state. Consider an oriented $J=3 / 2$ nucleus. The first-order quadrupolar Hamiltonian in the rotating frame has the form $\hat{H}_{Q}^{(1)}=\hbar \omega_{Q} \hat{T}_{2,0}$, and the $\left|J=\frac{3}{2}, m= \pm \frac{3}{2}\right\rangle$ states are degenerate eigenstates of $\hat{H}_{Q}^{(1)}$ with eigenvalues $+\hbar \omega_{Q} / 2$, whereas the degenerate $\left|J=\frac{3}{2}, m= \pm \frac{1}{2}\right\rangle$ states have eigenvalues $-\hbar \omega_{Q} / 2$. The central transition single-quantum coherence does not evolve under $\hat{H}_{Q}^{(1)}$, whereas the two satellite transition single-quantum coherences evolve at the quadrupolar splitting frequency $\omega_{Q}$. Therefore, when the rf frequency is tuned to resonance, the NMR spectrum has two lines with the central transition at zero frequency and the satellite transitions at frequency $\omega_{Q}$ (second-order quadrupolar and other offset effects have been neglected). If this is the case, one would expect that a long, weak radiofrequency pulse that is resonant with the 
central transition would cause a frequency-selective excitation of only that transition and be ineffective at exciting the satellite transitions. This selective excitation behavior of a weak resonant rf field in half-integer spin systems will be considered from a more theoretical standpoint below.

It is convenient to re-write the Hamiltonian and density operator in terms of fictitious Cartesian spin- $1 / 2$ single-transition operators $\hat{J}_{x}^{j-k}=\frac{1}{2}(|j\rangle\langle k|+| k\rangle\langle j|), \hat{J}_{y}^{j-k}=$ $-\frac{i}{2}(|j\rangle\langle k|-| k\rangle\langle j|)$, and $\hat{J}_{z}^{j-k}=\frac{1}{2}(|j\rangle\langle j|-| k\rangle\langle k|)$, where $|j\rangle,|k\rangle \in\{|J, m\rangle\}[168,169]$. If the $J=3 / 2$ magnetic sublevels are labeled $|1\rangle \equiv\left|J=\frac{3}{2}, m=+\frac{3}{2}\right\rangle,|2\rangle \equiv\left|J=\frac{3}{2}, m=+\frac{1}{2}\right\rangle$, $|3\rangle \equiv\left|J=\frac{3}{2}, m=-\frac{1}{2}\right\rangle$, and $|4\rangle \equiv\left|J=\frac{3}{2}, m=-\frac{3}{2}\right\rangle$, the Hamiltonian in Eq. 5.12 can be rewritten as:

$$
\begin{aligned}
\hat{H}_{\text {rot }} / \hbar & =\omega_{Q} \hat{T}_{2,0}-\omega_{1} \hat{J}_{x} \\
& =\omega_{Q}\left[\hat{J}_{z}^{1-2}-\hat{J}_{z}^{3-4}\right]-\omega_{1}\left[2 \hat{J}_{x}^{2-3}+\sqrt{3}\left(\hat{J}_{x}^{1-2}+\hat{J}_{x}^{3-4}\right)\right] .
\end{aligned}
$$

In the limit $\left|\omega_{Q}\right| \gg\left|\omega_{1}\right|$ it is convenient to transform into the interaction frame defined by the quadrupolar Hamiltonian using the transformation

$$
\hat{V}_{Q}(t)=e^{-i \omega_{Q} t \hat{T}_{2,0}}=\exp \left[-i \omega_{Q} t\left(\hat{J}_{z}^{1-2}-\hat{J}_{z}^{3-4}\right)\right]=\exp \left[-i \omega_{Q} t \hat{J}_{z}^{1-2}\right] \exp \left[+i \omega_{Q} t \hat{J}_{z}^{3-4}\right]
$$

since fictitious spin- $1 / 2$ operators that do not share a state index exist in orthogonal subspaces and therefore commute with each other. The transformed Hamiltonian in the 
quadrupolar interaction frame is:

$$
\hat{\widetilde{H}}_{\text {rot }} / \hbar=-2 \omega_{1} \hat{J}_{x}^{2-3}-\sqrt{3} \omega_{1}\left[\left(\hat{J}_{x}^{1-2}+\hat{J}_{x}^{3-4}\right) \cos \left(\omega_{Q} t\right)-\left(\hat{J}_{y}^{1-2}-\hat{J}_{y}^{3-4}\right) \sin \left(\omega_{Q} t\right)\right]
$$

The terms that rapidly oscillate at $\omega_{Q}$ may be neglected in the secular approximation (i.e., in an average Hamiltonian treatment they average to zero over the period $\left.\tau_{Q}=2 \pi / \omega_{Q}\right)$, so the effective Hamiltonian in the interaction frame is:

$$
\hat{\widetilde{H}}_{r o t} / \hbar \simeq-2 \omega_{1} \hat{J}_{x}^{2-3}
$$

where the large quadrupolar Hamiltonian has effectively truncated the rf Hamiltonian to a term that affects only the central transition $\left|J, m= \pm \frac{1}{2}\right\rangle$ states. After transforming back out of the quadrupolar interaction frame, the effective time development operator in the rotating frame is:

$$
\hat{U}_{r o t}(t) \simeq \exp \left[-i \omega_{Q} t\left(\hat{J}_{z}^{1-2}-\hat{J}_{z}^{3-4}\right)\right] \exp \left[+i 2 \omega_{1} t \hat{J}_{x}^{2-3}\right] .
$$

If the system is initially oriented along the $z$-axis, $\hat{\rho}(0) \propto \hat{J}_{z}=3 \hat{J}_{z}^{1-4}+\hat{J}_{z}^{2-3}$, and the density operator evolves under $\hat{U}_{\text {rot }}(t)$ as:

$$
\hat{\rho}(t)=\hat{U}_{\text {rot }}(t) \hat{J}_{z} \hat{U}_{\text {rot }}^{-1}(t) \simeq 3 \hat{J}_{z}^{1-4}+\left[\hat{J}_{z}^{2-3} \cos \left(2 \omega_{1} t\right)+\hat{J}_{y}^{2-3} \sin \left(2 \omega_{1} t\right)\right]
$$

where $\hat{J}_{z}^{1-4}$ commutes with both $\hat{T}_{2,0}$ and $\hat{J}_{x}^{2-3}$, and $\hat{J}_{y}^{2-3}$ commutes with $\hat{T}_{2,0}$. The "triplequantum $z$ magnetization", $3 \hat{J}_{z}^{1-4}$, is left invariant during the pulse. It is apparent that 
the quadrupolar-truncated rf field effectively nutates only the central transition fictitious spin- $1 / 2$ angular momentum at a rate $2 \omega_{1}$, i.e., twice the rate $\omega_{1}$ at which the complete orientation would nutate under the action of a rf field in the absence of a quadrupolar coupling. The result for general $J$ is that the central transition effectively nutates in the $\left|\omega_{1}\right| \ll\left|\omega_{Q}\right|$ limit at a rate that is $\left(J+\frac{1}{2}\right)$ times faster than the nutation rate in the $\left|\omega_{1}\right| \gg\left|\omega_{Q}\right|$ limit. This nutation behavior of quadrupolar nuclei is well known (e.g. Refs. $[270,271]$ and references therein) and stems from the fact that the nutation rate $\omega_{1}$ is independent of the spin quantum number $J$ for a pure rf rotation, whereas the matrix elements for rotation in the subspace of the central transition states do depend on $J$ as $\left\langle J, m= \pm \frac{1}{2}\left|\omega_{1} \hat{J}_{x}\right| J, m=\mp \frac{1}{2}\right\rangle=\frac{1}{2}\left(J+\frac{1}{2}\right) \omega_{1}$, which are $\left(J+\frac{1}{2}\right)$ times larger than the matrix elements for a $J=1 / 2$ spin.

Now consider the case when the initial density operator is $\hat{\rho}(0) \propto \hat{J}_{x}=2 \hat{J}_{x}^{2-3}+$ $\sqrt{3}\left(\hat{J}_{x}^{1-2}+\hat{J}_{x}^{3-4}\right)$. For simplicity, the time evolution will be evaluated only at $\tau_{Q}=2 \pi / \omega_{Q}$ (or at integer multiples of $\tau_{Q}$ that are stroboscopically synchronized with the quadrupolar evolution frequency). In this case, the rotating-frame evolution is:

$$
\begin{aligned}
\hat{\rho}\left(\tau_{Q}\right) & =\hat{U}_{\text {rot }}\left(\tau_{Q}\right) \hat{J}_{x} \hat{U}_{\text {rot }}^{-1}\left(\tau_{Q}\right) \\
& \simeq 2 \hat{J}_{x}^{2-3}+\sqrt{3}\left[\left(\hat{J}_{x}^{1-2}+\hat{J}_{x}^{3-4}\right) \cos \left(\omega_{1} \tau_{Q}\right)+\left(\hat{J}_{y}^{1-3}-\hat{J}_{y}^{2-4}\right) \sin \left(\omega_{1} \tau_{Q}\right)\right]
\end{aligned}
$$

where it can be seen that the $\hat{J}_{x}^{2-3}$ central transition term is invariant under the rf irradiation (and would also be invariant under quadrupolar evolution), whereas the satellite transition coherences evolve during the irradiation (and evolve further under the quadrupolar Hamiltonian). Therefore the central transition coherence, $\hat{J}_{x}^{2-3}$, is a constant of the 
motion and is said to be "spin-locked" by the rf irradiation. This result would also hold if the central transition coherence was excited selectively: for example, a $y$-directed $\omega_{1} t=\pi / 4$ pulse would transform $\hat{\rho}(0)=\hat{J}_{z}$ into $3 \hat{J}_{z}^{1-4}-\hat{J}_{x}^{2-3}$ as described above. Application of an $x$-directed rf pulse would spin-lock the central transition coherence $\hat{J}_{x}^{2-3}$. The spin locking behavior of the central transition coherence under rf irradiation is important because when the spin locking fails, central transition coherence can be transformed into different types of polarization, as is the case in NMR quadrupolar rotational adiabatic coherence transfer (§5.6.3) or rotary resonance experiments $(\S 5.6 .5)$.

\subsubsection{Adiabatic coherence transfer in half-integer spins via slow modula- tion of the quadrupolar interaction: RIACT}

The previous section examined the dynamics of the central transition under constant rf irradiation when the quadrupolar coupling was time-independent. However, the size of the quadrupolar coupling can be manipulated in high-field NMR experiments by spinning the sample. The angular momentum dynamics of half-integer quadrupolar nuclei under rf irradiation and static or magic-angle sample spinning conditions has been studied in detail by A. Vega [158]. As was discussed previously, magic-angle spinning at a frequency $\omega_{R}$ causes the periodically-modulated quadrupolar coupling to undergo multiple sign inversions/zero crossings over one rotor period $\tau_{R}=2 \pi / \omega_{R}$. Assuming that at its maximum the quadrupolar coupling frequency $\left|\omega_{Q}(t)\right|$ is much greater than the rf nutation frequency, the quadrupolar Hamiltonian dominates the rf Hamiltonian near the extrema of the $\omega_{Q}(t)$, and the rotating-frame eigenstates are very nearly the eigenstates of the quadrupolar Hamiltonian $\hat{H}_{Q}^{(1)} \propto \hat{T}_{2,0}$. These eigenstates are doubly-degenerate within the $\pm|m|$ manifolds, and 


\subsection{NMR METHODS OF MANIPULATING POLARIZATION IN QUADRUPOLAR NUCLEI WITH MAGNETIC FIELDS}

Vega writes the quadrupolar eigenstates as the superpositions $|C \pm\rangle=\frac{1}{\sqrt{2}}\left(\left|\frac{3}{2},+\frac{1}{2}\right\rangle \pm\left|\frac{3}{2},-\frac{1}{2}\right\rangle\right)$ and $|T \pm\rangle=\frac{1}{\sqrt{2}}\left(\left|\frac{3}{2},+\frac{3}{2}\right\rangle \pm\left|\frac{3}{2},-\frac{3}{2}\right\rangle\right)$, where $|C \pm\rangle$ represent the central transition singlequantum coherences, and $|T \pm\rangle$ represent the unique triple-quantum coherences. The instantaneous eigenenergies are $+\hbar \omega_{Q}(t) / 2$ for the $|T \pm\rangle$ states and $-\hbar \omega_{Q}(t) / 2$ for the $|C \pm\rangle$ states. ${ }^{14}$ The important point to note is that the $|C \pm\rangle$ states cross the $|T \pm\rangle$ states when $\omega_{Q}(t)=0$ since the rf perturbation is no longer negligible $\left(\left|\omega_{1}\right| \gg\left|\omega_{Q}(t)\right|\right)$, which results in strong mixing of the $|C \pm\rangle$ states with the $|T \pm\rangle$ states. In fact, when one considers the instantaneous eigenstates ${ }^{15}$ of the Hamiltonian $\hat{H}_{\text {rot }}(t) / \hbar=\omega_{Q}(t) \hat{T}_{2,0}-\omega_{1} \hat{J}_{x}$ over the full range of $\omega_{Q}(t)$, the state $|C+\rangle$ is converted smoothly to $|T+\rangle$ and $|C-\rangle$ is converted smoothly to $|T-\rangle$ (or vice versa) as $\omega_{Q}(t)$ varies from a large positive value to a large negative value (or vice versa). The system would remain in the $|C \pm\rangle$ states or $|T \pm\rangle$ during the course of the mechanical rotation if no rf perturbation were present.

As long as the variation of $\hat{H}_{\text {rot }}(t)$ is adiabatic, the mixing of the instantaneous eigenstates allows for the efficient conversion of central transition coherence to triplequantum coherence and back again as the quadrupolar coupling undergoes sign inversions during magic-angle spinning. Vega has defined an adiabaticity parameter $\alpha=\left|\omega_{1}^{2} /\left(\omega_{Q} \omega_{R}\right)\right|$, where $\alpha \gg 1$ marks the adiabatic passage regime and $\alpha \ll 1$ marks the sudden passage regime [158]. According to the well-known quantum mechanical adiabatic theorem $[272$, $\S \mathrm{XVII}]$, if the system is prepared in an eigenstate of the Hamiltonian, the system is main-

\footnotetext{
${ }^{14}$ When the rf perturbation $\hat{H}_{r} f=-\hbar \omega_{1} \hat{J}_{x}$ is included the zeroth-order eigenstates may be retained, but the $|C \pm\rangle$ states receive a first-order energy splitting of $2 \hbar \omega_{1}$, while the $|T \pm\rangle$ states remain degenerate.

${ }^{15} \mathrm{By}$ "instantaneous eigenstate" it is meant a state $|\psi(t)\rangle$ that satisfies the eigenvalue equation $\hat{H}(t)|\psi(t)\rangle=E(t)|\psi(t)\rangle$ for a particular value of $t$, although these are not truly eigenstates because the time-dependent system does not have a well-defined energy. In quantum chemistry the instantaneous eigenstates of a large time-independent system in the presence of a time-dependent perturbation are also known as the "adiabatic states", whereas the true eigenstates in the absence of the perturbation are known as the "diabatic states".
} 


\subsection{NMR METHODS OF MANIPULATING POLARIZATION IN QUADRUPOLAR NUCLEI WITH MAGNETIC FIELDS}

tained in the instantaneous eigenstate of the Hamiltonian as long as the Hamiltonian changes adiabatically. In the sudden passage regime $(\alpha \gg 1)$ the system remains in its original eigenstate before and after the crossing. Therefore one expects that adiabatic single-quantum to triple-quantum coherence conversions can be implemented in the limit $\alpha \gg 1$ where the rf pulse amplitude $\left|\omega_{1}\right|$ is high and the spinning speed $\omega_{R}$ is slow for relatively small quadrupolar couplings $\left|\omega_{Q}\right|$.

In fact, such adiabatic conversions have been demonstrated experimentally in the rotation-induced adiabatic coherence transfer (RIACT) experiment [273]. In this experiment, adiabaticity is ensured setting the rf power as high as possible with the sample spinning speed made as slow as possible while still retaining line-narrowing effects. The basic RIACT triple-quantum excitation scheme uses a $\pi / 4$ pulse of phase $\phi$ for the selective creation of central transition single-quantum coherence from longitudinal magnetization, followed by a spin-locking pulse of phase $\phi+\pi / 2$ and duration $\tau_{p}$, as described in the previous section. During the course of the spin-locking pulse the sample rotation induces adiabatic transfer of the central transition coherences to triple-quantum coherences. The crystallites do not undergo quadrupolar zero crossings all at the same time; in fact, crystallites in which $\omega_{Q}(t)$ is dominated by oscillation at the frequency $\omega_{R}$ undergo two zero crossings over one rotor period $\tau_{R}$ that cause the transfer $|C \pm\rangle \rightarrow|T \pm\rangle \rightarrow|C \pm\rangle$, whereas crystallites in which $\omega_{Q}(t)$ is dominated by oscillation at the frequency $2 \omega_{R}$ undergo four zero crossings that cause the transfer $|C \pm\rangle \rightarrow|T \pm\rangle \rightarrow|C \pm\rangle \rightarrow|T \pm\rangle \rightarrow|C \pm\rangle$ during $\tau_{R}$. These correspond to adiabatic demagnetization and remagnetization cycles of the central transition transverse magnetization. In a random powder, the choice of pulse width $\tau_{p}=\tau_{R} / 4$ maximizes the 
net transfer of central transition single-quantum coherence to triple-quantum coherence, as was determined numerically [273]. RIACT conversions of triple-quantum coherence to central transition single-quantum coherence during a single spin-locking pulse were found to be more efficient than triple-quantum excitation from longitudinal magnetization using a $\pi / 4$ pulse followed by a spin-locking pulse. The RIACT technique is somewhat limited by the presence of resonance offsets and second-order quadrupolar coupling effects (which have not been considered here) and is useful in systems where the quadrupolar coupling is not too large. RIACT also can be applied in higher spin systems, for instance to induce conversions between central transition single-quantum coherence and five-quantum coherence in $J=5 / 2$ systems [274].

The analog of the RIACT experiment cannot be applied efficiently to atomic systems if only amplitude modulation of the optical field is employed. A modulated Stark splitting frequency $\omega_{E}(t)$ depends on the absolute square of the modulated electric field, so it never changes sign and the types of adiabatic conversions described above cannot be completed. The best case would be a partial coherence transfer as $\underline{E}(t)$ is adiabatically reduced to zero, leaving the system in a superposition of central transition single-quantum and triple quantum-states. The quadratic Stark splitting is, however, an odd function of the detuning of the laser frequency from resonance [232]. It therefore may be possible to implement RIACT-type experiments in atomic systems using frequency sweeps of the laser through resonance. 


\subsubsection{Coherence transfer in half-integer spins via fast resonant modula- tion of the magnetic field: FAM}

The fast-amplitude modulation (FAM) technique was developed to induce more efficient transfers of multiple-quantum coherence to single-quantum coherence in quadrupolar nuclei in powdered solids [275]. The technique is most widely applied in magic-angle spinning experiments, but is also valid in static samples, which are considered here. As has been seen previously, a constant weak rf field applied to a static half-integer quadrupolar spin system is only effective at causing transitions within the central transition subspace, with any other conversions appearing in a higher-order perturbation theory treatment. The coherence transfer that will be considered is the conversion of triple-quantum coherence to central transition single-quantum coherence in a $J=3 / 2$ spin system.

As the FAM principle was originally conceived [151], a modulation of the rf field in the rotating frame at a frequency $\omega_{m}$ that is near the quadrupolar splitting frequency $\omega_{Q}$ is expected to be effective at selectively exciting the $\left|\frac{3}{2}, \pm \frac{3}{2}\right\rangle \longleftrightarrow\left|\frac{3}{2}, \pm \frac{1}{2}\right\rangle$ satellite transitions. This corresponds to a selective excitation at the satellite transition frequency in the rotating frame. Since the satellite transitions connect the $\left\{\left|\frac{3}{2}, \pm \frac{3}{2}\right\rangle\right\}$ manifold to the $\left\{\left|\frac{3}{2}, \pm \frac{1}{2}\right\rangle\right\}$ manifold, it might be expected that this excitation would be capable of converting triple-quantum coherence (a superposition of $\left|\frac{3}{2}, \pm \frac{3}{2}\right\rangle$ states) into central-transition single-quantum coherence (a superposition of $\left|\frac{3}{2}, \pm \frac{1}{2}\right\rangle$ states).

The theoretical formalism for FAM pulses in static and rotating single crystals and powders has been worked out in detail and has been compared to the continuous pulse and RIACT methods in the quadrupolar MQMAS experiment [276]. The treatment of a 
$J=3 / 2$ nucleus in a single static crystallite in the absence of second-order quadrupolar effects and resonance offsets is straightforward. The rotating-frame Hamiltonian in this case is:

$$
\hat{H}_{\text {rot }}(t) / \hbar=\omega_{Q} \hat{T}_{2,0}-\omega_{1} \cos \left(\omega_{m} t\right) \hat{J}_{x}
$$

where the laboratory-frame rf field is $\underline{B}_{1}(t)=B_{1} \cos \left(\omega_{m} t\right) \cos \left(\omega_{r} t\right)$, where $\omega_{m}$ is the amplitude modulation frequency, and $\omega_{r}$ is the rf carrier frequency (assumed to be set on resonance), and $2 \omega_{1}=\gamma B_{1}$. Methods of experimentally creating this type of amplitude modulation will be discussed later. It can be readily appreciated that the rotating-frame Hamiltonian 5.41 looks like the EFG PAS-frame NQR Hamiltonian in Eq. 5.10 in the case that the rf field is perpendicular to the axially-symmetric EFG principal axis (which is the geometry at which the NQR pulse is most efficient). So, in a sense, the problem of a rotating-frame FAM pulse is the same problem as that of an EFG PAS-frame NQR pulse applied to a $J=3 / 2$ nucleus for a static crystallite. The NQR pulse was seen to be efficient in $J=1$ nuclei when the carrier frequency was set on resonance to the NQR transition $\omega_{r} \simeq 3 \omega_{Q} / \sqrt{6}$; likewise, it is expected that the FAM pulse will be efficient in converting triple-quantum to single-quantum coherence in $J=3 / 2$ nuclei when the modulation frequency is nearly resonant with the rotating-frame quadrupolar splitting frequency $\omega_{m} \simeq \omega_{Q}$. In an analogy to the treatment of NQR pulses presented earlier, the behavior during the pulse can be better appreciated in the case of a resonant amplitude modulation $\omega_{m}=\omega_{Q}$ 
by transforming into the quadrupolar interaction frame according to Eq. $5.36:{ }^{16}$

$$
\begin{aligned}
\hat{\widetilde{H}}_{\text {rot }}(t) / \hbar= & -\omega_{1} \cos \left(\omega_{Q} t\right)\left\{2 \hat{J}_{x}^{2-3}+\sqrt{3}\left[\left(\hat{J}_{x}^{1-2}+\hat{J}_{x}^{3-4}\right) \cos \left(\omega_{Q} t\right)-\left(\hat{J}_{y}^{1-2}-\hat{J}_{y}^{3-4}\right) \sin \left(\omega_{Q} t\right)\right]\right\} \\
= & -2 \omega_{1} \cos \left(\omega_{Q} t\right) \hat{J}_{x}^{2-3}-\frac{\sqrt{3}}{2} \omega_{1}\left[\left(\hat{J}_{x}^{1-2}+\hat{J}_{x}^{3-4}\right)+\right. \\
& \left.\quad+\cos \left(2 \omega_{Q} t\right)\left(\hat{J}_{x}^{1-2}+\hat{J}_{x}^{3-4}\right)-\sin \left(2 \omega_{Q} t\right)\left(\hat{J}_{y}^{1-2}-\hat{J}_{y}^{3-4}\right)\right],
\end{aligned}
$$

which contains a time-independent piece, and all the other terms average to zero over the interval $\tau_{Q}=2 \pi / \omega_{Q}$. The remaining effective Hamiltonian in the quadrupolar interaction frame is:

$$
\hat{\widetilde{H}}_{\text {rot }}(t) / \hbar=-\frac{\sqrt{3}}{2} \omega_{1}\left(\hat{J}_{x}^{1-2}+\hat{J}_{x}^{3-4}\right)
$$

which selectively excites the satellite transitions by coupling the $\left|\frac{3}{2}, \pm \frac{3}{2}\right\rangle$ states to the $\left|\frac{3}{2}, \pm \frac{1}{2}\right\rangle$ states. The resonant $\mathrm{rf}$ amplitude modulation can be thought of as causing either a frequency-selective excitation of the satellite transitions, or a recoupling of terms in the rf Hamiltonian that are ordinarily truncated by the quadrupolar Hamiltonian. Upon transforming back into the rotating frame the time development operator becomes:

$$
\hat{U}_{\text {rot }}(t) \simeq \exp \left[-i \omega_{Q} t\left(\hat{J}_{z}^{1-2}-\hat{J}_{z}^{3-4}\right)\right] \exp \left[+i \frac{\sqrt{3}}{2} \omega_{1} t\left(\hat{J}_{x}^{1-2}+\hat{J}_{x}^{3-4}\right)\right]
$$

The evolution of a triple quantum coherence $\hat{\rho}(0)=\hat{J}_{x}^{1-4}=\frac{1}{2}\left(\left|\frac{3}{2},+\frac{3}{2}\right\rangle\left\langle\frac{3}{2},-\frac{3}{2}|+| \frac{3}{2},-\frac{3}{2}\right\rangle\left\langle\frac{3}{2},+\frac{3}{2}\right|\right)$

\footnotetext{
${ }^{16}$ Ref. [276] uses the quadrupolar modulation frame transformation $\hat{V}_{m}(t)=\exp \left[-i \omega_{m} t \hat{T}_{2,0}\right]$ for the nearlyresonant $\omega_{m} \simeq \omega_{Q}$ case, which is the more realistic situation in a powdered solid due to the broad distribution of quadrupolar frequencies $\omega_{Q}$. Also, it should be noted that the application of perturbation theory in the quadrupolar interaction frame is valid for $\left|\omega_{Q}\right| \gg\left|\omega_{1}\right|$, which may not be satisfied for all crystallites due to the orientation dependence of $\omega_{Q}$.
} 
under resonantly amplitude-modulated $\mathrm{rf}$ irradiation is most easily evaluated at $\tau_{Q}=2 \pi / \omega_{Q}$ (or an integer multiple thereof):

$$
\begin{aligned}
\hat{\rho}\left(\tau_{Q}\right) & \simeq e^{+i \frac{\sqrt{3}}{2} \omega_{1} \tau_{Q}\left(\hat{J}_{x}^{1-2}+\hat{J}_{x}^{3-4}\right)} \hat{J}_{x}^{1-4} e^{-i \frac{\sqrt{3}}{2} \omega_{1} \tau_{Q}\left(\hat{J}_{x}^{1-2}+\hat{J}_{x}^{3-4}\right)} \\
& =\cos ^{2}\left(\omega_{1}^{e} \tau_{Q}\right) \hat{J}_{x}^{1-4}+\sin ^{2}\left(\omega_{1}^{e} \tau_{Q}\right) \hat{J}_{x}^{2-3}+\cos \left(\omega_{1}^{e} \tau_{Q}\right) \sin \left(\omega_{1}^{e} \tau_{Q}\right)\left(\hat{J}_{y}^{1-3}-\hat{J}_{y}^{2-4}\right),(5 .
\end{aligned}
$$

where $\omega_{1}^{e}=\sqrt{3} \omega_{1} / 4$; the first term represents triple-quantum coherence, the second term represents central transition single-quantum coherence, and the third term includes doublequantum coherences. If the time evolution is not evaluated at $\tau_{Q}$ so that quadrupolar evolution is included, the (non-symmetric) double-quantum coherences evolve further under the quadrupolar Hamiltonian, but the other terms represent symmetric transitions that are invariant to first-order quadrupolar evolution. Therefore, it can be seen that FAM pulses can efficiently convert triple-quantum coherence to central-transition single-quantum coherence (and also back again) in $J=3 / 2$ systems.

In practice $[275]$ the FAM modulations are usually implemented by approximating the $\cos \left(\omega_{m} t\right)$ modulation by a train of $n$ binary pulse pairs $\left(\tau_{m} / 2\right)_{\phi}\left(\tau_{m} / 2\right)_{\bar{\phi}}$, where $\tau_{m}=2 \pi / \omega_{m}, \phi$ represents the $\mathrm{rf}$ phase, and $\bar{\phi}=\phi+\pi$. This causes a square-wave modulation of frequency $\omega_{m}$ between $+\omega_{1}$ and $-\omega_{1}$ in the rotating frame, where the $\pi$ phase shift of the rf carrier frequency switches the direction of the rf field in the rotating frame. This approach was chosen because for typical values of $\omega_{Q} / 2 \pi>1 \mathrm{MHz}, \tau_{m}$ can be on the order of hundreds of nanoseconds, and implementing a smooth amplitude modulation on a timescale much faster than this can be experimentally challenging when using highpower solid-state spectrometers. It should also be noted that when FAM pulses are applied 
to rotating powders, the situation again becomes a radiation- and rotation-mediated adiabatic coherence transfer. Additionally some of the efficiency of the FAM pulse is due to orientational-averaging effects [276].

When applied to AMO systems of half-integer angular momentum, similar weak magnetic field modulation techniques will be able to cause efficient conversions of centraltransition single-quantum coherence (which is present in a system that is oriented perpendicular to the applied electric field) into triple-quantum coherence in $J=3 / 2$ systems, where the quantization axis is along the electric field. Likewise, other coherence transfers could be facilitated in higher angular momentum systems, i.e., not only triple-quantum/singlequantum conversions in $J=5 / 2$ systems, but also five-quantum/triple-quantum conversions. The FAM-type conversions also have been implemented using adiabatic sweeps of the rf frequency to induce similar transfers between $|J, \pm| m|\rangle$ populations and $|J, \pm| m-1|\rangle$ populations $[277,278,279,280]$. These techniques would be the most useful in AMO experiments in which $\left|\omega_{E}\right| \gg\left|\omega_{B}\right|$. If the Stark and Larmor splittings are comparable, however, the dynamics become much more complicated.

\subsubsection{Coherence transfer in half-integer spins via matching the quadrupo- lar modulation frequency to the rotating frame Larmor frequency: rotary resonance}

A description of some rotary resonance effects in $J=5 / 2$ MQMAS experiments was given in $\S 3$. This discussion will include a schematic description of some rotary resonance effects in $J=3 / 2$ systems.

As was noted in $§ 5.6 .3$, under fast magic-angle sample spinning conditions and 
low-power $\mathrm{rf}$ spin locking the spin system is in the sudden-passage regime $\alpha \ll 1$, in which the central transition states $|C \pm\rangle$ and the triple-quantum states $|T \pm\rangle$ are expected to remain spin-locked during sample rotation, with no interconversions between single- and triple-quantum coherences. However, the conventional theory of the spin-locking of halfinteger quadrupolar nuclei under MAS conditions [158] does not take into account certain resonances that can occur when the rf nutation frequency is appropriately matched to the sample rotation frequency. Indeed, losses of spin-locking efficiency at these "rotary resonance" conditions were observed experimentally $[159,160]$ and a partial theory of the effects was advanced [159] before further experiments proved that these losses in spin locking efficiency amounted to gains in the efficiency of multiple-quantum coherence conversion [161], or before full theories of the rotary effects were developed [162, 163, 164].

Consider the rotating-frame Hamiltonian for a modulated quadrupolar interaction in the presence of a steady rf field, neglecting resonance offset and second-order quadrupolar effects:

$$
\hat{H}_{r o t}(t)=\hat{H}_{Q}^{(1)}(t)+\hat{H}_{r f}=\hbar \omega_{Q}(t) \hat{T}_{2,0}-\hbar \omega_{1} \hat{J}_{x}
$$

where $\omega_{Q}(t)$ is modulated at harmonics of the spinning frequency, $\omega_{R}$, according to Eq. 5.33. This is the Hamiltonian that was under consideration in the previous discussion of the RIACT technique, although here $\left|\omega_{1}\right| \sim \omega_{R}$ instead of the typical $\left|\omega_{1}\right| \gg \omega_{R}$ conditions in RIACT experiments. It is useful to separate the rf Hamiltonian as:

$$
\hat{H}_{r f} / \hbar=-\omega_{1} \hat{J}_{x}=-2 \omega_{1} \hat{J}_{x}^{2-3}-\sqrt{3} \omega_{1}\left(\hat{J}_{x}^{1-2}+\hat{J}_{x}^{3-4}\right),
$$


where the first term, $\hat{H}_{r f}^{C T} / \hbar=-2 \omega_{1} \hat{J}_{x}^{2-3}$, drives the central transition and the second term $\hat{H}_{r f}^{S T} / \hbar=-\sqrt{3} \omega_{1}\left(\hat{J}_{x}^{1-2}+\hat{J}_{x}^{3-4}\right)$ drives the satellite transitions. It is convenient to transform into the interaction frame of the modulated quadrupolar interaction plus the central transition rf Hamiltonian, defined by [162]:

$$
\begin{aligned}
\hat{V}_{Q, C T}(t) & =T\left\{\exp \left[-\frac{i}{\hbar} \int_{0}^{t} d t^{\prime}\left(\hat{H}_{Q}^{(1)}\left(t^{\prime}\right)+\hat{H}_{r f}^{C T}\right)\right]\right\} \\
& =\exp \left[-i\left(\Phi_{Q}(t) \hat{T}_{2,0}-2 \omega_{1} t \hat{J}_{x}^{2-3}\right)\right] \\
& \left.=\exp \left[-i \Phi_{Q}(t)\left(\hat{J}_{z}^{1-2}-\hat{J}_{z}^{3-4}\right)\right] \exp \left[+i 2 \omega_{1} t \hat{J}_{x}^{2-3}\right)\right]
\end{aligned}
$$

where $T$ is the Dyson time-ordering operator and the MAS quadrupolar dynamic phase is $\Phi_{Q}(t)=\int_{0}^{t} d t^{\prime} \omega_{Q}\left(t^{\prime}\right)$. The fact that $\left[\hat{H}_{Q}^{(1)}(t), \hat{H}_{r f}^{C T}\right]=0$ ensures that $\hat{H}_{Q}^{(1)}(t)+\hat{H}_{r f}^{C T}$ commutes with itself at all times so that the time ordering in Eq. 5.48 is unimportant. The quadrupolar-rf central transition interaction frame Hamiltonian is:

$$
\begin{aligned}
\hat{\widetilde{H}}_{r o t}(t) / \hbar= & \hat{V}_{Q, C T}^{-1}(t) \hat{H}_{r f}^{S T} \hat{V}_{Q, C T}(t) \\
= & -\sqrt{3} \omega_{1}\left\{\cos \left[\Phi_{Q}(t)\right]\left[\left(\hat{J}_{x}^{1-2}+\hat{J}_{x}^{3-4}\right) \cos \left(\omega_{1} t\right)-\left(\hat{J}_{y}^{1-3}-\hat{J}_{y}^{2-4}\right) \sin \left(\omega_{1} t\right)\right]\right. \\
& \left.\quad-\sin \left[\Phi_{Q}(t)\right]\left[\left(\hat{J}_{y}^{1-2}-\hat{J}_{y}^{3-4}\right) \cos \left(\omega_{1} t\right)+\left(\hat{J}_{x}^{1-3}+\hat{J}_{x}^{2-4}\right) \sin \left(\omega_{1} t\right)\right]\right\} \\
= & -\frac{\sqrt{3}}{2} \omega_{1}\left\{\cos \left(\omega_{1} t\right)\left[e^{+i \Phi_{Q}(t)}\left(\hat{J}_{+}^{1-2}+\hat{J}_{-}^{3-4}\right)+e^{-i \Phi_{Q}(t)}\left(\hat{J}_{-}^{1-2}+\hat{J}_{+}^{3-4}\right)\right]\right. \\
& \left.\quad+i \sin \left(\omega_{1} t\right)\left[e^{+i \Phi_{Q}(t)}\left(\hat{J}_{+}^{1-3}+\hat{J}_{-}^{2-4}\right)-e^{-i \Phi_{Q}(t)}\left(\hat{J}_{-}^{1-3}+\hat{J}_{+}^{2-4}\right)\right]\right\}, \quad
\end{aligned}
$$

where $\hat{J}_{ \pm}^{j-k}=\hat{J}_{x}^{j-k} \pm i \hat{J}_{y}^{j-k}$. Since the modulated quadrupolar frequency is periodic such that $\omega_{Q}\left(t+\tau_{R}\right)=\omega_{Q}(t)$ where $\tau_{R}=2 \pi / \omega_{R}$, the $\exp \left[ \pm i \Phi_{Q}(t)\right]$ are also periodic and therefore 
can be expanded in a Fourier series as:

$$
\begin{aligned}
\exp \left[+i \Phi_{Q}(t)\right] & =\sum_{n=-\infty}^{+\infty} A_{n} e^{i n \omega_{R} t} \\
\exp \left[-i \Phi_{Q}(t)\right] & =\sum_{n=-\infty}^{+\infty} A_{n}^{*} e^{-i n \omega_{R} t}=\sum_{n=-\infty}^{+\infty} A_{-n}^{*} e^{i n \omega_{r} t}
\end{aligned}
$$

where the coefficients $A_{n}$ are complex and depend on the quadrupolar parameters $C_{Q}$ and $\eta_{Q}$ and on crystallite orientation. The $\cos \left(\omega_{1} t\right)$ and $\sin \left(\omega_{1} t\right)$ terms also can be expanded in terms of exponential functions, such that:

$$
\begin{array}{r}
\hat{\widetilde{H}}_{r o t}(t) / \hbar=-\frac{\sqrt{3}}{4} \omega_{1} \sum_{n=-\infty}^{+\infty} \sum_{m= \pm 1} e^{i n \omega_{R} t} e^{i m \omega_{1} t}\left\{\left[A_{n}\left(\hat{J}_{+}^{1-2}+\hat{J}_{-}^{3-4}\right)+A_{-n}^{*}\left(\hat{J}_{-}^{1-2}+\hat{J}_{+}^{3-4}\right)\right]\right. \\
\left.+e^{i[\pi(m-1) / 2]}\left[A_{n}\left(\hat{J}_{+}^{1-3}+\hat{J}_{-}^{2-4}\right)-A_{-n}^{*}\left(\hat{J}_{-}^{1-3}+\hat{J}_{+}^{2-4}\right)\right]\right\},
\end{array}
$$

where $e^{i[\pi(m-1) / 2]}= \pm 1$ for $m= \pm 1$. This can be written in the form:

$$
\hat{\widetilde{H}}_{r o t}(t)=\sum_{n=-\infty}^{+\infty} \sum_{m= \pm 1} \hat{\widetilde{H}}_{n, m} e^{i n \omega_{R} t} e^{i m \omega_{1} t}
$$

where in terms of the $|C \pm\rangle,|T \pm\rangle$ basis states:

$$
\hat{\widetilde{H}}_{n, \pm 1} / \hbar=-\frac{\sqrt{3}}{2} \omega_{1}\left[A_{n}|T \pm\rangle\left\langle C \pm\left|+A_{-n}^{*}\right| C \mp\right\rangle\langle T \mp|\right] .
$$

The interaction frame Hamiltonian has a complicated time dependence, but when the nu- 
tation frequency $\omega_{1}$ is an integer multiple of the spinning frequency such that $\omega_{1}=N \omega_{R}$,

$$
\hat{\widetilde{H}}_{r o t}(t)=\sum_{n=-\infty}^{+\infty} \sum_{m= \pm 1} \hat{\widetilde{H}}_{n, m} e^{i(n+m N) \omega_{R} t}
$$

where terms with $n+m N=0$ are time-independent. In a lowest-order average Hamiltonian approach, the Hamiltonian is averaged over one rotor period $\tau_{R}$, resulting in an effective Hamiltonian in the interaction frame:

$$
\begin{aligned}
\hat{\widetilde{\tilde{H}}}_{\text {rot }}(t) & =\hat{\widetilde{H}}_{N,-1}+\hat{\widetilde{H}}_{-N,+1} \\
& =-\frac{\sqrt{3}}{2} \omega_{1}\left[\left(A_{N}|T-\rangle\left\langle C-\left|+A_{N}^{*}\right| C-\right\rangle\langle T-|\right)+\left(A_{-N}|T+\rangle\left\langle C+\left|+A_{-N}^{*}\right| T+\right\rangle\langle C+|\right)\right] .
\end{aligned}
$$

It may be readily appreciated that this Hamiltonian couples the central transition states $|C \pm\rangle$ to the triple-quantum transition states $|T \pm\rangle$, inducing efficient coherence transfers. The generalized rotary resonance condition for an arbitrary half-integer spin $J$ is $\omega_{1}=\frac{4 N}{2 J+1} \omega_{R}$. The lowest-order average Hamiltonian treatment is not sufficient to explain other interesting conversions of polarization that can occur at or in between rotary resonance conditions $[161,162,163,164,165]$; in fact, this method is suspect in the sudden passage regime because the $n^{t h}$-order average Hamiltonian term goes like $\sim\left(\omega_{1} / \omega_{R}\right)^{n}$, so the perturbation series may not converge (or at least not converge rapidly) when $\omega_{1}=N \omega_{R}$. All these problems have been addressed successfully by Floquet theoretical treatments [162, 163, 164].

This approach is very general and it is expected that these effects could be observed in AMO systems with crossed electric and magnetic fields if the Larmor frequency 
5.7. THE $J=1 \rightarrow J^{\prime}=0$ TRANSITION IN OPTICS EXPRESSED AS A SYSTEM OF TWO COUPLED SPINS-1/2 IN NMR

is appropriately matched to the Stark modulation frequency such that $\omega_{B}=\frac{4 N}{2 J+1} \omega_{R}$, in the limit that the Larmor splitting is much smaller than the Stark splitting. However, the theory as described above is only applicable to systems with half-integer angular momenta, as are the RIACT and FAM techniques.

\subsection{The $J=1 \rightarrow J^{\prime}=0$ transition in optics expressed as a system of two coupled spins-1/2 in NMR}

Thus far only angular momentum dynamics within a single $J$ manifold have been considered. However, in AMO experiments, it is also possible to excite optical transitions between levels of differing angular momentum, and this transition itself affects the angular momentum dynamics of both states. The simple example that will be considered here is the transition between a $J=1$ ground state and a $J^{\prime}=0$ excited state during optical pumping in the low light power limit while in the presence of a magnetic field. Radiationinduced $J \rightarrow J^{\prime}$ transitions are usually not considered in NMR, because excitations are usually performed within the nuclear ground state and the radiation does not directly couple different nuclei to each other. However, the $J=1, J^{\prime}=0$ system has four levels, and one might expect that this problem could be translated into the problem of two coupled spin$1 / 2$ angular momenta. In that case, it may be possible that the atomic angular momentum dynamics can be associated with the dynamics of an NMR system. This would represent another analogy between AMO and NMR and potentially provide more opportunities for new experimental design and cross-fertilization between these fields. 
5.7. THE $J=1 \rightarrow J^{\prime}=0$ TRANSITION IN OPTICS EXPRESSED AS A SYSTEM OF TWO COUPLED SPINS-1/2 IN NMR

\subsubsection{The optically-pumped atomic $J=1, J^{\prime}=0$ system}

Consider the atomic $J=1 \rightarrow J^{\prime}=0$ low-power optical transition in the presence of a magnetic field parallel to the light propagation axis; the quantum-mechanical formulation of this problem has been considered by Malakyan et al. [281]. Only the coherent dynamics will be considered here; i.e., relaxation will be neglected, because the mechanisms of relaxation can differ substantially between AMO and NMR systems (although these differences themselves would make interesting topics of study). The system is subject to the internal atomic Hamiltonian $\hat{H}_{0}$, the magnetic field (Zeeman) Hamiltonian $\hat{H}_{B}$, and the light-atom interaction (Stark) Hamiltonian $\hat{H}_{l}$ induced by the electric field of the radiation:

$$
\hat{H}(t)=\hat{H}_{0}+\hat{H}_{B}+\hat{H}_{l}(t)
$$

The $J=1$ triplet ground state has three magnetic sublevels $\left|T_{+}\right\rangle \equiv\left|\alpha_{g} ; J, m_{J}=+1\right\rangle,\left|T_{0}\right\rangle \equiv$ $\left|\alpha_{g} ; J, m_{J}=0\right\rangle$, and $\left|T_{-}\right\rangle \equiv\left|\alpha_{g} ; J, m_{J}=-1\right\rangle$, and the $J^{\prime}=0$ singlet excited state has one level $\left|S_{0}\right\rangle \equiv\left|\alpha_{e} ; J^{\prime}, m_{J^{\prime}}=0\right\rangle$. Here $\alpha_{g}$ and $\alpha_{e}$ represent the quantum numbers for the ground and excited states, respectively, other than the angular momentum quantum numbers, and the quantization axis $z$ is taken to be along the direction of light propagation. ${ }^{17}$

If the atomic ground state is assumed to have energy $E_{g}=0$ and the excited state

\footnotetext{
${ }^{17}$ This differs from the examples considered previously, in which the $z$-axis was taken to be along the electric field axis, which is the polarization axis in the case of a linearly-polarized optical field.
} 
has an energy $E_{e}=\hbar \Omega_{A}$, the laboratory-frame atomic Hamiltonian may be written as:

$$
\hat{H}_{0}=\hbar \Omega_{A}\left|S_{0}\right\rangle\left\langle S_{0}\right|=\hbar \Omega_{A}\left(\begin{array}{cccc}
0 & 0 & 0 & 0 \\
0 & 0 & 0 & 0 \\
0 & 0 & 1 & 0 \\
0 & 0 & 0 & 0
\end{array}\right)
$$

where the ordering of the basis states in the matrix has been taken to be $\left\{\left|T_{+}\right\rangle,\left|T_{0}\right\rangle,\left|S_{0}\right\rangle,\left|T_{-}\right\rangle\right\}$ in order to group the states by the value of their magnetic sublevel $m_{J}$ or $m_{J^{\prime}}$.

The Zeeman Hamiltonian in a $z$-directed magnetic field $\underline{B}=B \underline{\widetilde{z}}$ is:

$$
\hat{H}_{B}=-\underline{\mu}_{g} \cdot \underline{B}=-g_{g} \mu_{B} \hat{J}_{z} B / \hbar=-\hbar \omega_{B}\left(\begin{array}{cccc}
1 & 0 & 0 & 0 \\
0 & 0 & 0 & 0 \\
0 & 0 & 0 & 0 \\
0 & 0 & 0 & -1
\end{array}\right)
$$

where $\underline{\hat{\mu}}_{g}$ is the magnetic dipole moment operator of the $J=1$ ground state, $\omega_{B}=g_{g} \mu_{B} B$ is the Larmor frequency of the $J=1$ state, and $\mu_{0}$ is the Bohr magneton. ${ }^{18}$ The $\left|T_{0}\right\rangle$ and $\left|S_{0}\right\rangle$ states are not shifted by a magnetic field.

The atom-light interaction Hamiltonian with an oscillating light field $\underline{\mathcal{E}}=\mathcal{E}_{0} \cos (\omega t) \underline{\widetilde{x}}$ linearly polarized along $x$ is:

$$
\hat{H}_{l}(t)=-\underline{\hat{d}} \cdot \underline{\mathcal{E}}=-d_{x} \mathcal{E}_{0} \cos (\omega t)=-2 \hbar \omega_{l} \cos (\omega t)\left(\begin{array}{cccc}
0 & 0 & -1 & 0 \\
0 & 0 & 0 & 0 \\
-1 & 0 & 0 & 1 \\
0 & 0 & 1 & 0
\end{array}\right)
$$

where $\underline{\hat{d}}$ is the atomic electric dipole moment operator (with $\hat{d}_{x}=-\left(\hat{d}_{1,+1}-\hat{d}_{1,-1}\right) / \sqrt{2}$

\footnotetext{
${ }^{18}$ In this notation the electron $g$-factor is negative and the Bohr magneton is positive, so $\omega_{B}<0$ if $\underline{B}$ is in the $+z$-direction.
} 
5.7. THE $J=1 \rightarrow J^{\prime}=0$ TRANSITION IN OPTICS EXPRESSED AS A SYSTEM OF TWO COUPLED SPINS-1/2 IN NMR

in the spherical basis), $\omega_{l}=\mathcal{E}_{0}\left\langle\alpha^{\prime} ; J^{\prime}\left\|\hat{d}_{1}\right\| \alpha ; J\right\rangle /(2 \sqrt{2} \hbar)$ is the optical Rabi frequency, and $\left\langle\alpha^{\prime} ; J^{\prime}\left\|\hat{d}_{1}\right\| \alpha ; J\right\rangle$ is a reduced matrix element given by the Wigner-Eckart theorem $\left\langle\alpha^{\prime} ; J^{\prime}, m_{J^{\prime}}\left|\hat{d}_{k, q}\right| \alpha ; J, m_{J}\right\rangle=\left\langle J^{\prime}, M_{J^{\prime}} ; k, q \mid J, m_{J}\right\rangle\left\langle\alpha^{\prime} ; J^{\prime}\left\|\hat{d}_{k}\right\| \alpha ; J\right\rangle$, where $\left\langle\alpha^{\prime} ; J^{\prime}, m_{J^{\prime}}\left|\hat{d}_{k, q}\right| \alpha ; J, m_{J}\right\rangle$ is a Clebsch-Gordan coefficient. The laser frequency $\omega$ is near the optical resonance frequency $\Omega_{A}$, and potentially $\omega=\omega(t)$, i.e., the optical field is frequency-modulated. The quadratic Stark effect considered in previous sections may be neglected in the low light power limit where the optical pumping is not saturated.

The total Hamiltonian in the $\left\{\left|T_{+}\right\rangle,\left|T_{0}\right\rangle,\left|S_{0}\right\rangle,\left|T_{-}\right\rangle\right\}$basis is therefore:

$$
\hat{H}(t) / \hbar=\left(\begin{array}{cccc}
-\omega_{B} & 0 & 2 \omega_{l} \cos (\omega t) & 0 \\
0 & 0 & 0 & -2 \omega_{l} \cos (\omega t) \\
2 \omega_{l} \cos (\omega t) & 0 & \Omega_{A} & 0 \\
0 & 0 & -2 \omega_{l} \cos (\omega t) & \omega_{B}
\end{array}\right)
$$

and it is this Hamiltonian that will be used to try to make an analogy to an NMR system.

\subsubsection{The fictitious two-spin system}

The four-level system can be converted into a fictitious system of two spin- $1 / 2$ particles $J_{1}$ and $J_{2}$ through the associations $\left|T_{+}\right\rangle=|++\rangle,\left|T_{0}\right\rangle=(|+-\rangle+|-+\rangle) / \sqrt{2}$, $\left|T_{-}\right\rangle=|--\rangle$, and $\left|S_{0}\right\rangle=(|+-\rangle-|-+\rangle) / \sqrt{2} \cdot{ }^{19}$ The $J, J^{\prime}$ Hamiltonian can be converted into this fictitious two-spin basis through the transformation:

$$
\hat{H}^{\prime}(t)=\hat{U}_{T} \hat{H}(t) \hat{U}_{T}^{-1},
$$

\footnotetext{
${ }^{19}|++\rangle=\left|J_{1}=1 / 2, m_{1}=+1 / 2\right\rangle \otimes\left|J_{2}=1 / 2, m_{2}=+1 / 2\right\rangle,|+-\rangle=\left|J_{1}=1 / 2, m_{1}=+1 / 2\right\rangle \otimes \mid J_{2}=$ $\left.1 / 2, m_{2}=-1 / 2\right\rangle,|+-\rangle=\left|J_{1}=1 / 2, m_{1}=-1 / 2\right\rangle \otimes\left|J_{2}=1 / 2, m_{2}=+1 / 2\right\rangle$, and $|--\rangle=\mid J_{1}=1 / 2, m_{1}=$ $-1 / 2\rangle \otimes\left|J_{2}=1 / 2, m_{2}=-1 / 2\right\rangle$.
} 
where

$$
\hat{U}_{T}=\left(\begin{array}{cccc}
1 & 0 & 0 & 0 \\
0 & \frac{1}{\sqrt{2}} & \frac{1}{\sqrt{2}} & 0 \\
0 & \frac{1}{\sqrt{2}} & -\frac{1}{\sqrt{2}} & 0 \\
0 & 0 & 0 & 1
\end{array}\right)
$$

where the columns of $\hat{U}_{T}$ are the two-spin basis states written in the triplet-singlet basis.

It is instructive to write the Hamiltonian $\hat{H}^{\prime}$ in terms of basis operators of the fictitious two-spin system. A convenient choice of basis operators $\{\hat{B}\}$ is the set of sixteen dimensionless $\hat{J}_{1 i} \hat{J}_{2 j}$ angular momentum direct product operators, where $i, j \in\{x, y, z\}$ and the identity operators $\hat{1}_{1}$ and $\hat{1}_{2}$ have also been included in the single-spin basis sets. ${ }^{20}$ The Hamiltonian can be written:

$$
\hat{H}^{\prime}(t)=\sum_{i, j} b_{i j}(t) \hat{B}_{i j}
$$

where $b_{i j}=\operatorname{Tr}\left[\hat{B}_{i j}^{\dagger} \hat{H}^{\prime}(t)\right] / \operatorname{Tr}\left[\hat{B}_{i j}^{\dagger} \hat{B}_{i j}\right]$. It follows that:

$$
\begin{aligned}
\hat{H}^{\prime}(t) / \hbar & =\frac{\Omega_{A}}{4} \hat{1}+\left(-\omega_{B} \hat{J}_{1 z}-\omega_{B} \hat{J}_{2 z}\right)-\Omega_{A}\left(\hat{J}_{1} \cdot \underline{\hat{J}}_{2}\right)+\left[-\omega_{l}^{\prime} \cos (\omega t) \hat{J}_{1 x}+\omega_{l}^{\prime} \cos (\omega t) \hat{J}_{2 x}\right] \\
& =\left[\hat{H}_{0}^{\prime}+\hat{H}_{B}^{\prime}+\hat{H}_{l}^{\prime}(t)\right] / \hbar
\end{aligned}
$$

where

$$
\hat{H}_{B}^{\prime} / \hbar=-\omega_{B}\left(\hat{J}_{1 z}+\hat{J}_{2 z}\right)
$$

\footnotetext{
${ }^{20}$ For instance, $\hat{J}_{1 x} \hat{J}_{2 z}=\left(\frac{1}{2}|+\rangle\left\langle-\left|+\frac{1}{2}\right|-\right\rangle\langle+|\right) \otimes\left(\frac{1}{2}|+\rangle\left\langle+\left|-\frac{1}{2}\right|-\right\rangle\langle-|\right)$, or $\hat{J}_{y 2} \equiv \hat{1}_{1} \otimes \hat{J}_{y 2}=(|+\rangle\langle+|+$ $|-\rangle\langle-|) \otimes\left(\frac{-i}{2}|+\rangle\left\langle-\left|+\frac{i}{2}\right|-\right\rangle\langle+|\right)$.
} 
and

$$
\hat{H}_{0}^{\prime} / \hbar=\frac{\Omega_{A}}{4} \hat{1}-\Omega_{A}\left(\underline{J}_{1} \cdot \underline{\hat{J}}_{2}\right)
$$

and

$$
\hat{H}_{l}^{\prime}(t) / \hbar=-\omega_{l}^{\prime} \cos (\omega t)\left(\hat{J}_{1 x}-\hat{J}_{2 x}\right)
$$

with $\omega_{l}^{\prime}=2 \sqrt{2} \omega_{l}=\mathcal{E}_{0}\left\langle\alpha^{\prime} ; J^{\prime}\left\|\hat{d}_{1}\right\| \alpha ; J\right\rangle / \hbar$.

\subsubsection{The potential Hamiltonian analogy to NMR}

A spin-1 coupled to a spin-0 system does not often arise in NMR studies, so it is natural to try to represent such a system as two spin- $1 / 2$ systems that are coupled. The coupled spin-1/2 problem is ubiquitous in NMR. ${ }^{21}$ As will be seen, the Hamiltonian in Eq. 5.64 has some similarities to NMR Hamiltonians. The atomic Zeeman term, $\hat{H}_{B}^{\prime}$, looks exactly like the Zeeman Hamiltonian of two chemically equivalent spin- $1 / 2$ nuclei, upon identifying the $J=1$ atomic Larmor frequency with a spin-1/2 nuclear Larmor frequency. The internal atomic Hamiltonian $\hat{H}_{0}^{\prime}$ resembles the isotropic component of the NMR scalar coupling ( $J$-coupling) Hamiltonian with a $J$-coupling constant equal to $-\Omega_{A}$ in angular frequency units, plus a constant energy shift $\hbar \Omega_{A} / 4$ of all the levels that does not affect the angular momentum dynamics. ${ }^{22}$

There is one critical difference between the atomic Hamiltonian and an NMR

\footnotetext{
${ }^{21}$ In fact, in certain problems it is not unusual to write the two spin- $1 / 2$ system in a singlet-triplet basis.

${ }^{22}$ Note that $J$ is the conventional symbol for the NMR indirect spin-spin coupling constant, and does not refer to an angular momentum quantum number here.
} 
5.7. THE $J=1 \rightarrow J^{\prime}=0$ TRANSITION IN OPTICS EXPRESSED AS A SYSTEM OF TWO COUPLED SPINS-1/2 IN NMR

Hamiltonian that makes the analogy incomplete. The atom-light coupling Hamiltonian $\hat{H}_{l}^{\prime}$ looks similar to the laboratory-frame Hamiltonian for two nuclei in a radiofrequency magnetic field $\underline{B}_{1}(t)=B_{1} \cos (\omega t) \underline{\widetilde{x}}$ if $\omega_{l}^{\prime}$ is identified with the rf nuclear nutation frequency $\omega_{1}$, except that the first spin sees a field $+\underline{B}_{1}(t)$ and the second spin sees a field $-\underline{B}_{1}(t)$. This sign difference in the "rf Hamiltonian" is unphysical in real NMR experiments, which have no way of applying magnetic fields of different signs to two different nuclei. The other way to get a sign difference in the nutation frequency is if the nuclei had oppositelysigned gyromagnetic ratios, which again is unphysical, as no two nuclei have the same magnitude but opposite sign of gyromagnetic ratio. However, even if this were the case, this sign difference would also have to be reflected in the Zeeman Hamiltonian $\hat{H}_{B}^{\prime}$ in the form of a sign difference between the Larmor frequencies $\omega_{B}$ of the two nuclei, which it is not. Physically, this sign difference would mean that two equivalent nuclei could be distinguished by using rf fields to make them nutate in opposite directions. This behavior could also provide a means of decoupling two identical scalar-coupled nuclei using rf fields, which has never been done.

The Hamiltonian in Eq. 5.64 can also be considered to be somewhat unphysical from an NMR perspective if one considers the actual values of the physical constants. In an atomic system, the dominant Hamiltonian is the internal atomic Hamiltonian; i.e., $\Omega_{A} \gg \omega_{B}, \omega_{l} \cdot{ }^{23}$ However, the nuclear $J$-coupling is usually one of the smallest interactions in $\operatorname{NMR}\left(\sim 1-10^{2} \mathrm{~Hz}\right)$, whereas at high magnetic fields the nuclear Larmor frequency is $\sim 10^{8} \mathrm{~Hz}$, and rf nutation frequencies typically go up to $\sim 10^{4} \mathrm{~Hz}$ in liquid-state probes to

\footnotetext{
${ }^{23} \Omega_{A} / 2 \pi \sim 10^{14} \mathrm{~Hz}$ for a near-IR resonance; in the perturbative limit $\omega_{l} / 2 \pi \sim 10^{3} \mathrm{~Hz}$ is a reasonable value; and for $B$ in the $10^{-3} \mathrm{G}$ range, $\omega_{B} / 2 \pi \sim 10^{3} \mathrm{~Hz}$.
} 
$\sim 10^{5} \mathrm{~Hz}$ in solid-state probes.

Although the NMR $J$-coupling obviously does not dominate the dynamics in the laboratory frame, consider the following common liquid-state NMR Hamiltonian in the laboratory frame for two equivalent $J$-coupled spins in a static magnetic field $\underline{B}_{0}=B_{0} \underline{\tilde{z}}$, with an additional amplitude-modulated rf field $\underline{B}_{1}(t)=B_{1} \cos (\omega t) \underline{\widetilde{x}}$ :

$$
\hat{H}_{l a b} / \hbar=-\omega_{0}\left(\hat{I}_{z 1}+\hat{I}_{z 2}\right)+\omega_{J}\left(\hat{\underline{I}}_{z 1} \cdot \hat{\underline{I}}_{z 2}\right)-2 \omega_{1}(t) \cos \left(\omega_{r} t\right)\left(\hat{I}_{x 1}+\hat{I}_{x 2}\right),
$$

where $\omega_{0}=\gamma B_{0}, \omega_{J}=2 \pi J, \omega_{1}(t)=\omega_{1} \cos \left(\omega_{m} t\right), 2 \omega_{1}=\gamma B_{1}$, and $\omega_{r}$ is the rf carrier frequency. In the rotating frame defined by the transformation $\hat{V}_{\text {rot }}(t)=\exp \left[+i \omega_{r} t\left(\hat{I}_{z 1}+\right.\right.$ $\left.\left.\hat{I}_{z 1}\right)\right]$, the effective Hamiltonian becomes:

$$
\hat{H}_{\text {rot }} / \hbar \simeq-\delta\left(\hat{I}_{z 1}+\hat{I}_{z 2}\right)+\omega_{J}\left(\hat{I}_{z 1} \cdot \underline{I}_{z 2}\right)-\omega_{1} \cos \left(\omega_{m} t\right)\left(\hat{I}_{x 1}+\hat{I}_{x 2}\right)
$$

where $\delta=\omega_{0}-\omega_{r}$ is the resonance detuning and the rapidly-oscillating terms at frequencies $\omega_{m} \pm 2 \omega_{r}$ can be neglected if $\left|\omega_{m} \pm 2 \omega_{r}\right| \gg\left|\omega_{1}\right|$, since $\left|\omega_{1}\right|,\left|\omega_{m}\right| \ll\left|\omega_{r}\right|$. The resemblance of this rotating frame Hamiltonian to Eq. 5.64 should be apparent (aside from the relative signs of the $\hat{I}_{x 1}$ and $\hat{I}_{x 2}$ terms, which again is crucial), but the physical parameters have somewhat different meanings. In effect, the nuclear Larmor frequency has been reduced in the rotating frame to a small resonance offset $\delta$ that can be made arbitrarily small by tuning the rf carrier frequency $\omega_{r}$ close to the Larmor frequency $\omega_{0}$. If the resonance offset $\delta / 2 \pi$ is adjusted to be on the order of a few $\mathrm{Hz}$ and the $\mathrm{rf}$ nutation frequency $\omega_{1} / 2 \pi$ is made to be very weak and also of the order of only a few $\mathrm{Hz}$, then $|\delta|,\left|\omega_{1}\right| \ll\left|\omega_{J}\right|$ for large 
5.7. THE $J=1 \rightarrow J^{\prime}=0$ TRANSITION IN OPTICS EXPRESSED AS A SYSTEM OF TWO COUPLED SPINS-1/2 IN NMR

values of the $J$-coupling constant, and this situation resembles the atomic system. In order to make the analogy complete, the rf amplitude modulation frequency $\omega_{m}$ should be of the order of the $J$-coupling frequency $\omega_{J}$ (since $\omega \sim \Omega_{A}$ in the atomic system), and potentially $\omega_{m}=\omega_{m}(t)$.

The dynamics under the atomic Hamiltonian in Eq. 5.56 are usually calculated by making a "rotating wave approximation" in the rotating frame defined by the transformation $\hat{V}_{\text {rot }}(t)=\exp \left[-i \hat{H}_{0} t / \hbar\right]$. If the $J$-coupling Hamiltonian is truly dominant (an unusual case in NMR when an rf field is present) in Eq. 5.69 and if the modulation frequency is chosen such that $\omega_{m} \sim \omega_{J}$, then the equivalent procedure would be to approximate the Hamiltonian in the modulated frame defined by the transformation $\exp \left[-i \omega_{m} t\left(\hat{\underline{I}}_{z 1} \cdot \underline{\hat{I}}_{z 2}\right)\right]$, which is analogous to the $J$-coupling interaction frame transformation $\exp \left[-i \omega_{J} t\left(\hat{\underline{I}}_{z 1} \cdot \underline{\underline{I}}_{z 2}\right)\right] \cdot{ }^{24}$

\subsubsection{The observables}

As was discussed in $§ 5.4 .2$, there are many possible observable quantities in the atomic physics experiment. For example, the optical rotation of the light polarization in the atomic system is proportional to the polarization moment $P_{4}$, which yields [281]:

$$
\Delta \phi \propto \operatorname{Re}\left[\left\langle S_{0}|\hat{\rho}(t)| T_{-}\right\rangle+\left\langle T_{+}|\hat{\rho}(t)| S_{0}\right\rangle\right]
$$

where $\hat{\rho}(t)$ is the density operator for the system; its time dependence is determined by evolution under the Hamiltonian in Eq. 5.56 plus relaxation effects. The right-hand side of this equation is equal to $\operatorname{Re}\left[\operatorname{Tr}\left[\rho(t) \hat{\mathcal{O}}_{\phi}\right]\right]$, where $\hat{\mathcal{O}}_{\phi}=\left|T_{-}\right\rangle\left\langle S_{0}|+| S_{0}\right\rangle\left\langle T_{+}\right|$. If the non-

\footnotetext{
${ }^{24}$ It is possible to apply the second frame transformation to the approximate Hamiltonian in Eq. 5.69 if $\omega_{r} \gg \omega_{m}$, which will be the case since $\omega_{r} \sim \omega_{0} \sim 10^{8} \mathrm{~Hz}$ and $\omega_{m} \sim \omega_{J} \sim 10^{2} \mathrm{~Hz}$.
} 
5.7. THE $J=1 \rightarrow J^{\prime}=0$ TRANSITION IN OPTICS EXPRESSED AS A SYSTEM OF TWO COUPLED SPINS-1/2 IN NMR

Hermitian operator $\hat{\mathcal{O}}_{\phi}$ is written as $\hat{\mathcal{O}}_{\phi}=\hat{\mathcal{O}}_{\phi}^{+}+i \hat{\mathcal{O}}_{\phi}^{-}$, where $\hat{\mathcal{O}}_{\phi}^{+}=\frac{1}{2}\left(\hat{\mathcal{O}}_{\phi}+\hat{\mathcal{O}}_{\phi}^{\dagger}\right)$ and $\hat{\mathcal{O}}_{\phi}^{-}=\frac{1}{2 i}\left(\hat{\mathcal{O}}_{\phi}-\hat{\mathcal{O}}_{\phi}^{\dagger}\right)$ are Hermitian operators, then Eq. 5.70 can be rewritten as:

$$
\Delta \phi \propto \operatorname{Tr}\left[\hat{\rho}(t) \hat{\mathcal{O}}_{\phi}^{+}\right]
$$

where $\hat{\mathcal{O}}_{\phi}^{+}=\frac{1}{2}\left(\left|T_{-}\right\rangle\left\langle S_{0}|+| S_{0}\right\rangle\left\langle T_{-}|+| S_{0}\right\rangle\left\langle T_{+}|+| T_{+}\right\rangle\left\langle S_{0}\right|\right)$.

The optical rotation observable operator $\hat{\mathcal{O}}_{\phi}^{+}$can be written in terms of the fictitious two-spin operators as:

$$
\hat{\mathcal{O}}_{\phi}^{\prime}=\sqrt{2}\left(\hat{J}_{z 1} \hat{J}_{x 2}-\hat{J}_{x 1} \hat{J}_{z 2}\right)
$$

which is not a directly observable quantity in NMR (i.e., it does not correspond to nuclear magnetization), but it can be indirectly observed in a two-dimensional experiment. In twospin spherical tensor notation, $\hat{\mathcal{O}}_{\phi}^{+}=\sqrt{2}\left(\hat{T}_{1,+1}+\hat{T}_{1,-1}\right)$, where $\hat{T}_{1, \pm 1}=\frac{1}{2}\left(\hat{J}_{z 1} \hat{J}_{ \pm 2}-\hat{J}_{ \pm 1} \hat{J}_{z 2}\right)$. The observable $\hat{\mathcal{O}}_{\phi}^{+}$in NMR terminology corresponds to a particular combination of socalled antiphase magnetization.

As has been noted before, given a spin system prepared in some initial state, the NMR spectroscopist's game is to figure out how to manipulate the system into some other interesting state by implementing different Hamiltonians, usually by applying magnetic fields and/or sample rotations. As an interesting exercise, one may refer to Appendix D for some exact solutions of the time evolution of a $J$-coupled two-spin system in a magnetic field. Using the approach outlined above, these expressions can be converted into exact solutions of the $J=0, J^{\prime}=1$ atomic system in the presence of a static magnetic field but 
no optical field. For instance, if the initial density operator for a pair of identical spin-1/2 nuclei could be prepared as $\hat{\rho}^{\prime}(0) \propto \hat{J}_{x 1}-\hat{J}_{x 2}$ for a pair of identical spin-1/2 nuclei (ignoring the term proportional to the identity operator), then $\hat{\rho}(t)$ includes a term proportional to $-\frac{1}{2}\left\{\cos \left[\left(\omega_{J}+\delta\right) t\right]-\cos \left[\left(\omega_{J}-\delta\right) t\right]\right\}\left(\hat{J}_{z 1} \hat{J}_{x 2}-\hat{J}_{x 1} \hat{J}_{z 2}\right)=\sin \left(\omega_{J} t\right) \sin (\delta t)\left(\hat{J}_{z 1} \hat{J}_{x 2}-\hat{J}_{x 1} \hat{J}_{z 2}\right)$, where $\omega_{J}$ is the $J$-coupling constant and $\delta$ is the Larmor frequency in angular units. This term is proportional to the atomic optical rotation observable, $\hat{\mathcal{O}}_{\phi}{ }^{+}$. It averages to zero over times $t \gg \omega_{J}^{-1}$ in the limit of a strong $J$-coupling and weak magnetic field $\left|\omega_{J}\right| \gg|\delta|$. Upon making a transformation back into the singlet-triplet basis, one finds that $\hat{\rho}^{\prime}(0)= \pm\left(\hat{J}_{x 1}-\hat{J}_{x 2}\right)$ can be created upon equally populating the two states $\frac{1}{\sqrt{2}}\left(\left|T_{+}\right\rangle \mp\left|S_{0}\right\rangle\right)$ and $\frac{1}{\sqrt{2}}\left(\left|T_{-}\right\rangle \pm\left|S_{0}\right\rangle\right)$. If such a state could be prepared, one would expect that the system would develop a non-zero time-averaged optical rotation, but only on a timescale not much faster than the reciprocal optical frequency $\Omega_{A}^{-1}$, which is somewhat of an unrealistic case to measure.

\subsection{Conclusions}

The goal of this work has been to establish analogies between the angular momentum dynamics in atomic and molecular optics experiments in the presence of external fields and those in nuclear magnetic and quadrupole resonance experiments. In particular, the emphasis has been placed on the similarities between the coherent, quantum mechanical dynamics of these systems in the absence of relaxation effects, which can differ markedly between AMO and nuclear systems.

The analogies have been laid out to address the following questions: how can the two types of systems be polarized? What are the Hamiltonian operators that dictate 
their dynamics? What types of polarization can be measured? The Hamiltonian analogy focused on the fact that the effect of a magnetic field is the same in AMO and nuclear systems, but an electric field in $J>1 / 2$ AMO systems affects the dynamics through the quadratic Stark effect in nearly the same way as axially symmetric electric field gradients affect nuclear dynamics through the electric quadrupolar coupling. Several comparisons were made between NMR/NQR systems and AMO systems with magnetic and electric fields applied in different geometries. An exact solution was developed in the spherical tensor operator notation for the dynamics of a $J=1$ AMO system in crossed static electric and magnetic fields, which corresponds to the high-field NMR dynamics of a spin-1 nucleus with an axially-symmetric quadrupolar coupling during a steady rf pulse. The origin of signal in the nuclear quadrupolar resonance experiment was seen to be an example of an alignment-to-orientation conversion process that is familiar to AMO physicists, and a new analytical expression for the powder-averaged spin-1 NQR nutation signal was presented. The quadrupolar NMR analogy was extended to a review of techniques for manipulating polarization in high-field NMR studies of half-integer quadrupolar nuclei along with some comments on the potential applicability to AMO experiments.

An attempt was also made to draw an analogy between the dynamics of a resonant optical transition in the four-level $J=0 \rightarrow J^{\prime}=1$ system and NMR dynamics in the fourlevel system of two spin-1/2 nuclei. This analogy was found to be incomplete in that the AMO system could not be directly translated into a physical NMR system. However, it would be interesting to see what sort of dynamics could be induced in this system by coherent manipulation of the Hamiltonian. 
It is hoped that the formalism and analogies developed in this work can help to facilitate more interdisciplinary work that draws on the substantial expertise of the atomic and molecular physics and magnetic resonance communities. At a minimum, researchers could apply the insights and theoretical methods developed in other fields to their own work. Ideally, the ideas and techniques from one field could be applied to develop new experiments in the other. 


\section{Chapter 6}

\section{Distant dipolar field effects and}

\section{Xe-129 NMR "remote detection"}

\subsection{Remote detection NMR}

\subsubsection{Introduction to remote detection NMR}

Conventional NMR experiments are typically performed according to an "all-inone" paradigm in which the same coil that is used to apply rf pulses to manipulate the spin system is also used to detect the time-dependent nuclear magnetization by Faraday induction. This type of experiment is not only conceptually simple and easy to implement, it also makes good sense because the NMR reciprocity principle (§1.7) dictates that an rf coil that produces optimal (i.e., strong, homogeneous) pulses is also optimized for sensitive Faraday detection. For these reasons it has been sometimes overlooked that the information-encoding (usually coherent spin manipulation) portion of the NMR experiment 
can be considered separately from the magnetization-detection portion. In fact, some experiments exist for which the optimal conditions for NMR encoding are incompatible with the optimal conditions for NMR detection; therefore, it may prove advantageous to be able to separate and independently optimize the encoding and detection steps. For example, it may be desirable to perform experiments on powdered solids at zero magnetic field (i.e., spin encoding under an isotropic Hamiltonian) in order to obtain narrow lines, but Faraday detection is not applicable at zero field because the Larmor frequency is zero.

The original zero-field NMR experiment of Pines et al. shuttled the sample backand forth between high- and low-field environments for polarization, encoding, and detection $[282,283]$. Since the detection was not carried out directly during the evolution period, the zero-field evolution was incremented as the indirect time dimension of a two-dimensional Fourier NMR experiment. This experiment yielded good results but was somewhat mechanically challenging to implement.

Some twenty years after the first zero-field NMR work a general NMR encoding/detection modality dubbed "remote detection" NMR was introduced [284, 285, 286]. Rather than physically transporting the sample, the remote detection methodology relies on the use of a mobile "carrier" nucleus whose longitudinal magnetization can be encoded with NMR information point-by-point in one location and then transported to another location for detection. ${ }^{1}$ As opposed to an "all-in-one" encoding/detection experiment, the encoding and detection steps in a remote detection experiment are distant from each other in space and time. The experiment is modular in the sense that different combinations of

\footnotetext{
${ }^{1}$ Ref. [284] cites some examples of experiments that have used two-location encoding-detection schemes or other separate optimizations of the encoding and detection steps.
} 
encoding and detection environments can be employed as desired. For example, the NMR encoding could take place at high magnetic field or at low field, and the pulse sequence could encode the carrier nucleus with spectroscopic or image information [284, 287]. The carrier nucleus could be detected using Faraday induction at high field or by low-field atomic magnetometry [110], SQUID flux magnetometry [111], or spin-exchange optical detection [112]. In certain remote detection experiments there may be circumstances under which the individual optimization of the detection step can result in an increased sensitivity over the conventional NMR encoding/detection modality.

Perhaps the simplest remote detection encoding "module" is a two-pulse $90^{\circ}-t_{1}-$ $90^{\circ}$ spectroscopic encoding sequence, which will be discussed quantitatively in $\S 6.1 .2$. The first pulse converts initial longitudinal magnetization of the carrier nucleus to transverse magnetization, which evolves during the incremented indirect evolution time $t_{1}$. The second pulse converts the component of transverse magnetization that is orthogonal to the pulse phase into longitudinal magnetization. The magnitude of the stored longitudinal magnetization is an amplitude-modulated function of the evolution time $t_{1}$, where the modulation frequency is the rotating-frame frequency of the carrier nucleus. The longitudinal magnetization after the second rf pulse is thus encoded with a single indirect point $t_{1}$ of the time-domain NMR signal of the carrier nucleus.

Once an indirect point of the encoded signal is stored as longitudinal magnetization, the carrier nucleus can be safely transported to another location for detection as long as the transport time is shorter than the carrier longitudinal relaxation time. The longitudinal magnetization is preserved during transport as long as the external magnetic field 
along $z$ (i.e., the field parallel to the magnetization) dominates any transverse magnetic field components or any internal spin Hamiltonians. ${ }^{2}$ The transported magnetization can be detected by any means of choice; e.g., if Faraday detection is employed, a $90^{\circ}$ pulse at the beginning of the detection interval will convert the stored magnetization to transverse magnetization for detection. Note that the $90^{\circ}-t_{1}-90^{\circ}$ encoding sequence also leaves a $t_{1}$-dependent transverse magnetization component which is assumed to undergo complete dephasing on the way to the detector either through natural $T_{2}$ relaxation or by bulk dephasing during transport through magnetic field gradients. Any bulk transverse magnetization that is not dephased by the time it reaches the detector can add noise to the indirect signal, depending on the detection scheme. For example, if Faraday detection is employed, any surviving bulk transverse magnetization will in general possess a random component parallel to the direction of the detection pulse that will remain in the transverse plane after the pulse. As the indirect signal is recorded point-by-point, these random contributions to the signal will appear as noise in the indirect spectrum. A simple test to determine whether any transverse magnetization is reaching the detection coil is simply to turn on the receiver without applying a detection pulse.

The most important characteristics that the signal carrier nucleus should possess are mobility and a long longitudinal relaxation time. The carrier needs to be mobile in order to convey the encoded magnetization to the detector, and this magnetization should relax to equilibrium on a timescale that is long compared to the transport time in order to avoid signal loss. Xe-129 gas has been the mobile carrier of choice in remote detection

\footnotetext{
${ }^{2}$ The notion of storing indirectly-encoded magnetization along the $z$-axis during mechanical transport is not new; for example, this method is used in dynamic angle spinning experiments [135, 136, 137] to preserve the indirectly-encoded magnetization while the spinning rotor hops from one angle to another.
} 
experiments to date. The use of a fluid is ideal for continuous- or stopped-flow remote detection experiments in which the point-by-point encoding of NMR information takes place in subsequent volumes of the magnetized fluid. Xenon-129 gas has a longitudinal relaxation time on the order of tens of minutes or longer because it has relatively few efficient channels for nuclear magnetic relaxation, since it is monatomic, diamagnetic, dilute (low spin density), chemically inert, and possesses a spin-1/2 (non-quadrupolar) nucleus. Xenon also has the advantage that its nuclei can be hyperpolarized by spin-exchange optical pumping to levels that are $10^{3}-10^{5}$ times higher than the equilibrium polarization that can be achieved in a high magnetic field (on the order of $\sim 1-10$ Tesla) at room temperature. Note, however, that a gas has a low spin density (near atmospheric pressure) that results in a magnetization density that is two to three orders of magnitude lower than that of a similarly-polarized condensed phase sample. Nevertheless, the ability to produce nuclear polarization without having to use large superconducting magnets is convenient.

In addition to having properties that make it a good magnetization carrier, xenon also has properties that make it an interesting analyte [288], where "analyte" in the context of remote detection NMR means a nucleus whose NMR information-e.g., its spectrum or spatial distribution - is indirectly encoded. Xenon has a highly polarizable electron cloud, which results in a large range of potential chemical shifts, on the order of hundreds to thousands of ppm. This makes xenon a very sensitive probe of its local molecular environment. Xenon is also chemically inert, which means it can be introduced into other samples to "spy" on them without disrupting them chemically. Furthermore, xenon gas can be used for void-space imaging or fluid dynamics studies. 
In all the aforementioned examples xenon itself doubles as the analyte and as the mobile information carrier. The principal purpose of the work in this chapter, as discussed further in $\S 6.1 .3$, is to develop a remote detection experiment in which the carrier nucleus can be encoded with the NMR spectroscopic information of some different analyte nucleus. The mechanism by which is the spectroscopic information can be transmitted from the analyte to the carrier nucleus is the long range nuclear dipolar field.

It should be noted that helium-3 also can be hyperpolarized and may be superior to xenon-129 as remote detection carrier nucleus because it has a larger magnetic moment and a generally longer longitudinal relaxation time. On the other hand helium has a much smaller chemical shift range and therefore is not very useful as an analyte in spectroscopic encoding experiments, but would be ideal for void space imaging experiments. It also would be useful for dipolar field experiments in which the carrier nucleus does not also serve as the analyte. This work, however, used the pre-existing xenon polarization apparatus and expertise of the Pines laboratory.

\subsubsection{An example of remote detection NMR with spectroscopic encoding}

The concept of using an indirect point-by-point pulse sequence with physical transport of the spins to generate an NMR spectrum may seem somewhat unusual to the conventional NMR practitioner. The aim of this section is to give a pedagogical but quantitative account of how a simple two-pulse encoding remote detection experiment can be gently and gradually developed conceptually from a conventional one-pulse Fourier NMR experiment. Simpler descriptions of the remote detection experiment are available elsewhere $[284,286]$. It will be assumed that the analyte nucleus and the mobile information-carrier nucleus are 
one and the same, and that both NMR encoding and detection are achieved by use of rf coils (i.e., detection by Faraday induction is employed). Spin relaxation will be neglected, although its effects are easily added to this treatment. Fig. 6.1 shows a schematic of the simple pulse sequence toward which this discussion will build.

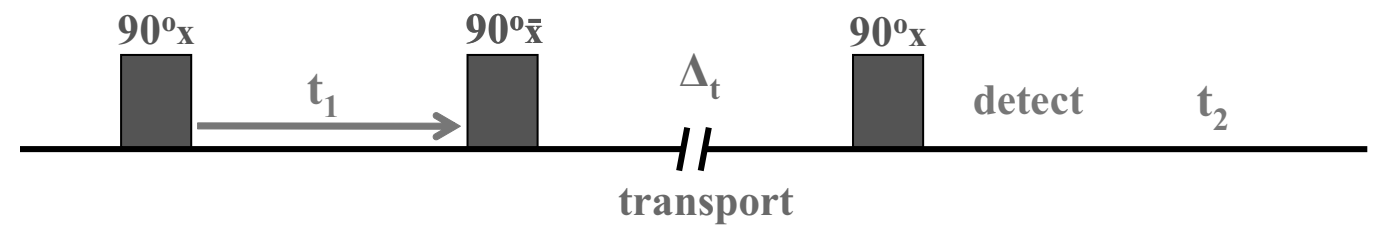

Figure 6.1: NMR remote detection experiment utilizing a two-pulse spectroscopic encoding sequence. After the sequence is applied the spin evolution during $t_{1}$ is stored as longitudinal magnetization of the carrier nucleus. This magnetization then can be transported during $\Delta_{t}$ to another location for detection. If inductive detection is to be used, a $90^{\circ}$ pulse will read out the stored magnetization for detection during $t_{2}$ as transverse magnetization.

Consider a one-pulse NMR experiment on a spin species $I$ whose spectrum contains only a single resonance. The rotating-frame Hamiltonian is time independent and may be written $\hat{H} / \hbar=\delta \hat{I}_{z}$, where $\delta$ is the resonance offset of the spin in the presence of some magnetic field directed along the $z$-axis. The spin system is assumed to be initially polarized along the $z$-axis, where the difference density operator is $\hat{\rho}(0) \propto \hat{I}_{z}$. A single $90^{\circ}$ pulse is applied in the $+x$-direction in the rotating frame. The precession of the resulting magnetization vector in the transverse plane is detected in quadrature. The pulse sequence and detected signal are:

$$
\begin{aligned}
& 90_{x}^{\circ}-t \text {-detect } \\
& S(t) \propto \operatorname{Tr}\left[\hat{\rho}(t) \hat{I}_{+}\right]=-\frac{i}{2} e^{i \delta t} .
\end{aligned}
$$

The numerical prefactor in the signal has been calculated assuming $I=1 / 2$, but the value 
of the spin quantum number does not actually play an important role in this discussion. The signal is not continuously sampled; rather, a total of $N$ points are discretely sampled from $t=0$ to $t=t^{\max }$ with an interval (dwell time) of $d t=t^{\max } / N$. The (discrete) Fourier transform of Eq. 6.2 obviously yields a peak at the frequency $\delta$. The encoding and detection steps in the conventional one-pulse NMR experiment overlap spatially; i.e., the same coil is used for excitation and for detection. They nearly overlap temporally; i.e., the spin precession at the resonance offset frequency is detected in real time, although one cannot detect at the same time a pulse is being applied. Here the excitation of the spins by the $90^{\circ}$ pulse is considered to be part of the encoding step.

Now imagine dividing the interval $t$ into two back-to-back intervals $t_{1}$ and $t_{2}$. In other words, the time variable $t_{1}=t$ is used only up until a certain point, after which the variable $t_{2}=t-t_{1}$ is used. This is only a mathematical split; nothing in the experiment has changed, so the detected signal is the same as Eq. 6.2 with renamed time variables:

$$
\begin{aligned}
& 90_{x}^{\circ}-t_{1}, t_{2} \text { - detect } \\
& S\left(t_{1}, t_{2}\right) \propto \operatorname{Tr}\left[\hat{\rho}\left(t_{1}, t_{2}\right) \hat{I}_{+}\right]=-\frac{i}{2} e^{i \delta t_{1}} e^{i \delta t_{2}} .
\end{aligned}
$$

A total of $N_{1}$ points are sampled during the first interval $t_{1}$ and $N_{2}$ points are sampled during $t_{2}$. If the signal of Eq. 6.4 is subjected to a two-dimensional Fourier transform with respect to $t_{1}$ and $t_{2}$, a diagonal peak is obtained in the $2 \mathrm{D}$ spectrum at frequency $\delta$ in both dimensions. Interestingly, the chemical information is obtained redundantly: the projection of the 2D spectrum onto either frequency axis gives the same NMR spectrum. In particular, the usual mathematical representation of the one-pulse NMR spectrum is recovered by 
setting $t_{2}=0$ in Eq. 6.5 (i.e., $t_{1}=t$ at all times) and taking the one-dimensional Fourier transform of the signal $S\left(t_{1}, 0\right)$.

This two time variable approach is easily extended to an indirect dimension/direct dimension two-dimensional NMR experiment. Rather than continuously detecting signal during the intervals $t_{1}$ and $t_{2}$, imagine shutting the detector off during $t_{1}$ and only recording signal during $t_{2}$. Instead of directly recording $N_{1}$ points during $t_{1}, N_{1}$ experiments are performed where the interval $t_{1}$ between the excitation pulse and the start of detection at $t_{2}=0$ is incremented experiment-by-experiment with an interval of $t_{1}^{\max } / N_{1}$. This is perhaps the most simple 2D NMR experiment: apply a pulse to create transverse magnetization, do not detect this magnetization over the course of a point-by-point incremented time $t_{1}$, and then measure the signal directly during the time $t_{2}$. Since the evolution during $t_{1}$ is not measured directly, $t_{1}$ is called an "indirect" time dimension, whereas $t_{2}$ is a "direct" time dimension. The spin evolution during $t_{1}$ has been switched from being directly detected to being indirectly detected, but the overall signal is the same as in Eq. 6.4:

$$
S\left(t_{1}, t_{2}\right) \propto \operatorname{Tr}\left[\hat{\rho}\left(t_{1}, t_{2}\right) \hat{I}_{+}\right]=-\frac{i}{2} e^{i \delta t_{1}} e^{i \delta t_{2}}
$$

Note that the NMR spectrum can be mapped out almost completely indirectly by measuring the signal at only a single point $t_{2}=0$ for every experimental increment of $t_{1}$, generating the one-dimensional data set $S\left(t_{1}, 0\right)$. Normally this would be an inefficient method of taking a one-dimensional spectrum because $N_{1}$ experiments are required rather than a single experiment. However, this indirect method may be worthwhile if the remote detection 
technique can offer an increase in sensitivity. ${ }^{3}$

When considering the two-dimensional NMR experiment described above, it is important to remember that when quadrature detection is employed (i.e., the observable is proportional to $\left.\hat{I}_{+}\right)$, only the evolution of the -1 -quantum coherence $(-1 \mathrm{QC})$ during the direct dimension contributes to the signal. During the indirect evolution the spin system is not observed, and no such automatic selection of the $-1 \mathrm{QC}$ occurs. Ordinarily, the application of a coherence pathway selection scheme can ensure that only the $-1 \mathrm{QC}$ is selected during $t_{1}$, resulting in a signal of the form of Eq. 6.5. However, since there are no mixing pulses between the $t_{1}$ and $t_{2}$ intervals that can change the coherence order of the system, the $-1 \mathrm{QC}$ is automatically selected during $t_{1}$. This is a consequence of the facts that the $-1 \mathrm{QC}$ is selected during direct detection and coherence order is preserved during free precession intervals.

The pulse sequence in Eq. 6.3 may be modified to include a back-to-back $\left[90_{\bar{\phi}}^{\circ}, 90_{\phi}^{\circ}\right]$ pulse pair between the $t_{1}$ and $t_{2}$ intervals, where $\bar{\phi}=\phi+\pi$. The pulse phase convention is defined such that a pulse with phase $\phi=0$ has a magnetic field in the $+x$ direction in the rotating frame, and the corresponding $\bar{\phi}=\pi$ pulse is in the $-x$ direction. Such a pair of pulses acts as a "do-nothing" element of the pulse sequence; i.e., the time development operator for a $\left[90_{\bar{x}}^{\circ}, 90_{x}^{\circ}\right]$ pair of ideal delta-function pulses (see $\left.\S 2.3 .3\right)$ is the identity operator, $\hat{U}_{90_{x}^{\circ}} \hat{U}_{90_{\bar{x}}^{\circ}}=e^{-i(\pi / 2) \hat{I}_{x}} e^{+i(\pi / 2) \hat{I}_{x}}=\hat{1}$. Since the pulses do nothing, the signal is the same

\footnotetext{
${ }^{3}$ Note that in principle, the $N_{1}$ indirect points may be encoded more rapidly in an NMR experiment that utilizes flow than in a conventional $2 \mathrm{D}$ NMR experiment. The rate at which indirect points can be encoded is limited in conventional 2D experiments by waiting for the equilibrium magnetization of the spins to recover via longitudinal relaxation. The next indirect point in a flow mode/remote detection experiment can be encoded as quickly as a fresh volume of carrier spins can be introduced into the encoding region.
} 
as in Eqs. 6.4 or 6.5:

$$
\begin{aligned}
& 90_{x}^{\circ}-t_{1}-\left[90_{\bar{x}}^{\circ}, 90_{x}^{\circ}\right]-t_{2} \text {-detect } \\
& S\left(t_{1}, t_{2}\right) \propto-\frac{i}{2} e^{i \delta t_{1}} e^{i \delta t_{2}}
\end{aligned}
$$

Coherence pathway selection is not necessary to obtain this signal even if the evolution during $t_{1}$ is detected indirectly, assuming that the pulse pair is perfect and causes no coherence transfers. For the sake of generality, one can also consider the situation where the system Hamiltonian changes suddenly at the division between the indirect and direct time intervals. The simplest case to consider is that of two time-independent Hamiltonians $\hat{H}_{1}$ and $\hat{H}_{2}$ that are active during the respective intervals $t_{1}$ and $t_{2}$ and commute with each other, $\left[\hat{H}_{1}, \hat{H}_{2}\right]=0$. If $\hat{H}_{1} / \hbar=\delta_{1} \hat{I}_{z}$ and $\hat{H}_{2} / \hbar=\delta_{2} \hat{I}_{z}$ represent two different rotating-frame resonance offset Hamiltonians, the signal due to the pulse sequence in Eq. 6.6 is found to be:

$$
S\left(t_{1}, t_{2}\right) \propto-\frac{i}{2} e^{i \delta_{1} t_{1}} e^{i \delta_{2} t_{2}}
$$

Such a situation could arise if the external magnetic field or the rf carrier frequency is suddenly changed after the end of the indirect evolution period. A two-dimensional Fourier transform of Eq. 6.8 gives a peak at the frequencies $\left(\delta_{1}, \delta_{2}\right)$ in the $2 \mathrm{D}$ spectrum. A projection of the $2 \mathrm{D}$ spectrum along either the indirect or direct frequency dimension yields the $1 \mathrm{D}$ NMR spectrum in the presence of the Hamiltonian $\hat{H}_{1}$ or $\hat{H}_{2}$, respectively. Note that in this manner the spectrum of the spin evolution during the interval $t_{1}$ can be recorded without 
making any direct measurements during this time. In particular, if only the first point $t_{2}=0$ is recorded for each of the $N_{1}$ transient experiments, the Fourier transform of the one-dimensional data set $S\left(t_{1}, 0\right) \propto-\frac{i}{2} e^{i \delta_{1} t_{1}}$ with respect to $t_{1}$ yields the indirectly-detected spectrum of the spin evolution under $\hat{H}_{1}$. The evolution under $\hat{H}_{2}$ during $t_{2}$ is immaterial for the purpose of determining the indirect spectrum, although the sensitivity of the detection can be improved by measuring for a longer period of time.

Now consider adding a delay $\Delta_{t}$ between the two back-to-back pulses. If the density operator after the first pulse but before the second, $\hat{\rho}\left(t_{1}^{+}, 0^{-}\right)$, commutes with the system Hamiltonian $\hat{H}_{t}(t)$ at all times $t$ during the delay $\Delta_{t}$, the density operator is preserved during the delay. Therefore, the signal calculated in Eq. 6.8 does not change with the addition of the delay:

$$
\begin{aligned}
& {\left[90_{x}^{\circ}-t_{1}-90_{\bar{x}}^{\circ}\right]-\Delta_{t}-\left[90_{x}^{\circ}-t_{2}-\text { detect }\right]} \\
& S\left(t_{1}, t_{2}\right) \propto-\frac{i}{2} e^{i \delta_{1} t_{1}} e^{i \delta_{2} t_{2}}
\end{aligned}
$$

A simple case when $\left[\hat{\rho}\left(t_{1}^{+}, 0^{-}\right), \hat{H}_{t}(t)\right]$ is guaranteed to be true is when $\hat{H}_{t}=0$. Since $\hat{H}_{t}$ is written in the rotating frame, the condition $\hat{H}_{t}=0$ holds when a constant $z$-directed magnetic field whose Larmor splitting is resonant with the rf carrier is applied during $\Delta_{t} \cdot{ }^{4}$ If $\hat{H}_{t} \neq 0$, it is difficult in practice to arrange a situation in which the Hamiltonian commutes with the density operator. This can be demonstrated by a more explicit calculation. Starting from initial $z$-magnetization, $\hat{\rho}(0) \propto \hat{I}_{z}$, the density operator immediately after the

\footnotetext{
${ }^{4}$ If separate encoding and detection coils are employed, the relevant rotating frame is that of the detection circuit.
} 
$\left[90_{x}^{\circ}-t_{1}-90_{\bar{x}}^{\circ}\right]$ sequence is:

$$
\hat{\rho}\left(t_{1}^{+}, 0^{-}\right) \propto \hat{I}_{z} \cos \left(\delta_{1} t_{1}\right)+\hat{I}_{x} \sin \left(\delta_{1} t_{1}\right)
$$

where the evolution during $t_{1}$ is under the Hamiltonian $\hat{H}_{1} / \hbar=\delta_{1} \hat{I}_{z}$. This density operator corresponds to magnetization in the direction of the unit vector $\underline{\widetilde{u}}=\cos \left(\delta_{1} t_{1}\right) \underline{\widetilde{z}}+\sin \left(\delta_{1} t_{1}\right) \underline{\widetilde{x}}$. This magnetization will be preserved during the delay $\Delta_{t}$ if the external magnetic field that gives rise to $\hat{H}_{t}$ is parallel to $\underline{\widetilde{u}}$ at all times. This is difficult to implement because the direction of $\underline{\widetilde{u}}$ depends on both $\delta_{1}$ and $t_{1}$, so the magnetic field direction would have to be adjusted for every increment of $t_{1}$ using a known value of $\delta_{1}$. The situation is even more complicated if the spectrum contains multiple resonances $\delta_{1}^{j}$. Furthermore, since the calculation takes place in the rotating frame, the $x$-component of $\underline{u}$ rotates with respect to laboratory-frame coordinates, so the complete preservation of the density operator during $\Delta_{t}$ would require the application of transverse $\mathrm{rf}$ fields. It would be almost hopeless to try to preserve the transverse magnetization component during $\Delta_{t}$ if $\hat{H}_{1}$ and $\hat{H}_{2}$ were due to two very different laboratory-frame magnetic fields. The question arises as to how the information about the evolution under $\hat{H}_{1}$ can be safely transmitted for detection through some environment that has a potentially arbitrary Hamiltonian $\hat{H}_{t}$.

Consider the situation encountered in a remote detection experiment that uses an rf coil for detection by Faraday induction. Here $\hat{H}_{1}$ and $\hat{H}_{2}$ are the resonance offset Hamiltonians in the encoding and detection coils, respectively, and $t_{1}$ and $t_{2}$ are the respective encoding and detection times. The encoding and detection coils are in separate locations, so the spins can experience external magnetic fields that differ between the coils. 
The Hamiltonian $\hat{H}_{t}$ corresponds to the interaction of the mobile carrier spins with some possibly varying external magnetic field during transport from the encoding coil to the detection coil. The travel time of the spins between the encoding and detection coils is $\Delta_{t}$. Each indirect point of the analyte spectrum is encoded in a volume $d V$ of the magnetized carrier. This information must be transmitted without loss as the volume is transported to the detection region.

Inspection of Eq. 6.11 reveals that there is a redundancy in the encoded magnetization: both the $z$-component and $x$-component of magnetization after the $\left[90_{x}^{\circ}-t_{1}-90_{\bar{x}}^{\circ}\right]$ indirect encoding sequence depend on the spin evolution during $t_{1}$ as the functions $\cos \left(\delta_{1} t_{1}\right)$ and $\sin \left(\delta_{1} t_{1}\right)$, respectively. If the transport Hamiltonian $\hat{H}_{t}$ is due to a magnetic field whose longitudinal component is much larger than its transverse component at all times, the $z$ component of the encoded magnetization will be very nearly preserved during transport. This is because this component of the magnetization is parallel to the magnetic field, i.e., that component of the density operator commutes with $\hat{H}_{t} \cdot{ }^{5}$ The transverse magnetization component will continue to precess during transport but decays with the intrinsic relaxation time $T_{2}$, and it will also experience bulk dephasing due to gradients of the longitudinal magnetic field. ${ }^{6}$ If the condition $1 / T_{1} \ll \Delta_{t} \ll 1 / T_{2}^{*}$ is satisfied, where $T_{1}$ is the longitudinal relaxation time of the carrier spins and $T_{2}^{*}$ is the effective transverse relaxation time including both intrinsic and bulk dephasing effects, the longitudinal component of the encoded magnetization will be preserved at the end of the travel time $\Delta_{t}$ but the transverse

\footnotetext{
${ }^{5}$ The requirement that the external magnetic field be directed along $z$ at all times during the transport is actually too restrictive. The spins in a magnetization volume experience a time-varying magnetic field as they are transported through a spatially inhomogeneous magnetic field. If this variation is adiabatic over the course of the transport the stored magnetization will follow the external field.

${ }^{6}$ The amount of gradient dephasing also depends on the rate at which the volume is transported through the gradient and on diffusion.
} 
component will be dephased:

$$
\hat{\rho}\left(t_{1}^{+}, 0^{+}\right) \propto \hat{I}_{z} \cos \left(\delta_{1} t_{1}\right)
$$

The signal recorded by the $\left[90_{x}^{\circ}\right.$ - detect $]$ quadrature detection sequence is:

$$
S\left(t_{1}, t_{2}\right) \propto-\frac{i}{2} \cos \left(\delta_{1} t_{1}\right) e^{i \delta_{2} t_{2}}
$$

This differs somewhat from Eq. 6.10 in that there is no frequency discrimination in the indirect spectrum between $+\delta_{1}$ and $-\delta_{1}$ due to the amplitude modulation of the signal $\cos \left(\delta_{1} t_{1}\right)=\frac{1}{2}\left(e^{+i \delta_{1} t}+e^{-i \delta_{1} t}\right)$; frequency discrimination can be restored by employing coherence pathway selection. The source of the difference between the signal in Eq. 6.13 and the signal in Eq. 6.10 that was built up by adding conceptual or "do-nothing" elements to the one-pulse NMR experiment is that the transverse magnetization is irreversibly dephased during the travel time $\Delta_{t}$.

A generalized remote detection NMR experiment may be represented as:

$$
\text { [encode } \left.t_{1}\right]-\Delta_{t}-[\text { detect }]
$$

i.e., the carrier is indirectly encoded point-by-point in one location as a function of the indirect time $t_{1}$ and transported to a second location for detection. In most cases of practical import the [encode] module can be further expanded as [prepare - evolve $t_{1}$-store]. In this case the remote detection experiment resembles the conceptual two-dimensional NMR experiment: [prepare $]-\left[\right.$ evolve $\left.t_{1}\right]-[\mathrm{mix}]-\left[\right.$ detect $\left.t_{2}\right]$ (see $\left.\S 1.8\right)$. One example of a remote 
detection encoding module is the $\left[90_{x}^{\circ}-t_{1}-90_{\bar{x}}^{\circ}\right]$ sequence, in which the first pulse prepares the system for evolution by converting initial $z$-magnetization to transverse magnetization, the delay $t_{1}$ allows the system to evolve, and the second pulse stores the information about this evolution as $z$-magnetization for transport during $\Delta_{t}$. The modular nature of the remote detection experiment allows this sequence to be replaced with sequences in which different types of NMR information are encoded, for example, a magnetic resonance image [287]. The nature of the [detect] module is also left purposely vague: the stored magnetization could be converted with a pulse to transverse magnetization for detection at high magnetic field using Faraday induction, or it could be detected directly using magnetometric techniques. One can conclude that the remote detection experiment is simply a generalization of the conventional NMR modality, the difference being that the NMR information is encoded indirectly point-by-point in the magnetization of a mobile carrier rather than being encoded directly, and the stored magnetization is then transported to another location to be detected by one of a variety of methods.

\subsubsection{Dipolar field-encoded remote detection NMR of an analyte}

Thus far NMR remote detection experiments have used encoding schemes in which the analyte (i.e., the sample of interest) is also the mobile carrier of the encoded longitudinal magnetization. For example, in the imaging or spectroscopy experiments involving xenon as the mobile carrier, it was the spatial distribution or chemical shift of the xenon itself that was encoded indirectly. It also would be useful to be able to encode information about a separate analyte in the magnetization of the mobile carrier. As an example, one could imagine flowing the carrier into or through a sample, encoding the NMR spectrum 
of a heteronucleus in that sample as stored carrier magnetization, and transporting the carrier to another location for sensitive detection. This chapter will explore the use of the long-range nuclear dipolar field for this purpose. Using this technique, the precession of the analyte can be encoded into longitudinal magnetization as in the conventional remote detection experiment, but then that encoded information can be transferred to a carrier nucleus which senses the magnetic field created by the analyte magnetization. This is a long-range (on the order of millimeters) interaction, so there is no need for the carrier ever to come in contact with the analyte and the technique is "non-invasive" even if the carrier is not chemically inert. ${ }^{7}$ However, as will be seen, the sensitivity of the technique is limited because the dipolar field effects are small, and the analyte needs to be concentrated and/or highly polarized.

\subsection{The distant dipolar field}

It has been known for some time that highly-magnetized nuclei in fluids can exhibit unusual spin dynamics due to the effect of their own bulk nuclear magnetic field. The strong, microscopically inhomogeneous local dipolar fields that drive the coherent dynamics in solids are averaged away by diffusion in fluids, leaving only the macroscopic mean field contribution of many distant dipoles [77]. The effects of the distant dipolar field (DDF, also known as the dipolar demagnetizing field) are weak but not negligible when the sample is highly magnetized, i.e., when the nuclei are highly polarized and/or there is a high spin

\footnotetext{
${ }^{7}$ A. Pines has noted that a similar information transfer from analyte to carrier nucleus can be induced by the nuclear Overhauser effect (NOE). However, NOE transfers are short range (i.e., they are on a molecular length scale), requiring the carrier to be mixed physically with the analyte. Under these circumstances the carrier needs to be chemically inert with respect to the analyte, and some mechanism needs to employed to extract the carrier from the analyte for transport.
} 
density.

The NMR effects of the DDF were first observed as a series of unexpected spin echoes in two-pulse experiments on condensed-phase He-3 $[289,290,291],{ }^{8}$ which has a large magnetization due to its high spin density and large thermal polarization at cryogenic temperatures and high magnetic field. DDF effects were later rediscovered in more conventional samples in the early 1990s. The DDF was observed to cause small resonance shifts and lineshape distortions after a single pulse in protic solvents [292]. Bowtell et al. used DDF theory to describe the multiple-echo effects that had been rediscovered in water [293, 294], multicomponent mixtures of protic solvents [295], and multicomponent heteronuclear systems [296]. Ref. [296] also proposed a method of indirectly imaging the DDF in heteronuclear systems and a two-dimensional technique for indirectly detecting the NMR spectrum of one spin species through the effect of its dipolar field on another (perhaps intermolecular) species. Morris et al. also investigated DDF-induced heteronuclear [297] and homonuclear [298] multiple spin echoes during this period.

At about the same time Warren's group independently began to study unusual peaks that appeared in the spectra of even very simple multidimensional NMR experiments on highly-magnetized systems $[299,300,301]$. The cause of these peaks was initially unclear and was originally ascribed to radiation damping effects, in which the precession of a highlymagnetized species induces an oscillating current in the receiver coil that is strong enough that the coil itself produces a transverse resonant magnetic field which causes a torque on the spins. However, it eventually became clear that radiation damping effects could

\footnotetext{
${ }^{8}$ The first experiment was in solid He-3 [289], in which DDF effects are observable because of an effective spin diffusion due to quantum-mechanical exchange.
} 
not explain all of the experimental results, particularly when gradient pulses that dephased the bulk magnetization were employed, and the consideration of additional DDF effects was necessary $[302,303]$. A great deal of the confusion about these multidimensional experiments was due to the fact that the additional peaks had the characteristics of multiple-quantum resonances. Unfortunately, the conventional quantum-mechanical theory of solution-state NMR had long before been stripped of its ability to describe coherent dipolar effects by the removal of the motionally-averaged dipolar Hamiltonian and the adoption of the singlemolecule reduced density operator. The discovery of coherent dipolar effects in solution lead to some rather dramatic descriptions of the generation of "impossible" cross peaks in $2 \mathrm{D}$ solution NMR and violation of the high-temperature approximation, as well as a pulse

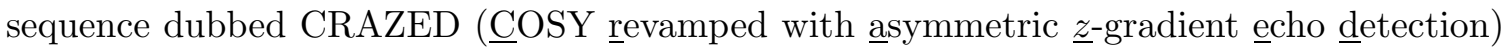
[303].

The quantum-mechanical theory was by then so integrated into the mainstream view of solution-state NMR that the DDF had to be re-justified as arising from long-range dipolar couplings that were not averaged away due to diffusion [302], despite its well-justified classical behavior in the multiple-echo experiments. Some workers even suspected that the classical DDF theory could not account for the complete behavior of the dipolar-induced intermolecular multiple-quantum coherences (iMQCs) [302]. At the time, a full quantum theory of iMQCs was not available and the connection between the multidimensional iMQC experiments and the multiple-echo DDF experiments hadn't been completed. Publications by Bowden et al. [304], Jeener et al. [75], and Levitt [305] helped to emphasize the correctness of the classical theory while also embracing the new ideas of the quantum-mechanical theory. 
Warren's group put forth a full quantum-mechanical theory of dipole-dipole interactions and iMQCs in liquids in an exhaustive work by Lee et al. in 1996 [76]. In this theory intermolecular multiple quantum coherences arise from multi-spin terms in the full $N \approx 10^{20}$-spin thermal equilibrium density operator that are converted into observable signal with the aid of rf pulses and short-time coherent dipolar coupling evolution. It is not a rigorous quantum-mechanical theory per se, in that it includes some very reasonable but non-quantum mechanical assumptions about the nature of molecular diffusion and the length of time over which dipole-coupled spin pairs can interact coherently in the presence of diffusion, which in turn restrict the number of coherence pathways available to the multi-spin system. These assumptions recover the classical DDF limit in which there are no correlated angular momentum dynamics between distant spins, as discussed in Ref. [76] and elsewhere [75, 306, 77, 307]. The multiple-echo DDF effects in homonuclear spin systems have since been explained in terms of the quantum formalism, where the $n$th echo arises due to $n$-spin terms in the full equilibrium density operator [308]. There have been many subsequent studies of CRAZED-type sequences and the nature, excitation, and dynamics of iMQCs in solution NMR, e.g. Refs. $[77,309,310,311,312,313,314,315,316]$. The long-range nature of iMQCs and DDF effects have made them useful probes of mesoscopic structure: e.g., as a contrast mechanism in magnetic resonance imaging [317, 318, 319, 320, 321, 322, 323, 324, 325]; in NMR multipleecho, scattering, and microscopy-type experiments [326, 327, 328, 329, 330, 331, 332, 333]; and even in the direct mapping of the dipolar field or magnetization distribution in solution $[334,335]$. Some of these experiments rely on the facts that the dipolar field is active only 
on the mesoscopic length scale $(\sim 10 \mu \mathrm{m}$ to $10 \mathrm{~mm})$ and that homonuclear intermolecular zero-quantum coherences are invariant to evolution under the $B_{0}$ field; these properties can be used to obtain narrow solution-state NMR spectra in the presence of a $B_{0}$ field that is inhomogeneous on the scale of sample dimensions but is relatively homogeneous on the length scale of the dipolar field [336, 337, 338, 339, 340, 341]. Recently, the DDF has been identified as a source of unusual turbulent/nonlinear spin dynamics in highly magnetized systems [342, 343, 344, 345, 346, 347, 348, 349, 350, 351]. Mukamel et al. have also tried to make analogies between intermolecular multiple-quantum coherences in NMR and certain nonlinear optics phenomena [352, 353, 354].

\subsubsection{Classical calculation of DDF effects}

The theory of calculating the dipolar field diverges into many seemingly-unrelated tentacles and this section attempts to review some of the common mean-field techniques. Some combination of these methods is employed in nearly all of the fast numerical algorithms now used for calculating classical DDF effects [355, 356, 357, 358, 359]. The discussion will

not include dynamic effects such as molecular diffusion (which can cause a redistribution of the sample magnetization) or radiation damping. Furthermore, the macroscopic mean-field approach is insufficient to describe the interesting dipole-dipole confinement effects that have been observed in fluid nanovolumes [360]. 


\section{Direct calculation of $\underline{B}_{d}(\underline{r})$}

The magnetic field created at the point $\underline{r}$ by $N$ non-overlapping classical magnetic dipoles $\underline{\mu}_{j}$ located at positions $\underline{r}_{j}$ can be found using Eq. $1.36:^{9}$

$$
\underline{b}_{d}(\underline{r})=\sum_{j=1}^{N} \frac{\mu_{0}}{4 \pi} \frac{1}{\left|\underline{r}-\underline{r}_{j}\right|^{3}}\left[\frac{3\left(\underline{r}-\underline{r}_{j}\right)\left[\left(\underline{r}-\underline{r}_{j}\right) \cdot \underline{\mu}_{j}\right]}{\left|\underline{r}-\underline{r}_{j}\right|^{2}}-\underline{\mu}_{j}\right],
$$

where $\underline{r}-\underline{r}_{j}$ is the vector from the location of $\underline{\mu}_{j}$ to the field point $\underline{r}$. It will be assumed that all the magnetic dipoles are of identical strength, $\left|\underline{\mu} \underline{j}_{j}\right|=\mu$. The lower-case notation $\underline{b}$ is used here to denote that this equation is valid microscopically, i.e., when matter is considered to be discrete ("granular") on an atomic level rather than continuous. The microscopic field can vary wildly on the atomic length scale, which is a true picture of the landscape that would be seen by a test dipole. Discussions of microscopic versus macroscopic fields may be found in Refs. [17, §6.6], [361, §1.2-1.3], and [362].

Eq. 6.15 can be rewritten as:

$$
\underline{b}_{d}(\underline{r})=\sum_{j=1}^{N} \underline{\underline{A}}_{D}^{j} \underline{\mu}_{j}
$$

where $\underline{\underline{A}}_{D}^{j}=\underline{\underline{A}}_{D}^{j}\left(\underline{r}-\underline{r}_{j}\right)$ is a second-rank tensor (see $\left.\S 1.3 .5\right)$. If the dipoles are fixed relative to each other in a molecular framework and $\underline{r}$ is static in the molecule-fixed frame (say $\underline{r}=\underline{r}_{i}$ marks the position of spin $i$ ), then $\underline{r}_{-} \underline{r}_{j}$ will randomly reorient in the laboratory frame due to rapid molecular tumbling in an isotropic fluid; the traceless tensor $\underline{\underline{A}}_{D}^{j}$ and hence the intramolecular dipolar field then averages to zero. The situation is different,

\footnotetext{
${ }^{9}$ Note that in this expression $\mu_{0}$ is a physical constant (the magnetic permeability of free space) rather than one of the classical dipoles.
} 
however, in the case of intermolecular interactions in a fluid. The vectors $\underline{r}_{j}$ point to spins on different molecules, and in a space-fixed frame $\underline{r}-\underline{r}_{j}$ wanders due to the diffusional motion of molecule $j$. Clearly, a molecule can diffuse only so far in a given amount of time, so in general the magnitude and direction of $\underline{r}-\underline{r}_{j}$ changes more during a given interval when $\left|\underline{r}-\underline{r}_{j}\right|$ is small than when $\left|\underline{r}-\underline{r}_{j}\right|$ is large. When the dipolar field is felt by a nucleus on some molecule $i$ such that $\underline{r}=\underline{r}_{i}$, the internuclear vector $\underline{r}_{i}-\underline{r}_{j}$ is more likely to undergo random isotropic reorientation while the molecules $i$ and $j$ are diffusing relative to each other when the molecules are initially close together than when they are far apart. The internuclear vector between nuclei on distant molecules hardly changes unless the diffusion time is very long, and $\underline{\underline{A}}_{D}^{j}$ does not orientationally-average to zero on a macroscopic length scale. ${ }^{10}$ This situation is described more quantitatively in Ref. [76]. The fact that the dipolar field is active on a macroscopic length scale should not be surprising; if it was not, a nucleus in a molecule undergoing diffusion could not produce a non-zero time-averaged magnetic field that could be felt by a test dipole (or a coil) external to the sample. Of course, the cases of a local dipolar field inside of and external to a magnetized medium are somewhat different, as will be discussed later.

It is difficult to work with a macroscopic number of $N \approx 10^{20}$ dipoles, and it is customary to take a simplifying mean-field approach. An average magnetization density $\underline{M}$

\footnotetext{
${ }^{10} \mathrm{As}$ is discussed in $\S 1.5 .4$, whether the orientational averaging is applied before or after secular truncation of the dipolar field (i.e., whether $\underline{\underline{A}}_{D}^{j}$ or just $\left(A_{D}^{j}\right)_{2,0}$ is averaged) depends on whether the internuclear vector reorients on a faster or slower timescale than the inverse Larmor frequency, which is determined by the intermolecular separation. However, since the truncated secular dipolar Hamiltonian also averages to zero under random reorientation of the internuclear vector, the arguments above still hold.
} 
may be introduced:

$$
\underline{M}(\underline{r})=\left\langle\sum_{j=1}^{N} \underline{\mu}_{j} \delta^{3}\left(\underline{r}-\underline{r}_{j}\right)\right\rangle,
$$

where the three-dimensional Dirac delta function $\delta^{3}\left(\underline{r}-\underline{r}_{j}\right)$ fixes the dipoles at $\underline{r}_{j}$. The angular brackets denote a "coarse-grained" spatial average such that enough dipoles are included in each small volume $d V$ that the average magnetization density $d \underline{M}$ is uniform across the volume. The delta function has units of inverse volume, so $\underline{M}$ has units of magnetic dipole moment per unit volume. The vector $\underline{M}$ is called the macroscopic magnetization density or sometimes just the macroscopic magnetization; it varies smoothly on a macroscopic length scale. The macroscopic magnetic dipolar field $\underline{B}_{d}(\underline{r})$ can be found by summing the contributions from each magnetization volume $d \underline{M}\left(\underline{r}^{\prime}\right)$ in the continuum limit:

$$
\underline{B}_{d}(\underline{r})=\frac{\mu_{0}}{4 \pi} \int d^{3} \underline{r}^{\prime} \frac{1}{\left|\underline{r}-\underline{r}^{\prime}\right|^{3}}\left[\frac{3\left(\underline{r}-\underline{r}^{\prime}\right)\left[\left(\underline{r}-\underline{r}^{\prime}\right) \cdot \underline{M}\left(\underline{r}^{\prime}\right)\right]}{\left|\underline{r}-\underline{r}^{\prime}\right|^{2}}-\underline{M}\left(\underline{r}^{\prime}\right)\right]
$$

This is a non-local equation, in the sense that the dipolar field at a single field point $\underline{r}$ depends on the magnetization density at every source point $\underline{r}^{\prime}$. Note that $\underline{B}_{d}(\underline{r})=\underline{B}_{d}\left[\underline{M}\left(\underline{r}^{\prime}\right)\right]$ is a functional of the magnetization $\underline{M}\left(\underline{r}^{\prime}\right)$.

High-field NMR studies are not sensitive to the entire dipolar field $\underline{B}_{d}$, only the part that affects the spin dynamics in the presence of a much larger applied magnetic field $\underline{B}_{0}=B_{0} \underline{\tilde{z}}$. The equation of motion of the magnetization $\underline{M}$ of a single spin species in the 
presence of a magnetic field $\underline{B}$ is:

$$
\begin{aligned}
\frac{d \underline{M}}{d t} & =\gamma \underline{M} \times \underline{B} \\
& =-\underline{\omega} \times \underline{M},
\end{aligned}
$$

where $\gamma$ is the gyromagnetic ratio characterizing the strength of the magnetic dipoles $\underline{\mu}_{j}$ that make up $\underline{M}$ and $\underline{\omega}=\gamma \underline{B}$. This equation is also valid in the rotating frame if $\underline{M}$ and $\underline{B}$ are replaced with the corresponding rotating frame variables. Eq. 6.19, when written in a component form that includes phenomenological relaxation effects, becomes the set of coupled Bloch equations:

$$
\begin{aligned}
\frac{d M_{x}}{d t} & =\gamma\left(M_{y} B_{z}-M_{z} B_{y}\right)-M_{x} / T_{2} \\
\frac{d M_{y}}{d t} & =-\gamma\left(M_{x} B_{z}-M_{z} B_{x}\right)-M_{y} / T_{2} \\
\frac{d M_{z}}{d t} & =\gamma\left(M_{x} B_{y}-M_{y} B_{x}\right)-\left(M_{z}-M_{e q}\right) / T_{1},
\end{aligned}
$$

where the equilibrium value of the magnetization is $M_{e q} \underline{\tilde{z}}$ and $T_{1}$ and $T_{2}$ are relaxation times for the components that are longitudinal and transverse to $z$, respectively. The distinction between longitudinal and transverse relaxation times is meaningful at high field. The Bloch equations may be solved trivially if $\underline{B}=\underline{B}_{0}$, but things are greatly complicated when the dipolar field is added $\left(\underline{B}=\underline{B}_{0}+\underline{B}_{d}\right)$, since $\underline{B}_{d}$ itself depends on $\underline{M}$. This turns Eq. 6.20 from a linear into a nonlinear set of equations. The Bloch equations are transformed in a 
frame rotating about $z$ at $\omega \approx \omega_{0}=\gamma B_{0}$, where the effective (secular) part of $\underline{B}_{d}$ is:

$$
\underline{B}_{d}^{s e c}(\underline{r})=\frac{\mu_{0}}{4 \pi} \int d^{3} \underline{r}^{\prime} \frac{1}{\left|\underline{r}-\underline{r}^{\prime}\right|^{3}}\left(\frac{3 \cos ^{2} \theta-1}{2}\right)\left[3 M_{z}\left(\underline{r}^{\prime}\right) \underline{\tilde{z}}-\underline{M}\left(\underline{r}^{\prime}\right)\right]
$$

where $\theta=\cos ^{-1}\left[\left(\underline{\widetilde{r}}-\underline{\widetilde{r}}^{\prime}\right) \cdot \underline{\widetilde{z}}\right]$ is the angle between $\underline{r}-\underline{r}^{\prime}$ and $\underline{z}$. Eq. 6.21 represents the effective dipolar field in a homonuclear system and is the direct generalization of Eq. 1.42.

Another situation of interest is the effect of a dipolar field created by the magnetization of a species $I$ on a heteronuclear species $S$. The effective field in a heteronuclear system can be found by taking the secular contribution in the doubly-rotating frame of $\omega_{0}^{I}=\gamma_{I} B_{0}$ and $\omega_{0}^{S}=\gamma_{S} B_{0}:$

$$
\underline{B}_{d}^{I, s e c}(\underline{r})=\frac{\mu_{0}}{4 \pi} \int d^{3} \underline{r}^{\prime} \frac{1}{\left|\underline{r}-\underline{r}^{\prime}\right|^{3}}\left(\frac{3 \cos ^{2} \theta-1}{2}\right)\left[2 M_{z}^{I}\left(\underline{r}^{\prime}\right) \underline{z}\right],
$$

where $\underline{M}^{I}$ is the spin- $I$ magnetization, and it is understood that this equation represents the effect of $\underline{B}_{d}^{I, s e c}(\underline{r})$ on the spin-S magnetization $\underline{M}^{S}$. The field point $\underline{r}$ represents a location of spin- $S$ magnetization and the source point $\underline{r}^{\prime}$ represents locations of spin- $I$ magnetization. The transverse terms of $\underline{B}_{d}^{s e c}$ in Eq. 6.21 disappear in Eq. 6.22 because they rapidly oscillate at $\left|\omega_{0}^{I}-\omega_{0}^{S}\right|$ in the frame of the spin $S$ and time-average to zero. Note that although the effective dipolar field in Eq. 6.22 is along the $z$-axis, it does not correspond to the $z$-component of $\underline{B}_{d}^{I}$ given by Eq. 6.18 . This is because some of the $z$-component of $\underline{B}_{d}$ is non-secular (also in the homonuclear case). Eq. 6.22 would correspond to the $z$-component of the full spin- $I$ dipolar field if the full field $\underline{B}_{d}$ was evaluated using only the $z$-component of $\underline{M}^{I}$. 
The "homonuclear" and "heteronuclear" labels are associated with the "strong dipolar coupling" and "weak dipolar coupling" limits of the secular dynamics (see §1.3.5). As will be discussed in $§ 6.2 .3$, even the collective effect of the distant dipolar couplings is very weak. In practice, almost any two homonuclear species satisfy the weak coupling condition where it is appropriate to use the "heteronuclear" secular dipolar field (Eq. 6.22) to describe the dynamics of one species due to the dipolar field of the other. The use of the "homonuclear" secular dipolar field (Eq. 6.21) will be limited mostly to studies of the self-dynamics of a spin species in the presence of its own dipolar field.

Eqs. 6.21 and 6.22 are difficult to evaluate analytically even for many symmetric distributions of the magnetization $\underline{M}(r)$ because of the $P_{2}(\cos \theta)$ dependence in the integrand. The $\left|\underline{r}-\underline{r}^{\prime}\right|^{-3}$ term also can cause difficulties in certain coordinate systems. It is more convenient to evaluate these equations in a conjugate Fourier space [289]. The spatial Fourier transforms of the magnetization $\underline{M}(\underline{r})$ and secular dipolar field $\underline{B}_{d}(\underline{r})$ in Eq. 6.21 (the secular label "sec" will be dropped for convenience) are [289]:

$$
\begin{aligned}
& \underline{\widetilde{M}}(\underline{k})=\int d^{3} \underline{r} e^{i \underline{k} \cdot \underline{\underline{M}}} \underline{\underline{M}}(\underline{r}) \\
& \underline{\widetilde{B}}_{d}(\underline{k})=\int d^{3} \underline{r} e^{i \underline{k} \cdot \underline{r}} \underline{B}_{d}(\underline{r}) \\
& =\frac{\mu_{0}}{4 \pi} \frac{4 \pi}{3} \frac{1}{2}\left[3(\underline{\widetilde{k}} \cdot \underline{\widetilde{z}})^{2}-1\right]\left[3 \widetilde{M}_{z}(\underline{k})-\underline{\widetilde{M}}(\underline{k})\right],
\end{aligned}
$$

where $\underline{k}=k \underline{\widetilde{k}}$ is the Fourier conjugate variable to $\underline{r}$ and has units of reciprocal length. ${ }^{11}$ Note that Eq. 6.24 is local in $\underline{k}$-space: there are no integrals over $\underline{k}^{\prime}$ involved, and only a

\footnotetext{
${ }^{11}$ This definition of $\underline{k}$ differs by a factor of $2 \pi$ from the definition that has been adopted by the magnetic resonance imaging community, which uses a convention of $\exp (i 2 \pi \underline{k} \cdot \underline{r})$ in spatial Fourier transforms.
} 
trivial algebraic computation is necessary to find the dipolar field in $\underline{k}$-space. An inverse Fourier transformation yields the dipolar field in coordinate space. Certain edge-effect errors that occur during numerical discrete Fourier transforms can be avoided by including empty grid space around the magnetization volume [355]. Some analytical calculations can be facilitated by making Fourier series expansions of the magnetization if the longitudinal and transverse components are each modulated with a single characteristic wavelength [291, 293].

A simple analytical expression for the dipolar field in position space may be obtained if the magnetization is strongly modulated along a single coordinate $\underline{s}$. This may be accomplished, for example, by employing magnetic field gradients $\partial B_{z} / \partial s$, as will be discussed in $\S 6.2 .3$. In such a case the homonuclear secular dipolar field in position space becomes [289]:

$$
\underline{B}_{d}(s)=\mu_{0} \Delta_{s}\left[M_{z}(s) \underline{\widetilde{z}}-\frac{1}{3} \underline{M}(s)\right]
$$

where $\Delta_{s}=\frac{1}{2}\left[3(\underline{\widetilde{s}} \cdot \underline{\widetilde{z}})^{2}-1\right]$. The heteronuclear secular dipolar field is:

$$
\underline{B}_{d}(s)=\mu_{0} \Delta_{s}\left[\frac{2}{3} M_{z}(s)\right] \underline{\widetilde{z}} .
$$

These equations are valid only when the magnetization is strongly modulated compared to the smallest sample dimensions, i.e., when the spectrum of $\underline{\widetilde{M}}(\underline{k})$ contains only high$|k|$ components such that all $|k L| \gg 1$, where $L$ is the distance to the nearest edge of the sample [363]. The magnetization is not required to be modulated at only a single characteristic wavelength, just that it is modulated along only one direction. Refs. [363] 
and [76] make important corrections to Eqs. 6.25 and 6.26 when the magnetization contains a $k=0$ (unmodulated) component, which never satisfies the condition $|k L| \gg 1$; the case of completely uniform magnetization in simple sample geometries is best treated by magnetostatic methods (see the next section). Note that the presence of unmodulated or weakly modulated magnetization is requisite for the detection of any signal since in that case $\underline{M}$ does not average to zero over the sample volume. The $k=0$ pathology of the Fourier transform technique has been noted in studies of classical dipole lattices [364]. Note that Eqs. 6.25 and 6.26 are local and depend only on the direction of $\underline{s}$ relative to $\underline{B}_{0}$ and the displacement in the $\underline{s}$-direction. The field does not depend on the geometry of the sample: when the magnetization is modulated on a length scale that is much shorter than the size of the sample, the dipolar field effectively does not "know" about the shape of the sample except at points near the edges.

\section{Calculation of $\underline{B}_{d}(\underline{r})$ by the magnetostatic method}

Recall that in normal NMR experiments the macroscopic nuclear magnetization $\underline{M}$ is a result of the thermal polarization of the nuclei in the presence of the magnetic field $\underline{B}_{0}$. The study of magnetic fields produced in magnetized media belongs to the realm of classical magnetostatics $[17, \S 5],[47, \S 6]$. The magnetostatic approach to calculating the nuclear dipolar field has been described clearly and with many illuminating examples by Levitt [305], Vlassenbroek et al. [365], and Augustine [366]. Magnetic fields inside of matter 
are usually calculated with the aid of an auxiliary field $\underline{H},{ }^{12}$ defined by:

$$
\underline{B}=\mu_{0}(\underline{H}+\underline{M}) .
$$

The rationale for the introduction of this new field is based on the classical view that all magnetic fields are produced from charged currents; some of these currents can be "free" (e.g., the electron supercurrent in a superconducting magnet that surrounds the sample), and some are "bound" within the matter itself (e.g., the induced currents of circulating atomic electrons) $[47, \S 6.2],[368, \S 10.8]$. The field $\underline{H}$ is the free current contribution to the magnetic field. Where there is no matter present, $\underline{M}=0$ and all currents are free, so $\underline{B}$ and $\underline{H}$ are simply proportional by a factor of $\mu_{0}$, the vacuum permeability.

Solutions of $\underline{B}$ or $\underline{H}$ in terms of $\underline{M}$ can be obtained using the Maxwell equations. If there are no time-varying macroscopic electric fields inside the sample, the relevant Maxwell equations for $\underline{B}$ are:

$$
\begin{gathered}
\underline{\nabla} \cdot \underline{B}=0 \\
\underline{\nabla} \times \underline{B}=\mu_{0} \underline{J},
\end{gathered}
$$

where $\underline{J}$ is the macroscopic volume current density. If $\underline{J}$ is separated into free and bound currents $\underline{J}=\underline{J}_{f}+\underline{J}_{b}$, then Eqs. 6.28 and 6.29 in conjunction with Eq. 6.27 yield the

\footnotetext{
${ }^{12}$ In the classic literature $\underline{H}$ is called the magnetic field and $\underline{B}$ is the magnetic flux density or magnetic induction field. The distinction between $\underline{B}$ and $\underline{H}$ has caused much confusion in the past [367]. Although the use of $\underline{H}$ is often more convenient for the experimentalist, the field $\underline{B}$ is the more fundamental quantity; following Griffiths $[47, \S 6.3], \underline{B}$ will be referred to as the magnetic field and $\underline{H}$ will remain nameless.
} 
differential equations for $\underline{H}$ :

$$
\begin{aligned}
\underline{\nabla} \cdot \underline{H} & =-\underline{\nabla} \cdot \underline{M} \\
\underline{\nabla} \times \underline{H} & =\underline{J}_{f},
\end{aligned}
$$

where the bound current is defined by

$$
\underline{J}_{b}=\underline{\nabla} \times \underline{M} .
$$

A medium in which the induced magnetization is proportional to the applied magnetic field is called a linear magnetic medium, which satisfies the relation:

$$
\underline{M}=\underline{\underline{\chi}} \underline{H},
$$

where $\underline{\underline{\chi}}$ is the magnetic susceptibility tensor and $\underline{\mathrm{H}}$ represents the applied field. Often the induced magnetization is parallel to the applied field, in which case:

$$
\underline{M}=\chi \underline{H},
$$

where $\chi$ is a constant called the magnetic susceptibility of the medium. Eq. 6.27 yields:

$$
\underline{B}=\mu_{0}(1+\chi) \underline{H}=\mu \underline{H},
$$

where $\underline{B}$ and $\underline{H}$ are proportional by a factor of $\mu=\mu_{0}(1+\chi)$, the magnetic permeability 
of the material. ${ }^{13}$ In vacuum there is no matter and hence no magnetic susceptibility or magnetization, so $\mu=\mu_{0}$, the permeability of free space.

The usual case considered in NMR is of a polarization field $\underline{H}_{0}=\underline{B}_{0} / \mu_{0}$ generated by a superconducting magnet which magnetizes the sample according to:

$$
\underline{M}_{t o t}=\left(\chi_{e}+\chi_{n}\right) \underline{H}_{0},
$$

where $\chi_{e}$ represents the electronic contribution to the magnetic susceptibility and $\chi_{n}$ represents the nuclear contribution. The electronic magnetization in a diamagnetic substance comes from induced currents in the atomic electrons. ${ }^{14}$ An electronically paramagnetic substance will also have a contribution to $\chi_{e}$ through the magnetic alignment of the electron magnetic moments. Nuclear paramagnetism, i.e., the magnetic alignment of the nuclear magnetic moments, is responsible for the nuclear susceptibility. The bulk nuclear susceptibility per unit volume can be calculated by statistical mechanical means in the high temperature limit according to the Curie law (Eq. 1.119):

$$
\chi_{n} \simeq C \mu_{0} \frac{\gamma^{2} \hbar^{2} I(I+1)}{3 k_{B} T}
$$

where $C=N / V$ is the concentration of a spin- $I$ nucleus with gyromagnetic ratio $\gamma$ and $k_{B} T$ is the thermal energy. Materials whose magnetization is due to spin paramagnetism act as linear magnetic media in the high temperature limit because in this limit the Curie law states that the thermal equilibrium magnetization is proportional to the applied magnetic field. An

\footnotetext{
${ }^{13}$ Note that here the symbols $\mu_{0}$ and $\mu$ represent magnetic permeabilities, not dipole moments.

${ }^{14}$ Although the diamagnetic susceptibility is a macroscopic quantity, its microscopic source is the same type of atomic-scale interactions that cause the chemical shielding [5, §4.5].
} 
$I=1 / 2$ nucleus at $T=298 \mathrm{~K}$ has a Curie susceptibility of $\chi_{n} \simeq\left(2.0 \times 10^{-14}\right) \cdot C \cdot(\gamma / 2 \pi)^{2}$ if $C$ is measured in molarity (moles per liter) and $\gamma / 2 \pi$ is measured in $\mathrm{MHz} /$ Tesla. Water $\left(\mathrm{H}_{2} \mathrm{O}\right)$ at room temperature has a concentration of $\sim 55.5 \mathrm{M}$; using a proton concentration of $C \sim$ $111 \mathrm{M}$ and gyromagnetic ratio $\gamma_{H 1} / 2 \pi=42.6 \mathrm{MHz} /$ Tesla, the bulk nuclear susceptibility of room temperature water is $\chi_{\mathrm{H} 1}^{\mathrm{H}_{2} \mathrm{O}}=+4.0 \times 10^{-9}$. The bulk electronic susceptibility of water, which is diamagnetic, is $-1.3 \times 10^{-5}$. $^{15}$ The electronic susceptibility usually dominates the nuclear susceptibility so completely that the total magnetic susceptibility of a substance is approximately equal to the electronic susceptibility.

The combination of Eq. 6.36 and Eq. 6.27 gives:

$$
\begin{aligned}
\underline{B} & =\mu_{0}\left(1+\chi_{e}+\chi_{n}\right) \underline{H}_{0} \\
& =\underline{B}_{0}+\underline{B}_{e}+\underline{B}_{n},
\end{aligned}
$$

where $\underline{B}_{e}=\mu_{0} \underline{M}_{e}=\mu_{0} \chi_{e} \underline{H}_{0}$ can be considered to be the magnetic field generated by the electrons and $\underline{B}_{d}=\mu_{0} \underline{M}_{n}=\mu_{0} \chi_{n} \underline{H}_{0}$ is the magnetic field generated by the magnetized nuclei. This equation holds only if the matter is uniformly magnetized and characterized by only one type of nuclear and electronic susceptibility; i.e., the problem has no boundary conditions. Any real sample, however, is finite and has physical boundaries: e.g., a liquid constrained in a cylindrical sample tube. In this case one must be concerned with how changes in susceptibility (e.g. from liquid to glass to air) affect the magnetic fields inside

\footnotetext{
${ }^{15}$ One generally expects the bulk electronic susceptibility of a diamagnetic compound to be negative, since the induced electronic currents generate magnetic fields that oppose the applied field, as is observed on the atomic level in the form of the chemical shielding. Conversely, bulk paramagnetic susceptibilities tend to be positive since the net alignment of nuclear or electronic dipoles (and hence the magnetization) is parallel to the applied field. Note that the net spin angular momentum aligns parallel to the applied field for nuclei with positive gyromagnetic ratios and antiparallel to the applied field for electrons and negative- $\gamma$ nuclei.
} 
these media. Once boundary conditions are added to the problem, e.g., in the case of a finite-length cylinder with uniform magnetization inside and zero magnetization outside, the bulk electronic and nuclear fields can become dependent on position:

$$
\underline{B}=\underline{B}_{0}+\underline{B}_{e}(\underline{r})+\underline{B}_{n}(\underline{r}) .
$$

The electronic and nuclear fields $\underline{B}_{e}(\underline{r})$ and $\underline{B}_{n}(\underline{r})$ also would become dependent on position if the electronic or nuclear magnetization were to be made non-uniform. The electronic magnetization is typically uniform in a homogeneous medium of constant $\chi_{e}$, but is not uniform in an inhomogeneous medium. The nuclear magnetization is uniform in a homogeneous medium but can be shaped under the combination of resonant radiofrequency and gradient pulses. The bulk electronic magnetization is not manipulated in an NMR experiment and the electronic susceptibility field $\underline{B}_{e}(\underline{r})$ is constant (in time) from the perspective of the nuclei, particularly since the electrons adjust themselves to perturbations of the nuclear magnetization on a timescale that is much faster than the nuclear dynamics. For this reason the electronic susceptibility field is often included as a contribution to inhomogeneity of the $\underline{B}_{0}$ field:

$$
\underline{B}=\underline{B}_{0}(\underline{r})+\underline{B}_{n}(\underline{r}),
$$

where $\underline{B}_{0}(\underline{r})=\underline{B}_{0}+\underline{B}_{e}(\underline{r})$. The dependence of $\underline{B}_{e}(\underline{r})$ on position indicates that even if the external field $\underline{B}_{0}$ is completely homogeneous (in practice, it never is), magnetic susceptibility boundaries inside or outside of the sample can contribute to magnetic field inhomogeneities 
that are felt by the nuclei. ${ }^{16}$ This causes a distribution of resonance frequencies across the sample, which leads to deleterious line broadening effects. Magnetic field inhomogeneities can be partially compensated by employing shim coils, if the inhomogeneities slowly vary over distance. As was mentioned previously, intermolecular zero-quantum coherences can also be used to obtain narrow lines in the presence of inhomogeneous fields.

As may be apparent, the bulk nuclear susceptibility field $\underline{B}_{n}\left[\underline{M}_{n}(\underline{r})\right]$ is the same entity as the distant (nuclear) dipolar field $\underline{B}_{d}[\underline{M}(\underline{r})]$ that has been discussed in previous sections. The most common magnetostatic method of calculating the dipolar field in terms of the magnetization comes from ignoring the polarization field $\underline{B}_{0}$ and considering the induced magnetization $\underline{M}(\underline{r})$ to be "frozen" into the medium, as in a ferromagnet. In this model the nuclear magnetization is no longer "induced" by $\underline{H}_{0}$, and one can define an internal field $\underline{H}_{d}$ such that:

$$
\underline{B}_{d}=\mu_{0}\left(\underline{H}_{d}+\underline{M}\right) .
$$

The only real free current $\underline{J}_{f}$ that generates $\underline{H}_{0}$ is zero in this model; the entire effective current is then due to only the bound current $\underline{J}_{b}=\underline{\nabla} \times \underline{M}$ created by the magnetization. Eqs. 6.30 and 6.43 become:

$$
\begin{aligned}
\underline{\nabla} \cdot \underline{H}_{d} & =-\underline{\nabla} \cdot \underline{M} \\
\underline{\nabla} \times \underline{H}_{d} & =0 .
\end{aligned}
$$

\footnotetext{
${ }^{16}$ Strictly speaking, the nuclei only feel the secular contribution of $\underline{B}_{e}(\underline{r})$, and $\underline{B}_{e}(\underline{r})$ must be modified to be microscopically correct, as will be discussed later.
} 
Since $\underline{\nabla} \times \underline{H}_{d}=0$, an effective magnetic "scalar potential" can be defined as in electrostatics, despite the fact that there are no real magnetic charges. ${ }^{17}$ The auxiliary field may be written in terms of the scalar potential $\Phi_{M}$ as:

$$
\underline{H}_{d}=-\underline{\nabla} \Phi_{M}
$$

Inserting Eq. 6.44 and Eq. 6.27 into Eq. 6.28 yields:

$$
\nabla^{2} \Phi_{M}=-\rho_{M}
$$

where $\rho_{M}=-\underline{\nabla} \cdot \underline{M}$ is the effective magnetic charge density that generates the nuclear field $\underline{H}_{d}$. Eq. 6.45 is an effective magnetostatic Poisson equation, which may be solved given suitable boundary conditions. The boundary conditions used here will be defined by a magnetization density $\underline{M}(\underline{r})$ that may be non-zero within some volume $V^{\prime}$ bounded by the surface $S^{\prime}$, but falls sharply to zero outside of $S^{\prime}$. The Poisson equation may be integrated with these boundary conditions to yield $[17, \S 5.9 . \mathrm{C}]$ :

$$
\Phi_{M}(\underline{r})=-\frac{1}{4 \pi} \int_{V^{\prime}} d^{3} \underline{r}^{\prime} \frac{\underline{\nabla}^{\prime} \cdot \underline{M}\left(\underline{r}^{\prime}\right)}{\left|\underline{r}-\underline{r}^{\prime}\right|}+\frac{1}{4 \pi} \int_{S^{\prime}} d^{2} \underline{r}^{\prime} \frac{\tilde{\tilde{n}}^{\prime} \cdot \underline{M}\left(\underline{r}^{\prime}\right)}{\left|\underline{r}-\underline{r}^{\prime}\right|},
$$

where primed coordinates are source coordinates and unprimed coordinates are field coordinates; $\underline{\widetilde{n}}$ represents the unit vector outwardly normal from the surface $S^{\prime}$. The first term is a volume integral over source points within $V^{\prime}$ and the second term is a surface integral over

\footnotetext{
${ }^{17}$ Note that the $\underline{E}$ and $\underline{B}$ fields of physical classical electric and magnetic dipoles are very different on a microscopic level (Fig. 10.8 of Ref. [368]), and the fields inside of polarized/magnetized media also differ (Fig. 10.21 of Ref. [368]).
} 
$S^{\prime}$ that arises due to the discontinuity in $\underline{M}$ at $S^{\prime}$. Note that the volume integral vanishes if the magnetization is uniform within the volume since $\underline{\nabla} \cdot \underline{M}=0$. Also note that both integrals involve powers of $\left|\underline{r}-\underline{r}^{\prime}\right|^{-1}$ instead of $\left|\underline{r}-\underline{r}^{\prime}\right|^{-3}$ as in Eq. 6.18. This may simplify the calculation if $\left|\underline{r}-\underline{r}^{\prime}\right|$ is expanded in a coordinate system.

The dipolar field may be calculated by the magnetostatic potential method using Eqs. 6.41, 6.44, and 6.46:

$$
\underline{B}_{d}(\underline{r})=\mu_{0}\left[\underline{M}(\underline{r})-\underline{\nabla} \Phi_{M}(\underline{r})\right]
$$

The secular part of $\underline{B}_{d}(\underline{r})$ gives the desired dipolar field contribution to the spin dynamics, after one important modification is made. The macroscopic dipolar field $\underline{B}_{d}$ is obtained by averaging the microscopic dipolar field $\underline{b}_{d}$ over some length scale $d V^{1 / 3}$ that is large compared to molecular dimensions. A nucleus at some position $\underline{r}$, however, is sensitive not only to the long-range environment, but also to its local environment. Consider the contribution of nearby dipoles within a volume $\delta V$ centered at $\underline{r}$ that is smaller than the the coarse-graining volume $d V$ over which $\underline{B}_{d}$ is uniform, but is large enough to include many dipoles. If this volume is spherical and contains many dipoles that are randomly distributed but aligned parallel to each other, the magnetic field contribution of these nearby dipoles is nearly zero $[305,365,362]$. Furthermore, when molecular diffusion is considered, the contributions of the nearest dipoles average to zero on an NMR timescale due to random reorientation of the internuclear vector. From a local perspective, the dipole at $\underline{r}$ does not see a net field from the nearby dipoles and appears to reside within an empty cavity $\delta V$. 
The local field felt by the dipole at $\underline{r}$ is:

$$
\underline{B}_{d}^{l}(\underline{r})=\underline{B}_{d}(\underline{r})-\underline{B}_{d}^{\delta}(\underline{r})
$$

where the field $\underline{B}_{d}^{\delta}(\underline{r})$ of a uniformly-magnetized spherical volume $\delta V$ at $\underline{r}$ has been subtracted from the macroscopic field to give the effective field inside the "cavity". The magnetic field inside a sphere of uniform magnetization $\underline{M}$ is $\frac{\mu_{0}}{4 \pi} \frac{8 \pi}{3} \underline{M}$, so the correct local field experienced by a nucleus is:

$$
\underline{B}_{d}^{l}(\underline{r})=\underline{B}_{d}(\underline{r})-\frac{2}{3} \mu_{0} \underline{M}(\underline{r})
$$

This approximation to the local field is called the Lorentz sphere correction and is commonly employed in magnetostatic treatments of the dipolar field [369, 305, 365, 362]. This correction obviously is not necessary if the nuclei that are the sources of the macroscopic dipolar field are completely external to the nuclei of interest, i.e., in the case of a solution containing "analyte" nuclei that is physically separated from the "probe" nuclei that sense the analyte dipolar field. Note that since a mean field approximation is still being used, the local field $\underline{B}_{d}^{l}$ is only an approximation to the exact microscopic field $\underline{b}_{d}$ in Eq. 6.15. Romalis et al. [370] have taken an approach that appears to be equivalent to saying that the macroscopic dipolar field $\underline{B}_{d}(\underline{r})$ is normally calculated using an average contribution of $\frac{\mu_{0}}{4 \pi} \frac{8 \pi}{3} \underline{M}(\underline{r})$ due to the contact term in Eq. 1.36 and that this the contact term should be 
subtracted out because the nuclei do not overlap. In that case Eq. 6.41 becomes:

$$
\underline{B}_{d}(\underline{r})=\mu_{0}\left[\underline{H}_{d}(\underline{r})+\frac{1}{3} \underline{M}(\underline{r})\right]
$$

The physical reasoning is somewhat different from that of the Lorentz sphere approach, but the result is the same.

The literature contains many magnetostatic calculations of the fields inside of magnetized media; some of these calculations were made in the context of electronic diamagnetic susceptibility or ferromagnetic susceptibility problems, but the methods are equally applicable to nuclear susceptibility problems. One useful result gives the internal field of a uniformly magnetized ellipsoidal volume [371, 365]:

$$
\underline{H}_{d}=-\underline{\underline{D}} \underline{M}
$$

where $\underline{\underline{D}}$ is a geometrical factor; it depends on only the shape of the sample, not on position. The tensor $\underline{\underline{D}}$ is easily specified in the principal axis system of the ellipsoid; i.e., by the components $D_{X X}, D_{Y Y}$, and $D_{Z Z}$, where $X, Y$, and $Z$ are along the three ellipsoid semiaxes. The trace of $\underline{\underline{D}}$ is $D_{X X}+D_{Y Y}+D_{Z Z}=1$. The dipolar field is given by Eqs. 6.41 and 6.49:

$$
\underline{B}_{d}=\mu_{0}(-\underline{\underline{D}} \underline{M}+\underline{M})
$$

The contribution of $\hat{H}_{d}$ to $\underline{B}_{d}$ often (but not always) opposes the contribution of $\underline{M}$ itself; for 
this reason, $\underline{H}_{d}$ is sometimes called the dipolar demagnetizing field and $\underline{\underline{D}}$ is a demagnetizing factor. Demagnetization field effects have been known in NMR for a long time, although not necessarily due to the nuclear susceptibility $[369,372,373]$. When the Lorentz sphere correction added to Eq. 6.52, the local dipolar field is:

$$
\begin{aligned}
\underline{B}_{d}^{l} & =\mu_{0}(-\underline{\underline{D}} \underline{M}+\underline{M})-\frac{2}{3} \mu_{0} \underline{M} \\
& =-\frac{\mu_{0}}{3}(3 \underline{\underline{D}}-\underline{\underline{1}}) \underline{M} .
\end{aligned}
$$

Note the formal similarity of this equation to Eq. 1.36 for the field of a magnetic dipole without the contact contribution, which can be written as $\underline{B}(\underline{r})=\underline{\underline{A}}_{D} \underline{\mu}$, where $\underline{\underline{A}}_{D}=$ $\frac{\mu_{0}}{4 \pi r^{3}}\left[3 \underline{\underline{r}} / r^{2}-\underline{\underline{1}}\right]$. Note, however, that $\underline{\underline{A}}_{D}$ is axially symmetric whereas $\underline{\underline{D}}$ is not axially symmetric unless the ellipsoid is axially symmetric, i.e., unless the semiminor axes along $X$ and $Y$ are equal. ${ }^{18}$ Note that $\left(3 \underline{\underline{r}} / r^{2}-\underline{\underline{1}}\right)$ and $(3 \underline{\underline{D}}-\underline{\underline{1}})$ are both traceless, since $\operatorname{Tr}[\underline{\underline{r}}]=r^{2}$, $\operatorname{Tr}[\underline{D}]=1$, and $\operatorname{Tr}[\underline{\underline{1}}]=3$. If the ellipsoid is axially symmetric, i.e., it is a spheroid with symmetry axis $\underline{\widetilde{Z}}$ along the direction $\underline{\widetilde{s}}$ in the laboratory frame, the secular contribution to $\underline{B}_{d}^{l}$ may be calculated in exact analogy to the calculation of the homonuclear secular dipolar Hamiltonian:

$$
\underline{B}_{d}^{l, s e c}=\mu_{0} \alpha_{s}\left[M_{z} \underline{\underline{z}}-\frac{1}{3} \underline{M}\right]
$$

\footnotetext{
${ }^{18}$ Here the ellipsoid is axially symmetric about the $Z$ axis; Refs. [371] and [365] have the symmetry axis along $X$.
} 
and in the heteronuclear case:

$$
\underline{B}_{d}^{l, s e c}=\mu_{0} \alpha_{s}\left[\frac{2}{3} M_{z}\right] \underline{\widetilde{z}},
$$

where the spheroid shape factor is $\alpha_{s}=-\frac{1}{2}\left(3 D_{Z Z}-1\right) \frac{1}{2}\left[3(\underline{\widetilde{s}} \cdot \underline{\widetilde{z}})^{2}-1\right] ; D_{Z Z}$ is the principal component of $\underline{\underline{D}}$ along $\underline{\widetilde{Z}} \cdot{ }^{19}$ Eqs. 6.54 and 6.55 still hold if the magnetized volume is a general ellipsoid but not a spheroid, but $\alpha_{s}$ then depends on additional elements of the $\underline{\underline{D}}$ tensor. Note the similarity between Eqs. 6.54 and 6.55 and the secular magnetic dipole fields given by Eqs. 1.42 and 1.43. Care should be taken to remember that Eqs. 6.54 and 6.55 do not represent the full local dipolar field, only its secular contribution.

The secular dipolar field given by Eqs. 6.54 and 6.55 for a uniformly magnetized ellipsoid also has the same form as the dipolar field given by Eqs. 6.25 and 6.26 for a unidirectional strongly modulated magnetization in an arbitrary volume. The interpretation, however, is entirely different. The geometric factor $\Delta_{s}$ in Eqs. 6.25 and 6.26 depends only on the orientation of the modulation direction relative to the $\underline{B}_{0}$ field; it does not depend on the shape or size of the sample as long as the modulation is on a much shorter length scale than any sample dimension. Conversely, the shape factor $\alpha_{s}$ in Eqs. 6.54 and 6.55 depends only on the shape of the sample (although not its size) and on the orientation of the sample (but not of the magnetization) relative to the $\underline{B}_{0}$ field. These properties can be appreciated by considering several useful special cases of the spheroid. A spherical sample has $D_{Z Z}=1 / 3$ and $\alpha_{s}=0$, and the local dipolar field vanishes everywhere inside a sphere that is uniformly magnetized along any direction. This holds not only for the secular

\footnotetext{
${ }^{19}$ This definition of $\alpha_{s}$ differs from the definition in Ref. [346] by a factor of -1 .
} 
contribution but for the whole dipolar field, because the Lorentz sphere correction exactly cancels the internal macroscopic field of the sphere. Often the dipolar field is small due to the symmetry of a uniformly-magnetized sample; this is why gradient pulses are often employed to create a strong modulation of the magnetization that breaks this symmetry (see §6.2.3). Two other special cases of the uniformly-magnetized spheroid are the extreme prolate spheroid tending toward an infinite cylinder with $D_{Z Z} \rightarrow 0$ and the extreme oblate spheroid tending toward a flat disk with $D_{Z Z} \rightarrow 1$. If the infinite cylinder has its axis along $\underline{B}_{0}$ such that $(\underline{\widetilde{s}} \cdot \underline{\widetilde{z}})=1$, then $\alpha_{s}=1 / 2$. A disk whose plane is perpendicular to $\underline{B}_{0}$ has $(\underline{\widetilde{s}} \cdot \underline{\widetilde{z}})=1$ and $\alpha_{s}=-1 ;$ a disk whose plane includes $\underline{B}_{0}$ has $(\underline{\widetilde{s}} \cdot \underline{\widetilde{z}})=0$ and $\alpha_{s}=1 / 2$. Any uniformly magnetized spheroid whose axis is at the magic angle with respect to $\underline{B}_{0}$ has $\left[3(\underline{\widetilde{s}} \cdot \underline{\widetilde{z}})^{2}-1\right]=0$, in which case the local secular dipolar field vanishes everywhere (independent of the direction of magnetization). This result has been used to average the inhomogeneous bulk (electronic) susceptibility field via magic-angle spinning experiments on liquids [374].

The magnetostatic calculations become more complicated if the magnetized volume is not ellipsoidal. Some simple cases have been solved or approximated. Levitt [305] has considered the problem of the dipolar field in a volume that has strongly-modulated magnetization in a single direction; the secular part of the field agrees with the predictions of Eqs. 6.25 and 6.26. The problem of the susceptibility field in finite-length cylinders of uniform but arbitrarily-directed magnetization has been studied by direct numerical evaluation of the magnetostatic surface integral [375] and via an expansion of the magnetostatic potential in a series of spherical harmonics [376]. Susceptibility effects in some other simple 
geometries were considered in Refs. [377] and [378]. The magnetostatic calculations become difficult when the magnetization is not uniform because the volume integral in Eq. 6.46 cannot be neglected. A simplification of Eq. 6.46 for cylindrical samples is discussed in $\S 6.5$.

\subsubsection{Quantum-mechanical calculation of intermolecular multiple quan- tum coherences}

The starting point for any quantum-mechanical calculation is the determination of the initial density operator and Hamiltonian. The quasi-motionally-averaged laboratoryframe Hamiltonian in solution is:

$$
\hat{H}_{l a b}=\hat{H}_{Z}+\hat{H}_{c s}+\hat{H}_{J}+\hat{H}_{D}(t)
$$

where the Zeeman, chemical shielding, $J$-coupling, and dipole-dipole coupling Hamiltonians have been included. All anisotropic interactions have been considered to be averaged to zero by rapid molecular tumbling except for the dipole-dipole interaction, which is retained but remains time dependent because of molecular diffusion. At high magnetic fields the Zeeman interaction dominates all other spin interactions determines the thermal equilibrium polarization. The homonuclear thermal equilibrium density operator for $N$ molecules $j$ that each include an $I=1 / 2$ nucleus is:

$$
\begin{aligned}
\hat{\rho}_{e q} & \simeq Q^{-1} \exp \left[-\beta \hat{H}_{Z}\right] \\
& =\prod_{j=1}^{N}\left(\frac{1}{2} \hat{1}_{j}+\zeta \hat{I}_{z j}\right)
\end{aligned}
$$


where Eq. 1.120 has been used and the thermal polarization of the nuclei is $\zeta=\tanh \left(\beta \hbar \gamma B_{0} / 2\right)$. Here $\beta=\left(k_{B} T\right)^{-1}$ is the reciprocal thermal energy, $\gamma$ is the gyromagnetic ratio, $\underline{B}_{0}=B_{0} \underline{\tilde{z}}$ is the polarizing magnetic field, and $N \approx 10^{20}$ for a macroscopic sample. Eq. 6.57 is exact for spin- $1 / 2$ nuclei and there is no need to apply the weak high temperature approximation Eq. 1.112 using $\zeta \approx \beta \hbar \gamma B_{0} / 2$. Eq. 6.57 is also general in that it could represent any sample magnetized along $z$ with polarization $\zeta$, regardless of the source of polarization.

The spin dynamics are best calculated in the nearly-resonant rotating frame where the Zeeman interaction is mostly subtracted out. For the sake of simplicity $J$-couplings will not be considered. The rotating-frame Hamiltonian is:

$$
\begin{aligned}
\hat{H} / \hbar & =\sum_{j=1}^{N} \delta_{j} \hat{I}_{z j}+\sum_{j=1}^{N} \sum_{k>j}^{N} \hat{H}_{D}^{j k} \\
& =\sum_{j=1}^{N} \delta_{j} \hat{I}_{z j}+\frac{1}{2} \sum_{j=1}^{N} \sum_{k \neq j}^{N} \omega_{D}^{j k}(t)\left[3 \hat{I}_{z j} \hat{I}_{z k}-\underline{\hat{I}}_{j} \cdot \underline{\hat{I}}_{k}\right],
\end{aligned}
$$

where the resonance detuning and chemical shift of nucleus $j$ has been included in the frequency $\delta_{j}$ and only the secular dipole-dipole coupling $\hat{H}_{D}^{j k}$ (Eq. 1.40) has been retained. The factor of $\frac{1}{2}$ before the dipolar Hamiltonian in the second line corrects for the doublecounting of couplings $\omega_{D}^{j k}(t)=\omega_{D}^{k j}(t)$. The secular dipolar coupling constant $\omega_{D}^{j k}(t)=$ $-\frac{\mu_{0}}{4 \pi} \gamma^{2} \hbar\left|\underline{r}_{j k}(t)\right|^{-3} P_{2}\left[\cos \theta_{j k}(t)\right]$ includes the dependence on the angle $\theta_{j k}(t)=\cos ^{-1}\left[\underline{\widetilde{r}}_{j k}(t) \cdot \underline{\widetilde{z}}\right]$ between the internuclear vector $\underline{r}_{j k}(t)=\underline{r}_{j}(t)-\underline{r}_{k}(t)$ and $\underline{B}_{0}$; the second-rank Legendre polynomial is $P_{2}(x)=\left(3 x^{2}-1\right) / 2$. The internuclear vector is explicitly time dependent due to molecular diffusion.

The density operator for two $z$-magnetized spin-1/2 heteronuclear species $I$ and $S$ 
is:

$$
\hat{\rho}_{e q}=\prod_{j=1}^{N_{I}} \prod_{k=1}^{N_{S}}\left(\frac{1}{2} \hat{1}_{j}^{I}+\zeta^{I} \hat{I}_{z j}\right)\left(\frac{1}{2} \hat{1}_{k}^{S}+\zeta^{S} \hat{S}_{z k}\right)
$$

where the polarizations are $\zeta^{I}=\tanh \left(\beta \hbar \gamma_{I} B_{0} / 2\right)$ and $\zeta^{S}=\tanh \left(\beta \hbar \gamma_{S} B_{0} / 2\right)$ at thermal equilibrium. The heteronuclear secular Hamiltonian is:

$$
\hat{H} / \hbar=\sum_{j=1}^{N_{I}} \delta_{j}^{I} \hat{I}_{z j}+\sum_{k=1}^{N_{S}} \delta_{k}^{S} \hat{S}_{z k}+\frac{1}{2} \sum_{j=1}^{N_{I}} \sum_{k=1}^{N_{S}} \omega_{D}^{j k}(t)\left[2 \hat{I}_{z j} \hat{S}_{z k}\right]
$$

where $\omega_{D}^{j k}(t)=-\frac{\mu_{0}}{4 \pi} \gamma_{I} \gamma_{S} \hbar\left|\underline{r}_{j k}(t)\right|^{-3} P_{2}\left[\cos \theta_{j k}(t)\right]$. Note that the secular dipolar Hamiltonian commutes with the resonance offset Hamiltonian in both the homonuclear and heteronuclear cases, so the evolution under these two Hamiltonians can be calculated separately and sequentially.

The central assumption of Warren's theory of intermolecular multiple-quantum coherences [76] is that the time $t_{c}$ over which each dipolar coupling can act coherently is short enough that $\underline{r}_{j k}(t)$ is approximately constant and:

$$
\left|\omega_{D}^{j k}\right| t_{c} \ll 1
$$

An estimate of the coherence time is the time over which $\underline{r}_{j k}(t)$ changes appreciably due to diffusion; after this time the spins have moved apart or the internuclear vector has reoriented randomly. The chief consequence of this assumption is that only the short time evolution under each dipolar coupling need be considered. Integrating the lowest-order contribution 
to Eq. 2.14 gives:

$$
\hat{\rho}(t) \simeq-\frac{i}{\hbar} t\left[\hat{H}_{D}^{j k}, \hat{\rho}(0)\right]
$$

where $t>t_{c}$. This approximation is valid when $\left\|\hat{H}_{D}^{j k} / \hbar\right\| t \ll 1$, i.e., $\left|\omega_{D}^{j k}\right| t \ll 1$. Warren et al. have shown that each dipolar coupling is able to act only once on the density operator through a single commutator; evolution pathways in which the same coupling $\omega_{D}^{j k}$ acts twice or more are negligibly small.

Another result of Warren et al. is that the $\underline{\hat{I}}_{j} \cdot \underline{\hat{I}}_{k}$ terms in Eq. 6.58 can be omitted if all the spins in the system are identical (i.e., they belong to the same chemical species) because they do not cause evolution that leads to observable signal. This is tantamount to neglecting the $\underline{M}\left(\underline{r}^{\prime}\right)$ term in Eq. 6.21. The assumption that the spins are identical implies $\delta_{j}=\delta$, i.e., all the spins have the same chemical shift and resonance offset. Note, however, that if the spins are subject to an inherently or susceptibility-induced inhomogeneous $\underline{B}_{0}$ field, their resonance frequencies will become dependent on position: $\delta_{j}=\delta_{j}(\underline{r})$. The same situation applies during a magnetic field gradient pulse. In these cases the spins are distinguishable through a position-dependent shift of their Larmor frequencies, and the assumption that the $\underline{\hat{I}}_{j} \cdot \underline{\hat{I}}_{k}$ terms (or the $\underline{M}\left(\underline{r}^{\prime}\right)$ term in the classical picture) can be neglected is suspect. This point will be discussed further in $\S 6.2 .3$.

If the $\underline{\hat{I}}_{j} \cdot \underline{\hat{I}}_{k}$ terms can be neglected, all the two-spin homonuclear dipolar Hamiltonians $\hat{H}_{D}^{j k} / \hbar=3 \omega_{D}^{j k} \hat{I}_{z j} \hat{I}_{z k}$ commute with each other, so the dipolar evolution can be calculated by sequential single commutations of the density operator with two-spin dipolar Hamiltonians. All the heteronuclear two-spin dipolar Hamiltonians $\hat{H}_{D}^{j k} / \hbar=2 \omega_{D}^{j k} \hat{I}_{z j} \hat{S}_{z k}$ 
commute with each other as well. The full equilibrium density operator in Eq. 6.57 may be expanded in a manner similar to Eq. 1.112:

$$
\hat{\rho}_{e q}=2^{-N}\left\{\hat{1}+\zeta \sum_{j}^{N} \hat{I}_{z j}+\zeta^{2} \sum_{j<k}^{N} \hat{I}_{z j} \hat{I}_{z k}+\zeta^{3} \sum_{j<k<l}^{N} \hat{I}_{z j} \hat{I}_{z k} \hat{I}_{z l}+\ldots\right\},
$$

where the $n$th sum in the series contains products only of $n$ single-spin operators $\hat{I}_{z j}$ (the product of $N-n$ single-spin identity operators is implicit in these terms). Eq. 6.63 is exact for a spin- $1 / 2$ system if the series is not truncated; no high-temperature approximation has been applied, although the weak high temperature approximation is certainly appropriate. As was discussed in $\S 1.4 .3$, the $n \neq 1$ terms do not correspond to observable (single-spin) magnetization. Evolution due to resonance offsets or rf pulses causes pure rotations of the single-spin operators, but it does not remove or add non-identity single-spin operators from an $n$-spin term in the density operator. At best, a combination of rf pulses and offset evolution can convert an $n$-spin term $\hat{B}_{n}^{e q}=2^{-N} \zeta^{n} \prod_{j=1}^{n} \hat{I}_{z j}$ in the equilibrium density operator into an arbitrary $n$-spin term:

$$
\hat{B}_{n}=\prod_{j^{\prime}=1}^{n} \hat{I}_{\alpha j^{\prime}}
$$

where $\alpha \in\{x, y, z\}$. The primed notation indicates that the index $j^{\prime}$ need not be taken sequentially from 1 to $n$, just as long as $n$ terms are taken in the product. Consider what happens when a two-spin density operator term $\hat{I}_{\alpha j} \hat{I}_{z k}$ evolves for a short time under the homonuclear dipolar coupling Hamiltonian $\hat{H}_{D}^{j k}$ (neglecting the $\underline{\hat{I}}_{j} \cdot \underline{\hat{I}}_{k}$ Hamiltonian term) 
according to Eq. 6.62:

$$
\begin{aligned}
& \hat{B}_{n=2}=\hat{I}_{x j} \hat{I}_{z k} \longrightarrow-\frac{i}{\hbar} t\left[3 \hbar \omega_{D}^{j k} \hat{I}_{z j} \hat{I}_{z k}, \hat{I}_{x j} \hat{I}_{z k}\right]=\frac{3}{4} \omega_{D}^{j k} t \hat{I}_{y j} \\
& \hat{B}_{n=2}=\hat{I}_{y j} \hat{I}_{z k} \longrightarrow-\frac{i}{\hbar} t\left[3 \hbar \omega_{D}^{j k} \hat{I}_{z j} \hat{I}_{z k}, \hat{I}_{y j} \hat{I}_{z k}\right]=-\frac{3}{4} \omega_{D}^{j k} t \hat{I}_{x j} \\
& \hat{B}_{n=2}=\hat{I}_{z j} \hat{I}_{z k} \longrightarrow-\frac{i}{\hbar} t\left[3 \hbar \omega_{D}^{j k} \hat{I}_{z j} \hat{I}_{z k}, \hat{I}_{z j} \hat{I}_{z k}\right]=0 .
\end{aligned}
$$

The term involving two longitudinal spin operators does not evolve under the dipolar coupling, but the terms containing a transverse spin operator on spin $j$ and a longitudinal spin operator on spin $k$ are reduced to transverse single-spin operators on spin $j$. This type of mechanism is well-known in solution-state NMR in the context of the conversion of unobservable intramolecular two-spin density operator (antiphase magnetization) terms into observable single-spin (in-phase) transverse magnetization under weak $J$-coupling evolution. Two-spin transverse terms $\hat{I}_{(x / y) j} \hat{I}_{(x / y) k}$ are not converted to single-spin terms after a single commutation with the dipolar Hamiltonian.

It should be clear by induction that an $n$-spin term containing $n-1$ longitudinal spin operators and a single transverse spin operator $j$ can be converted to observable singlespin transverse magnetization after commutation with $n-1$ separate dipolar couplings $\omega_{D}^{j k}$, e.g.:

$\hat{B}_{n=3}=\hat{I}_{x j} \hat{I}_{z k} \hat{I}_{z l} \longrightarrow-\frac{i}{\hbar} t\left[3 \hbar \omega_{D}^{j k} \hat{I}_{z j} \hat{I}_{z k},-\frac{i}{\hbar} t\left[3 \hbar \omega_{D}^{j l} \hat{I}_{z j} \hat{I}_{z l}, \hat{I}_{x j} \hat{I}_{z k} \hat{I}_{z l}\right]\right]=-\frac{9}{16} \omega_{D}^{j k} \omega_{D}^{j l} t^{2} \hat{I}_{x j}$.

It is in this manner that the multi-spin terms in the full $N$-spin density operator are con- 
verted into observable magnetization by long-range dipolar couplings. When summed over all spins, three spin terms like the one in Eq. 6.66 contribute a factor of $\zeta^{2} \sum_{k, l} \omega_{D}^{j k} \omega_{D}^{k l}=$ $\left(\zeta \sum_{k} \omega_{D}^{j k}\right)^{2}$ to the observable signal. An $n$-spin term with one transverse spin operator and $n-1$ longitudinal spin operators makes a contribution to the observable signal on the order of $\left(\zeta \sum_{k} \omega_{D}^{j k}\right)^{n-1}$. Note that if only single-spin terms were retained in the equilibrium density operator in the "strong" high temperature approximation (Eq. 1.114), the distant dipolar couplings would not contribute to any observable signal. Any commutation of a single-spin operator with a dipolar coupling either leaves the operator invariant (in the case of longitudinal magnetization) or creates an unobservable two-spin operator (in the case of transverse magnetization). The two-spin operator could only be converted back into singlespin transverse magnetization by commutation with the same dipolar coupling. Warren et al. have shown that this is a negligibly weak process because it goes as $\zeta \sum_{j}\left(\omega_{j k} t\right)^{2}$, and $\omega_{j k} t$ is already known from Eq. 6.61 to be very small. The abandonment of the strong high temperature approximation in favor of making the weak high temperature approximation or keeping the full $N$-spin density operator is what Warren et al. have called "violation of the high temperature approximation."

The details of the Warren iMQC calculations can be quite complex, particularly if spatial inhomogeneities such as magnetic field gradient pulses are included. Furthermore, only the classical theory is suitable for including radiation damping and molecular diffusion dynamics in a tractable manner. The Warren theory has been presented only very schematically and most of the remaining discussion will focus on the classical description, but schematic quantum calculations often provide the quickest insight into the results of a 
particular pulse sequence.

\subsubsection{DDF effects in homonuclear systems: resonance shifts and multiple echoes}

The effects of the distant dipolar field are best understood by considering a few simple examples of one- and two-pulse experiments in a homogeneous, homonuclear system (e.g., proton NMR experiments on pure water).

\section{Resonance shift under the dipolar field after the action of a single rf pulse}

Consider a infinite cylinder aligned parallel to $\underline{B}_{0}$ with uniform magnetization $\underline{M}_{0}=M_{0} \underline{\tilde{z}}$ also along $\underline{B}_{0}$. This could, for example, represent a thermally-polarized homogeneous sample in a long NMR tube. The magnetization immediately after a $\theta_{y}$ rf pulse is:

$$
\underline{M}_{\theta}=M_{0} \cos \theta \underline{\widetilde{z}}+M_{0} \sin \theta \underline{\widetilde{x}} .
$$

It is assumed that the pulse tips the magnetization uniformly across the sample, rather than the more realistic case that only the small region of magnetization near the rf coil is tipped. It is also assumed that the $\underline{B}_{0}$ field is perfectly homogeneous across the sample, so that the transverse magnetization does not spatially dephase. Relaxation effects are neglected. The secular contribution of the magnetization in Eq. 6.67 to the local dipolar field can be 
calculated according to Eq. 6.54 as:

$$
\begin{aligned}
\underline{B}_{d}^{l, s e c} & =\frac{1}{2} \mu_{0}\left[M_{0} \cos \theta \underline{\widetilde{z}}-\frac{1}{3} \underline{M}\right] \\
& =\frac{1}{3} \mu_{0} M_{0} \cos \theta \underline{\widetilde{z}}-\frac{1}{6} \mu_{0} M_{0} \sin \theta \underline{\widetilde{x}},
\end{aligned}
$$

where the value $\alpha_{s}=\frac{1}{2}$ has been used for an infinite cylinder whose symmetry axis is

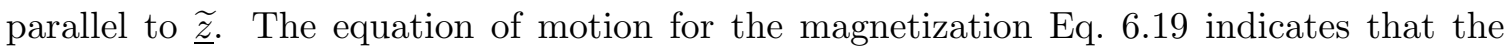
term proportional to $\underline{M}$ in Eq. 6.68 has no effect on the spin dynamics because $\underline{M} \times \underline{M}=0$, and the total effective magnetic field felt by the nuclei is:

$$
\underline{B}^{e f f}=\left(B_{0}+\frac{1}{2} \mu_{0} M_{0} \cos \theta\right) \underline{\widetilde{z}}
$$

where the chemical shift has been included in the definition of $B_{0}$. The spins therefore see an increase (if $M_{0}>0$ ) in their resonance frequency by the factor:

$$
\Delta \omega_{\theta}=\frac{1}{2} \mu_{0} \gamma M_{0} \cos \theta
$$

This shift in resonance frequency is most pronounced when the tip angle $\theta$ is small and most of the initial magnetization remains along $\underline{\underline{z}}$, but $\theta$ must be large enough to create an observable amount of transverse magnetization. No dipolar field-induced resonance shift is observed after a $\theta=\pi / 2$ pulse. The results of the $\theta$-tip angle experiment could be compared with an experiment using a $\theta_{y}^{\prime} \mathrm{rf}$ tip pulse, where $\theta^{\prime}=\pi-\theta$. This would cause a shift in resonance frequency by $-\Delta \omega_{\theta}$ due to dipolar field effects, or a $-2 \Delta \omega_{\theta}$ decrease in frequency 
from the $\theta$-tip experiment. One could imagine using these two single-pulse experiments to perform a nuclear self-magnetometry determination of $M_{0}$; a more detailed study would $\operatorname{map} \Delta \omega_{\theta}$ versus $\theta$. Of course, this determination would requires Eq. 6.68 to be accurate, i.e., the initial magnetization is uniform and the rf pulse uniformly tips the magnetization over the length of a long cylinder. The two-pulse determination would be the most accurate if $\theta$ is small since the secular dipolar field is maximized and the transverse magnetization (which induces radiation damping effects if the detection is by Faraday induction) is minimized. Note that the resonance shift is expected to be small; protons in room temperature water in a $700 \mathrm{MHz}$ magnet have $\frac{1}{2} \mu_{0} \gamma M_{0}=\frac{1}{2} \chi^{H_{2} O} \gamma B_{0}=\frac{1}{2}\left(4.0 \times 10^{-9}\right) \cdot\left(2 \pi \times 700 \times 10^{6}\right.$ $\mathrm{Hz})=2 \pi \times 1.4 \mathrm{~Hz}$. Therefore, the maximum frequency difference between the $\theta \approx 0$ and $\theta^{\prime} \approx \pi$ experiments is about $2.8 \mathrm{~Hz}$ in a $700 \mathrm{MHz}$ magnet, which may be not too much larger than the width of the water resonance.

Edzes [292] made the first measurements of self-precession and cross-precession in homonuclear systems after the application of a single pulse. One effect that was observed during these experiments was a "frequency chirp" of the NMR line; this effect was ascribed to the transient change in $M_{z}$ as the longitudinal magnetization was restored to its equilibrium value after the action of the $\theta$-pulse. The resulting time-dependent frequency shift of the NMR line was found to interfere with the establishment of an internal frequency lock because the $T_{1}$ relaxation time was faster than the lock time constant.

The effects of the dipolar field are slightly different when two spin species are present rather than just one. Consider a mixture of two homonuclear species $I_{A}$ and $I_{B}$ (of the same gyromagnetic ratio $\gamma$ ) in a long cylindrical sample with uniform magnetization 
$\underline{M}_{0}=\underline{M}_{0}^{A}+\underline{M}_{0}^{B}=M_{0}^{A} \underline{\widetilde{z}}+M_{0}^{B} \underline{\widetilde{z}}$. For instance, one species could be water and the other could be acetone. The magnetization after a non-selective uniform $\theta_{y}$-tip pulse is:

$$
\underline{M}_{\theta}=\left(M_{0}^{A} \cos \theta \underline{\widetilde{z}}+M_{0}^{A} \sin \theta \underline{\widetilde{x}}\right)+\left(M_{0}^{B} \cos \theta \underline{\widetilde{z}}+M_{0}^{B} \sin \theta \underline{\widetilde{x}}\right) .
$$

As per Eq. 6.70, species $I_{A}$ sees a resonance shift of $\Delta \omega_{\theta}^{A A}=\frac{1}{2} \mu_{0} M_{0}^{A} \cos \theta$ due to the dipolar field of its own magnetization and species $I_{A}$ sees a self-resonance shift of $\Delta \omega_{\theta}^{B B}=$ $\frac{1}{2} \mu_{0} M_{0}^{B} \cos \theta$. However, each species also precesses in the dipolar field of the other. A simple analysis of the coupled Bloch equations for $\underline{M}_{A}$ and $\underline{M}_{B}$ indicates that the strongcoupling "homonuclear" limit of the secular dipolar field crosses over to the weak-coupling "heteronuclear" limit when $\left|\omega_{0}^{A}-\omega_{0}^{B}\right| \gg\left|\gamma B_{d}\right|[363,305,365]$, i.e., when the difference in resonance frequency between the two species becomes much greater than the shifts in resonance frequency caused by the action of the dipolar field of one species on the other. These shifts are expected to be on the order of $\sim 1 \mathrm{~Hz}$ at best for concentrated roomtemperature protic solvents at high field; most homonuclear species have lines that are spaced by much more than this. Therefore, it is more appropriate to use the "heteronuclear" expression Eq. 6.55 for the secular dipolar field:

$$
\underline{B}_{d}^{l, s e c}=\frac{1}{2} \mu_{0}\left[\frac{2}{3} M_{0} \cos \theta\right] \underline{\widetilde{z}}
$$

where it is implicitly understood that this secular contribution is felt by one species in the presence of the dipolar field of the other species. In addition to the self-dipolar field precession, the spin species experience cross-precessional resonance shifts of $\Delta \omega_{\theta}^{A B}=$ 
$\frac{1}{3} \mu_{0} \gamma M_{0}^{B} \cos \theta$ and $\Delta \omega_{\theta}^{B A}=\frac{1}{3} \mu_{0} \gamma M_{0}^{A} \cos \theta$, where the $\Delta \omega_{\theta}^{X Y}$ notation indicates the dipolar field felt by species $X$ due to the dipolar field of species $Y$. The total resonance shifts are:

$$
\begin{aligned}
\Delta \omega_{\theta}^{A} & =\frac{1}{2} \mu_{0} \gamma\left(M_{0}^{A}+\frac{2}{3} M_{0}^{B}\right) \cos \theta \\
\Delta \omega_{\theta}^{B} & =\frac{1}{2} \mu_{0} \gamma\left(M_{0}^{B}+\frac{2}{3} M_{0}^{A}\right) \cos \theta .
\end{aligned}
$$

An interesting result that has been noted by several workers $[365,306]$ is that when $\theta<$ $\pi / 2$, even if the two species have the same magnetization $M_{0}^{A}=M_{0}^{B}=M_{0}$, dipolar field cross-precession when causes a small redistribution between the Zeeman energy and $z$-magnetization such that the two lines have unequal intensities, although their sum is conserved.

The case of two heteronuclear species $I$ and $S$ at high field is similar except pulses of different tip angles can be individually applied to each species using different $\mathrm{rf}$ channels. This is only possible in the homonuclear case by the use of frequency-selective pulses. Consider the case in which no pulse is applied to spin $I$ and a $90^{\circ}$ pulse is applied to spin $S$ $\left(\theta^{I}=0, \theta^{S}=\pi / 2\right)$. The self-dipolar field precession of spin $S$ is zero since the magnetization is purely transverse; the precession of $S$ due to the dipolar field of $I$ is maximized since all the $I$ magnetization remains along $z$. The resonance shift of spin $S$ due to the dipolar field of $\operatorname{spin} I$ is:

$$
\Delta \omega^{S I}=\frac{1}{3} \mu_{0} \gamma_{S} M_{0}^{I}
$$

In this manner spin $S$ can be used as a nuclear magnetometer to determine the magneti- 
zation of spin $I$. Again, this result is valid for a uniformly-magnetized, homogeneous, long cylindrical system that is uniformly affected by rf pulses.

One final point to consider about the single-pulse experiment is what happens when the $\underline{B}_{0}$ field is not homogeneous (i.e., due to magnetic susceptibility effects) or when a pulsed magnetic field gradient of $\underline{B}_{z}$ is applied. In both cases the total $z$-directed magnetic field $B_{0}(\underline{r}) \underline{z}$ (excluding the dipolar field) becomes dependent on position and the resonance frequency varies from point to point in the sample. As a consequence, even if the sample is initially uniformly magnetized at thermal equilibrium and that uniformity is preserved after the application of a homogeneous rf pulse, the transverse magnetization will spatially dephase as it evolves under the inhomogeneous $\underline{B}_{0}$ field. The integrand of Eq. 6.21 for the secular homonuclear dipolar field contains a term proportional to $\underline{M}\left(\underline{r}^{\prime}\right)$; call this component of the field $\underline{B} \frac{M}{d}$. Clearly $\underline{B} \frac{M}{d} \propto \underline{M}$ if the magnetization $\underline{M}\left(\underline{r}^{\prime}\right)=\underline{M}$ is uniform. When the magnetization is spatially inhomogeneous, however, $\underline{B} \underline{\underline{M}}(\underline{r})$ may not be proportional to $\underline{M}(\underline{r})$ because it depends on the size and direction of $\underline{M}\left(\underline{r}^{\prime}\right)$ everywhere. The $\underline{B} \frac{M}{d}$ contribution to the secular dipolar field was neglected in Eq. 6.68 when the magnetization was uniform because $\underline{M} \times \underline{B} \frac{M}{d}=0$ in the Bloch equations; the result was that the effective homonuclear secular dipolar field was entirely longitudinal. This contribution of $\underline{B} \frac{\underline{M}}{d}(\underline{r})$ cannot be neglected when it is not proportional to $\underline{M}(\underline{r})$ because the cross product of the two vectors is not zero. Therefore $\underline{M}(\underline{r})$ precesses about $\underline{B} \frac{M}{d}(\underline{r})$ (and simultaneously about the purely $z$-directed dipolar field contribution) such that transverse magnetization can be tipped out of the transverse plane. In this case dipolar field effects cannot be ignored even if the magnetization is initially purely transverse. If the $\underline{B}_{0}$ inhomogeneities 
are strong, however (perhaps due to a strong gradient pulse), it will be assumed that the strong dephasing of transverse magnetization across the sample will lead to a small contribution to $\underline{B} \frac{M}{d}(\underline{r})$ from the magnetization at distant points $\underline{r}^{\prime}$; in this case $\underline{B} \frac{M}{d}(\underline{r})$ may be nearly parallel to $\underline{M}(\underline{r})$ in which case the effects of $\underline{B} \underline{d}$ can be neglected. ${ }^{20}$ These transverse field effects would not be observed in heteronuclear or most multicomponent homonuclear systems because the secular dipolar field (Eq. 6.22) does not depend on any transverse components of $\underline{M}\left(\underline{r}^{\prime}\right)$, and hence is invariant to spatial dephasing of transverse magnetization during evolution under an inhomogeneous $\underline{B}_{0}$ field.

\section{Two-pulse experiments and multiple echoes}

It is well-known that the two-pulse $90^{\circ}-\tau-90^{\circ}$ pulse sequence generates a spin echo at time $\tau$ after the second pulse. In fact, this is the original sequence that Hahn used when he discovered the spin echo phenomenon [379]. The Hahn echo occurs after the second pulse initiates a rephasing of transverse magnetization that has dephased due to evolution in an inhomogeneous $\underline{B}_{0}$ field during the interval between the two pulses. The dephasing of transverse magnetization due to $T_{2}$ relaxation is caused by random magnetic field fluctuations on the molecular level and cannot be reversed to form an echo. A $90^{\circ}$ $\tau-180^{\circ}$ pulse sequence completely refocuses the inhomogeneous dephasing, but the $90^{\circ}$ $\tau-90^{\circ}$ sequence induces only a partial refocusing. Nevertheless, both sequences produce a single spin echo at a time $t=\tau$ after the second pulse. Therefore, it was very surprising when multiple echoes were observed by Deville et al. [289] and later workers after applying

\footnotetext{
${ }^{20}$ In this case the transverse magnetization becomes strongly modulated due to dephasing and one might think that Eq. 6.25 would be applicable, which indicates that the $\underline{B} \frac{M}{d}$ term is parallel to $\underline{M}$. However, here the magnetization is not strongly modulated along a single direction, and Eq. 6.25 does not apply.
} 
two-pulse sequences to highly magnetized systems. The appearance of multiple echoes was linked to the effects of the distant dipolar field; these effects will be illustrated here with the results of some simple two-pulse H-1 NMR experiments (with and without pulsed field gradients) in room temperature water in a cylindrical sample tube at $B_{0}=7.04 \mathrm{~T}$.

Fig. 6.2 shows the pulse sequences and experimental spectra that will be discussed. The simplest sequences to calculate are the ones that involve strong magnetic field gradient pulses because the sample geometry will not be important in determining the dipolar field. Relaxation and molecular diffusion effects will be neglected, and all calculations will be performed in the rotating frame. The only magnetic fields considered during free precession periods will be the local secular dipolar field, a $z$-directed static resonance offset field $\underline{B}_{\delta}(\underline{r})=\left[\left(1-\sigma_{i s o}\right) B_{0}(\underline{r})-\omega_{r} / \gamma\right]$ that is possibly inhomogeneous, and pulsed magnetic field $z$-gradients $G\left(z-z_{0}\right) \underline{\widetilde{z}}$, where $G=\partial B_{z} / \partial z$. The variable $z_{l}=z-z_{0}$ will be introduced as a matter of convenience.

Continuous gradients. Fig. 6.2(a) is the continuous-gradient version of the two pulse experiment. The initial thermal equilibrium magnetization is $\underline{M}(0)=M_{0} \underline{\tilde{z}}$. An initial $90_{x}^{\circ}$ pulse creates transverse magnetization

$$
\underline{M}\left(0^{+}\right)=-M_{0} \underline{\widetilde{y}}
$$

in an approximately $1 \mathrm{~cm}$-high portion of the sample within the active region of the $\mathrm{rf}$ coil. The rest of the magnetization in the sample tube is considered to be unaffected by the rf pulse and contributes no signal; it also will be assumed to generate no significant 

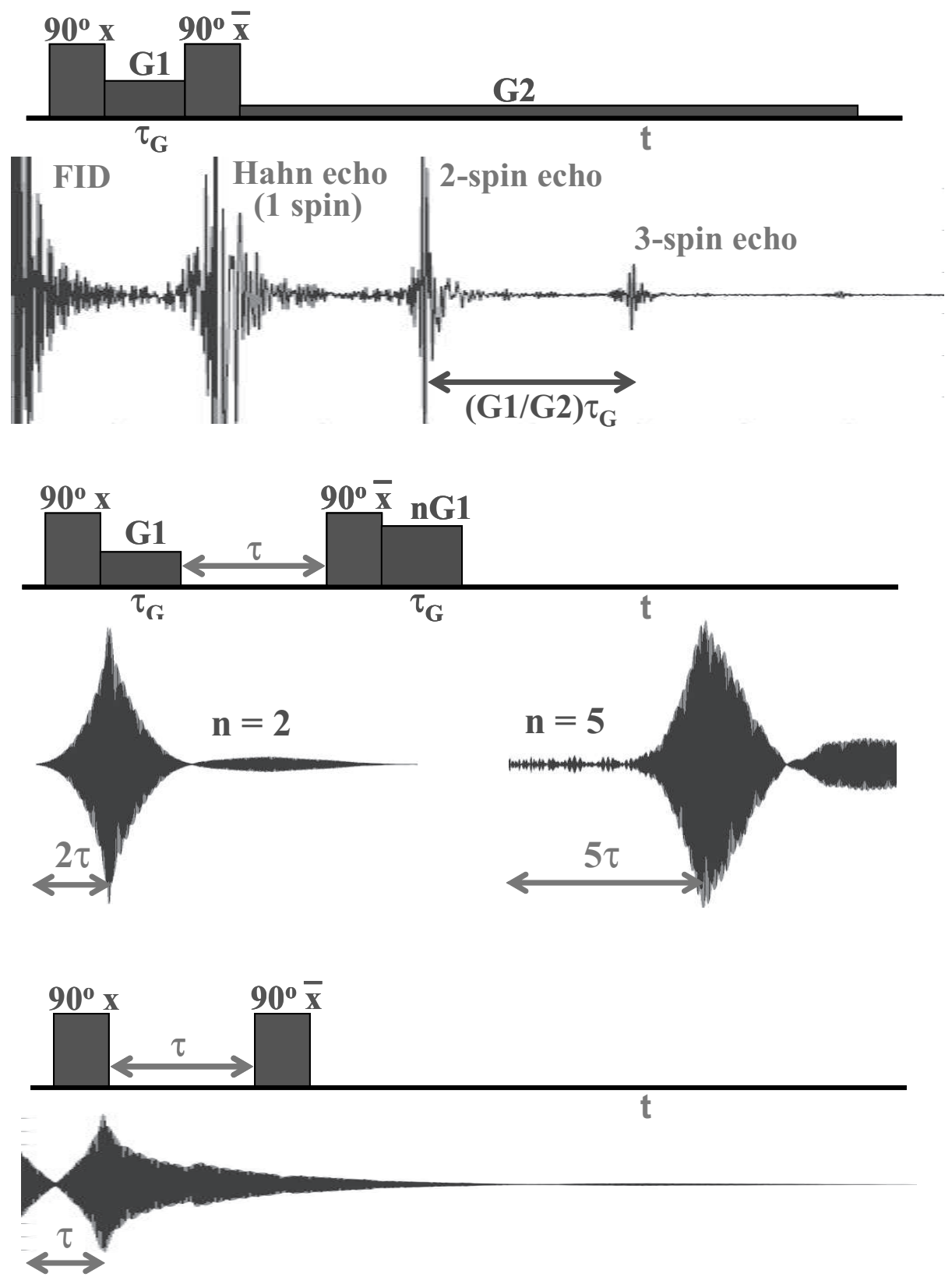

Figure 6.2: Dipolar field-induced multiple echoes in water observed after two-pulse sequences.

dipolar field in the active region. ${ }^{21}$ Immediately after the first rf pulse a $z$-gradient pulse

\footnotetext{
${ }^{21}$ In actuality, the longitudinal magnetization outside the active region generates at least some small dipolar field within the active region near the top and bottom edges. Also, molecular diffusion is expected to transport some small amount of magnetization across the "boundaries" of the active region.
} 
of strength $G_{1}$ is applied for duration $\tau_{G}$. The gradient is assumed to be large enough that the inhomogeneities in $\underline{B}_{0}$ can be neglected by comparison; dipolar field effects will also be neglected because the magnetization is purely transverse and strongly dephased and the contribution of $\underline{B} \frac{M}{d}$ (discussed in the section on single-pulse experiments) is considered to be small. Therefore, just before the second pulse the transverse magnetization is:

$$
\underline{M}\left(z_{l}, \tau_{G}^{-}\right)=-M_{0} \cos \left[\left(\delta-\gamma G_{1} z_{l}\right) \tau_{G}\right] \underline{\underline{y}}+M_{0} \sin \left[\left(\delta-\gamma G_{1} z_{l}\right) \tau_{G}\right] \underline{\widetilde{x}}
$$

where $\delta=-\gamma B_{\delta}$. At time $\tau_{G}^{-}$the magnetization is purely transverse and is wound in a helix of pitch $2 \pi / k_{1}=2 \pi /\left(\gamma G_{1} \tau_{G}\right)$ over the length of the active region of the sample. ${ }^{22}$ There are $k_{1} L / 2 \pi$ windings of the helix over the length of the active region $L$. The $90_{\bar{x}}^{\circ}$ pulse converts the magnetization to:

$$
\underline{M}\left(z_{l}, \tau_{G}^{+}\right)=M_{0} \cos \left[\left(\delta-\gamma G_{1} z_{l}\right) \tau_{G}\right] \underline{\widetilde{z}}+M_{0} \sin \left[\left(\delta-\gamma G_{1} z_{l}\right) \tau_{G}\right] \underline{\widetilde{x}} .
$$

The two-pulse sequence has created a purely sinusoidally-modulated $z$-magnetization that will create a secular dipolar field during the detection interval $t$. It will be convenient to track only the circularly-polarized component of magnetization density $M_{+}=M_{x}+i M_{y}$ during the detection interval since it is the integral of $M_{+}(\underline{r})$ over the sample that is measured in a quadrature-detected NMR experiment. If the helix pitch $\left|k_{1}\right|$ is large compared to the length of the active region $L$ and the diameter of the tube $d$, Eq. 6.25 can be used to calculate the secular contribution of the dipolar field felt by the spins during the detection

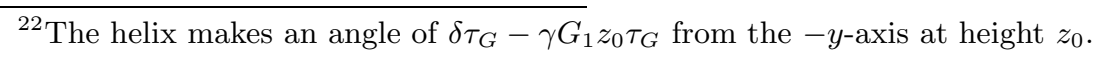


interval:

$$
\underline{B}_{d}=\mu_{0} M_{0} \cos \left[\left(\delta-\gamma G_{1} z_{l}\right) \tau_{G}\right] \underline{\tilde{z}}
$$

where the direction of modulation is $\underline{\widetilde{s}}=\underline{\widetilde{z}}$ and the contribution to $\underline{B}_{d}(\underline{r})$ due to $\underline{M}\left(\underline{r}^{\prime}\right)$ is assumed to be parallel to $\underline{M}(\underline{r})$ and has been neglected. Immediately after the second rf pulse a continuous $z$-gradient of strength $G_{2}$ is applied. The evolution of transverse magnetization under the second gradient, resonance offset field, and secular dipolar field is:

$$
M_{+}\left(z_{l}, t ; \tau_{G}\right)=M_{0} \sin \left[\left(\delta-\gamma G_{1} z_{l}\right) \tau_{G}\right] \exp \left\{i\left[\delta-\gamma G_{2} z_{l}-\mu_{0} \gamma M_{0} \cos \left[\left(\delta-\gamma G_{1} z_{l}\right) \tau_{G}\right]\right] t\right\}
$$

The winding of the second gradient pulse at time $t$ can be characterized by a wavenumber $k_{2}=\gamma G_{2} t$. The exponential function in Eq. 6.79 can be expanded using a Jacobi-Anger relation $[170, \S 2 \cdot 22]$ :

$$
e^{i Z \cos \theta}=\sum_{m=-\infty}^{+\infty} i^{m} J_{m}(Z) e^{i m \theta}
$$


where $J_{m}(Z)$ is the Bessel function of the first kind of order $m$. The result is:

$$
\begin{aligned}
M_{+}\left(z_{l}, t ; \tau_{G}\right) & =M_{0} \sin \left[\left(\delta-\gamma G_{1} z_{l}\right) \tau_{G}\right] e^{i\left(\delta-\gamma G_{2} z_{l}\right) t} \sum_{m=-\infty}^{+\infty} i^{m} J_{m}\left(-\mu_{0} \gamma M_{0} t\right) e^{i m\left(\delta-\gamma G_{1} z_{l}\right) \tau_{G}} \\
& =-\frac{1}{2} M_{0} \sum_{m=-\infty}^{+\infty} i^{m}\left[J_{m-1}\left(-t / \tau_{d}\right)+J_{m+1}\left(-t / \tau_{d}\right)\right] e^{i\left[\left(\delta-\gamma G_{2} z_{l}\right) t+m\left(\delta-\gamma G_{1} z_{l}\right) \tau_{G}\right]} \\
& =M_{0} \sum_{m=-\infty}^{+\infty} i^{m}\left(\frac{m \tau_{d}}{t}\right) J_{m}\left(-t / \tau_{d}\right) e^{i\left[\left(\delta-\gamma G_{2} z_{l}\right) t+m\left(\delta-\gamma G_{1} z_{l}\right) \tau_{G}\right]},
\end{aligned}
$$

where the Bessel function recursion identity $J_{n-1}(x)+J_{n+1}(x)=2 n J_{n}(x) / x$ has been used and $\tau_{d}=\mu_{0} \gamma M_{0}$ is known as the characteristic dipolar demagnetizing time; its value is $\left|\tau_{d}\right|=\left|\chi^{H_{2} O} \omega_{0}\right|^{-1} \approx 130 \mathrm{~ms}$ for thermally-polarized protons in room temperature water at $B_{0}=7.04 \mathrm{~T}$. The bulk NMR signal depends on average transverse magnetization density $M_{+}\left(t ; \tau_{G}\right)=\frac{1}{L} \int_{0}^{L} d z_{l} M_{+}\left(z_{l}, t ; \tau_{G}\right)$. In general, the transverse magnetization density is dephased as a function of $z$ and integrates to a small value. Note, however, that Eq. 6.81 has a component that is independent of position when $t=-n\left(G_{1} / G_{2}\right) \tau_{G}$ :

$$
\begin{aligned}
M_{+}\left(z_{l}, t=-n \frac{G_{1}}{G_{2}} \tau_{G}\right)= & i^{n} M_{0}\left(\frac{n \tau_{d}}{t}\right) J_{n}\left(-t / \tau_{d}\right) e^{i\left[n\left(1-G_{1} / G_{2}\right) \delta \tau_{G}\right]} \\
& \quad+M_{0} \sum_{m \neq n} i^{m}\left(\frac{m \tau_{d}}{t}\right) J_{m}\left(-t / \tau_{d}\right) e^{i\left[\left(m-n G_{1} / G 2\right) \delta \tau_{G}-(m-n) G_{1} z_{l} \tau_{G}\right]} .
\end{aligned}
$$

Assuming that the integral over the sum of position-dependent terms is small, the average 
magnetization density at this time is: ${ }^{23}$

$$
M_{+}\left(t=-n \frac{G_{1}}{G_{2}} \tau_{G}\right)=i^{n} M_{0}\left(\frac{n \tau_{d}}{t}\right) J_{n}\left(-t / \tau_{d}\right) e^{i\left[n\left(1-G_{1} / G_{2}\right) \delta \tau_{G}\right]} .
$$

This result is demonstrated in Fig. 6.2(a): transverse magnetization is dephased by the gradients is rephased at times $t=-n \frac{G_{1}}{G_{2}} \tau_{G}$ due to evolution under the gradients and positiondependent dipolar field. Since $t>0$, the rephasing occurs only for the $n<0$ terms when the two gradients have the same sign and only for the $n>0$ terms when the gradients have opposite signs. The $n$ th-order echo is due to the $|n|$ th term in the sum Eq. 6.81. The higher-order echoes are damped, partly due to $T_{2}$ relaxation. Note, however, that there is also a contribution due to the $J_{n}$ intensity factors; for instance, at short times $x=t / \tau_{d} \ll 1$ these functions go like $J_{n}(x) \approx x^{n} /\left(2^{n} n\right.$ !), so $J_{n+1}(x) / J_{n}(x) \approx x /[2(n+1)] \ll 1$. This gives the damping at the echo maxima; the actual echo envelopes are attenuated due to the action of the gradient $G_{2}$ and natural $\underline{B}_{0}$ inhomogeneities. Note that the magnitude of the dipolar field and hence the intensities of the echo maxima are independent of the gradient strength as long as the gradients are strong enough to put the system in the strong modulation limit of Eq. 6.25.

If no dipolar field were present, Eq. 6.79 would read $M_{+}\left(z_{l}, t ; \tau_{G}\right)=M_{0} \sin [(\delta-$ $\left.\left.\gamma G_{1} z_{l}\right) \tau_{G}\right] \exp \left[i\left(\delta-\gamma G_{2} z_{l}\right) t\right]$, where the dephasing of transverse magnetization due to $G_{1}$ is rephased under the gradient $G_{2}$ to form a single echo at $t=\left|G_{1} / G_{2}\right| \tau_{G}$ with average magnetization $M_{+}\left(t=\frac{G_{1}}{G_{2}} \tau_{G}\right)=\frac{i}{2} M_{0} \exp \left[-i\left(1-G_{1} / G_{2}\right) \delta \tau_{G}\right]$ if $G_{1} / G_{2}>0$ and

\footnotetext{
${ }^{23}$ Note that when the transverse magnetization is detected by the induction current generated in a coil, the NMR signal is proportional to $\partial M_{+}(t) / \partial t$ (in the laboratory frame) rather than to the net magnetization $M_{+}(t)$. This point is considered further in $\S 6.3$.
} 
$M_{+}\left(t=-\frac{G_{1}}{G_{2}} \tau_{G}\right)=-\frac{i}{2} M_{0} \exp \left[i\left(1-G_{1} / G_{2}\right) \delta \tau_{G}\right]$ if $G_{1} / G_{2}<0$. The case $G_{1}=G_{2}$ is the familiar Hahn echo in the presence of a steady inhomogeneous $z$-directed magnetic field. The magnetization at the time of the echo $t=\tau_{G}$ after the second rf pulse is $M_{+}\left(t=\frac{G_{1}}{G_{2}} \tau_{G}\right)=-\frac{i}{2} M_{0}$; the resonance offset/chemical shift has also been refocused, and the factor of $\frac{1}{2}$ indicates a loss of half the signal intensity due to an incomplete echo. ${ }^{24}$ The Hahn echo originates from single-spin terms in the initial density operator; the $n$th DDF-induced echo originates from $|n|$-spin terms in the initial density operator [308]. This point will be discussed further in the next section.

Pulsed field gradients with an $n$-quantum filter. Fig. 6.2(b) shows a pulse sequence that is capable of cleanly selecting out only the $n$th echo by use of properly-matched pulsed field gradients. The analysis is similar to the case of continuous gradients, except here the gradient pulses will be considered to be strong enough that the gradient time $\tau_{G}$ can be chosen to be short enough that evolution under the resonance offset can be neglected during $\tau_{G}$. Furthermore, the $\underline{B}_{0}$ field is considered to be inhomogeneous such that $\delta=\delta(\underline{r})$ dominates the reversible dephasing during the $\tau$ and $t$ intervals, although this position dependence will be suppressed in the notation for convenience.

After the $90_{x}^{\circ}-G_{1}\left(\tau_{G}\right)-\tau-90_{\bar{x}}^{\circ}$ two-pulse sequence, the initial uniform magnetization $\underline{M}(0)=M_{0} \underline{\tilde{z}}$ becomes:

$$
\underline{M}\left(z_{l}, \tau^{+}\right)=M_{0} \cos \left(\delta \tau-\gamma G_{1} z_{l} \tau_{G}\right) \underline{\widetilde{z}}+M_{0} \sin \left(\delta \tau-\gamma G_{1} z_{l} \tau_{G}\right) \underline{\widetilde{x}} .
$$

\footnotetext{
${ }^{24}$ The other half of the signal is the portion that is refocused at $\tau_{G}$ when $G_{1}=-G_{2}$; this gradient "anti-echo" retains chemical shift information.
} 
The $z$-magnetization is strongly modulated with a characteristic wavenumber $G_{1} \tau_{G}$. The $x$-magnetization is also strongly modulated and can be represented as the superposition of two oppositely-wound helices. The second gradient pulse $G_{2}=n G_{1}$ of duration $\tau_{G}$ causes an additional winding of the transverse magnetization:

$$
\begin{aligned}
M_{+}\left(z_{l}, \tau^{+}\right) & =M_{0} \sin \left(\delta \tau-\gamma G_{1} z_{l} \tau_{G}\right) \exp \left(-i \gamma n G_{1} z_{l} \tau_{G}\right) \\
& =-\frac{i}{2} M_{0}\left[e^{i\left[\delta \tau-(n+1) \gamma G_{1} z_{l} \tau_{G}\right]}-e^{-i\left[\delta \tau+(n-1) \gamma G_{1} z_{l} \tau_{G}\right]}\right]
\end{aligned}
$$

When $n= \pm 1$ the second gradient pulse exactly unwinds one or the other of the oppositelywound helices and that part of the transverse magnetization is rephased. The secular dipolar field is during the detection interval due to the $z$-magnetization is:

$$
\underline{B}_{d}=\mu_{0} M_{0} \cos \left(\delta \tau-\gamma G_{1} z_{l} \tau_{G}\right) \underline{\widetilde{z}} .
$$

The transverse magnetization during the detection interval $t$ evolves under the resonance offset field and dipolar field:

$$
\begin{aligned}
M_{+}\left(z_{l}, \tau, t\right) & =M_{0} \sin \left(\delta \tau-\gamma G_{1} z_{l} \tau_{G}\right) e^{-i \gamma n G_{1} z_{l} \tau_{G}} \exp \left\{i\left[\delta-\mu_{0} \gamma M_{0} \cos \left(\delta \tau-\gamma G_{1} z_{l} \tau_{G}\right)\right] t\right\} \\
& =-\frac{i}{2} M_{0} e^{i \delta t} \sum_{m=-\infty}^{+\infty} i^{m} J_{m}\left(-\mu_{0} \gamma M_{0} t\right) \\
& \times\left[e^{i\left[(m+1) \delta \tau-(m+n+1) \gamma G_{1} \tau_{G}\right]}-e^{i\left[(m-1) \delta \tau-(m+n-1) \gamma G_{1} \tau_{G}\right]}\right] \\
& =-\frac{1}{2} M_{0} e^{i \delta t} \sum_{m=-\infty}^{+\infty} i^{m}\left[J_{m-1}\left(-\mu_{0} \gamma M_{0} t\right)+J_{m+1}\left(-\mu_{0} \gamma M_{0} t\right)\right] e^{i m \delta \tau} e^{-i(m+n) \gamma G_{1} z_{l} \tau_{G}} \\
& =\sum_{m=-\infty}^{+\infty} i^{m} M_{0}\left(\frac{m \tau_{d}}{t}\right) J_{m}\left(-t / \tau_{d}\right) e^{-i(m+n) \gamma G_{1} z_{l} \tau_{G}} e^{i m \delta \tau} e^{i \delta t}
\end{aligned}
$$


where $\tau_{d}=\left(\mu_{0} \gamma M_{0}\right)^{-1}$. The $m=-n$ term in the sum is independent of position, and the integrated average magnetization density is:

$$
M_{+}(\tau, t)=-i^{n} M_{0}\left(\frac{n \tau_{d}}{t}\right) J_{n}\left(-t / \tau_{d}\right) e^{-i n \delta(\underline{r}) \tau} e^{i \delta(\underline{r}) t}
$$

where the relation $J_{-n}(x)=(-1)^{n} J_{n}(x)$ for integer $n$ has been used. ${ }^{25}$ The results of this experiment on protons in room temperature water at $B_{0}=7.04 \mathrm{~T}$ are shown in Fig. 6.2(b) for $n=+2$ and $n=+5$. Eq. 6.89 indicates that dephasing of transverse magnetization due evolution in an inhomogeneous $\underline{B}_{0}$ field during the interval $\tau$ is refocused into a single echo at time $t=n \tau$. Unlike the continuous gradient version of the experiment (Fig. 6.2(a)), the pulsed gradient version (Fig. 6.2(b)) refocuses all of the spatial inhomogeneity of the magnetization before $t=0$, but only for the $n$th echo. The other echoes that are observed in the continuous gradient experiment never form because the magnetization components that give rise to them are dephased at the beginning of the detection interval and are never rephased by another gradient. The echo envelopes in Fig. 6.2(b) are much more robust than those in Fig. 6.2(a) because the dephasing is due to weak natural $\underline{B}_{0}$ inhomogeneities rather than strong applied magnetic field gradients. Note that if $n<0$ (i.e., the gradient pulses $G_{1}$ and $G_{2}$ have opposite signs), although the gradient dephasing is refocused at $t=0$, the $\underline{B}_{0}$ inhomogeneities do not form an echo during $t$ because the phase factor $e^{i|n| \delta(\underline{r}) \tau} e^{i \delta(\underline{r}) t}$ is never independent of $\delta(\underline{r})$ for $\tau>0, t>0$. This is the "anti-echo" experiment, whereas the $n>0$ condition (i.e., gradient pulses of the same sign) selects the echo pathway.

\footnotetext{
${ }^{25}$ The evolution under the inhomogeneous $z$-field has been represented explicitly through the positiondependent frequency $\delta=\delta(\underline{r})$. This notation is not actually correct: the spatial averaging requires quantities like $\exp [i \delta(\underline{r}) t]$ to be spatially integrated. The inhomogeneous evolution due to $\underline{B}_{\delta}(\underline{r})$ should really considered at the level of Eq. 6.87 before the spatial integration is performed.
} 
If the interval between the two rf pulses is incremented as an indirect dimension, Eq. 6.89 can be used to calculate the average transverse magnetization:

$$
M_{+}\left(t_{1}, t_{2}\right)=-i^{n} M_{0}\left(\frac{n \tau_{d}}{t_{2}}\right) J_{n}\left(-t_{2} / \tau_{d}\right) e^{-i n \delta t_{1}} e^{i \delta t_{2}}
$$

where $t_{1}$ is the time variable in the indirect dimension and $t_{2}$ is the direct time. The spin system evolves at the frequency $-n \delta$ during the indirect dimension and $\delta$ during the direct dimension; this behavior is characteristic of a multiple-quantum correlation experiment in which an $n$-quantum coherence in the indirect dimension is converted to -1-quantum coherence for direct detection. ${ }^{26}$ In fact, the $90^{\circ}-G_{1}\left(\tau_{G}\right)-t_{1}-90^{\circ}-n G_{1}\left(\tau_{G}\right)-t_{2}$ pulse sequence is exactly the $n$-quantum CRAZED experiment of Warren et al., which indirectly detects $n$-quantum intermolecular multiple quantum coherence evolution. The multiplequantum NMR interpretation is that only one coherence in the indirect dimension is selected via an $n$-quantum filter using standard pulsed field gradient coherence pathway selection. The Fourier-transformed $n$-quantum CRAZED spectrum exhibits a cross peak at frequency $-n \delta$ in the indirect dimension with frequency $\delta$ in the direct dimension. It is also possible to generate cross peaks between spins in different molecules [302], e.g., protons in water with protons in acetone. Note that the use of the weak coupling expression for the secular dipolar field is appropriate when the chemical shift difference between the two spin species exceeds $\left|\gamma B_{d}\right| \sim\left|\tau_{d}\right|^{-1}$; the Bessel function arguments then go as $J_{n}\left(\frac{2}{3} t / \tau_{d}\right)$.

The multiple-quantum nature of the CRAZED experiment can be appreciated by some simple density operator calculations. Starting with the initial density operator in Eq.

\footnotetext{
${ }^{26} \mathrm{~A} p$-quantum coherence evolves at frequency $-p \delta$ under the resonance offset Hamiltonian $\hat{H}_{\delta} / \hbar=\delta \hat{I}_{z}$.
} 
6.57 , the $N$-spin density operator at the end $t_{1}$ before the second $\mathrm{rf}$ pulse is:

$$
\hat{\rho}\left(t_{1}\right)=\prod_{j=1}^{N}\left[\frac{1}{2} \hat{1}_{j}-\zeta \hat{I}_{y j} \cos \left(\delta_{j} t_{1}-\gamma G_{1} z_{j} \tau_{G}\right)+\zeta \hat{I}_{x j} \sin \left(\delta_{j} t_{1}-\gamma G_{1} z_{j} \tau_{G}\right)\right],
$$

where $\gamma_{j}=\gamma$ for a homonuclear species and Warren's argument that evolution under the dipole-dipole Hamiltonian can be neglected during $t_{1}$ is in effect. The resonance offsets $\delta_{j}$ can vary from spin to spin through the chemical shift if multiple chemical species are present; even if there is only one species, the $\delta_{j}$ can still vary if $\underline{B}_{0}$ is inhomogeneous. The vertical position $z_{j}$ of course varies from spin to spin. Consider only a single 3 -spin term from Eq. 6.90:

$\hat{B}_{3}\left(t_{1}\right)=2^{-(N-3)} \zeta^{3} \hat{I}_{x j} \sin \left(\delta_{j} t_{1}-\gamma G_{1} z_{j} \tau_{G}\right) \hat{I}_{y k} \cos \left(\delta_{k} t_{1}-\gamma G_{1} z_{k} \tau_{G}\right) \hat{I}_{y l} \cos \left(\delta_{l} t_{1}-\gamma G_{1} z_{l} \tau_{G}\right)$

This term arose from the term $\hat{B}_{3}(0)=-2^{-(N-3)} \zeta^{3} \hat{I}_{z j} \hat{I}_{z k} \hat{I}_{z l}$ in the initial density operator. This term contains \pm 3 - and \pm 1 -quantum coherences. Only the +3 -quantum coherence will end up passing a $n=+3$-quantum filter $\left(G_{2}=3 G_{1}\right)$ filter:

$$
\hat{B}_{3}^{(3)}\left(t_{1}\right) \propto \hat{I}_{+j} \hat{I}_{+k} \hat{I}_{+l} e^{i\left[3 \delta t_{1}-\gamma G_{1}\left(z_{j}+z_{k}+z_{l}\right) \tau_{G}\right]}
$$

where the relation $\delta_{j}+\delta_{k}+\delta_{l}=3 \delta$ in the case of equivalent spins in a homogeneous magnetic field has been used to emphasize the triple-quantum nature of the evolution. Returning to 
Eq. 6.91, the total density operator after the second $\mathrm{rf}$ and $G_{2}=3 G_{1}$ gradient pulse is:

$$
\begin{aligned}
\hat{\rho}\left(t_{1}, t_{2}=0\right)=\prod_{j=1}^{N} & {\left[\frac{1}{2} \hat{1}_{j}+\zeta \hat{I}_{z j} \cos \left(\delta_{j} t_{1}-\gamma G_{1} z_{j} \tau_{G}\right)\right.} \\
& +\zeta \hat{I}_{x j} \sin \left(\delta_{j} t_{1}-\gamma G_{1} z_{j} \tau_{G}\right) \cos \left(-3 \gamma G_{1} z_{j} \tau_{G}\right) \\
& \left.+\zeta \hat{I}_{y j} \sin \left(\delta_{j} t_{1}-\gamma G_{1} z_{j} \tau_{G}\right) \sin \left(-3 \gamma G_{1} z_{j} \tau_{G}\right)\right] .
\end{aligned}
$$

The 3-spin term of Eq. 6.91 has evolved to contain the component:

$$
\begin{gathered}
\hat{B}_{3}\left(t_{1}, t_{2}=0\right)=2^{-(N-3)} \zeta^{3} \hat{I}_{x j} \hat{I}_{z k} \hat{I}_{z l} \sin \left(\delta_{j} t_{1}-\gamma G_{1} z_{j} \tau_{G}\right) \cos \left(\delta_{k} t_{1}-\gamma G_{1} z_{k} \tau_{G}\right) \\
\times \cos \left(\delta_{l} t_{1}-\gamma G_{1} z_{l} \tau_{G}\right) \cos \left(-3 \gamma G_{1} z_{j} \tau_{G}\right) .
\end{gathered}
$$

The discussion of $\S 6.2 .2$ showed how a density operator term like $\hat{I}_{x j} \hat{I}_{z k} \hat{I}_{z l}$ could be converted into single-spin transverse magnetization $\hat{I}_{x j}$ after two successive commutations with the dipole-dipole Hamiltonians $\hat{H}_{D}^{j l}$ and $\hat{H}_{D}^{j k}$. Quadrature detection selects out the -1-quantum coherence which evolves at $\hat{I}_{-j} e^{i \delta_{j} t_{2}}$ in the direct dimension. The spatial averaging required to find the position-independent terms in the density operator is somewhat complicated [76], but the term that passes the triple-quantum filter is the +3 -quantum coherence that evolved as $\hat{I}_{+j} \hat{I}_{+k} \hat{I}_{+l} e^{-i 3 \delta t_{1}}$ during the indirect evolution time. Note that $(n+1)$ - and higher-order spin terms in the density operator also generate $n$-quantum coherences during $t_{1}$ but require two or more commutators using the same dipolar coupling to produce observable signal, which is a negligibly small pathway. The general result is that $n$-spin terms in the initial density operator lead to the $n$th multiple echo which gives the $n$-quantum CRAZED signal. When multi-spin terms in the density operator or dipolar couplings are neglected, only the 
usual $n=1$ Hahn echo is predicted.

No gradients. The experiment shown in Fig. 6.2(c) is an ordinary Hahn echo experiment (or COSY NMR experiment if the delay $\tau$ is instead incremented as an indirect dimension $t_{1}$ ). These data were taken with the sample arranged such that only the tip of the NMR tube was in the active region of the rf coil; the water-glass-air susceptibility boundary at the bottom of the tube makes a very inhomogeneous $\underline{B}_{0}$ field within the water. This inhomogeneity manifests itself as a line broadening effect in a conventional one-pulse NMR experiment. During the interval $\tau$ the transverse magnetization evolves in the presence of the inhomogeneous offset field $B_{\delta}(\underline{r})$. This will eventually lead to a complicated magnetization distribution from which it is hard to calculate the dipolar field. A large simplifying assumption is made here, which is that the local secular dipolar field is of the form:

$$
\underline{B}_{d}(\underline{r})=\mu_{0} \alpha_{\delta}\left[3 M_{z}(\underline{r}) \underline{\tilde{z}}-\underline{M}(\underline{r})\right]
$$

where $\alpha_{\delta}$ is a position-independent proportionality constant. There is no real justification for this assumption, except it is true in a uniformly-magnetized ellipsoid or in a sample in which the magnetization is strongly modulated along one direction; it is not true in a sample with an arbitrary geometry or arbitrary magnetization. Starting from initially uniform magnetization $\underline{M}(0)=M_{0} \underline{\underline{z}}$, the magnetization after the two rf pulses is:

$$
\underline{M}\left(\underline{r}, \tau_{+}\right)=M_{0} \cos [\delta(\underline{r}) \tau] \underline{\widetilde{z}}+M_{0} \sin [\delta(\underline{r}) \tau] \underline{\widetilde{x}},
$$


where Eq. 6.95 guarantees that the effect of the local secular dipolar field is zero during this time because the magnetization is transverse and $\underline{M}(\underline{r}) \times \underline{B}_{d}(\underline{r})=0$. The magnetization dephases during $\tau$ due to the inhomogeneous $\underline{B}_{0}$ field; if this dephasing is strong enough, perhaps $\underline{B}(\underline{r})$ sees only a local contribution from the magnetization and Eq. 6.95 may be valid. Assuming that the effective part of the local secular dipolar field during the detection time $t$ is:

$$
\underline{B}_{d}(\underline{r})=\mu_{0} \alpha_{\delta} M_{0} \cos [\delta(\underline{r}) \tau] \underline{\widetilde{z}}
$$

the transverse magnetization density at time $t$ is:

$$
\begin{aligned}
\underline{M}_{+}(\underline{r}, t ; \tau) & =M_{0} \sin [\delta(\underline{r}) \tau] \exp \left\{i\left[\delta(\underline{r})-\mu_{0} \gamma \alpha_{\delta} M_{0} \cos [\delta(\underline{r}) \tau]\right] t\right\} \\
& =\sum_{m=-\infty}^{+\infty} i^{m} M_{0}\left(\frac{m \tau_{d}}{\alpha_{\delta} t}\right) J_{m}\left(-\alpha_{\delta} t / \tau_{d}\right) e^{i m \delta(\underline{r}) \tau} e^{i \delta(\underline{r}) t}
\end{aligned}
$$

where $\tau_{d}=\left(\mu_{0} \gamma M_{0}\right)^{-1}$. Evolution under $\underline{B}_{0}$ inhomogeneity causes a dephasing of the transverse magnetization which is refocused to form echoes at times $t=|m| \tau$ for $m<0$.

\subsubsection{A comment on DDF effects in quadrupolar spin systems}

Chapters 3, 4, and 5 deal almost exclusively with quadrupolar nuclei and their dynamics and it is reasonable to consider how the analysis of such systems can be extended to include distant dipolar field effects. DDF effects can be described in $I=1 / 2$ systems using a purely classical model because the average nuclear polarization can be described entirely in terms of the classical magnetization vector. Quadrupolar $(I>1 / 2)$ nuclei, 
however, have more degrees of freedom and can support types of polarization other than dipole polarization. A correct description of the quadrupolar nuclear dynamics must include the effects of the nuclear dipolar field on these degrees of freedom. Quantum-mechanical or semiclassical descriptions of distant dipolar field effects in multilevel systems have been developed by other workers in the context of $J$-coupled spin-1/2 systems [306, 380, 381]. It seems that DDF effects in $I>1 / 2$ systems have not been considered thus far in the literature. At the least there has been one report of using Li-7 $(I=3 / 2)$ to detect the dipolar field of a protonated solvent [297], although the data were not included and no mention of the quadrupolar nature of $\mathrm{Li}-7$ was made.

The analysis must be done using a quantum-mechanical or semiclassical formalism since not all of the degrees of freedom of an $I>1 / 2$ nucleus can be described classically. It is convenient to use a basis of spherical tensor operators (see Appendix A) to represent the nuclear angular momentum polarization. The density operator of a spin $I$ nucleus can be written in terms of $(2 I+1)^{2}$ spherical tensor operator components $\hat{T}_{k, q}$ of integer rank $0 \leq k \leq 2 I$ and order $q=-k, \cdots,+k$. Rank $k=1$ operators correspond to nuclear magnetization ( $q=0$ for longitudinal magnetization, $q= \pm 1$ for transverse magnetization).

In the Warren quantum mechanical picture multispin terms in the full $N \approx 10^{20}$-spin density operator can be converted to observable single-spin terms by short-time evolution under non-repeated intermolecular dipolar couplings (see §6.2.2). Mathematically speaking, density operator terms like $\hat{I}_{x j} \hat{I}_{z k}$ are converted to terms like $\hat{I}_{y j}$ by a single commutation with the dipolar Hamiltonian $\hat{H}_{D}^{j k} \propto \hat{I}_{z j} \hat{I}_{z k}$; the spin operator $\hat{I}_{z k}$ is "removed" from the density operator term (i.e., it is converted to $\hat{1}_{k}$ ). It is easy to show that in the general 
$I \geq 1 / 2, S \geq 1 / 2$ case only $\hat{I}_{z i}$ or $\hat{S}_{z j}$ terms can be removed by a single commutation with the heteronuclear dipolar Hamiltonian. ${ }^{27}$ Therefore one expects that heteronuclear DDF effects will manifest themselves in systems containing at least one quadrupolar nucleus in the same way as in spin-1/2 heteronuclear systems, if the quadrupolar nucleus is prepared only in a state of magnetization (rank-1 polarization). Normal DDF H-1-H-2 $(I=1 / 2$, $S=1$ ) correlation experiments at $B_{0}=7.05 \mathrm{~T}$ in an $80 \% / 20 \% \mathrm{H}_{2} \mathrm{O} / \mathrm{D}_{2} \mathrm{O}$ mixture exhibited no unexpected results (data not shown). Note that higher rank polarization terms will also evolve under the dipolar field, but not into observable rank-1 polarization.

The situation is different in homonuclear systems. Here the term "homonuclear system" will be taken to mean a single-species system in the strong-dipolar coupling limit; dipolar field effects are so weak that the truncated weak-coupling ("heteronuclear") dipolar Hamiltonian applies to most multiple-component homonuclear systems. The full homonuclear secular dipolar Hamiltonian $\hat{H}_{D}^{j k} / \hbar=\omega_{D}^{j k}\left(3 \hat{I}_{z j} \hat{I}_{z k}-\underline{\hat{I}}_{j} \cdot \underline{\hat{I}}_{k}\right)$ contains extra "flip-flop" terms that the heteronuclear secular dipolar Hamiltonian does not. The entire $\underline{\hat{I}}_{j} \cdot \underline{\hat{I}}_{k}$ term is usually neglected in the Warren treatment of homonuclear DDF experiments [76]. This is justified when the spins are truly equivalent - i.e., when a single spin- $1 / 2$ species is considered and the spins are not "labeled" by their position in an inhomogeneous magnetic field - because the multi-spin terms enter symmetrically into the equilibrium density operator and this symmetry is not broken by non-selective pulses. This is equivalent to neglecting the term proportional to $\underline{M}$ in Eq. 6.21 in the absence of magnetic field gradients (see the

\footnotetext{
${ }^{27}$ The commutator $\left[\hat{B}_{n=2}, \hat{H}_{D}^{j k}\right]=\left[\hat{T}_{l, m}^{I i} \hat{T}_{k, q}^{S j}, 2 \hbar \omega_{D}^{i j} \hat{I}_{z i} \hat{S}_{z j}\right]$ contains products like $\hat{T}_{k, q}^{S j} \hat{S}_{z j}=\hat{T}_{k, q}^{S j} \hat{T}_{1,0}^{S j} ;$ since the multiplication of spherical tensor operators follows the rules of angular momentum addition, the spin- $S$ term of this product can only equal $\hat{1}_{S}^{j}=\hat{T}_{0,0}^{S j}$ if the Clebsch-Gordan coefficient $\langle k, q ; 1,0 \mid 0,0\rangle \neq 0$, i.e., $k-1=0$ and $q+0=0$ such that $\hat{T}_{k, q}^{S j}=\hat{T}_{1,0}^{S j}=\hat{S}_{z j}$. An analogous argument may be used when removing spin- $I$ terms.
} 
discussion of DDF effects in the one-pulse experiment in $§ 6.2 .3)$. The commutator of density operator terms like $\left(\hat{I}_{\alpha j} \hat{I}_{\beta k}+\hat{I}_{\alpha k} \hat{I}_{\beta j}\right)$ with the $\underline{\hat{I}}_{j} \cdot \underline{\hat{I}}_{k}$ Hamiltonian term does not remove a spin operator when $\alpha, \beta \in\{x, y, z\}$. Since only the short-time (single commutator) dynamics are relevant to describe DDF effects in solution, the $\underline{\hat{I}}_{j} \cdot \underline{\hat{I}}_{k}$ terms in the dipolar Hamiltonian do not lead to the development of observable single-spin terms in the density operator, and therefore can be neglected. This is no longer the case in quadrupolar spin systems because a single commutation with $\underline{\underline{I}}_{i} \cdot \underline{\hat{I}}_{j}$ can remove a spin operator from certain symmetric density operator terms of the form $\left(\hat{T}_{l, m ; \phi}^{i} \hat{T}_{k, q ; \phi^{\prime}}^{j}+\hat{T}_{l, m ; \phi}^{j} \hat{T}_{k, q ; \phi^{\prime}}^{i}\right)$, where $\hat{T}_{l, m ; \phi}$ is a Hermitian combination of spherical tensor operators of a given $l$ and $|m|$ (see Eq. 5.2).

As an example, consider a spin $I=1$ homonuclear system in the absence of all interactions except chemical shifts and long-range dipolar couplings. An analysis of the relevant commutators shows that when the density operator contains only rank-1 (magnetization) spin operators the dipolar field dynamics proceed in the same manner as in a spin-1/2 system, i.e., the $\underline{\underline{I}}_{i} \cdot \underline{\hat{I}}_{j}$ Hamiltonian terms can be neglected, which is equivalent to neglecting the transverse dipolar field. A normal homonuclear Li-7 $(I=3 / 2)$ CRAZED experiment at $B_{0}=7.05 \mathrm{~T}$ in nearly-saturated $\mathrm{LiBr}(\mathrm{aq})$ exhibited no unexpected results (data not shown). However, the presence of the $\underline{\hat{I}}_{i} \cdot \underline{\hat{I}}_{j}$ terms in the Hamiltonian can affect the dynamics when higher-rank polarization is present. These dynamics are not due to evolution of the spins in some sort of "distant multipolar field"; rather, they are due to the evolution of rank $k>1$ polarization in the presence of the usual transverse secular dipolar field. For example, DDF-mediated conversions of the type $q=0 \leftrightarrow|q|=1 \leftrightarrow|q|=2$ are possible for $k=2$ polarization in the $I=1$ system. ${ }^{28}$ This of course requires the system to

\footnotetext{
${ }^{28}$ This is a simplification because the phase of the transverse dipolar field $(x$ or $y)$ determines which
} 
be prepared in some mixture of transverse magnetization and rank-2 polarization. However, these effects would be difficult to observe because rank- 2 polarization is not directly measurable. It can be shown for the general spin $I$ case that no secular DDF effects can convert $k>1$ polarization into observable $k=1$ magnetization. Therefore one would have to perform a 2D experiment that involves both a multiple-quantum excitation and a conversion to observable signal; looking for small DDF effects during multiple-quantum spectroscopy of a potentially low- $\gamma$ quadrupolar nucleus is a difficult proposition.

Thus far the discussion of DDF effects in quadrupolar nuclei have focused on the ability of $I>1 / 2$ nuclei to support rank $k>1$ polarization; it would be interesting to see what would happen if a quadrupolar coupling was included. The motionally-averaged electric quadrupolar coupling is zero in isotropic but is non-zero in general in an anisotropic medium. For example, the spectrum of an $I=1$ nucleus in an oriented medium presents a quadrupolar-split doublet (see $\S 4$ ). Goldman and Desvaux have investigated the crossprecession of the components of a $J$-split doublet $\left(I_{1}=1 / 2, I_{2}=1 / 2\right)$ in the presence of each other's dipolar fields in a variable tip-angle experiment [306]. Their analysis could be applied to an $I=1$ system to determine whether such a DDF cross-precession effect can be observed between the components of a quadrupolar-split doublet. ${ }^{29}$ The DDF effect in the $J$-coupled system was quite small even in a concentrated protic solvent: a small shift of the resonance frequencies was observed and the intensity ratio of the two doublet components deviated from unity by a maximum of $8-9 \%$. The dipolar field would be even smaller order- $q$ components can convert into each other, e.g., $\hat{T}_{2,0}$ polarization in an $x$-directed DDF evolves as $\left(\hat{I}_{x i} \hat{T}_{2,0}^{j}+\hat{I}_{x j} \hat{T}_{2,0}^{i}\right) \rightarrow\left(\hat{T}_{2,1 ; \pi / 2}^{i}+\hat{T}_{2,1 ; \pi / 2}^{j}\right)$ under $\hat{H}_{D}^{i j}$.

${ }^{29}$ The theoretical analysis might best be done in a fictitious spin-1/2 operator basis [150] rather than in a spherical tensor operator basis. 
in the case of a dilute nucleus that had a smaller gyromagnetic ratio than that of $\mathrm{H}-1$. Furthermore, it is important that the doublet splitting not exceed by too much the Larmor frequency of the nucleus due to its own dipolar field; this may be related to the truncation of the secular dipolar field by the frequency difference between the doublet components. The maximum cross-precession effect was observed at $\mathrm{rf}$ pulse tip angles near $\theta=0$ and $\theta=\pi$; unfortunately these are the tip angles for which the transverse magnetization and hence the observed signal is the smallest.

When investigating DDF effects in homonuclear quadrupolar systems it is difficult to find a physical system in which the DDF is large, i.e., one that has a relatively high- $\gamma$ nucleus present at high concentration. For example, the $I=1$ nuclei $\mathrm{H}-2$ and N-14 can be found at high concentrations in neat liquids but have low gyromagnetic ratios (recall that the thermal equilibrium magnetization is quadratic in $\gamma$ and the low Larmor frequency is also a disadvantage when inductive detection is employed). It might be advantageous to try to observe a DDF cross-precession effect in the $I=3 / 2$ nucleus Li- 7 , which has a relatively high gyromagnetic ratio, $\gamma_{L i 7} / 2 \pi=16.55 \mathrm{MHz} / \mathrm{T}$. It also has a small quadrupolar coupling in $\mathrm{Li}^{+}$salts (e.g., the $\mathrm{Li}-7$ linewidth is only a few $\mathrm{Hz}$ in $\mathrm{LiBr}(\mathrm{aq})$ ), which leads to sharp, intense lines. The small coupling also would result in a small averaged quadrupolar coupling in an anisotropic medium, which is good for cross-precession investigations. It is possible to find systems that contain Li in high concentrations, e.g., molten lithium acetate $\left(\mathrm{Li}\left[\mathrm{O}(\mathrm{CO}) \mathrm{CH}_{3}\right] \cdot 2 \mathrm{H}_{2} \mathrm{O}\right.$, m.p. $\left.=70^{\circ} \mathrm{C}\right)$, although this would be an isotropic fluid. It might be difficult to prepare an oriented system with a high Li concentration: a polar salt would not dissolve in an organic liquid crystal, and high concentrations of $\mathrm{Li}^{+}$may disrupt the 
liquid crystalline phase of an aqueous bicellar solution. A concentrated aqueous solution of a lithium salt prepared in a stretched gel may be feasible.

\subsection{Indirect detection with the distant dipolar field}

The homonuclear dipolar field experiments potentially could be modified so that structural or contrast-enhanced image information about a mobile carrier nucleus in a spatially inhomogeneous environment could be encoded via the carrier's self-DDF. Some of the experiments are discussed in a non-remote detection modality in Refs. [317, 318, 319, $320,321,322,323,324,325,326,327,328,329,330,331,332,333,334,335]$. Another situation that might commonly arise in remote detection experiments is when some of the carrier nucleus is bound and some is mobile, e.g., xenon in solution with some sample of interest versus free xenon gas. In this case the distant dipolar field could be used to trans-

fer spectral information from the "bound" nuclei through space to the mobile nuclei via a CRAZED-type experiment, which would obviate the need to take the xenon out of solution before transport. However, many analytes of interest do not contain xenon, so when xenon is the mobile carrier of choice it would make more sense to use a heteronuclear indirect detection experiment in which the spectrum of an analyte heteronucleus is transferred via its DDF to the xenon carrier. The DDF is significant on length scales of up to millimeters, so there is no need for the analyte and carrier ever to come in contact with each other. This section will introduce the basic DDF indirect detection experiment and explain how it could be modified for use as an encoding module in remote detection experiments. Much of the discussion is also applicable to homonuclear experiments. 


\subsection{INDIRECT DETECTION WITH THE DISTANT DIPOLAR FIELD 408}

\subsubsection{The basic indirect detection experiment}

Bowtell [296] was the first to propose using the distant dipolar field to transfer the indirectly-encoded NMR spectroscopic information of an analyte nucleus to a sensor heteronucleus on a different molecule for direct detection. He suggested that this method could be used to enhance the detection sensitivity of a low- $\gamma$ or dilute analyte nucleus (i.e., one with low bulk magnetization) if the sensor nucleus was highly polarized, although some signal per unit time was sacrificed by incrementing the analyte indirect time dimension point-by-point rather than detecting in direct time. Intramolecular heteronuclear polarization transfers are routine in solution-state NMR using the $J$-coupling, but the ability to perform intermolecular transfers through the DDF is a great advantage because the experimenter has the freedom to add a probe molecule which contains high- $\gamma$ nuclei that are not present in the analyte molecule or are present in much higher concentration. In the context of remote detection experiments, it is useful to have a probe that can act as a mobile carrier of the NMR information and which is not required to come into physical contact with the analyte.

Bowtell's three-pulse experiments studied heteronuclear multiple spin echoes, but he suggested a means by which frequency-domain spectra could be acquired. Augustine and Zilm used slightly-modified versions of Bowtell's pulse sequence to demonstrate P-31detected intermolecular heteronuclear correlation spectra of $\mathrm{H}-1$ in solution $[366,382]$ and gas-phase Xe-129-detected spectra of H-1 in methane [366]. Bachiller et al. [383] introduced a four-pulse experiment that used heteronuclear double- and zero-quantum coherences to encode heteronuclear sum- and difference-frequency spectra in the indirect dimension. Warren 


\subsection{INDIRECT DETECTION WITH THE DISTANT DIPOLAR FIELD 409}

et al. [77] have also considered a Bowtell-type three-pulse experiment from a theoretical perspective and critiqued its ability to provide significant sensitivity enhancement. Ardelean

et al. [384] used similar sequences to investigate the attenuation of heteronuclear DDFinduced multiple echoes by diffusion. All these studies considered homogeneous mixtures of the sensor and analyte, although He et al. have demonstrated homonuclear correlations between two separated chemical species [302].

The basic version of the DDF heteronuclear correlation experiment considered here uses the following three-pulse sequence (Fig. 6.3):

$$
\left[90_{\phi_{1}}^{\circ I}\right]-G_{1}\left(\tau_{G}\right)-t_{1}-\left[90_{\phi_{2}}^{\circ I}, 90_{\phi_{3}}^{\circ S}\right]-G_{2}\left(\tau_{G}\right)-t_{2}-\operatorname{detect} S\left(\phi_{r}\right),
$$

where $I$ is the indirectly-detected analyte nucleus and $S$ is the directly-detected sensor nucleus. The two spin- $I$ rf pulses have phases $\phi_{1}$ and $\phi_{2}$, the spin- $S$ pulse has phase $\phi_{3}$, and the receiver phase is $\phi_{r}$. There are two $z$-gradient pulses of strength $G_{1}$ and $G_{2}$ and duration $\tau_{G}$; these pulses are employed in a specific ratio $G_{2}=\kappa G_{1}$ that will be discussed later. Since this is a two-dimensional experiment, it is sometimes desirable to include a strong, long "crusher" $z$-gradient pulse before the recycle delay at the start of the experiment to dephase any leftover transverse magnetization from the last transient acquisition.

The evolution of the system under the pulse sequence in Fig. 6.3 will be calculated including relaxation effects but excluding molecular diffusion effects, which are considered in Ref. [296]. The sample will be considered to be a homogeneous mixture of the molecules that contain spins $I$ and $S$; the case in which the analyte and sensor are physically separated is discussed in $\S 6.4 .2$. The resonance offsets of spins $I$ and $S$ are denoted $\delta_{I}$ and 
6.3. INDIRECT DETECTION WITH THE DISTANT DIPOLAR FIELD 410

I
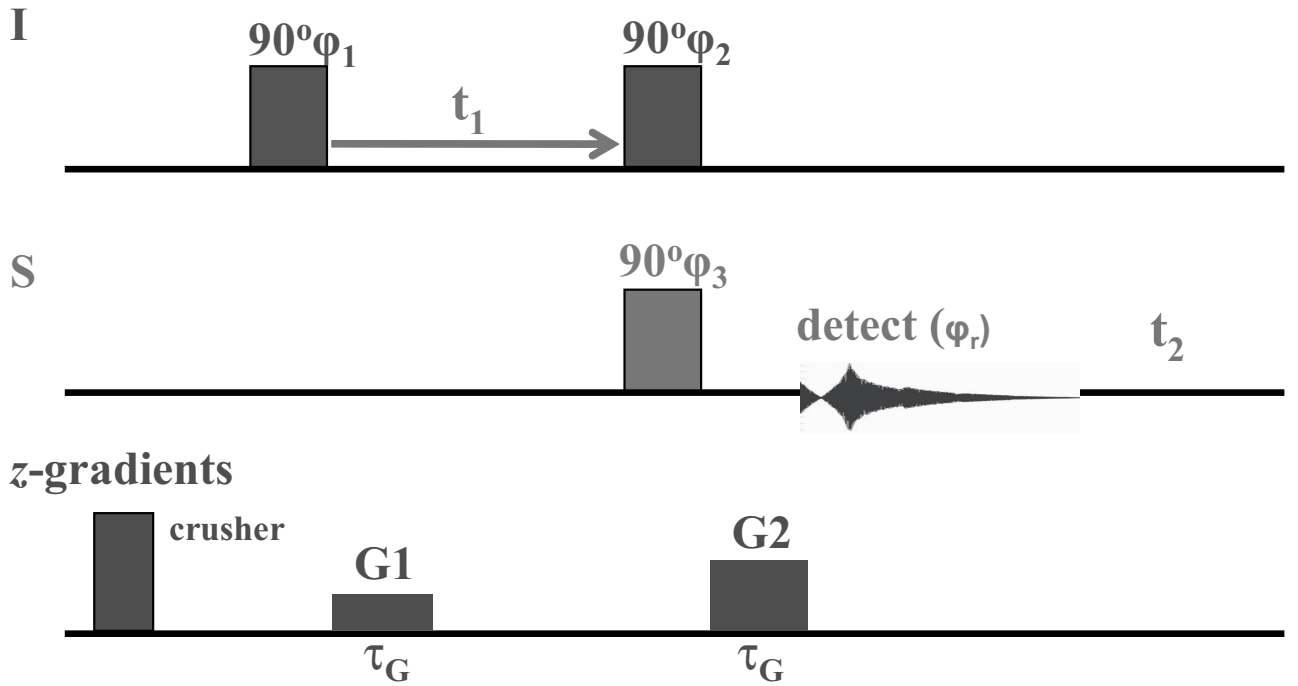

Figure 6.3: Pulse sequence for DDF indirect detection.

$\delta_{S}$, respectively. The offset corresponds to the sum of the resonance detuning, chemical shielding, and any intrinsic or susceptibility-induced spatial inhomogeneity of the $\underline{B}_{0}$ field. It will be assumed that $\phi_{1}=\phi_{2}=\phi_{3}=\phi_{r}=0$. The initial total magnetization density is $\underline{M}=\underline{M}^{I}(0)+\underline{M}^{S}(0)=M_{0}^{I} \underline{\tilde{z}}+M_{0}^{s} \underline{\widetilde{z}}$, where $M_{0}^{I}$ and $M_{0}^{S}$ are only the initial (not necessarily equilibrium) magnetizations. The magnetization of spin $I$ after the rf and gradient pulses have been applied but before acquisition begins is:

$$
\begin{aligned}
\underline{M}^{I}\left(\underline{r}, t_{1}, 0\right)=- & M_{0}^{I} \cos \left(\delta_{I} t_{1}-\gamma_{I} G_{1} z_{l} \tau_{G}\right) e^{-t_{1} / T_{2}^{I}} \underline{\widetilde{z}} \\
& +M_{0}^{I} \sin \left(\delta_{I} t_{1}-\gamma_{I} G_{1} z_{l} \tau_{G}\right) e^{-t_{1} / T_{2}^{I}}\left[\cos \left(-\gamma_{I} G_{2} z_{l} \tau_{G}\right) \underline{\widetilde{x}}+\sin \left(-\gamma_{I} G_{2} z_{l} \tau_{G}\right) \underline{\widetilde{y}}\right]
\end{aligned}
$$

where the gyromagnetic ratio of $\operatorname{spin} I$ is $\gamma_{I}$; the variable $z_{l}=z-z_{0}$ is the vertical position within the sample, where $z_{0}$ marks the bottom of the active region of the rf coil. The gradient 


\subsection{INDIRECT DETECTION WITH THE DISTANT DIPOLAR FIELD411}

pulse length $\tau_{G}$ is assumed to be short enough that resonance offset evolution and relaxation effects can be neglected during the gradient pulses. The transverse relaxation time of spin $I$ is $T_{2}^{I}$; any inhomogeneous dephasing that is normally included in the $T_{2}^{I *}$ relaxation time has been implicitly included in the position-dependent offset frequency $\delta_{I}(\underline{r})$. It has been assumed that the evolution of the purely-transverse magnetization of spin $I$ during $t_{1}$ is not influenced by its self-dipolar field.

Note that the magnetization of the spin $S$ at the same time is:

$$
\underline{M}^{S}\left(\underline{r}, t_{1}, 0\right)=-M_{0}^{S}\left[\cos \left(-\gamma_{S} G_{2} z_{l} \tau_{G}\right) \underline{\tilde{y}}+\sin \left(-\gamma_{S} G_{2} z_{l} \tau_{G}\right) \underline{\widetilde{x}}\right],
$$

which corresponds to a helix of pitch $2 \pi /\left(\gamma_{S} G_{2} \tau_{G}\right)$. The $S$-spin transverse magnetization is completely dephased at the beginning of the detection interval, so this pulse sequence would lead to no net signal if the dipolar field of spin $I$ did not rephase the magnetization at some point. In the absence of dipolar field effects the analyte and sensor nuclei are uncoupled and the indirect detection sequence would give the same result as a one-pulse experiment on spin $S$.

The $z$-component of $I$ spin magnetization relaxes during $t_{2}$ to its equilibrium value $M_{e q}^{I}$ with the longitudinal relaxation time $T_{1}^{I}$ as:

$$
M_{z}^{I}\left(\underline{r}, t_{1}, t_{2}\right)=-M_{0}^{I} \cos \left(\delta_{I} t_{1}-\gamma_{I} G_{1} z_{l} \tau_{G}\right) e^{-t_{1} / T_{2}^{I}} e^{-t_{2} / T_{1}^{I}}+M_{e q}^{I}\left(1-e^{-t_{2} / T_{1}^{I}}\right) .
$$

If the spin system is initially prepared at thermal equilibrium, then $M_{0}^{I}=M_{e q}^{I}$. During $t_{2}$ the longitudinal component of spin- $S$ magnetization that develops due to $T_{1}^{S}$ relaxation creates 


\subsection{INDIRECT DETECTION WITH THE DISTANT DIPOLAR FIELD 412}

a secular dipolar field felt by spin $I$ that is parallel to the $z$-axis, and thus does not affect the evolution of $M_{z}^{I}$. It could cause some precession of the transverse magnetization of spin $I$, but this magnetization is not observed and does not contribute to the secular dipolar field felt by spin $S$. The $z$-magnetization in Eq. 6.102 contains a cosine-modulated component and an unmodulated component $\left\langle M_{z}^{I}\right\rangle=M_{e q}^{I}\left(1-e^{-t_{2} / T_{1}^{I}}\right)$. Assuming that the modulation length $\left|2 \pi /\left(\gamma_{I} G_{1} \tau_{G}\right)\right|$ is small compared to any sample dimension, the secular dipolar field of spin $I$ felt by spin $S$ can be calculated using Eq. 6.26 upon first subtracting the unmodulated component of magnetization $\left\langle M_{z}^{I}\right\rangle$ from $M_{z}^{I}$ according to Warren's correction [363]:

$$
\underline{B}_{d}^{I}=-\frac{2}{3} \mu_{0} M_{0}^{I} \cos \left(\delta_{I} t_{1}-\gamma_{I} G_{1} z_{l} \tau_{G}\right) e^{-t_{1} / T_{2}^{I}} e^{-t_{2} / T_{1}^{I}} \underline{\widetilde{z}} .
$$

This correction makes the implicit assumption that the dipolar field created by the uniform (unmodulated) magnetization is small compared to the field created by the strongly modulated magnetization. If the contribution of the unmodulated magnetization is to be included the result will depend on the sample geometry. In the case of an infinite cylinder this contribution is $\frac{1}{3} \mu_{0} M_{e q}\left(1-e^{-t_{2} / T_{1}^{I}}\right) \underline{\widetilde{z}}$ (see Eq. 6.55). This factor is small for times $t_{2} \ll T_{1}^{I}$. At longer times it may not have negligible magnitude, but it is independent of the parameter $t_{1}$ and will cause only a small chirped resonance shift of the $S$ spins, which will be neglected here. Eq. 6.103 depends on the direct time $t_{2}$, so the $S$ spins will evolve in the presence of the DDF of the $I$ spins with the dynamic phase:

$$
\begin{aligned}
\Phi_{d}\left(t_{2} ; 0\right) & =-\gamma_{S} \int_{0}^{t_{2}} d t\left[-\frac{2}{3} \mu_{0} M_{0}^{I} \cos \left(\delta_{I} t_{1}-\gamma_{I} G_{1} z_{l} \tau_{G}\right) e^{-t_{1} / T_{2}^{I}} e^{-t / T_{1}^{I}}\right] \\
& =\frac{2}{3} \gamma_{S} \mu_{0} M_{0}^{I} T_{1}^{I} \cos \left(\delta_{I} t_{1}-\gamma_{I} G_{1} z_{l} \tau_{G}\right) e^{-t_{1} / T_{2}^{I}}\left(1-e^{-t_{2} / T_{1}^{I}}\right) .
\end{aligned}
$$




\subsection{INDIRECT DETECTION WITH THE DISTANT DIPOLAR FIELD 413}

See Eq. 2.8 for a description of dynamic phases in the context of quantum-mechanical time evolution.

If quadrature detection is employed in the direct dimension, only the transverse magnetization component $M_{+}^{S}=M_{x}^{S}+i M_{y}^{S}$ is of interest. The transverse magnetization evolves during $t_{2}$ under a combination of the spin- $S$ resonance offset and the spin- $I$ dipolar field as:

$$
\begin{gathered}
M_{+}^{S}\left(\underline{r}, t_{1}, t_{2}\right)=-i M_{0}^{S} e^{i\left[\delta_{S} t_{2}-\gamma_{S} G_{2} z_{l} \tau_{G}+\frac{2}{3} \mu_{0} \gamma_{S} M_{0}^{I} T_{1}^{I} \cos \left(\delta_{I} t_{1}-\gamma_{I} G_{1} z_{l} \tau_{G}\right) e^{-t_{1} / T_{2}^{I}}\left(1-e^{\left.-t_{2} / T_{1}^{I}\right)}\right]\right.} e^{-t_{2} / T_{2}^{S}} \\
=-i M_{0}^{S} \sum_{m=-\infty}^{+\infty}\left\{i^{m} J_{m}\left[\frac{2}{3} \frac{T_{1}^{I}}{\tau_{d}^{S}} e^{-t_{1} / T_{2}^{I}}\left(1-e^{-t_{2} / T_{1}^{I}}\right)\right] e^{i m \delta_{I} t_{1}} e^{i \delta_{S} t_{2}} e^{-t_{2} / T_{2}^{S}}\right. \\
\left.\times e^{-i\left(m \gamma_{I} G_{1}+\gamma_{S} G_{2}\right) z_{l} \tau_{G}}\right\}
\end{gathered}
$$

where the Jacobi-Anger expansion (Eq. 6.80) has been used and $\tau_{d}^{S}=\left(\mu_{0} \gamma_{S} M_{0}^{I}\right)^{-1}$ is the characteristic dipolar demagnetizing time of spin $S$ in the presence of the dipolar field of spin $I$. The helix of spin $S$ magnetization wound by the gradient pulse $G_{2}=\kappa G_{1}$ is unwound by the action of the spin $I$ dipolar field if the relative gradient strengths are chosen such that $\kappa=n \gamma_{I} / \gamma_{S}$, where $n$ is an integer. ${ }^{30}$ In that case the spatially-averaged magnetization density is equal to the $m=-n$ position-independent component of Eq. 6.105:

$$
M_{+}^{S}\left(t_{1}, t_{2}\right)=-i^{n+1} M_{0}^{S} J_{n}\left[\frac{2}{3} \frac{T_{1}^{I}}{\tau_{d}^{S}} e^{-t_{1} / T_{2}^{I}}\left(1-e^{-t_{2} / T_{1}^{I}}\right)\right] e^{-i n \delta_{I} t_{1}} e^{i \delta_{S} t_{2}} e^{-t_{2} / T_{2}^{S}}
$$

The relation $J_{-n}(x)=(-1)^{n} J_{n}(x)$ for integer $n$ has been used. Eq. 6.106 is valid in a homogeneous $\underline{B}_{0}$ field; if the evolution frequencies $\delta_{I}$ and $\delta_{S}$ depend on position, the factor

\footnotetext{
${ }^{30}$ In this case the pitch of the helix of spin $S$ magnetization after the second gradient pulse is an integer submultiple of the wavelength of modulated spin $I$ magnetization.
} 


\subsection{INDIRECT DETECTION WITH THE DISTANT DIPOLAR FIELD 414}

$e^{-i n \delta_{I} t_{1}} e^{i \delta_{S} t_{2}}$ must be replaced by its spatially-averaged value. The choice of $n$ selects the $n$-quantum spectrum of spin $I$ in the indirect dimension. The indirect spectrum corresponds to the normal quadrature-detected spectrum of $I$ when $n=-1$ and $G_{2}=-\left(\gamma_{I} / \gamma_{S}\right) G_{1}$ :

$$
M_{+}^{S}\left(t_{1}, t_{2}\right)=M_{0}^{S} J_{1}\left[\frac{2}{3} \frac{T_{1}^{I}}{\tau_{d}^{S}} e^{-t_{1} / T_{2}^{I}}\left(1-e^{-t_{2} / T_{1}^{I}}\right)\right] e^{i \delta_{I} t_{1}} e^{i \delta_{S} t_{2}} e^{-t_{2} / T_{2}^{S}}
$$

This is the standard version of the DDF indirect detection sequence, which transmits the information about the single-quantum spectrum of the analyte nucleus $I$ to the sensor nucleus $S$ through the distant dipolar field. If the rotating magnetization of spin $S$ is detected by the current it inductively generates in an rf coil, the quadrature-detected signal is $S_{+}^{S} \propto \frac{\partial M_{+}^{l a b}\left(t_{2}\right)}{\partial t_{2}}$. The laboratory-frame rotating magnetization $M_{+}^{l a b}$ can be generated from Eq. 6.107 simply by replacing the offset frequency $\delta_{S}$ with the Larmor frequency $\omega_{0}^{S}$, which includes the chemical shielding but excludes the resonance detuning. The signal after transformation back into the rotating frame (i.e., after mixing the laboratory-frame signal with the rf carrier frequency) is:

$$
\begin{aligned}
S_{+}^{S} \propto & M_{0}^{S} \frac{2}{3 \tau_{d}^{S}} J_{0}\left[\frac{2}{3} \frac{T_{1}^{I}}{\tau_{d}^{S}} e^{-t_{1} / T_{2}^{I}}\left(1-e^{-t_{2} / T_{1}^{I}}\right)\right] e^{i \delta_{I} t_{1}} e^{-t_{1} / T_{2}^{I}} e^{i \delta_{S} t_{2}} e^{-\left(t_{2} / t_{1}^{I}+t_{2} / T_{2}^{S}\right)} \\
& +M_{0}^{S}\left(i \omega_{0}^{S}-\frac{1}{T_{2}^{S}}-\frac{1}{T_{1}^{I}} \frac{e^{-t_{2} / T_{1}^{I}}}{1-e^{-t_{2} / T_{1}^{I}}}\right) J_{1}\left[\frac{2}{3} \frac{T_{1}^{I}}{\tau_{d}^{S}} e^{-t_{1} / T_{2}^{I}}\left(1-e^{-t_{2} / T_{1}^{I}}\right)\right] e^{i \delta_{I} t_{1}} e^{i \delta_{S} t_{2}} e^{-t_{2} / T_{2}^{S}}
\end{aligned}
$$

where the relation $\frac{d J_{1}(x)}{d x}=J_{0}(x)-J_{1}(x) / x$ has been used. At high magnetic fields the Larmor frequency $\left|\omega_{0}^{S}\right| \approx 10^{8} \mathrm{~Hz}$ is orders of magnitude larger than $\left|\tau_{d}^{S}\right|^{-1},\left|T_{2}^{S}\right|^{-1}$, or 
$\left|T_{1}^{I}\right|^{-1}$, so the signal is approximately:

$$
S_{+}^{S} \propto i \omega_{0}^{S} M_{0}^{S} J_{1}\left[\frac{2}{3} \frac{T_{1}^{I}}{\tau_{d}^{S}} e^{-t_{1} / T_{2}^{I}}\left(1-e^{-t_{2} / T_{1}^{I}}\right)\right] e^{i \delta_{I} t_{1}} e^{i \delta_{S} t_{2}} e^{-t_{2} / T_{2}^{S}}
$$

which is proportional to $M_{+}^{S}\left(t_{1}, t_{2}\right)$ in Eq. 6.107. This proportionality is usually taken for granted in single-pulse NMR spectroscopy where the signal $S_{+} \propto \partial M_{+} / \partial t$ due to a rotating magnetization component of the form $M_{+}(t)=M_{0} e^{i \delta t}$ is obviously proportional to $M_{+}(t)$ itself. The additional time modulation due to the dipolar field makes the proportionality less obvious, but it is still valid.

The amplitude of the signal in Eq. 6.109 goes like $M_{0}^{S} J_{1}\left[\frac{2}{3} \frac{T_{1}^{I}}{\tau_{d}^{S}} e^{-t_{1} / T_{2}^{I}}\left(1-e^{-t_{2} / T_{1}^{I}}\right)\right]$, where the argument of the Bessel function depends on the dipolar demagnetizing time $\tau_{d}^{S}$ and the analyte spin relaxation parameters $T_{1}^{I}$ and $T_{2}^{I}$. In the absence of relaxation this amplitude is $M_{0}^{S} J_{1}\left[\frac{2}{3} t_{2} / \tau_{d}^{S}\right]$, which grows to be on the order of $M_{0}^{S}$ for $t_{2} \sim \tau_{d}^{S}$. In this limit the signal of a single point of the spin- $I$ indirect spectrum is on the order of the single-shot direct spin- $S$ signal, which would be a great enhancement if the analyte is dilute or has low polarization and the sensor is highly magnetized. In practice, the signal is rarely able to evolve to its full relaxation-free value. Relaxation effects help to ensure that the Bessel function argument is small. The dipolar demagnetizing time can also be quite long-e.g., $\tau_{d}^{S} \approx 10^{5} \mathrm{~s}$ for $\mathrm{H}-1$ detection of a $1 \mathrm{mM} \mathrm{C}-13$ labeled compound thermally polarized at room temperature with $B_{0}=16.45 \mathrm{~T}$-so even in the absence of relaxation it may not be desirable to wait until the full signal develops. These factors and others that will be described later limit the amount of signal that can be collected per shot, and most physical systems will be in the limit where the Bessel function argument is small compared to unity. 
The Bessel function power series

$$
J_{n}(x)=\sum_{k=0}^{+\infty} \frac{(-1)^{k} x^{2 k+n}}{2^{2 k+n} k !(n+k) !},
$$

can be used to approximate the Bessel function in the $x \ll 1$ limit:

$$
J_{n}(x) \approx \frac{x^{n}}{2^{n} n !}
$$

where only the leading $k=0$ term has been retained. The short-time approximation to the signal in Eq. 6.109 is:

$$
S_{+}^{S} \propto \frac{1}{3} i \omega_{0}^{S} \gamma_{S} \mu_{0} M_{0}^{I} M_{0}^{S} T_{1}^{I} e^{i \delta_{I} t_{1}} e^{-t_{1} / T_{2}^{I}} e^{i \delta_{S} t_{2}} e^{-t_{2} / T_{2}^{S}}\left(1-e^{-t_{2} / T_{1}^{I}}\right)
$$

which is bilinear in the analyte and sensor nuclear magnetizations. The signal is scaled down by a factor on the order of $\frac{T_{1}^{I}}{\tau_{d}^{S}} e^{-t_{1} / T_{2}^{I}}$ relative to the spin- $S$ direct signal, and in addition to the normal $T_{2}$ relaxation in the direct dimension there is a factor of $1-e^{-t_{2} / T_{1}^{I}}$ that goes like $t_{2} / T_{1}^{I}$ at the beginning of the acquisition but grows to unity over a time long compared to $T_{1}^{I}$. It is not surprising that a large sensor magnetization $M_{0}^{S}$ and long lifetime $T_{1}^{I}$ of the modulated analyte magnetization are desirable, but in the short time limit the size of the analyte magnetization $M_{0}^{I}$ also determines the magnitude of the signal.

Bowtell [296] has made a detailed analysis of the prospects of sensitivity enhancement using this type of indirect detection sequence. Although the direct detection of spin $I$ has the advantage that $N_{1}$ signal averages could be performed in about the time it takes to collect $N_{1}$ spin- $S$ transients indirectly without signal averaging, some of the advantage 


\subsection{INDIRECT DETECTION WITH THE DISTANT DIPOLAR FIELD 417}

of direct detection is offset by the fact that in the indirect method the detection bandwidth need be only wide enough to accommodate the spectrum of the sensor, which potentially could be only a single line. The direct detection method collects signal across a bandwidth that must accommodate the entire spectrum of spin $I$ and therefore collects a commensurate amount of noise. On the other hand, indirect detection sequences are subject to multiplicative noise termed " $t_{1}$ noise", in which fluctuations in the experimental conditions from transient to transient manifest themselves as noise in the indirect dimension of the spectrum $[7, \S 6.8 .2]$. Bowtell has suggested on the basis of sensitivity considerations that intermolecular indirect detection may best be implemented through the nuclear Overhauser effect [385], although the NOE does not act over a mesoscopic distance and the sensor and analyte are required to be intermingled on a molecular scale.

Warren et al. [77] have raised some additional concerns about sensitivity enhancement that were not included in Bowtell's estimates. If the sensor nucleus is highly magnetized - which is desirable for sensitivity enhancement experiments - and if inductive detection is employed, radiation damping can be significant as the transverse magnetization of the sensor rephases under the action of the analyte dipolar field. The NMR signal is reduced as the radiation damping torque returns the transverse magnetization to its equilibrium orientation along $z$. A simple way to suppress radiation damping is to prepare only a small amount of sensor transverse magnetization with a small tip-angle pulse; unfortunately, less sensor magnetization results in less signal. Detuning the rf detection circuit also reduces radiation damping but adversely affects the detection sensitivity. Radiation damping effects also can be largely suppressed by active electronic feedback techniques $[386,387]$ 


\subsection{INDIRECT DETECTION WITH THE DISTANT DIPOLAR FIELD418}

or by using quality factor-switching probes $[388,389]$. As will be discussed in $\S 6.3 .2$, the use of remote detection methodology also can circumvent the radiation damping problem. A more serious problem is the action of the transverse self-dipolar field of the sensor nucleus. As the sensor magnetization rephases under the action of the analyte dipolar field, it creates its own dipolar field which is not necessarily parallel to the magnetization. This field can rotate the magnetization out of the transverse plane and cause a reduction of the NMR signal. Warren et al. have suggested that these effects may be suppressed by employing e.g. homonuclear dipolar decoupling sequences as is routine in solid-state NMR experiments. The effects of molecular diffusion may also have a deleterious effect on the sensitivity of the DDF indirect detection experiment, particularly if a rapidly-diffusing species such as a gas is used (e.g., Xe-129 as a sensor/mobile carrier for remote detection applications). For example, the sensitivity enhancement that can be achieved by using gradient-modulated magnetization distributions would be destroyed if the sensor diffuses over a distance larger than the length scale of the magnetization modulation during the DDF encoding interval.

The two-dimensional Fourier transform of Eq. 6.112 with respect to the time variables $t_{1}$ and $t_{2}$ clearly generates cross peaks $\left(\delta_{I}, \delta_{S}\right)$ at the offset frequencies of the analyte and sensor nuclei, some examples of which will be presented in $\S 6.4 .3$. The projection along the indirect dimension of the spectrum is just the normal one-dimensional NMR spectrum of the analyte. In fact, the pulse sequence of Fig. 6.3 is often used as a basic element of heteronuclear correlation sequences in $J$-coupled systems. The similarity between the standard heteronuclear correlation spectroscopy and the DDF version is perhaps evident upon considering that the heteronuclear $J$-coupling and dipolar coupling Hamiltonians have the 


\subsection{INDIRECT DETECTION WITH THE DISTANT DIPOLAR FIELD419}

same form. Of course, the DDF version of the experiment yields a much weaker signal because it arises from only the short-time contribution of small distant dipolar couplings. In the context of remote detection the spectral information of the sensor nucleus is generally not of interest, and recording only one point $t_{2}=T$ of the signal is sufficient to generate a one-dimensional data set $S_{+}\left(t_{1} ; T\right)$ that maps out the analyte signal in an indirect, pointby-point fashion. The time $T$ can be determined experimentally in order to maximize the dipolar field-induced signal.

The choice of relative gradient strengths $G_{2} / G_{1}=\kappa=-\gamma_{I} / \gamma_{S}$ selects the -1 quantum coherence in the indirect dimension (see Eq. 6.107). In some cases it may be advantageous to select another coherence. For instance, the sign of the coherence $(+n$ versus $-n)$ can be important. If the analyte and sensor molecules are mixed homogeneously and the $\underline{B}_{0}$ field is inhomogeneous on a length scale that is larger than the scale over which the dipolar field is active, one expects that any given small volume of sensor molecules experiences the same external field as does the nearby small volume of analyte molecules responsible for creating the DDF felt by the sensor. Eq. 6.105 indicates that the inhomogeneous dephasing of an analyte $n$-quantum coherence during $t_{1}$ can be transmitted through the DDF to the sensor nucleus at time $t_{2}$ with a total phase equal to:

$$
\begin{aligned}
-n \delta_{I} t_{1}+\delta_{S} t_{2} & =n \gamma_{I} B_{\delta}(\underline{r}) t_{1}-\gamma_{S} B_{\delta}(\underline{r}) t_{2} \\
& =n \gamma_{I}\left[\left(1-\sigma_{i s o}^{I}\right) B_{0}(\underline{r})-\omega_{r}^{I} / \gamma_{I}\right] t_{1}-\gamma_{S}\left[\left(1-\sigma_{i s o}^{S}\right) B_{0}(\underline{r})-\omega_{r}^{S} / \gamma_{S}\right] t_{2} \\
& \simeq-n \delta_{I}^{0} t_{1}+\delta_{S}^{0} t_{2}+\left(n \gamma_{I} t_{1}-\gamma_{S} t_{2}\right) \Delta B_{0}(\underline{r})
\end{aligned}
$$

where $\underline{B}_{0}(\underline{r})=\left[B_{0}+\Delta B_{0}(\underline{r})\right] \underline{\tilde{z}}$ is the inhomogeneous external field and $\delta_{I}^{0}=-\gamma_{I}[(1-$ 


\subsection{INDIRECT DETECTION WITH THE DISTANT DIPOLAR FIELD420}

$\left.\left.\sigma_{i s o}^{I}\right) B_{0}-\omega_{r}^{I} / \gamma_{I}\right]$ and $\delta_{S}^{0}=-\gamma_{S}\left[\left(1-\sigma_{i s o}^{S}\right) B_{0}-\omega_{r}^{S} / \gamma_{S}\right]$ are the inhomogeneity-free resonance offsets of spins $I$ and $S$ respectively. The right-hand side of the last line of Eq. 6.113 has used the approximation $1-\sigma_{i s o} \approx 1$ because chemical shieldings are usually only $\sim 10^{3}$ ppm at best. Eq. 6.113 assumes that only analyte and sensor molecules at the same point $\underline{r}$ are correlated through the dipolar field and hence experience the same field inhomogeneity $\Delta B_{0}(\underline{r})$, which is a reasonable assumption if $\underline{B}_{0}(\underline{r})$ varies on a longer length scale than the dipolar field. ${ }^{31}$ The field inhomogeneities are refocused in a coherence transfer echo [390] at $t_{2}=n\left(\gamma_{I} / \gamma_{S}\right) t_{1}=\kappa t_{1}$ if the sign of $n$ is chosen properly. Coherence transfer echoes of the inhomogeneous dephasing are well-known in intramolecular $J$-coupled systems in which the two spins $I$ and $S$ obviously experience the same external field $\underline{B}_{0}(\underline{r})$. The sign of the coherence order $n$ determines whether the inhomogeneous dephasing is refocused: since the -1-quantum coherence is detected on spin $S$, a $+|n|$-quantum coherence on spin $I$ must be used to form the echo if $\gamma_{I} / \gamma_{S}>1$ and a $-|n|$-quantum coherence on spin $I$ must be used if $\gamma_{I} / \gamma_{S}<1$. The echo is formed at a time during the acquisition that is proportional to the indirect time $t_{1}$, so the echo travels in the acquisition window as $t_{1}$ is incremented. This leads to a characteristic shearing $[7, \S 6.6 .1]$ of the $2 \mathrm{D}$ NMR spectrum along a skew axis of slope $\kappa$ with a projection along the indirect dimension that is not free of the inhomogeneous dephasing. The echo and anti-echo pathways have opposite signs of $\kappa$ and the respective cross peaks fall on opposite sides of zero frequency in the indirect dimension; the peak that corresponds to the echo pathway is easily distinguished by the shearing effect. A narrow, inhomogeneity-free line can be obtained in the indirect dimension after applying a mathematical shearing transformation to the echo pathway peak.

\footnotetext{
${ }^{31}$ Note that if the analyte and sensor are physically separated, this assumption may not hold.
} 


\subsection{INDIRECT DETECTION WITH THE DISTANT DIPOLAR FIELD421}

Alternatively, the pulse sequence could be modified to include a delayed acquisition period or to realign the coherence transfer echo such that it forms at a constant time in the acquisition window [391, 392]; these techniques are heteronuclear predecessors of the more recent split$t_{1}$ technique [142] used to remove the shearing effect in the MQMAS echo experiment (see $\S 3)$. If the sign of the coherence in the indirect dimension is chosen such that the echo pathway is selected, the use of these methods may increase the resolution of the indirect spectrum by removing the inhomogeneous broadening.

The resolution of the indirect spectrum can also be increased by choosing a coherence order $|n|>1$. A spin- $I n$-quantum coherence evolves during $t_{1}$ at the frequency $-n \delta_{I}$, so the resolution of an $n$-quantum spectrum is $|n|$ times that of the single-quantum spectrum, assuming that the coherence relaxation rates (and hence the linewidths) are the same. This phenomenon is discussed in $\S 4$ in the context of double-quantum line narrowing in spin-1 systems. Note, however, that the intensity of a multiple-quantum line is less than that of a single-quantum line, as can be seen from the $J_{n}$-dependence of Eq. 6.105. Since sensitivity seems to be the major concern in the indirect detection experiments, it may not be worthwhile to seek higher spectral resolution with analyte multiple-quantum coherences.

\section{Technical issues}

Several issues regarding the implementation of the DDF indirect detection sequence should be discussed. The pulse sequence as diagrammed in Fig. 6.3 places the first gradient pulse $G_{1}$ immediately after the rf pulse, but the signal as calculated above is the same regardless of where the gradient pulse is placed within the $t_{1}$ interval. It is advantageous to place the gradient pulse at the beginning of the $t_{1}$ interval if the analyte has 


\subsection{INDIRECT DETECTION WITH THE DISTANT DIPOLAR FIELD 422}

a high enough magnetization that radiation damping may be significant. However, since the rf channel of spin $I$ is used only for excitation and not for detection, the rf circuit can be safely detuned to suppress radiation damping without loss of detection signal. The disadvantage of putting the gradient pulse at the beginning of the indirect evolution time is that the magnetization helix it creates can be partially destroyed by diffusion of the analyte molecules if $t_{1}$ is long and the pitch of the helix $2 \pi /\left(\gamma G_{1} \tau_{G}\right)$ is small. Furthermore, if the dipolar field of the analyte is made large by the spatial symmetry-breaking of the gradient pulse, the interaction between the analyte DDF and radiation damping in the excitation coil may in time induce turbulent spin dynamics [351]. For these reasons, it may be more desirable to place the gradient pulse at the end of the $t_{1}$ interval just before the second spin- $I$ rf pulse. Alternatively, a compromise can be made where the gradient pulse $G_{1}$ is split into two parts that are placed at the beginning and the end of the $t_{1}$ interval [363].

The signal in Eq. 6.112 gives an undesirable phase-twist lineshape [7, §6.5.1],[4, §4.3.4] upon Fourier transformation. This lineshape contains dispersive components with broad wings that can interfere with the resolution of closely-spaced resonances. Pure absorptive-phase lineshapes can be obtained e.g. by the States hypercomplex method [143] or other techniques $[7, \S 6.5 .3]$. These methods require the collection of both the echo and antiecho pathways, at the expense of including the inhomogeneous broadening inherent in the antiecho signal $[7, \S 6.5 .2],[145]$.

The use of pulsed field gradients $G_{2} / G_{1}=\kappa=n \gamma_{I} / \gamma_{S}$ automatically selects the spin- $I n$-quantum coherence in the indirect dimension, but if no gradients are used all coherences pass and are observed in the spectrum. This can be appreciated from a schematic 


\subsection{INDIRECT DETECTION WITH THE DISTANT DIPOLAR FIELD423}

density operator description of the pulse sequence of Fig. 6.3. If $\phi_{1}=\phi_{2}=\phi_{3}=\phi_{r}=0$ (i.e., all $\mathrm{rf}$ pulses are along the rotating frame $x$-axis) and no gradient pulses are employed, the $I=1 / 2, S=1 / 2$ initial density operator in Eq. 6.59 has evolved by the beginning of the acquisition into:

$$
\hat{\rho}\left(t_{1}, t_{2}=0\right)=\prod_{j=1}^{N_{I}} \prod_{k=1}^{N_{S}}\left[\frac{1}{2} \hat{1}_{j}^{I}-\zeta^{I} \hat{I}_{z j} \cos \left(\delta_{I} t_{1}\right)-\zeta^{I} \hat{I}_{x j} \sin \left(\delta_{I} t_{1}\right)\right]\left[\frac{1}{2} \hat{1}_{k}^{S}-\zeta^{S} \hat{S}_{y k}\right] .
$$

The multi-spin terms in this product that contain $|n|$ longitudinal spin- $I$ operators $\left\{\hat{I}_{z j}\right\}$ and a single transverse spin- $S$ operator $\hat{S}_{y k}$ evolve as spin- $I|n|$-quantum coherences during $t_{1}$ and are converted into transverse magnetization on spin $S$ after the action of $n$ dipolar couplings $\hat{H}_{D}^{j k}$ during $t_{2}$. For instance, single-quantum spin- $I$ signal arises from the evolution of terms like

$$
2^{-\left(N_{I}+N_{S}-2\right)} \zeta^{I} \zeta^{S} \cos \left(\delta_{I} t_{1}\right) \hat{I}_{z j} \hat{S}_{y k} \longrightarrow-2^{-\left(N_{I}+N_{S}-1\right)} \zeta^{I} \zeta^{S} \cos \left(\delta_{I} t_{1}\right) \omega_{D}^{j k} t_{2} \hat{S}_{x k}
$$

under the action of $\hat{H}_{D}^{j k}$ at short times $t_{2}$ according to Eq. 6.62. Triple-quantum spin- $I$ signal can arise from evolution of terms like:

$$
\begin{aligned}
2^{-\left(N_{I}+N_{S}-4\right)}\left(\zeta^{I}\right)^{3} \zeta^{S} \cos ^{3}\left(\delta_{I} t_{1}\right) \hat{I}_{z j} \hat{I}_{z l} \hat{I}_{z m} \hat{S}_{y k} \longrightarrow \\
2^{-\left(N_{I}+N_{S}-1\right)}\left(\zeta^{I}\right)^{3} \zeta^{S} \cos ^{3}\left(\delta_{I} t_{1}\right) \omega_{D}^{j k} \omega_{D}^{l k} \omega_{D}^{m k} t_{2}^{3} \hat{S}_{y k}
\end{aligned}
$$

under the action of the dipolar couplings $\omega_{D}^{j k}, \omega_{D}^{l k}$, and $\omega_{D}^{m k}$. Processes like these occur for all coherence orders $n$ leading to peaks at frequencies $-n \delta_{I}$ in the indirect dimension of the 


\subsection{INDIRECT DETECTION WITH THE DISTANT DIPOLAR FIELD424}

spectrum, although the higher-order peaks are less intense.

When the indirect detection experiment is performed without gradient pulses or with only weak gradients that incompletely suppress undesired coherences, an alternative method of coherence pathway selection must be used. It is particularly important to suppress the strong direct free induction decay of the $S$ spins. This FID is the usual signal that would follow a single rf pulse on the $S$ spins and is not produced by DDF effects; therefore, it is not modulated by evolution of the analyte spins during the indirect time dimension and leads to a large zero-frequency artifact in the indirect spectrum. The obvious choice of coherence pathway selection in the absence of pulsed field gradients is phase cycling of the rf pulses. The direct FID is easily suppressed using a two-step phase cycle. This can be appreciated by examining the evolution of the terms in the initial density operator that evolve into observable heteronuclear DDF-induced spin- $S$ signal or the direct spin- $S$ FID. The set of phases that will be considered is $\phi_{1}=\phi_{2}=\phi_{3}=\phi_{r}=0$ (see Fig. 6.3), i.e., all pulses are along the $x$-direction in the rotating frame. Neglecting relaxation, the spin- $S$ transverse magnetization (corresponding to the direct FID) evolves from initial $z$-magnetization $\hat{\rho}(0) \propto \hat{S}_{z}$ according to:

$$
\hat{S}_{z} \stackrel{90_{x}^{\circ I}}{\longrightarrow} \hat{S}_{z} \stackrel{t_{1}}{\longrightarrow} \hat{S}_{z} \frac{90_{x}^{\circ I}}{90_{x}^{\circ S}}-\hat{S}_{y} \underset{\phi_{r}}{\longrightarrow}-\frac{i}{2} e^{i \delta_{S} t_{2}}
$$

where the final expression is the quadrature-detected signal in the direct dimension proportional to $e^{i \phi_{r}} \operatorname{Tr}\left[\hat{\rho} \hat{S}_{+}\right]$(see $\left.\S 1.7\right)$. Using the same set of pulse and receiver phases, the spin- $I$ indirect evolution detected on spin $S$ evolves from initial two-spin terms like $\hat{\rho}(0) \propto \hat{I}_{z j} \hat{S}_{z k}$ 
according to:

$\hat{I}_{z j} \hat{S}_{z k} \stackrel{90_{x}^{\circ I}}{\longrightarrow}-\hat{I}_{y j} \hat{S}_{z k} \stackrel{t_{1}}{\longrightarrow}-\hat{I}_{y j} \hat{S}_{z k} \cos \left(\delta_{I} t_{1}\right) \underset{90_{x}^{\circ S}}{\stackrel{90_{x}^{\circ I}}{\longrightarrow}} \hat{I}_{z j} \hat{S}_{y k} \cos \left(\delta_{I} t_{1}\right) \underset{\phi_{r}}{\longrightarrow}-\frac{1}{4} \omega_{D}^{j k} t_{2} \cos \left(\delta_{I} t_{1}\right) e^{i \delta_{S} t_{2}}$

where density operator terms that do not lead to observable transverse magnetization on spin $S$ have been omitted and only the short time evolution $\hat{I}_{z j} \hat{S}_{y k} \rightarrow-\frac{1}{2} \omega_{D}^{j k} t_{2} \hat{S}_{x k}$ under the heteronuclear dipolar coupling Hamiltonian $\hat{H}_{D}^{j k}$ has been included. If a second experiment is performed in which the phases of the simultaneous spin $I$ and spin $S 90^{\circ}$ pulses are shifted by $\pi$ radians so that they are in the $-x$ direction in the rotating frame (i.e., $\phi_{1}=0^{\circ}$, $\left.\phi_{2}=\phi_{3}=180^{\circ}, \phi_{r}=0^{\circ}\right)$, then the direct FID is:

$$
\hat{S}_{z} \stackrel{90_{x}^{\circ I}}{\longrightarrow} \hat{S}_{z} \stackrel{t_{1}}{\longrightarrow} \hat{S}_{z} \underset{90_{\bar{x}}^{\circ S}}{\stackrel{90_{x}^{\circ}}{\longrightarrow}} \hat{S}_{y} \underset{\phi_{r}}{\stackrel{t_{2}}{\longrightarrow}} \frac{i}{2} e^{i \delta_{S} t_{2}}
$$

and the indirectly-detected signal comes from terms like:

$\hat{I}_{z j} \hat{S}_{z k} \stackrel{90_{x}^{\circ I}}{\longrightarrow}-\hat{I}_{y j} \hat{S}_{z k} \stackrel{t_{1}}{\longrightarrow}-\hat{I}_{y j} \hat{S}_{z k} \cos \left(\delta_{I} t_{1}\right) \underset{90_{\bar{x}}}{\stackrel{90 \bar{x}}{\longrightarrow}} \hat{I}_{z j} \hat{S}_{y k} \cos \left(\delta_{I} t_{1}\right) \underset{\phi_{r}}{\stackrel{t_{2}}{\longrightarrow}}-\frac{1}{4} \omega_{D}^{j k} t_{2} \cos \left(\delta_{I} t_{1}\right) e^{i \delta_{S} t_{2}}$

A comparison of the two experiments shows that the pulse phase shifts invert the sign of the 


\subsection{INDIRECT DETECTION WITH THE DISTANT DIPOLAR FIELD426}

direct FID but do not change the indirect signal. Coaddition of the signals from these two experiments therefore suppresses the direct FID but retains the indirect heteronuclear signal. This result holds when relaxation is neglected; if relaxation is included, the $90_{x}^{\circ I}-t_{1}-90_{x}^{\circ I}$ sequence stores spin- $I$ magnetization in the $-z$ direction and the $90_{x}^{\circ I}-t_{1}-90_{\bar{x}}^{\circ I}$ sequence stores spin- $I$ magnetization in the $+z$ direction, and these two longitudinal magnetization components relax differently.

The indirect signal in Eqs. 6.118 and 6.120 becomes amplitude-modulated as $\cos \left(\delta_{I} t_{1}\right)=\frac{1}{2}\left(e^{+i \delta_{I} t_{1}}+e^{-i \delta_{I} t_{1}}\right)$ by incrementation of the indirect time variable and contains the frequency components $\pm \delta_{I}$ in the indirect spectrum. This lack of frequency discrimination is a result of passing both the +1 -quantum and -1-quantum coherences during $t_{1}$, and results in an indirect spectrum that corresponds to the superposition of the normal quadrature-detected (-1QC) spectrum with its mirror image. As has been discussed already, pulsed field gradient coherence pathway selection naturally chooses either the $+1 \mathrm{QC}$ or the $-1 \mathrm{QC}$ during $t_{1}$ depending on the relative sign of the gradient pulses; this frequency discrimination is also easily implemented via a proper phase cycling scheme. Consider the choice of pulse and receiver phases $\phi_{1}=0^{\circ}, \phi_{2}=\phi_{3}=90^{\circ}, \phi_{r}=0^{\circ}$; i.e., the simultaneous spin- $I$ and spin- $S 90^{\circ}$ pulses are along the $y$-direction in the rotating frame. The indirectly-detected signal develops according to:

$\hat{I}_{z j} \hat{S}_{z k} \stackrel{90_{x}^{\circ I}}{\longrightarrow}-\hat{I}_{y j} \hat{S}_{z k} \stackrel{t_{1}}{\longrightarrow} \hat{I}_{x j} \hat{S}_{z k} \sin \left(\delta_{I} t_{1}\right) \frac{90_{y}^{\circ I}}{90_{y}^{\circ S}}-\hat{I}_{z j} \hat{S}_{x k} \sin \left(\delta_{I} t_{1}\right) \underset{\phi_{r}}{\stackrel{t_{2}}{\longrightarrow}}-\frac{i}{4} \omega_{D}^{j k} t_{2} \sin \left(\delta_{I} t_{1}\right) e^{i \delta_{S} t_{2}}$ 


\subsection{INDIRECT DETECTION WITH THE DISTANT DIPOLAR FIELD427}

where again only density operator terms that eventually develop into observable spin- $S$ transverse magnetization have been retained, and the short time evolution under the heteronuclear dipolar coupling Hamiltonian $\hat{H}_{D}^{j k}$ is $\hat{I}_{z j} \hat{S}_{x k} \rightarrow \frac{1}{2} \omega_{D}^{j k} t_{2} \hat{S}_{y k}$. Coaddition of the signals in Eqs. 6.118 and 6.121 gives a net signal from the arbitrary spin pair $j-k$ that is proportional to $-\frac{1}{4} \omega_{D}^{j k} t_{2} e^{i \delta_{I} t_{1}} e^{i \delta_{S} t_{2}}$, a phase-modulated signal which corresponds to the selection of the pure -1-quantum coherence on spin $I$ during $t_{1}$. Selection of the +1 -quantum coherence during $t_{1}$ can be achieved with the choice of phases $\phi_{1}=0^{\circ}, \phi_{2}=\phi_{3}=90^{\circ}$, $\phi_{r}=180^{\circ}$, where the $180^{\circ}$ shift of the receiver phase corresponds to a multiplication of the signal in Eq. 6.121 by $e^{i \pi}=-1$; when this signal is added to the signal in Eq. 6.118 , the net

result is $-\frac{1}{4} \omega_{D}^{j k} t_{2} e^{-i \delta_{I} t_{1}} e^{i \delta_{S} t_{2}}$. The two-step phase cycle that achieves frequency discrimination in the indirect dimension does not suppress the direct FID; however, the two-step FID-suppression and frequency discrimination phase cycles always can be combined into a larger four-step phase cycle.

It may be concluded that when pulsed field gradients are absent or weak, suppression of the direct spin- $S$ FID can be achieved along with frequency discrimination of the indirect spin- $I$ signal by using a four-step phase cycle in which the first spin- $I 90^{\circ}$ pulse phase and the receiver phase are held constant at $\phi_{1}=\phi_{r}=0^{\circ}, 0^{\circ}, 0^{\circ}, 0^{\circ}$ while the phases of the simultaneous spin- $I$ and spin- $S 90^{\circ}$ pulses are incremented together through $\phi_{2}=\phi_{3}=0^{\circ}, 90^{\circ}, 180^{\circ}, 270^{\circ}$. This choice of phases selects the spin- $I-1 \mathrm{QC}$ during $t_{1}$; the $+1 \mathrm{QC}$ can be selected by inverting the receiver phase according to $\phi_{r}=0^{\circ}, 180^{\circ}, 0^{\circ}, 180^{\circ}$. The spin- $I+1 \mathrm{QC}$ and $-1 \mathrm{QC}$ carry the same spectral information, but only one coherence will result in the formation of a coherence transfer echo of the inhomogeneous broadening 


\subsection{INDIRECT DETECTION WITH THE DISTANT DIPOLAR FIELD428}

during $t_{2}$, depending on the relative signs of $\gamma_{I}$ and $\gamma_{S}$. Note that signals of the form $e^{ \pm i \delta_{I} t_{1}} e^{i \delta_{S} t_{2}}$ do not yield pure absorptive-phase lineshapes upon Fourier transformation, as was discussed earlier. The elimination of broad dispersive components from the twodimensional lineshape can be effected by adopting e.g. a hypercomplex acquisition scheme, resulting in enhanced spectral resolution.

\subsubsection{Proposed modification of the indirect detection experiment for re- mote detection}

The pulse sequence in Fig. 6.3 was developed for use with a conventional NMR detection scheme. This sequence is easily modified to serve as an encoding module for a remote indirect detection sequence:

$$
\left[90_{\phi_{1}}^{\circ I}\right]-t_{1}-G_{1}\left(\tau_{G}\right)-\left[90_{\phi_{2}}^{\circ I}, 90_{\phi_{3}}^{\circ}\right]-G_{2}\left(\tau_{G}\right)-\frac{T}{2}-\left[180_{\phi_{4}}^{\circ I}, 180_{\phi_{5}}^{\circ}\right]-\frac{T}{2}-\left[90_{\phi_{S}}^{\circ S}\right]
$$

as shown in Fig. 6.4. The analyte nucleus is designated as spin $I$ and the mobile sensor nucleus is designated as spin $S$. This sequence is similar to the basic DDF heteronuclear correlation sequence, except that the sensor spin magnetization is not detected during a direct dimension $t_{2}$; rather, the dipolar field-mediated signal — which is zero immediately after the creation of the analyte dipolar field at the end of the indirect time interval $t_{1}$-is allowed to develop for a time $T$ into transverse magnetization on spin $S$ before being stored as longitudinal magnetization for transport and subsequent remote detection. The delay $T$ is chosen to maximize the DDF-encoded spin- $S$ magnetization at the point when it is stored for transport; the value of $T$ can be optimized experimentally. 
I

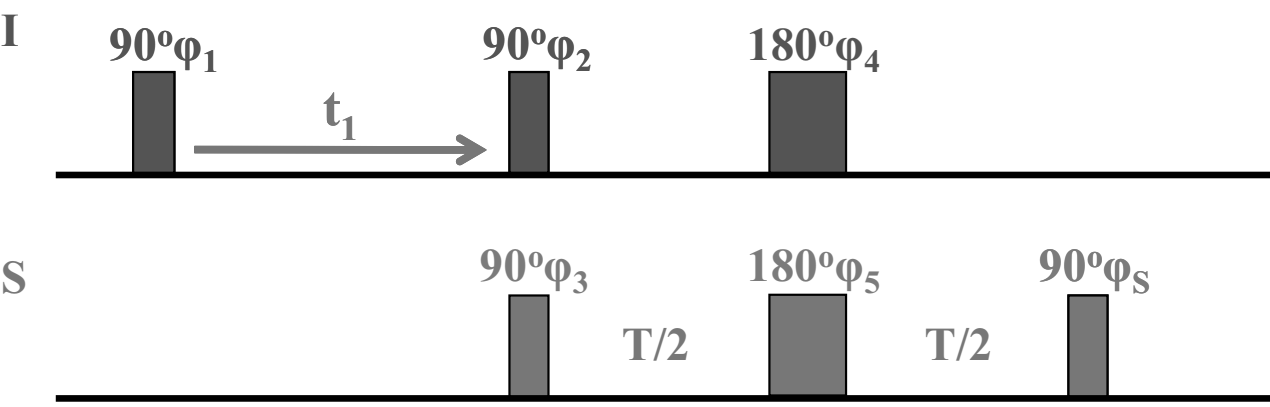

z-gradients

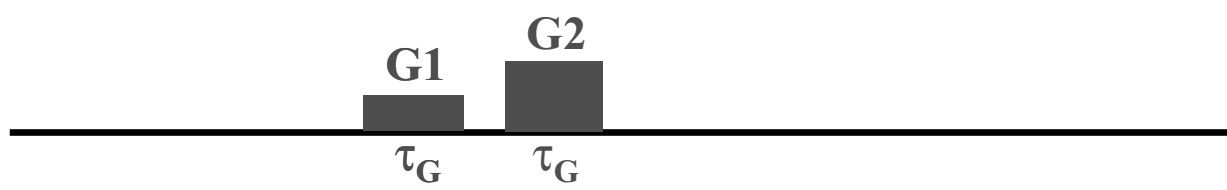

Figure 6.4: Modified DDF indirect detection pulse sequence for use as an encoding module for remote detection NMR.

Simultaneous $180^{\circ}$ pulses are applied to both spins at a time $t=T / 2$ that is halfway between the creation of the encoded analyte dipolar field and the storage of the sensor magnetization. As will be seen, these $180^{\circ}$ pulses refocus the chemical shift evolution of spin $S$ at the storage time $t=T$ while retaining the dipolar evolution. The gradient pulses are optional and may be used for coherence pathway selection or to enhance the dipolar field for certain sample configurations, as will be discussed later. Phase cycling may be employed if no gradient pulses are used or if they are too weak to provide complete coherence pathway selection. The first gradient pulse is placed at the end of the indirect evolution period $t_{1}$ to minimize the effects of molecular diffusion of the analyte and turbulent spin dynamics, as is discussed in the "technical issues" portion of $\S 6.3 .1$.

The magnitude of the stored longitudinal magnetization of spin $S$ at $t=T$ may be calculated using the usual techniques. Consider the experiment in which all pulse phases are equal to zero: $\phi_{1}=\phi_{2}=\phi_{3}=\phi_{4}=\phi_{5}=\phi_{S}=0$. The analyte longitudinal magnetization 
that contributes to the heteronuclear secular dipolar field when $t<T / 2$ is given by Eq. 6.102 , and the $180_{x}^{\circ I}$ pulse at $t=T / 2$ inverts this magnetization, such that:

$$
\begin{aligned}
& M_{z}^{I}\left(\underline{r}, t_{1}, t<\frac{T}{2}\right)=-M_{0}^{I} \cos \left(\delta_{I} t_{1}-\gamma_{I} G_{1} z_{l} \tau_{G}\right) e^{-t_{1} / T_{2}^{I}} e^{-t / T_{1}^{I}}+M_{e q}^{I}\left(1-e^{-t / T_{1}^{I}}\right), \\
& M_{z}^{I}\left(\underline{r}, t_{1}, t>\frac{T}{2}\right)=M_{0}^{I} \cos \left(\delta_{I} t_{1}-\gamma_{I} G_{1} z_{l} \tau_{G}\right) e^{-t_{1} / T_{2}^{I}} e^{-t / T_{1}^{I}}+M_{e q}^{I}\left(1+e^{-t / T_{1}^{I}}-2 e^{-\left(t-\frac{T}{2}\right) / T_{1}^{I}}\right),
\end{aligned}
$$

where the solution of the Bloch equations (Eq. 6.20) for the relaxation of longitudinal magnetization $M_{z}(t)=M_{e q}+\left[M_{z}\left(t_{0}\right)-M_{e q}\right] e^{-\left(t-t_{0}\right) / T_{1}}$ has been used. The longitudinal relaxation may be neglected if it so happens that $T \ll T_{1}^{I}$; if not, at least the constantmagnetization component contribution to the dipolar field presumably can be neglected, as was discussed in $\S 6.3 .1$. In the limit that the gradient modulation length $\left|2 \pi /\left(\gamma_{I} G_{1} \tau_{G}\right)\right|$ is small compared to any sample dimension, the secular heteronuclear dipolar field of spin $I$ felt by spin $S$ is:

$$
\begin{aligned}
& \underline{B}_{d}^{I}\left(t<\frac{T}{2}\right)=-\frac{2}{3} \mu_{0} M_{0}^{I} \cos \left(\delta_{I} t_{1}-\gamma_{I} G_{1} z_{l} \tau_{G}\right) e^{-t_{1} / T_{2}^{I}} e^{-t / T_{1}^{I}} \underline{\widetilde{z}} \\
& \underline{B}_{d}^{I}\left(t>\frac{T}{2}\right)=+\frac{2}{3} \mu_{0} M_{0}^{I} \cos \left(\delta_{I} t_{1}-\gamma_{I} G_{1} z_{l} \tau_{G}\right) e^{-t_{1} / T_{2}^{I}} e^{-t / T_{1}^{I}} \underline{\widetilde{z}} .
\end{aligned}
$$

The respective dynamic phases acquired by spin $S$ during evolution during the intervals 


\subsection{INDIRECT DETECTION WITH THE DISTANT DIPOLAR FIELD431}

$[0, T / 2]$ and $[T / 2, T]$ in the presence of the dipolar field of spin $I$ are:

$$
\begin{aligned}
\Phi_{d}(T / 2 ; 0) & =-\gamma_{S} \int_{0}^{T / 2} d t\left[-\frac{2}{3} \mu_{0} M_{0}^{I} \cos \left(\delta_{I} t_{1}-\gamma_{I} G_{1} z_{l} \tau_{G}\right) e^{-t_{1} / T_{2}^{I}} e^{-t / T_{1}^{I}}\right] \\
& =+\frac{2}{3} \gamma_{S} \mu_{0} M_{0}^{I} T_{1}^{I} \cos \left(\delta_{I} t_{1}-\gamma_{I} G_{1} z_{l} \tau_{G}\right) e^{-t_{1} / T_{2}^{I}}\left(1-e^{-T / 2 T_{1}^{I}}\right), \\
\Phi_{d}(T ; T / 2) & =-\gamma_{S} \int_{T / 2}^{T} d t\left[+\frac{2}{3} \mu_{0} M_{0}^{I} \cos \left(\delta_{I} t_{1}-\gamma_{I} G_{1} z_{l} \tau_{G}\right) e^{-t_{1} / T_{2}^{I}} e^{-t / T_{1}^{I}}\right] \\
& =-\frac{2}{3} \gamma_{S} \mu_{0} M_{0}^{I} T_{1}^{I} \cos \left(\delta_{I} t_{1}-\gamma_{I} G_{1} z_{l} \tau_{G}\right) e^{-t_{1} / T_{2}^{I}} e^{-T / 2 T_{1}^{I}}\left(1-e^{-T / 2 T_{1}^{I}}\right) .
\end{aligned}
$$

If only the dipolar field was inverted at $t=T / 2$ due to the inversion of the longitudinal magnetization of spin $I$ by the $180_{x}^{\circ I}$ pulse, the total phase accumulated by the $S$ spins at $t=$ $T$ under the dipolar evolution would be $\Phi_{d}(T / 2 ; 0)+\Phi_{d}(T ; T / 2)=\frac{2}{3} \gamma_{S} \mu_{0} M_{0}^{I} T_{1}^{I} \cos \left(\delta_{I} t_{1}-\right.$ $\left.\gamma_{I} G_{1} z_{l} \tau_{G}\right) e^{-t_{1} / T_{2}^{I}}\left(1-e^{-T / 2 T_{1}^{I}}\right)^{2}$, which would equal zero if the longitudinal relaxation of spin $I$ could be neglected $\left(T_{1}^{I} \rightarrow \infty\right)$; i.e., the inversion of the dipolar field at $t=T / 2$ would refocus the dipolar evolution at time $t=T$. However, not only is the dipolar field inverted at $t=T / 2$, but at the same time the magnetization of $\operatorname{spin} S$ is rotated by $180^{\circ}$ around the $x$-axis by the $180_{x}^{\circ S}$ pulse. It can be shown that at $t=T / 2$ the dipolar evolution contributes a phase equal to $\Phi_{d}(T / 2 ; 0)-\Phi_{d}(T ; T / 2)=\frac{2}{3} \gamma_{S} \mu_{0} M_{0}^{I} T_{1}^{I} \cos \left(\delta_{I} t_{1}-\right.$ $\left.\gamma_{I} G_{1} z_{l} \tau_{G}\right) e^{-t_{1} / T_{2}^{I}}\left(1-e^{-T / T_{1}^{I}}\right)$ (which goes to $\frac{2}{3} \gamma_{S} \mu_{0} M_{0}^{I} T \cos \left(\delta_{I} t_{1}-\gamma_{I} G_{1} z_{l} \tau_{G}\right) e^{-t_{1} / T_{2}^{I}}$ as $\left.T_{1}^{I} \rightarrow \infty\right)$; i.e., the dipolar evolution of spin $S$ continues for $t>T / 2$ without refocusing. As usual, the $180_{x}^{\circ S}$ pulse refocuses any chemical shift/resonance offset evolution of the spin $S$ transverse magnetization in a spin echo at $t=T$; the $180_{x}^{\circ I}$ pulse also creates an echo of the spin $I$ transverse magnetization, but this component does not contribute to 


\subsection{INDIRECT DETECTION WITH THE DISTANT DIPOLAR FIELD 432}

the secular dipolar field and therefore does not affect the observed signal. The fact that the simultaneous $180^{\circ}$ pulses refocus the resonance offset evolution but retain the dipolar evolution is easily appreciated from a quantum-mechanical perspective by transforming into the toggling frame $(\S 2.3 .3)$ of the $180^{\circ}$ pulses. Since a $180_{x}^{\circ}$ rotation of spin $I$ or spin $S$ takes $\hat{I}_{z} \rightarrow-\hat{I}_{z}$ or $\hat{S}_{z} \rightarrow-\hat{S}_{z}$, respectively, the resonance offset Hamiltonians $\hat{H}_{\delta}^{I} / \hbar=\delta_{I} \hat{I}_{z}$ and $\hat{H}_{\delta}^{S} / \hbar=\delta_{S} \hat{S}_{z}$ are inverted by the $180^{\circ}$ pulses, but the heteronuclear

dipolar Hamiltonian $\hat{H}_{D}^{I S} / \hbar=\sum_{j, k} 2 \omega_{D}^{j k} \hat{I}_{z j} \hat{S}_{z k}$ is unchanged. Since the resonance offset and dipolar Hamiltonians commute with each other, it is easily appreciated that the inversion of the resonance offset Hamiltonians leads to an apparent time reversal of the offset evolution, while the dipolar evolution proceeds as usual.

A detailed calculation in the absence of molecular diffusion, radiation damping, and sensor nucleus self-DDF effects shows that when all the pulse phases are equal to zero, at $t=T$ the DDF-encoding sequence stores the cosine-modulated component of sensor transverse magnetization as longitudinal magnetization according to:

$$
\begin{aligned}
& M_{z}^{S}\left(\underline{r}, t_{1}, T\right)= \\
& M_{0}^{S} \cos \left[-\gamma_{S} G_{2} z_{l} \tau_{G}+\frac{2}{3} \mu_{0} \gamma_{S} M_{0}^{I} T_{1}^{I} \cos \left(\delta_{I} t_{1}-\gamma_{I} G_{1} z_{l} \tau_{G}\right) e^{-t_{1} / T_{2}^{I}}\left(1-e^{-T / T_{1}^{I}}\right)\right] e^{-T / T_{2}^{S}} \\
& =\frac{M_{0}^{S}}{2}\left\{e^{-i \gamma_{S} G_{2} z_{l} \tau_{G}} e^{i\left[\frac{2}{3} \mu_{0} \gamma_{S} M_{0}^{I} T_{1}^{I} e^{-t_{1} / T_{2}^{I}}\left(1-e^{-T / T_{1}^{I}}\right) \cos \left(\delta_{I} t_{1}-\gamma_{I} G_{1} z_{l} \tau_{G}\right)\right]}\right. \\
& \left.\quad+e^{i \gamma_{S} G_{2} z_{l} \tau_{G}} e^{-i\left[\frac{2}{3} \mu_{0} \gamma_{S} M_{0}^{I} T_{1}^{I} e^{-t_{1} / T_{2}^{I}}\left(1-e^{-T / T_{1}^{I}}\right) \cos \left(\delta_{I} t_{1}-\gamma_{I} G_{1} z_{l} \tau_{G}\right)\right]}\right\} e^{-T / T_{2}^{S}} \\
& =\frac{M_{0}^{S}}{2} \sum_{m=-\infty}^{+\infty}\left\{i^{m} J_{m}\left[\frac{2}{3} \frac{T_{1}^{I}}{\tau_{d}^{S}}\left(1-e^{-T / T_{1}^{I}}\right) e^{-t_{1} / T_{2}^{I}}\right] e^{-T / T_{2}^{S}} e^{i m \delta_{I} t_{1}} e^{-i\left(m \gamma_{I} G_{1}+\gamma_{S} G_{2}\right) z_{l} \tau_{G}}\right. \\
& \left.\quad+i^{m} J_{m}\left[-\frac{2}{3} \frac{T_{1}^{I}}{\tau_{d}^{S}}\left(1-e^{-T / T_{1}^{I}}\right) e^{-t_{1} / T_{2}^{I}}\right] e^{-T / T_{2}^{S}} e^{i m \delta_{I} t_{1}} e^{-i\left(m \gamma_{I} G_{1}-\gamma_{S} G_{2}\right) z_{l} \tau_{G}}\right\},
\end{aligned}
$$




\subsection{INDIRECT DETECTION WITH THE DISTANT DIPOLAR FIELD 433}

where $\tau_{d}^{S}=\left(\mu_{0} \gamma_{S} M_{0}^{I}\right)^{-1}$. If the storage pulse phase is shifted to $\phi_{S}=90^{\circ}$, the sinemodulated component of transverse magnetization is stored, and a similar Bessel function expansion of the magnetization can be performed.

If the ratio of gradient pulse amplitudes is chosen to be $\kappa=G_{2} / G_{1}=n \gamma_{I} / \gamma_{S}$, only the following position-independent $m=-n$ and $m=+n$ components of Eq. 6.126 contribute to the net magnetization density:

$$
\begin{aligned}
M_{z}^{S}\left(t_{1} ; T\right)=\frac{M_{0}^{S}}{2}\left\{i^{-n} J_{-n}\left[\frac{2}{3} \frac{T_{1}^{I}}{\tau_{d}^{S}}\left(1-e^{-T / T_{1}^{I}}\right) e^{-t_{1} / T_{2}^{I}}\right] e^{-T / T_{2}^{S}} e^{-i n \delta_{I} t_{1}}\right. \\
\left.\quad+i^{n} J_{n}\left[-\frac{2}{3} \frac{T_{1}^{I}}{\tau_{d}^{S}}\left(1-e^{-T / T_{1}^{I}}\right) e^{-t_{1} / T_{2}^{I}}\right] e^{-T / T_{2}^{S}} e^{i n \delta_{I} t_{1}}\right\} \\
=M_{0}^{S} J_{n}\left[\frac{2}{3} \frac{T_{1}^{I}}{\tau_{d}^{S}}\left(1-e^{-T / T_{1}^{I}}\right) e^{-t_{1} / T_{2}^{I}}\right] e^{-T / T_{2}^{S}}\left\{\frac{(-1)^{n}}{2}\left[i^{n} e^{i n \delta_{I} t_{1}}+i^{-n} e^{-i n \delta_{I} t_{1}}\right]\right\},
\end{aligned}
$$

where the relation $J_{-n}(x)=J_{n}(-x)=(-1)^{n} J_{n}(x)$ for integer $n$ has been used. The expression in braces is equal to $(-1)^{(n-1) / 2} \sin \left(n \delta_{I} t_{1}\right)$ when $n$ is an odd integer and is equal to $(-1)^{n / 2} \cos \left(n \delta_{I} t_{1}\right)$ when $n$ is an even integer. The single-quantum spectrum of the analyte is selected by choosing $|n|=1$. When $n=1$ the stored net magnetization equals:

$$
M_{z}^{S}\left(t_{1} ; T\right)=M_{0}^{S} J_{1}\left[\frac{2}{3} \frac{T_{1}^{I}}{\tau_{d}^{S}}\left(1-e^{-T / T_{1}^{I}}\right) e^{-t_{1} / T_{2}^{I}}\right] e^{-T / T_{2}^{S}} \sin \left(\delta_{I} t_{1}\right)
$$

When the argument of the Bessel function is small either due to relaxation effects or by simply not waiting until $T \sim \tau_{d}^{S}$, Eq. 6.128 may be approximated using Eq. 6.111:

$$
M_{z}^{S}\left(t_{1} ; T\right) \approx M_{0}^{S} f(T) \sin \left(\delta_{I} t_{1}\right) e^{-t_{1} / T_{2}^{I}}
$$




\subsection{INDIRECT DETECTION WITH THE DISTANT DIPOLAR FIELD434}

where the dimensionless amplitude factor $f(T)=\frac{T_{1}^{I}}{3 \tau_{d}^{S}}\left(1-e^{-T / T_{1}^{I}}\right) e^{-T / T_{2}^{S}} \ll 1$ explicitly depends on the value of the delay $T$, which may be optimized experimentally to maximize $f(T)$. Eq. 6.129 clearly demonstrates that the DDF remote detection encoding module Eq. 6.122 encodes the amplitude-modulated free induction decay of the analyte spins $I$ in the longitudinal magnetization of the sensor spins $S$. This magnetization may then be transported for remote detection.

Some significant differences arise between the basic DDF indirect detection experiment and the remote detection version due to the fact that whereas the basic experiment employs quadrature detection in the direct dimension $t_{2}$, the remote detection experiment does not detect the sensor magnetization during the $T$ delay and does not select a magnetization component in quadrature. This latter factor is responsible for the lack of frequency discrimination in the indirect dimension even when pulsed field gradients are employed, i.e., both the $+n \delta_{I}$ and the $-n \delta_{I}$ Fourier components are selected regardless of the relative signs of the gradient pulse amplitudes. By convention, when quadrature detection is used only the evolution of the $-1 \mathrm{QC}$ is measured, and the $+1 \mathrm{QC}$ is disregarded. The application of the linear gradients $G_{1}$ (during the indirect time interval) and $G_{2}=\kappa G_{1}=n\left(\gamma_{I} / \gamma_{S}\right)$ (during detection) selects the coherence pathway $p_{1}=-n p_{2}$, where $p_{1}$ is the coherence order selected in the indirect dimension and $p_{2}$ is the coherence order selected in the direct dimension. Quadrature detection ensures $p_{2}=-1$, so the $p_{1}=+n$ coherence is selected in the indirect dimension. However, the magnetization is not detected during the remote detection DDF encoding sequence and both the $p_{2}=+1$ and $p_{2}=-1$ coherences contribute during the interval $T$. Therefore two coherence pathways are selected: $+n \rightarrow-1$ 


\subsection{INDIRECT DETECTION WITH THE DISTANT DIPOLAR FIELD 435}

and $-n \rightarrow+1$. The gradient pulses do filter out the two additional pathways $+n \rightarrow+1$

and $-n \rightarrow-1$, as well as any additional pathways that would be present in the absence of coherence pathway selection. The selection of a single coherence pathway can be enforced by a proper phase cycling scheme, with or without concurrent pulsed field gradient coherence pathway selection. Note that the analyte $+1 \mathrm{QC}$ and $-1 \mathrm{QC}$ carry the same spectral information, but the selection of one or the other in the conventional version of the DDF indirect detection experiment can result in the formation of a coherence transfer echo due to cancellation of the analyte inhomogeneous evolution during $t_{1}$ by the sensor inhomogeneous evolution during $t_{2}$. In the remote detection version of the experiment, however, the $180^{\circ}$ pulse on spin $S$ refocuses the inhomogeneous evolution of the sensor at time $T$, which therefore cannot be used to cancel the inhomogeneous evolution of the analyte during $t_{1}$.

The fact that the magnetization is not detected over the course of the DDF indirect detection encoding sequence (Eq. 6.122) is a consequence of the key feature of the NMR remote detection modality, namely that the encoding and detection steps are separated. The DDF encoding module provides an example of the potential advantage of the remote detection technique over the conventional all-in-one NMR encoding-detection scheme. If the conventional DDF indirect detection experiment (see §6.3.1) is performed using a highly magnetized sensor nucleus - as is desirable for high detection sensitivity - the action of the analyte dipolar field may create transverse magnetization of the sensor nucleus that is so large that the radiation damping interaction with the rf coil may significantly torque the magnetization out of the transverse plane. This results in a reduction of the observed signal. Radiation damping effects can be suppressed by detuning the resonant circuit of 


\subsection{INDIRECT DETECTION WITH THE DISTANT DIPOLAR FIELD 436}

the detection channel of the rf probe because the oscillating nuclear magnetization induces a smaller current in the coil, which in turn creates a smaller magnetic field acting back upon the spins. The disadvantage of detuning the detection circuit is that this decreases the detection efficiency, which again results in reduced signal. However, if the experiment is performed in a remote detection modality, the $\mathrm{rf}$ channel that serves the sensor spins can be safely detuned without a loss of detection efficiency because this channel is not used to detect the sensor magnetization. ${ }^{32}$ Rather, the DDF-encoded sensor magnetization is stored as longitudinal magnetization, which then may be transported to a detector that is not susceptible to radiation damping effects, such as an atomic magnetometer or SQUID flux magnetometer. One adverse effect of detuning the sensor spin channel during the DDF remote detection encoding sequence is a decrease of the $S$-spin rf pulse strength that necessitates the use of increased pulse lengths, but this is only a minor problem if the reduction of rf field strength is not too severe. The remote detection modality's ability to optimize the NMR encoding and detection steps separately may provide an answer to the objection [77] (see §6.3.1) that radiation damping makes the DDF indirect detection experiment infeasible, although this experiment still must contend with the deleterious effects of the sensor spin transverse self-dipolar field.

The use of stopped-flow remote detection methodology also may be able to improve the sensitivity of the DDF indirect detection experiment in the case that the analyte longitudinal relaxation is slow. If the analyte longitudinal relaxation time $T_{1}^{I}$ is long com-

\footnotetext{
${ }^{32}$ This is similar in spirit to techniques that use quality factor-switching probes, as discussed in Refs. $[388,389]$ and references therein. The $Q$-switching experiments require special hardware, as do remote detection NMR experiments. However, remote detection can be implemented using standard NMR probes for encoding and using standard detectors; the extra hardware is necessary to transport the informationbearing nucleus. $Q$-switching techniques require modification of the probe itself.
} 


\subsection{INDIRECT DETECTION WITH THE DISTANT DIPOLAR FIELD 437}

pared to the DDF encoding interval $T$, the analyte dipolar field is long-lived enough that it should be possible to transport several volumes of sensor magnetization past the analyte for encoding before the analyte dipolar field decays away. The use of several volumes of sensor magnetization to encode each indirect point of the analyte spectrum could be advantageous, i.e., the signals recorded from each volume could be coadded to enhance the sensitivity of the experiment. When all other things are equal it is better simply to encode for a longer interval $T$ since the DDF signal increases linearly with $T$ under the conditions considered in this work. However, it might be preferable to encode several volumes using a shorter value of $T$ in order to minimize the amount of time that the effects of diffusion, radiation damping, or the transverse self-dipolar field of the sensor could act. All of these factors tend to decrease the observed signal. Note that it would also be possible to perform multiple acquisitions per indirectly encoded point of the analyte spectrum in the case when the sensor magnetization was static (i.e., not refreshed by flow), but only if the sensor magnetization returned to its initial value between each acquisition. This would require $T_{2}^{S}, T_{1}^{S} \ll T_{1}^{I}$. Whether or not flow is used, it also would be beneficial to encode multiple sensor volumes per indirect point to enhance sensitivity anytime when the sensor transverse relaxation time $T_{2}^{S}$ was very short. In that case no advantage could be gained by encoding for a time $T$ that is much longer than $T_{2}^{S}$ because the sensor transverse magnetization would have decayed away. 


\subsection{HETERONUCLEAR DDF INDIRECT DETECTION EXPERIMENTS USING XE-129 AS A SENSOR

\subsection{Heteronuclear DDF indirect detection experiments using Xe-129 as a sensor}

The discussion in $\S 6.1 .1$ outlined how the properties of Xe-129 gas make it an ideal mobile carrier of NMR information in remote detection experiments. Some experimental studies were performed to examine the suitability of hyperpolarized Xe-129 gas for use as a sensor nucleus in distant dipolar field indirect detection experiments. These experiments were not implemented in a remote detection mode; rather, they were conducted in a single location with the pulse sequence in Fig. 6.3 to be used as a proof-of-principle for the DDFencoding module. Augustine et al. have already demonstrated hyperpolarized Xe-129 DDF indirect detection using the protons of methane gas as an analyte [366]. Romalis et al. have investigated dipolar field effects in hyperpolarized liquid Xe-129 [393, 348, 394, 359] and gas-phase He-3 [370] but have not indirectly encoded NMR spectra using the dipolar field. Augustine's experiments were performed on a homogeneous mixture of xenon and methane gases, but a homogeneous mixture is not ideal for remote detection experiments because the carrier cannot easily be extracted from the analyte for transport. The experiments described in this work were conducted in a coaxial tube-within-a-tube geometry, i.e., xenon gas in the inner cylindrical tube was used to detect an analyte in the outer tube. In such a geometry the analyte dipolar field at the location of the sensor spins falls off with distance from the analyte magnetization; some of the subtleties of experiments that use this sample geometry are discussed in $\S 6.4 .2$.

The characteristics other than sample geometry that distinguish DDF-encoded xenon remote detection experiments from the heteronuclear DDF experiments described 


\subsection{HETERONUCLEAR DDF INDIRECT DETECTION EXPERIMENTS USING XE-129 AS A SENSOR

above have to do with the mechanisms of producing and maintaining hyperpolarization and the fast diffusion rate of a gas-phase carrier. The production of hyperpolarized xenon nuclei by spin-exchange optical pumping is discussed in $\S 6.4 .1$. The use of hyperpolarized nuclei presents special problems if point-by-point indirect encoding is to be performed on the same sample volume because the initial polarization is depleted by multiple rf pulses as the polarization relaxes toward its comparatively small thermal equilibrium value; this problem is discussed further in $\S 6.6$. Furthermore, the high diffusion rate of xenon gas can potentially cause difficulties for DDF encoding since the remote detection sensor nucleus ideally should remain static during its evolution in the presence of the spatially inhomogeneous analyte dipolar field. This problem is discussed in $\S 6.4 .1$.

\subsubsection{Introduction to Xe-129 NMR}

The many NMR studies using xenon, primarily in the gas phase, have established the utility of this nucleus as a chemically inert probe of its local environment. Xenon spectroscopy has proved useful in the study of such diverse topics as the gas flow in and the structure of nanoporous materials and the specific or non-specific interactions of xenon with hydrophobic sites in proteins. Xenon gas has also been used successfully in void-space imaging and visualization of macroscopic gas flow. The review by Goodson [288] discusses all of these experiments as well as many more applications of xenon NMR; a comprehensive review of xenon NMR experiments will not be attempted here.

Due to its high electronic polarizability xenon has a large chemical shift range, which makes it a useful probe for spectroscopic studies. A more striking and widely utilized property of xenon is that its nuclei (as well as those of other noble gases) can be polarized 


\subsection{HETERONUCLEAR DDF INDIRECT DETECTION EXPERIMENTS}

to a substantial fraction of unity via spin-exchange interactions with the highly-polarized electrons of optically-pumped alkali atoms. The ability to create nuclear hyperpolarization establishes the feasibility of NMR detection of gases, which have inherently low thermal equilibrium nuclear magnetization due to the low number density of nuclei. Furthermore, this hyperpolarization persists for a long time due to the typically long longitudinal relaxation times of noble gas nuclei.

Xenon has two NMR-active isotopes: the spin-1/2 Xe-129 isotope at $26.4 \%$ natural abundance $\left(\gamma_{X e 129} / 2 \pi=-11.86 \mathrm{MHz} / \mathrm{T}\right)$ and the spin-3/2 Xe-131 isotope at $21.2 \%$ natural abundance $\left(\gamma_{X e 131} / 2 \pi=3.52 \mathrm{MHz} / \mathrm{T}\right)$. Both nuclei can be hyperpolarized, but in remote detection applications it is more practical to measure only the spin- $1 / 2 \mathrm{Xe}-129$ isotope because of its larger gyromagnetic ratio and because the Xe-131 hyperpolarization is quickly dissipated into its efficient electric quadrupolar relaxation channel [395]. Xe-131 also cannot be hyperpolarized as efficiently as Xe-129 due to its lower spin-exchange cross section [396]. The Xe-129 hyperpolarization can persist for a long time due to the typically long longitudinal relaxation times times of spin-1/2 noble gas nuclei; consequently, xenon polarization can be maintained during transport over relatively long distances in remote detection experiments. It is also possible to perform many NMR experiments on the same volume of xenon by using up the polarization bit-by-bit if the length of the experiments are much shorter than the longitudinal relaxation time.

\section{Diffusion of xenon gas}

Gases have much higher diffusion rates than liquids and it is necessary to consider

whether xenon gas diffusion is significant enough to destroy the sensor magnetization helix 
created in a DDF indirect detection experiment over the timescale during which the sensor evolves in the presence of the analyte dipolar field. It is also necessary to consider the effects of diffusion when multiple experiments are performed on the same batch of xenon in the case where the excitation coil is too small to excite the entire xenon volume. The characteristic diffusion coefficient of free xenon gas (90\%-enriched Xe-129) at a pressure of 1 atm has been determined to be [397]:

$$
D_{X e}=5.7 \times 10^{-2} \mathrm{~cm}^{2} \mathrm{~s}^{-1}
$$

The diffusion coefficient of Xe-129 in a xenon mixture of natural isotopic abundance will differ from the value for pure Xe-129, but this difference should be small since the masses of the naturally-occurring isotopes of xenon differ from 129 by a relatively small fraction. Ref. [398] has obtained a comparable result for the xenon self-diffusion coefficient.

The diffusion coefficient is ideally inversely proportional to the gas pressure, so at room temperature it will be assumed to be:

$$
D_{X e}=\left(5.7 \times 10^{-2} \mathrm{~cm}^{2} \mathrm{~s}^{-1}\right) / P,
$$

where $P$ is the pressure measured in atmospheres.

Spin-exchange optical pumping of xenon is typically performed in the presence of a buffer gas. As will be discussed later, the experimentalist has the option of using this xenon-buffer gas mixture directly or of freezing out pure xenon. If the mixture is used the xenon diffusion rate will be affected. The buffer gas mixture often contains a large amount 


\subsection{HETERONUCLEAR DDF INDIRECT DETECTION EXPERIMENTS USING XE-129 AS A SENSOR}

of helium, which is a light atom with a high diffusion coefficient, and its presence increases the diffusion coefficient of the mixture. The mutual diffusion coefficient of a xenon-helium binary mixture is easily calculated in terms of the diffusion coefficients of the individual components and such quantities have been measured long ago [399]. However, the quantity that is actually of interest in xenon NMR experiments is not the mutual diffusion coefficient of the mixture, but rather the self-diffusion coefficient of xenon in the presence of the buffer gas. This coefficient may be calculated according to [400]:

$$
D_{X e / B G}(x)=\left[\frac{x}{D_{X e}}+\frac{1-x}{{ }^{0} D_{X e / B G}}\right]^{-1},
$$

where $x$ is the molar fraction of Xe in the mixture and $1-x$ is the molar fraction of the buffer gas; $D_{X e}$ is the diffusion coefficient of xenon alone and ${ }^{0} D_{X e / B G}$ is the diffusion coefficient of xenon at infinite dilution in the buffer gas. Unfortunately, the diffusion coefficient of a species at infinite dilution is not as commonly measured as the diffusion coefficient of the pure species or the mutual diffusion coefficient of a binary mixture.

The effects of diffusion on dipolar field experiments can be considerable, particularly if gradient pulses are employed [296, 401, 384, 402, 403, 404, 405]. Assuming that the diffusion of the analyte can be neglected over the course of an encoding experiment, the diffusion of the sensor nucleus (i.e., xenon) can be roughly estimated to be non-negligible when the encoding interval $T$ is long enough that $\sqrt{D T} \sim d$, where $D$ is the diffusion coefficient and $d$ is a characteristic distance over which the analyte dipolar field varies. In an experiment without gradient pulses $d$ might be the distance over which the rf coil excites the sample. In an indirect detection experiment with gradient pulses the characteristic distance 


\subsection{HETERONUCLEAR DDF INDIRECT DETECTION EXPERIMENTS USING XE-129 AS A SENSOR

can be taken to equal the pitch of modulation of the analyte and sensor magnetization, $d=2 \pi /\left|\gamma_{I} G_{1} \tau_{G}\right|=2 \pi /\left|\gamma_{S} G_{2} \tau_{G}\right|$. The diffusion over $T=1 \mathrm{~s}$ is on the order of $\sqrt{D T}=0.24$ cm for pure Xe gas under standard conditions (Eq. 6.130), which is not negligible compared to an $\mathrm{rf}$ excitation region of $\sim 1 \mathrm{~cm}$. The diffusion can be made slower by using higher gas pressures.

\section{Spin-exchange optical pumping of Xe-129}

The electronic polarization of alkali metals in atomic vapors can be enhanced almost to $100 \%$ via optical pumping with circularly polarized laser light [406, 407, 408]. The enhanced electronic polarization can be transferred to the nuclei of noble gas atoms via dipole coupling-mediated spin exchange collisions. This means of producing nuclear hyperpolarization is called spin-exchange optical pumping [409, 407, 410, 411, 251, 412, $413,414]$, which is quite effective when applied to xenon [410] and has been used in recent years $[415,416,417,418]$ to produce Xe-129 polarizations approaching $70 \%[416,417,418]$. The hyperpolarized xenon subsequently can be removed from the optical pumping mixture if desired and used on its own (see e.g., Ref. [419] and many later studies by various workers). Some good introductory references on spin-exchange optical pumping include the review by Walker and Happer [412], the monograph by Knize et al. [251], the monograph by Appelt [414], the brief review in Ref. [420] by Brunner, and the dissertation by Long [421]. The review by Happer [407] is authoritative but advanced, as is the review by Appelt et al. [413]. This section will describe spin-exchange optical pumping technique as well as the experimental conditions that were used in this work. The experimental apparatus will be described first in order to be used as an illustrative example in the subsequent discussion 


\subsection{HETERONUCLEAR DDF INDIRECT DETECTION EXPERIMENTS USING XE-129 AS A SENSOR

of the general technique. No effort will be made to discuss the variety of optical pumping techniques; rather, the specific technique used here will be described.

Xenon hyperpolarization was achieved using a commercial continuous-flow spinexchange polarizer (MITI IGI 9800 Xe, Magnetic Imaging Technologies, Inc. (Polarean), Durham, NC). Optical pumping of the $\mathrm{D}_{1}$ transition of natural isotopic abundance rubidium vapor was performed in a $\sim 70 \mathrm{~cm}^{3}$ cylindrical pyrex optical cell heated to $155-185{ }^{\circ} \mathrm{C}$ (corresponding to an estimated number density of $\sim 1-5 \times 10^{14}$ atoms $/ \mathrm{cm}^{3}[414,420]$ ) using circularly polarized light from a $60 \mathrm{~W}$ c.w. diode laser array operating at $794.8 \mathrm{~nm}$ (FWHM $1.35 \mathrm{~nm}$ ). A magnetic field of $\sim 20 \mathrm{G}$ was applied parallel to the direction of laser propagation using a Helmholtz coil pair. The cell was pressurized to $\sim 70$ psig $(5.8 \mathrm{~atm})$ with a natural isotopic abundance mixture of xenon with helium/nitrogen buffer gas in a 1:10:89 $\mathrm{Xe}: \mathrm{N}_{2}:$ He ratio, which corresponds to a total Xe partial pressure of $\sim 44$ Torr $(11$ Torr Xe129). Pure hyperpolarized xenon was isolated from the optical pumping mixture by freezing in a liquid nitrogen-cooled cold finger in a $\sim 500 \mathrm{G}$ magnetic field. The remaining buffer gas was evacuated from the polarizer manifold and pure xenon gas was recovered by thawing the xenon ice. The polarizer was operated under conditions such that approximately $1 \mathrm{~atm}$ of pure xenon could be produced in a $5 \mathrm{~mm}$ o.d. J-Young NMR tube per 15 minutes of optical pumping and xenon collection. Final xenon polarizations were in the range of $1-10 \%$ with $4-8 \%$ being typical.

The rubidium $\mathrm{D}_{1}$ transition is between the $\left|g=5{ }^{2} \mathrm{~S}_{1 / 2}\right\rangle$ ground state and an excited state $\left|e=5{ }^{2} \mathrm{P}_{1 / 2}\right\rangle$. The ground state is characterized by the electronic angular momentum quantum numbers $S_{g}=1 / 2, L_{g}=0$, and $J_{g}=1 / 2$, where $\underline{J}_{g}=\underline{L}_{g}+\underline{S}_{g}$ is the 


\subsection{HETERONUCLEAR DDF INDIRECT DETECTION EXPERIMENTS USING XE-129 AS A SENSOR

total ground state electronic angular momentum vector and $\underline{L}_{g}$ and $\underline{S}_{g}$ are the ground state orbital and intrinsic spin angular momenta, respectively. The excited state is characterized by $S_{e}=1 / 2, L_{e}=1$, and $J_{e}=1 / 2$. Rubidium has two stable isotopes: $\operatorname{Rb}-85(I=5 / 2)$ and $\operatorname{Rb}-87(I=3 / 2)$, and the total atomic angular momenta in the ground and excited states are $\underline{F}_{g}=\underline{J}_{g}+\underline{I}$ and $\underline{F}_{e}=\underline{J}_{e}+\underline{I}$, respectively. The values of $F$ can range from $|J+I|$ to $|J-I|$ in integer steps. The electronic angular momentum couples to the nuclear angular momentum through the hyperfine interaction, which is dominated by a magnetic dipole-dipole contact coupling Hamiltonian of the form $\hat{H}_{h f} / \hbar=A(\underline{\hat{J}} \cdot \underline{\hat{I}})$. The typical magnetic field applied to the $\mathrm{Rb}$ cell in a spin-exchange optical pumping experiment is not strong enough to decouple the electronic and nuclear angular momenta. ${ }^{33}$ In this case the good eigenstates of the atomic system are the eigenstates $\left\{\left|F, m_{F}\right\rangle\right\}$ of $\hat{F}^{2}$ and the ground and excited states have $\left(2 F_{g}+1\right)$ and $\left(2 F_{e}+1\right)$ magnetic sublevels, respectively. Ordinarily this magnetic hyperfine structure would have to be included in any treatment of optical pumping, but the presence of He buffer gas at a pressure of several atmospheres causes enough pressure broadening of the $\mathrm{Rb}$ lines that the hyperfine structure is unresolved and the optical pumping can be considered to occur between states of well-defined $J_{g}$ and $J_{e}$.

This neglect of the hyperfine structure can be justified more physically. Collisions between the polarized rubidium atoms and spinless buffer gas atoms (in this case the nearly $100 \%$ naturally-abundant He-4 isotope) perturb the Rb electron cloud and cause a ran-

\footnotetext{
${ }^{33}$ The Zeeman plus hyperfine coupling Hamiltonian of the atomic system is $\hat{H}_{Z, h f} / \hbar=-\left[\omega_{J} \hat{J}_{z}+\omega_{I} \hat{I}_{z}\right]+$ $A(\underline{\hat{J}} \cdot \underline{\hat{I}})$, where $\omega_{J}$ and $\omega_{I}$ are the respective electronic and nuclear Larmor frequencies. This Hamiltonian has the same form as the NMR scalar coupling Hamiltonian (see Appendix D). The strong coupling limit $|A| \gg\left|\omega_{J}-\omega_{I}\right|$ has approximate eigenstates $\left|F, m_{F} ; J, I\right\rangle$ and the weak coupling limit $|A| \ll\left|\omega_{J}-\omega_{I}\right|$ has approximate eigenstates $\left|J, m_{J} ; I, m_{I}\right\rangle$. Since $\left|\omega_{J}-\omega_{I}\right| / 2 \pi \approx\left|\omega_{J}\right| / 2 \pi$ is $\sim 56 \mathrm{MHz}$ in a magnetic field of 20 $\mathrm{G}\left(\gamma_{e^{-}} / 2 \pi=-28 \mathrm{GHz} / \mathrm{T}\right)$ and $|A| / 2 \pi$ for the $\mathrm{Rb}$ isotopes is on the order of $\mathrm{GHz}$ in the $5{ }^{2} \mathrm{~S}_{1 / 2}$ ground state and hundreds of $\mathrm{MHz}$ in the $5^{2} \mathrm{P}_{1 / 2}$ excited state [414], this system is located well inside the strong hyperfine coupling regime.
} 


\subsection{HETERONUCLEAR DDF INDIRECT DETECTION EXPERIMENTS USING XE-129 AS A SENSOR

domization of the total angular momentum vector $\underline{J}_{e}$ of the excited state $[422,423]$. The perturbations due to these collisions are sudden with respect to the reorientation of the alkali nuclear angular momentum vector $\underline{I}$, which therefore remains unchanged by the collisions. Consequently the excited-state electronic angular momentum vector changes rapidly with respect to the nuclear angular momentum vector. If the correlation time for electron randomization is much shorter than the reciprocal excited-state hyperfine coupling constant, $\tau_{e} \ll\left|A_{e}\right|^{-1}$, then the excited-state hyperfine coupling Hamiltonian $\hat{H}_{h f}^{e}(t) / \hbar=A_{e}\left[\underline{J}_{e}(t) \cdot \underline{\hat{I}}\right]$ effectively averages to zero over the characteristic timescale of the electron-nucleus coupling dynamics. ${ }^{34}$ Collisional de-excitation of the alkali atoms (which will be discussed later) is also sudden with respect to the nuclear angular momentum dynamics, and under these conditions the alkali nuclear polarization is conserved in the interval between electronic excitation by a pumping photon and collisional de-excitation. Since the optical pumping only directly affects the electrons and not the nucleus, the collision-induced decoupling of the hyperfine interaction largely removes the nuclear spin as an actor in the optical pumping cycle. The hyperfine interaction that is averaged away by fast electron randomization does re-enter when considering spin relaxation; he alkali nucleus serves as a reservoir of angular momentum and the recoupling of the hyperfine interaction in between alkali-alkali spinexchange collisions (which redistribute angular momentum among the hyperfine levels) is known to help establish an internal spin temperature quasi-equilibrium among the hyperfine states [424]. The establishment of a spin-temperature distribution has been observed to occur even in high-pressure spin-exchange optical pumping cells in which alkali-alkali

\footnotetext{
${ }^{34}$ This concept is similar to NMR heteronuclear decoupling of dipolar or indirect spin-spin couplings by continuous-wave irradiation of one partner of the spin pair, although c.w. decoupling does not completely randomize the nuclear angular momentum.
} 


\subsection{HETERONUCLEAR DDF INDIRECT DETECTION EXPERIMENTS USING XE-129 AS A SENSOR

spin exchange rates are smaller than the optical pumping or electron randomization rates $[425,426,414]$. For further discussions of electron randomization, spin temperature distribution, and the neglect of hyperfine structure in certain optical pumping experiments, the reader is referred to Refs. [407, 427, 251, 412, 413, 414] (general), [428, 429, 422, 423],[32, $\S 5.6]$ (electron randomization), [424],[32, §5.5] (spin temperature), and [430],[215, §5.4] (spin temperature and neglect of hyperfine structure).

The neglect of hyperfine structure results in a much-simplified picture of optical pumping, and pumping of the alkali $\mathrm{D}_{1}$ transition is particularly simple. In the case of rubidium the process may be viewed as a selective excitation followed by de-excitation of the valence electron in the system of four magnetic sublevels of the $5^{2} \mathrm{~S}_{1 / 2}=[\mathrm{Kr}] 5 s^{1}$ and $5{ }^{2} \mathrm{P}_{1 / 2}=[\mathrm{Kr}] 5 p^{1}$ electronic states (Fig. 6.5). Both rubidium isotopes can be considered to be equivalent in this picture that neglects hyperfine structure. Following a relatively standard notation, $\underline{S} \equiv \underline{S}_{g}=\underline{J}_{g}$ denotes the ground state electronic angular momentum vector and $\underline{J} \equiv \underline{J}_{e}$ denotes the excited state electronic angular momentum vector. The alkali $\mathrm{D}_{1}$ optical pumping cycle can be reduced to three steps [412, 414]: (1) selective depopulation of one of the two ground state magnetic sublevels by absorption of circularly polarized light (longitudinal depopulation pumping); (2) equalization of the excited-state sublevel populations by collisional mixing ( $\underline{J}$-randomization) mainly due to the buffer gas (primarily helium); and (3) non-radiative quenching from the excited state by collisions with nitrogen molecules, after which the ground state magnetic sublevels are repopulated equally. Each of these three processes will be described in turn; the complete process is diagrammed schematically in Fig. $6.5 .^{35}$ The net effect of the optical pumping cycle is to

\footnotetext{
${ }^{35}$ It has been assumed that the nitrogen quenching distributes population to the ground state magnetic
} 


\subsection{HETERONUCLEAR DDF INDIRECT DETECTION EXPERIMENTS USING XE-129 AS A SENSOR

transfer population from one ground state sublevel to another, which results in a polarization of the electronic angular momentum. This cycle is continually repeated during a continuous pumping experiment, resulting in an accumulation of nearly all of the population in one ground state magnetic sublevel and a ground state electronic polarization near $100 \%$. This electronic polarization then can be partially transferred to a noble gas nucleus through spin-exchange collisions, which will be discussed later.

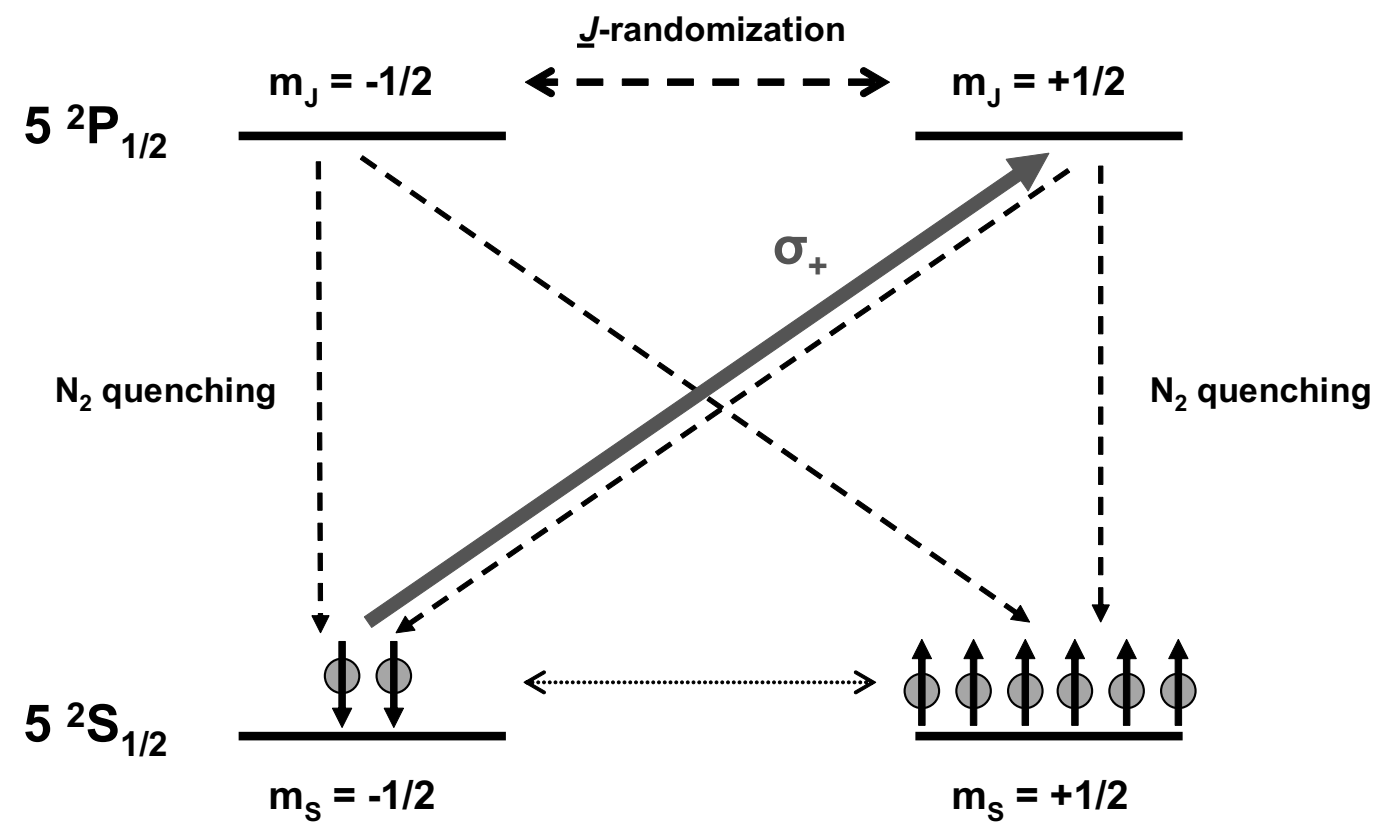

Figure 6.5: Schematic of optical pumping of the $\mathrm{Rb} \mathrm{D}_{1}$ transition using circularly-polarized light. The $\sigma_{+}$pumping light causes a selective $m_{S}=-1 / 2 \rightarrow m_{J}=+1 / 2$ transition. The excited-state sublevel populations are equalized by electron-randomization collisions with the buffer gas. Collisions with nitrogen non-radiatively quench the atoms from the excited state; de-excitation populates the ground state sublevels equally. The net effect is an accumulation of population in the $m_{S}=+1 / 2$ ground state sublevel. If the relaxation of the ground state polarization is slow, significant spin polarization can accumulate.

Circularly-polarized light has a well-defined component of intrinsic spin angular

sublevels equally rather than conserving the magnetic quantum number. It does not in actuality matter which process occurs as far as the optical pumping is concerned if the excited-state sublevel populations are equalized before the quenching collision occurs. 


\subsection{HETERONUCLEAR DDF INDIRECT DETECTION EXPERIMENTS USING XE-129 AS A SENSOR

momentum along the direction of light propagation: $+\hbar\left(m_{s}=+1\right)$ for left-circularly polarized $\left(\sigma_{+}\right)$light and $-\hbar\left(m_{s}=-1\right)$ for right-circularly polarized $\left(\sigma_{-}\right)$light [431],[432, Appendix 1]. Absorption of $\sigma_{+}$light resonant with the $\mathrm{Rb} \mathrm{D}_{1}$ transition selectively transfers population from the $\left|5{ }^{2} \mathrm{~S}_{1 / 2}, m_{S}=-\frac{1}{2}\right\rangle$ magnetic sublevel of the electronic ground state to the $\left|5^{2} \mathrm{P}_{1 / 2}, m_{J}=+\frac{1}{2}\right\rangle$ excited state sublevel in accordance with one of the wellknown atomic selection rules [414],[433, §15.2]: $\Delta L=+1, \Delta S=0$ with $\Delta J=0$ (not $0 \rightarrow 0), \Delta m_{J}=+1 .{ }^{36}$ This process is called longitudinal depopulation pumping because it is performed with the direction of light propagation parallel to an applied magnetic field. The application of a magnetic field that is larger than stray inhomogeneous fields across the pumping cell ensures that all atoms are polarized along the axis of circular light polarization. The absorption of a pumping photon by the atom thus can be seen to conserve the longitudinal component of angular momentum $\left(\Delta m_{s}=-1\right.$ for photon destruction combined with $\Delta m_{J}=+1$ for the electron spin-flip). This transfer of photon spin to the atom is the basic mechanism by which the alkali electronic polarization is increased. If a broadband laser source such as a diode array is used, buffer gas collisions can also play an important role in the pumping process by broadening the $\mathrm{D}_{1}$ absorption line to a width that is comparable to that of the laser source, which allows the efficient absorption of laser power [434, 422, 435].

The $\mathrm{Rb} 5^{2} \mathrm{P}_{1 / 2}$ excited state is initially nearly unpopulated at thermal equilibrium, but optical pumping with $\sigma_{+}$light selectively creates population in the $m_{J}=+1 / 2$ magnetic sublevel. This population, however, is rapidly distributed nearly evenly between the two excited state sublevels due to destruction of the ensemble spin polarization by electron

\footnotetext{
${ }^{36}$ Optical pumping with $\sigma_{-}$light works equally well and selects the transition $\left|5{ }^{2} \mathrm{~S}_{1 / 2} ; S, m_{S}=+\frac{1}{2}\right\rangle \rightarrow$ $\left|5^{2} \mathrm{P}_{1 / 2}, m_{J}=-\frac{1}{2}\right\rangle$.
} 


\subsection{HETERONUCLEAR DDF INDIRECT DETECTION EXPERIMENTS USING XE-129 AS A SENSOR

randomization collisions with He- $4[428,429,422,423]$. This process is also called collisional mixing. The collisional depolarization of the excited state ensures that the ground state sublevels are equally repopulated by de-excitation. This de-excitation may include spontaneous radiative emission (fluorescence): the $\left|e, m_{J}=+\frac{1}{2}\right\rangle \rightarrow\left|g, m_{S}=-\frac{1}{2}\right\rangle$ emission in a $S=1 / 2, J=1 / 2$ system occurs at twice the rate as the $\left|e, m_{J}=+\frac{1}{2}\right\rangle \rightarrow\left|g, m_{S}=+\frac{1}{2}\right\rangle$ emission [407],[32, §3.8]. This repopulation pumping would ordinarily reduce the optical pumping efficiency by preferentially returning excited-state population to the ground-state sublevel from which it originated. If, however, the excited-state collisional mixing occurs on a faster timescale than the fluorescence lifetime, the photon emissions from the two equallypopulated excited-state sublevels balance each other and the two ground-state sublevels are repopulated equally. If the electron randomization is slow, a quenching buffer gas can be added in order to ensure that the ground-state sublevels are populated equally by nonradiative (collisional) de-excitation, as is discussed below. When the system is de-excited without a preferential repopulation of the ground state magnetic sublevels, the ground state polarization is created solely by the population imbalance produced by depopulation pumping. ${ }^{37}$

The role of nitrogen molecules in the buffer gas mixture is to non-radiatively de-

\footnotetext{
${ }^{37}$ Several sources provide various descriptions of the "average efficiency" of alkali- $\mathrm{D}_{1}$ optical pumping over one cycle of excitation and de-excitation (neglecting hyperfine structure) [408, 436, 412]. Imagine an initially unpolarized atomic ensemble represented by four atoms, with two atoms populating each magnetic sublevel of the $5^{2} \mathrm{~S}_{1 / 2}$ ground state. Two $\sigma_{+}$photons will pump the two $m_{S}=-\frac{1}{2}$ atoms into the $m_{S}=+\frac{1}{2}$ sublevel of the $5^{2} \mathrm{P}_{1 / 2}$ excited state, and electron randomization will distribute (in an average sense) one atom into each excited-state magnetic sublevel. The ground state sublevels are repopulated with one atom each upon de-excitation, so the average effect of the two pumping photons is to leave one atom in the $m_{S}=-\frac{1}{2}$ ground state sublevel and three in the $m_{S}=+\frac{1}{2}$ sublevel, which is a net addition of one unit of angular momentum (measured in $\hbar$ ) and a population imbalance of two atoms. Therefore it can be said that on average it takes two circularly-polarized photons to deposit one unit of angular momentum in the alkali vapor, or that each photon deposits an average of a half unit of angular momentum with the other half of the angular momentum being lost to translational motion after electron randomization. Alternatively, it takes an average of one photon to polarize one atom fully (i.e., it takes two photons to create a population excess of two atoms).
} 


\subsection{HETERONUCLEAR DDF INDIRECT DETECTION EXPERIMENTS USING XE-129 AS A SENSOR

excite (quench) Rb atoms from the excited state via collisions [437, 438, 407, 439, 435]. Molecular nitrogen makes an ideal inelastic collision partner (as opposed to helium atoms) because it can carry away a variable amount of energy in its vibrational and rotational degrees of freedom. This energy is eventually thermalized by equilibration with the nitrogen translational degrees of freedom. Nitrogen quenching is used to reduce the effects of radiation trapping. In the absence of rapid quenching the $\mathrm{Rb}$ atoms can return to the ground state by fluorescence emission, which is not completely polarized and is resonant with the $\mathrm{D}_{1}$ transition. The multiple scattering and reabsorption of these photons in an atomic vapor that is sufficiently optically thick is called radiation trapping [407, 440]. Since these photons are not fully polarized and they scatter in various directions, their reabsorption reduces the efficiency of the optical pumping, which relies on the atoms absorbing light of a well-defined circular polarization and propagation direction. Radiation trapping can be suppressed by adjusting the concentration of nitrogen in the pumping cell so that the $\mathrm{Rb}$ excited state lifetime is reduced to a value that is less than the fluorescence lifetime, i.e., the atoms are non-radiatively quenched before they can fluoresce. When spin-exchange optical pumping is performed in cells that have a high noble gas density, the effect of quenching gas on the final noble gas nuclear polarization has been found to be diminished because the destruction of electronic polarization via alkali-noble gas collisions is fast enough to compete with the depolarization due to radiation trapping [441].

The ground state electronic spin polarization can relax by several mechanisms, the most notable of which are spin-exchange collisions with species that bear a nuclear spin $[407,408,251,413],[32, \S 5.4],[442,443,444] .{ }^{38}$ A spin-exchange collision conserves the total

\footnotetext{
${ }^{38}$ There also exist so-called "spin destruction" interactions, e.g., anisotropic dipolar interactions during
} 


\subsection{HETERONUCLEAR DDF INDIRECT DETECTION EXPERIMENTS USING XE-129 AS A SENSOR

angular momentum of the system but not necessarily the individual angular momentum components; the transfer of electronic polarization to another angular momentum reservoir serves as a relaxation mechanism for the electron spin. Non-negligible cross sections exist in typical spin-exchange optical pumping cells for both two- and three-body spin exchange collisions.

The mechanism of three-body spin exchange is well understood [448, 449, 411, 450] and involves the formation of an alkali-atom-noble gas van der Waals complex in the presence of a third body (typically a buffer gas atom or molecule) that carries away the energy that is liberated upon complex formation. Collisions with molecular nitrogen have been found to be efficient at initiating and terminating the formation of alkali-noble gas van der Waals complexes, but other species in the gas mixture also can serve this role. Spin exchange in long-lived alkali atom-heavy noble gas van der Waals complexes dominates the contribution of binary collisions if the gas pressure is not too high $[451,452,453] .{ }^{39}$ However, in the work described here the total gas pressure is on the order of several atmospheres and binary collisions dominate the spin exchange because frequent collisions with the buffer gas severely limit the lifetime (and hence the $\mathrm{Rb}-\mathrm{Xe}$ spin-exchange contact time) of the van der Waals complexes $[452,454,455]{ }^{40}$ The duration of a binary collision is very short (on

alkali-alkali atom collisions that do not conserve total spin angular momentum [445, 446, 447],[32, §5.8].

${ }^{39}$ Heavy noble gases (such as Xe) form these complexes more easily than light ones because they experience longer-range van der Waals forces due to their larger electronic polarizabilities.

${ }^{40}$ The determination of spin exchange rates - particularly the relative contributions of binary and threebody collisions - is not simple (see e.g., [450, 442, 454, 456, 455, 457, 458]) and it is difficult to find experiments that were conducted both at high buffer gas pressure and low magnetic field. Cates et al. [454] have estimated that spin exchange due to binary collisions dominates three-body spin exchange at greater than $\sim 350$ Torr total Xe pressure (compared to $\sim 44$ Torr in this work), but these experiments were performed in the absence of buffer gas where the breakup of $\mathrm{Rb}-\mathrm{Xe}$ van der Waals complexes is caused by collisions with Xe itself. Rice and Raftery's [455] high field experiments clearly demonstrate the dominance of binary spin exchange collisions at buffer gas pressures of an atmosphere or more, and their results indicate that their high field binary spin exchange rates are actually smaller than those at low field, which is consistent with Ref. [457]. 


\subsection{HETERONUCLEAR DDF INDIRECT DETECTION EXPERIMENTS USING XE-129 AS A SENSOR

the order of ps) but the collisions are frequent and the net spin transfer can be significant. Most of the Rb electronic polarization lost in spin-exchange collisions with Xe is transferred to rotational angular momentum of the relative motion of the two atoms [459, 452, 460], largely due to a spin-orbit interaction of the alkali valence electron within the electron core of the heavy noble gas atom. ${ }^{41}$ This spin-rotation exchange manifests itself as spin relaxation because the rotational angular momentum vectors of the $\mathrm{Rb}-\mathrm{Xe}$ pairs are randomly oriented within gas, which causes an ensemble-averaged depolarization of the Rb electrons.

The electronic polarization of the rubidium ground state can also be transferred to polarization of the nucleus of its collision partner, in this case xenon. From the perspective of the rubidium electron this is a mechanism of spin relaxation, but from the perspective of the xenon nucleus it is the mechanism by which nuclear hyperpolarization can be produced. The mechanism of spin exchange is a hyperfine interaction between the rubidium electron and the xenon nucleus during the collision. The hyperfine interaction is due to the direct dipoledipole coupling of the electron spin with the nuclear spin. Unlike the dipolar field effects discussed elsewhere in this chapter which arise from the anisotropic long-range contribution of the dipole field, the electron-nucleus hyperfine coupling is dominated $[462,412,411]$ by the isotropic contact contribution to the dipole field (see Eq. 1.37) since the probability of finding the rubidium valence electron at the location of the xenon nucleus is quite high (particularly due to exchange correlation effects) [463, 464, 465, 251, 412]. The theory of spin exchange between polarized alkali atom electrons and noble gas nuclei is well understood [463, 464, 465, 412], and the Hamiltonian for this type of spin exchange can be written as

\footnotetext{
${ }^{41}$ The mechanism of alkali-noble gas spin-rotation coupling is somewhat different when light noble gas atoms such as He are involved [461].
} 
[412]:

$$
\begin{aligned}
\hat{H}_{\text {s.e. }} / \hbar & =\alpha(R)[\underline{\hat{S}} \cdot \underline{\hat{K}}] \\
& =\alpha(R)\left[\hat{S}_{z} \hat{K}_{z}+\frac{1}{2}\left(\hat{S}_{+} \hat{K}_{-}+\hat{S}_{-} \hat{K}_{+}\right)\right]
\end{aligned}
$$

where $\underline{\hat{S}}$ is the electron spin angular momentum operator for the Rb ground state, $\underline{\hat{K}}$ is the Xe nuclear spin angular momentum operator, and $\alpha(R)$ is the $\mathrm{Rb}-\mathrm{Xe}$ hyperfine coupling constant parameterized by the Rb-Xe interatomic distance $R$. The "flip-flop" terms $\frac{1}{2}\left(\hat{S}_{+} \hat{K}_{-}+\hat{S}_{-} \hat{K}_{+}\right)$in Eq. 6.133 induce transfers of angular momentum between the Rb electron and Xe nucleus $[32, \S 5.4]$; e.g., the $\hat{S}_{-} \hat{K}_{+}$term can induce the spin-exchange transfer $\left|m_{S}=+\frac{1}{2} ; m_{K}=-\frac{1}{2}\right\rangle \rightarrow\left|m_{S}=-\frac{1}{2} ; m_{K}=+\frac{1}{2}\right\rangle \cdot{ }^{42}$

Not only is the Xe nuclear relaxation slow enough to allow substantial polarization to build after numerous spin exchange collisions, but once the xenon is separated from the rubidium its longitudinal relaxation can be on the order of minutes to hours (depending on the external magnetic field strength and whether the cell is coated or otherwise designed to protect against depolarizing wall collisions). This is enough time to allow the xenon to be transported or for its hyperpolarization to be used up bit-by-bit in consecutive NMR experiments. Xenon gas relaxation has been studied at high and low magnetic fields [466, $456,467,468,455]$; the dominant sources of relaxation in the absence of $\mathrm{Rb}$ tend to be $\mathrm{Xe}-$ wall collisions as well as binary $\mathrm{Xe}-\mathrm{Xe}$ collisions at high gas pressures and the formation of Xe-Xe van der Waals complexes at low pressures. Xenon relaxation by diffusion through magnetic field inhomogeneities also can be significant [464, 469, 470, 471],[32, §2.8]; this

\footnotetext{
${ }^{42}$ Spin-independent quantum numbers have been neglected.
} 


\subsection{HETERONUCLEAR DDF INDIRECT DETECTION EXPERIMENTS USING XE-129 AS A SENSOR

mechanism may be important in low-field NMR applications but is negligible in the highly homogeneous fields of modern high-field superconducting magnets.

\section{Batch mode xenon NMR experiments}

Cates et al. [472] first demonstrated that xenon could be frozen out of the optical pumping gas mixture while still retaining its hyperpolarization. If a continuous-flow spinexchange hyperpolarization apparatus is used the freezing can be accomplished by flowing the xenon/buffer gas mixture through a liquid nitrogen-cooled $(T=77 \mathrm{~K})$ cold finger until sufficient xenon ice has accumulated. The remaining buffer gas is then evacuated from the cold finger and a "batch" of pure hyperpolarized xenon gas can be recovered by thawing the ice $[473,416]$. The 1:10:89 Xe: $\mathrm{N}_{2}:$ He gas ratio of the optical pumping mixture has been chosen to optimize xenon polarization so that the magnetization of pure xenon in batch mode experiments is maximized.

The freeze-thaw batch method should be implemented with an awareness of the relaxation properties of xenon polarization in the solid state $[472,473,474,475]$. A natural isotopic abundance mixture of xenon contains both the Xe-129 and Xe-131 isotopes; the latter nucleus possess an electric quadrupole moment which couple to local electric field gradients caused by lattice defects [476]. These quadrupolar couplings can be quite large and cause rapid relaxation of the Xe-131 nucleus. If there exists a degeneracy between the Xe-129 energy levels and the levels of an appropriate Xe-131 transition, cross relaxation [477, 478] of Xe-129 with fast-relaxing Xe-131 can significantly increase the relaxation rate of the Xe129. In fact, this mechanism may dominate the Xe-129 relaxation at temperatures below $20 \mathrm{~K}[473,474,479]$. The experiments that are described in this chapter were performed 


\subsection{HETERONUCLEAR DDF INDIRECT DETECTION EXPERIMENTS USING XE-129 AS A SENSOR

with a $\sim 500 \mathrm{G}$ magnetic field applied to the xenon cold finger. This field corresponds to a Xe-129 Larmor frequency of $\sim 0.6 \mathrm{MHz}$, which should be large enough to render the cross relaxation inefficient by breaking the degeneracy of the Xe-129 levels with the continuum ${ }^{43}$ of Xe-131 levels, assuming that the the Xe-129 Larmor splitting is greater than the Larmor splitting plus quadrupolar splitting of Xe-131. Between temperatures of $20 \mathrm{~K}$ and $120 \mathrm{~K}$ and at magnetic fields greater than $500 \mathrm{G}$ the Xe-129 relaxation is dominated by nuclear spinflip scattering of lattice phonons mediated by the spin-rotation interaction [473, 474, 475]. However, at temperatures above $120 \mathrm{~K}$ as the xenon nears its melting point at $161.4 \mathrm{~K}$ the Xe-129 relaxation appears to be dominated by fluctuations of the Xe-Xe dipolar couplings due to vacancy diffusion, which can result in longitudinal relaxation times on the order of seconds [475]. For this reason it is advantageous to thaw the xenon ice as rapidly as possible to minimize the time it spends at temperatures near the phase transition. ${ }^{44}$

Nuclear relaxation times can be drastically reduced if the xenon comes into contact with paramagnetic or ferromagnetic species, due to the large dipole couplings to unpaired electrons. For this reason it is important to ensure that the hyperpolarization system and sample cell are free of oxygen and that the joints in the vacuum lines are not made out of steel. Furthermore, wall collisions can also serve as a major relaxation mechanism depending on the composition of the sample cell. Most of the experiments described here used glass sample tubes whose inner surfaces were coated with $\operatorname{SurfaSil}{ }^{\circledR}$, which is a siliconizing fluid that has been found to reduce nuclear relaxation due to wall collisions [450, 480], probably

\footnotetext{
${ }^{43}$ There exists a continuum of levels over a finite range of quadrupolar couplings due to the random distribution of the orientations of local electric field gradients in the lattice.

${ }^{44}$ The triple point of xenon is at $T=161.4 \mathrm{~K}$ and $P=0.8 \mathrm{~atm}$, which indicates that the thawing of xenon ice under vacuum at liquid nitrogen temperature is actually a sublimation process. However, if enough xenon ice is collected to produce gas at a pressure of multiple atmospheres, the pressure during the thaw can rapidly exceed $0.8 \mathrm{~atm}$, causing the xenon ice to melt rather than sublime.
} 


\subsection{HETERONUCLEAR DDF INDIRECT DETECTION EXPERIMENTS USING XE-129 AS A SENSOR

by covering open oxygen sites on the glass surface and by reducing the duration of xenon adsorption.

The batch method allows the creation of a finite volume of hyperpolarized xenon gas which can then be used for a variety of purposes, including conducting DDF indirect detection experiments. The indirect detection experiments are multidimensional experiments that require an rf pulse on the xenon sensor nucleus for every indirectly detected point. The hyperpolarization is continuously depleted over the course of the experiment because not only is longitudinal magnetization lost after every pulse due to conversion to transverse magnetization, but the non-equilibrium xenon hyperpolarization also relaxes toward its thermal equilibrium value in the interval between pulses rather than return to its initial value. A $90^{\circ} \mathrm{rf}$ pulse applied to a static volume of xenon would use up all of the hyperpolarization, leaving only the relatively tiny thermal equilibrium polarization (which grows in slowly with the time constant $T_{1}$ ) for the acquisition of the indirect point; this necessitates the use of small tip-angle pulse arrays which result in reduced signals. The consequences of small tip-angle experiments are considered in detail in $\S 6.6$.

\section{Flow mode xenon NMR experiments}

Instead of using the freeze-thaw batch mode method, it is possible to use the output of a continuous flow xenon polarizer directly. Such experiments in continuous- and stoppedflow mode have been conducted in the Pines lab, but they were not used in this work. Only dilute xenon gas is available in the current Pines lab flow mode experiments due to the presence of buffer gases in the optical pumping mixture, as opposed to the batch method which produces pure xenon gas. If flow mode experiments are to be performed it may be 


\subsection{HETERONUCLEAR DDF INDIRECT DETECTION EXPERIMENTS USING XE-129 AS A SENSOR

possible to maximize the xenon magnetization by increasing the fraction of xenon in the mixture somewhat beyond the $1 \%$ level used in batch mode experiments; this may sacrifice some polarization, but the magnetization is proportional not only to xenon polarization but also to xenon concentration.

Since a fresh volume of xenon can be used for each indirectly encoded point, flow mode experiments have the advantage that long, multiple-pulse experiments with large pulse tip angles can be implemented without worrying about the hyperpolarization being depleted by pulses or relaxation. The use of large tip angle (i.e., $90^{\circ}$ ) pulses allows an increase in signal over the small tip angle schemes that must be employed in multi-pulse batch mode experiments; however, this gain can be more than offset by the reduction in magnetization caused by using a dilute xenon mixture. In practice, batch mode experiment of reasonable length should yield a somewhat larger signal than a flow mode experiment using a $1 \%$ xenon mixture; however, the situation may become comparable for very long experiments in which the batch mode tip angle must be made very small. The flow method (and in particular the stopped flow method) is by far ideal for remote detection experiments because flow is a natural means of transporting encoded volumes of magnetization, as opposed to creating a new batch of xenon for each indirectly encoded point. It should also be possible to create a single large batch of pure xenon and then flow it until the batch is depleted, but thus far in this laboratory the flow experiments have exploited the continuous-flow polarizer in order to produce indefinite amounts of hyperpolarized xenon.

Perhaps the biggest disadvantage of using dilute xenon mixtures from the perspective of conducting distant dipolar field experiments is the increase in the xenon diffusion 
rate due to the large fraction of helium buffer gas. Dipolar field encoding experiments require the sensor nucleus to be relatively stationary on the length scale over which the analyte dipolar field varies during the course of the encoding. The use of stopped flow can ensure that there is no bulk transport of the gas during the encoding step, but a large amount of xenon diffusion into, out of, or within the encoding volume during this time can effectively destroy the desired signal. Diffusion during the encoding step is likely a major source of diminished signal even in the experiments on pure xenon discussed in this work (for example, no signal was observed in preliminary DDF experiments involving xenon-He buffer gas mixtures); any increase in the diffusion rate would only exacerbate the problem.

\subsubsection{Geometric considerations for dipolar field encoding: coaxial sample tube configuration}

The examples and calculations of dipolar field encoding experiments presented thus far have considered only the case in which the analyte and sensor spins are mixed homogeneously. This configuration is not ideal for remote detection NMR experiments because it is difficult to remove the sensor from the analyte for subsequent transport and detection. It may be particularly convenient to encode the sensor nucleus while flowing it past the analyte without the two ever coming into contact with each other. Fortunately the nuclear dipolar field acts through space and there is no requirement for the sensor and analyte to be mixed. This feature of dipolar field encoding was first demonstrated by Warren et al. $[302,317]$ in homonuclear CRAZED experiments that correlated the NMR signals of separated species in a two-phase system of immiscible fluids and in a coaxial sample tube configuration. 


\subsection{HETERONUCLEAR DDF INDIRECT DETECTION EXPERIMENTS USING XE-129 AS A SENSOR

The calculations of the heteronuclear DDF indirect detection NMR signals in $\S 6.3 .1$

and $\S 6.3 .2$ were based on the assumption that the sensor nucleus sampled the analyte dipolar field over the same volume as the magnetization distribution that created the field, i.e., that the sensor and analyte were intermixed. These calculations need to be modified in the case that the sensor nucleus samples the analyte dipolar field over a volume that is external to the analyte magnetization. A detailed modification of these calculations is not attempted here, but the problem of the separation of analyte and sensor nuclei is explored qualitatively via experiments and magnetic field calculations.

The pulsed field gradient version of the dipolar field encoding experiment relies on the winding of the sensor magnetization into a helix with a pitch that is matched to the wavelength of modulation of the analyte longitudinal magnetization; observable signal arises as the sensor magnetization helix is unwound under the action of the analyte dipolar field. Since it is this distant dipolar field that transmits the analyte spectral information to the sensor nuclei, what really matters for the experiment is that the modulation wavelength of the analyte secular dipolar field matches the pitch of the sensor magnetization helix after the second gradient pulse. It is therefore crucial that the modulation of the analyte secular dipolar field mirror the modulation of the analyte magnetization. This is easily shown to be the case in homogeneous mixtures if the analyte magnetization is strongly modulated, i.e., when the modulation wavelength is small compared to any sample dimension (see e.g. Refs. [305, 366] and Eqs. 6.25 and 6.26). Furthermore, as long as the strong modulation condition is satisfied the size of the signal in homogeneous mixtures depends only weakly on the actual value of the modulation wavelength [303, 317], at least until molecular diffusion 


\subsection{HETERONUCLEAR DDF INDIRECT DETECTION EXPERIMENTS USING XE-129 AS A SENSOR

can no longer be neglected as the characteristic diffusion length over the timescale of the DDF encoding becomes comparable to the magnetization modulation wavelength.

The xenon-sensor DDF indirect detection experiments that were performed here (see $§ 6.4 .3$ ) used a coaxial tube geometry where a xenon sensor sealed in the inner tube was placed in an analyte solution held in the outer tube. A schematic of this sample geometry is shown in Fig. 6.6. At the beginning of the detection interval in the indirect detection pulse sequence (Fig. 6.3) the combination of rf and gradient pulses has created in the coil region a sinusoidally-modulated analyte $z$-magnetization in the outer tube and a sensorspin magnetization helix in the inner tube. The chemical shift evolution during the indirect dimension affects the phase of the $z$-modulation but otherwise does not affect its spatial distribution (neglecting $B_{0}$ inhomogeneity) and will not be considered here. The question to be resolved is, does the $z$-modulated analyte magnetization create a secular dipolar field in the inner tube with a matching $z$-modulation? If so, the gradient dephasing of the sensor transverse magnetization would rephase in the presence of the analyte dipolar field as usual, producing an observable signal.

DDF indirect detection experiments with gradient pulses were performed using a simple model system: the $\mathrm{H}-1$ nucleus in benzene $\left(\mathrm{C}_{6} \mathrm{H}_{6}\right)$ was used as the analyte in the outer tube $10 \mathrm{~mm}$ o.d. outer tube and the P-31 nucleus in concentrated phosphoric acid $\left(\mathrm{H}_{3} \mathrm{PO}_{4}(\mathrm{aq})\right)$ was used as the sensor in the $5 \mathrm{~mm}$ o.d. inner tube (Fig. 6.6). The geometry of this system simulated that of the xenon experiments but it was advantageous here to avoid the use of hyperpolarized xenon gas both for reasons of convenience and to minimize the effects of diffusion of the modulated magnetization, which can be rapid in the case of a gas. 


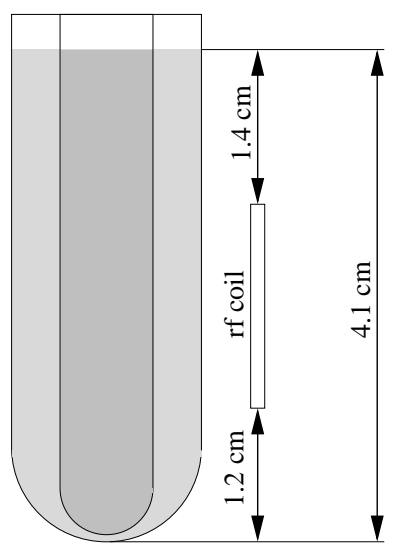

Figure 6.6: Schematic of typical coaxial tube geometry used in distant dipolar field investigations. The analyte was placed in the outer tube and the sensor was placed in the inner tube. The outer diameters of the inner and outer tubes were $5 \mathrm{~mm}$ and $10 \mathrm{~mm}$, respectively. The wall of the inner tube was $0.9 \mathrm{~mm}$ thick.

The experiments were performed at 7.05 Tesla on a Varian Unity Inova spectrometer using a Varian 10-mm broadband solution-state NMR probe. The customized 10-mm probe had three rf channels: H-1/F-19, broadband, and Xe-129. The P-31-detected benzene spectra were recorded for several values of the gradient-induced modulation using the DDF indirect detection pulse sequence in Fig. 6.3. A plot of the integrated intensity of the indirect dimension projection of the cross peak between benzene (H-1) and phosphoric acid (P-31) versus the number of magnetization modulation wavelengths over the coil region is shown in Fig. 6.7. The signal is seen to be attenuated when the magnetization modulation is large, contrary to the the results obtained in homogeneous mixtures in which the signal levels off to a constant non-zero value as the magnetization becomes strongly modulated [303, 317].

The source of this attenuation can be understood by considering the spatial distribution of the secular dipolar field of the analyte within the inner tube where the sensor resides. Eq. 6.26 for the heteronuclear secular dipolar field is not valid for slowly varying magnetization distributions or in regions outside the source magnetization volume; there- 


\subsection{HETERONUCLEAR DDF INDIRECT DETECTION EXPERIMENTS USING XE-129 AS A SENSOR

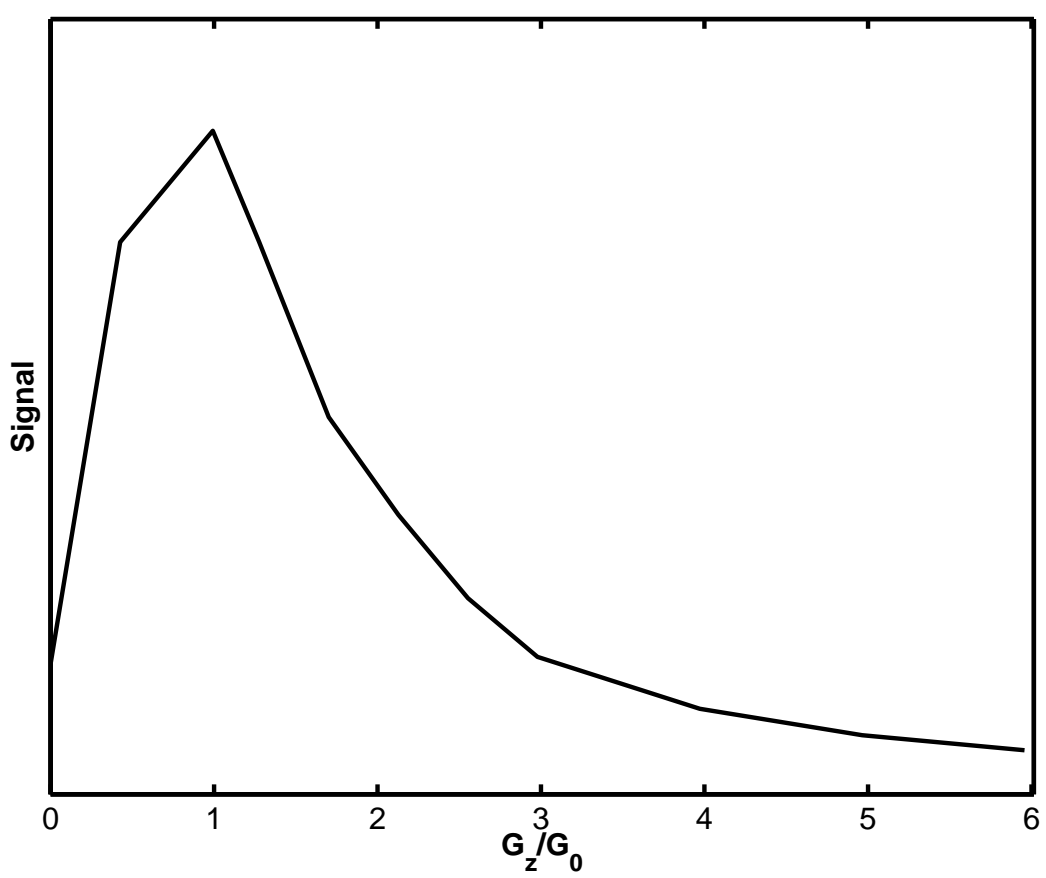

Figure 6.7: Plot of the intensity of the DDF-encoded H-1 spectrum of benzene as a function of magnetization modulation wavelength using a separated P-31 sensor in a coaxial tube geometry. The pulse sequence in Fig. 6.3 was used with 256 points in the indirect dimension; the signal reported here (in arbitrary units) was the sum of the three points nearest the maximum of the indirect dimension projection of the benzene $(\mathrm{H}-1) /$ phosphoric acid (P-31) cross peak. $G_{z}$ denotes the strength of a $\tau_{G}=1 \mathrm{~ms} z$-gradient pulse, which represents either the first gradient pulse $G_{1}$ which modulated the $\mathrm{C}_{6} \mathrm{H}_{6} / \mathrm{H}-1$ magnetization or the second gradient pulse $G_{2}$ which modulated the $\mathrm{H}_{3} \mathrm{PO}_{4} / \mathrm{P}-31$ magnetization. $G_{0}=2 \pi /\left|\gamma \tau_{G} d\right|$ represents the $z$-gradient strength that would modulate either the $\mathrm{H}-1$ or $\mathrm{P}-31$ magnetization by one wavelength over the $\mathrm{rf}$ coil length $(d=1.5 \mathrm{~cm}): G_{0}^{1}=0.157 \mathrm{G} / \mathrm{cm}$ or $G_{0}^{2}=0.387$ $\mathrm{G} / \mathrm{cm}$. The ratio $G_{2} / G_{1}=G_{0}^{2} / G_{0}^{1}$ was fixed to the value $\kappa=-\gamma_{H 1} / \gamma_{P 31}=-2.47$. The ratio $G_{z} / G_{0}$ equals the number of modulation wavelengths of $\mathrm{H}-1$ longitudinal magnetization and the number of turns of the P-31 magnetization helix over the distance $d$. A four-step phase cycle was employed to suppress the direct P-31 FID and to ensure frequency discrimination in the indirect dimension. A recycle delay of $6 \mathrm{~s}$ between transient acquisitions was used and a $4 \mathrm{~ms}, 22 \mathrm{G} / \mathrm{cm} z$-gradient spoiler pulse preceded each delay. This done to ensure that the analyte and sensor magnetizations had returned nearly to their equilibrium values by the time of the next acquisition; otherwise spectral artifacts can occur [481].

fore, simulations of this field were performed by numerically evaluating Eq. 6.157 in $§ 6.5$.

The integrals in Eq. 6.157 were evaluated using the MATLAB ${ }^{\circledR}$ [482] code in Appendix E.

The integration was not optimized; Riemann sums were performed over the entire range 


\subsection{HETERONUCLEAR DDF INDIRECT DETECTION EXPERIMENTS USING XE-129 AS A SENSOR}

of radial and axial coordinates (only sums over points within the analyte magnetization distribution are actually necessary). The coaxial tubes were taken to be of infinitesimal thickness; the inner and outer tubes had diameters of $5 \mathrm{~mm}$ and $10 \mathrm{~mm}$, respectively, and the coil region was taken to be $15 \mathrm{~mm}$ high. The analyte magnetization volume was defined by $2.5 \mathrm{~mm}<R<5.0 \mathrm{~mm}$ and $-7.5 \mathrm{~mm}<z<7.5 \mathrm{~mm}$, where $R$ and $z$ are the radial and axial coordinates, respectively. The analyte magnetization distribution was taken to be symmetric about the $z$-axis. In the real system the analyte magnetization above and below the coil region is uniform, but this magnetization was neglected here. The secular dipolar field was determined on a $30 \mathrm{~mm}$ high by $20 \mathrm{~mm}$ wide grid in the $x-z$ plane made up of squares $0.1 \mathrm{~mm}$ on a side; only points with $x>0$ were calculated and the $x<0$ points were generated by mirror reflection. The secular dipolar field was evaluated at the center of the grid squares and its amplitude was plotted in arbitrary units.

The field distribution in a vertical cross section of the tube ( $x-z$ plane) is shown in Figs. 6.8 and 6.9 in the case of an analyte magnetization modulation of a half-wavelength over the length of the coil region and in Figs. 6.10 and 6.11 in the case of a modulation of three wavelengths over the coil region. The Lorentz sphere correction to the secular dipolar field was not made in the region of the analyte magnetization and was not necessary elsewhere, since this correction only yields the local field at positions that coincide with magnetization source points. As can be seen, in both cases the overall vertical distribution of dipolar field modulation matches the $z$-modulation of the analyte magnetization, and one expects that a matched-pitch magnetization helix of the sensor spins in the inner tube would successfully rephase under the action of the analyte field. The dependence on gradient 


\subsection{HETERONUCLEAR DDF INDIRECT DETECTION EXPERIMENTS USING XE-129 AS A SENSOR}

strength enters because of the cancellation and attenuation of the dipolar field in regions external to the analyte volume. The dipolar field falls off rapidly with distance from the magnetization source. Furthermore, the volumes of analyte magnetization of alternating sign may be approximated as adjacent uniformly-magnetized volumes of opposite sign whose external dipolar fields tend to cancel each other. The result is that the analyte dipolar field becomes attenuated near the center of the inner tube when the analyte magnetization modulation wavelength (which characterizes the length scale of the magnetization volumes of opposite sign) becomes comparable to or smaller than the distance from the analyte volume to the center of the tube. In that case the parts of the sensor spin magnetization helix near the center of the inner tube will not properly rephase because they do not feel the analyte dipolar field, and will not contribute to the net signal. When the analyte magnetization modulation wavelength becomes very short the analyte dipolar field hardly penetrates into the sensor volume. This problem is only exacerbated upon accounting for the finite thickness of the inner tube, which further separates the sensor from the analyte. Note that the calculation of the signal in the DDF experiment is no longer as straightforward as it was in $\S 6.3 .1$; the time evolution of the sensor magnetization must be calculated for every point in the sensor volume and the net signal is proportional to the integral of the transverse magnetization over this volume.

One interesting point to note is that the dipolar field simulations indicate that the sign of the analyte secular dipolar field in the inner tube is opposite that of the analyte magnetization at the same vertical position $z$. This is easily understood by considering the full dipolar field distribution; the field lines outside a volume magnetized in the $+z$-direction 


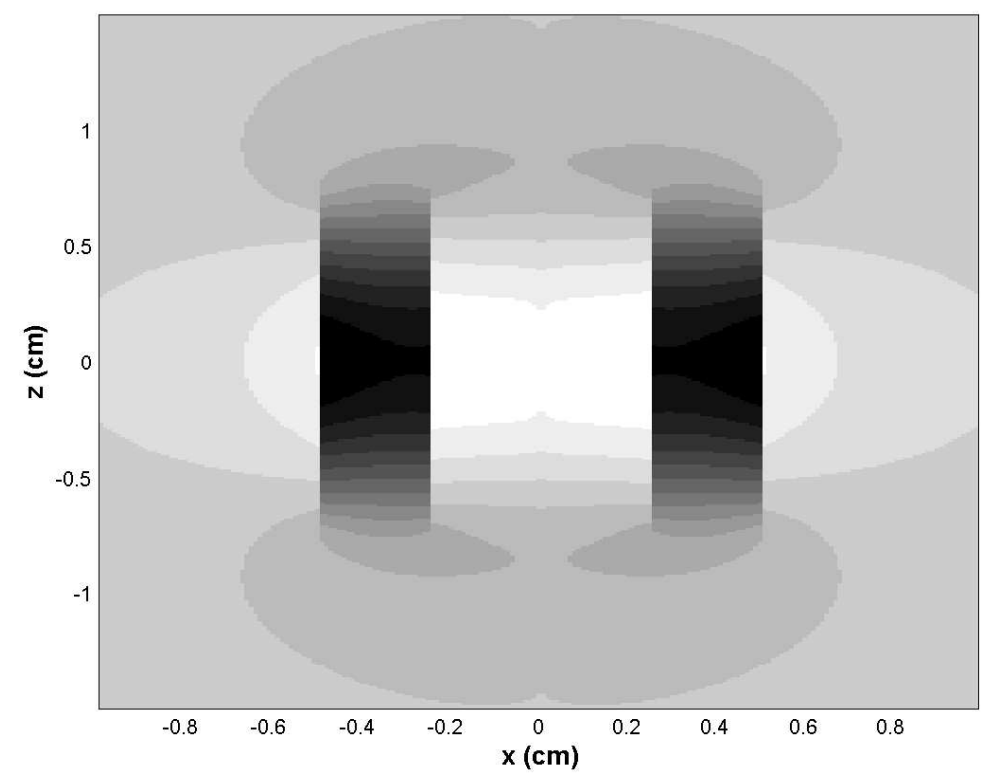

Figure 6.8: Plot (pseudocolor) of a vertical cross section of the secular dipolar field generated by modulated analyte magnetization in the outer tube in a coaxial tube geometry $(0.5$ modulation wavelengths over the coil region).

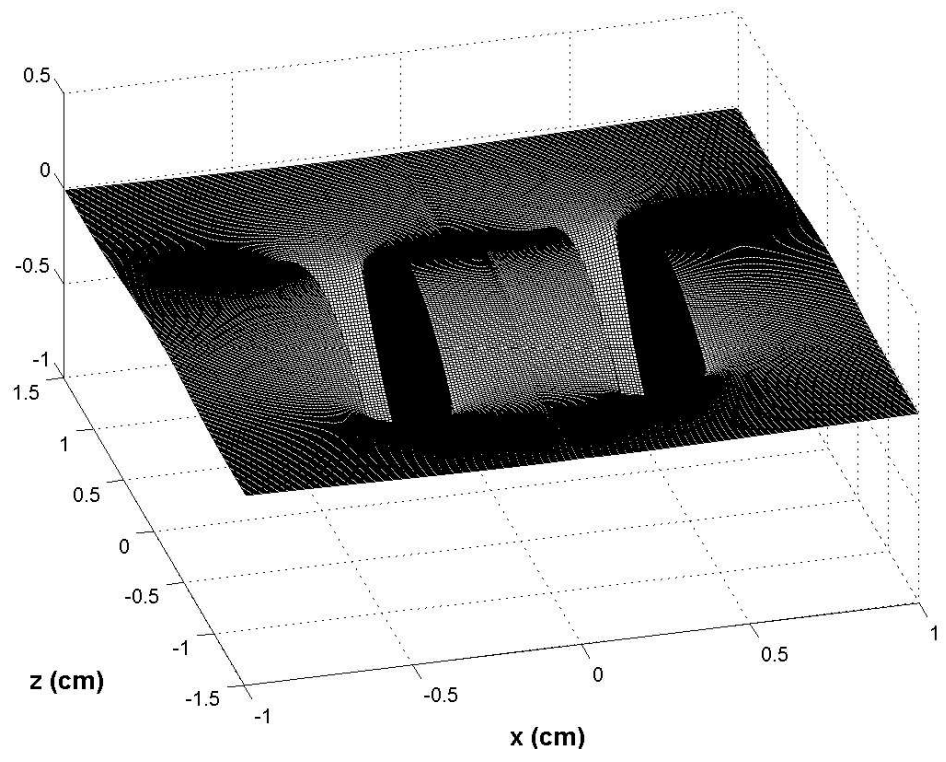

Figure 6.9: Plot (mesh) of a vertical cross section of the secular dipolar field generated by modulated analyte magnetization in the outer tube in a coaxial tube geometry $(0.5$ modulation wavelengths over the coil region). 


\subsection{HETERONUCLEAR DDF INDIRECT DETECTION EXPERIMENTS

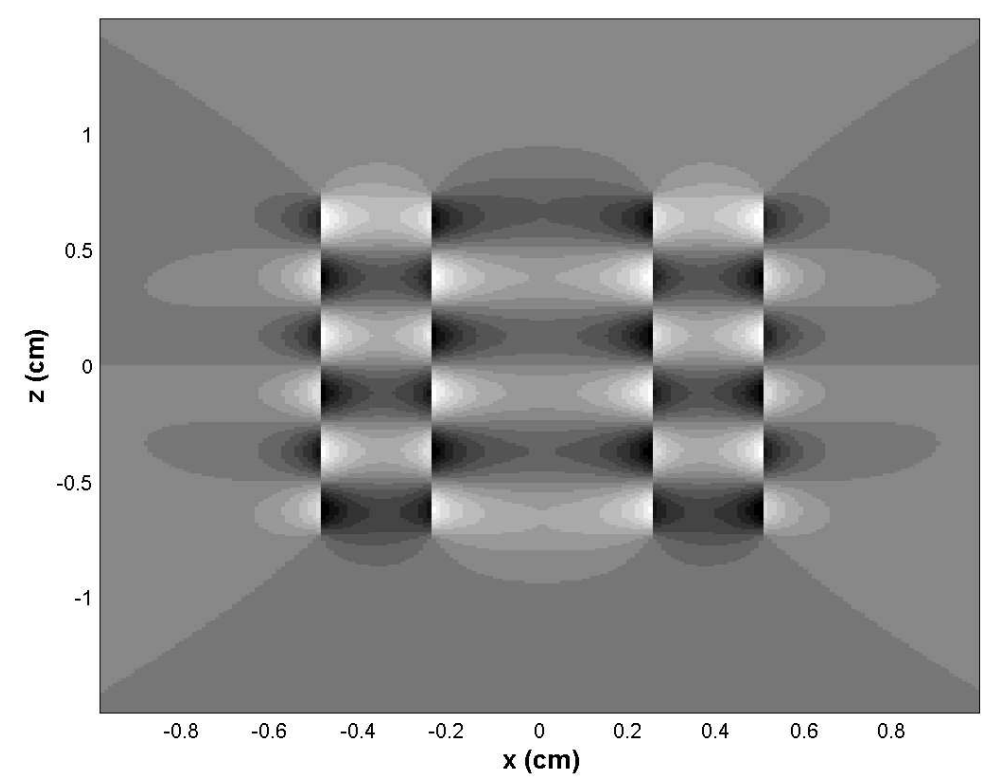

Figure 6.10: Plot (pseudocolor) of a vertical cross section of the secular dipolar field generated by modulated analyte magnetization in the outer tube in a coaxial tube geometry ( 3 modulation wavelengths over the coil region).

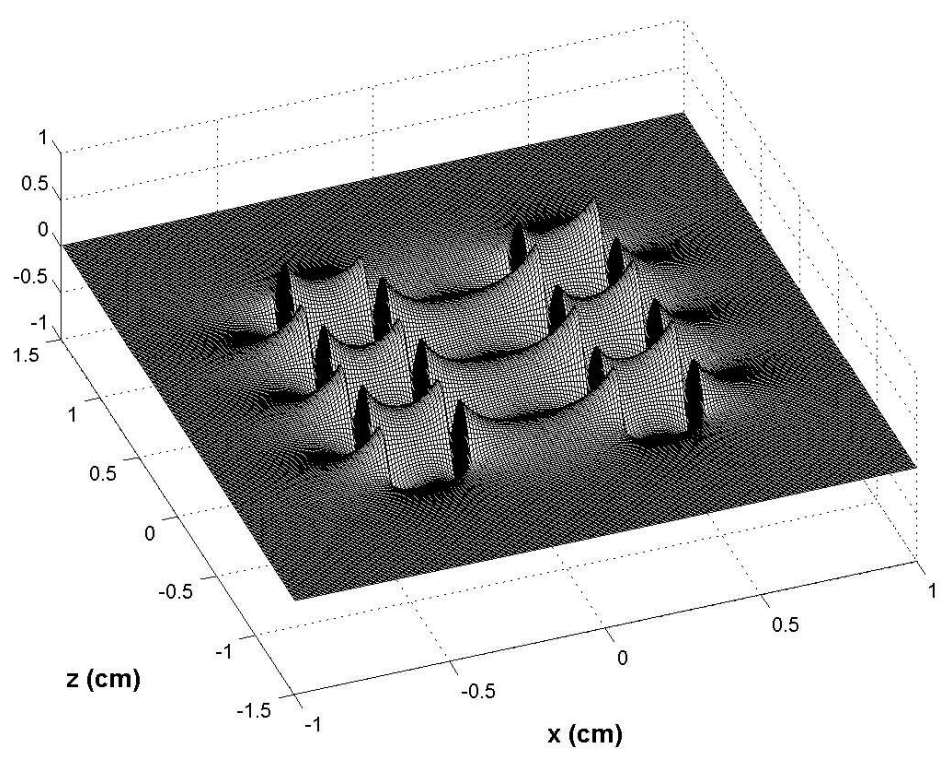

Figure 6.11: Plot (mesh) of a vertical cross section of the secular dipolar field generated by modulated analyte magnetization in the outer tube in a coaxial tube geometry ( 3 modulation wavelengths over the coil region). 


\subsection{HETERONUCLEAR DDF INDIRECT DETECTION EXPERIMENTS USING XE-129 AS A SENSOR

start out parallel to the magnetization at the top of the volume but curve around so that they are in the $-z$-direction next to the volume. The sign of the dipolar field within the inner tube is the opposite of what it would be if the analyte was magnetized in the same direction but the analyte and sensor were homogeneously mixed. The analyses in $§ 6.3 .1$ and 6.2.3 predict that this would result in a reversal of the sign of the indirectly detected spectrum in a phase-sensitive experiment and a reversal of the sign of the odd-order echoes (since $J_{n}(-x)=(-1)^{n} J_{n}(x)$ for integer $n$ ) in a DDF multiple echo experiment.

\subsubsection{Experimental DDF indirect spectra using the coaxial tube geome- try. Results and future prospects.}

Distant dipolar field indirect detection was implemented using a hyperpolarized Xe-129 gas sensor in the coaxial tube geometry using the pulse sequence in Fig. 6.3. These experiments were performed to investigate the suitability of Xe-129 for dipolar field encoding and were not incorporated into a complete remote detection experiment. The hyperpolarized Xe-129 gas was produced by the batch-mode (freeze-thaw) spin-exchange optical pumping method (see $\S 6.4 .1$ ) at polarization levels of $1-10 \%$ and was stored in a SurfaSil ${ }^{\circledR}$-coated J-Young tube. The tube had an outer diameter of $5 \mathrm{~mm}$ and the length of the tube up to the bottom of the valve was about $20 \mathrm{~cm}$. This tube was filled with pure natural-abundance xenon to a total xenon gas pressure of 3-5 atm and was placed in a 10-mm outer diameter NMR tube that was partially filled with the analyte solution to be studied. The NMR experiments were performed at 7.05 Tesla on a Varian Unity Inova spectrometer using a Varian 10-mm broadband solution-state NMR probe. The custom-made 10-mm probe had three rf channels: H-1/F-19, broadband, and Xe-129; the broadband channel was used for 


\subsection{HETERONUCLEAR DDF INDIRECT DETECTION EXPERIMENTS USING XE-129 AS A SENSOR

Xe-129 detection due to its higher sensitivity. The batch mode experiments provide only a finite reservoir of hyperpolarized gas; therefore, Xe-129 detection rf pulses of less than $90^{\circ}$ were used in order not to use up all the hyperpolarization at once by converting it to transverse magnetization.

The ideal analyte for a proof-of-principle experiment would have a high magnetization, i.e., a high spin density and polarization. Fig. 6.12 shows the indirect-dimension projection of the Xe-129-detected DDF indirect $\mathrm{H}-1$ spectrum of water $\left(\mathrm{H}_{2} \mathrm{O}\right)$ in the coaxial tube geometry at $7.05 \mathrm{~T} .{ }^{45}$ The indirectly-detected spectrum reproduces the normal directly-detected water $\mathrm{H}-1$ spectrum at a resonance offset of $\sim 200 \mathrm{~Hz}$. It does, however, exhibit a distorted (non-Lorentzian) lineshape with a broadened base. This distortion is likely primarily due to the depletion of the hyperpolarized (non-equilibrium) Xe-129 longitudinal magnetization over the course of multiple acquisitions due to conversion to transverse magnetization by rf pulses and to a lesser extent $T_{1}$ relaxation. This gradual depletion of magnetization causes a non-exponential damping of the time-domain signal in the indirect dimension, which leads to distorted lineshapes. This problem is considered further in $\S 6.6$ along with a proposed remedy. Since Xe-129 rf tip pulses of less than $90^{\circ}$ were used there was always longitudinal magnetization present during the experiment; part of the lineshape distortion in the indirect dimension could also be due to a chirp of the Larmor frequency of Xe-129 in the presence of its own gradually diminishing longitudinal self-dipolar field. Note that this effect is not present in normal DDF indirect detection experiments that use $90^{\circ}$ sensor-spin pulses.

\footnotetext{
${ }^{45}$ The H-1 thermal equilibrium magnetization density at this field can be calculated according to Eq. 1.102 for $I=1 / 2$ nuclei using $\gamma_{H 1} / 2 \pi=42.6 \mathrm{MHz} / \mathrm{T}$ and a total $\mathrm{H}-1$ concentration of $\sim 111 \mathrm{M}: \mu_{0}\left|M_{e q}^{H 1}\right| \approx 286$ $\mu \mathrm{G}$ at $7.05 \mathrm{~T}$ and room temperature. The Xe-129 magnetization is calculated in $\S 6.7 .2$ to be $\left|M^{X e 129}\right| \approx 80$
} 


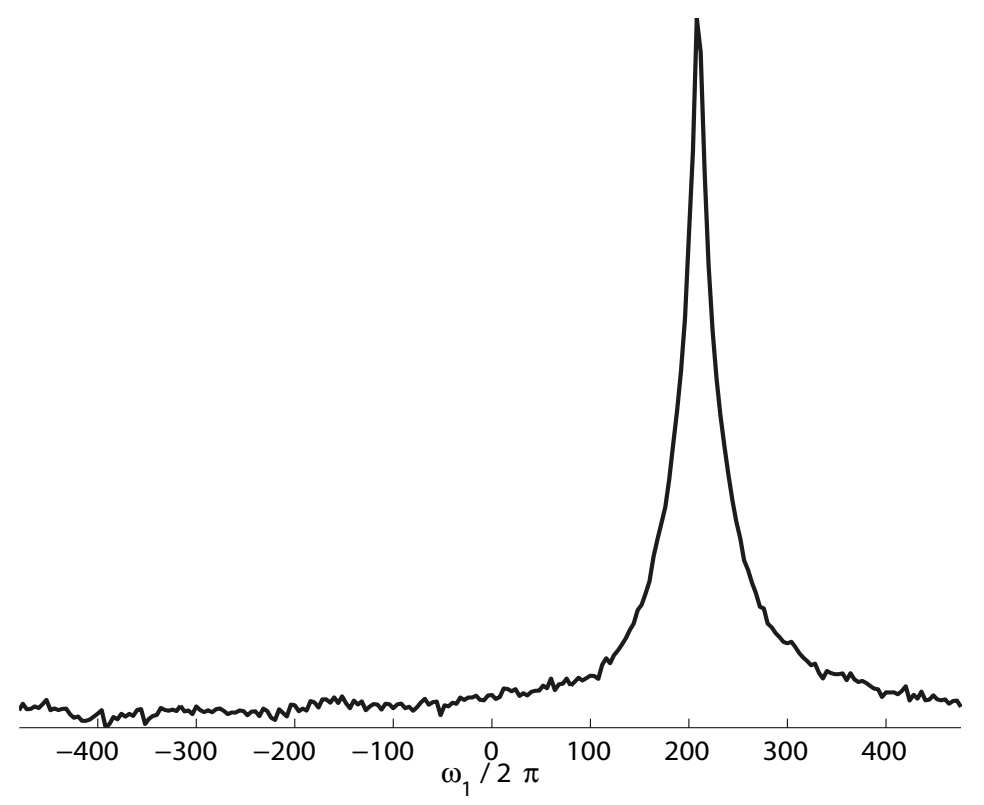

Figure 6.12: H-1 spectrum of water at $7.05 \mathrm{~T}$ detected indirectly via the distant dipolar field using a separated Xe-129 sensor. Xe-129 small-tip pulses of $\theta \approx \pi / 8$ were used. A coaxial sample geometry was used. A total of 512 points were taken in the indirect dimension with a spectral window of $2048 \mathrm{~Hz}$; a four-step phase cycle per indirect point was employed to suppress the Xe-129 direct FID and to ensure frequency discrimination in the indirect dimension. The sequence employed $\tau_{G}=1 \mathrm{~ms} z$-gradient pulses at strengths $G_{1}=0.33$ $\mathrm{G} / \mathrm{cm}$ and $G_{2}=1.18 \mathrm{G} / \mathrm{cm}$ in the ratio $\kappa=G_{2} / G_{1}=-\gamma_{H 1} / \gamma_{X e 129}=3.59$. Xe- 129 was detected at $82.92 \mathrm{MHz}$ during an acquisition time of $1 \mathrm{~s}$ with a spectral window of 2000 Hz. Residual Xe-129 net transverse magnetization was suppressed before each transient acquisition by using a recycle delay of $2 \mathrm{~s}$ between acquisitions preceded by a $4 \mathrm{~ms}, 22$ $\mathrm{G} / \mathrm{cm}$ z-gradient spoiler pulse.

Another inherent disadvantage of batch-mode Xe-129 NMR experiments is the restriction to small tip-angle rf pulses on the xenon channel, which leads to diminished Xe-129 transverse magnetization and an accompanying reduction in signal, as well as to the lineshape distortions caused by depletion of the reservoir of hyperpolarization. These limitations may be overcome in the future by performing experiments in flow mode. The use of flow-preferably stopped flow so that the bulk xenon gas is static during the DDF $\mu \mathrm{G}$ under conditions that were similar (perhaps somewhat more favorable) than those used here. 


\subsection{HETERONUCLEAR DDF INDIRECT DETECTION EXPERIMENTS USING XE-129 AS A SENSOR

encoding interval - would allow the gas to be refreshed after each transient acquisition so that full $90^{\circ} \mathrm{Xe}-129 \mathrm{rf}$ pulses can be applied without destroying the hyperpolarization for the next acquisition. Flowing the sensor nucleus is also the most natural mode of transport in remote detection experiments; the alternative in batch mode is to encode one indirect point in a fixed volume of gas and transport the whole sample to another point for detection. However, flow mode experiments can have their own disadvantages, as was discussed in §6.4.1. As implemented in the Pines lab using the commercial continuous-flow spin-exchange polarizer, the Xe-129 optical pumping mixture contains mostly inert buffer gases that are removed during freeze-thaw batch mode operation but are present in flow mode when using the direct output of the polarizer. This dilution of the Xe-129 causes a reduction of the xenon magnetization and hence of the signal. The Xe-129 magnetization can be greatly increased by performing spin-exchange optical pumping at high xenon concentrations and/or by using isotopically-enriched Xe-129 mixtures. The spin-exchange optical pumping of high pressure xenon can result in enhanced magnetization due to an increase in spin density even as the nuclear polarization decreases [441]. Another important reason to eliminate buffer gases from the xenon mixture is the increase of the Xe-129 diffusion rate in the presence of lowmolecular weight species (see Eq. 6.132). Fast xenon diffusion during dipolar field encoding can destroy any enhancements of the signal due to the use of gradient modulation of the magnetization.

The distant dipolar field can be used to record indirect analyte spectra that have a more complicated structure than just a single resonance; e.g., it is possible to observe a $J$ coupled (scalar-coupled) analyte indirect spectrum. Other workers have considered the case 


\subsection{HETERONUCLEAR DDF INDIRECT DETECTION EXPERIMENTS USING XE-129 AS A SENSOR

of heteronuclear correlation spectroscopy in which two spin species interact with each other through both intramolecular $J$-couplings and distant intermolecular dipolar couplings [380, 381]. The problem considered here is somewhat simpler, i.e., two intramolecular analyte spin species that interact with each other through $J$-couplings and with a third sensor nucleus through distant intermolecular dipolar couplings. The analysis of this system proceeds analogously to the analysis in $§ 6.3 .1$ except the analyte magnetization is modulated by the $J$-coupling as well as by the chemical shift. Some difficulty was encountered when trying to resolve $J$-modulated analyte spectra using a Xe-129 sensor due to the line broadening caused by the gradual depletion of xenon hyperpolarization (see $\S 6.6$ ); therefore, the thermallypolarized P-31 nucleus in concentrated phosphoric acid $\left(85 \% \mathrm{H}_{3} \mathrm{PO}_{4}\right.$ in water) was used as a sensor to acquire a proof-of-principle $J$-resolved spectrum. Fig. 6.13 shows the indirectdimension projection of the P-31-detected DDF indirect F-19 spectrum as well as the 2D spectrum of 2,2,2-trifluoroethanol $\left(\mathrm{CF}_{3} \mathrm{CH}_{2} \mathrm{OH}\right)$ at $7.05 \mathrm{~T}$. The same type of coaxial sample tube geometry that was used in the xenon experiments was used here with the P-31 sensor in the inner tube and the analyte in the outer tube. As can be seen, the lineshapes and spectral resolution are better than those in the Xe-129-detected DDF indirect spectrum of water. The signal-to-noise ratio was smaller in this experiment than it was in the DDF Xe129 detection of water (Fig. 6.12); both the analyte and sensor magnetization densities were larger in the $\mathrm{H}_{2} \mathrm{O} / \mathrm{Xe}-129$ experiment than they were in the $\mathrm{CF}_{3} \mathrm{CH}_{2} \mathrm{OH} / \mathrm{H}_{3} \mathrm{PO}_{4}$ experiment, although the Xe-129 Larmor frequency was only $68 \%$ that of the P-31 Larmor frequency. ${ }^{46}$ The spectral intensity of the fluorine nuclei is distributed over a 1:2:1 triplet split by the

\footnotetext{
${ }^{46}$ The F-19 thermal equilibrium magnetization density can be calculated according to Eq. 1.102 using $\gamma_{F 19} / 2 \pi=-40.1 \mathrm{MHz} / \mathrm{T}$ and a total F-19 concentration of $\sim 42 \mathrm{M}$ : total $\mu_{0}\left|M_{e q}^{F 19}\right| \approx 96 \mu \mathrm{G}$ at $7.05 \mathrm{~T}$ and room temperature. The P-31 magnetization density can be calculated using $\gamma_{P 31} / 2 \pi=17.25 \mathrm{MHz} / \mathrm{T}$ and a P-31 concentration of $\sim 15 \mathrm{M}: \mu_{0}\left|M_{e q}^{P 31}\right| \approx 6 \mu \mathrm{G}$ at $7.05 \mathrm{~T}$ and room temperature.
} 


\subsection{HETERONUCLEAR DDF INDIRECT DETECTION EXPERIMENTS

F-19-H-1 scalar coupling constant ${ }^{3} J_{F H}$, as expected.
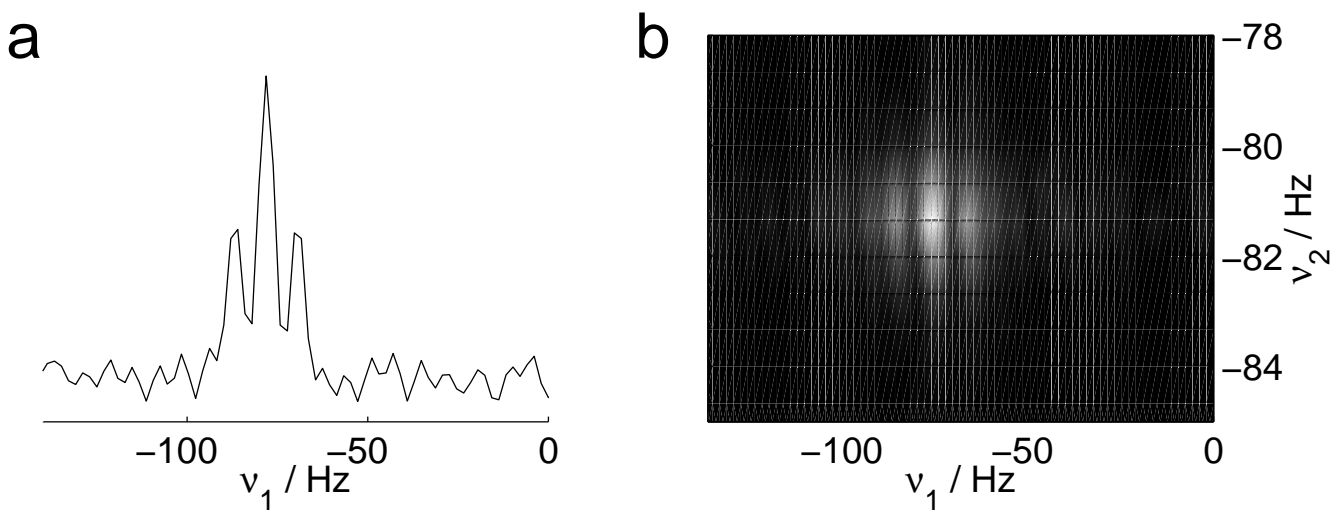

Figure 6.13: F-19 spectrum of 2,2,2-trifluoroethanol at $7.05 \mathrm{~T}$ detected indirectly via the distant dipolar field using a separated P-31 sensor: (a) is a trace of the indirect dimension and (b) is a portion of the $2 \mathrm{D}$ spectrum $\left(\nu_{1}\right.$ is the indirect dimension (F-19) frequency and $\nu_{2}$ is the direct dimension (P-31) frequency). A coaxial sample geometry was used. A total of 512 points were taken in the indirect dimension with a spectral window of $1000 \mathrm{~Hz}$; a two-step phase cycle per indirect point was employed to suppress the P-31 direct FID. The sequence employed $\tau_{G}=1 \mathrm{~ms} z$-gradient pulses at strengths $G_{1}=0.38 \mathrm{G} / \mathrm{cm}$ and $G_{2}=0.88 \mathrm{G} / \mathrm{cm}$. An experimentally-optimized ratio of $G_{2} / G_{1}=2.29$ was used instead of the predicted ideal value $G_{2} / G_{1}=\kappa=\gamma_{H 1} / \gamma_{P 31}=2.47$. P-31 was detected at $121.35 \mathrm{MHz}$ during an acquisition time of $1.5 \mathrm{~s}$ with a spectral window of $500 \mathrm{~Hz}$. A recycle delay of $5 \mathrm{~s}$ between transient acquisitions was used and a $4 \mathrm{~ms}, 22 \mathrm{G} / \mathrm{cm}$-gradient spoiler pulse preceded each delay.

It is clear from Fig. 6.12 that the signal-to-noise ratio in the Xe-129 DDF indirect detection experiment is much less than what is routinely recorded in high-field directdetection NMR experiments. Some loss of signal is of course expected due to the fact that the magnetization of the Xe-129 sensor was smaller than the $\mathrm{H}-1$ magnetization of the $\mathrm{H}_{2} \mathrm{O}$ analyte; ideally a sensitivity enhancement would be sought by using a sensor that was more highly magnetized than the analyte. However, the DDF indirect detection signal is even much less than the direct Xe-129 free induction decay. As has been discussed previously in various sections, the reduction of signal of DDF indirect detection compared to direct de- 


\subsection{HETERONUCLEAR DDF INDIRECT DETECTION EXPERIMENTS USING XE-129 AS A SENSOR

tection can come from several sources, which are summarized below. Some of these effects cannot be compensated by or even become worse upon using a more highly magnetized sensor. In a remote detection experiment it is also possible to use a more sensitive detector, but even the most sensitive magnetometers may not be able to compensate for the signal losses.

The DDF indirect detection experiment is susceptible to a number of signalreducing factors. Molecular diffusion of the sensor during DDF encoding can destroy spatial modulations of the magnetization that are desirable for coherence pathway selection and creating large signals. This effect can be minimized by reducing the diffusion rate of the sensor, e.g., by reducing the amount of light buffer gases in the Xe-129 gas mixture. Another alternative would be to use a solution of Xe-129 in a nonpolar solvent (xenon is hydrophobic) as the sensor; this would decrease the xenon diffusion rate but also would decrease the Xe-129 relaxation times, which may be undesirable in remote detection experiments if the sensor transport period is long. The efficiency of DDF encoding can be reduced by the effects of the sensor transverse self-dipolar field, which can rotate the sensor magnetization out of the transverse plane. Radiation damping interactions between a highly polarized sensor and a resonantly-tuned rf coil can have similar deleterious effects. Radiation damping did not appear to be a significant problem in the experiments conducted here, but at any rate its effects may be reduced in a remote detection experiment by using a detuned rf coil for the encoding of the sensor. Radiation damping and self-dipolar field effects will become larger if a more highly magnetized sensor is used in an attempt to increase sensitivity, e.g., an isotopically-enriched Xe-129 mixture at a higher polarization or pressure than was used 


\subsection{HETERONUCLEAR DDF INDIRECT DETECTION EXPERIMENTS USING XE-129 AS A SENSOR

here. The effects of diffusion, radiation damping, and self-DDF interactions take time to develop; if the analyte $T_{1}$ happens to be long, it may be possible to reduce these effects on the DDF encoding in a remote detection experiment by using a short encoding time to encode several volumes of sensor magnetization per indirect point of the analyte spectrum. The signals from each of these volumes could be coadded to enhance sensitivity. Another potentially serious problem in DDF encoding experiments is the effect of $t_{1}$ noise, i.e., fluctuations of the environmental conditions from experiment to experiment that show up as noise in the indirect dimension $[7, \S 6.8 .2]$. There is currently no good solution for the $t_{1}$ noise or transverse self-DDF problems. One final problem is that the dipolar field may have a long range a the molecular length scale but actually has a short range on a macroscopic length scale; if the sensor and analyte are physically separated the sensor will not be able to sample the full strength of the analyte dipolar field. This effect can be minimized by using a thin-walled container to separate the sensor from the analyte.

Note that the Xe-129 experiments reported here were conducted in batch mode. Xenon-129 remote detection experiments will likely be performed in flow mode, for which several additional factors affecting the sensitivity need to be considered. Flow mode experiments are able to provide an indefinite supply of hyperpolarized Xe-129 gas, whereas batch mode experiments are limited to a finite reservoir of gas. Flow mode experiments can be conducted using full $90^{\circ}$ pulses on the Xe-129 without worrying about depleting the magnetization reservoir or about $T_{1}$ relaxation losses over multiple transient acquisitions. This will lead to larger signals than in the batch mode small tip-angle scheme. However, the xenon spin density is lower in a flow mode experiment if the buffer gases in the optical 


\subsection{HETERONUCLEAR DDF INDIRECT DETECTION EXPERIMENTS USING XE-129 AS A SENSOR

pumping mixture are not removed first, and this will reduce the signal as compared to a batch mode experiment using pure xenon gas. It may however be possible to prepare a batch of hyperpolarized Xe-129 gas by the freeze-thaw method and then use it for a (finite duration) flow experiment. Note that if the direct output of the continuous flow polarizer is used, the length of the indirect detection experiment is indefinite and signal can be accumulated by using extra acquisitions (at the expense of performing a longer experiment). Also note that radiation damping and self-DDF effects would be expected to be larger in a flow mode experiment compared to a batch mode experiment if larger tip angles were used at the same Xe-129 spin density.

The DDF indirect detection experiments described above used analytes that were thermally polarized in a high magnetic field on the order of Tesla. This restricts the remote detection DDF encoding to take place in a high field environment unless a magnetic-field prepolarization is performed or the analyte polarization can be enhanced past its thermal equilibrium value, e.g., by direct optical pumping or spin exchange-optical pumping. Barros et al. have performed intermolecular double-quantum coherence imaging of the protons in water in a relatively low field of $16 \mathrm{mT}(160 \mathrm{G})$ by using an Overhauser polarization transfer from the electrons of a dissolved organic free radical, a technique that also requires the ability to irradiate EPR transitions [483]. 


\subsection{Calculation of the distant dipolar field for cylindrically symmetric geometries}

Eq. 6.26 for the heteronuclear secular dipolar field is simple and local in coordinate space but is not valid when the magnetization distribution varies slowly over the magnetization volume or at points exterior to this volume. The most efficient of the methods discussed in $§ 6.2 .1$ for calculating the secular dipolar field of an arbitrary magnetization distribution seems to be the evaluation of the local equation in Fourier space (the heteronuclear counterpart of Eq. 6.24), where the conversion between coordinate space and $\underline{k}$-space and back again can be implemented via numerical Fourier transforms. However, the sample geometries investigated in this work either involved a cylindrical tube or two coaxial tubes, and the resulting magnetization distributions - or at least their secular contributions - also possessed cylindrical symmetry. It is therefore worthwhile to investigate to what degree the calculation of the secular dipolar field can be simplified in a system that has cylindrical symmetry.

A continuous magnetization distribution $\underline{M}(\underline{r})$ inside a volume $V^{\prime}$ bounded by a surface $S^{\prime}$ creates a macroscopic magnetic field that is given everywhere by Eqs. 6.46 and 6.47 (reproduced here):

$$
\underline{B}_{d}(\underline{r})=\mu_{0}\left[\underline{M}(\underline{r})-\underline{\nabla} \Phi_{M}(\underline{r})\right]
$$


where the effective magnetostatic potential is:

$$
\Phi_{M}(\underline{r})=-\frac{1}{4 \pi} \int_{V^{\prime}} d^{3} \underline{r}^{\prime} \frac{\underline{\nabla}^{\prime} \cdot \underline{M}\left(\underline{r}^{\prime}\right)}{\left|\underline{r}-\underline{r}^{\prime}\right|}+\frac{1}{4 \pi} \int_{S^{\prime}} d^{2} \underline{r}^{\prime} \frac{\underline{\tilde{n}}^{\prime} \cdot \underline{M}\left(\underline{r}^{\prime}\right)}{\left|\underline{r}-\underline{r}^{\prime}\right|} .
$$

Primed coordinates are source coordinates and unprimed coordinates are field coordinates; $\underline{\tilde{n}}$ represents the unit vector outwardly normal from the surface $S^{\prime}$. Note that the dipolar field in Eq. 6.134 can be written as a functional of the magnetization density:

$$
\underline{B}_{d}(\underline{r})=\underline{B}_{d}[\underline{M}(\underline{r})]
$$

The quantity of interest in NMR dipolar field experiments is not the full dipolar field, but rather only its secular portion. In particular, the DDF remote detection experiments primarily concern the heteronuclear secular dipolar field created by a magnetization distribution $\underline{M}^{I}(\underline{r})$ of the analyte spins and experienced by the sensor spins. The discussion after Eq. 6.22 indicates that the heteronuclear secular dipolar field is equal to the $z$-component of the full dipolar field evaluated as a functional of only the $z$-component of the source magnetization vector:

$$
\underline{B}_{d}^{I, s e c}(\underline{r})=\left(\underline{B}_{d}^{I}\left[M_{z}^{I}(\underline{r}) \underline{\widetilde{z}}\right] \cdot \underline{\widetilde{z}}\right) \underline{\widetilde{z}} .
$$

Note that this prescription for determining $\underline{B}_{d}^{I, s e c}(\underline{r})$ is not equivalent to evaluating Eq. 6.134 via Eq. 6.135 and then retaining only the $z$ component of the resultant field. Eq. 6.137 can be used in conjunction with Eqs. 6.134 and 6.135 to write expressions for the 
heteronuclear secular dipolar field:

$$
\underline{B}_{d}^{I, s e c}(\underline{r})=\mu_{0}\left[M_{z}^{I}(\underline{r})-\frac{\partial}{\partial z} \Phi_{M}^{I, z}(\underline{r})\right] \underline{\widetilde{z}}
$$

where

$$
\Phi_{M}^{I, z}(\underline{r})=-\frac{1}{4 \pi} \int_{V^{\prime}} d^{3} \underline{r}^{\prime}\left[\frac{1}{\left|\underline{r}-\underline{r}^{\prime}\right|} \frac{\partial M_{z}^{I}\left(\underline{r}^{\prime}\right)}{\partial z^{\prime}}\right]+\frac{1}{4 \pi} \int_{S^{\prime}} d^{2} \underline{r}^{\prime} \frac{M_{z}^{I}\left(\underline{r}^{\prime}\right)\left(\underline{\widetilde{n}}^{\prime} \cdot \underline{\widetilde{z}}^{\prime}\right)}{\left|\underline{r}-\underline{r}^{\prime}\right|} .
$$

It will be useful to expand the magnetization density in a cylindrical coordinate system:

$$
\underline{M}^{I}(\underline{r})=M_{R}^{I}(R, \phi, z) \underline{\widetilde{R}}+M_{\phi}^{I}(R, \phi, z) \underline{\widetilde{\phi}}+M_{z}^{I}(R, \phi, z) \underline{\widetilde{z}},
$$

where $\underline{\widetilde{R}}, \underline{\widetilde{\phi}}$, and $\underline{\widetilde{z}}$ are unit vectors in the direction of increasing $R=\sqrt{x^{2}+y^{2}}, \phi=$ $\tan ^{-1}(y / x)$, and $z$, respectively. If the analyte magnetization distribution is axially symmetric about $z$, it is independent of the azimuthal angle:

$$
\underline{M}^{I}(\underline{r})=\underline{M}^{I}(R, z)
$$

and furthermore the surface normal unit vector $\underline{\widetilde{n}}^{\prime}$ is also independent of $\phi^{\prime}$. In that case the evaluation of Eq. 6.139 involves the evaluation of integrals of the form

$$
\mathcal{I}=\int_{V^{\prime}} d^{3} \underline{r}^{\prime} \frac{f\left(R^{\prime}, z^{\prime}\right)}{\left|\underline{r}-\underline{r}^{\prime}\right|}
$$

The surface integral in Eq. 6.139 is just a special case of Eq. 6.142 with a constraint on the 


\subsection{CALCULATION OF THE DISTANT DIPOLAR FIELD FOR CYLINDRICALLY SYMMETRIC GEOMETRIES}

integration boundaries. The volume integral can be written in cylindrical coordinates as

$$
\int_{V^{\prime}} d^{3} \underline{r}^{\prime}=\int d R^{\prime} R^{\prime} \int d \phi^{\prime} \int d z^{\prime}
$$

where the limits of integration constrain $\underline{r}^{\prime}=\left(R^{\prime}, \phi^{\prime}, z^{\prime}\right)$ to be within the volume $V^{\prime}$.

The key to evaluating Eq. 6.142 lies in determining an appropriate expansion for $\left|\underline{r}-\underline{r}^{\prime}\right|^{-1}$. This function is a type of Green function, which is a class of functions that satisfy the differential equation $\nabla^{\prime 2} G\left(\underline{r}, \underline{r}^{\prime}\right)=-4 \pi \delta^{3}\left(\underline{r}-\underline{r}^{\prime}\right)[17, \S 1.10]$. The function $\left|\underline{r}-\underline{r}^{\prime}\right|^{-1}$ may be written exactly in terms of cylindrical coordinates as:

$$
\frac{1}{\left|\underline{r}-\underline{r}^{\prime}\right|}=\frac{1}{\sqrt{R^{2}+R^{\prime 2}-2 R R^{\prime} \cos \left(\phi-\phi^{\prime}\right)+\left(z-z^{\prime}\right)^{2}}} .
$$

This is not a particularly simple form; even the integration of $\left|\underline{r}-\underline{r}^{\prime}\right|^{-1}$ alone with respect to $\phi^{\prime}$ would be difficult. A standard technique is to expand the Green function as a sum of spherical harmonic functions $[17, \S 3.9]$ :

$$
\frac{1}{\left|\underline{r}-\underline{r}^{\prime}\right|}=4 \pi \sum_{l=0}^{\infty} \sum_{m=-l}^{l} \frac{1}{2 l+1} \frac{r_{<}^{l}}{r_{>}^{l+1}} Y_{l, m}^{*}\left(\theta^{\prime}, \phi^{\prime}\right) Y_{l, m}(\theta, \phi),
$$

where $r=\sqrt{x^{2}+y^{2}+z^{2}}, \theta=\cos ^{-1}(z / r)$, and $\phi=\tan ^{-1}(y / x)$ are the usual spherical polar coordinates. The symbol $r_{<}\left(r_{>}\right)$denotes the lesser (greater) of $r$ and $r^{\prime}$. Eq. 6.145 becomes particularly simple in the case of axial symmetry about $z$ because only the $m=0$ term survives the integration in Eq. 6.142 with respect to $\phi^{\prime}$; therefore, the double sum is reduced to a single sum over $l$. This is, however, still an infinite sum, which may not converge very quickly, particularly in the case of a magnetization distribution that differs 
significantly from a spherical geometry (such as a long cylinder). A common Green function expansion in cylindrical coordinates is $[17, \S 3.11]$ :

$$
\frac{1}{\mid \underline{r}-\underline{r^{\prime} \mid}}=\frac{2}{\pi} \sum_{m=-\infty}^{\infty} \int_{0}^{\infty} d k e^{i m\left(\phi-\phi^{\prime}\right)} \cos \left[k\left(z-z^{\prime}\right)\right] I_{m}\left(k R_{<}\right) K_{m}\left(k R_{>}\right)
$$

where $I_{m}$ and $K_{m}$ are the modified Bessel functions of the first and second kind, respectively, of order $m[484, \S 11.5],[241, \S 9.6]$. The symbol $R_{<}\left(R_{>}\right)$denotes the lesser (greater) of $R$ and $R^{\prime}$. In the case of axial symmetry about $z$ only the $m=0$ term survives the integration in Eq. 6.142 with respect to $\phi^{\prime}$, which eliminates the sum over $m$. However, Eq. 6.146 is still somewhat unsatisfactory because it involves an extra integral with respect to the variable $k$ over a product of trigonometric and Bessel functions.

Although it does not seem to have achieved wide use, there exists another compact representation of the Green function in cylindrical coordinates [485, 486]:

$$
\frac{1}{\left|\underline{r}-\underline{r}^{\prime}\right|}=\frac{1}{\pi \sqrt{R R^{\prime}}} \sum_{m=-\infty}^{\infty} e^{i m\left(\phi-\phi^{\prime}\right)} Q_{m-\frac{1}{2}}(\chi)
$$

where $Q_{m-\frac{1}{2}}$ is a Legendre function of the second kind ${ }^{47}$ of half-integer order $m-\frac{1}{2}[484$, $\S 12.10],[241, \S 8]$ and the variable $\chi$ is defined by:

$$
\chi=\frac{R^{2}+R^{\prime 2}+\left(z-z^{\prime}\right)^{2}}{2 R R^{\prime}}
$$

\footnotetext{
${ }^{47}$ These functions can be implemented in Mathematica ${ }^{\circledR}[242]$ for $\chi \geq 1$ as the Type 3 LegendreQ functions.
} 
where it can be seen that $\chi \geq 1$. Eq. 6.147 can be used to rewrite Eq. 6.142 as

$$
\begin{aligned}
\mathcal{I} & =\frac{1}{\pi \sqrt{R}} \int_{V^{\prime}} d^{3} \underline{r}^{\prime} \frac{1}{\sqrt{R^{\prime}}} \sum_{m=-\infty}^{\infty} e^{i m\left(\phi-\phi^{\prime}\right)} Q_{m-\frac{1}{2}}(\chi) f\left(R^{\prime}, z^{\prime}\right) \\
& =\frac{2}{\sqrt{R}} \iint_{V^{\prime}} d R^{\prime} d z^{\prime} \sqrt{R^{\prime}} Q_{-\frac{1}{2}}(\chi) f\left(R^{\prime}, z^{\prime}\right)
\end{aligned}
$$

where the definite integration with respect to $\phi^{\prime}$ from 0 to $2 \pi$ returns $2 \pi$ times the $m=0$ component, according to the relation $\int_{0}^{2 \pi} d \phi^{\prime} e^{-i m \phi^{\prime}}=2 \pi \delta_{m, 0}$, where $m$ is an integer.

According to Eq. 6.138 it is not the integral $\mathcal{I}$ (proportional to the effective magnetostatic potential) that matters when calculating the secular dipolar field, but rather its derivative $\frac{\partial \mathcal{I}}{\partial z}$ with respect to the field coordinate $z$. The limits of integration in Eq. 6.149 are independent of the coordinate $z$ and therefore it is possible to differentiate under the integral sign, where the only $z$-dependence of the integrand is within $\chi$ :

$$
\begin{aligned}
\frac{\partial \mathcal{I}}{\partial z} & =\frac{\partial}{\partial z}\left[\frac{2}{\sqrt{R}} \iint_{V^{\prime}} d R^{\prime} d z^{\prime} \sqrt{R^{\prime}} Q_{-\frac{1}{2}}(\chi) f\left(R^{\prime}, z^{\prime}\right)\right] \\
& =\frac{2}{\sqrt{R}} \iint_{V^{\prime}} d R^{\prime} d z^{\prime} \sqrt{R^{\prime}}\left[\frac{\partial}{\partial z} Q_{-\frac{1}{2}}(\chi)\right] f\left(R^{\prime}, z^{\prime}\right) .
\end{aligned}
$$

The derivative may be found more simply after re-writing the Legendre function in terms of an elliptic integral $[241, \S 8.13]:^{48}$

$$
Q_{-\frac{1}{2}}(\chi)=\mu K(\mu)
$$

\footnotetext{
${ }^{48}$ The symbols $\chi$ and $\mu$ are used only in order to be consistent with Ref. [485] and do not represent the magnetic susceptibility or magnetic dipole moment.
} 
where $K$ is the complete elliptic integral of the first kind [484, §5.8],[241, §17.3], and

$$
\mu=\sqrt{\frac{2}{\chi+1}}=\sqrt{\frac{4 R R^{\prime}}{\left(R+R^{\prime}\right)^{2}+\left(z-z^{\prime}\right)^{2}}},
$$

where $\mu$ is on the interval $(0,1]$. The derivative of the Legendre function may then be written

$$
\begin{aligned}
\frac{\partial}{\partial z} Q_{-\frac{1}{2}}(\chi) & =\frac{\partial \mu}{\partial z} K(\mu)+\mu \frac{\partial K(\mu)}{\partial \mu} \frac{\partial \mu}{\partial z}=\frac{\partial \mu}{\partial z}\left[K(\mu)+\mu \frac{\partial K(\mu)}{\partial \mu}\right] \\
& =-\frac{1}{8} \frac{z-z^{\prime}}{R R^{\prime}} \mu^{3}\left[K(\mu)+\frac{E(\mu)}{1-\mu}\right]
\end{aligned}
$$

where $E$ is the complete elliptic integral of the second kind $[484, \S 5.8],[241, \S 17.3]$. The derivative of $\mu$ follows from its definition (Eq. 6.152):

$$
\frac{\partial \mu}{\partial z}=-\frac{z-z^{\prime}}{4 R R^{\prime}} \mu^{3}
$$

and the derivative of $K$ follows from the identity [242]:

$$
\frac{\partial K(Z)}{\partial Z}=\frac{E(Z)-(1-Z) K(Z)}{2(1-Z) Z}
$$

Eq. 6.153 may be used to re-write Eq. 6.150 as

$$
\frac{\partial \mathcal{I}}{\partial z}=-\frac{1}{4 R^{3 / 2}} \iint_{V^{\prime}} d R^{\prime} d z^{\prime} \frac{1}{\sqrt{R^{\prime}}}\left(z-z^{\prime}\right) \mu^{3}\left[K(\mu)+\frac{E(\mu)}{1-\mu}\right] f\left(R^{\prime}, z^{\prime}\right) .
$$

This expression allows for a more direct evaluation of the dipolar field in a system that 


\subsection{CALCULATION OF THE DISTANT DIPOLAR FIELD FOR CYLINDRICALLY SYMMETRIC GEOMETRIES}

has symmetry about the $z$ axis; the three dimensional integration over source coordinates has been reduced to a two dimensional integration. Note that an expansion of the Green function in Eq. 6.22 would lead to an expression that is more complicated than the one that is obtained here due to the cube root of $\left|\underline{r}-\underline{r}^{\prime}\right|$ in the integrand.

As a concrete example, consider the case of two coaxial cylinders (tube-within-atube geometry) with the analyte filling the space between the inner and outer cylinders. The $z$ axis defines the symmetry axis. The inner cylinder has radius $R_{1}$ and the outer cylinder has radius $R_{2}$; the analyte magnetization $\underline{M}^{I}\left(\underline{r}^{\prime}\right)$ is distributed from height $z_{1}$ to height $z_{2}$ and is axially symmetric about $z$ (Eq. 6.141). The volume $V^{\prime}$ is defined by the simultaneous constraints $R_{1} \leq R^{\prime} \leq R_{2}$ and $z_{1} \leq z^{\prime} \leq z_{2}$. The boundary surface $S^{\prime}$ can be split into four parts: an inner cylindrical surface at $R^{\prime}=R_{1}$ with $\underline{\widetilde{n}}^{\prime}=-\underline{\widetilde{R}}$, an outer cylindrical surface at $R^{\prime}=R_{2}$ with $\underline{\widetilde{n}}^{\prime}=+\underline{\widetilde{R}}$, a bottom annulus at $z^{\prime}=z_{1}$ with $\underline{\widetilde{n}}^{\prime}=-\underline{\widetilde{z}}$, and a top annulus at $z^{\prime}=z_{2}$ with $\underline{\tilde{n}}^{\prime}=+\underline{\widetilde{z}}$. According to Eq. 6.139 the magnetization at the cylindrical surfaces does not contribute to the surface integral because the vector $\underline{\widetilde{n}}^{\prime}$ normal to these surfaces is perpendicular to $\underline{\widetilde{z}}$. However, the magnetization at the top and bottom surfaces does contribute. If the discussion is limited to the so-called "exterior problem" in which the magnetic field is evaluated only at points $\underline{r}$ that are exterior to the volume $V^{\prime}$ — which includes points within the inner cylinder where the sensor nucleus is contained in the tube-within-a-tube geometry - then $\underline{M}^{I}(\underline{r})$ and hence $M_{z}^{I}(R, z)$ in Eq. 6.138 is zero at the location of every field point. Eq. 6.156 can then be used in conjunction with Eqs. 6.138 and 6.139 to write the following expression for the secular dipolar field exterior to the 
spins $I$ in the tube-within-a-tube geometry:

$$
\begin{aligned}
\underline{B}_{d}^{I, s e c}(R, z)=- & \frac{\mu_{0}}{4 \pi} \frac{1}{4 R^{3 / 2}} \int_{R_{1}}^{R_{2}} d R^{\prime} \int_{z_{1}}^{z_{2}} d z^{\prime}\left\{\frac{1}{\sqrt{R^{\prime}}}\left(z-z^{\prime}\right) \mu^{3}\left[K(\mu)+\frac{E(\mu)}{1-\mu}\right] \frac{\partial M_{z}^{I}\left(R^{\prime}, z^{\prime}\right)}{\partial z^{\prime}}\right\} \\
& -\frac{\mu_{0}}{4 \pi} \frac{1}{4 R^{3 / 2}} \int_{R_{1}}^{R_{2}} d R^{\prime}\left\{\frac{1}{\sqrt{R^{\prime}}}\left(z-z_{1}\right) \mu_{1}^{3}\left[K\left(\mu_{1}\right)+\frac{E\left(\mu_{1}\right)}{1-\mu_{1}}\right] M_{z}^{I}\left(R^{\prime}, z_{1}\right)\right\} \\
& +\frac{\mu_{0}}{4 \pi} \frac{1}{4 R^{3 / 2}} \int_{R_{1}}^{R_{2}} d R^{\prime}\left\{\frac{1}{\sqrt{R^{\prime}}}\left(z-z_{2}\right) \mu_{2}^{3}\left[K\left(\mu_{2}\right)+\frac{E\left(\mu_{2}\right)}{1-\mu_{2}}\right] M_{z}^{I}\left(R^{\prime}, z_{2}\right)\right\},
\end{aligned}
$$

where $\mu$ is defined by Eq. 6.152 and $\mu_{1}$ and $\mu_{2}$ are equal to $\mu$ when evaluated at $z^{\prime}=z_{1}$ or $z^{\prime}=z_{2}$, respectively. The first integral is the volume integral over $V^{\prime}$ and the second and third integrals are those portions of the surface integral over $S^{\prime}$ that include only the bottom or top annular surfaces, respectively. Eq. 6.157 is probably about as far as this problem can be simplified analytically because the integrals are quite difficult. Unlike Eq. 6.26 which is valid only when the magnetization is strongly modulated in one direction, Eq. 6.157 is generally applicable for any axially-symmetric magnetization distribution. However, this technique still seems to remain disadvantageous when compared to the numerical Fourier transform-inverse transform technique because the equation is still nonlocal, i.e., a double integral must be performed over all points $\left(R^{\prime}, z^{\prime}\right)$ within $V^{\prime}$ just to find the field at a single point $(R, z)$. These double integrations can be quite computationally expensive if performed on a finely-discretized grid. One should also note that numerical integrations in cylindrical coordinates are sometimes sensitive to the placement of the grid boundary. ${ }^{49}$ Ref. [485] found the compact Green function expansion (Eq. 6.147) to be insensitive to these

\footnotetext{
${ }^{49}$ Additional errors can occur in the numerical integration if the magnetization distribution is axially symmetric but spheroidal instead of cylindrical, due to the difficulty of properly representing the spheroidal surface in a discretized cylindrical coordinate system.
} 


\subsection{CALCULATION OF THE DISTANT DIPOLAR FIELD FOR CYLINDRICALLY SYMMETRIC GEOMETRIES}

integration errors when calculating potential functions, but Eq. 6.157 actually utilized the gradient of the potential (see Eqs. 6.134, 6.138), and the process of taking the derivative may amplify these errors.

The computation can be made slightly easier if the analyte magnetization distribution is independent of the distance $R$ from the $z$-axis, i.e., $M_{z}^{I}(\underline{r})=M_{z}^{I}(z)$, as is the case in the DDF-encoding experiments described above where transverse magnetization is first modulated by a linear $z$-gradient pulse and then one component is stored along the $z$-axis. In this case the integration over $R^{\prime}$ in Eq. 6.157 can be performed ahead of time given a particular geometry, and then only a single integration need be performed for any further choice of $M_{z}^{I}(z)$. As an example, consider the case of DDF encoding where the magnetization distribution is sinusoidally modulated along $z$, which can be represented generally

as

$$
M_{z}^{I}(z)=M_{0}^{I} \cos (k z+\varphi)
$$

For the purposes of this example the lower and upper limits of the reach of the rf and gradient coils will be taken to be $z_{1}=0$ and $z_{2}=d$, respectively. For the sake of simplicity, further assume that the parameters of the gradient pulse are chosen such that the $z$-component of analyte magnetization vanishes at $z_{1}$ and $z_{2}$, such that

$$
M_{z}^{I}(z)=M_{0}^{I} \sin \left(k_{n} z\right),
$$


where

$$
k_{n}=\frac{n \pi}{d}
$$

and $n$ is an integer. In this case the $z$-magnetization vanishes at $z_{1}$ and $z_{2}$ and so do the surface integrals in Eq. 6.157. The exterior solution of the analyte dipolar field then becomes

$$
\underline{B}_{d}^{I, s e c}(R, z)=-\frac{\mu_{0}}{4 \pi} M_{0}^{I} \frac{k_{n}}{4 R^{3 / 2}} \int_{0}^{d} d z^{\prime} \cos \left(k_{n} z^{\prime}\right) F\left(z^{\prime} ; z\right)
$$

where the relation $\frac{\partial M_{z}^{I}\left(z^{\prime}\right)}{\partial z^{\prime}}=k_{n} M_{0}^{I} \cos \left(k_{n} z\right)$ has been used, and

$$
F\left(z^{\prime} ; z\right)=\left(z-z^{\prime}\right) \int_{R_{1}}^{R_{2}} d R^{\prime} \frac{1}{\sqrt{R^{\prime}}} \mu^{3}\left[K(\mu)+\frac{E(\mu)}{1-\mu}\right]
$$

Note that Eq. 6.161 can be re-written as

$$
\underline{B}_{d}^{I, s e c}(R, z)=-\frac{\mu_{0}}{4 \pi} M_{0}^{I} \frac{k_{n}}{4 R^{3 / 2}} \int_{-\infty}^{\infty} d z^{\prime} \cos \left(k_{n} z^{\prime}\right) F\left(z^{\prime} ; z\right)\left[h\left(z^{\prime}\right)-h\left(z^{\prime}-d\right)\right]
$$

where the term in square brackets is a boxcar function defined in terms of the Heaviside step function, $h\left(x-x_{0}\right)$, which equals 1 when $x>x_{0}$ and equals 0 when $x<x_{0}$. The boxcar function equals 1 when $0<z^{\prime}<d$ and equals 0 otherwise. The integral in Eq. 6.163 is just the Fourier cosine transform with respect to $z^{\prime}$ (and with fixed $k_{n}$ ) of the product of $F\left(z^{\prime} ; z\right)$ and the boxcar function. The Fourier convolution theorem $[484, \S 15.5]$ states that this integral is just the convolution of the Fourier transform of $F\left(z^{\prime} ; z\right)$ with the Fourier 


\subsection{DEPLETION AND DIFFUSION OF XENON MAGNETIZATION}

DURING MULTI-PULSE BATCH MODE EXPERIMENTS

transform of the boxcar function. The Fourier transform of a boxcar function is known to be a sinc function, but the Fourier transform of $F\left(z^{\prime} ; z\right)$ is not readily calculated.

\subsection{Depletion and diffusion of xenon magnetization during multi-pulse batch mode experiments}

The key difference between an ordinary point-by-point (or multidimensional) NMR experiment and a batch-mode experiment on a hyperpolarized sample is that the hyperpolarization does not return to its initial value during the recycle delay between the transient acquisitions. Rather, this non-equilibrium longitudinal magnetization is continuously depleted both by relaxation to its comparatively small thermal equilibrium value and by conversion to transverse magnetization by rf pulses. Consequently, only a finite number of indirectly-encoded points can be acquired before the magnetization returns to its thermal equilibrium value, which in the case of xenon gas is too small to be detected efficiently. Furthermore, any decay of the magnetization over the course of a point-by-point experiment results in a damping of the indirect time-domain signal that manifests itself as line broadening in the frequency domain that decreases spectral resolution. The imaging community has developed variable tip-angle rf pulse schemes that counter these types of effects by progressively increasing the fraction of longitudinal magnetization that is tipped into the transverse plane in order to maintain the transverse magnetization after each pulse at a constant value $[487,488,489]$. These schemes, however, have relied on the assumption that the rf pulses always act on the same volume of magnetization, i.e., either the entire sample resides within the $\mathrm{rf}$ coil or magnetization is not transported into and out of the coil 


\subsection{DEPLETION AND DIFFUSION OF XENON MAGNETIZATION DURING MULTI-PULSE BATCH MODE EXPERIMENTS}

region by molecular diffusion. Diffusion effects are expected to be considerable in gas phase samples; a proper model of these effects can lead to the development of variable tip-angle pulse schemes that can lead to line narrowing in the indirect dimension in point-by-point batch-mode xenon NMR experiments. This section explores some simple analytic models of the effects of one-dimensional diffusion on multiple-pulse experiments in cylindrical tubes; the experiment considered here consists of an array of evenly-spaced pulses of constant tip angle. Another possible approach to the problem of depleted magnetization might be to use a model-free feedback experiment to generate the variable tip angles, but this approach is not considered here. Note that this section only discusses the diffusion of xenon during intervals in between the encoding of indirect points; it does not consider the substantial effects of diffusion during the encoding step itself in DDF indirect detection experiments.

\subsubsection{Limit of extremely slow diffusion}

An ideal rf coil generates a homogeneous magnetic field near the coil in what will be referred to as the "active" region of the sample and a negligible field outside this volume. It may be assumed that only spins within the active region are affected by rf pulses, and reciprocally, only the net magnetization of the spins within this region is subsequently detected by the coil. The simplest situation to consider is the case in which the diffusion of magnetized spins in and out of the active region is negligible over the timescale of the NMR experiment. In other words, over the course of the point-by-point experiment only the same set of spins is pulsed and detected. This is the same situation as would be encountered if there was significant diffusion but the rf coil was large enough to include the entire sample in the region of homogeneous rf field, because transport of magnetization between different 


\subsection{DEPLETION AND DIFFUSION OF XENON MAGNETIZATION DURING MULTI-PULSE BATCH MODE EXPERIMENTS}

parts of the sample would not change the net magnetization within the active region. The variable tip angle scheme has already been solved for this case [489] and the result is reviewed here.

Consider an experiment that consists of an array of $N$ rf pulses with the same phase and inter-pulse delay $\Delta$. This situation could be considered to correspond to a series of detection pulses in a point-by-point encoding experiment separated by a constant recycle delay, where the maximum indirect evolution time is assumed to be negligible compared to the recycle delay. The theoretical treatment is easily modified to include variable delays between the pulses. The pulses are labeled by an index $n$ ranging from 0 to $N-1$ and the $n$th pulse has a tip angle $\theta_{n}$. Initially it will be assumed that all the tip angles are equal, $\theta_{n}=\theta$, as is the case in a normal point-by-point/multidimensional encoding experiment. It will be assumed that $\theta$ is on the interval $[0, \pi / 2]$. Let $M_{z}^{A}$ represent the longitudinal magnetization within the active region of the sample at time $t$. The notation

$$
t_{n}=n \Delta
$$

will denote the time of the $n$th pulse and $t_{n}^{-}$and $t_{n}^{+}$will denote the times just before and just after the application of that pulse, respectively.

The initial longitudinal magnetization is:

$$
M_{z}^{A}\left(t_{0}^{-}\right)=M_{0} .
$$

After the first rf pulse of tip angle $\theta$ is applied a fraction $\cos \theta$ of the initial magnetiza- 


\subsection{DEPLETION AND DIFFUSION OF XENON MAGNETIZATION} DURING MULTI-PULSE BATCH MODE EXPERIMENTS

tion remains along the $z$-axis and a fraction $\sin \theta$ is converted to transverse magnetization which is assumed to be irreversibly dephased by $T_{2}$ relaxation before the next pulse. The longitudinal magnetization just after the first pulse is:

$$
M_{z}^{A}\left(t_{0}^{+}\right)=M_{0} \cos \theta .
$$

The general relation between the longitudinal magnetization just before and just after an rf pulse of tip angle $\theta$ is:

$$
M_{z}^{A}\left(t_{n}^{+}\right)=M_{z}^{A}\left(t_{n}^{-}\right) \cos \theta .
$$

If longitudinal relaxation is neglected for the moment, the longitudinal magnetization following the delay $\Delta$ between the first and second pulses is:

$$
M_{z}^{A}\left(t_{1}^{-}\right)=M_{0} \cos \theta .
$$

After the second pulse:

$$
M_{z}^{A}\left(t_{1}^{+}\right)=\left[M_{0} \cos \theta\right] \cos \theta=M_{0} \cos ^{2} \theta
$$

For future convenience, make the definition:

$$
\alpha=\cos \theta
$$


It is easy to see that a general recursion relationship can be written:

$$
M_{z}^{A}\left(t_{n+1}^{-}\right)=\alpha M_{z}^{A}\left(t_{n}^{-}\right)
$$

such that:

$$
M_{z}^{A}\left(t_{n}^{-}\right)=\alpha^{n} M_{z}^{A}\left(t_{0}^{-}\right)=[\cos \theta]^{n} M_{0}
$$

If longitudinal relaxation during the inter-pulse delay $\Delta$ is to be included, Eq. 6.171 must be modified:

$$
M_{z}^{A}\left(t_{n+1}^{-}\right)=\alpha M_{z}^{A}\left(t_{n}^{-}\right) e^{-\Delta / T_{1}}+M_{e q}\left[1-e^{-\Delta / T_{1}}\right]
$$

where $T_{1}$ is the longitudinal relaxation time and $M_{e q}$ is value of the thermal equilibrium magnetization. For experiments with hyperpolarized nuclei it can be assumed that the initial magnetization is much larger than the thermal equilibrium magnetization, i.e., $M_{0} \gg M_{e q}$; in fact, $M_{e q}$ will be assumed to be so small as to be below the measurement threshold. In that case:

$$
M_{z}^{A}\left(t_{n+1}^{-}\right) \approx \alpha M_{z}^{A}\left(t_{n}^{-}\right) e^{-\Delta / T_{1}}
$$

and

$$
M_{z}^{A}\left(t_{n}^{-}\right)=\alpha^{n} M_{z}^{A}\left(t_{0}\right) e^{-t_{n} / T_{1}}
$$


where $\left[e^{-\Delta / T_{1}}\right]^{n}=e^{-n \Delta / T_{1}}=e^{-t_{n} / T_{1}}$. The magnitude of the transverse magnetization immediately following each pulse is:

$$
\left|M_{+}^{A}\left(t_{n}^{+}\right)\right|=M_{z}^{A}\left(t_{n}^{-}\right) \sin \theta
$$

The signal detected after each pulse is therefore equal to:

$$
S\left(t_{n}^{+}\right)=\left[S_{0} \sin \theta\right] \alpha^{n} e^{-t_{n} / T_{1}},
$$

where $S_{0}$ is the signal that would be detected if the entire initial magnetization $M_{0}$ was tipped into the transverse plane. This signal can be written as

$$
S\left(t_{n}^{+}\right)=\left[S_{0} \sin \theta\right] e^{-t_{n} / \tau}
$$

where

$$
\begin{aligned}
\tau & =\left[\frac{1}{T_{1}}+\frac{-\Delta}{\ln \alpha}\right]^{-1} \\
& =\left[\frac{1}{T_{1}}+\frac{-\Delta}{\ln (\cos \theta)}\right]^{-1}
\end{aligned}
$$

where the relation $\alpha^{n}=\alpha^{t_{n} / \Delta}=\left[e^{\ln \alpha}\right]^{t_{n} / \Delta}=\exp \left[\frac{\ln \alpha}{\Delta} t_{n}\right]$ has been used. The second term in brackets in Eq. 6.179 is greater than zero since $0<\theta<\pi / 2$ and $\Delta>0$. It follows that Eq. 6.178 predicts the signal after each evenly-spaced pulse decays exponentially with an effective relaxation time $\tau<T_{1}$ that approaches $T_{1}$ for small tip angles. The decay is faster than the longitudinal relaxation rate is due to the fact that longitudinal magnetization is 


\subsection{DEPLETION AND DIFFUSION OF XENON MAGNETIZATION DURING MULTI-PULSE BATCH MODE EXPERIMENTS}

continuously being depleted not only by relaxation but also by the succession of $\mathrm{rf}$ pulses. If the array of rf pulses represents the series of detection pulses in a point-by-point experiment, the exponential decay of Eq. 6.178 multiplies the indirect time-domain signal, resulting in an effective indirect spectrum that is the convolution of the desired spectrum with a Lorentzian of half-width at half-maximum $\tau$.

Fig. 6.14 shows a comparison of the simulated depletion of the signal observed immediately after each pulse in the case of a negligibly small tip angle (solid line), which exhibits pure longitudinal relaxation, and in the case of a constant tip angle of $\theta=22.5^{\circ}$ in the presence of longitudinal relaxation with no diffusion (dotted line).

The extra line broadening caused by longitudinal relaxation and depletion of magnetization by rf pulses may be removed by adopting a variable tip-angle scheme. The generalization of Eq. 6.171 to the case of variable tip angles $\theta_{n}$ is:

$$
M_{z}^{A}\left(t_{n+1}^{-}\right)=\alpha_{n} M_{z}^{A}\left(t_{n}^{-}\right)
$$

where

$$
\alpha_{n}=\cos \theta_{n} .
$$

The generalization of Eq. 6.175 is:

$$
M_{z}^{A}\left(t_{n}^{-}\right)=\left[\prod_{j=0}^{n-1} \alpha_{j}\right] M_{z}^{A}\left(t_{0}^{-}\right) e^{-t_{n} / T_{1}}
$$

The product in brackets reduces to $\alpha^{n}$ when all $\alpha_{j}=\alpha($ for $n>0)$ and will be assumed to 


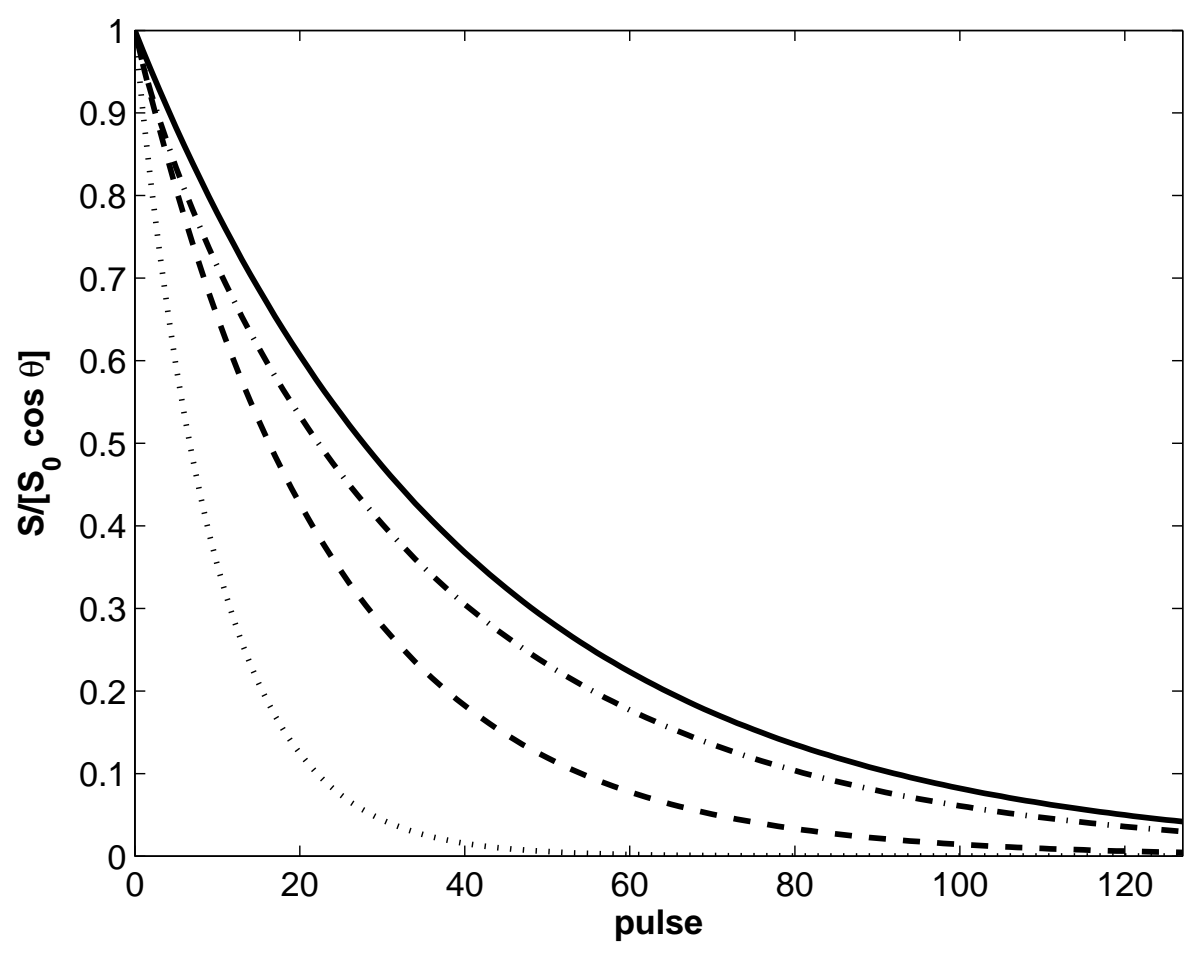

Figure 6.14: Simulated decay of observed signal in multiple-pulse, constant tip-angle batch Xe NMR experiments in a long tube. All decay curves were normalized to an initial value of 1 and calculated using a longitudinal relaxation time of $T_{1}=40 \mathrm{~min}$. A total of $N=128$ pulses equally spaced by the interval $\Delta=60 \mathrm{~s}$ were used. The solid line indicates the limit of negligibly small tip angles where the decay of signal is purely due to exponential longitudinal relaxation. The rest of the curves were calculated using a constant tip angle of $\theta=\pi / 8=22.5^{\circ}$. The dotted line indicates the limit of no gas diffusion. The dashed line indicates the limit of fast diffusion with local equilibration of magnetization in the infinite tube approximation using an active region length of $2 d=1.5 \mathrm{~cm}$ and a Xe diffusion coefficient of $D=5.7 \times 10^{-2} \mathrm{~cm}^{2} \mathrm{~s}^{-1}$ at $1 \mathrm{~atm}$ and room temperature $\left(k_{1}=0.23\right)$. The dot-dash line indicates the limit of fast diffusion with extended diffusion of magnetization using the same values of $d$ and $D$.

equal 1 when $n=0$. The generalization of Eq. 6.177 for the signal immediately after the $n$th pulse is:

$$
S\left(t_{n}^{+}\right)=\left[S_{0} \sin \theta_{n}\right]\left[\prod_{j=0}^{n-1} \alpha_{j}\right] e^{-t_{n} / T_{1}}
$$




\subsection{DEPLETION AND DIFFUSION OF XENON MAGNETIZATION}

The signal immediately after the first pulse is $S\left(t_{0}^{+}\right)=S_{0} \sin \theta_{0}$; the signal immediately after every subsequent pulse can be maintained at this value by choosing the tip angles to be solutions of $S\left(t_{n}^{+}\right)=S_{0} \sin \theta_{0}[489]$ :

$$
\theta_{n}=\sin ^{-1}\left[\frac{\sin \theta_{0}}{e^{-t_{n} / T_{1}} \prod_{j=0}^{n-1} \alpha_{j}}\right]=\sin ^{-1}\left[\frac{\sin \theta_{0}}{e^{-n \Delta / T_{1}} \prod_{j=0}^{n-1} \cos \theta_{j}}\right]
$$

If there is no longitudinal relaxation $\left(T_{1} \rightarrow \infty\right)$, Eq. 6.184 reduces to

$$
\theta_{n}=\tan ^{-1}\left[\frac{1}{\sqrt{(N-1)-n}}\right]
$$

Note that Eq. 6.185 predicts $\theta_{N-1}=\pi / 2$, i.e., the final rf pulse uses up the remaining longitudinal magnetization by tipping it $90^{\circ}$ into the transverse plane.

Note that in any variable tip angle scheme there is a restriction on the number of pulses that can be used depending on how much signal is desired. If the signal $S_{0} \cos \theta_{0}$ is large, relatively few pulses can be applied before the magnetization is used up.

The preceding discussion only gave consideration to the situation in which the xenon diffusion plays no role over the course of the experiment. The rest of the discussion will focus on simple models of the role of diffusion. 


\subsubsection{Limit of extremely fast diffusion with global equilibration of mag- netization}

Consider a cylindrical NMR tube of length $2 L$ filled with xenon, with an active rf coil region of length $2 d$. The ratio of the two lengths is

$$
R=d / L
$$

which is also the ratio of the tube volume affected by the rf coil to the total tube volume. It will be useful to define a diffusion length $\lambda_{t}$ that characterizes the distance over which gas diffuses during some time $t$. The usual definition of the one-dimensional diffusion length is [490]:

$$
\lambda_{t}=2 \sqrt{D t}
$$

where $D$ is the diffusion coefficient. If $t$ is discretized into increments of $\Delta$, the following quantity can be defined:

$$
\lambda_{n}=2 \sqrt{D(n \Delta)}
$$

which corresponds to the diffusion length over the time $t_{n}=n \Delta$. An "extremely fast diffusion" regime can be roughly defined by the condition $L \ll \lambda_{1}$, i.e., the gas diffuses completely over the length of the tube during the interval $\Delta$ between pulses. This condition actually is not expected to hold for typical xenon experiments: using a value of $D=$ 


\subsection{DEPLETION AND DIFFUSION OF XENON MAGNETIZATION} DURING MULTI-PULSE BATCH MODE EXPERIMENTS

$5.7 \times 10^{-2} \mathrm{~cm}^{2} \mathrm{~s}^{-1}$ for $1 \mathrm{~atm}$ of xenon gas at room temperature (Eq. 6.130) and a long delay $\Delta=60 \mathrm{~s}$ yields $\lambda_{1}=3.7 \mathrm{~cm}$, which is smaller than the length of a typical NMR tube, $2 L=15 \mathrm{~cm}$. However, the "extremely fast diffusion" regime is the simplest to treat theoretically, so it is worth mentioning briefly.

The same case as before will be considered: $N$ rf pulses of constant tip angle $\theta$ labeled from $n=0$ to $N-1$ that are evenly separated by intervals $\Delta$. All net transverse magnetization is assumed to be irreversibly dephased between pulses. Let $M_{z}^{A}$ represent the longitudinal magnetization in the active region of the tube and $M_{z}^{R}$ represent the longitudinal magnetization in the rest of the tube. Only $M_{z}^{A}$ is depleted by the rf pulses and Eq. 6.167 still holds:

$$
M_{z}^{A}\left(t_{n}^{+}\right)=M_{z}^{A}\left(t_{n}^{-}\right) \cos \theta
$$

The magnetization outside the active region is unaffected by the pulses:

$$
M_{z}^{R}\left(t_{n}^{+}\right)=M_{z}^{R}\left(t_{n}^{-}\right)
$$

If the diffusion is extremely fast the longitudinal magnetization at time $t_{n}^{+}$completely equilibrates throughout the tube by time $t_{n+1}^{-}$. This magnetization density is the volumeweighted average of the magnetization density inside and outside of the active region. Let $V_{A}=(2 d) A$ and $V_{R}=(2 L) A-V_{A}$ be the volumes inside and outside of the active region, respectively, where $A$ is the area of the cross section of the tube. The following relation can 
be written:

$$
\begin{aligned}
M_{z}^{A}\left(t_{n+1}^{-}\right)=M_{z}^{R}\left(t_{n+1}^{-}\right) & =\frac{M_{z}^{A}\left(t_{n}^{+}\right) V_{A}+M_{z}^{R}\left(t_{n}^{+}\right) V_{R}}{V_{A}+V_{R}} \\
& =\left[M_{z}^{A}\left(t_{n}^{-}\right) \cos \theta\right] R+\left[M_{z}^{R}\left(t_{n}^{-}\right)\right](1-R),
\end{aligned}
$$

where Eqs. 6.189 and 6.190 have been used to write the last line. Since the extremely fast diffusion ensures that $M_{z}^{A}\left(t_{n}^{-}\right)=M_{z}^{R}\left(t_{n}^{-}\right)$at the point just before each rf pulse, it is possible to write:

$$
M_{z}^{A}\left(t_{n+1}^{-}\right)=\alpha M_{z}^{A}\left(t_{n}^{-}\right)
$$

where

$$
\begin{aligned}
\alpha & =R \cos \theta+(1-R) \\
& =1-2 R \sin ^{2}(\theta / 2) .
\end{aligned}
$$

The recursion relation in Eq. 6.192 is the same as in Eq. 6.171 and all the subsequent results that follow from that equation hold here. Once longitudinal relaxation is included, the signal immediately after each pulse is found to be:

$$
S\left(t_{n}^{+}\right)=\left[S_{0} \sin \theta\right] e^{-t_{n} / \tau}
$$


where

$$
\begin{aligned}
\tau & =\left[\frac{1}{T_{1}}+\frac{-\Delta}{\ln \alpha}\right]^{-1} \\
& =\left[\frac{1}{T_{1}}+\frac{-\Delta}{\ln \left[1-2 R \sin ^{2}(\theta / 2)\right]}\right]^{-1}
\end{aligned}
$$

Since $R$ is on the interval [0,1] and $\theta$ is on the interval [0, $\pi / 2]$, the value of $\alpha$ in Eq. 6.193 is on the interval $[0,1]$ and is always greater or equal to the value of $\alpha$ in Eq. 6.170. Therefore the effective exponential damping of the signal in the extremely fast diffusion case (Eq. 6.194) has a longer time constant (i.e., closer to the intrinsic relaxation time $T_{1}$ ) than in the case where there is no diffusion (Eq. 6.178). This is to be expected: the longitudinal magnetization within the active region that is depleted by rf pulses is partially replenished by diffusional equilibration with fresh magnetization in the rest of the tube.

Note that $\tau \rightarrow T_{1}$ in Eq. 6.195 as $\theta \rightarrow 0$. This indicates that an array of evenlyspaced rf pulses of sufficiently small tip angle can be used to determine the intrinsic longitudinal relaxation time of the sample, $T_{1}$, independent of the sample geometry (as long as the extremely fast diffusion limit holds). The results of two or more constant- $\theta$ array experiments with different finite values of $\theta$ can be used to fit Eq. 6.195 to obtain $T_{1}$ and $R$. If these values are known, a variable tip angle scheme analogous to the one given by Eq. 6.184 can be implemented using the modified definition:

$$
\alpha_{n}=1-2 R \sin ^{2}\left(\theta_{n} / 2\right)
$$




\subsection{DEPLETION AND DIFFUSION OF XENON MAGNETIZATION DURING MULTI-PULSE BATCH MODE EXPERIMENTS}

\subsubsection{Limit of fast diffusion with local equilibration of magnetization}

Although the "extremely fast diffusion" model is simple and indicates how molecular diffusion can extend the "lifetime" of hyperpolarized signal, the diffusion rate of xenon gas under normal conditions is not fast enough to place the system in this limit. If the diffusion is somewhat slower, one can consider the "fast diffusion limit." This limit is roughly characterized by the condition $d \ll \lambda_{1} \ll L$ where the diffusion between pulses causes the magnetization to equilibrate across the active region of the tube but not across the whole tube. It will be necessary to consider the effects of diffusion on the magnetization distribution in more detail. Once again the problem will be to follow the distribution of the longitudinal magnetization $M_{z}^{A}(z, t)$ in the active region of the coil over the course of the tip angle array, where $z$ denotes the position along the tube axis. The longitudinal magnetization in the rest of the tube (i.e., outside the active region) will be denoted $M_{z}^{R}(z, t)$. The initial magnetization distribution is assumed to be uniform over the tube, $M_{z}^{A}(z, 0)=M_{z}^{R}(z, 0)=M_{0}$.

Before tackling the diffusion problem head-on, imagine the case in which the longitudinal magnetization has some constant value $M_{z}^{A}(z, 0)=M_{z}^{A}$ inside the active region of the tube and another constant value $M_{z}^{R}(z, 0)=M_{z}^{R}$ outside the active region. Assume for now that the active region is at the center of the tube so that its position defined by $-d<z<d$ ) and the external region is defined by $d<z<L$ and $-L<z<-d$. The solution of the one-dimensional diffusion equation for the magnetization distribution 
everywhere in the tube at time $t$ is known analytically [490, §2.2.4]:

$$
M_{z}(z, t)=M_{z}^{R}+\frac{1}{2}\left(M_{z}^{A}-M_{z}^{R}\right) \sum_{m=-\infty}^{+\infty}\left[\operatorname{erf}\left(\frac{d+2 m L-z}{\lambda_{t}}\right)+\operatorname{erf}\left(\frac{d-2 m L+z}{\lambda_{t}}\right)\right]
$$

where $\operatorname{erf}(\mathrm{x})$ is the error function and $\lambda_{t}$ is given by Eq. 6.187. If the diffusion is not "extremely" fast, i.e., it is slow enough that the condition $\lambda_{t} \ll L$ holds, the magnetization gradient between the active region and the rest of the tube will not have time to fully equilibrate over the length of the tube, $2 L$, during the time $t$. In this case the tube can be considered to be effectively "infinitely" long. In this limit it does not matter whether or not the active region is at the center of the tube. Under the same initial conditions the magnetization everywhere in an infinitely long tube is:

$$
M_{z}(z, t)=M_{z}^{R}+\frac{1}{2}\left(M_{z}^{A}-M_{z}^{R}\right)\left[\operatorname{erf}\left(\frac{d-z}{\lambda_{t}}\right)+\operatorname{erf}\left(\frac{d+z}{\lambda_{t}}\right)\right]
$$

If the diffusion is fast enough that $\lambda_{t} \gg d$, the argument of the error function is small near the active region of the tube where $|z| \sim d$. In the limit $x \ll 1$ the error function may be approximated as:

$$
\operatorname{erf}(x) \approx \frac{2}{\sqrt{\pi}} x
$$


In this limit Eq. 6.198 becomes:

$$
M_{z}(z, t) \approx M_{z}^{R}+\frac{2 d}{\sqrt{\pi} \lambda_{t}}\left(M_{z}^{A}-M_{z}^{R}\right)=\left(1-k_{t}\right) M_{z}^{R}+k_{t} M_{z}^{A}
$$

where

$$
k_{t} \equiv \frac{2 d}{\sqrt{\pi} \lambda_{t}}
$$

Eq. 6.200 states that if the diffusion is fast on a timescale $t$ over the length $d$, the resulting magnetization distribution is approximately uniform (i.e., independent of $z$ ) in regions near the boundaries of the active region of the tube. In other words, in the fast diffusion limit all of the active region is assumed to be "near" the boundary, and $M_{z}^{R}$ is then taken to represent the magnetization outside of but close to the active region. It will be assumed that the diffusion of magnetization in parts of the tube that are far from the active region does not contribute to the magnetization distribution near the active region. This assumption results from the infinite tube approximation and breaks down when the pulse array is long enough that the characteristic diffusion length over the course of the array is on the order of the half-length of the tube, $\lambda_{N-1}=2 \sqrt{D(N-1) \Delta} \sim L$, in which case the full Eq. 6.197 must be used.

Now consider what happens when $\theta$-tip angle pulses are applied at the times $t_{n}=n \Delta$, where $n$ ranges from 0 to $N-1$. Relaxation effects will be neglected for now. The condition of "fast diffusion" over the inter-pulse interval $\Delta$, previously defined as $\lambda_{1} \ll L$, 
is more accurately defined to be:

$$
k_{1}=\frac{2 d}{\sqrt{\pi} \lambda_{1}} \ll 1
$$

where $\lambda_{1}=2 \sqrt{D \Delta}$. This defines the criterion under which the diffusion distributes magnetization approximately uniformly across the active region of the tube. The value of this constant for a sample of xenon gas at 1 atm pressure and room temperature with an active region of $2 d=1.5 \mathrm{~cm}$ is $k_{1}=0.23$ for $\Delta=60 \mathrm{~s}$ and $k_{1}=0.46$ for $\Delta=15 \mathrm{~s}$.

The longitudinal magnetization before the first pulse in the array is:

$$
M_{z}^{A}\left(z, t_{0}^{-}\right)=M_{z}^{R}\left(z, t_{0}^{-}\right)=M_{0}
$$

After the first pulse:

$$
\begin{aligned}
& M_{z}^{A}\left(z, t_{0}^{+}\right)=M_{0} \cos \theta \\
& M_{z}^{R}\left(z, t_{0}^{+}\right)=M_{0},
\end{aligned}
$$

i.e., the pulse tips the magnetization inside the active region of the tube but does not affect the magnetization outside the tube. Eq. 6.200 for the local magnetization in the fast diffusion limit can be used to calculate the magnetization near the tube at time $t_{1}^{-}=\Delta$ before the next pulse:

$$
\begin{aligned}
& M_{z}^{A}\left(z, t_{1}^{-}\right) \approx\left(1-k_{1}\right) M_{0}+k_{1} M_{0} \cos \theta \\
& M_{z}^{R}\left(z, t_{1}^{-}\right) \approx\left(1-k_{1}\right) M_{0}+k_{1} M_{0} \cos \theta
\end{aligned}
$$




\subsection{DEPLETION AND DIFFUSION OF XENON MAGNETIZATION} DURING MULTI-PULSE BATCH MODE EXPERIMENTS

$M_{z}^{R}\left(z, t_{1}^{-}\right)$is only defined for locations $|z| \sim d$ near the active region. The position label $z$ will be suppressed from now on. It is apparent from these equations that magnetization that is depleted in the active region of the coil by the pulse is partially replenished by local equilibration with magnetization outside of but near to the active region.

After the second pulse the longitudinal magnetization distribution is approximately equal to:

$$
\begin{aligned}
& M_{z}^{A}\left(t_{1}^{+}\right)=\left[\left(1-k_{1}\right) M_{0}+k_{1} M_{0} \cos \theta\right] \cos \theta \\
& M_{z}^{R}\left(t_{1}^{+}\right)=\left(1-k_{1}\right) M_{0}+k_{1} M_{0} \cos \theta,
\end{aligned}
$$

and after diffusion over the interval $\Delta$ :

$$
\begin{aligned}
& M_{z}^{A}\left(t_{2}^{-}\right)=\left(1-k_{1}\right)\left[\left(1-k_{1}\right) M_{0}+k_{\Delta} M_{0} \cos \theta\right]+k_{1}\left\{\left[\left(1-k_{1}\right) M_{0}+k_{1} M_{0} \cos \theta\right] \cos \theta\right\} \\
& M_{z}^{R}\left(t_{2}^{-}\right)=\left(1-k_{1}\right)\left[\left(1-k_{1}\right) M_{0}+k_{1} M_{0} \cos \theta\right]+k_{1}\left\{\left[\left(1-k_{1}\right) M_{0}+k_{1} M_{0} \cos \theta\right] \cos \theta\right\} .
\end{aligned}
$$

The recursive solution for the longitudinal magnetization inside the active region at time $t_{n+1}^{-}$in terms of $t_{n}^{-}$is:

$$
\begin{aligned}
M_{z}^{A}\left(t_{n+1}^{-}\right) & =\left(1-k_{1}\right) M_{z}^{R}\left(t_{n}^{+}\right)+k_{1} M_{z}^{A}\left(t_{n}^{+}\right) \\
& =\left(1-k_{1}\right) M_{z}^{R}\left(t_{n}^{-}\right)+k_{1} M_{z}^{A}\left(t_{n}^{-}\right) \cos \theta \\
& =\left[k_{1}(\cos \theta-1)+1\right] M_{z}^{A}\left(t_{n}^{-}\right) \\
& \equiv \alpha M_{z}^{A}\left(t_{n}^{-}\right)
\end{aligned}
$$


where the relation $M_{z}^{A}\left(t_{n}^{-}\right)=M_{z}^{R}\left(t_{n}^{-}\right)$has been used and

$$
\begin{aligned}
\alpha & =k_{1}(\cos \theta-1)+1 \\
& =1-2 k_{1} \sin ^{2}(\theta / 2) .
\end{aligned}
$$

The inequality $0<\alpha<1$ holds in the fast diffusion limit where $d / \lambda_{1} \ll 1$.

The recursion relation in Eq. 6.208 is familiar (see Eqs. 6.171, 6.192) and once longitudinal relaxation is included, the signal immediately after the $n$th pulse is found to be:

$$
S\left(t_{n}^{+}\right)=\left[S_{0} \sin \theta\right] e^{-t_{n} / \tau}
$$

where the effective decay constant of the signal is defined by:

$$
\begin{aligned}
\tau & =\left[\frac{1}{T_{1}}+\frac{-\Delta}{\ln \alpha}\right]^{-1} \\
& =\left[\frac{1}{T_{1}}+\frac{-\Delta}{\ln \left[1-\frac{2 d}{\sqrt{\pi D \Delta}} \sin ^{2}(\theta / 2)\right]}\right]^{-1}
\end{aligned}
$$

Since $\alpha$ may be presumed to be on the interval $[0,1]$ in the fast diffusion limit, $\ln \alpha$ is on $(-\infty, 0]$ and $\tau<T_{1}$. The rf pulses still cause an effective exponential decay of the signal that is faster than pure $T_{1}$ relaxation alone, but diffusion again increases the effective relaxation time of the magnetization somewhat by replenishing magnetization in the active region of the tube that has been depleted by the rf pulses. Unlike the "extremely fast" diffusion model, the "fast diffusion/local equilibration" model depends on parameters such as the 


\subsection{DEPLETION AND DIFFUSION OF XENON MAGNETIZATION}

DURING MULTI-PULSE BATCH MODE EXPERIMENTS

diffusion coefficient and the absolute length of the active region of the tube. Note, however, that these two models predict the same result if the tube length $2 L$ in the extremely fast diffusion model is replaced in the local equilibration model by the quantity $\sqrt{\pi} \lambda_{1}$, which is on the order of the diffusion length $\lambda_{1}$ over the inter-pulse delay $\Delta$. The physical content of these two models is the same, the only difference is that the first model assumes that the magnetization equilibrates over the entire length of the tube during $\Delta$ and the second model predicts that the gas equilibrates only over the characteristic length $\sqrt{\pi} \lambda_{1}<2 L$ during this time.

Fig. 6.14 shows the simulated depletion of the signal observed immediately after each pulse in the case of a constant tip angle of $\theta=22.5^{\circ}$ in the presence of longitudinal relaxation with fast diffusion $\left(k_{1}=0.23\right)$ in the local equilibration model (dashed line). The signal decay — which is exponential — is slower than in the case of no diffusion (dotted line) because the magnetization in the active region of the tube is continually refreshed by an external-but local - reservoir of magnetization that is not depleted by rf pulses.

\subsubsection{Limit of fast diffusion with extended diffusion of magnetization}

The fast diffusion/local equilibration model predicts an apparent exponential damping of the signal in the periodic multi-pulse experiment. However, as will be discussed later in this section, experiments performed in the fast diffusion regime indicate that the true decay of the signal is non-exponential, and perhaps is biexponential. The failure of the local equilibration model is most likely due to its inability to capture the long-time behavior of the diffusion. In this model the magnetization distribution near the active region is always uniform; as a result, as the magnetization near this region becomes more and more 


\subsection{DEPLETION AND DIFFUSION OF XENON MAGNETIZATION DURING MULTI-PULSE BATCH MODE EXPERIMENTS}

depleted, a magnetization gradient is established between this region and parts of the tube that are somewhat farther away. Over the course of a long experiment with many pulses this magnetization gradient may become quite large and physically one expects diffusion to help to "average" this gradient. In order to fully explain the diffusion in the tube it is necessary to develop a model that includes some features of long-time/long-range diffusion throughout the tube.

The restriction that the diffusion after each pulse is local and occurs only over a short time $\Delta$ will now be lifted. The diffusion will still be assumed to be slow enough that the tube can be considered to be infinite, i.e., there will be no "reflections" of diffusing magnetization that is incident on the top or bottom of the tube. It will be assumed that in the fast diffusion limit the magnetization is approximately uniform across the active region of the tube at the discrete times $t_{n}=n \Delta$. An rf pulse applied at $t_{n}$ therefore will be assumed to "burn" a rectangular hole in the longitudinal magnetization over the length of the active region of the tube, and the magnetization at the time of detection will be assumed to be constant over the active region.

Eq. 6.198 can be used to track the diffusion of an initial square "plug" of magnetization over time in an infinitely long tube. Define a function $g_{n}(z)$ to be:

$$
g_{n}(z)=\frac{1}{2}\left[\operatorname{erf}\left(\frac{d-z}{\lambda_{n}}\right)+\operatorname{erf}\left(\frac{d+z}{\lambda_{n}}\right)\right]
$$

where $\lambda_{n}$ is defined according to Eq. 6.188. Define another function $\sigma(z)$ to be:

$$
\sigma(z)=h(z+d)-h(z-d)
$$




\subsection{DEPLETION AND DIFFUSION OF XENON MAGNETIZATION} DURING MULTI-PULSE BATCH MODE EXPERIMENTS

where the Heaviside step function, $h\left(x-x_{0}\right)$, equals 1 when $x>x_{0}$ and equals 0 when $x<x_{0}$. The boxcar function $\sigma(z)$ equals 1 when $-d<z<d$ and equals 0 otherwise.

Since the magnetization distribution is to be tracked as a function of the incremented time $t_{n}=n \Delta$, Eq. 6.198 can be used to describe the evolution in increments of $\Delta$ of an initial rectangular magnetization distribution $M_{z}(z, 0)=c_{0} \sigma(z)$ :

$$
c_{0} \sigma(z) \stackrel{\Delta}{\longrightarrow} c_{0} g_{1}(z) \stackrel{\Delta}{\longrightarrow} c_{0} g_{2}(z) \stackrel{\Delta}{\longrightarrow} c_{0} g_{3}(z) \stackrel{\Delta}{\longrightarrow} \ldots
$$

The preceding results can be used to follow the diffusion of magnetization while relaxing the local equilibration condition. The inside/outside-the-active-region variables $M_{z}^{A}(z)$ and $M_{z}^{R}(z)$ may be dropped in favor of using a function $M_{z}(z)$ that represents the entire distribution. The evolution proceeds as follows. Initially,

$$
M_{z}\left(z, t_{0}^{-}\right)=M_{0}
$$

The effect of any rf pulse is to reduce the longitudinal magnetization that was in the active region before the pulse by an amount $\cos \theta$ but leave the magnetization outside this region unchanged. This action can be represented by the equation:

$$
M_{z}\left(z, t_{n}^{+}\right)=M_{z}\left(z, t_{n}^{-}\right)-(1-\cos \theta) M_{z}\left(z, t_{n}^{-}\right) \sigma(z)
$$

where the first term represents the magnetization distribution before the pulse and the second term represents the "hole" burned in that distribution by the pulse. Therefore, after 
the first pulse the magnetization distribution becomes

$$
M_{z}\left(z, t_{0}^{+}\right)=M_{0}-M_{0}(1-\cos \theta) \sigma(z)
$$

After diffusion for time $\Delta$ :

$$
M_{z}\left(z, t_{1}^{-}\right)=M_{0}-M_{0}(1-\cos \theta) g_{1}(z)
$$

After the second pulse:

$$
M_{z}\left(z, t_{1}^{+}\right)=\left[M_{0}-M_{0}(1-\cos \theta) g_{1}(z)\right]-(1-\cos \theta)\left[M_{0}-M_{0}(1-\cos \theta) g_{1}(z)\right] \sigma(z)
$$

Thus far the treatment has been exact (in the infinite tube limit). An approximation must be invoked in order to treat the diffusion analytically over the next interval $\Delta$. The first term in Eq. 6.219 can be propagated using Eq. 6.214. The second term representing the magnetization hole is not easily propagated in a closed mathematical form. In the fast diffusion limit it will be assumed that all positions $-d<z<d$ inside the active region are near enough to the region boundaries that the distribution $g_{n}(z)$ within the active region can be treated as a constant using Eq. 6.199:

$$
g_{n}(z) \sigma(z) \approx k_{n} \sigma(z)=\frac{2 d}{\sqrt{\pi} \lambda_{n}} \sigma(z)
$$

and $k_{n}$ is defined in terms of $\lambda_{n}$ according to Eq. 6.201. Eq. 6.219 can be approximated 
using Eq. 6.220 as:

$$
M_{z}\left(z, t_{1}^{+}\right) \approx\left[M_{0}-M_{0}(1-\cos \theta) g_{1}(z)\right]-(1-\cos \theta)\left[M_{0}-M_{0}(1-\cos \theta) k_{1}\right] \sigma(z)
$$

The physical assumption is that the magnetization is "flat" across the active region before the pulse is applied and consequently the $\cos \theta$ reduction of magnetization in that region also results in a "flat" distribution, i.e., a "rectangular hole" is burned. Eq. 6.222 is easily propagated through a time $\Delta$ according to Eq. 6.214:

$$
M_{z}\left(z, t_{2}^{-}\right)=\left[M_{0}-M_{0}(1-\cos \theta) g_{2}(z)\right]-(1-\cos \theta)\left[M_{0}-M_{0}(1-\cos \theta) k_{1}\right] g_{1}(z)
$$

After the third pulse the magnetization distribution becomes:

$$
\begin{aligned}
& M_{z}\left(z, t_{2}^{+}\right)= \\
& \left\{\left[M_{0}-M_{0}(1-\cos \theta) g_{2}(z)\right]-(1-\cos \theta)\left[M_{0}-M_{0}(1-\cos \theta) k_{1}\right] g_{1}(z)\right\} \\
& -(1-\cos \theta)\left\{\left[M_{0}-M_{0}(1-\cos \theta) g_{2}(z)\right]-(1-\cos \theta)\left[M_{0}-M_{0}(1-\cos \theta) k_{1}\right] g_{1}(z)\right\} \sigma(z) .
\end{aligned}
$$


This may be approximated as:

$$
\begin{aligned}
& M_{z}\left(z, t_{2}^{+}\right)=\left\{\left[M_{0}-M_{0}(1-\cos \theta) g_{2}(z)\right]-(1-\cos \theta)\left[M_{0}-M_{0}(1-\cos \theta) k_{1}\right] g_{1}(z)\right\} \\
& \quad-(1-\cos \theta)\left\{\left[M_{0}-M_{0}(1-\cos \theta) k_{2}\right]-(1-\cos \theta)\left[M_{0}-M_{0}(1-\cos \theta) k_{1}\right] k_{1}\right\} \sigma(z) .
\end{aligned}
$$

After diffusion for time $\Delta$ the distribution becomes:

$$
\begin{aligned}
& M_{z}\left(z, t_{3}^{-}\right)=\left\{\left[M_{0}-M_{0}(1-\cos \theta) g_{3}(z)\right]-(1-\cos \theta)\left[M_{0}-M_{0}(1-\cos \theta) k_{1}\right] g_{2}(z)\right\} \\
& \quad-(1-\cos \theta)\left\{\left[M_{0}-M_{0}(1-\cos \theta) k_{2}\right]-(1-\cos \theta)\left[M_{0}-M_{0}(1-\cos \theta) k_{1}\right] k_{1}\right\} g_{1}(z) .
\end{aligned}
$$

The general procedure is clear: the diffusion of the holes burned in the magnetization distribution by each pulse is treated exactly, but each new hole is initially approximated as being rectangular.

If the notation $M_{z}^{n}\left(z ;\left\{g_{m}\right\}\right)$ is used to denote the dependence of the magnetization distribution on the various $g_{m}(z)$ functions at time $t_{n}^{-}$, a general recursive relationship for the magnetization distribution at successive intervals can be written as:

$$
M_{z}^{n+1}\left(z ;\left\{g_{m}\right\}\right)=M_{z}^{n}\left(z ;\left\{g_{m+1}\right\}\right)-(1-\cos \theta) M_{z}^{n}\left(z ;\left\{k_{m}\right\}\right) g_{1}(z)
$$

Only the magnetization inside the active region of the tube, $M_{z}^{A}(z)=M_{z}(z) \sigma(z)$, is de- 
tected. This magnetization distribution is:

$$
M_{z}^{n+1}\left(z ;\left\{g_{m}\right\}\right) \sigma(z)=M_{z}^{n}\left(z ;\left\{g_{m+1}\right\}\right) \sigma(z)-(1-\cos \theta) M_{z}^{n}\left(z ;\left\{k_{m}\right\}\right) g_{1}(z) \sigma(z)
$$

Again approximate the magnetization within the active region as a constant using Eq. 6.220:

$$
\begin{aligned}
M_{z}^{n+1}\left(z ;\left\{g_{m}\right\}\right) \sigma(z) & \approx M_{z}^{n+1}\left(z ;\left\{k_{m}\right\}\right) \sigma(z) \\
& =M_{z}^{n}\left(z ;\left\{k_{m+1}\right\}\right) \sigma(z)-(1-\cos \theta) M_{z}^{n}\left(z ;\left\{k_{m}\right\}\right) k_{1} \sigma(z)
\end{aligned}
$$

The magnetization within the active region alone is:

$$
M_{z}^{n+1}\left(\left\{k_{m}\right\}\right)=M_{z}^{n}\left(\left\{k_{m+1}\right\}\right)-(1-\cos \theta) k_{1} M_{z}^{n}\left(\left\{k_{m}\right\}\right) .
$$

This is not yet a practical recursion relation since $M_{z}^{n}\left(\left\{k_{m+1}\right\}\right)$ and $M_{z}^{n}\left(\left\{k_{m}\right\}\right)$ are different functions. The proper recursion relation can be obtained by working in a linear vector space of the coefficients $\left\{k_{m}\right\}$. Define two $N$-dimensional column vectors $\underline{g}(z)$ and $\underline{k}$ :

$$
\underline{g}(z)=\left(\begin{array}{c}
1 \\
g_{1}(z) \\
g_{2}(z) \\
g_{3}(z) \\
\vdots \\
g_{N-1}(z)
\end{array}\right) \quad \underline{k}=\left(\begin{array}{c}
1 \\
k_{1} \\
k_{2} \\
k_{3} \\
\vdots \\
k_{N-1}
\end{array}\right)
$$

The magnetization distribution in Eqs. 6.215, 6.218, and 6.222 at the respective times $t_{0}^{-}$, 
$t_{1}^{-}$, and $t_{2}^{-}$can be written as:

$$
\begin{aligned}
& M_{z}\left(z, t_{0}^{-}\right)=\underline{g}^{T}(z) \cdot \underline{M}_{z}^{0} \\
& M_{z}\left(z, t_{1}^{-}\right)=\underline{g}^{T}(z) \cdot \underline{M}_{z}^{1} \\
& M_{z}\left(z, t_{2}^{-}\right)=\underline{g}^{T}(z) \cdot \underline{M}_{z}^{2},
\end{aligned}
$$

where $\underline{g}^{T}(z)$ is the transpose of $\underline{g}(z)$ (i.e., a row vector). The vectors $\underline{M}_{z}^{0}, \underline{M}_{z}^{1}$, and $\underline{M}_{z}^{2}$ are:

$$
\underline{M}_{z}^{0}=\left(\begin{array}{c}
M_{0} \\
0 \\
0 \\
0 \\
\vdots \\
0
\end{array}\right) \quad \underline{M}_{z}^{1}=\left(\begin{array}{c}
M_{0} \\
-(1-\cos \theta) M_{0} \\
0 \\
0 \\
\vdots \\
0
\end{array}\right) \quad \underline{M}_{z}^{2}=\left(\begin{array}{c}
M_{0} \\
-(1-\cos \theta)\left[1-(1-\cos \theta) k_{1}\right] M_{0} \\
-(1-\cos \theta) M_{0} \\
0 \\
\vdots \\
0
\end{array}\right)
$$

The next step is to seek a way to relate the vectors $\left\{\underline{M}_{z}^{n}\right\}$ to each other. The necessary relations are made through by Eqs. 6.226-6.229. Eq. 6.226, which approximates the magnetization distribution at time $t_{n+1}^{-}$in terms of distributions at time $t_{n}$, may be written in the vector notation as:

$$
\underline{g}^{T}(z) \cdot \underline{M}_{z}^{n+1}=\left[\underline{g}^{T}(z) \cdot \underline{\underline{G}}^{+} \cdot \underline{M}_{z}^{n}\right]-(1-\cos \theta)\left(\underline{k}^{T} \cdot \underline{M}_{z}^{n}\right)\left[\underline{g}^{T}(z) \cdot \underline{u}_{1}\right],
$$


where the $N \times N$-dimensional matrix $\underline{\underline{G}}^{+}$that takes every $g_{m}(z) \rightarrow g_{m+1}(z)$ is defined by:

$$
\underline{\underline{G}}^{+}=\left(\begin{array}{cccccc}
1 & 0 & 0 & 0 & 0 & \cdots \\
0 & 0 & 0 & 0 & 0 & \\
0 & 1 & 0 & 0 & 0 & \\
0 & 0 & 1 & 0 & 0 & \\
0 & 0 & 0 & 1 & 0 & \\
\vdots & & & & & \ddots
\end{array}\right)
$$

and the vector $\underline{u}_{1}$ is given by:

$$
\underline{u}_{1}=\left(\begin{array}{c}
0 \\
1 \\
0 \\
0 \\
\vdots \\
0
\end{array}\right)
$$

Eq. 6.235 may be written as:

$$
\underline{g}^{T}(z) \cdot \underline{M}_{z}^{n+1}=\underline{g}^{T}(z) \cdot \underline{\alpha} \cdot \underline{M}_{z}^{n}
$$

with

$$
\underline{\underline{\alpha}}=\underline{\underline{G}}^{+}-(1-\cos \theta) \underline{u}_{1} \underline{k}^{T}
$$

where $\underline{u}_{1} \underline{k}^{T}$ is the outer product of $\underline{u}_{1}$ and $\underline{k}^{T}$. The $N \times N$-dimensional matrix representation 
of $\underline{\underline{\alpha}}$ is: ${ }^{50}$

$\underline{\underline{\alpha}}=\left(\begin{array}{cccccc}1 & 0 & 0 & 0 & 0 & \cdots \\ -(1-\cos \theta) & -(1-\cos \theta) k_{1} & -(1-\cos \theta) k_{2} & -(1-\cos \theta) k_{3} & -(1-\cos \theta) k_{4} & \\ 0 & 1 & 0 & 0 & 0 & \\ 0 & 0 & 1 & 0 & 0 & \\ 0 & 0 & 0 & 1 & 0 & \ddots \\ \vdots & & & & & \ddots\end{array}\right)$

The definition of $\underline{\underline{\alpha}}$ can be modified to include longitudinal relaxation over the interval $\Delta$ :

$$
\underline{\underline{\alpha}}^{\prime}=\left[\underline{\underline{G}}^{+}-(1-\cos \theta) \underline{u}_{1} \underline{k}^{T}\right] e^{-\Delta / T_{1}}
$$

It is clear from Eqs. 6.238 and 6.241 that:

$$
\begin{aligned}
\underline{M}_{z}^{n+1} & =\underline{\underline{\alpha}}^{\prime} \underline{M}_{z}^{n}=\left(\underline{\underline{\alpha}}^{\prime}\right)^{n} \underline{M}_{z}^{0} \\
& =(\underline{\underline{\alpha}})^{n} e^{-t_{n} / T_{1}} \underline{M}_{z}^{0}
\end{aligned}
$$

where $\left(e^{-\Delta / T_{1}}\right)^{n}=e^{-t_{n} / T_{1}}$. These results may be used in conjunction with Eq. 6.229 to write a recursion relation in the vector notation for the approximation to the longitudinal

\footnotetext{
${ }^{50}$ The matrix $\underline{\underline{\alpha}}$ becomes very large if the number of pulses in the array, $N$, is very large; therefore it may be difficult to manipulate $\underline{\underline{\alpha}}$ on a computer because of memory constraints. However, $\underline{\underline{\alpha}}$ is a sparse matrix (i.e., most of its elements are zero) and sparse matrices can be implemented computationally with drastically reduced memory requirements. If the implementation is, for example, performed in MATLAB ${ }^{\circledR}[482]$ the matrix $\underline{\underline{\alpha}}$ can be defined by the following commands: $\mathrm{n}=1: \mathrm{N}-1 ; \mathrm{k}=\left[1, \mathrm{~d} . / \operatorname{sqrt}\left(\mathrm{pi}^{*} \mathrm{D}^{*} \mathrm{n}^{*} \text { Delta }\right)\right]^{\prime} ;$ alpha $=$ $(\operatorname{sparse}(1,1,1, \mathrm{~N}, \mathrm{~N})+\operatorname{sparse}(3: \mathrm{N}, 2: \mathrm{N}-1,1, \mathrm{~N}, \mathrm{~N}))-(1-\cos (\text { theta }))^{*} \operatorname{sparse}\left(2,1: \mathrm{N}, \mathrm{k} .{ }^{\prime}, \mathrm{N}, \mathrm{N}\right)$.
} 
magnetization within the active region at time $t_{n+1}$ :

$$
\begin{aligned}
M_{z}^{n+1}\left(\left\{k_{n}\right\}\right) & =\underline{k}^{T} \cdot \underline{M}_{z}^{n+1}=\underline{k}^{T} \cdot \underline{\underline{\alpha}}^{\prime} \cdot \underline{M}_{z}^{n}=\underline{k}^{T} \cdot\left(\underline{\underline{\alpha}}^{\prime}\right)^{n} \cdot \underline{M}_{z}^{0} \\
& =M_{0}\left[\underline{k}^{T} \cdot(\underline{\underline{\alpha}})^{n} \cdot \underline{u}_{0}\right] e^{-t_{n} / T_{1}}
\end{aligned}
$$

where

$$
\underline{u}_{0}=\left(\begin{array}{c}
1 \\
0 \\
0 \\
0 \\
\vdots \\
0
\end{array}\right) .
$$

The signal after each pulse is:

$$
S\left(t_{n}^{+}\right)=S_{0} \sin \theta\left[\underline{k}^{T} \cdot(\underline{\underline{\alpha}})^{n} \cdot \underline{u}_{0}\right] e^{-t_{n} / T_{1}}
$$

where $S_{0}$ is the magnitude of the signal that would be detected after a $90^{\circ}$ pulse applied to the initial $z$-magnetization.

Fig. 6.14 shows the simulated depletion of the signal observed immediately after each pulse in the case of a constant tip angle of $\theta=22.5^{\circ}$ in the presence of longitudinal relaxation with fast diffusion $\left(k_{1}=0.23\right)$ in the extended diffusion model (dash-dot line). The signal decay is non-exponential and is slower than in the case of fast diffusion with local equilibration (dashed line) because the magnetization in the active region of the tube is continually refreshed by a possibly distant external reservoir of undepleted magnetization. 


\subsection{DEPLETION AND DIFFUSION OF XENON MAGNETIZATION DURING MULTI-PULSE BATCH MODE EXPERIMENTS}

Note that under the conditions considered here the diffusion nearly nullifies the depletion of signal due to the action of rf pulses that have a fairly large tip angle, i.e., the pure longitudinal relaxation limit (solid line) is almost recovered. The depletion of signal in the limit of no diffusion (dotted line) is much more rapid.

Note that the extended diffusion model presented here can fail if the "infinite tube" approximation fails, i.e., the total length of the experiment is long enough that the diffusion of the magnetization gradient created in the active region of the tube diffuses far enough to reflect off the top or bottom of the tube. This occurs when $\lambda_{N-1}=2 \sqrt{D(N-1) \Delta} \sim L$ if the active region is at the center of the tube. If the infinite tube approximation fails a full treatment using Eq. 6.197 is necessary.

Eq. 6.245 can be used to generate the a variable tip angle scheme to generate a constant signal over the pulse array if the tip angles $\theta_{n}$ are chosen according to:

$$
\theta_{n}=\sin ^{-1}\left[\frac{\sin \theta_{0}}{e^{-t_{n} / T_{1}}\left[\underline{k}^{T} \cdot \prod_{j=0}^{n-1} \underline{\underline{\alpha}}_{j} \cdot \underline{u}_{0}\right]}\right],
$$

where

$$
\underline{\underline{\alpha}}_{n}=\underline{\underline{G}}^{+}-\left(1-\cos \theta_{n}\right) \underline{u}_{1} \underline{k}^{T},
$$

and the product is time-ordered such that $\underline{\underline{\alpha}}_{j}$ matrices with lower indices $j$ act first:

$$
\prod_{j=0}^{n-1} \underline{\underline{\alpha}}_{j}=\underline{\underline{\alpha}}_{n-1} \underline{\underline{\alpha}}_{n-2} \cdots \underline{\underline{\alpha}}_{1} \underline{\underline{\alpha}}_{0} .
$$




\subsection{DEPLETION AND DIFFUSION OF XENON MAGNETIZATION DURING MULTI-PULSE BATCH MODE EXPERIMENTS}

The predictions of the fast diffusion/extended equilibration model (Eq. 6.245) were checked against an experimental measurement of the decay of Xe-129 signal in a long $(\sim 20 \mathrm{~cm})$ tube. The length of the active region of the tube near the rf coil was $2 d \approx 1.5$ cm. Fig. 6.15 shows the measured signal decay versus the predicted decay of Xe-129 at a pressure of $P=0.9$ atm during an array of 200 constant tip-angle pulses of $\theta \simeq \pi / 8$ spaced by $60 \mathrm{~s}\left(k_{1}=0.217\right)$. The Xe-129 longitudinal relaxation time in this tube was measured to be $T_{1}=32.4$ minutes in an experiment that immediately preceded the diffusion experiment. The experimental measurement of the signal decay including diffusion effects agrees reasonably well with the predictions of the model; e.g., both predict similar nonexponential decays. The agreement between the experiment and the model becomes very good if an effective relaxation time of $T_{1}^{*}=25-26$ minutes is used in the model. One possible explanation for the inability of the model to describe the experimental results completely is that the "infinite tube" approximation failed because the bottom of the tube is only $2 \mathrm{~cm}$ from the center of the active region of the tube (i.e., the distance to the bottom was only about the size of the active region itself). In that case "reflections" of the magnetization off the bottom of the tube during diffusion must be considered. It would then be possible for a volume of depleted magnetization to diffuse out of the active region of the tube and reflect off the bottom of tube to return once again to the active region, which would accelerate the observed signal decay as compared to the case in which boundary reflections can be neglected. If the accelerated decay manifested itself as an effective exponential relaxation, this may explain the discrepancy between the model and the experiments.

It is interesting to observe that a Fourier transform of the experimentally-observed 


\subsection{DEPLETION AND DIFFUSION OF XENON MAGNETIZATION} DURING MULTI-PULSE BATCH MODE EXPERIMENTS

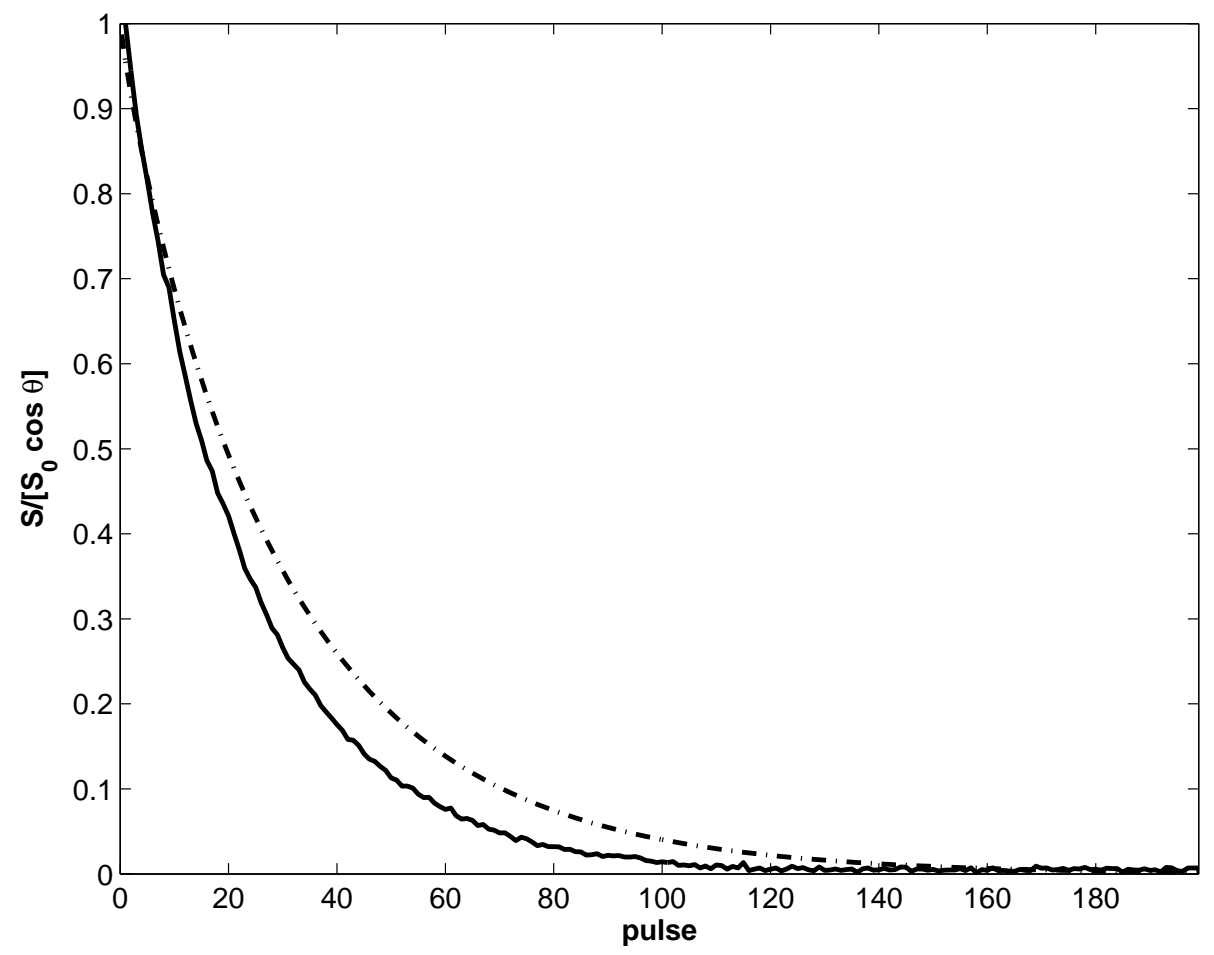

Figure 6.15: Measured decay of signal in a multiple-pulse, constant tip-angle batch Xe NMR experiment in a long tube compared to a model that incorporates fast diffusion effects causing an extended equilibration of magnetization. The experimental data are plotted with a solid line and the results of the model (Eq. 6.245) are plotted with a dash-dot line; the initial signals are normalized to 1 . The $5 \mathrm{~mm}$ o.d. J-Young tube was $\sim 20 \mathrm{~cm}$ long and the rf coil (about $1.5 \mathrm{~cm}$ high) was centered $\sim 2 \mathrm{~cm}$ from the bottom of the tube. The pulse array used constant tip-angle pulses of $\theta \approx \pi / 8$ spaced by 60 s. The Xe-129 longitudinal relaxation time was measured to be $T_{1}=34.2 \mathrm{~min}$ in a prior experiment that used fewer, more widely spaced pulses with smaller tip angles in order to minimize the depletion of longitudinal magnetization.

signal decay in Fig. 6.15 reproduces the same type of distorted (non-Lorentzian) lineshape observed in the Xe-129-detected DDF indirect H-1 spectrum of water in Fig. $6.12 .{ }^{51}$ The distorted lineshape in Fig. 6.12 appears to be primarily due to the depletion of non-equilibrium

\footnotetext{
${ }^{51}$ Some care should be taken when comparing the Fourier transform (FT) of the data in Fig. 6.15 with the indirect spectrum in Fig. 6.12. The FT of the data in Fig. 6.15 has width of much less than $1 \mathrm{~Hz}$ because the decay occurs over the timescale of tens of minutes, whereas the width of the $\mathrm{H}_{2} \mathrm{O}$ resonance in Fig. 6.12 is on the order of tens of $\mathrm{Hz}$. It is important to remember that the spectrum in Fig. 6.12 was acquired indirectly (point-by-point), i.e., an increment of $t_{1}$ between two indirect points that might be on the order of milliseconds could correspond to seconds or minutes of experimental time while a transient acquisition (or multiple acquisitions if phase cycling is employed) plus recycle delay is performed. Therefore the slow relaxation of Xe-129 can manifest itself as a much more rapid damping of the indirectly-detected signal.
} 
Xe-129 longitudinal magnetization due to $T_{1}$ relaxation and conversion to transverse magnetization by rf pulses.

\subsection{Detection of nuclear magnetization by atomic magne- tometry}

This section reports the results of a measurement of the nuclear magnetization of Xe-129 with a frequency-modulated nonlinear magneto-optical rotation (FM NMOR) atomic magnetometer. Unlike the other parts of this chapter, the work described here is not related to developing a dipolar field encoding module for remote detection NMR. It is, however, related to the possibility of developing an optical atomic magnetometry detection module for remote detection NMR, and thus is appropriately included in this chapter. Furthermore, atomic magnetometry as applied to the detection of nuclear magnetization may be viewed as the use of atomic electrons to measure the distant dipolar field of the target nuclei. In this case the electrons and nuclei are not coherently manipulated using rf pulses as the nuclei are in the DDF encoding experiments described above, but a discussion of magnetometric techniques is certainly germane to a discussion of the potential applications of the nuclear dipolar field.

Atomic magnetometers have existed for a long time; thirty-five years ago CohenTannoudji et al. first used atomic magnetometry to detect nuclear magnetization in an experiment that utilized a $\mathrm{Rb}-87$ magnetometer to detect the Larmor precession of opticallypumped He-3 gas in a separate cell [491]. The work reported here [492] used the FM NMOR atomic magnetometer built by the Budker group in the UC Berkeley physics department, 
which is further described in Refs. [493, 232, 494, 281]. The Romalis group at Princeton has also constructed a sensitive atomic magnetometer using a small-volume $\left(0.3 \mathrm{~cm}^{3}\right)$ magnetometer cell [495]. Atomic magnetometers compete well with SQUID flux magnetometers [111] in terms of sensitivity to small magnetic fields and do not require expensive electronics or cryogenic temperatures for operation. Modern atomic magnetometers can achieve sensitivities of better than $10^{-11} \mathrm{G} / \sqrt{\mathrm{Hz}}$ (see Refs. [493, 110, 495] and references therein), i.e., in the sub-femtotesla range for a measurement time of one second.

\subsubsection{The Berkeley FM NMOR optical pumping magnetometer}

The Budker lab magnetometer measures the rotation of the plane of linearlypolarized laser light passed through an alkali-vapor magnetometer cell. The angle of optical rotation in the FM NMOR experiment is simply proportional to the magnetic field when that field is along the direction of the light propagation. Optical rotation due to the presence of a magnetic field component along the direction of light propagation is also known as Faraday rotation or the Faraday effect. The Berkeley technique takes advantage of ultra-narrow dispersion-lineshape resonances in the magnetic field dependence of the optical rotation [496, 493]; several such resonances in the frequency-modulated version of the experiment are shown in Fig. 6.16. As can be seen from Fig. 6.16 the magnetometer response has a linear dependence on deviations of the magnetic field from its value at the center of a resonance; changes in the magnetic field on the order of $\mu \mathrm{G}$ can result in optical rotations of $10^{-3}-10^{-2} \mathrm{rad}$

The version of the magnetometer used in this work utilized a 10-cm diameter paraffin-coated spherical magnetometer cell containing Rb-87 at room temperature $\left(\sim 10^{10}\right.$ 


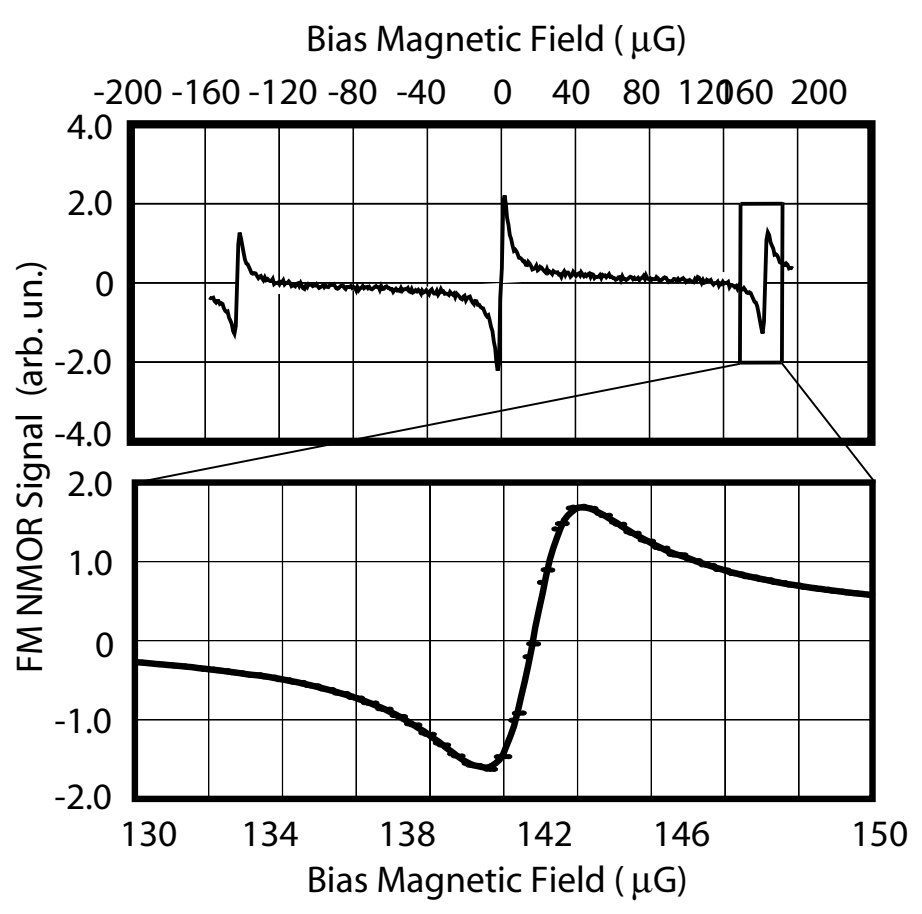

Figure 6.16: Measured dispersive FM NMOR resonances in the $\mathrm{Rb}-87 \mathrm{D}_{1}$ line as a function of applied bias field at a laser modulation frequency of $\Omega_{m}=200 \mathrm{~Hz}$. The amplitude of the longitudinal bias field equals $\Omega_{L} /\left(g_{F} \mu_{B}\right)$, where $\Omega_{L}$ is the Rb electronic Larmor frequency in the presence of the bias field, $g_{F}$ is the $g$-factor of the $F=2$ sublevel of the $5^{2} \mathrm{~S}_{1 / 2} \mathrm{Rb}$ - 87 ground state, and $\mu_{B}$ is the Bohr magneton. The central resonance occurs at $\Omega_{L}=0$ and the "satellite" resonances occur at $\Omega_{L}= \pm \Omega_{m} / 2$.

atoms $/ \mathrm{cm}^{3}$ number density) and no buffer gas. ${ }^{52}$ The apparatus is shown in Fig. 6.17.

The atoms were subjected to frequency-modulated $4 \mu \mathrm{W}$ diode laser light whose mean frequency $\Omega_{0}$ was locked to the $F_{g}=2 \rightarrow F_{e}=1$ hyperfine transition within the $\mathrm{Rb}_{1}$ $\left(5^{2} \mathrm{~S}_{1 / 2} \rightarrow 5^{2} \mathrm{P}_{1 / 2}\right)$ line using a technique involving an auxiliary $\mathrm{Rb}$ vapor cell (not shown).

The optical rotation was measured using a polarizer and analyzer oriented at $\sim 45^{\circ}$ relative

\footnotetext{
${ }^{52}$ The low $\mathrm{Rb}$ density keeps the atomic medium optically thin under resonance conditions, allowing for the transmission of most of the incident laser light. It also reduces the influence of depolarizing $\mathrm{Rb}-\mathrm{Rb}$ spin-exchange collisions which interfere with the magnetic precession of atomic alignment (see the discussion below). The use of spherical optical cells is advantageous in magnetometry applications because the selfdipolar field due to a uniform magnetization of the Rb atoms would be zero in this geometry (after making the Lorentz sphere correction, see the magnetostatic calculations in $\S 6.2 .1$ ). The use of spherical cells may not be as important for the Berkeley FM NMOR magnetometry technique because the optical pumping primarily creates atomic alignment (see the discussion below) which does not correspond to magnetization. Additionally, any macroscopic magnetization would be proportional the Rb density, which is is low.
} 
to each other (a balanced polarimeter); the transmission through the analyzer was recorded using two photodiodes in a scheme that allows the acquisition of in-phase and quadrature $\left(90^{\circ}\right.$ out-of-phase) signals. The magnetometer cell was surrounded by a four-layer magnetic shield that suppressed external magnetic fields by a factor of $\sim 10^{6}$.

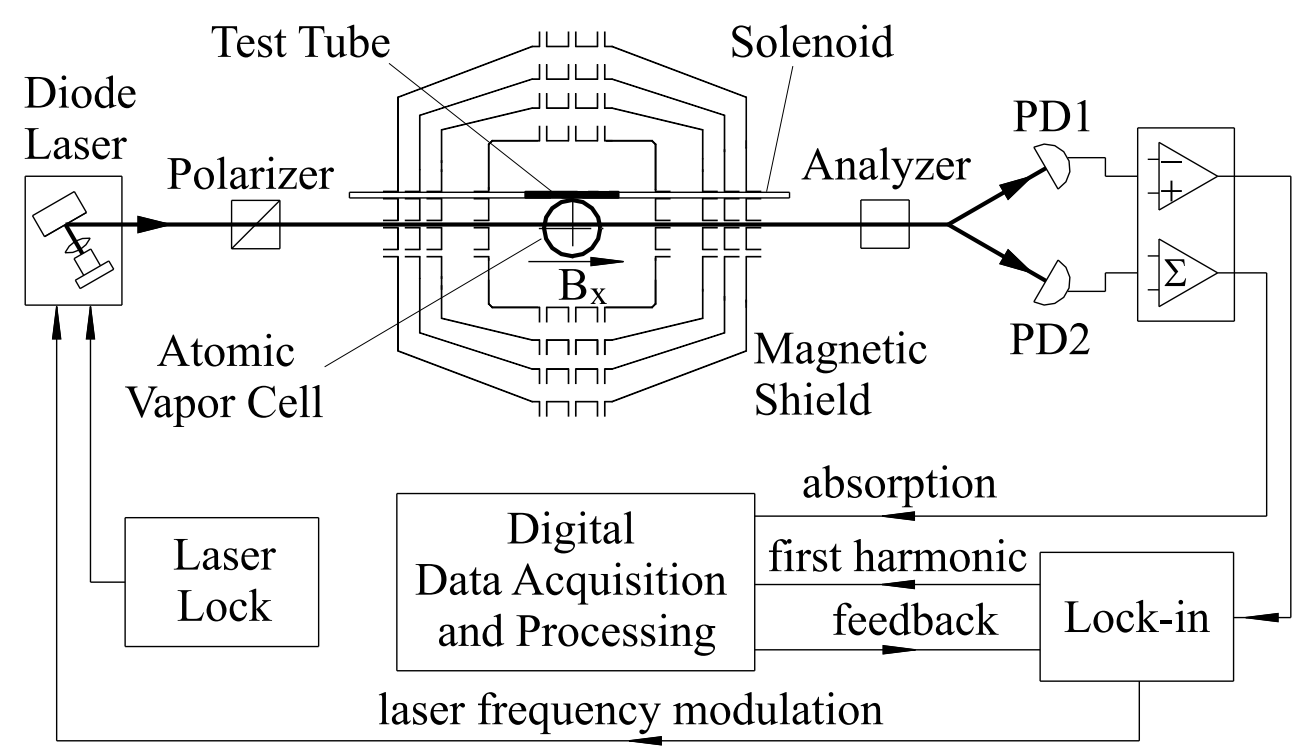

Figure 6.17: FM NMOR atomic magnetometer used for detecting Xe-129 nuclear magnetization. The abbreviation $B_{x}$ refers to the bias magnetic field applied across the Rb vapor cell and PD1 and PD2 refer to photodiodes used to detect the optical rotation.

The source of the nonlinear magneto-optical rotation (NMOR) can be understood as follows (see also Ref. [110]). A nonlinear optical effect involves at least two light-atom interactions, e.g., the absorption of a photon changes the atomic polarization and hence changes the optical properties of the medium, which are subsequently probed by a second atom-photon interaction. In the case of the NMOR magnetometer the linearly polarized laser light tuned to the $F_{g}=2 \rightarrow F_{e}=1$ transition optically pumps the Rb atoms into an aligned state, where the axis of alignment is along the direction of light polarization. Atomic alignment is the analog of what is known as quadrupolar order in NMR (see §5). 


\subsection{DETECTION OF NUCLEAR MAGNETIZATION BY ATOMIC}

Define the direction of light propagation to be in the $+z$-direction and the light polarization to be along the $x$-axis. Atoms that are aligned in a particular direction (e.g., along the $x$ axis) will not further absorb light polarized in that direction, but will absorb the orthogonal polarization (i.e., along the $y$-axis). ${ }^{53}$ This differential between absorption coefficients of orthogonal light polarizations is called linear dichroism. If there is a magnetic field in the $z$-direction (parallel to the light propagation) the atomic alignment axis will precess in the $x-y$ plane around the magnetic field. This causes a rotation of the axis of linear dichroism, which in turn rotates the plane of polarization of the light during its transmission through the atomic vapor [221]. At higher light powers the combination of the Zeeman effect due to the magnetic field along $z$ and the quadratic Stark effect due to the light field along $x$ causes periodic interconversion of the atomic polarization of alignment along the $x$-axis into orientation (analogous to magnetization in NMR) along the $z$-axis and back again $[232,221,240]$ (see $\S 5)$. The presence of atomic orientation parallel to the direction of light propagation creates circular birefringence, i.e., the indices of refraction and hence the phase velocities of right- or left-circularly polarized light components differ. This causes a relative phase shift of the two components as the light passes through the atomic medium, which rotates the plane of linear polarization. The NMOR due to both linear dichroism and circular birefringence requires the creation of ground state polarization (coherence) via optical pumping. ${ }^{54}$

\footnotetext{
${ }^{53}$ This is a generalization; the actual effects can be more complicated.

${ }^{54}$ In addition to these two mechanisms of nonlinear optical rotation there is also a mechanism of Faraday rotation that is linear, i.e., the atoms need not first be polarized (aligned or oriented) by the light in order to exhibit optical rotation when probed [110]. The presence of a magnetic field along $z$ causes a splitting of the hyperfine sublevels due to the Zeeman effect, and the resultant detuning of the components from the zero-field resonance causes a difference in the indices of refraction for right- and left-circularly polarized light. This circular birefringence leads to a rotation of the plane of light polarization; there is also a linear circular dichroism effect that induces ellipticity of the light. The light power used in the magnetometry
} 


\subsection{DETECTION OF NUCLEAR MAGNETIZATION BY ATOMIC}

MAGNETOMETRY

The NMOR magnetometer described above uses the same laser beam to continuously optically pump (polarize) the system while simultaneously probing the optical rotation. Once an atom is optically pumped the paraffin coating of the magnetometer cell enables the polarization to survive many wall collisions before its polarization is probed as it transits the laser beam again. The long lifetime of the ground-state polarization is the source of the ultra-narrow resonances in the magnetic field dependence of the optical rotation. The extreme narrowness of these resonances is what makes the magnetometer so sensitive to small changes in the magnetic field; a sharp line results in a large change in optical rotation given a relatively small change in magnetic field. Alternatively, the long lifetimes of the ground state coherences allow them to evolve for a long time in the magnetic field and therefore cause the light to acquire a relatively large angle of rotation when the atomic polarization is probed.

The latest generation of the Berkeley NMOR magnetometer utilizes a frequency modulation of the laser in the presence of a bias magnetic field applied parallel to the direction of light propagation. Fig. 6.16 depicts the measured FM NMOR resonances as a function of bias field when the modulation frequency is set to $\Omega_{m} / 2 \pi=200 \mathrm{~Hz}$. The central resonance is the zero field resonance and the "satellite" resonances occur at the condition $\Omega_{m}=2\left|\Omega_{L}\right|$, where $\Omega_{L}$ is the Larmor frequency of the ground state electrons in the presence of the bias field. ${ }^{55}$ The source of this resonance in the case of linear dichroism is the modulation of the optical properties of the atomic medium at frequency $2 \Omega_{L}$ due to the experiments is strong enough that the NMOR dominates the linear magneto-optical rotation.

${ }^{55}$ The Larmor frequency is $\Omega_{L}=g_{F} \mu_{B} B$, where $B$ is the value of the bias magnetic field, the Landé $g$-factor of an electron in the $F_{g}=2$ hyperfine level of the Rb-87 $5{ }^{2} \mathrm{~S}_{1 / 2}$ ground state is $g_{F}=1 / 2$, and the Bohr magneton is $\mu_{B} / 2 \pi=1.4 \mathrm{MHz} / \mathrm{G}$ (or $14 \mathrm{GHz} / \mathrm{T}$ ). Therefore it can be seen that the condition $\Omega_{m}=2\left|\Omega_{L}\right|$ is satisfied when $B \approx \pm 140 \mu \mathrm{G}$ for $\Omega_{m} / 2 \pi=200 \mathrm{~Hz}$. 


\subsection{DETECTION OF NUCLEAR MAGNETIZATION BY ATOMIC}

Larmor precession of atomic alignment. ${ }^{56}$ The theory $[232,281]$ of alignment-to-orientation conversion (the source of circular birefringence in NMOR) has not been fully developed yet; this effect may not contribute to the FM resonances. During the magnetometry experiments the bias field was fixed to $B_{z}=140 \mu \mathrm{G}$ and the laser modulation frequency was locked to the center of the $\Omega_{m}=2 \Omega_{L}$ resonance, leading to values of $\Omega_{m} \approx 200 \mathrm{~Hz}$ in the presence of the net field inside the magnetic shield. The net field was a combination of the dominant bias field, the field to be measured, and any other residual magnetic field contributions. The optical rotation was measured using lock-in detection at the first harmonic of the modulation frequency $\Omega_{m}$. The optical rotation was not measured on the in-phase channel that exhibits dispersive lineshapes - for which the optical rotation is zero at the centers of the resonances - but rather on a $\pi / 2$ phase-shifted (quadrature) channel that exhibits absorptive lineshapes, for which the optical rotation is maximal at the centers of the FM resonances [494]. The FM technique is useful because it can extend the dynamic range of the magnetometer so that it can detect magnetic fields below the $\mu \mathrm{G}$ range on top of a background field (such as Earth's field) on the order of even $\sim 1 \mathrm{G}$, rather than just detecting $\mu \mathrm{G}$ or smaller deviations from zero field. The use of a dominant bias field also renders the scalar NMOR magnetometer linearly sensitive to magnetic fields parallel to the direction of light propagation (Faraday effect) and relatively insensitive to transverse fields (Voigt effect) [493]. The ability of the FM technique to measure resonances away from the zero-frequency resonance also can help to avoid some low-frequency noise.

\footnotetext{
${ }^{56}$ Atomic alignment, having the transformation properties of a rank-2 spherical tensor, returns to its original state after every half-Larmor period during rotation about the orthogonal magnetic field, which causes the modulation at frequency $2 \Omega_{L}$.
} 


\subsubsection{Detection of hyperpolarized Xe-129 gas by atomic magnetometry}

The FM NMOR magnetometer was used to detect the nuclear magnetization of hyperpolarized xenon gas produced by the batch spin-exchange optical pumping method described in $\S 6.4 .1$. The basic magnetometer apparatus had to be modified slightly to accommodate the xenon experiments. The hole in the magnetic shielding permitted the introduction of a custom sapphire sample tube closed with a spring-loaded titanium valve; the overall dimensions of the tube were $6.4 \mathrm{~mm}$ outer- $/ 4.8 \mathrm{~mm}$ inner-diameter by $140 \mathrm{~mm}$ long. The tube was designed to operate at gas pressures of up to $30 \mathrm{~atm}$ and the tube materials were chosen to ensure a long xenon relaxation time [497]. A piercing solenoid was built to accommodate the sample tube (Fig. 6.17) in order to avoid depolarization of the xenon spins as they were moved from the laboratory magnetic environment (Earth field $\sim 0.5 \mathrm{G})$ to the zero field environment within the magnetic shield. The tube was inserted into the part of the solenoid that was outside the magnetic shield and the DC solenoid field was increased to $\sim 2 \mathrm{G}$ (large enough to dominate any Earth field contributions) before the tube was pushed inside the shield. Once the sample tube was in place next to the magnetometer cell the solenoid field was reduced to $0.45 \mathrm{G}$, which was small enough to induce an almost negligible magnetic field due to the magnetic susceptibility polarization of the tube (primarily from the titanium valve), but large enough that the xenon spins remained magnetized along the tube axis during the course of the measurement. The

ability to apply a magnetic field to the xenon while it is within the magnetic shielding is important for magnetic resonance experiments (not reported here). The constant magnetic field produced by the solenoid at the position of the magnetometer cell (i.e., the "leakage 


\subsection{DETECTION OF NUCLEAR MAGNETIZATION BY ATOMIC}

field") was reduced by a factor of $\approx 2 \times 10^{5}$ (as determined by an auxiliary measurement) compared to the field inside the solenoid. This leakage field was smaller than and mostly transverse to the $140 \mu \mathrm{G}$ bias field at the position of the magnetometer cell and therefore the FM NMOR measurement was largely insensitive to it. An internal coil set was used to null the leakage field during the xenon experiment in order to make sure it did not contribute to the measurement.

The version of the FM NMOR magnetometer described here was not designed with the intent to measure xenon nuclear magnetization, so it is not geometrically optimized for maximum sensitivity. Even when the sample tube is optimally placed the xenon dipolar field falls off rapidly over the $10-\mathrm{cm}$ diameter cell. A calibration solenoid of the same dimensions as the sample tube that generated a known current was used to measure a geometric suppression factor of $\sim 5000$ that is equal to the ratio of the macroscopic dipolar field inside the sample tube to the average field sensed by the magnetometer.

Hyperpolarized Xe-129 gas in the range of $4-8 \%$ polarization and $\sim 5$ atm total xenon pressure was produced in a natural abundance xenon mixture using the Pines lab MITI spin-exchange polarizer by the batch mode (freeze-thaw) method. NMR investigations of the xenon in the custom sample tube were performed at 7.05 Tesla on a Varian Unity Inova spectrometer using a Varian $10 \mathrm{~mm}$ broadband solution-state NMR probe. The custom $10 \mathrm{~mm}$ probe had three rf channels, H-1/F-19, broadband, and Xe-129, where the broadband channel was used for Xe-129 detection due to its higher sensitivity. The longitudinal relaxation time in the sapphire tube was measured to be $T_{1} \sim 45$ minutes at $7.05 \mathrm{~T}$. The Earth-field $T_{1}$ was estimated to be $\lesssim 15$ minutes by allowing the xenon magnetization 


\subsection{DETECTION OF NUCLEAR MAGNETIZATION BY ATOMIC}

to relax outside the magnet and periodically returning it to the magnet for measurement. ${ }^{57}$

When the FM NMOR measurements were conducted, immediately after thawing the hyperpolarized xenon gas was carried by hand from the chemistry complex to the magnetometer in the physics building ( $\sim 5$ minute transport time) ${ }^{58}$ Fig. 6.18 shows an example of the measurement of the longitudinal relaxation of Xe-129 magnetization by atomic magnetometry. The measurement time was about $3 \mathrm{~s}$ per data point and the calculated initial average magnetic field across the magnetometer cell was 10-20 nG with a signal-to-noise ratio of about 10. An exponential decay of the signal was observed with a time constant of $13.9 \pm 1.5$ minutes that was attributed to longitudinal relaxation in the near-zero-field environment. The sample tube was moved in and out of position near the magnetometer cell (always remaining within the magnetic shield) in intervals of about one minute near the cell and one minute removed from it. The geometric suppression factor was about two orders of magnitude higher when the tube was away from the cell than when the tube was positioned optimally, which rendered the observed signal effectively zero during these times. This movement of the sample tube caused a modulation of the magnetization signal which made the signal easier to discriminate from slow background drifts (which were subtracted from the data in Fig. 6.18). Fig. 6.18 exhibits a small $(<1 \mathrm{nG})$ average offset from zero field due to the magnetic susceptibility contribution of the sample tube to the observed field.

An estimate of the size of the dipolar field of the Xe-129 sample is consistent with the measured value. The macroscopic magnetic field inside a long cylinder of uniform

\footnotetext{
${ }^{57}$ Care should be taken not to accidentally depolarize the sample, e.g., by bringing it too close to an active computer monitor.

${ }^{58}$ Speedy transport was ensured by the efforts of J. Granwehr.
} 


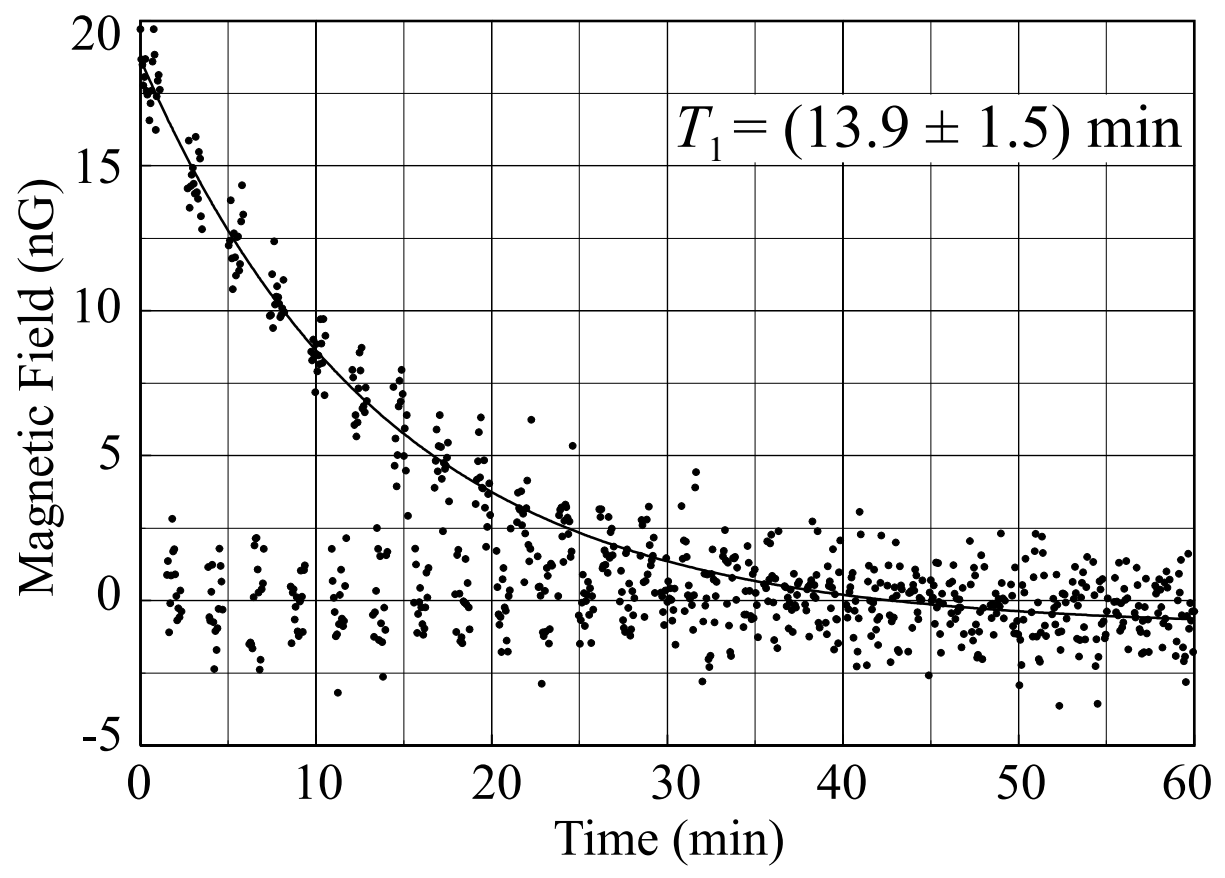

Figure 6.18: Measurement of the nuclear magnetization of Xe-129 gas as a function of time by atomic magnetometry. The discrimination of the Xe-129 longitudinal relaxation from the background signal was aided by moving the sample approximately every minute to a position of near-zero sensitivity of the magnetometer $14 \mathrm{~cm}$ away from the position of optimum sensitivity. The slow drift of the background signal due to temperature-related changes of the residual magnetization of the magnetic shielding was subtracted from the data. The small $<1 \mathrm{nG}$ offset of the decay curve from zero magnetic field was due to the diamagnetic susceptibility contribution of the sample tube.

magnetization $\underline{M}=M \underline{\widetilde{z}}$ is $\underline{B}=\mu_{0} M \underline{\tilde{z}}$, where the local Lorentz field correction has been neglected. The macroscopic magnetization of $N$ identical spin- $1 / 2$ nuclei may be calculated according to Eq. 1.104 using $\gamma_{X e 129} / 2 \pi=-11.86 \mathrm{MHz} / \mathrm{T}$ (or $-1.186 \mathrm{kHz} / \mathrm{G}$ ) and typical values of the polarization $(\zeta=0.05)$ and spin density $\left(N / V=x P / k_{B} T=3.2 \times 10^{25}\right.$ atoms $/ \mathrm{m}^{3}$ for an ideal gas with pressure $P=5 \mathrm{~atm}$, temperature $T=298 \mathrm{~K}$, and Xe-129 mole fraction $x=0.264$ ), resulting in a magnetic field of $|B| \simeq 80 \mu \mathrm{G}$ inside the sample tube. Therefore the average field sensed by the magnetometer is estimated to be $\approx 15 \mathrm{nG}$ when the factor of $\sim 5000$ geometric suppression factor is accounted for. This calculation 
used the value of the magnetization immediately after polarization; the value to which the magnetization has relaxed by the time it has been carried to the magnetometer is expected to be somewhat smaller, perhaps $\approx 10 \mathrm{nG}$ depending on the travel time. A $10 \%$ initial polarization would correspond to an average field of $\approx 20 \mathrm{nG}$. These values are consistent with the experimental results (Fig. 6.18).

As was noted earlier, the FM NMOR magnetometer used in this work was not optimized for xenon measurements. In particular, the geometric suppression factor can be increased by some three orders of magnitude by using a smaller magnetometer cell located as close as possible to the xenon sample. Furthermore, the magnetic noise observed within the magnetic shield in this work was some two orders of magnitude higher than the projected intrinsic sensor noise of the instrument of $10^{-11} \mathrm{G} / \sqrt{\mathrm{Hz}}$ or less $[493,494]$. This noise can be suppressed by employing a gradiometric arrangement of magnetic sensors. A next-generation FM NMOR gradiometer is already under construction; it employs two small (1-cm diameter by 1-cm length) cylindrical magnetometer cells in gradiometric mode within five-layer $\mu$-metal magnetic shielding. It is hoped that Xe-129 remote detection experiments (not necessarily dipolar field-encoded) can be carried out with this new atomic magnetometer.

\subsection{Conclusions}

This chapter reviewed the effects of the nuclear distant dipolar field (DDF) in solution-state NMR and examined the feasibility of performing NMR remote detection ex-

periments that used the DDF to encode the spectral information of an analyte of interest 
in the magnetization of a nearby mobile sensor nucleus. The principle of the recently developed remote detection detection method is to separate the NMR encoding and detection steps both physically and temporally so that each step can be optimized independently; the NMR information is transported from the encoding region to the detection region by means of a mobile carrier nucleus such as hyperpolarized Xe-129 gas. The original version of the remote detection experiment was not able to encode spectral information of an analyte other than the carrier nucleus itself. The use of a DDF encoding module would for the first time allow remote detection spectroscopy to be performed on a potentially arbitrary analyte; the long-range nature of the dipolar field permits this encoding to take place without having to mix the mobile carrier with the analyte. Remote detection experiments were not actually performed in this work, but a detailed theoretical and experimental analysis of the prospects of DDF-encoding remote detection was conducted.

The theory of the distant dipolar field in solution was reviewed using both the classical and the quantum-mechanical formalism. The DDF phenomena were interpreted classically as the dynamics of the bulk nuclear magnetization in the presence of a macroscopic magnetic field created by the nuclear paramagnetism. The quantum-mechanical interpretation ascribes these dynamics to the evolution of the nuclear polarization due to the short-lived coherent action of a macroscopic number of nuclear dipole-dipole couplings. DDF effects in highly-magnetized samples were seen to be the cause of some interesting nuclear spin dynamics such as resonance shifts and multiple spin echoes, which were described theoretically and illustrated with the results of some H-1 NMR experiments on water.

An analysis of the basic version of the heteronuclear DDF indirect detection ex- 
periment (invented by previous workers) was conducted; the predicted signal was calculated and the limitations of this technique were reviewed. The DDF indirect detection experiment forms the cornerstone of the DDF remote detection encoding module; it is the means by which the spectral information of an analyte nucleus is transferred through the analyte dipolar field to a sensor heteronucleus. A modification of the basic DDF indirect experiment for use as an encoding module in remote detection NMR experiments was proposed and its advantages and limitations were discussed.

The remote detection experiments that have been conducted to date have used hyperpolarized Xe-129 gas as the mobile information carrier, so an investigation of the use of xenon in DDF experiments was conducted. The process of spin-exchange optical pumping of Xe-129 was reviewed and the relative merits of the batch-mode xenon NMR method (which was used in the proof-of-principle experiments here) versus the flow-mode method (which has been used in remote detection experiments) were compared. Some new insights were developed about the nature of DDF experiments in which the analyte and sensor are physically separated (i.e., a coaxial tube geometry). The interpretation of experimental measurements of the DDF indirect detection signal as a function of magnetization modulation wavelength in the coaxial tube configuration was aided by a qualitative analysis using a computational method for calculating the dipolar field that is applicable to cylindrically-symmetric geometries. This method has not been used thus far in other NMR DDF studies. A new theoretical model incorporating the effects of rf pulses and gas diffusion was developed to explain an unexpected decay profile of xenon hyperpolarization in batch-mode experiments in a long sample tube. The model was compared to experimental 
results with some success and a technique for increasing the indirect-dimension resolution of DDF-encoded xenon spectra in such samples was proposed.

The H-1 NMR spectrum of water was detected through the proton dipolar field in a batch-mode experiment using a separated hyperpolarized Xe-129 gas sensor in a coaxial tube configuration at a magnetic field of $7.05 \mathrm{~T}$. The linewidth of the indirect spectrum of water was unfavorably broad due to the depletion of xenon hyperpolarization in the batch mode experiment, so a P-31 sensor was used in a proof-of-principle acquisition of a $J$-resolved F-19 spectrum of 2,2,2-trifluorethanol in the coaxial tube configuration. Although in principle the use of a highly magnetized sensor nucleus such as hyperpolarized Xe-129 gas could permit the sensitive detection of a weakly-magnetized analyte, the observed sensitivity of the DDF indirect detection experiments was quite low when compared to direct NMR detection. The DDF encoding method is limited by the long times that are required to encode indirect spectra of dilute analytes with full sensitivity. The experiment was also predicted to be susceptible to the effects of diffusion of the sensor, self-dipolar field nutation of the sensor, radiation damping of the sensor, multiplicative noise in the indirect dimension, and the limited range of the dipolar field. All of these effects tend to reduce the sensitivity of the experiment; a few but not all of them could likely be eliminated or reduced by the use of remote detection methodology and careful experimental design. One other drawback of the DDF encoding method is that it requires not only the sensor but also the analyte to be highly magnetized, which means that the DDF encoding must be conducted by polarizing the analyte at high magnetic fields unless another source of creating high nuclear polarizations is available. At this time it is difficult to foresee an application in which DDF-encoded 
remote detection spectroscopy would be superior to direct NMR detection. The DDF encoding method proposed here does however remain as a novel extension of the remote detection NMR methodology.

In addition to the DDF remote detection encoding module described above, experiments were conducted to assess the ability of an optical atomic magnetometer built by a physics group at Berkeley to detect nuclear magnetization. Atomic magnetometry has applicability as a detection module for the remote detection NMR experiment; in particular, it is performed at low magnetic fields, obviating the need for high-field superconducting magnets if the nucleus can be sufficiently polarized by an alternate means. The Berkeley magnetometer was successfully used to measure the $T_{1}$ decay of $\sim 5 \%$-polarized Xe-129 gas at $\sim 5$ atm total xenon pressure with a signal-to-noise ratio of $\sim 10$ using a detection time of $3 \mathrm{~s}$ per point. It is estimated that this demonstrated sensitivity can be improved by two to three orders of magnitude by using an optimized gradiometer; the prospects of implementing a remote detection experiment using this type of detection module are promising.

Finally, a brief digression on the theory of DDF effects on quadrupolar (spin $I>$ $1 / 2$ ) nuclei was made. This topic has not been explored in the literature. It was found that DDF effects in these systems should be identical to those observed in spin- $1 / 2$ systems when the only nuclear polarization present is in the form of magnetization, but some interesting dynamics may occur when the quadrupolar nuclei are prepared in a state of polarization that corresponds to a combination of classical magnetization and higher-rank polarization. Some speculations were made about the feasibility of observing these effects experimentally; the prospects are daunting. 


\section{Bibliography}

[1] Marvin Chester. Primer of Quantum Mechanics. Krieger Publishing Company, Malabar, FL (1992).

[2] Maurice Goldman. Quantum Description of High-Resolution NMR in Liquids. Oxford University Press, New York, NY (1988 (reprinted 1991 with corrections)).

[3] Malcolm H. Levitt. Spin Dynamics: Basics of Nuclear Magnetic Resonance. John Wiley \& Sons, Inc., Chichester, UK (2001).

[4] John Cavanagh, Wayne J. Fairbrother, Arthur G. Palmer III, and Nicholas J. Skelton. Protein NMR Spectroscopy: Principles and Practice. Academic Press, San Diego, CA (1996).

[5] C.P. Slichter. Principles of Magnetic Resonance. Springer series in solid-state science; 1. Springer-Verlag, Berlin, Germany, 3rd edition (1990 (corrected 1996)).

[6] A. Abragam. Principles of Nuclear Magnetism. International series of monographs on physics; 32. Oxford University Press, New York, NY (1961).

[7] Richard R. Ernst, Geoffrey Bodenhausen, and Alexander Wokaun. Principles of 
Nuclear Magnetic Resonance in One and Two Dimensions. International series of monographs on chemistry; 14. Oxford University Press, New York, NY (1987 (reprinted 1991 with corrections)).

[8] M. Mehring. Principles of High Resolution NMR in Solids. Springer-Verlag, Berlin, Germany, 2nd revised enlarged edition (1983).

[9] Klaus Schmidt-Rohr and Hans Wolfgang Spiess. Multidimensional Solid-State NMR and Polymers. Academic Press, San Diego, CA (1994).

[10] Melinda J. Duer, editor. Solid-State NMR Spectroscopy: Principles and Applications. Blackwell Science, Ltd., Oxford, UK (2002).

[11] Aage Bohr and Ben R. Mottelson. Nuclear Structure, volume I (Single-Particle Motion). W.A. Benjamin, Inc., New York, NY (1969).

[12] Aage Bohr and Ben R. Mottelson. Nuclear Structure, volume II (Nuclear Deformations). W.A. Benjamin, Inc., Reading, MA (1975).

[13] B. Castel and I.S. Towner. Modern Theories of Nuclear Moments. Oxford studies in nuclear physics; 12. Oxford University Press, New York, NY (1990).

[14] Kenneth S. Krane. Introductory Nuclear Physics. John Wiley \& Sons, Inc., New York, NY (1988).

[15] Hans Frauenfelder and Ernest M. Henley. Subatomic Physics. Prentice-Hall, Inc., Upper Saddle River, NJ, 2nd edition (1991). 
[16] John M. Blatt and Victor F. Weisskopf. Theoretical Nuclear Physics. John Wiley \& Sons, Inc., New York, NY (1958).

[17] John David Jackson. Classical Electrodynamics. John Wiley \& Sons, Inc., New York, NY, 3rd edition (1999).

[18] C.G. Gray. "Spherical tensor approach to multipole expansions. I. Electrostatic interactions." Can. J. Phys. 54, 505 (1976).

[19] C.G. Gray and P.J. Stiles. "Spherical tensor approach to multipole expansions. II. Magnetostatic interactions." Can. J. Phys. 54, 513 (1976).

[20] Richard N. Zare. Angular Momentum: Understanding Spatial Aspects in Chemistry and Physics. John Wiley \& Sons, Inc., New York, NY (1988).

[21] M.A. Preston and R.K. Bhaduri. Structure of the Nucleus. Addison-Wesley Publishing Company, Inc., Reading, MA (1975).

[22] Vladislav Gerginov, Andrei Derevianko, and Carol E. Tanner. "Observation of the nuclear magnetic octupole moment of Cs-133." Phys. Rev. Lett. 91, 072501 (2003).

[23] P.J. Unsworth. "Nuclear dipole, quadrupole and octupole moments of Gd-155 by atomic beam magnetic resonance." J. Phys. B 2, 122 (1969).

[24] E. Stachowska, M. Fabiszisky, and J. Dembczynski. "Upper limits of higher nuclear moments of Ti-47 and Ti-49." Zeit. Phys. D 32, 27 (1994).

[25] Ming-Yuan Liao and Gerard S. Harbison. "The nuclear hexadecapole interaction 
of iodine-127 in cadmium iodide measured using zero-field two dimensional nuclear magnetic resonance." J. Chem. Phys. 100, 1895 (1994).

[26] J. Cederberg, D. Olson, A. Nelson, D. Laine, P. Zimmer, M. Welge, M. Feig, T. Höft, and N. London. "Evidence for a nuclear hexadecapole interaction in the hyperfine spectrum of LiI." J. Chem. Phys. 110, 2431 (1999).

[27] S.L. Segel. "Nuclear electric hexadecapole interactions in solids." J. Chem. Phys. 69, $2434(1978)$.

[28] Richard P. Feynman, Robert B. Leighton, and Matthew Sands. The Feynman Lectures on Physics, volume III. Addision-Wesley Publishing Company, Reading, MA (1965).

[29] R. Shankar. Principles of Quantum Mechanics. Plenum Press, New York, NY, 2nd edition (1994).

[30] Scott A. Smith, William E. Palke, and J.T. Gerig. "The Hamiltonians of NMR: Part I.” Concepts Magn. Reson. 4, 107 (1992).

[31] L.D. Landau and E.M. Lifshitz. Quantum Mechanics: Non-Relativistic Theory. Course of theoretical physics; 3. Pergamon Press, Ltd., Oxford, UK, 2nd revised enlarged edition (1965).

[32] Dmitry Budker, Derek F. Kimball, and David P. DeMille. Atomic Physics: An Exploration Through Problems and Solutions. Oxford University Press, Oxford, UK (2004). 
[33] Malcolm H. Levitt. "The signs of frequencies and phases in NMR." J. Magn. Reson. 126, $164(1997)$.

[34] Malcolm H. Levitt and Ole G. Johannessen. "Signs of frequencies and phases in NMR: The role of radiofrequency mixing." J. Magn. Reson. 142, 190 (2000).

[35] Frank A.L. Anet and Daniel J. O'Leary. "The shielding tensor. Part I: Understanding its symmetry properties." Concepts Magn. Reson. 3, 193 (1991).

[36] Jozef Kowalewski and Lawrence Werbelow. "Evaluation of spin relaxation induced by chemical shielding anisotropy: A comment on the importance of the antisymmetric component." J. Magn. Reson. 128, 144 (1997).

[37] Sungsool Wi and Lucio Frydman. "Quadrupolar-shielding cross-correlations in solid state nuclear magnetic resonance: Detecting antisymmetric components in chemical shift tensors." J. Chem. Phys. 116, 1551 (2002).

[38] M. Goldman, P.J. Grandinetti, A. Llor, Z. Olejniczak, J.R. Sachleben, and J.W. Zwanziger. "Theoretical aspects of higher-order truncations in solid-state nuclear magnetic resonance." J. Chem. Phys. 97, 8947 (1992).

[39] A. Llor. "Equivalence between dynamical averaging methods of the Schrödinger equation: average Hamiltonian, secular averaging, and Van Vleck transformation." Chem. Phys. Lett. 199, 383 (1992).

[40] A. Llor. "Erratum and Addendum: 'Equivalence between dynamical averaging methods of the Schrödinger equation: average Hamiltonian, secular averaging and Van 
Vleck transformation' (Chem. Phys. Letters 199 (1992) 383)." Chem. Phys. Lett. 204, 217 (1993).

[41] Michael Mehring and Volker A. Weberru $\beta$. Object-Oriented Magnetic Resonance:

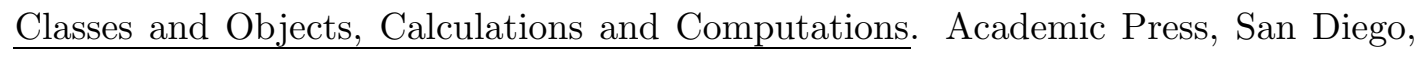
CA (2001).

[42] M. Matti Maricq and J.S. Waugh. "NMR in rotating solids." J. Chem. Phys. 70, 3300 (1979).

[43] Sungsool Wi and Lucio Frydman. "Heteronuclear recoupling in solid-state magicangle-spinning NMR via overtone irradiation.” J. Am. Chem. Soc. 123, 10354 (2001).

[44] Alexej Jerschow. "From nuclear structure to the quadrupolar NMR interaction and high-resolution spectroscopy." Prog. NMR Spectrosc. (2005).

[45] J.J. Sakurai. Modern Quantum Mechanics. Addison-Wesley Publishing Company, Inc., Reading, MA, revised edition (1994).

[46] Cynthia J. Jameson. "Understanding NMR chemical shifts." Annu. Rev. Phys. Chem. 47, 135 (1996).

[47] David J. Griffiths. Introduction to Electrodynamics. Prentice-Hall, Inc., Englewood Cliffs, NJ, 2nd edition (1989).

[48] D.J. Griffiths. "Hyperfine splitting in the ground state of hydrogen." Am. J. Phys. 50, 698 (1982). 
[49] J.M. Millar, A.M. Thayer, D.B. Zax, and A. Pines. "Zero-field NMR of smallamplitude motions in a polycrystalline solid.” J. Am. Chem. Soc. 108, 5113 (1986).

[50] A.M. Thayer, M. Luzar, and A. Pines. "Zero-field N.M.R. study of the biaxial smectic E phase." Liq. Cryst. 2, 241 (1987).

[51] Ann M. Thayer. Pulsed Zero Field NMR of Solids and Liquid Crystals. Ph.D. dissertation, UC Berkeley (February 1987).

[52] Gerald L. Pollack and Daniel R. Stump. "Two magnets oscillating in each other's fields." Can. J. Phys. 75, 313 (1997).

[53] T.P. Das and E.L. Hahn. "Nuclear Quadrupole Resonance Spectroscopy." In Frederick Seitz and David Turnbull, editors, "Solid State Physics: Supplement 1," page 1. Academic Press, Inc., New York, NY (1958).

[54] M.H. Cohen and F. Reif. "Quadrupole Effects in Nuclear Magnetic Resonance Studies of Solids." In Frederick Seitz and David Turnbull, editors, "Solid State Physics: Advances in Research and Applications," volume 5, page 321. Academic Press, Inc., New York, NY (1957).

[55] Dieter Freude. "Quadrupolar nuclei in solid-state nuclear magnetic resonance." In R.A. Meyers, editor, "Encyclopedia of Analytical Chemistry," page 12188. John Wiley \& Sons, Ltd., Chichester, UK (2000).

[56] P.P. Man. "Quadrupolar Interactions." In David M. Grant and Robin K. Harris, editors, "Encyclopedia of Nuclear Magnetic Resonance," volume 6, page 3838. John Wiley \& Sons, Inc., Chichester, UK (1996). 
[57] R. Sternheimer. "On nuclear quadrupole moments." Phys. Rev. 80, 102 (1950).

[58] R. Sternheimer. "On nuclear quadrupole moments." Phys. Rev. 84, 244 (1951).

[59] Thomas Meersmann and Mathias Haake. "Magnetic field dependent Xenon-131 quadrupolar splitting in gas and liquid phase NMR." Phys. Rev. Lett. 81, 1211 (1998).

[60] Freddie R. Salsbury Jr. and Robert A. Harris. "The origin of the magnetic-fielddependent quadrupolar splitting." J. Chem. Phys. 109, 8338 (1998).

[61] Zhehong Gan, Parthasarathy Srinivasan, John R. Quine, Stefan Steuernagel, and Benno Knott. "Third-order effect in solid-state NMR of quadrupolar nuclei." Chem. Phys. Lett. 367, 163 (2003).

[62] Alex D. Bain. "Exact calculation, using angular momentum, of combined Zeeman and quadrupolar interactions in NMR." Mol. Phys. 101, 3163 (2003).

[63] Philip J. Grandinetti. "Does phase cycling work for nuclei experiencing strong quadrupolar couplings?" Solid State NMR 23, 1 (2003).

[64] J. Jeener and F. Henin. "A presentation of pulsed nuclear magnetic resonance with full quantization of the radio frequency magnetic field." J. Chem. Phys. 116, 8036 (2002).

[65] Lyndon Emsley and Alexander Pines. Lectures on Pulsed NMR. Italian Physical Society, International School of Physics Enrico Fermi; Course CXXIII; Nuclear Magnetic Double Resonance, 2nd edition (1993). 
[66] Moritz Andreas Kälin. Multiple Photon Processes in Electron Paramagnetic Resonance Spectroscopy. Ph.D. dissertation, ETH Zürich (Diss. ETH No. 15142) (2003).

[67] Chang Jae Lee. NMR with Generalized Dynamics of Spin and Spatial Coordinates. Ph.D. dissertation, UC Berkeley (November 1987).

[68] D.I. Hoult and B. Bhakar. "NMR signal reception: Virtual photons and coherent spontaneous emission." Concepts Magn. Reson. 9, 277 (1997).

[69] Karl Blum. Density Matrix Theory and Applications. Physics of atoms and molecules series. Plenum Press, New York, NY, 2nd edition (1996).

[70] Thomas C. Farrar and John E. Harriman. Density Matrix Theory and its Applications in NMR Spectroscopy. Farragut Press, Madison, WI, 2nd edition (1995).

[71] U. Fano. "Description of states in quantum mechanics by density matrix and operator techniques." Rev. Mod. Phys. 29, 74 (1957).

[72] D. Giulini, E. Joos, C. Kiefer, J. Kupsch, I.-O. Stamatescu, and H.D. Zeh. Decoherence and the Appearance of a Classical World in Quantum Theory. SpringerVerlag, Berlin, Germany (1996).

[73] Richard C. Tolman. The Principles of Statistical Mechanics. Dover Publications, Inc., New York, NY (1979 (reproduced from original 1938 edition)).

[74] Richard P. Feynman. Statistical Mechanics: A Set of Lectures. Advanced Book Classics. Perseus Books, Reading, MA (1972). 
[75] J. Jeener. "Unified derivation of the dipolar field and relaxation terms in the BlochRedfield equations of liquid NMR." J. Chem. Phys. 103, 1309 (1995).

[76] S. Lee, W. Richter, S. Vathyam, and W.S. Warren. "Quantum treatment of the effects of dipole-dipole interactions in liquid nuclear magnetic resonance." J. Chem. Phys. 105, $874(1996)$.

[77] Warren S. Warren and Sangdoo Ahn. "The boundary between liquidlike and solidlike behavior in magnetic resonance." J. Chem. Phys. 108, 1313 (1998).

[78] A.G. Redfield. "The theory of relaxation processes." Adv. Magn. Reson. 1, 1 (1965).

[79] Maurice Goldman. "Formal theory of spin-lattice relaxation." J. Magn. Reson. 149, $160(2001)$.

[80] Nagarajan Murali and V.V. Krishnan. "A primer for nuclear magnetic relaxation in liquids." Concepts Magn. Reson. A 17A, 86 (2003).

[81] Peter Luginbühl and Kurt Wüthrich. "Semi-classical nuclear spin relaxation theory revisited for use with biological macromolecules." Prog. NMR Spectrosc. 40, 199 (2002).

[82] Kwaku T. Dayie, Gerhard Wagner, and Jean-François Lefèvre. "Theory and practice of nuclear spin relaxation in proteins." Annu. Rev. Phys. Chem. 47, 243 (1996).

[83] R.K. Wangsness and F. Bloch. "The dynamical theory of nuclear induction." $\underline{\text { Phys. }}$ Rev. 89, 728 (1953). 
[84] Claude Cohen-Tannoudji, Bernard Diu, and Franck Laloë. Quantum Mechanics, volume I. Hermann, Paris, France (1977).

[85] Claude Cohen-Tannoudji, Bernard Diu, and Franck Laloë. Quantum Mechanics, volume II. Hermann, Paris, France (1977).

[86] Uzi Eliav and Gil Navon. "Criteria for multiexponential relaxation of exchanging spin-3/2 nuclei." J. Magn. Reson. 88, 223 (1990).

[87] Lawrence G. Werbelow. "Adiabatic nuclear magnetic resonance linewidth contributions for central transitions of $I>1 / 2$ nuclei." J. Chem. Phys. 104, 3457 (1996).

[88] Lawrence Werbelow and Robert E. London. "Dynamic frequency shift." Concepts Magn. Reson. 8, 325 (1996).

[89] Lawrence G. Werbelow. "Dynamic frequency shift." In D.M. Grant and R.K. Harris, editors, "Encyclopedia of Nuclear Magnetic Resonance," volume 3, page 1776. John Wiley \& Sons, Inc., Chichester, England (1996).

[90] Lawrence G. Werbelow. "Relaxation processes: cross correlation and interference terms." In D.M. Grant and R.K. Harris, editors, "Encyclopedia of Nuclear Magnetic Resonance," volume 6, page 4072. John Wiley \& Sons, Inc., Chichester, England (1996).

[91] Anil Kumar, R. Christy Rani Grace, and P.K. Madhu. "Cross-correlations in NMR." Prog. NMR Spectrosc. 37, 191 (2000). 
[92] Lawrence G. Werbelow. "NMR dynamic frequency shifts and the quadrupolar interaction." J. Chem. Phys. 70, 5381 (1979).

[93] Uzi Eliav, Hadassah Shinar, and Gil Navon. "An observation of Na-23 NMR triplequantum dynamic shift in solution.” J. Magn. Reson. 94, 439 (1991).

[94] A.D. McLachlan. "Line widths of electron resonance spectra in solution." Proc. Roy. Soc. A (London) 280A, 271 (1964).

[95] Richard L. Hilt and Paul S. Hubbard. "Nuclear magnetic relaxation of three spin systems undergoing hindered rotations." Phys. Rev. 134, A392 (1964).

[96] Lawrence G. Werbelow and Alan G. Marshall. "Internal rotation and nonexponential methyl nuclear relaxation for macromolecules." J. Magn. Reson. 11, 299 (1973).

[97] Norbert Müller. "Observation of multi-exponential longitudinal and transverse relaxation in two-dimensional NMR." Chem. Phys. Lett. 131, 218 (1986).

[98] Norbert Müller, Geoffrey Bodenhausen, and Richard R. Ernst. "Relaxation-induced violations of coherence transfer selection rules in nuclear magnetic resonance." $\mathrm{J}$. Magn. Reson. 75, 297 (1987).

[99] Paul S. Hubbard. "Nonexponential nuclear magnetic relaxation by quadrupole interactions." J. Chem. Phys. 53, 985 (1970).

[100] Guy Jaccard, Stephen Wimperis, and Geoffrey Bodenhausen. "Multiple-quantum NMR spectroscopy of $\mathrm{S}=3 / 2$ spins in isotropic phase: A new probe for multiexponential relaxation." J. Chem. Phys. 85, 6282 (1986). 
[101] James Pekar and John S. Leigh Jr. "Detection of biexponential relaxation in sodium23 facilitated by double-quantum filtering." J. Magn. Reson. 69, 582 (1986).

[102] Lawrence Werbelow and Guy Pouzard. "Quadrupolar relaxation. The multiquantum coherences." J. Chem. Phys. 85, 3887 (1981).

[103] Lawrence G. Werbelow. "Relaxation theory for quadrupolar nuclei." In D.M. Grant and R.K. Harris, editors, "Encyclopedia of Nuclear Magnetic Resonance," volume 6, page 4092. John Wiley \& Sons, Inc., Chichester, England (1996).

[104] Uzi Eliav, Hadassah Shinar, and Gil Navon. "The formation of a second-rank tensor in Na-23 double-quantum filtered NMR as an indicator for order in a biological tissue." J. Magn. Reson. 98, 223 (1992).

[105] F. Bloch and A. Siegert. "Magnetic resonance for nonrotating fields." Phys. Rev. 57, $522(1940)$

[106] Paul A. Keifer. " $90^{\circ}$ pulse width calibrations: How to read a pulse width array." Concepts Magn. Reson. 11, 165 (1999).

[107] Richard P. Feynman, Frank L. Vernon, and Robert W. Hellwarth. "Geometric representation of the Schrödinger equation for solving maser equations." J. Appl. Phys. 28, 49 (1957).

[108] Eiichi Fukushima and Stephen B.W. Roeder. Experimental Pulse NMR: A Nuts and Bolts Approach. Addison-Wesley Publishing Company, Reading, MA (1981). 
[109] D.I. Hoult and R.E. Richards. "The signal-to-noise ratio of the nuclear magnetic resonance experiment." J. Magn. Reson. 24, 71 (1976).

[110] D. Budker, W. Gawlik, D.F. Kimball, S.M. Rochester, V.V. Yashchuk, and A. Weis. "Resonant nonlinear magneto-optical effects in atoms." Rev. Mod. Phys. 74, 1153 (2002).

[111] Ya.S. Greenberg. "Application of superconducting quantum interference devices to nuclear magnetic resonance." Rev. Mod. Phys. 70, 175 (1998).

[112] D. Raftery, H.W. Long, D. Shykind, P.J. Grandinetti, and A. Pines. "Multiple-pulse nuclear magnetic resonance of optically pumped xenon in a low magnetic field." $\underline{\text { Phys. }}$ Rev. A 50, 567 (1994).

[113] D.I. Hoult. "The principle of reciprocity in signal strength calculations-A mathematical guide." Concepts Magn. Reson. 12, 173 (2000).

[114] J.J. van der Klink. "The NMR reciprocity theorem for arbitrary probe geometry." Concepts Magn. Reson. 148, 147 (2001).

[115] W.P. Aue, E. Bartholdi, and R.R. Ernst. "Two-dimensional spectroscopy. Application to nuclear magnetic resonance." J. Chem. Phys. 64, 2229 (1976).

[116] W.P. Aue, J. Karhan, and R.R. Ernst. "Homonuclear broad band decoupling and two-dimensional J-resolved NMR spectroscopy.” J. Chem. Phys. 64, 4226 (1976).

[117] L. Emsley, D.D. Laws, and Alexander Pines. Lectures on Pulsed NMR. Proceed- 
ings of the International School of Physics Enrico Fermi; Course CXXXIX; Magnetic Resonance and Brain Function: Approaches from Physics, 3rd edition (1998).

[118] Ray Freeman and Eriks Kupče. "New methods for fast multidimensional NMR." J. Biomol. NMR 27, 101 (2003).

[119] A.M. Portis. "Electronic structure of $F$ centers: Saturation of the electronic spin resonance." Phys. Rev. 91, 1071 (1953).

[120] M.H. Levitt, D.P. Raleigh, F. Creuzet, and R.G. Griffin. "Theory and simulations of homonuclear spin pair systems in rotating solids." J. Chem. Phys. 92, 6347 (1990).

[121] Mattias Edén and Lucio Frydman. "Quadrupolar-driven recoupling of homonuclear dipolar interactions in the nuclear magnetic resonance of rotating solids." Phys. 114, 4116 (2001).

[122] F.J. Dyson. "The radiation theories of Tomonaga, Schwinger, and Feynman." $\underline{\text { Phys. }}$

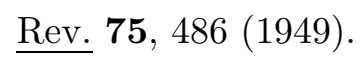

[123] Ulrich Haeberlen. "High resolution NMR in solids: Selective averaging." Adv. Magn. Reson.: Supplement 1 (1976).

[124] Philip Pechukas and John C. Light. "On the exponential form of time-displacement operators in quantum mechanics." J. Chem. Phys. 44, 3897 (1966).

[125] M. Matti Maricq. "Convergence of the Magnus expansion for time dependent two level systems." J. Chem. Phys. 86, 5647 (1987). 
[126] M. Matti Maricq. "Application of average Hamiltonian theory to the NMR of solids." Phys. Rev. B 25, 6622 (1982).

[127] Jon H. Shirley. "Solution of the Schrödinger equation with a Hamiltonian periodic in time." Phys. Rev. 138, B979 (1965).

[128] U. Haeberlen and J.S. Waugh. "Coherent averaging effects in magnetic resonance." Phys. Rev. 175, 453 (1968).

[129] Richard P. Feynman. "An operator calculus having applications in quantum electrodynamics." Phys. Rev. 84, 108 (1951).

[130] Alfred G. Redfield. "Nuclear magnetic resonance saturation and rotary saturation in solids." Phys. Rev. 98, 1787 (1955).

[131] Moses Lee and Walter I. Goldburg. "Nuclear-magnetic-resonance line narrowing by a rotating rf field." Phys. Rev. 140, A1261 (1965).

[132] D.E. Demco, J. Tegenfeldt, and J.S. Waugh. "Dynamics of cross relaxation in nuclear magnetic double resonance." Phys. Rev. B 11, 4133 (1975).

[133] Jamie Dale Walls. New NMR Structural Methods and Dynamical Models for

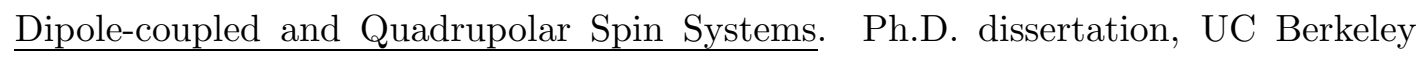
(Spring 2003).

[134] A. Samoson, E. Lippmaa, and A. Pines. "High resolution solid-state N.M.R. Averaging of second-order effects by means of a double-rotor." Mol. Phys. 65, 1013 (1988). 
[135] A. Llor and J. Virlet. "Towards high-resolution NMR of more nuclei in solids: Sample spinning with time-dependent spinner axis angle." Chem. Phys. Lett. 152, 248 (1988).

[136] B.F. Chmelka, K.T. Mueller, A. Pines, J. Stebbins, Y. Wu, and J.W. Zwanziger. "Oxygen-17 NMR in solids by dynamic-angle spinning and double rotation." Nature 339, 42 (1989).

[137] K.T. Mueller, B.Q. Sun, G.C. Chingas, J.W. Zwanziger, T. Terao, and A. Pines. "Dynamic-angle spinning of quadrupolar nuclei." J. Magn. Reson. 86, 470 (1990).

[138] Lucio Frydman and John S. Harwood. "Isotropic spectra of half-integer quadrupolar spins from bidimensional magic-angle spinning." J. Am. Chem. Soc. 117, 5367 (1995).

[139] Ales Medek, John S. Harwood, and Lucio Frydman. "Multiple-quantum magic-angle spinning NMR: A new method for the study of quadrupolar nuclei in solids." J. Am. Chem. Soc. 117, 12779 (1995).

[140] Amir Goldbourt and Perunthiruthy K. Madhu. "Multiple-quantum magic-angle spinning: High resolution solid state NMR spectroscopy of half-integer quadrupolar nuclei." Monatsh. Chem. 133, 1497 (2002).

[141] Kevin J. Pike, Reena P. Malde, Sharon E. Ashbrook, Jamie McManus, and Stephen Wimperis. "Multiple-quantum MAS NMR of quadrupolar nuclei. Do five-, seven-, and nine-quantum experiments yield higher resolution than the three-quantum experiment?" Solid State NMR 16, 203 (2000).

[142] Steven P. Brown, Stephen J. Heyes, and Stephen Wimperis. "Two-dimensional MAS 
multiple-quantum NMR of quadrupolar nuclei. Removal of inhomogeneous secondorder broadening." J. Magn. Reson. A 119, 280 (1996).

[143] D.J. States, R.A. Haberkorn, and D.J. Ruben. "A two-dimensional nuclear Overhauser experiment with pure absorption phase in four quadrants." J. Magn. Reson. 48, 286 (1982).

[144] D. Massiot, B. Touzo, D. Trumeau, J.P. Coutures, J. Virlet, P. Florian, and P.J. Grandinetti. "Two-dimensional magic-angle spinning isotropic reconstruction sequences for quadrupolar nuclei." Solid State NMR 6, 73 (1996).

[145] P.J. Grandinetti, J.H. Baltisberger, A. Llor, Y.K. Lee, U. Werner, M.A. Eastman, and A. Pines. "Pure-absorption-mode lineshapes and sensitivity in two-dimensional dynamic-angle spinning NMR." J. Magn. Reson. A 103, 72 (1993).

[146] Steven P. Brown and Stephen Wimperis. "Two-dimensional multiple-quantum MAS NMR of quadrupolar nuclei. Acquisition of the whole echo." J. Magn. Reson. 124, 279 (1997).

[147] Steven P. Brown and Stephen Wimperis. "Two-dimensional multiple-quantum MAS NMR of quadrupolar nuclei. A comparison of methods." J. Magn. Reson. 128, 42 (1997).

[148] C. Fernandez and J.P. Amoureux. "Triple-quantum MAS-NMR of quadrupolar nuclei." Solid State NMR 5, 315 (1996).

[149] S. Vega, T.W. Shattuck, and A. Pines. "Fourier-transform double-quantum NMR in solids." Phys. Rev. Lett. 37, 43 (1976). 
[150] S. Vega and A. Pines. "Operator formalism for double quantum NMR." J. Chem. Phys. 66, 5624 (1977).

[151] S. Vega and Y. Naor. "Triple quantum NMR with $I=3 / 2$ in solids." J. Chem. Phys. 75, 75 (1981).

[152] Geoffrey Bodenhausen, Herbert Kogler, and R.R. Ernst. "Selection of coherencetransfer pathways in NMR pulse experiments." J. Magn. Reson. 58, 370 (1984).

[153] Malcolm H. Levitt, P.K. Madhu, and Colan E. Hughes. "Cogwheel Phase Cycling." J. Magn. Reson. 155, 300 (2002).

[154] Colan E. Hughes, Marina Carravetta, and Malcolm H. Levitt. "Some conjectures for cogwheel phase cycling." J. Magn. Reson. 167, 259 (2004).

[155] C.A. Fyfe, J. Skibsted, H. Grondey, and H. Meyer zu Altenschildesche. "Pulsed field gradient multiple-quantum MAS NMR spectroscopy of half-integer spin quadrupolar nuclei." Chem. Phys. Lett. 281, 44 (1997).

[156] Alexej Jerschow, John W. Logan, and Alexander Pines. "High-resolution NMR of quadrupolar nuclei using mixed multiple-quantum coherences.” J. Magn. Reson. 149, 268 (2001).

[157] Alexej Jerschow and Norbert Müller. "Efficient simulation of coherence transfer pathway selection by phase cycling and pulsed field gradients in NMR.” J. Magn. Reson. 134, 17 (1998). 
[158] Alexander J. Vega. "MAS NMR spin locking in half-integer quadrupolar nuclei." J. Magn. Reson. 96, 50 (1992).

[159] W. Sun, J.T. Stephen, L.D. Potter, and Y. Wu. "Rotation-induced resonance and second-order quadrupolar effects on spin locking of half-integer quadrupolar nuclei." J. Magn. Reson. A 116, 181 (1995).

[160] Susan M. De Paul, Matthias Ernst, Jay S. Shore, Jonathan F. Stebbins, and Alexander Pines. "Cross-polarization from quadrupolar nuclei to silicon using low-radiofrequency amplitudes during magic-angle spinning." J. Phys. Chem. B 101, 3240 (1997).

[161] Thomas Vosegaard, Pierre Florian, Dominique Massiot, and Philip J. Grandinetti. "Multiple quantum magic-angle spinning using rotary resonance excitation." J. Chem. Phys. 114, 4618 (2001).

[162] Jamie D. Walls, Kwang Hun Lim, and Alexander Pines. "Theoretical studies of the spin dynamics of quadrupolar nuclei at rotational resonance conditions." J. Chem. Phys. 116, 79 (2002).

[163] Zhehong Gan and Philip Grandinetti. "Rotary resonance in multiple-quantum magicangle spinning." Chem. Phys. Lett. 352, 252 (2002).

[164] Jamie D. Walls, Kwang Hun Lim, John W. Logan, Jeffry T. Urban, Alexej Jerschow, and Alexander Pines. "Theoretical investigations of $I=5 / 2$ quadrupolar spin dynamics in the sudden-passage regime." J. Chem. Phys. 117, 518 (2002). 
[165] John W. Logan, Jeffry T. Urban, Jamie D. Walls, Kwang Hun Lim, Alexej Jerschow, and Alexander Pines. "Investigations of low-amplitude radio frequency pulses at and away from rotary resonance conditions for $I=5 / 2$ nuclei." Solid State NMR 22, 97 $(2002)$.

[166] John William Logan. Gas Dynamics Studied via Gas-Phase NMR and Solid-State NMR Studies of Quadrupolar Nuclei. Ph.D. dissertation, UC Berkeley (Spring 2003).

[167] Sharon E. Ashbrook, Steven P. Brown, and Stephen Wimperis. "Multiple-quantum cross-polarization in MAS NMR of quadrupolar nuclei." Chem. Phys. Lett. 288, 509 (1998).

[168] A. Wokaun and R.R. Ernst. "Selective excitation and detection in multilevel spin systems: Application of single transition operators." J. Chem. Phys. 67, 1752 (1977).

[169] Shimon Vega. "Fictitious spin 1/2 operator formalism for multiple quantum NMR." J. Chem. Phys. 68, 5518 (1978).

[170] G.N. Watson. A Treatise on the Theory of Bessel Functions. Cambridge Univeristy Press, Cambridge, UK, 2nd edition (1944).

[171] Malcolm H. Levitt. "Why do spinning sidebands have the same phase?" J. Magn. Reson. 82, 427 (1989).

[172] Mattias Edén. "Computer simulations in solid-state NMR. III. Powder averaging." Concepts Magn. Reson. 18A, 24 (2003). 
[173] Mads Bak and Niels Chr. Nielsen. "REPULSION, a novel approach to efficient powder averaging in solid-state NMR." J. Magn. Reson. 125, 132 (1997).

[174] Tania Giavani, Henrik Bildsøe, Jørgen Skibsted, and Hans J. Jakobsen. “A solid-state N-14 magic-angle spinning NMR study of some amino acids.” J. Magn. Reson. 166, $262(2004)$

[175] Klaus Eichele, Michael D. Lumsden, and Roderick E. Wasylishen. "Nitrogen-14 coupled dipolar-chemical shift C-13 NMR spectra of the amide fragment of peptides in the solid state." J. Phys. Chem. 97, 8909 (1993).

[176] Marciel Torrent, Danielle Mansour, Edmund P. Day, and Keiji Morokuma. "Quantum chemical study on oxygen-17 and nitrogen-14 nuclear quadrupole coupling parameters of peptide bonds in $\alpha$-helix and $\beta$-sheet proteins." J. Phys. Chem. A 105, 4546 (2001).

[177] Andrew L. Lee and A. Joshua Wand. "Assessing potential bias in the determination of rotational correlation times of proteins by NMR relaxation.” J. Biomol. NMR $\mathbf{1 3}$, 101 (1999).

[178] Ad Bax, Lewis E. Kay, Steven W. Sparks, and Dennis A. Torchia. "Line narrowing of amide proton resonances in 2D NMR spectra of proteins." J. Am. Chem. Soc. 111, 408 (1989).

[179] Jeffrey R. Brender, Deanne M. Taylor, and A. Ramamoorthy. "Orientation of amidenitrogen-15 chemical shift tensors in peptides: A quantum chemical study." J. Am. Chem. Soc. 123, 914 (2001). 
[180] Daniel Canet, Philippe Barthe, Pierre Mutzenhardt, and Christian Roumestand. "A comprehensive analysis of multifield N-15 relaxation parameters in proteins: Determination of N-15 chemical shift anisotropies.” J. Am. Chem. Soc. 123, 4567 (2001).

[181] M. Goldman. "Interference effects in the relaxation of a pair of unlike spin- $\frac{1}{2}$ nuclei." J. Magn. Reson. 60, 437 (1984).

[182] Konstantin Pervushin, Roland Riek, Gerhard Wider, and Kurt Wüthrich. "Attenuated $T_{2}$ relaxation by mutual cancellation of dipole-dipole coupling and chemical shift anisotropy indicates an avenue to NMR structures of very large biological macromolecules in solution." Proc. Natl. Acad. Sci. 94, 12366 (1997).

[183] Giovanni Lipari and Attila Szabo. "Model-free approach to the interpretation of nuclear magnetic resonance relaxation in macromolecules. 1. Theory and range of validity." J. Am. Chem. Soc. 104, 4546 (1982).

[184] Giovanni Lipari and Attila Szabo. "Model-free approach to the interpretation of nuclear magnetic resonance relaxation in macromolecules. 2. Analysis of experimental results." J. Am. Chem. Soc. 104, 4559 (1982).

[185] Nico Tjandra, Scott E. Feller, Richard W. Pastor, and Ad Bax. "Rotational diffusion anisotropy of human ubiquitin from N-15 NMR relaxation.” J. Am. Chem. Soc. 117, $12562(1995)$

[186] Larry G. Werbelow, Alain Allouche, and Guy Pouzard. "Multiplet structure in coupled spin systems containing high-spin nuclei.” J. Chem. Soc. Farad. Trans. 2 83, 871 (1987). 
[187] R.E. London, D.M. LeMaster, and L.G. Werbelow. "Unusual NMR multiplet structures of spin-1/2 nuclei coupled to spin-1 nuclei." J. Am. Chem. Soc. 116, 8400 (1994).

[188] Lawrence G. Werbelow and Robert E. London. "Dynamic nuclear magnetic resonance frequency shifts for spin $1 / 2$ nuclei coupled to efficiently relaxed spin $>1 / 2$ nuclei." J. Chem. Phys. 102, 5181 (1995).

[189] Lawrence G. Werbelow and Jozef Kowalewski. "Nuclear spin relaxation of spin onehalf nuclei in the presence of neighboring higher-spin nuclei." J. Chem. Phys. 107, 2775 (1997)

[190] Stephan Grzesiek and Ad Bax. "Interference between dipolar and quadrupolar interactions in the slow tumbling limit: A source of line shift and relaxation in H-2-labeled compounds." J. Am. Chem. Soc. 116, 10196 (1994).

[191] Nico Tjandra, Stephan Grzesiek, and Ad Bax. "Magnetic field dependence of nitrogen-proton $J$ splittings in N-15-enriched human ubiquitin resulting from relaxation interference and residual dipolar coupling." J. Am. Chem. Soc. 118, 6264 (1996).

[192] Nico Tjandra, Attila Szabo, and Ad Bax. "Protein backbone dynamics of N-15 chemical shift anisotropy from quantitative measurement of relaxation interference effects." J. Am. Chem. Soc. 118, 6986 (1996).

[193] Stephan Grzesiek, Jacob Anglister, Hao Ren, and Ad Bax. "C-13 line narrowing by 
H-2 decoupling in $\mathrm{H}-2 / \mathrm{C}-13 / \mathrm{N}-15$-enriched proteins. Application to triple resonance 4D $J$ connectivity of sequential amides.” J. Am. Chem. Soc. 115, 4369 (1993).

[194] M. Llinás, M.P. Klein, and K. Wüthrich. "Amide proton spin-lattice relaxation in polypeptides." Biophys. J. 24, 849 (1978).

[195] Andy C. LiWang and Ad Bax. "Solution NMR characterization of hydrogen bonds in a protein by indirect measurement of deuterium quadrupole couplings." J. Magn. Reson. 127, 54 (1997).

[196] L.J. Burnett and B.H. Muller. "Deuteron quadrupole coupling constants in three solid deuterated paraffin hydrocarbons: $\mathrm{C}_{2} \mathrm{D}_{6}, \mathrm{C}_{4} \mathrm{D}_{10}, \mathrm{C}_{6} \mathrm{D}_{14}$." J. Chem. Phys. 55, 5829 (1971).

[197] D. Petit, J.-P. Korb, A. Delville, J. Grandjean, and P. Laszlo. "Theory of nuclear spin relaxation in heterogeneous media and application to the cross correlation between quadrupolar and dipolar fluctuations of deuterons in clay gels.” J. Magn. Reson. 96, 252 (1992).

[198] G.J. Bowden and W.D. Hutchison. "Tensor operator formalism for multiple-quantum NMR. 1. Spin-1 nuclei." J. Magn. Reson. 67, 403 (1986).

[199] G.L. Hoatson and K.J. Packer. "The creation, interconversion and observation of states of zero-, single-, and double-quantum order in the N.M.R. spectroscopy of spin-1 nuclei using non-selective r.f. pulses.” Mol. Phys. 40, 1153 (1980).

[200] B.C. Sanctuary, T.K. Halstead, and P.A. Osment. "Multipole N.M.R. IV. Dynamics of single spins." Mol. Phys. 49, 753 (1983). 
[201] N. Chandrakumar. "High-resolution multiple quantum spectra of spin-1 systems in the isotropic phase." J. Am. Chem. Soc. 115, 3780 (1993).

[202] Y.S. Yen and D.P. Weitekamp. "Indirect detection of spin-1 double-quantum coherence in liquids." J. Magn. Reson. 47, 476 (1982).

[203] Gareth A. Morris and Ray Freeman. "Enhancement of nuclear magnetic resonance signals by polarization transfer." J. Am. Chem. Soc. 101, 760 (1979).

[204] Geoffrey Bodenhausen and David J. Ruben. "Natural abundance nitrogen-15 NMR by enhanced heteronuclear spectroscopy." Chem. Phys. Lett. 69, 185 (1980).

[205] A. Minoretti, W.P. Aue, M. Reinhold, and R.R. Ernst. "Coherence transfer by radiofrequency pulses for heteronuclear detection of multiple-quantum transitions." $\underline{\mathrm{J}}$. Magn. Reson. 40, 175 (1980).

[206] Sungsool Wi, Sharon E. Ashbrook, Stephen Wimperis, and Lucio Frydman. "Secondorder quadrupole-shielding effects in magic-angle spinning solid-state nuclear magnetic resonance." J. Chem. Phys. 118, 3131 (2003).

[207] Sungsool Wi and Lucio Frydman. "Residual dipolar couplings between quadrupolar nuclei in high resolution solid state NMR: Description and observations in the highfield limit." J. Chem. Phys. 112, 3248 (2000).

[208] Sungsool Wi, Veronica Frydman, and Lucio Frydman. "Residual dipolar couplings between quadrupolar nuclei in solid state nuclear magnetic resonance at arbitrary fields." J. Chem. Phys. 114, 8511 (2001). 
[209] Charles R. Sanders II and James P. Schwonek. "Characterization of magnetically orientable bilayers in mixtures of dihexanoylphosphatidylcholine and dimyristoylphosphatidylcholine by solid-state NMR." Biochemistry 31, 8898 (1992).

[210] Marcel Ottiger and Ad Bax. "Characterization of magnetically oriented phospholipid micelles for measurement of dipolar couplings in macromolecules." J. Biomol. NMR 12, 361 (1998).

[211] Giorgia Zandomeneghi, Marco Tomaselli, Philip T.F. Williamson, and Beat H. Meier. "NMR of bicelles: orientation and mosaic spread of the liquid-crystal director under sample rotation.” J. Biomol. NMR 25, 113 (2003).

[212] Ulf Henriksson, Lars Ödberg, Jan Christer Eriksson, and Lennart Westman. "Nitrogen-14 nuclear magnetic relaxation in aqueous micellar solutions of $n$ hexadecyltrimethylammonium bromide and chloride." J. Phys. Chem. 81, 76 (1977).

[213] Andrew E. Derome and Michael P. Williamson. "Rapid-pulsing artifacts in doublequantum-filtered COSY." J. Magn. Reson. 88, 177 (1990).

[214] Marcis Auzinsh and Ruvin Ferber. Optical Polarization of Molecules. Cambridge monographs on atomic, molecular, and chemical physics; 4. Cambridge University Press, Cambridge, UK (1995).

[215] Dieter Suter. The Physics of Laser-Atom Interactions. Cambridge studies in modern optics. Cambridge University Press, Cambridge, UK (1997).

[216] E.B. Alexandrov, M.P. Chaika, and G.I. Khvostenko. Interference of Atomic States. Springer series on atoms and plasmas; 7. Springer-Verlag, Berlin, Germany (1993). 
[217] James D. Macomber. The Dynamics of Spectroscopic Transitions: Illustrated by Magnetic Resonance and Laser Effects. Wiley-Interscience monographs in chemical physics. John Wiley \& Sons, Inc., New York (1976).

[218] I.I. Sobelman. Atomic Spectra and Radiative Transitions. Springer series on atoms and plasmas; 12. Springer-Verlag, Berlin, Germany, 2nd edition (1992).

[219] L. Allen and J.H. Eberly. Optical Resonance and Two-Level Atoms. Dover Publications, Inc., New York, NY (1987 (reproduced from original 1975 edition)).

[220] R. Kemp-Harper, D.J. Philp, and P.W. Kuchel. "Nuclear magnetic resonance of $J$ coupled quadrupolar nuclei: Use of the tensor operator product basis." J. Chem. Phys. 115, 2908 (2001).

[221] S.M. Rochester and D. Budker. "Atomic polarization visualized." Am. J. Phys. 69, $450(2001)$.

[222] Marcis Auzinsh. "Angular momentum dynamics in magnetic and electric field: Classical and quantum approach.” Can. J. Phys. 75, 853 (1997).

[223] T.K. Halstead, P.A. Osment, and B.C. Sanctuary. "Multipole N.M.R. IX. Polar graphical representation of nuclear spin polarizations." J. Magn. Reson. 60, 382 (1984).

[224] E.B. Alexandrov, M. Auzinsh, D. Budker, D.F. Kimball, S.M. Rochester, and V.V. Yashchuk. "Dynamic effects in nonlinear magneto-optics of atoms and molecules." $\underline{J}$ Opt. Soc. Am. B (2005). 
[225] Y.K. Lee. "Spin-1 nuclear quadrupole resonance theory with comparisons to nuclear magnetic resonance." Concepts Magn. Reson. 14, 155 (2002).

[226] V.V. Yashchuk, D. Budker, W. Gawlik, D.F. Kimball, Yu.P. Malakyan, and S.M. Rochester. "Selective addressing of high-rank polarization moments." $\underline{\text { Phys. Rev. }}$ Lett. 90, 253001 (2003).

[227] Giovanni Moruzzi and Franco Strumia, editors. The Hanle Effect and Level-Crossing Spectroscopy. Plenum Press, New York, NY (1991).

[228] G. Campolieti, B.C. Sanctuary, and H.B.R. Cole. "Multipole theory of soft pulses in NMR of quadrupolar solids.” J. Magn. Reson. 88, 457 (1990).

[229] B.C. Sanctuary. "Correction: 'Multipole theory of soft pulses in NMR of quadrupolar solids'." J. Magn. Reson. A 104, 242 (1993).

[230] M. Lombardi. "Création d'orientation par combinaison de deux alignements. Alignement et orientation des niveaux excités d'une décharge haute fréquence." J. Physique (Paris) 30, 631 (1969).

[231] Robert C. Hilborn, Larry R. Hunter, Kent Johnson, Stephen K. Peck, Alison Spencer, and John Watson. "Atomic barium and cesium alignment-to-orientation conversion in external electric and magnetic fields." Phys. Rev. A 50, 2467 (1994).

[232] D. Budker, D.F. Kimball, S.M. Rochester, and V.V. Yashchuk. "Nonlinear magnetooptical rotation via alignment-to-orientation conversion." Phys. Rev. Lett. 85, 2088 (2000). 
[233] Janis Alnis and Marcis Auzinsh. "Angular-momentum spatial distribution symmetry breaking in Rb by an external magnetic field." Phys. Rev. A 63, 023407 (2001).

[234] Matthew C. Kuntz, Robert C. Hilborn, and Alison M. Spencer. "Dynamic Stark shift and alignment-to-orientation conversion." Phys. Rev. A 65, 023411 (2002).

[235] Y.K. Lee, H. Robert, and D.K. Lathrop. "Circular polarization excitation and detection in N-14 NQR." J. Magn. Reson. 148, 355 (2001).

[236] J.C. Pratt. "Nuclear quadrupole resonance in the interaction representation." Mol. Phys. 34, 539 (1977).

[237] M.S. Krishnan and B.C. Sanctuary. "Evolution of spin density matrix in pure NQR." Z. Naturforsch. 42a, 907 (1987).

[238] Mangala S. Krishnan, Francis P. Temme, and B.C. Sanctuary. "Theory of pulses in nuclear quadrupole resonance spectroscopy. I. Physical picture.” Mol. Phys. 78, 1385 (1993).

[239] B.C. Sanctuary and Mangala S. Krishnan. "Theory of NQR pulses." Z. Naturforsch. 49a, 71 (1994).

[240] D. Budker, D.F. Kimball, S.M. Rochester, and J.T. Urban. "Alignment-to-orientation conversion and nuclear quadrupole resonance." Chem. Phys. Lett. 378, 440 (2003).

[241] Milton Abramowitz and Irene A. Segun, editors. Handbook of Mathematical Functions, with Formulas, Graphs, and Mathematical Tables. Dover Publications, Inc., New York, NY (1965 (reprinted 1968 with corrections)). 
[242] Mathematica v. 4.1. Wolfram Research, Inc, Champaign, IL (2001).

[243] N. Bloembergen. "Linear Stark effect in magnetic resonance spectra." Science 133, $1363(1961)$.

[244] Toshimoto Kushida and A.H. Silver. "Electrically induced nuclear resonance in $\mathrm{Al}_{2} \mathrm{O}_{3}$ (ruby)." Phys. Rev. 130, 1692 (1963).

[245] E. Brun, R. Hann, W. Pierce, and W.H. Tanttila. "Spin transitions induced by external rf electric field in GaAs." Phys. Rev. Lett. 8, 365 (1962).

[246] E. Brun, R.J. Mahler, H. Mahon, and W.L. Pierce. "Electrically induced nuclear quadrupole spin transitions in a GaAs single crystal." Phys. Rev. 129, 1965 (1963).

[247] Hiroshi Hatanaka. "Thermal mixing between spin systems in $\mathrm{Al}_{2} \mathrm{O}_{3}$ by a resonant $\mathrm{rf}$ electric field.” Phys. Rev. B 40, 289 (1989).

[248] Hiroshi Hatanaka and Masahiro Takahama. "Adiabatic demagnetization of quadrupolar nuclear spins in a rotating frame by use of an rf electric field." Phys. Rev. B 47, $3213(1993)$.

[249] Abbas Khadjavi, Allen Lurio, and W. Happer. "Stark effect in the excited states of Rb, Cs, Cd, and Hg." Phys. Rev. 167, 128 (1968).

[250] J.R.P. Angel and P.G.H. Sandars. "The hyperfine structure Stark effect. I. Theory." Proc. Roy. Soc. A (London) 305, 125 (1968).

[251] R.J. Knize, Z. Wu, and W. Happer. "Optical pumping and spin exchange in gas cells." 
In David Bates and Benjamin Bederson, editors, "Advances in Atomic and Molecular Physics," volume 24, page 223. Academic Press, Inc., San Diego, CA (1988).

[252] J.P. Barrat and C. Cohen-Tannoudji. "Étude du pompage optique dans le formalisme de la matrice densité." J. Physique Radium 22, 329 (1961).

[253] J.P. Barrat and C. Cohen-Tannoudji. "Élargissement et déplacement des raies de résonance magnétique causés par une excitation optique." J. Physique Radium 22, $443(1961)$

[254] W. Happer and B.S. Mathur. "Effective operator formalism in optical pumping." Phys. Rev. 163, 12 (1967).

[255] B.S. Mathur, H. Tang, and W. Happer. "Light shifts in alkali atoms." $\underline{\text { Phys. Rev. }}$ 171, 11 (1968).

[256] B.R. Bulos, A. Marshall, and W. Happer. "Light shifts due to real transitions in optically pumped alkali atoms." Phys. Rev. A 4, 51 (1971).

[257] Robert C. Hilborn. "Atoms in orthogonal electric and magnetic fields: A comparison of quantum and classical models." Am. J. Phys. 63, 330 (1995).

[258] C.H. Townes and A.L. Schawlow. Microwave Spectroscopy. Dover Publications, Inc., New York, NY (1975 (reproduced from original 1955 edition)).

[259] S. Vega, E.T. Olejniczak, and R.G. Griffin. "Rotor frequency lines in the nuclear magnetic resonance spectra of rotating solids." J. Chem. Phys. 80, 4832 (1984). 
[260] C.P. Slichter and William C. Holton. "Adiabatic demagnetization in a rotating reference frame." Phys. Rev. 122, 1701 (1961).

[261] A.N. Garroway and G.C Chingas. "Creation of $J$-ordered magnetic states by adiabatic demagnetization in the rotating frame." J. Magn. Reson. 38, 179 (1980).

[262] Kenneth J. Packer and Kevin M. Wright. "J order and cross polarization in scalarcoupled spin systems.” J. Magn. Reson. 41, 268 (1980).

[263] G.C. Chingas, A.N. Garroway, W.B. Moniz, and R.D. Bertrand. "Adiabatic J crosspolarization in liquids for signal enhancement in NMR." J. Am. Chem. Soc. 102, $2526(1980)$.

[264] Colan E. Hughes, Richard Kemp-Harper, and Stephen Wimperis. "NMR excitation of quadrupolar order using adiabatic demagnetization in the rotating frame." J. Chem. Phys. 108, 876 (1998).

[265] J. Jeener and P. Broekaert. "Nuclear magnetic resonance in solids: Thermodynamic effects of a pair of rf pulses." Phys. Rev. 157, 232 (1967).

[266] Thomas M. Barbara, Robert L. Vold, and Regitze R. Vold. "A modified JeenerBroekaert sequence for relaxation measurements in liquid crystals." J. Magn. Reson. 59, 478 (1984).

[267] Stephen Wimperis and Geoffrey Bodenhausen. "Broadband excitation of quadrupolar order by modified Jeener-Broekaert sequences." Chem. Phys. Lett. 132, 194 (1986).

[268] Richard Kemp-Harper, Steven P. Brown, Colan E. Hughes, Peter Styles, and Stephen 
Wimperis. "Na-23 NMR methods for selective observation of sodium ions in ordered environments." Prog. NMR Spectrosc. 30, 157 (1997).

[269] Colan E. Hughes and Stephen Wimperis. "Bounds on spin dynamics and the design of multiple-pulse NMR experiments." J. Chem. Phys. 106, 2105 (1997).

[270] A. Samoson and E. Lippmaa. "Excitation phenomena and line intensities in highresolution NMR powder spectra of half-integer quadrupolar nuclei.” $\underline{\text { Phys. Rev. B }}$ 28, 6567 (1983).

[271] A.P.M. Kentgens, J.J.M. Lemmens, F.M.M. Geurts, and W.S. Veeman. "Twodimensional solid-state nutation NMR of half-integer quadrupolar nuclei." J. Magn. Reson. 71, 62 (1987).

[272] Albert Messiah. Quantum Mechanics: Two Volumes Bound as One. Dover Publications, Inc., Mineola, NY (1999).

[273] Gang Wu, David Rovnyak, and Robert G. Griffin. "Quantitative multiple-quantum magic-angle-spinning NMR spectroscopy of quadrupolar nuclei in solids." $\underline{\text { J. Am. }}$ Chem. Soc. 118, 9326 (1996).

[274] T. Mildner, M.E. Smith, and R. Dupree. "2D five quantum MAS NMR using rotationally induced coherence transfer." Chem. Phys. Lett. 306, 297 (1999).

[275] P.K. Madhu, Amir Goldbourt, Lucio Frydman, and Shimon Vega. "Sensitivity enhancement of the MQMAS NMR experiment by fast amplitude modulation of the pulses." Chem. Phys. Lett. 307, 41 (1999). 
[276] P.K. Madhu, Amir Goldbourt, Lucio Frydman, and Shimon Vega. "Fast radiofrequency amplitude modulation in multiple-quantum magic-angle-spinning nuclear magnetic resonance: Theory and experiments." J. Chem. Phys. 112, 2377 (2000).

[277] A.P.M. Kentgens and R. Verhagen. "Advantages of double frequency sweeps in static, MAS and MQMAS NMR of spin $I=3 / 2$ nuclei." Chem. Phys. Lett. 300, 435 (1999).

[278] Dinu Iuga, Hartmut Schäfer, Rieko Verhagen, and Arno P.M. Kentgens. "Population and coherence transfer induced by double frequency sweeps in half-integer quadrupolar spin systems." J. Magn. Reson. 147, 192 (2000).

[279] Hartmut Schäfer, Dinu Iuga, Rieko Verhagen, and Arno P.M. Kentgens. "Population and coherence transfer in half-integer quadrupolar spin systems induced by simultaneous rapid passages of the satellite transitions: A static and spinning single crystal nuclear magnetic resonance study." J. Chem. Phys. 114, 3073 (2001).

[280] Dinu Iuga and Arno P.M. Kentgens. "Influencing the satellite transitions of halfinteger quadrupolar nuclei for the enhancement of magic angle spinning spectra." J. Magn. Reson. 158, 65 (2002).

[281] Yu.P. Malakyan, S.M. Rochester, D. Budker, D.F. Kimball, and V.V. Yashchuk. "Nonlinear magneto-optical rotation of frequency-modulated light resonant with a low- $J$ transition." Phys. Rev. A 69, 013817 (2004).

[282] D.P. Weitekamp, A. Bielecki, D. Zax, K. Zilm, and A. Pines. "Zero-field nuclear magnetic resonance.” Phys. Rev. Lett. 50, 1807 (1983). 
[283] Ann M. Thayer and Alexander Pines. "Zero-field NMR." Acc. Chem. Res. 20, 47 (1987).

[284] Adam J. Moulé, Megan M. Spence, Song-I Han, Juliette A. Seeley, Kimberly L. Pierce, Sunil Saxena, and Alexander Pines. "Amplification of xenon NMR and MRI by remote detection." Proc. Natl. Acad. Sci. 100, 9122 (2003).

[285] Adam James Moulé. Ex-situ and Remote Detection Nuclear Magnetic Resonance. Ph.D. dissertation, UC Berkeley (Spring 2003).

[286] Juliette Anne Seeley. Remote Detection for More Sensitive NMR and MRI. Ph.D. dissertation, UC Berkeley (Spring 2004).

[287] Juliette A. Seeley, Song-I Han, and Alexander Pines. "Remotely detected high-field MRI of porous samples.” J. Magn. Reson. 167, 282 (2004).

[288] Boyd M. Goodson. "Nuclear magnetic resonance of laser-polarized noble gases in molecules, materials, and organisms." J. Magn. Reson. 155, 157 (2002).

[289] G. Deville, M. Bernier, and J.M. Delrieux. "NMR multiple echoes observed in solid He-3." Phys. Rev. B 19, 5666 (1979).

[290] G. Eska, H.-G. Willers, B. Amend, and W. Wiedemann. "Spin echo experiments in superfluid He-3." Physica B \& C 108B, 1155 (1981).

[291] D. Einzel, G. Eska, Y. Hirayoshi, T. Kopp, and P. Wölfle. "Multiple spin echoes in a normal Fermi liquid.” Phys. Rev. Lett. 53, 2312 (1984). 
[292] Hommo T. Edzes. "The nuclear magnetization as the origin of transient changes in the magnetic field in pulsed NMR experiments." J. Magn. Reson. 86, 293 (1990).

[293] R. Bowtell, R.M. Bowley, and P. Glover. "Multiple spin echoes in liquids in a high magnetic field.” J. Magn. Reson. 88, 643 (1990).

[294] Harald Körber, Elmar Dormann, and Georg Eska. "Multiple spin echoes for protons in water." J. Magn. Reson. 93, 589 (1991).

[295] A.S. Bedford, R. Bowtell, and R.M. Bowley. "Multiple spin echoes in multicomponent liquids." J. Magn. Reson. 93, 516 (1991).

[296] R. Bowtell. "Indirect detection via the dipolar demagnetizing field." J. Magn. Reson. 100, 1 (1992).

[297] John C. Waterton, Richard P.O. Jones, and Gareth A. Morris. "A spatial editing of NMR spectra by use of heteronuclear multiple spin echoes.” J. Magn. Reson. 97, 218 (1992).

[298] Richard P.O. Jones, Gareth A. Morris, and John C. Waterton. "Multiple spin echoes in a high-resolution spectrometer." J. Magn. Reson. 98, 115 (1992).

[299] M.A. McCoy and W.S. Warren. "Three-quantum nuclear magnetic resonance spectroscopy of liquid water: Intermolecular multiple-quantum coherence generated by spin-cavity coupling." J. Chem. Phys. 93, 858 (1990).

[300] Daniel Abergel, Marc A. Delsuc, and Jean-Yves Lallemand. "Comment on: Is multiple 
quantum nuclear magnetic resonance spectroscopy of liquid water real?" J. Chem. Phys. 96, 1657 (1992).

[301] W.S. Warren, Qiuhong He, M. McCoy, and F.C. Spano. "Reply to the Comment on: Is multiple quantum nuclear magnetic resonance of water real?" $\underline{\text { J. Chem. Phys. 96, }}$ 1659 (1992).

[302] Qiuhong He, Wolfgang Richter, Sujatha Vathyam, and Warren S. Warren. "Intermolecular multiple-quantum coherences and cross correlations in solution nuclear magnetic resonance." J. Chem. Phys. 98, 6779 (1993).

[303] Warren S. Warren, Wolfgang Richter, Amy Hamilton Andreotti, and Bennett T. Farmer II. "Generation of impossible cross-peaks between bulk water and biomolecules in solution NMR." Science 262, 2005 (1993).

[304] G.J. Bowden, T. Heseltine, and M.J. Prandolini. "Double quantum and cross correlation peaks in COSY NMR spectra." Chem. Phys. Lett. 233, 639 (1995).

[305] Malcolm H. Levitt. "Demagnetization field effects in two-dimensional solution NMR." Concepts Magn. Reson. 8, 77 (1995).

[306] M. Goldman and H. Desvaux. "Cross-precession induced by the average dipolar field in high-resolution NMR." Chem. Phys. Lett. 256, 497 (1996).

[307] J. Jeener. "Equivalence between the 'classical' and the 'Warren' approaches for the effects of long range dipolar couplings in liquid nuclear magnetic resonance." J. Chem. Phys. 112, 5091 (2000). 
[308] E.D. Minot, P.T. Callaghan, and N. Kaplan. "Multiple echoes, multiple quantum coherence, and the dipolar field: Demonstrating the significance of higher order terms in the equilibrium density matrix.” J. Magn. Reson. 140, 200 (1999).

[309] Sangdoo Ahn and Warren S. Warren. "Effects of intermolecular dipolar couplings in solution NMR in separated time intervals: the competition for coherence transfer pathways." Chem. Phys. Lett. 291, 121 (1998).

[310] Sangdoo Ahn, Natalia Lisitza, and Warren S. Warren. "Intermolecular zero-quantum coherences of multi-component spin systems in solution NMR." J. Magn. Reson. 133, 266 (1998).

[311] Sangdoo Ahn, Sanghyuk Lee, and Warren S. Warren. "The competition between intramolecular $J$ couplings, radiation damping, and intermolecular dipolar couplings in two-dimensional solution nuclear magnetic resonance.” Mol. Phys. 95, 769 (1998).

[312] Huiming Zhang, Natalia Lizitsa [sic], Robert G. Bryant, and Warren S. Warren. "Experimental characterization of intermolecular multiple-quantum coherence pumping efficiency in solution NMR.” J. Magn. Reson. 148, 200 (2001).

[313] Chandrasekhar Ramanathan and Richard Bowtell. "Dynamics of the nuclear magnetic resonance COSY-revamped by asymmetric z-gradients (CRAZED) experiment." $\underline{\text { J. }}$ Chem. Phys. 114, 10854 (2001).

[314] Zhong Chen, Shaokuan Zheng, and Jianhui Zhong. "Optimal RF flip angles for multiple spin-echoes and iMQCs of different orders with the CRAZED pulse sequence." Chem. Phys. Lett. 347, 143 (2001). 
[315] W.S. Warren, S.Y. Huang, S. Ahn, and Y.Y. Lin. "Understanding third-order dipolar effects in solution nuclear magnetic resonance: Hahn echo decays and intermolecular triple-quantum coherences." J. Chem. Phys. 116, 2075 (2002).

[316] Zhong Chen, Zhi-Wei Chen, Dennis W. Hwang, Jianhui Zhong, and Lian-Pin Hwang. "Separation and characterization of different signals from intermolecular three-spin orders in solution NMR." J. Magn. Reson. 171, 244 (2004).

[317] Wolfgang Richter, Sanghyuk Lee, Warren S. Warren, and Qiuhong He. "Imaging with intermolecular multiple-quantum coherences in solution nuclear magnetic resonance." Science 267, 654 (1995).

[318] Warren S. Warren, Sangdoo Ahn, Marlene Mescher, Michael Garwood, Kamil Ugurbil, Wolfgang Richter, Rahim R. Rizi, Jeff Hopkins, and John S. Leigh. "MR imaging contrast enhancement based on intermolecular zero quantum coherences." 281, 247 (1998).

[319] Wolfgang Richter, Marlene Richter, Warren S. Warren, Hellmut Merkle, Peter Andersen, Gregor Adriany, and Kamil Ugurbil. "Functional magnetic resonance imaging with intermolecular multiple-quantum coherences.” Magn. Reson. Imag. 18, 489 $(2000)$.

[320] Jianhui Zhong, Zhong Chen, and Edmund Kwok. "New image contrast mechanisms in intermolecular double-quantum coherence human MR imaging." J. Magn. Reson. Imag. 12, 311 (2000).

[321] S. Sendhil Velan, P.T. Narasimhan, and Russell E. Jacobs. "MR imaging with phase 
encoding of intermolecular multiple quantum coherences." J. Magn. Reson. 152, 189 (2001).

[322] Louis-Serge Bouchard, Rahim R. Rizi, and Warren S. Warren. "Magnetization structure contrast based on intermolecular multiple-quantum coherences." Magn. Reson. Med. 48, 973 (2002).

[323] Xiaoping Tang, Henry Ong, Kerry Shannon, and Warren S. Warren. "Simultaneous acquisition of multiple orders of intermolecular multiple-quantum coherence images." Magn. Reson. Imag. 21, 1141 (2003).

[324] Chih-Liang Chin, Xiaoping Tang, Louis-S. Bouchard, Punam K. Saha, Warren S. Warren, and Felix W. Wehrli. "Isolating quantum coherences in structural imaging using intermolecular double-quantum coherence MRI." J. Magn. Reson. 165, 309 (2003).

[325] T. Hou, Z. Chen, D.W. Hwang, J.H. Zhong, and L.P. Hwang. "Intermolecular doublequantum coherence MR microimaging of pig tail with unique image contrast." Magn. Reson. Imag. 22, 543 (2004).

[326] R. Bowtell and P. Robyr. "Structural investigations with the dipolar demagnetizing field in solution NMR." Phys. Rev. Lett. 76, 4971 (1996).

[327] P. Robyr and R. Bowtell. "Nuclear magnetic resonance microscopy in liquids using the dipolar field." J. Chem. Phys. 106, 467 (1997).

[328] P. Robyr and R. Bowtell. "Measuring Patterson functions of inhomogeneous liquids using the nuclear dipolar field." J. Chem. Phys. 107, 702 (1997). 
[329] S. Capuani, M. Alesiani, F.M. Alessandri, and B. Maraviglia. "Characterization of porous media structure by non linear NMR methods." Magn. Reson. Imag. 19, 319 (2001).

[330] S.M. Brown, P.N. Sen, and D.G. Cory. "Nuclear magnetic resonance scattering across interfaces via the dipolar demagnetizing field." J. Chem. Phys. 116, 295 (2002).

[331] S.M. Brown, P.N. Sen, and D.G. Cory. "Scaling laws in NMR scattering via dipolar fields.” J. Magn. Reson. 154, 154 (2002).

[332] F.M. Alessandri, S. Capuani, and B. Maraviglia. "Multiple spin echoes in heterogeneous systems: Physical origins of the observed dips.” J. Magn. Reson. 156, 72 $(2002)$.

[333] X.-P. Tang, C.-L. Chin, L.-S. Bouchard, F.W. Wehrli, and W.S. Warren. "Observing

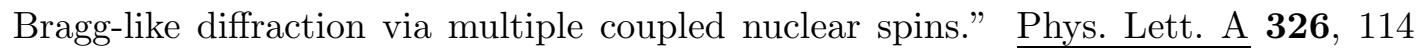
(2004).

[334] R. Bowtell, S. Gutteridge, and C. Ramanathan. "Imaging the long-range dipolar field in structured liquid state samples." J. Magn. Reson. 150, 147 (2001).

[335] S. Gutteridge, C. Ramanathan, and R. Bowtell. "Mapping the absolute value of $M_{0}$ using dipolar field effects." Magn. Reson. Med. 47, 871 (2002).

[336] Sujatha Vathyam, Sanghyuk Lee, and Warren S. Warren. "Homogeneous NMR spectra in inhomogeneous fields." Science 272, 92 (1996). 
[337] S. Vathyam. "Correction to 'Homogeneous NMR spectra in inhomogeneous fields' (vol. 272, pg. 92, 1996)." Science 273, 564 (1996).

[338] Y.-Y. Lin, N. Murali, W. Brey, C.R. Bowers, and W.S. Warren. "High-resolution, > 1 GHz NMR in unstable magnetic fields.” Phys. Rev. Lett. 85, 3732 (2000).

[339] Cornelius Faber, Eberhard Pracht, and Axel Haase. "Resolution enhancement in in vivo NMR spectroscopy: detection of intermolecular zero-quantum coherences." $\underline{\text { J. }}$ Magn. Reson. 161, 127 (2003).

[340] Zhong Chen, Zhiwei Chen, and Jianhui Zhong. "High-resolution NMR spectra in inhomogeneous fields via IDEAL (Intermolecular Dipolar-interaction Enhanced All Lines) method." J. Am. Chem. Soc. 126, 446 (2004).

[341] Zhong Chen, Ting Hou, Zhi-Wei Chen, Dennis W. Hwang, and Lian-Pin Hwang. "Selective intermolecular zero-quantum coherence in high-resolution NMR under inhomogeneous fields." Chem. Phys. Lett. 386, 200 (2004).

[342] J. Jeener. "Dynamical effects of the dipolar field inhomogeneities in high-resolution NMR: Spectral clustering and instabilities." Phys. Rev. Lett. 82, 1772 (1999).

[343] Bérengère Villard and Pierre-Jean Nacher. "NMR spectral clustering and instabilities in polarised liquid He-3." Physica B 284, 180 (2000).

[344] Yung-Ya Lin, Natalia Lisitza, Sangdoo Ahn, and Warren S. Warren. "Resurrection of crushed magnetization and chaotic dynamics in solution NMR spectroscopy." 290, 118 (2000). 
[345] K.L. Sauer, F. Marion, P.J. Nacher, and G. Tastevin. "NMR instabilities and spectral clustering in laser-polarized liquid xenon.” Phys. Rev. B 63, 184427 (2001).

[346] J. Jeener. "Dynamical instabilities in liquid nuclear magnetic resonance experiments with large nuclear magnetization, with and without pulsed field gradients." J. Chem. Phys. 116, 8439 (2002).

[347] Susie Y. Huang, Yung-Ya Lin, Natalia Lisitza, and Warren S. Warren. "Signal interferences from turbulent spin dynamics in solution nuclear magnetic resonance spectroscopy." J. Chem. Phys. 116, 10325 (2002).

[348] M.P. Ledbetter and M.V. Romalis. "Nonlinear effects from dipolar interactions in hyperpolarized liquid Xe-129.” Phys. Rev. Lett. 89, 287601 (2002).

[349] Pierre-Jean Nacher, François Marion, Bérengère Villard, and Geneviève Tastevin. "NMR dynamics with large dipolar interactions in liquid Helium-3 films." $\underline{\text { J. Low }}$ Temp. Phys. 126, 85 (2002).

[350] Pierre-Jean Nacher, Nathalie Piegay, François Marion, and Geneviève Tastevin. "NMR instabilities in highly magnetised liquid helium solutions." J. Low Temp. Phys. 126, 145 (2002).

[351] Susie Y. Huang, Jamie D. Walls, You Wang, Warren S. Warren, and Yung-Ya Lin. "Signal irreproducibility in high-field solution magnetic resonance experiments caused by spin turbulence." J. Chem. Phys. 121, 6105 (2004).

[352] Shaul Mukamel and Andreas Tortschanoff. "Multiple quantum coherences in liquid 
state NMR and nonlinear optics: collective vs local origin." Chem. Phys. Lett. 357, 327 (2002).

[353] J. Jeener. "Comment on 'Multiple quantum coherences in liquid state NMR and nonlinear optics: collective vs local origin' [Chem. Phys. Lett. 357 (2002) 327]." Chem. Phys. Lett. 368, 247 (2003).

[354] S. Mukamel. "Reply to comment on 'Multiple quantum coherences in liquid state NMR and nonlinear optics; collective vs. local origin' [Chem. Phys. Lett. 357 (2002) 327]." Chem. Phys. Lett. 368, 249 (2003).

[355] Tilman Enss, Sangdoo Ahn, and Warren S. Warren. "Visualizing the dipolar field in solution NMR and MR imaging: three-dimensional structure simulations." Chem. Phys. Lett. 305, 101 (1999).

[356] Sean Garrett-Roe and Warren S. Warren. "Numerical studies of intermolecular multiple quantum coherences: High-resolution NMR in inhomogeneous fields and contrast enhancement in MRI." J. Magn. Reson. 146, 1 (2000).

[357] Wyndham B. Blanton. "BlochLib: a fast NMR C++ tool kit." J. Magn. Reson. 162, 269 (2003).

[358] Wyndham Bolling Blanton. High Performance Computations in NMR. Ph.D. dissertation, UC Berkeley (Fall 2002).

[359] M.P. Ledbetter, I.M. Savukov, L.-S. Bouchard, and M.V. Romalis. "Numerical and experimental studies of long-range magnetic dipolar interactions." J. Chem. Phys. 121, 1454 (2004). 
[360] Jonathan Baugh, Alfred Kleinhammes, Daxing Han, Qi Wang, and Yue Wu. "Confinement effect on dipole-dipole interactions in nanofluids." Science 294, 1505 (2001).

[361] D.P. Craig and T. Thirunamachandran. Molecular Quantum Electrodynamics: An

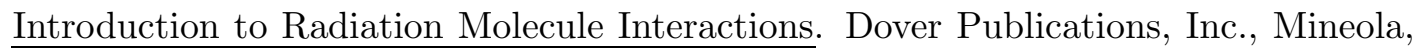
NY (1984 (reprinted 1998 with corrections)).

[362] C.J. Durant, M.P. Hertzberg, and P.W. Kuchel. "Magnetic Susceptibility: Further insights into macroscopic and microscopic fields and the sphere of Lorentz." Concepts Magn. Reson. A 18A, 72 (2003).

[363] W.S. Warren, S. Lee, W. Richter, and S. Vathyam. "Correcting the classical dipolar demagnetizing field in solution NMR.” Chem. Phys. Lett. 247, 207 (1995).

[364] M.H. Cohen and F. Keffer. "Dipolar sums in the primitive cubic lattices." $\underline{\text { Phys. Rev. }}$ 99, 1128 (1955).

[365] A. Vlassenbroek, J. Jeener, and P. Broekaert. "Macroscopic and microscopic fields in high-resolution liquid NMR." J. Magn. Reson. A 118, 234 (1996).

[366] Matthew Paul Augustine. Theoretical Treatment of Time Dependent Phenomena and The Investigation of Highly Magnetized Systems. Ph.D. dissertation, Yale University (May 1995).

[367] John J. Roche. " $B$ and $H$, the intensity vectors of magnetism: A new approach to resolving a century-old controversy." Am. J. Phys. 68, 438 (2000). 
[368] Edward M. Purcell. Electricity and Magnetism. Berkeley Physics Course; vol. 2. McGraw-Hill Book Company, New York, NY (1965).

[369] W.C. Dickinson. "The time average magnetic field at the nucleus in nuclear magnetic resonance experiments." Phys. Rev. 81, 717 (1951).

[370] M.V. Romalis and W. Happer. "Inhomogeneously broadened spin masers." $\underline{\text { Phys. }}$

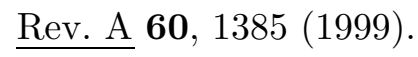

[371] J.A. Osborn. "Demagnetizing factors of the general ellipsoid." Phys. Rev. 67, 351 (1945).

[372] J.R. Zimmerman and M.R. Foster. "Standardization of N.M.R. high resolution spectra." J. Phys. Chem. 61, 282 (1957).

[373] L.E. Drain. "The broadening of magnetic resonance lines due to field inhomogeneities in powdered samples." Proc. Phys. Soc. 80, 1380 (1962).

[374] A.N. Garroway. "Magic-angle sample spinning of liquids." J. Magn. Reson. 49, 168 (1982).

[375] George Mozurkewich, H.I. Ringermacher, and D.I. Bolef. "Effect of demagnetization on magnetic resonance line shapes in bulk samples: Application to tungsten." $\underline{\text { Phys. }}$

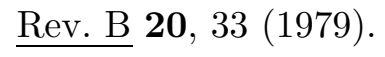

[376] Thomas M. Barbara. "Cylindrical demagnetization effects and microprobe design in high-resolution NMR.” J. Magn. Reson. A 109, 265 (1994). 
[377] C.H. Durney, J. Bertolina, D.C. Ailion, R. Christman, A.G. Cutillo, A.H. Morris, and S. Hashemi. "Calculation and interpretation of inhomogeneous line broadening in models of lungs and other heterogeneous structures.” J. Magn. Reson. 85, 554 (1989).

[378] L.F. Fuks, F.S.C. Huang, C.M. Carter, W.A. Edelstein, and P.B. Roemer. "Susceptibility, lineshape, and shimming in high-resolution NMR." J. Magn. Reson. 100, 229 (1992).

[379] E.L. Hahn. "Spin echoes." Phys. Rev. 80, 580 (1950).

[380] Sangdoo Ahn, Warren S. Warren, and Sanghyuk Lee. "Quantum treatment of intermolecular multiple-quantum coherences with intramolecular $J$-coupling in solution NMR.” J. Magn. Reson. 128, 114 (1997).

[381] Elke Kossel, Rainer Kimmich, and Ioan Ardelean. "Influence of $J$-coupling on heteronuclear nonlinear (or multiple) spin echoes." Chem. Phys. Lett. 347, 157 (2001).

[382] Matthew P. Augustine and Kurt W. Zilm. "Observation of bulk susceptibility effects in high-resolution nuclear magnetic resonance." J. Magn. Reson. A 123, 145 (1996).

[383] Patricia R. Bachiller, Sangdoo Ahn, and Warren S. Warren. "Detection of intermolecular heteronuclear multiple-quantum coherences in solution NMR.” J. Magn. Reson. 122, 94 (1996).

[384] Ioan Ardelean, Elke Kossel, and Rainer Kimmich. "Attenuation of homo- and heteronuclear multiple spin echoes by diffusion." J. Chem. Phys. 114, 8520 (2001). 
[385] Joseph H. Noggle and Roger E. Schirmer. The Nuclear Overhauser Effect. Academic Press, Inc., New York, NY (1971).

[386] P. Broekaert and J. Jeener. "Suppression of radiation damping in NMR in liquids by active electronic feedback." J. Magn. Reson. A 113, 60 (1995).

[387] Alain Louis-Joseph, Daniel Abergel, and Jean-Yves Lallemand. "Neutralization of radiation damping by selective feedback on a $400 \mathrm{MHz}$ NMR spectrometer.” J. Biomol.

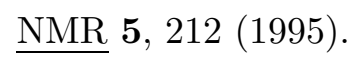

[388] W.E. Maas, F.H. Laukien, and D.G. Cory. "Suppression of radiation damping by $Q$ switching during acquisition.” J. Magn. Reson. A 113, 274 (1995).

[389] C. Anklin, M. Rindlisbacher, G. Otting, and F.H. Laukien. "A probehead with switchable quality factor. Suppression of radiation damping." J. Magn. Reson. B 106, 199 (1995).

[390] A.A. Maudsley, A. Wokaun, and R.R. Ernst. "Coherence transfer echoes." $\underline{\text { Chem. }}$ Phys. Lett. 55, 9 (1978).

[391] Philip H. Bolton and Geoffrey Bodenhausen. "Resolution enhancement in heteronuclear two-dimensional spectroscopy by realignment of coherence transfer echoes." J. Magn. Reson. 46, 306 (1982).

[392] M. Gochin, D.P. Weitekamp, and A. Pines. "A SHARP method for high-resolution NMR of heteronuclear spin systems in inhomogeneous fields." J. Magn. Reson. 53, $431(1985)$. 
[393] M.V. Romalis and M.P. Ledbetter. "Transverse spin relaxation in liquid Xe-129 in the presence of large dipolar fields." Phys. Rev. Lett. 87, 067601 (2001).

[394] J.J. Heckman, M.P. Ledbetter, and M.V. Romalis. "Enhancement of SQUID-detected NMR signals with hyperpolarized liquid Xe-129 in a $1 \mu \mathrm{T}$ magnetic field." $\underline{\text { Phys. Rev. }}$ Lett. 91, 067601 (2003).

[395] Frank J. Adrian. "Quadrupolar relaxation of Xe-131 in xenon gas." Phys. Rev. 138, A403 (1965).

[396] C.H. Volk, T.M. Kwon, J.G. Mark, Y.B. Kim, and J.C. Woo. "Measurement of the Rb-Xe-131 spin-exchange cross section in Xe-131 relaxation studies." Phys. Rev. Lett. 44, $136(1980)$.

[397] Ross W. Mair, David G. Cory, Sharon Peled, Ching-Hua Tseng, Samuel Patz, and Ronald L. Walsworth. "Pulsed-field-gradient measurements of time-dependent gas diffusion." J. Magn. Reson. 135, 478 (1998).

[398] P.W.E. Peereboom, H. Luigjes, and K.O. Prins. "An NMR spin-echo study of selfdiffusion in xenon." Physica A 156, 260 (1989).

[399] R.J.J. van Heijningen, J.P. Harpe, and J.J.M. Beenakker. "Determination of the diffusion coefficients of binary mixtures of the noble gases as a function of temperature and concentration." Physica 38, 1 (1968).

[400] Rodolfo H. Acosta, Peter Blümler, Luis Agulles-Pedrós, Andreas E. Morbach, Jörg Schmiedeskamp, Annette Herweling, Ursula Wolf, Alexander Scholz, Wolfgang G. 
Schrieber, Werner Heil, Manfred Thelen, and Hans-Wolfgang Spiess. "Controlling diffusion of He-3 by buffer gases: A structural contrast agent in lung MRI.” J. Magn. Reson. Imag. (2005).

[401] Ioan Ardelean and Rainer Kimmich. "Diffusion measurements with the pulsed gradient nonlinear spin echo method." J. Chem. Phys. 112, 5275 (2000).

[402] J. Jeener. "Comment on "Diffusion measurements with the pulsed gradient nonlinear spin echo method" [J. Chem. Phys. 112, 5275 (2000)].” J. Chem. Phys. 116, 1204 $(2002)$.

[403] Ioan Ardelean and Rainer Kimmich. "Response to "Comment on 'Diffusion measurements with the pulsed gradient nonlinear spin echo method' " [J. Chem. Phys. 116, 1204 (2002)]." J. Chem. Phys. 116, 1206 (2002).

[404] J. Jeener. "Macroscopic molecular diffusion in liquid NMR, revisited." Concepts Magn. Reson. 14, 79 (2002).

[405] M. Engelsberg, Wilson Barros Jr., and Fernando Hallwass. "Intermolecular doublequantum coherences in two-dimensional spectra of binary mixtures in solution. The role of diffusion." J. Chem. Phys. 120, 10659 (2004).

[406] A. Kastler. "Quelques suggestions concernant la production optique et la détection optique d'une inégalité de population des niveaux de quantification spatiale des atomes. Application a l'expérience de Stern et Gerlach et la résonance magnétique." J. Phys.

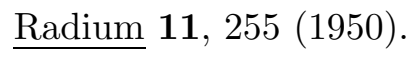

[407] William Happer. "Optical pumping." Rev. Mod. Phys. 44, 169 (1972). 
[408] W. Happer and W.A. van Wijngaarden. "An optical pumping primer." Hyperfine Interact. 38, 435 (1987).

[409] M.A. Bouchiat, T.R. Carver, and C.M. Varnum. "Nuclear polarization in He-3 gas induced by optical pumping and dipolar exchange." Phys. Rev. Lett. 5, 373 (1960).

[410] B.C. Grover. "Noble-gas NMR detection through noble-gas-rubidium hyperfine contact interaction." Phys. Rev. Lett. 40, 391 (1978).

[411] W. Happer, E. Miron, S. Schaefer, D. Schreiber, W.A. van Wijngaarden, and X. Zeng. "Polarization of the nuclear spins of noble-gas atoms by spin exchange with optically pumped alkali-metal atoms." Phys. Rev. A 29, 3092 (1984).

[412] Thad G. Walker and William Happer. "Spin-exchange optical pumping of noble-gas nuclei." Rev. Mod. Phys. 69, 629 (1997).

[413] S. Appelt, A. Ben-Amar Baranga, C.J. Erickson, M.V. Romalis, A.R. Young, and W. Happer. "Theory of spin-exchange optical pumping of He-3 and Xe-129." Phys.

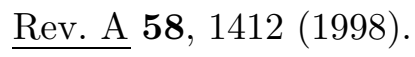

[414] Stephan Appelt. From Photon Spin to Magnetic Resonance Imaging (2004). Habilitationsschrift, RWTH-Aachen.

[415] B. Driehuys, G.D. Cates, E. Miron, K. Sauer, D.K. Walter, and W. Happer. "Highvolume production of laser-polarized Xe-129.” Appl. Phys. Lett. 69, 1668 (1996).

[416] U. Ruth, T. Hof, J. Schmidt, D. Fick, and H.J. Jänsch. "Production of nitrogen-free, hyperpolarized Xe-129 gas." Appl. Phys. B 68, 93 (1999). 
[417] Anthony L. Zook, Bhavin B. Adhyaru, and Clifford R. Bowers. "High capacity production of $>65 \%$ spin polarized xenon-129 for NMR spectroscopy and imaging." J. Magn. Reson. 159, 175 (2002).

[418] Kevin Knagge, Jonathan Prange, and Daniel Raftery. "A continuously recirculating optical pumping apparatus for high xenon polarization and surface NMR studies." Chem. Phys. Lett. 397, 11 (2004).

[419] D. Raftery, H. Long, T. Meersmann, P.J. Grandinetti, L. Reven, and A. Pines. "Highfield NMR of adsorbed xenon polarized by laser pumping." Phys. Rev. Lett. 66, 584 (1991).

[420] Eike Brunner. "Enhancement of surface and biological magnetic resonance using laser-polarized noble gases." Concepts Magn. Reson. 11, 313 (1999).

[421] Henry W. Long. Applications of Highly Spin-Polarized Xenon in NMR. Ph.D. dissertation, UC Berkeley (September 1993).

[422] Alan Gallagher. "Collisional depolarization of the Rb $5 p$ and Cs $6 p$ doublets." $\underline{\text { Phys. }}$ Rev. 157, 68 (1967).

[423] B.R. Bulos and W. Happer. "Nuclear-spin inertia and pressure broadening of ${ }^{2} P_{1 / 2}$ Hanle-effect signals.” Phys. Rev. A 4, 849 (1971).

[424] L. Wilmer Anderson, Francis M. Pipkin, and James C. Baird Jr. "N-14-N-15 hyperfine anomaly." Phys. Rev. 116, 87 (1959).

[425] A.R. Young, S. Appelt, A. Ben-Amar Baranga, C. Erickson, and W. Happer. "Three- 
dimensional imaging of spin polarization of alkali-metal vapor in optical pumping cells." Appl. Phys. Lett. 70, 3081 (1997).

[426] A. Ben-Amar Baranga, S. Appelt, C.J. Erickson, A.R. Young, and W. Happer. "Alkali-metal-atom polarization imaging in high-pressure optical-pumping cells." Phys. Rev. A 58, 2282 (1998).

[427] N.D. Bhaskar, J. Camparo, W. Happer, and A. Sharma. "Light narrowing of magnetic resonance lines in dense, optically pumped alkali-metal vapor." Phys. Rev. A 23, 3048 (1981).

[428] A. Omont. "Relaxation par collisions des états excités dun atome." J. Physique 26, $26(1965)$.

[429] Frank A. Franz and Judy R. Franz. "Excited-state mixing in the optical pumping of alkali-metal vapors." Phys. Rev. 148, 82 (1966).

[430] Dieter Suter. "Optically excited Zeeman coherences in atomic ground states: Nuclearspin effects." Phys. Rev. A 46, 344 (1992).

[431] Richard A. Beth. "Mechanical detection and measurement of the angular momentum of light." Phys. Rev. 50, 115 (1936).

[432] W. Heitler. The Quantum Theory of Radiation. Dover Publications, Inc., New York, NY, 3rd edition (1984 (reproduced from 1954 Oxford 3rd edition)).

[433] Robert G. Mortimer. Physical Chemistry. Benjamin/Cummings Publishing Company, Inc., Redwood City, CA (1993). 
[434] Shang yi Ch'en and Makoto Takeo. "Broadening and shift of spectral lines due to the presence of foreign gases." Rev. Mod. Phys. 29, 20 (1957).

[435] M.E. Wagshul and T.E. Chupp. "Optical pumping of high-density Rb with a broadband dye laser and GaAlAs diode laser arrays: Application to He-3 polarization." Phys. Rev. A 40, 4447 (1989).

[436] T.E. Chupp, M.E. Wagshul, K.P. Coulter, A.B. McDonald, and W. Happer. "Polarized, high-density, gaseous He-3 targets." Phys. Rev. C 36, 2244 (1987).

[437] F.A. Franz. "Enhancement of alkali optical pumping by quenching." $\underline{\text { Phys. Lett. A }}$ 27, 457 (1968).

[438] J.A. Bellisio, P. Davidovits, and P.J. Kindlmann. "Quenching of rubidium resonance radiation by nitrogen and the noble gases." J. Chem. Phys. 48, 2376 (1968).

[439] E.S. Hrycyshyn and L. Krause. "Inelastic collisions between excited alkali atoms and molecules. VII. Sensitized fluorescence and quenching in mixtures of rubidium with $\mathrm{H}_{2}, \mathrm{HD}, \mathrm{D}_{2}, \mathrm{~N}_{2}, \mathrm{CH}_{4}, \mathrm{CD}_{4}, \mathrm{C}_{2} \mathrm{H}_{4}$, and $\mathrm{C}_{2} \mathrm{H}_{6}$." Can. J. Phys. 48, 2761 (1970).

[440] D. Tupa, L.W. Anderson, D.L. Huber, and J.E. Lawler. "Effect of radiation trapping

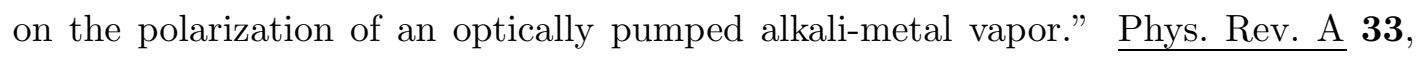
$1045(1986)$.

[441] Muhammad G. Mortuza, Satyanarayana Anala, Galina E. Pavlovskaya, Todd J. Dieken, and Thomas Meersmann. "Spin-exchange optical pumping of high-density xenon-129." J. Chem. Phys. 118, 1581 (2003). 
[442] Thad G. Walker. "Estimates of spin-exchange parameters for alkali-metal-noble-gas pairs." Phys. Rev. A 40, 4959 (1989).

[443] S.R. Schaefer, G.D. Cates, and W. Happer. "Determination of spin-exchange parameters between optically pumped rubidium and Kr-83." Phys. Rev. A 41, 6063 (1990).

[444] I.A. Nelson and T.G. Walker. "Rb-Xe relaxation in dilute Xe mixtures." Phys. Rev. A 65, $012712(2001)$.

[445] N.D. Bhaskar, J. Pietras, J. Camparo, W. Happer, and J. Liran. "Spin destruction in collisions between cesium atoms." Phys. Rev. Lett. 44, 930 (1980).

[446] C.J. Erickson, D. Levron, W. Happer, S. Kadlecek, B. Chann, L.W. Anderson, and T.G. Walker. "Spin relaxation resonances due to the spin-axis interaction in dense rubidium and cesium vapor." Phys. Rev. Lett. 85, 4237 (2000).

[447] S. Kadlecek, T. Walker, D.K. Walter, C. Erickson, and W. Happer. "Spin-axis relaxation in spin-exchange collisions of alkali-metal atoms." Phys. Rev. A 63, 052717 (2001).

[448] C.C. Bouchiat, M.A. Bouchiat, and L.C.L. Pottier. "Evidence for Rb-rare-gas molecules from the relaxation of polarized Rb atoms in a rare gas. Theory." Rev. 181, 144 (1969).

[449] M.A. Bouchiat, J. Brossel, and L.C. Pottier. "Evidence for Rb-rare-gas molecules from the relaxation of polarized Rb atoms in a rare gas. Experimental results." J. Chem. Phys. 56, 3703 (1972). 
[450] X. Zeng, Z. Wu, T. Call, E. Miron, D. Schreiber, and W. Happer. "Experimental determination of the rate constants for spin exchange between optically pumped $\mathrm{K}, \mathrm{Rb}$, and Cs atoms and Xe-129 nuclei in alkali-metal-noble-gas van der Waals molecules." Phys. Rev. A 31, 260 (1985).

[451] C.H. Volk, T.M. Kwon, and J.G. Mark. "Measurement of the Rb-87-Xe-129 spinexchange cross section.” Phys. Rev. A 21, 1549 (1980).

[452] N.D. Bhaskar, W. Happer, and T. McClelland. "Efficiency of spin exchange between rubidium spins and Xe-129 nuclei in a gas." Phys. Rev. Lett. 49, 25 (1982).

[453] N.D. Bhaskar, W. Happer, M. Larsson, and X. Zeng. "Slowing down of rubidiuminduced nuclear spin relaxation of Xe-129 gas in a magnetic field." Phys. Rev. Lett. 50, 105 (1983).

[454] G.D. Cates, R.J. Fitzgerald, A.S. Barton, P. Bogorad, M. Gatzke, N.R. Newbury, and B. Saam. "Rb-Xe-129 spin-exchange rates due to binary and three-body collisions at high Xe pressures.” Phys. Rev. A 45, 4631 (1992).

[455] Charles V. Rice and Daniel Raftery. "Rubidium-xenon spin exchange and relaxation rates measured at high pressure and high magnetic field." J. Chem. Phys. 117, 5632 $(2002)$.

[456] Matthew P. Augustine and Kurt W. Zilm. "Optical pumping magnetic resonance in high magnetic fields: Measurement of high field spin exchange cross sections." $\underline{\text { Chem. }}$ Phys. Lett. 280, 24 (1997). 
[457] Yuan-Yu Jau, Nicholas N. Kuzma, and William Happer. "Magnetic decoupling of Xe-129-Rb and Xe-129-Cs binary spin exchange.” Phys. Rev. A 67, 022720 (2003).

[458] Yuan-Yu Jau, Nicholas N. Kuzma, and William Happer. "Measurement of Xe-129-Cs binary spin-exchange rate coefficient." Phys. Rev. A 69, 061401(R) (2004).

[459] Robert A. Bernheim. "Spin relaxation in optical pumping." J. Chem. Phys. 36, 135 (1962).

[460] Z. Wu, T.G. Walker, and W. Happer. "Spin-rotation interaction of noble-gas alkalimetal atom pairs." Phys. Rev. Lett. 54, 1921 (1985).

[461] Thad G. Walker, Joseph H. Thywissen, and William Happer. "Spin-rotation interaction of alkali-metal-He-atom pairs." Phys. Rev. A 56, 2090 (1997).

[462] D.K. Walter, W. Happer, and T.G. Walker. "Estimates of the relative magnitudes of the isotropic and anisotropic magnetic-dipole hyperfine interactions in alkali-metalnoble-gas systems." Phys. Rev. A 58, 3642 (1998).

[463] R.M. Herman. "Theory of spin exchange between optically pumped rubidium and foreign gas nuclei." Phys. Rev. 137, A1062 (1965).

[464] Rodger L. Gamblin and Thomas R. Carver. "Polarization and relaxation processes in He-3 gas." Phys. Rev. 138, A946 (1965).

[465] T.G. Walker, K. Bonin, and W. Happer. "Electron-noble gas spin-flip scattering at low energy." Phys. Rev. A 35, 3749 (1987). 
[466] E.R. Hunt and H.Y. Carr. "Nuclear magnetic resonance of Xe-129 in natural xenon." Phys. Rev. 130, 2302 (1963).

[467] I.L. Moudrakovski, S.R. Breeze, B. Simard, C.I. Ratcliffe, J.A. Ripmeester, T. Seideman, J.S. Tse, and G. Santyr. "Gas-phase nuclear magnetic relaxation in Xe-129 revisited." J. Chem. Phys. 114, 2173 (2001).

[468] B. Chann, I.A. Nelson, L.W. Anderson, B. Driehuys, and T.G. Walker. "Xe-129-Xe molecular spin relaxation." Phys. Rev. Lett. 88, 113201 (2002).

[469] L.D. Schearer and G.K. Walters. "Nuclear Spin-Lattice Relaxation in the Presence of Magnetic-Field Gradients.” Phys. Rev. 139, A1398 (1965).

[470] G.D. Cates, S.R. Schaefer, and W. Happer. "Relaxation of spins due to magnetic field inhomogeneities in gaseous samples at low magnetic fields and low pressures." $\underline{\text { Phys. }}$

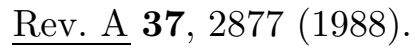

[471] K.C. Hasson, G.D. Cates, K. Lerman, P. Bogorad, and W. Happer. "Spin relaxation due to magnetic field inhomogeneities: Quartic dependence and diffusion-constant measurements." Phys. Rev. A 41, 3672 (1990).

[472] G.D. Cates, D.R. Benton, M. Gatzke, W. Happer, K.C. Hasson, and N.R. Newbury. "Laser production of large nuclear-spin polarization in frozen xenon." $\underline{\text { Phys. Rev. }}$ Lett. 65, 2591 (1990).

[473] M. Gatzke, G.D. Cates, B. Driehuys, D. Fox, W. Happer, and B. Saam. "Extraordinarily slow nuclear spin relaxation in frozen laser-polarized Xe-129.” Phys. Rev. Lett. 70, 690 (1993). 
[474] R.J. Fitzgerald, M. Gatzke, David C. Fox, G.D. Cates, and W. Happer. "Xe-129 spin relaxation in frozen xenon." Phys. Rev. B 59, 8795 (1999).

[475] N.N. Kuzma, B. Patton, K. Raman, and W. Happer. "Fast nuclear spin relaxation in hyperpolarized solid Xe-129.” Phys. Rev. Lett. 88, 147602 (2002).

[476] William W. Warren Jr. and R.E. Norberg. "Multiple-pulse nuclear-magneticresonance transients of Xe-129 and Xe-131 in solid xenon." Phys. Rev. 154, 277 (1967).

[477] N. Bloembergen, S. Shapiro, P.S. Pershan, and J.O. Artman. "Cross-relaxation in spin systems." Phys. Rev. 114, 445 (1959).

[478] Harold T. Stokes and David C. Ailion. "Zeeman-quadrupole cross relaxation between two nuclear spin species." J. Chem. Phys. 70, 3572 (1979).

[479] Stephen Lang, Igor L. Moudrakovski, Christopher I. Ratcliffe, John A. Ripmeester, and Giles Santyr. "Increasing the spin-lattice relaxation time of hyperpolarized xenon ice at 4.2 K.” Appl. Phys. Lett. 80, 886 (2002).

[480] B. Driehuys, G.D. Cates, and W. Happer. "Surface relaxation mechanisms of laserpolarized Xe-129.” Phys. Rev. Lett. 74, 4943 (1995).

[481] C.A. Corum and A.F. Gmitro. "Spatially varying steady state longitudinal magnetization in distant dipolar field-based sequences." J. Magn. Reson. 171, 131 (2004).

[482] MATLAB v. 7. The MathWorks, Inc., Natick, MA (2004). 
[483] Wilson Barros Jr., Paulo Loureiro de Sousa, and M. Engelsberg. "Low field intermolecular double-quantum coherence imaging via the Overhauser effect." J. Magn. Reson. 165, 175 (2003).

[484] George B. Arfken and Hans J. Weber. Mathematical Methods for Physicists. Academic Press, Inc., San Diego, CA, 4th edition (1995).

[485] Howard S. Cohl and Joel E. Tohline. "A compact cylindrical Green's function expansion for the solution of potential problems." Astrophys. J. 527, 86 (1999).

[486] Howard S. Cohl, A.R.P. Rau, Joel E. Tohline, Dana A. Browne, John E. Cazes, and Eric I. Barnes. "Useful alternative to the multipole expansion of $1 / r$ potentials." Phys. Rev. A 64, 052509 (2001).

[487] Michael K. Stehling. "Improved signal in "snapshot" FLASH by variable flip angles." Magn. Reson. Imag. 10, 165 (1992).

[488] J.P. Mugler III, F.H. Epstein, and J.R. Brookeman. "Shaping the signal response during the approach to steady-state in 3-dimensional magnetization-prepared rapid gradient-echo imaging using variable flip angles." Magn. Reson. Med. 28, 165 (1992).

[489] Lei Zhao, Robert Mulkern, Ching-Hua Tseng, Daniel Williamson, Samuel Patz, Robert Kraft, Ronald L. Walsworth, Ferenc A. Jolesz, and Mitchell S. Albert. "Gradient-echo imaging considerations for hyperpolarized Xe-129 MR." ․ Magn. Reson. B 113, 179 (1996).

[490] J. Crank. The Mathematics of Diffusion. Oxford University Press, Oxford, UK, 2nd edition (1975). 
[491] C. Cohen-Tannoudji, J. DuPont-Roc, S. Haroche, and F. Laloë. "Detection of the static magnetic field produced by the oriented nuclei of optically pumped He-3 gas." Phys. Rev. Lett. 22, 758 (1969).

[492] V.V. Yashchuk, J. Granwehr, D.F. Kimball, S.M. Rochester, A.H. Trabesinger, J.T. Urban, D. Budker, and A. Pines. "Hyperpolarized xenon nuclear spins detected by optical atomic magnetometry." Phys. Rev. Lett. 93, 160801 (2004).

[493] D. Budker, D.F. Kimball, S.M. Rochester, V.V. Yashchuk, and M. Zolotorev. "Sensi-

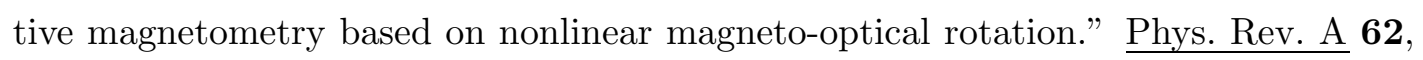
$043403(2000)$.

[494] D. Budker, D.F. Kimball, V.V. Yashchuk, and M. Zolotorev. "Nonlinear magnetooptical rotation with frequency-modulated light." Phys. Rev. A 65, 055403 (2002).

[495] I.K. Kominis, T.W. Kornack, J.C. Allred, and M.V. Romalis. "A subfemtotesla multichannel atomic magnetometer." Nature 422, 596 (2003).

[496] Dmitry Budker, Valeriy Yashchuk, and Max Zolotorev. "Nonlinear magneto-optic effects with ultranarrow widths.” Phys. Rev. Lett. 81, 5788 (1998).

[497] Mathias Haake, Boyd M. Goodson, David D. Laws, Eike Brunner, Michelle C. Cyrier, Robert H. Havlin, and Alexander Pines. "NMR of supercritical laser-polarized xenon." Chem. Phys. Lett. 292, 686 (1998).

[498] D.A. Varshalovich, A.N. Moskalev, and V.K. Khersonskii. Quantum Theory of Angular Momentum. World Scientific Publishing Company, Inc., Singapore (1988). 
[499] Eugene D. Commins. "Physics 221A Lecture notes, UC Berkeley." (Fall 1999). Unpublished.

[500] Gordon Baym. Lectures on Quantum Mechanics. Lecture Notes and Supplements in Physics. Addison-Wesley Publishing Company, Inc., Reading, MA (1990).

[501] Robert L. Cook and Frank C. de Lucia. "Application of the theory of irreducible tensor operators to molecular hyperfine structure." Am. J. Phys. 39, 1433 (1971).

[502] A.J. Stone. "Transformation between cartesian and spherical tensors." Mol. Phys. 29, $1461(1975)$.

[503] G.J. Bowden, W.D. Hutchison, and J. Khachan. "Tensor operator formalism for multiple-quanum NMR. 2. Spins $\frac{3}{2}, 2$, and $\frac{5}{2}$ and general I." J. Magn. Reson. 67, 415 (1986).

[504] F. Marinelli, G. Pouzard, and L. Werbelow. "Simplified closed form expressions for

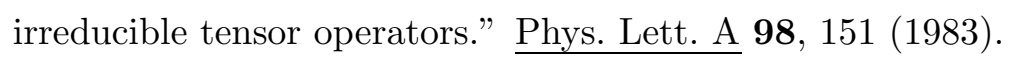

[505] B.C. Sanctuary. "Multipole operators for an arbitrary number of spins." J. Chem. Phys. 64, 4352 (1976).

[506] James D. Bjorken and Sidney D. Drell. Relativistic Quantum Fields. International Series in Pure and Applied Physics. McGraw-Hill, Inc., New York, NY (1965). 


\section{Appendix A}

\section{Spherical tensor operators and the}

\section{Wigner-Eckart theorem}

The spherical tensor operator formalism is very useful in the quantum theory of angular momentum, particularly when rotations are concerned. The concept of spherical tensors will be introduced in a series of abstractions from the more familiar concept of Cartesian tensors, and this formalism will be extended to describe quantum-mechanical spherical tensor operators. Afterward, the Wigner-Eckart theorem will be described as an example of the utility of the spherical tensor operator formalism. Appendix B will discuss the properties of spherical tensors under rotations. 


\section{A.1 Cartesian Tensors and Dyadics}

As a first introduction to tensors, we first consider the example of the classical energy of a magnetic dipole moment interacting with a locally induced magnetic field. This energy may be written as:

$$
U_{\sigma}=-\underline{\mu}^{T} \cdot \underline{\underline{\sigma}} \cdot \underline{B}_{0}=-\underline{\mu}^{T} \cdot \underline{B}_{i n d}^{\sigma}
$$

where $\underline{\mu}$ is the magnetic dipole moment vector (represented by a column vector, where the row vector $\underline{\mu}^{T}$ is its transpose), $\underline{B}_{0}$ is the vector of the externally applied magnetic field, and $\underline{\underline{\sigma}}$ is a tensor that describes the ability of $\underline{B}_{0}$ to cause the local environment induce a magnetic field $\underline{B}_{i n d}^{\sigma}=\underline{\underline{\sigma}} \cdot \underline{B}_{0}$ at the position of the magnetic dipole. Eq. A.1 is the completely classical version of the usual NMR chemical shielding Hamiltonian. This equation does not make reference to any particular coordinate system, but it can be cast into a representation in terms of its Cartesian components if some Cartesian coordinate system $\{x, y, z\}$ is chosen:

$$
U_{\sigma}=-\sum_{i, j \in\{x, y, z\}} \mu_{i} \sigma_{i j} B_{0 j}
$$

where the vector and tensor components are defined by $\mu_{i}=\underline{\tau}^{T} \cdot \underline{\mu}, B_{0 j}=\underline{\tilde{j}}^{T} \cdot \underline{B}_{0}$, and $\sigma_{i j}=\underline{\underline{i}}^{T} \cdot \underline{\underline{\sigma}} \cdot \underline{\widetilde{j}}$. The vectors $\underline{\widetilde{i}}$ and $\underline{\tilde{j}}$ are Cartesian unit basis vectors from the set $\{\underline{\widetilde{x}}, \underline{\widetilde{y}}, \underline{z}\}$. 
Using this notation the vectors and tensors can be written in terms of their components as:

$$
\begin{gathered}
\underline{\mu}^{T}=\sum_{i} \mu_{i} \underline{\tilde{i}}^{T} \\
\underline{B}_{0}=\sum_{j} B_{0 j} \underline{\tilde{j}} \\
\underline{\underline{\sigma}}=\sum_{i, j} \sigma_{i j} \underline{\tilde{i}}^{T} \underline{\tilde{j}},
\end{gathered}
$$

where $\underline{\widetilde{i}}^{T} \widetilde{j}$ is the outer product between the vectors $\underline{\tilde{i}}^{T}$ and $\underline{\widetilde{j}}$. When the tensor $\underline{\underline{\sigma}}$ is written in this way, it takes in a vector from either side and returns a scalar (i.e., a number), which in this case is the energy $U_{\sigma}$. This is due to the direct product structure of this definition of $\underline{\underline{\sigma}}$. Note that the tensor $\underline{\underline{\sigma}}$ is described by two independent Cartesian indices, the vectors $\underline{\mu}$ and $\underline{B}_{0}$ take one Cartesian index each, and the scalar quantity $U_{\sigma}$ has no Cartesian indices. These entities are referred to as rank-2, rank-1, and rank-0 Cartesian tensors, respectively. A rank- $n$ Cartesian tensor has $n$ Cartesian indices, and as a rule can be combined with $n$ vectors (rank-1 tensors) to form a scalar (rank-0 tensor) through a process called tensor contraction. Eqs. A.1 and A.2 demonstrate how a second-rank Cartesian tensor $\underline{\underline{\sigma}}$ can take in two vectors $\underline{\mu}$ and $\underline{B}$ to form a scalar $U_{\sigma}$ by contraction. In fact, Eq. A.1 also shows how a rank-1 tensor and another rank-1 tensor can be combined to give a scalar through a special case of tensor contraction: i.e., the ordinary dot product $\underline{\mu}^{T} \cdot \underline{B}_{i n d}^{\sigma}$.

There is another way to reach Eq. A.2, which begins with a definition of $\sigma$ in terms of direct products (or dyad products) of the Cartesian basis vectors rather than in terms of outer products. The direct product of $\underline{\widetilde{i}}$ and $\underline{\widetilde{j}}[29, \S 10.1]$ is written $\underline{\widetilde{i}} \otimes \underline{\tilde{j}} \equiv \underline{\underline{\tilde{i}}} \underline{\underline{j}} .{ }^{1}$

\footnotetext{
${ }^{1}$ If the reader is more familiar with linear algebra manipulations through the Dirac notation of quantum mechanics, the following associations may be helpful: $\underline{\widetilde{i}} \rightarrow|i\rangle, \underline{\widetilde{i}} \rightarrow\langle i|, \underline{\widetilde{i}} \underline{\underline{j}} \rightarrow| i\rangle\langle j|, \underline{\underline{\sigma}} \rightarrow \hat{\sigma}, \underline{\widetilde{i}}^{T} \cdot \underline{\widetilde{j}}=\langle i \mid j\rangle$,
} 
The double-underline notation is to show that this product can be treated as a second-rank Cartesian tensor, since it has two Cartesian indices. A second-rank Cartesian tensor formed from two vectors is called a dyadic. Two second-rank Cartesian tensors can be contracted to give a scalar; for instance, the sums over two unit dyadics in three-dimensional space can be contracted as $\sum_{m, n}(\underline{\widetilde{m}} \otimes \underline{\widetilde{r}})^{T}: \sum_{i, j}(\underline{\widetilde{i}} \otimes \underline{\tilde{j}})=\sum_{m, n ; i, j}\left(\underline{\widetilde{m}}^{T} \cdot \underline{\widetilde{i}}\right)\left(\underline{\widetilde{n}}^{T} \cdot \underline{\tilde{j}}\right)=\sum_{m, n ; i, j} \delta_{m, i} \delta_{n, j}=3$, which is just a number. The colon represents a generalization of the dot product between two vectors to a scalar product between two second-rank tensors. Returning to the equations for $U_{\sigma}$, we write $\underline{\underline{\sigma}}$ (which is a second-rank tensor but not necessarily a dyadic) as a linear combination of the Cartesian basis dyadics $\underline{\underline{i}} \underline{\tilde{j}}$, and make a dyadic out of the two vectors $\underline{\mu}$ and $\underline{B}_{0}$ :

$$
\begin{aligned}
\underline{\underline{B_{0} \mu}} & =\underline{B}_{0} \otimes \underline{\mu}=\left(\sum_{i} B_{0 i} \underline{\tilde{i}}\right) \otimes\left(\sum_{j} \mu_{j} \underline{\tilde{j}}\right)=\sum_{i, j} B_{0 i} \mu_{j}(\underline{\widetilde{i}} \otimes \underline{\tilde{j}}) \\
& =\sum_{i, j} B_{0 i} \mu_{j} \underline{\underline{\tilde{i} \widetilde{j}}} \\
\underline{\underline{\sigma}} & =\sum_{i, j} \sigma_{i j} \underline{\underline{\tilde{i} \tilde{j}}}
\end{aligned}
$$

where the vector and tensor components are defined as usual. This is an alternative definition of the quantities in Eq. A.3. Now Eq. A.1 can be rewritten as:

$$
U_{\sigma}=-\left(\underline{\underline{B_{0} \mu}}\right)^{T}: \underline{\underline{\sigma}}
$$

$\overline{\underline{\widetilde{i}}^{T} \cdot \underline{\underline{\sigma}} \cdot \underline{\tilde{j}} \rightarrow\langle i|\hat{\sigma}| j\rangle, \underline{\widetilde{i}} \otimes \underline{\tilde{j}}=\underline{\underline{\tilde{i}} \tilde{j}} \rightarrow|i\rangle \otimes|j\rangle}=|i j\rangle$. This analogy is not meant to be taken literally because these Cartesian tensors are objects in real space, not in a quantum-mechanical Hilbert space. Furthermore, quantum Hilbert spaces have some properties such as the dual-space relation between bra and ket vectors that do not apply to vectors in real space. 
which of course recovers Eq. A.2:

$$
U_{\sigma}=-\sum_{i, j} \mu_{i} B_{0 j} \sigma_{i j}
$$

where the following relations hold:

$$
\begin{aligned}
& \underline{\underline{B}}^{T}: \underline{\underline{A}}=\sum_{i, j} B_{j i} A_{i j}, \\
& \underline{\underline{B}}^{T}=\sum_{i, j} B_{j i}(\tilde{\underline{i}} \otimes \widetilde{j})^{T}
\end{aligned}
$$

where in this case $\underline{\underline{B}}=-\underline{\underline{B_{0} \mu}}$.

There is no difference between Eq. A.2 and Eq. A.6, since the vector or tensor components are just numbers and may be taken in any order within the sum. The direct product method of writing Cartesian tensors is more general than the outer product method because it can be generalized to tensors with ranks higher than two. For instance, in this way a product of $n$ vectors on the left can be contracted with a rank- $n$ tensor on the right-much like the dot product between two vectors - whereas the outer product definition of a secondrank tensor only accepts one vector from the left and one from the right, and there is no good way to deal with how a third- or higher-rank tensor accepts vectors. This ambiguity about how to form Cartesian tensors suggests that it might be more straightforward to work directly with the tensor components, since the component equations A.2 and A.6 are equivalent regardless of how the tensors were constructed. In fact, it makes sense to define a vector (first-rank Cartesian tensor) $\underline{v}$ as the set of three components $\left\{v_{i}\right\}(i \in\{x, y, z\})$ and to define a second-rank Cartesian tensor $\underline{\underline{A}}$ as the set of nine components $\left\{A_{i j}\right\}$, where 
the actual numeric values of the components depend on the choice of coordinate system.

In order to pursue this idea further, consider the components $\left\{u_{i}\right\}$ of a vector (firstrank Cartesian tensor) $\underline{u}$, and in particular how these components respond to a rotation of the system. For instance, a positive $90^{\circ}$ rotation about the $z$-axis takes the unit basis vectors $\underline{\widetilde{x}} \rightarrow \underline{\widetilde{y}}, \underline{\widetilde{y}} \rightarrow-\underline{\widetilde{x}}$, and $\underline{\widetilde{z}} \rightarrow \underline{\widetilde{z}}$, and thus $u_{x} \rightarrow u_{y}, u_{y} \rightarrow-u_{x}$, and $u_{z} \rightarrow u_{z}$. Therefore it may be concluded that the components $\left\{u_{i}\right\}$ of a first-rank tensor $\underline{u}$ transform under rotations in the same way as do the unit basis vectors $\{\widetilde{\underline{i}}\}$. Next, consider some dyadic (second-rank Cartesian tensor) $\underline{\underline{A}}=\underline{u} \otimes \underline{v}$, where $\underline{u}$ and $\underline{v}$ are both vectors. The Cartesian components

of this dyadic are $A_{i j}=(\underline{\underline{\tilde{i}}})^{T}: \underline{\underline{A}}=\underline{\tilde{i}}^{T} \cdot \underline{\underline{A}} \cdot \underline{\tilde{j}}=u_{i} v_{j}$. Under the same $90^{\circ}$ rotation about the $z$-axis the unit dyadics transform as $\underline{\underline{\tilde{x} \widetilde{x}}} \rightarrow \underline{\underline{\widetilde{y}}}, \underline{\underline{\widetilde{x}}} \rightarrow-\underline{\underline{\widetilde{y} \widetilde{x}}}, \underline{\underline{\widetilde{x}}} \rightarrow \underline{\underline{\widetilde{y} \widetilde{z}}}$, etc. It can easily be seen from the definition of the $\left\{A_{i j}\right\}$ in terms of $u_{i}$ and $v_{j}$ that $A_{x x} \rightarrow A_{y y}, A_{x y} \rightarrow-A_{y x}$ and $A_{x z} \rightarrow A_{y z}$, etc., which mimics the form of the transformations of the unit dyadics $\{\underline{\underline{\tilde{i}}} \tilde{j}=(\underline{i} \otimes \underline{j})\}$. These transformation rules hold even for second-rank tensors $\underline{\underline{A}}$ that are not dyadics (i.e., that are not necessarily made from two vectors). Upon extending the analogy, the Cartesian components of a third-rank tensor are found to transform under rotations as do the basis tensors $\{(\underline{\tilde{i}} \otimes \underline{j} \otimes \underline{\underline{k}})\}$, and so on for higher-rank Cartesian tensors. Therefore a rank- $n$ tensor in three-dimensional Cartesian space can be thought of as a collection of $3^{n}$ components that transform into each other as do the rank- $n$ unit basis tensors.

\section{A.2 Spherical Tensors and Tensor Operators}

The problem of representing a Cartesian tensor in terms of its Cartesian components has already been considered, and the discussed now turns to the problem of generating 
the spherical components of Cartesian tensors. These components can be grouped to form sets of spherical tensors, and it will be seen how the spherical tensors can be considered to be stand-alone objects defined by their rotation properties, without making reference to a definition in terms of Cartesian tensors. Finally, the concept of spherical tensors will be extended to include spherical tensor operators, which are sets of quantum-mechanical operators that obey the same transformation rules under rotations in the quantum Hilbert space as do the spherical tensors under rotations in real space. The theory of these operators is deeply rooted in the quantum theory of angular momentum $[20, \S 5],[498],[45, \S 3.10],[29$, $\S 15.3],[499, \S 11],[500, \S 17],[484, \S 4.4]$. A good, concise description of spherical tensors and tensor operators as they apply to NMR is given in Appendix A of the text by Mehring [8]; see also Appendix B.5 of Tl text by Schmidt-Rohr and Spiess [9].

\section{A.2.1 Spherical Tensors}

Section A.1 discussed the projection of Cartesian tensors $\underline{\underline{A}}$ onto a set of Cartesian basis vectors, thus generating the Cartesian tensor components. The same procedure can be used to project a Cartesian tensor onto a set of spherical basis vectors in order to generate the spherical components of that tensor. The spherical basis vectors are:

$$
\begin{aligned}
\underline{\tilde{e}}_{1,0} & =\underline{\widetilde{z}} \\
\underline{\widetilde{e}}_{1, \pm 1} & =\mp \frac{1}{\sqrt{2}}(\underline{\widetilde{x}} \pm i \underline{\tilde{y}}),
\end{aligned}
$$

where here $i=\sqrt{-1}$ is the imaginary factor. The three spherical components of a vector $\underline{u}$

(a rank-1 Cartesian tensor) can be written as $u_{1,0}=\underline{\tilde{e}}_{1,0}^{\dagger} \cdot \underline{u}$ and $u_{1, \pm 1}=\underline{\widetilde{e}}_{1, \pm 1}^{\dagger} \cdot \underline{u}$, in analogy 
to the definition of the Cartesian components of $\underline{u}$. The transpose has been generalized to a conjugate transpose since the spherical unit vectors are complex. Eq. A.9 can be used to relate the three spherical components of $\underline{u}$ to the three Cartesian components of $\underline{u}: u_{1,0}=u_{z}$ and $u_{1, \pm 1}=\mp \frac{1}{\sqrt{2}}\left(u_{x} \pm i u_{y}\right)$. Note that the spherical basis vectors in Eq. A.9 transform into each other under rotations as do the spherical harmonics $Y_{1,0}$ and $Y_{1, \pm 1}$, and so do the spherical components of $\underline{u} .^{2}$ For example, a positive $90^{\circ}$ rotation about the $y$-axis takes $\underline{\widetilde{z}}=\underline{\widetilde{e}}_{1,0} \rightarrow \underline{\widetilde{x}}=-\frac{1}{\sqrt{2}}\left(\underline{\widetilde{e}}_{1,+1}-\underline{\widetilde{e}}_{1,-1}\right)$ and $u_{1,0} \rightarrow-\frac{1}{\sqrt{2}}\left(u_{1,+1}-u_{1,-1}\right)$, and the same rotation takes $Y_{1,0} \rightarrow-\frac{1}{\sqrt{2}}\left(Y_{1,+1}-Y_{1,-1}\right)$.

In order to project out the spherical components of a second-rank Cartesian tensor, the spherical basis tensors in the direct product space must first be formed. The spherical harmonics $Y_{l, m}$ can be considered to be objects that bear total (orbital) angular momentum $l$ and a component of angular momentum $m$ along some quantization axis, as may be verified by applying the angular momentum operators $\hat{l}^{2}$ and $\hat{l}_{z}$ in the coordinate representation to a spherical harmonic function. Therefore, the spherical basis vectors can be considered to be objects that carry angular momentum $l=1$ with $m=0, \pm 1$. A set of spherical basis tensors $\stackrel{\widetilde{\widetilde{e}}}{L, M}_{L, \mathrm{~m}}$ can be created by combining two of these $l=1$ basis vectors according to the

\footnotetext{
${ }^{2}$ In fact, the $l=1$ spherical harmonic functions $Y_{1, m}$ are just proportional to the spherical components of the position vector $\underline{r}=x \underline{\widetilde{x}}+y \underline{\tilde{y}}+z \underline{\widetilde{z}}$ (Ref. [500] $\S 17)$.
} 
quantum-mechanical rules of angular momentum addition [501, Appendix A]:

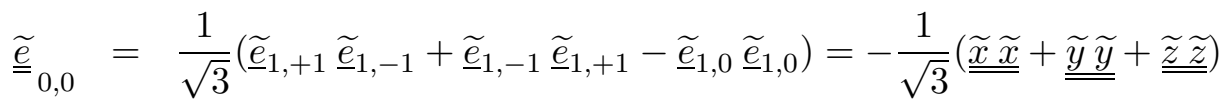

$$
\begin{aligned}
& \underline{\underline{\widetilde{e}}}_{1,0}=\frac{1}{\sqrt{2}}\left(\underline{\underline{\widetilde{e}}}_{1,+1} \underline{\widetilde{\underline{e}}}_{1,-1}-\underline{\underline{\widetilde{e}}}_{1,-1} \underline{\underline{\widetilde{e}}}_{1,+1}\right)=-\frac{i}{\sqrt{2}}(\underline{\underline{\widetilde{x} \widetilde{y}}}-\underline{\underline{\widetilde{y} \widetilde{x}}})
\end{aligned}
$$

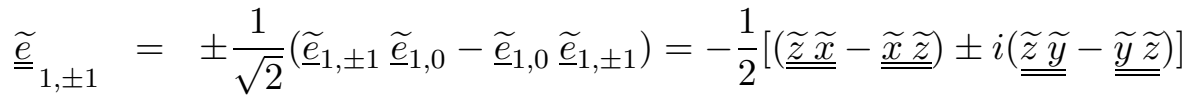

$$
\begin{aligned}
& \underline{\underline{\underline{e}}}_{2,0}=\sqrt{\frac{1}{6}}\left(2 \underline{\widetilde{e}}_{1,0} \underline{\widetilde{e}}_{1,0}-\underline{\widetilde{e}}_{1,+1} \underline{\widetilde{\widetilde{e}}}_{1,-1}-\underline{\widetilde{e}}_{1,-1} \underline{\widetilde{e}}_{1,+1}\right)=\sqrt{\frac{1}{6}}[3 \underline{\underline{\tilde{z} \widetilde{z}}}-(\underline{\underline{\widetilde{x} \tilde{x}}}+\underline{\underline{\widetilde{y}}}+\underline{\underline{\widetilde{z} \tilde{z}}})]
\end{aligned}
$$

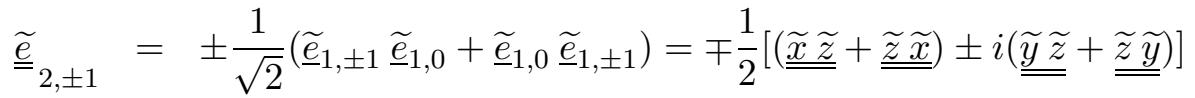

$$
\begin{aligned}
& \underline{\underline{\widetilde{e}}}_{2, \pm 2}=\underline{\underline{\widetilde{e}}}_{1, \pm 1} \underline{\underline{\widetilde{e}}}_{1, \pm 1}=\frac{1}{2}[(\underline{\underline{\underline{\widetilde{x}}}}-\underline{\underline{\widetilde{y} \widetilde{y}}}) \pm i(\underline{\underline{\widetilde{x} \widetilde{y}}}+\underline{\underline{\widetilde{y} \widetilde{x}}}],
\end{aligned}
$$

where $\underline{\widetilde{\widetilde{e}}}_{l 1, m 1} \underline{\widetilde{\widetilde{e}}}_{l 2, m 2} \equiv \underline{\widetilde{\widetilde{e}}}_{l 1, m 1} \otimes \underline{\underline{\widetilde{e}}}_{l 2, m 2}$, and the indices $L=\left|l_{1}+l_{2}\right|, \ldots,\left|l_{1}-l_{2}\right|, M=$ $+L, \ldots,-L$ obey the rules for the addition of two angular momenta $l_{1}=1$ and $l_{2}=1$. Not surprisingly, the nine basis spherical tensors $\stackrel{\widetilde{e}}{=}_{L, M}$ can be written as linear combinations of the nine basis Cartesian tensors $\underline{\underline{\widetilde{i}}} \underline{\underline{j}}$. The $\left\{\underline{\underline{\widetilde{e}}}_{L, M}\right\}$ transform into each other under rotations as do the spherical harmonics $\left\{Y_{L, M}\right\}$. The $L=1$ spherical basis tensors in Eq. A.10 have a different form from the $l=1$ basis vectors in Eq. A.9 because the tensors are written in the combined $\underline{l}_{1} \otimes \underline{l}_{2}$ direct product space.

The spherical components of an arbitrary second rank Cartesian tensor $\underline{\underline{A}}$ may be generated by projecting $\underline{\underline{A}}$ onto the spherical basis tensors: $A_{L, M}=\underline{\underline{\widetilde{e}}}_{L, M}^{\dagger}: \underline{\underline{A}}$, in direct analogy to defining the Cartesian components as $A_{i j}=(\underline{\underline{i} \widetilde{j}})^{T}: \underline{\underline{A}}$. The spherical components 
are found to be:

$$
\begin{aligned}
A_{0,0} & =-\frac{1}{\sqrt{3}}\left(A_{x x}+A_{y y}+A_{z z}\right) \\
A_{1,0} & =-\frac{i}{\sqrt{2}}\left(A_{x y}-A_{y x}\right) \\
A_{1, \pm 1} & =-\frac{1}{2}\left[\left(A_{z x}-A_{x z}\right) \pm i\left(A_{z y}-A_{y z}\right)\right] \\
A_{2,0} & =\sqrt{\frac{1}{6}}\left[3 A_{z z}-\left(A_{x x}+A_{y y}+A_{z z}\right)\right] \\
A_{2, \pm 1} & =\mp \frac{1}{2}\left[\left(A_{x z}+A_{z x}\right) \pm i\left(A_{y z}+A_{z y}\right)\right] \\
A_{2, \pm 2} & =\frac{1}{2}\left[\left(A_{x x}-A_{y y}\right) \pm i\left(A_{x y}+A_{y x}\right)\right] .
\end{aligned}
$$

These nine spherical components of $\underline{\underline{A}}$ are written in terms of the nine Cartesian components. The $\left\{A_{L, M}\right\}$ transform under rotations as do the spherical basis tensors in Eq. A.10 and also as do the spherical harmonics $\left\{Y_{L, M}\right\}$. In fact, in the case that $\underline{\underline{A}}=\underline{r} \otimes \underline{r}$ (such that $A_{i j}=i j$, where $\left.i, j \in\{x, y, z\}\right)$, then $A_{L, M} \propto Y_{L, M}(\theta, \phi)$, where the spherical polar coordinates $(\theta, \phi)$ are those that describe the orientation of the position vector $\underline{r}$ itself. Note that if $\underline{\underline{A}}$ is a dyadic, the $\left\{A_{L, M}\right\}$ act like the $\left\{Y_{L, M}\right\}$ only under rotations in the combined direct product space. For example, if $\underline{\underline{A}}=\underline{r}_{1} \otimes \underline{r}_{2}$, then the $A_{L, M}$ acts like $Y_{L, M}(\theta, \phi)$ only if $\underline{r}_{1}$ and $\underline{r}_{2}$ are rotated together, not independently. In this case $(\theta, \phi)$ are coordinates that describe the joint system, since the $\left(\theta_{1}, \phi_{1}\right)$ and $\left(\theta_{2}, \phi_{2}\right)$ of $\underline{r}_{1}$ and $\underline{r}_{2}$ are not independent.

Reiterating the ongoing theme, the generalized definition of a spherical tensor is a set of components $\left\{T_{k, q}\right\}$ that transform into each other under rotations as do the spherical harmonics $\left\{Y_{k, q}\right\}$. The rank of the spherical harmonic (or spherical tensor component) $k$ can range from 0 to $\infty$ in integer steps, and a spherical tensor of a given rank $k$ has 
$(2 \mathrm{k}+1)$ spherical components of order $q$ ranging from $-k$ to $+k$ in integer steps. It is important to distinguish between the use of the word "rank" as applied to Cartesian tensors and the word "rank" as applied to spherical tensors. The rank $n$ of a Cartesian tensor indicates how many Cartesian indices are needed to describe the tensor, or how many vectors can be contracted with the tensor to form a (rank-0) scalar. The word rank as applied to spherical tensors is terminology borrowed from the language of spherical harmonics, since the spherical harmonics $Y_{k, q}(\theta, \phi)$ are defined in terms of the Legendre polynomials $P_{k}(\cos \theta)$ of rank $k$. A rank-0 (scalar) Cartesian tensor has one Cartesian component and can be written in terms of a the single component of a rank-0 spherical tensor, and the three components of a rank-1 (vector) Cartesian tensor can be written in terms of the three components of a rank-1 spherical tensor. However, Eq. A.11 showed how a rank-2 Cartesian tensor can be decomposed into one rank-0, three rank-1, and five rank-2 spherical components, ${ }^{3}$ and here the meaning of the word "rank" begins to diverge. The situation is more complicated when relating higher-rank Cartesian tensors to the spherical tensors [502]. However, it should be stressed that the definition of spherical tensors in terms of their rotation properties is internally self-consistent, and no reference to Cartesian tensors (dyadics or otherwise) need be made, unless the Cartesian representation is convenient. Some interesting properties of spherical tensors will be mentioned at the end of $\S$ A.2.2, and the transformation properties of spherical tensors under rotations will be discussed further in Appendix B.

\footnotetext{
${ }^{3}$ In the language of group theory, a spherical tensor of rank $k$ transforms as an irreducible representation of the rotation group. In other words, a group of spherical tensor components of a given rank is the smallest set of components that obey all the symmetries of the rotation group. Cartesian tensors transform reducibly, since they can be broken down into irreducible sets of (rank- $k$ spherical) components, cf. Eq. A.11.
} 


\section{A.2.2 Spherical Tensor Operators}

The discussion of spherical tensors has so far alluded several times to the quantum theory of angular momentum. For instance, if the spherical harmonics $Y_{l, m}(\theta, \phi)=$ $\langle\theta, \phi \mid l, m\rangle$ were considered to be wavefunctions, they would be eigenstates of the orbital angular momentum operator. In fact, the eigenstates of orbital angular momentum $|l, m\rangle$ can be shown to transform into each other under quantum-mechanical rotations in the Hilbert space as do the spherical harmonics $Y_{l, m}(\theta, \phi)$ under rotations in real space. Now, consider an operator in the state space of some quantized angular momentum $\underline{\hat{I}}$, where the eigenstates of $\underline{\hat{I}}^{2}$ are $|I, m\rangle$ with $m$ being the projection of the angular momentum onto the quantization axis. The angular momentum quantum number $I$ can be integral or half-integral (compare to the spherical harmonics, which have integer rank). The eigenstates of angular momentum form a complete basis set in the state space of $\underline{\hat{I}}^{2}$, in the sense that any operator in this space can be written in the general form $\hat{A}=\sum_{m, n} A_{m n}|I, m\rangle\langle I, n|$. By now, it should not be surprising that linear combinations of the $|I, m\rangle\langle I, n|$ can be found that transform into each other under rotations as do the spherical harmonics, in light of the fact that the individual angular momentum eigenstates transform this way. In fact, $|I, m\rangle\langle I, n|$ represents an operator that can be decomposed using the rules of angular momentum addition into a linear combination of operators $\hat{T}_{k, q}$ of rank $k=|I-I|, \ldots,|I+I|=0, \ldots, 2 I$ and order $q=-k, \ldots,+k$, with $m=n+q$. The $\left\{\hat{T}_{k, q}\right\}$ have integer $k$ and $q$ and are called spherical tensor operators ${ }^{1}$; they transform into each other under rotations as do the spherical harmonic functions $\left\{Y_{k, q}\right\}$. The state space of an angular momentum $I$ can be completely

\footnotetext{
${ }^{1}$ More accurately, they are the order- $q$ components of the rank- $k$ spherical tensor operators.
} 
described by the $(2 I+1)^{2}$ spherical tensor operators that range from rank $k=0$ to $k=2 I$ with $q$ ranging from $-k$ to $+k$. These operators are often conveniently written using the set of operators $\left\{\hat{1}, \hat{I}_{z}, \hat{I}_{+}, \hat{I}_{-}\right\}$[8],[198],[503],[504]. A few such operators are listed below:

$$
\begin{aligned}
\hat{T}_{0,0} & =\hat{1} \\
\hat{T}_{1,0} & =\hat{I}_{z} \\
\hat{T}_{1, \pm 1} & =\mp \sqrt{\frac{1}{2}} \hat{I}_{ \pm} \\
\hat{T}_{2,0} & =\sqrt{\frac{1}{6}}\left[3 \hat{I}_{z}^{2}-I(I+1) \hat{1}\right] \\
\hat{T}_{2, \pm 1} & =\mp \frac{1}{2}\left(\hat{I}_{z} \hat{I}_{ \pm}+\hat{I}_{ \pm} \hat{I}_{z}\right) \\
\hat{T}_{2, \pm 2} & =\frac{1}{2} \hat{I}_{ \pm}^{2} \\
\hat{T}_{3,0} & =\sqrt{\frac{1}{10}}\left[5 \hat{I}_{z}^{3}-\{3 I(I+1)-1\} \hat{I}_{z}\right] \\
\hat{T}_{3, \pm 1} & =\mp \frac{1}{4} \sqrt{\frac{3}{10}}\left[5\left(\hat{I}_{z}^{2} \hat{I}_{ \pm}+\hat{I}_{ \pm} \hat{I}_{z}^{2}\right)-\{2 I(I+1)+1\} \hat{I}_{ \pm}\right] \\
\hat{T}_{3, \pm 2} & =\frac{1}{2} \sqrt{\frac{3}{4}}\left(\hat{I}_{z} \hat{I}_{ \pm}^{2}+\hat{I}_{ \pm}^{2} \hat{I}_{z}\right) \\
\hat{T}_{3, \pm 3} & =\mp \frac{1}{2} \sqrt{\frac{1}{2}} \hat{I}_{ \pm}^{3}
\end{aligned}
$$

where these operators can be normalized via $\hat{T}_{k, q} \leftarrow \frac{1}{k !}\left[\frac{(2 k+1)(2 I-k) ! 2^{k}(2 k) !}{(2 I+k+1) !}\right]^{1 / 2} \hat{T}_{k, q}$, such that $\operatorname{Tr}\left[\hat{T}_{k, q}^{\dagger} \hat{T}_{k, q}\right]=1[198]$. The $\hat{T}_{k, q}$ are also orthogonal such that $\operatorname{Tr}\left[\hat{T}_{k^{\prime}, q^{\prime}}^{\dagger} \hat{T}_{k, q}\right]=0$ (where $k \neq k^{\prime}, q \neq q^{\prime}$ ), so that the spherical tensor operators form a complete set of basis operators in the state space of the angular momentum $\underline{I}$, and any operator in this space can be written in the form $\hat{A}=\sum_{k, q} c_{k, q} \hat{T}_{k, q}$. The similarity between the spherical tensors in Eq. A.11 and the spherical tensor operators in Eq. A.12 may be observed by noting that if $\underline{\underline{A}}=\underline{u} \otimes \underline{u}$, then e.g. $A_{2,0}=\sqrt{\frac{1}{6}}\left[3 u_{z} u_{z}-\left(u_{x} u_{x}+u_{y} u_{y}+u_{z} u_{z}\right)\right]=\sqrt{\frac{1}{6}}\left[3 u_{z}^{2}-\underline{u} \cdot \underline{u}\right]$, whereas 
$\hat{T}_{2,0}=\sqrt{\frac{1}{6}}\left[3 \hat{I}_{z}^{2}-\underline{\hat{I}} \cdot \underline{\hat{I}}\right]$ for an angular momentum $\underline{I}$, where $\underline{\hat{I}} \cdot \underline{\hat{I}}=I(I+1) \hat{1}$.

The concept of spherical tensor operators can be extended to systems of multiple angular momenta by forming higher rank tensor operators from angular momentum vector operators through the formalism of angular momentum addition, in much the same way as was used for the spherical tensors of $\S$ A.2.1. This formalism is the most useful in the case of two coupled angular momenta due to the difficulty of coupling multiple angular momenta, but attempts at the general case have been made [505]. Only the case of two angular momenta will be considered here. The second-rank Cartesian tensor operator ${ }^{2} \underline{\underline{T}}=\underline{\hat{I}}_{2} \otimes \underline{\hat{I}}_{1}$ can be decomposed into nine rank $k \leq 2$ spherical tensor operators $\hat{T}_{k, q}^{(1,2)}[8]$ :

$$
\begin{aligned}
\hat{T}_{0,0}^{(1,2)} & =-\sqrt{\frac{1}{3}}\left(\underline{\hat{I}}_{1} \cdot \underline{\underline{I}}_{2}\right) \\
\hat{T}_{1,0}^{(1,2)} & =-\frac{1}{2} \sqrt{\frac{1}{2}}\left(\hat{I}_{1+} \hat{I}_{2-}-\hat{I}_{1-} \hat{I}_{2+}\right) \\
\hat{T}_{1, \pm 1}^{(1,2)} & =\frac{1}{2}\left(\hat{I}_{z 1} \hat{I}_{ \pm 2}-\hat{I}_{ \pm 1} \hat{I}_{z 2}\right) \\
\hat{T}_{2,0}^{(1,2)} & =\sqrt{\frac{1}{6}}\left(3 \hat{I}_{z 1} \hat{I}_{z 2}-\hat{I}_{1} \cdot \underline{I}_{2}\right) \\
\hat{T}_{2, \pm 1}^{(1,2)} & =\mp \frac{1}{2}\left(\hat{I}_{z 1} \hat{I}_{ \pm 2}+\hat{I}_{ \pm 1} \hat{I}_{z 2}\right) \\
\hat{T}_{2, \pm 2}^{(1,2)} & =\frac{1}{2} \hat{I}_{ \pm 1} \hat{I}_{ \pm 2}
\end{aligned}
$$

The analogy to Eq. A.11 should be clear upon substituting $\hat{I}_{ \pm}=\hat{I}_{x} \pm i \hat{I}_{y}$ and $\underline{\underline{I}}_{1} \cdot \hat{\underline{I}}_{2}=$ $\hat{I}_{x 1} \hat{I}_{x 2}+\hat{I}_{y 1} \hat{I}_{y 2}+\hat{I}_{z 1} \hat{I}_{z 2}$. However, the $\hat{T}_{k, q}^{(1,2)}$ operators do not form a complete basis set in the $\left(2 I_{1}+1\right)\left(2 I_{2}+1\right)$-dimensional joint Hilbert space, even upon including operators of up to rank $k=2\left(I_{1}+I_{2}\right)$. It is often more convenient to use the basis of spherical tensor

\footnotetext{
${ }^{2}$ Here the direct product combines two Cartesian vectors into a second-rank Cartesian tensor.
} 
product operators $\left\{\hat{T}_{k, q}^{(1)} \hat{T}_{k^{\prime}, q^{\prime}}^{(2)}\right\}$, or products of the symmetric/antisymmetric combinations of the single-spin tensor operators [220]. However, in the special case of two coupled spin$1 / 2$ nuclei, the $\left[\left(2 I_{1}+1\right)\left(2 I_{2}+1\right)\right]^{2}=16$ operator matrix elements can be written in a basis of the nine $\hat{T}_{k, q}^{(1,2)}$ joint operators of rank $k \leq 2$, plus the seven distinct single-spin operators $\hat{T}_{k, q}^{(1)}$ and $\hat{T}_{k, q}^{(2)}$ of rank $k \leq 1$ (noting that $\left.\hat{T}_{0,0}^{(1)}=\hat{T}_{0,0}^{(2)}=\hat{1}^{(1,2)}\right)[7, \S 2.1 .10] .^{3}$

The spherical tensor operators have many interesting properties. The most important properties are treated in separate sections: the Wigner-Eckart theorem is discussed in $\S A .3$, and the behavior of spherical tensor operators under rotations is discussed in Appendix B. Additionally, the following commutation relations hold:

$$
\begin{aligned}
{\left[\hat{I}_{z}, \hat{T}_{k, q}\right] } & =q \hat{T}_{k, q} \\
{\left[\hat{I}_{ \pm}, \hat{T}_{k, q}\right] } & =\sqrt{k(k+1)-q(q \pm 1)} \hat{T}_{k, q \pm 1}
\end{aligned}
$$

Here it is understood that if the joint spherical tensors $\hat{T}_{k, q}^{(1,2)}$ are used, then $\hat{I}_{z}=\hat{I}_{z 1}+\hat{I}_{z 2}$ and $\hat{I}_{ \pm}=\hat{I}_{ \pm 1}+\hat{I}_{ \pm 2}$. The spherical tensor operators also obey the relation:

$$
\left(\hat{T}_{k, q}\right)^{\dagger}=(-1)^{q} \hat{T}_{k,-q}
$$

i.e., they are not Hermitian.

Some other useful identities apply to both spherical tensors and spherical tensor operators. The spherical tensors couple according to the rules of angular momentum

\footnotetext{
${ }^{3}$ The convention $\hat{A}^{(1)}=\hat{A}^{(1)} \otimes \hat{1}^{(2)}, \hat{B}^{(2)}=\hat{1}^{(1)} \otimes \hat{B}^{(2)}$, and $\hat{A}^{(1)} \hat{B}^{(2)} \equiv \hat{A}^{(1)} \otimes \hat{B}^{(2)}$ is employed, where here the direct product combines the Hilbert spaces of the two angular momenta.
} 
addition:

$$
\begin{aligned}
T_{k, q} & =\sum_{m_{1}, m_{2}}\left\langle l_{1}, m_{1} ; l_{2}, m_{2} \mid k, q\right\rangle A_{l_{1}, m_{1}} B_{l_{2}, m_{2}} \\
A_{l_{1}, m_{1}} B_{l_{2}, m_{2}} & =\sum_{k, q}\left\langle l_{1}, m_{1} ; l_{2}, m_{2} \mid k, q\right\rangle T_{k, q}
\end{aligned}
$$

where $\left\langle l_{1}, m_{1} ; l_{2}, m_{2} \mid k, q\right\rangle$ is a Clebsch-Gordan coefficient. Eq. A.17 can be used to generate Eq. A.13 from Eq. A.12. Two second-rank tensors can be contracted according to:

$$
\underline{\underline{B}}^{T}: \underline{\underline{A}}=\sum_{k=0}^{2} \sum_{q=-k}^{+k}(-1)^{q} B_{k, q} A_{k,-q}
$$

where we have already seen in $\S$ A.1 that $\underline{\underline{B}}^{T}: \underline{\underline{A}}=\sum_{i, j} B_{j i} A_{i j}$ in Cartesian coordinates. Note that the labels $A$ and $B$ can be exchanged without changing the sum.

\section{A.3 Wigner-Eckart Theorem}

The Wigner-Eckart theorem is used to aid in the evaluation of the matrix elements of spherical tensor operators in the basis of the eigenstates of angular momentum. Let $|\alpha ; I, m\rangle$ be an eigenstate of a quantized angular momentum $\underline{\hat{I}}$, where $\alpha$ denotes all the quantum numbers that label the state other than those of angular momentum. As usual, the order- $q$ component of some rank- $k$ spherical tensor operator $\hat{T}_{k}$ is denoted $\hat{T}_{k, q}$. The Wigner-Eckart theorem states:

$$
\left\langle\alpha^{\prime} ; I^{\prime}, m^{\prime}\left|\hat{T}_{k, q}\right| \alpha ; I, m\right\rangle=\left\langle I^{\prime}, m^{\prime} ; k, q \mid I, m\right\rangle\left\langle\alpha^{\prime} ; I^{\prime}\left\|\hat{T}_{k}\right\| \alpha ; I\right\rangle
$$


where $\left\langle I^{\prime}, m^{\prime} ; k, q \mid I, m\right\rangle$ is a Clebsch-Gordan coefficient and and the quantity $\left\langle\alpha^{\prime} ; I^{\prime}\left\|\hat{T}_{k}\right\|\right.$ $\alpha ; I\rangle$ is called the reduced matrix element of $\hat{T}_{k} \cdot{ }^{4}$ The physical content of the WignerEckart theorem is as follows. The left side of Eq. A.20 is a matrix element connecting two (possibly different) angular momentum states via the operator $\hat{T}_{k, q}$. The right side of the equation is a product of two terms. The reduced matrix element $\left\langle\alpha^{\prime} ; I^{\prime}\left\|\hat{T}_{k}\right\| \alpha ; I\right\rangle$ depends on $\alpha$ and $\alpha^{\prime}$ (and also on $I, I^{\prime}$ and $k$ ) and carries the dynamical information of the system, i.e. one must typically solve the Schrödinger Equation to determine the part of the eigenstate that depends on the variables $\alpha$ and $\alpha^{\prime} .^{5}$ Also, any physical constants that distinguish one vector operator from another can be included in the definition of $\hat{T}_{k}$. The reduced matrix element carries no dependence on $m, m^{\prime}$, or $q$, which bear the geometric information. Here "geometric information" refers to the components of the angular momenta $I$ and $I^{\prime}$ with respect to a particular direction (e.g., $m=0$ specifies the $I_{z}$ component of angular momentum, if $z$ is the quantization axis), and also to the directional components of $\hat{T}_{k}$.

All the geometric information on the right side of Eq. A.20 - the information about the angular distribution - is contained in the Clebsch-Gordan coefficient (which, conversely, does not depend on $\alpha$ ). The Wigner-Eckart theorem that within a particular manifold of angular momentum states the matrix elements of all operators $\hat{T}_{k, q}$ of a given rank and order are proportional to each other, where the proportionality constant is determined by the reduced matrix elements. For instance the angular momentum operator $\hat{I}$ and the magnetic moment operator $\underline{\hat{\mu}}$ are proportional to each other within some manifold of states $\{|\alpha ; I, m\rangle\}$, because both can be represented by rank $k=1$ vector operators. Also, since

\footnotetext{
${ }^{4}$ Some statements of the Wigner-Eckart theorem differ in their definition of the reduced matrix element by a factor of $(2 I+1)^{1 / 2}$.

${ }^{5}$ For instance, $\alpha$ could label the nuclear ground state, which must be found by solving the appropriate multi-nucleon Schrödinger Equation in an appropriate nuclear potential.
} 
many Hamiltonian operators can be written simply in terms of Cartesian tensors, a spherical tensor decomposition of the Hamiltonian (e.g. via Eq. A.19) followed by the application of the Wigner-Eckart theorem rapidly allows the Hamiltonian to be written in terms of matrix elements of the angular momentum operator. Some examples are the magnetic dipoledipole coupling Hamiltonian, in which the two nuclear magnetic moments may be related to angular momentum operators; or the electric quadrupolar Hamiltonian, in which the electric quadrupole moment of the nucleus may be related to its angular momentum. 


\section{Appendix B}

\section{Rotations and spherical tensors}

The theory of rotations is very important in NMR for several interrelated reasons: the evolution of nuclear polarization in the presence of a static magnetic field is formally equivalent to a time-dependent rotation about the field at the Larmor frequency; the rotating and tilted frame transformations that are widely used in calculations involve time-dependent and static rotations, respectively; rotations are used to write anisotropic interactions expressed in a molecule-fixed coordinate system in terms of laboratory-fixed coordinates; techniques such as magic angle spinning utilize an experimentally-induced sample rotation to average away certain spin interactions; etc. The spherical tensor formalism is truly powerful in two contexts: they provide an elegant means of expressing the geometric dependence of the matrix elements of operators when used in conjunction with the Wigner-Eckart theorem (see Appendix A), and their transformations under rotations can be expressed extremely simply. A brief summary of rotations and their effects on spherical tensors is presented here. The quantum-mechanical theory of rotations is quite elegant by 
itself, but the emphasis here is on their practical use. Most of the references cites in Appendix A contain discussions of rotation theory; specialized texts on angular momentum theory such as the one by Zare [20] typically have the most detailed descriptions.

The angular momentum operator component $\underline{\hat{I}} \cdot \underline{\widetilde{n}}$ is the generator of infinitesimal rotations about $\underline{\tilde{}}$ in the Hilbert space of the spin $I[29, \S 12],[45, \S 3.6],[28, \S 17-3],[20, \S \S],[499$, $\S 8]$. The quantum mechanical operator for a finite rotation by angle $\theta$ around $\underline{\tilde{n}}$ may be written in terms of the angular momentum operator as

$$
\hat{R}_{\underline{\tilde{n}}}(\chi)=\exp [-i \chi(\underline{\hat{I}} \cdot \underline{\tilde{n}})]
$$

As usual, the angular momentum operator $\underline{\hat{I}}$ has been taken to be dimensionless. All rotations will be assumed to be counterclockwise about $+\underline{\tilde{\tilde{n}}}$ when $\chi>0$. For example, the operator $\exp \left(-i \pi \hat{I}_{x} / 2\right)$ generates a $90^{\circ}$ counterclockwise rotation about $+\underline{\widetilde{x}}$. Rotations are assumed to be active, i.e., a system is rotated while the coordinate axes remain fixed; it is also possible to achieve the same physical result by making a passive rotation of the coordinate axes while the system is fixed. Rotation operators are unitary:

$$
\hat{R} \hat{R}^{-1}=\hat{1}
$$

a property which is related to the fact that the norm of a vector (or of any other operator that describes the system) is preserved under rotation and a rotation can be undone by a 
subsequent "reverse rotation." Quantum-mechanical states $|\psi\rangle$ are rotated according to

$$
\left|\psi^{\prime}\right\rangle=\hat{R}_{\underline{\underline{n}}}(\chi)|\psi\rangle
$$

and operators are rotated according to

$$
\hat{A}^{\prime}=\hat{R}_{\underline{\widetilde{n}}}(\chi) \hat{A} \hat{R}_{\underline{\underline{r}}}^{-1}(\chi)
$$

For example, the Cartesian components $\hat{I}_{x}, \hat{I}_{y}$, and $\hat{I}_{z}$ of the angular momentum vector operator $\hat{\underline{I}}$ transform under rotations as

$$
\begin{aligned}
& \hat{R}_{x}(\chi) \hat{I}_{x} \hat{R}_{x}^{-1}(\chi)=\hat{I}_{x} \\
& \hat{R}_{y}(\chi) \hat{I}_{x} \hat{R}_{y}^{-1}(\chi)=\hat{I}_{x} \cos \chi-\hat{I}_{z} \sin \chi \\
& \hat{R}_{z}(\chi) \hat{I}_{x} \hat{R}_{z}^{-1}(\chi)=\hat{I}_{x} \cos \chi+\hat{I}_{y} \sin \chi \\
& \hat{R}_{x}(\chi) \hat{I}_{y} \hat{R}_{x}^{-1}(\chi)=\hat{I}_{y} \cos \chi+\hat{I}_{z} \sin \chi \\
& \hat{R}_{y}(\chi) \hat{I}_{y} \hat{R}_{y}^{-1}(\chi)=\hat{I}_{y} \\
& \hat{R}_{z}(\chi) \hat{I}_{y} \hat{R}_{z}^{-1}(\chi)=\hat{I}_{y} \cos \chi-\hat{I}_{x} \sin \chi \\
& \hat{R}_{x}(\chi) \hat{I}_{z} \hat{R}_{x}^{-1}(\chi)=\hat{I}_{z} \cos \chi-\hat{I}_{y} \sin \chi \\
& \hat{R}_{y}(\chi) \hat{I}_{z} \hat{R}_{y}^{-1}(\chi)=\hat{I}_{z} \cos \chi+\hat{I}_{x} \sin \chi \\
& \hat{R}_{z}(\chi) \hat{I}_{z} \hat{R}_{z}^{-1}(\chi)=\hat{I}_{z},
\end{aligned}
$$

which are exactly the relations one expects for the transformation of the components of a vector under rotations. Eq. B.5 can be proven by multiplying the matrix representa- 
tions of these operators or by using a theorem for the rotation of spherical tensor operator components that will be presented later.

Three coordinates are necessary to specify a rotation in three-dimensional space, e.g., the rotation angle $\chi$ and the polar coordinates $(\theta, \phi)$ that describe the orientation of $\underline{\tilde{n}}$. It is often more convenient to parameterize the rotation operator by the three Euler rotation angles. Any rotation can be characterized in terms of three successive rotations that each involve one angular coordinate. These so-called Euler angles may be used to relate a coordinate system $(x, y, z)$ that is fixed in space (e.g., the laboratory frame) to a coordinate system $(X, Y, Z)$ that is fixed to the system that is rotated (i.e., the body-fixed frame, which for example could be a molecule-fixed frame). ${ }^{1}$ The three Euler rotations that carry the space-fixed frame into the body-fixed frame are $[20, \S 3.2]$ : (1) a rotation by angle $\alpha$ about $x$ that carries the $(x, x, z)$ frame into a new frame $\left(x^{\prime}, y^{\prime}, z^{\prime}\right) ;(2)$ a rotation by $\beta$ about the new coordinate $y^{\prime}$ that carries $\left(x^{\prime}, y^{\prime}, z^{\prime}\right)$ into $\left(x^{\prime \prime}, y^{\prime \prime}, z^{\prime \prime}\right)$; and (3) a rotation by $\gamma$ about $z^{\prime \prime}$ that carries $\left(x^{\prime \prime}, y^{\prime \prime}, z^{\prime \prime}\right)$ into $(X, Y, Z)$. Note that $z$ coincides with $z^{\prime}$ and that $z^{\prime \prime}$ coincides with $Z$. Each of the three Euler rotations can be written in the form of Eq. B.1 and the net rotation is their product:

$$
\hat{R}(\alpha, \beta, \gamma)=\exp \left(-i \gamma \hat{I}_{z^{\prime \prime}}\right) \exp \left(-i \beta \hat{I}_{y^{\prime}}\right) \exp \left(-i \alpha \hat{I}_{z}\right)
$$

where, as usual, the operations are ordered such that operators that act first go on the right side of the expression. Eq. B.6 is an alternative parameterization of Eq. B.1. The ordering of the operators in the product is crucial because in general rotations do not commute with

\footnotetext{
${ }^{1}$ Zare $[20]$ uses the opposite convention of $(X, Y, Z)$ for the space-fixed frame and $(x, y, z)$ for the body-fixed frame.
} 
each other. It is often more convenient to write the rotation in terms of space-fixed axes $[20, \S 3.2]:$

$$
\hat{R}(\alpha, \beta, \gamma)=\exp \left(-i \alpha \hat{I}_{z}\right) \exp \left(-i \beta \hat{I}_{y}\right) \exp \left(-i \gamma \hat{I}_{z}\right)
$$

This expression can be derived from Eq. B.6 with the aid of an identity involving unitary rotations of functions of operators:

$$
\hat{U} f(\hat{A}) \hat{U}^{-1}=f\left(\hat{U} \hat{A} \hat{U}^{-1}\right)
$$

which can be derived by inserting appropriate factors of $\hat{U} \hat{U}^{-1}$ into a power series expansion of $f(\hat{A})$. This identity may also be used to rewrite Eq. B.1 in terms of the polar angles $(\theta, \phi)$ that specify the direction of $\underline{\tilde{n}}$ in a space-fixed coordinate system:

$$
\begin{aligned}
\hat{R}_{\underline{\underline{n}}}(\chi) & =\hat{R}_{x}(\phi) \hat{R}_{y}(\theta) \hat{R}_{z}(\chi) \hat{R}_{y}^{-1}(\theta) \hat{R}_{x}^{-1}(\phi) \\
& =e^{-i \phi \hat{I}_{z}} e^{-i \theta \hat{I}_{y}} e^{-i \chi \hat{I}_{z}} e^{+i \theta \hat{I}_{y}} e^{+i \phi \hat{I}_{z}}
\end{aligned}
$$

where the relation $\underline{\tilde{r}}=\sin \theta \cos \phi \underline{\widetilde{x}}+\sin \theta \sin \phi \underline{\tilde{y}}+\cos \theta \underline{\widetilde{z}}$ has been used. Zare's book [20] contains useful $(\phi, \theta, \chi)$-parameterized matrix representations (Eqs. 3.36, 3.37) of the rotation operator which relates the space-fixed coordinate system to the body-fixed coordinate system in real space (i.e., as opposed to spin space).

The fundamental relation that describes the Euler angle-parameterized rotation 
of a spherical tensor operator $\hat{T}_{k, q}$ is

$$
\hat{R}(\alpha, \beta, \gamma) \hat{T}_{k, q} \hat{R}^{-1}(\alpha, \beta, \gamma)=\sum_{q^{\prime}=-k}^{k} D_{q^{\prime}, q}^{(k)}(\alpha, \beta, \gamma) \hat{T}_{k, q^{\prime}}
$$

The Wigner rotation matrix element $D_{q^{\prime}, q}^{(k)}(\alpha, \beta, \gamma)$ is

$$
D_{q^{\prime}, q}^{(k)}(\alpha, \beta, \gamma)=\left\langle k, q^{\prime}|\hat{R}(\alpha, \beta, \gamma)| k, q\right\rangle
$$

where the $\{|k, q\rangle\}$ are normalized angular momentum eigenstates. Eq. B.7 may be used to write Eq. B.11 as

$$
D_{q^{\prime}, q}^{(k)}(\alpha, \beta, \gamma)=e^{-i\left(q^{\prime} \alpha+q \gamma\right)} d_{q^{\prime}, q}^{(k)}(\beta),
$$

where the reduced Wigner rotation matrix element is

$$
d_{q^{\prime}, q}^{(k)}(\beta)=\left\langle k, q^{\prime}\left|e^{-i \beta \hat{I}_{y}}\right| k, q\right\rangle .
$$

The reduced Wigner rotation matrix elements are tabulated in many sources, e.g., Table 3.1 of Ref. [20].

Eq. B.10 holds for any spherical tensor, although the representations of the spherical tensor and the Wigner rotation matrix will vary according to the dimensionality of the Hilbert spin space or whether the rotations take place in a Cartesian space (real space) instead of spin space. In fact, Eq. B.10 may be considered to be the definition of the components of a rank- $k$ spherical tensor $T_{k}$. This equation is extremely useful because of this 
generality: e.g., the expression for the rotation of a rank-1 spherical tensor is the same no matter whether that tensor is written in the space of a spin- $1 / 2$ particle, a spin- 1 particle, two coupled spin-1/2 particles, or even represents a rank-1 spherical harmonic function. Only the representations of the spherical tensor and Wigner rotation matrices are needed to determine the effects of the rotation.

An important result of Eq. B.10 is that the spherical tensor rank $k$ is conserved under rotations; only the components $q$ are interconverted. This has an important consequence: rank- $k$ polarization of a spin $I$ cannot be converted to any other rank under the action of a magnetic field alone. This can be appreciated from the following argument. Imagine that a magnetic field $\underline{B}=B \underline{\tilde{n}}$ is applied to a spin $I$ that initially possesses rank- $k$ polarization, i.e., take the difference density operator to be $\hat{\rho}^{I}(0) \propto \hat{T}_{k, q}^{I}$. The evolution in the presence of the magnetic field is given by

$$
\begin{aligned}
\hat{\rho}(t) & =e^{-i \hat{H}_{Z} t / \hbar} \hat{\rho}(0) e^{-i \hat{H}_{Z} t / \hbar} \\
& =\exp \left[i \gamma_{I} B t(\underline{\hat{I}} \cdot \underline{\widetilde{n}})\right] \hat{T}_{k, q}^{I} \exp \left[-i \gamma_{I} B t(\underline{\hat{I}} \cdot \underline{\widetilde{n}})\right] \\
& =\hat{R}_{\underline{\underline{n}}}\left(-\omega_{I} t\right) \hat{T}_{k, q}^{I} \hat{R}_{\underline{\tilde{n}}}^{-1}\left(-\omega_{I} t\right),
\end{aligned}
$$

where $\hat{H}_{Z}=-\hbar \gamma_{I} B(\underline{\hat{I}} \cdot \underline{\widetilde{n}})$ is the Zeeman Hamiltonian and the Larmor frequency is $\omega_{I}=\gamma_{I} B$. Eq. B.14 corresponds to a time-dependent rotation of the polarization about direction of the magnetic field $\underline{\tilde{n}}$ at the Larmor frequency $\omega_{I}$. According to Eq. B.10, this evolution conserves the spherical tensor rank of the polarization. This is a general result for the angular momentum dynamics of any system subjected to a Hamiltonian that contains only rank-1 spherical tensor operators (i.e., components of $\underline{\underline{I}}$ ). This conservation is exemplified 
by Eq. B.5, where the components $\hat{I}_{x}, \hat{I}_{y}$, and $\hat{I}_{z}$ of the rank-1 vector operator $\underline{\hat{I}}$ - which can be written as linear combinations of spherical tensor operators $\hat{T}_{1, q}^{I}$ —were seen to transform into each other (and only each other) under rotations. Another consequence of Eq. B.10 is that rf pulses cannot be used to excite multiple-quantum coherences (MQCs) from initial magnetization in a spin $I$ system if the only other interactions present are Zeeman/chemical shift interactions. This is because all the Hamiltonians contain only rank-1 spin operators, because magnetization is proportional to $\langle\underline{\underline{I}}\rangle$ and thus corresponds to rank-1 terms in the density operator, and because MQCs by definition correspond to $\hat{T}_{k, q}$ polarization terms with $|q|>1$, which in turn implies $k>1$. However, the rank of the polarization can be changed if the spin evolution takes place under a combination of the magnetic field of an rf pulse and a Hamiltonian that contains spin operators higher than rank 1 such as the quadrupolar Hamiltonian. This problem is discussed in detail in terms of MQC conversion and orientation-to-alignment conversion in $\S 5$ and in other chapters of this dissertation. Multiple quantum coherences can also be created in coupled spin systems by the use of rf pulses in conjunction with dipolar or scalar coupling interactions.

Eq. B.10 results in some other important relations involving $z$-rotations. For example,

$$
\hat{R}_{z}(\chi) \hat{T}_{k, q}^{I} \hat{R}_{z}^{-1}(\chi)=e^{-i \chi \hat{I}_{z}} \hat{T}_{k, q}^{I} e^{+i \chi \hat{I}_{z}}=e^{-i \chi q} \hat{T}_{k, q}^{I}
$$

where $\hat{R}_{z}(\chi)=\hat{R}_{z}(\chi, 0,0)=\hat{R}_{z}(0,0, \chi)$ in the Euler angle parameterization and $d_{q^{\prime}, q}^{(k)}(0)=1$ according to Eq. B.13. If $\hat{T}_{k, q}^{I}$ represents a density operator element for rank- $k$, coherence order $q$ polarization in the Zeeman basis, it can be seen that a $z$-rotation conserves not 
only polarization rank but also coherence order. The coherence order-dependent phase factor $\exp (-i \chi q)$ that is gained upon rotation is what allows coherence pathway selection to be implemented via phase cycling and pulsed $z$-gradient selection techniques. Eq. B.15 also holds for a non-selective $z$-rotation $\hat{R}_{z}(\chi)=\exp \left(-i \chi \hat{I}_{Z}\right)$ acting on a product operator spherical tensor operator $\hat{T}_{k, q}^{(1,2)}$ in the joint spin space of $I_{1}$ and $I_{2}$ (see Appendix A.2.2), where here $\hat{I}_{Z}=\hat{I}_{z 1}+\hat{I}_{z 2}$ is the $z$-component of total angular momentum $\underline{\hat{I}}=\underline{\underline{I}}_{1}+\underline{\hat{I}}_{2}$. A special case of Eq. B.10 is the invariance of $\hat{T}_{k, 0}$ spherical tensor components to $z$-rotations, i.e.,

$$
\hat{R}_{z}(\chi) \hat{T}_{k, 0} \hat{R}_{z}^{-1}(\chi)=e^{-i \chi \hat{I}_{z}} \hat{T}_{k, 0} e^{+i \chi \hat{I}_{z}}=\hat{T}_{k, 0}
$$

This is a consequence of the relation $\left[\hat{I}_{z}, \hat{T}_{k, 0}\right]=0$ (see Eq. A.14). The convenient interpretation is that a $\hat{T}_{k, 0}$ term in the density operator represents a generalized "longitudinal polarization" that does not evolve in the presence of a $z$-directed magnetic field.

Eq. B.10 also finds use in NMR when relating the spatial tensors of some interaction (e.g., electric field gradient tensor for the quadrupolar coupling, chemical shielding tensor, etc.) in the molecular (crystallite) frame to tensors in the laboratory frame:

$$
A_{k, q}^{\mathrm{LAB}}=\sum_{m=-k}^{k} D_{m, q}^{(k)}\left(\alpha^{\prime}, \beta^{\prime}, \gamma^{\prime}\right) A_{k, m}^{\mathrm{MOL}}
$$

where the laboratory and molecular frames are related through the Euler angle-parameterized rotation operator $R\left(\alpha^{\prime}, \beta^{\prime}, \gamma^{\prime}\right)$. This type of relation is useful in solid state NMR when studies on random powders or single crystals are performed. The Cartesian spatial tensors may 
be diagonalized in their own so-called principal axis system (PAS); the spherical components in this frame take especially simple forms. If multiple interactions are present per molecule, e.g., a nucleus that experiences simultaneous chemical shielding and quadrupolar coupling interactions, it is useful to transform the spatial tensors in the various PAS frames into a common molecular frame such that

$$
\begin{aligned}
A_{l, m}^{\mathrm{MOL}} & =\sum_{p=-k}^{k} D_{p, m}^{(k)}(\alpha, \beta, \gamma) A_{k, p}^{\mathrm{PAS}} \\
A_{k, q}^{\mathrm{LAB}} & =\sum_{m=-k}^{k} \sum_{p=-k}^{k} D_{m, q}^{(k)}\left(\alpha^{\prime}, \beta^{\prime}, \gamma^{\prime}\right) D_{p, m}^{(k)}(\alpha, \beta, \gamma) A_{k, p}^{\mathrm{PAS}},
\end{aligned}
$$

where Eq. B.17 has been used to write the last line via the two consecutive rotations PAS $\stackrel{(\alpha, \beta, \gamma)}{\longrightarrow}$ MOL $\stackrel{\left(\alpha^{\prime}, \beta^{\prime}, \gamma^{\prime}\right)}{\longrightarrow}$ LAB. This type of procedure is reviewed in Mehring's text $[8, \S 2.3]$. The molecular and PAS frames can be taken to be coincident if there is only one relevant interaction per molecule. Further rotations can be added; e.g., in the case of sample spinning a third time-dependent rotation can be added: $\mathrm{PAS} \rightarrow \mathrm{MOL} \rightarrow \mathrm{ROT} \rightarrow \mathrm{LAB}$, where ROT can be written in terms of Euler rotations that relate the molecular frame to the rotor-fixed frame $[8, \S 2.6]$. Similar transformations between frames of spin tensor operators can be made, although in NMR a frame in which the spin quantization axis is along $z$ is almost always chosen. Note that some care must be taken when relating spin tensor operators in different frames to each other; i.e., the components of the angular momentum operator in a body-fixed frame exhibit anomalous commutation relations $[20, \S 3.4, \S 5.1]$.

A few other useful identities involving rotations are included here. The Wigner 
rotation matrix elements satisfy the following relations (see e.g. [45, §3.6]):

$$
\begin{aligned}
d_{m^{\prime}, m}^{(l) *}(\theta) & =d_{m^{\prime}, m}^{(l)}(\theta) \\
d_{m^{\prime}, m}^{(l)}(\theta) & =(-1)^{m-m^{\prime}} d_{m, m^{\prime}}^{(l)}(\theta)=d_{-m,-m^{\prime}}^{(l)}(\theta) \\
D_{m, 0}^{(l)}(\phi, \theta, 0) & =\sqrt{\frac{4 \pi}{2 l+1}} Y_{l, m}^{*}(\theta, \phi) \\
d_{m, 0}^{(l)}(\theta) & =\sqrt{\frac{4 \pi}{2 l+1}} Y_{l, m}(\theta, \phi) e^{-i m \phi} \\
D_{0,0}^{(l)}(0, \theta, 0) & =d_{0,0}^{(l)}(\theta)=P_{l}(\cos \theta)
\end{aligned}
$$

where $Y_{l, m}(\theta, \phi)$ is a spherical harmonic function and $P_{l}(\cos \theta)$ is a Legendre polynomial.

The matrix representation of the $I=1 / 2$ rotation operator in the $\left|I=\frac{1}{2}, m= \pm \frac{1}{2}\right\rangle$ basis is $[45, \S 3.5]$ :

$$
D^{\left(\frac{1}{2}\right)}(\alpha, \beta, \gamma)=\left(\begin{array}{cc}
e^{-i(\alpha+\gamma) / 2} \cos (\beta / 2) & -e^{-i(\alpha-\gamma) / 2} \sin (\beta / 2) \\
e^{+i(\alpha-\gamma) / 2} \sin (\beta / 2) & e^{+i(\alpha+\gamma) / 2} \cos (\beta / 2)
\end{array}\right)
$$




\section{Appendix C}

\section{Time-dependent perturbation}

\section{theory via a power series expansion of the time development operator}

A common quantum mechanical problem is the solution of the time evolution of a system subject to a small time-dependent interaction in the presence of a large, timeindependent interaction. This solution can only be approximated if the total Hamiltonian is homogeneous $(\S 2)$, and typically a perturbative solution is sought. In some texts the generation of the expressions for transition amplitudes can be quite mathematical and complicated, involving sets of differential equations for the coefficients of the wavefunction projected onto a particular basis set [85, XIII.B]. Methods based on the Dyson series of the time development operator in the interaction frame are more elegant $[45, \S 5.6],[272$, XVII.1,2]. The Dyson series is usually obtained by the iterative solution of an integral equation. At the 
beginning of $\S 2.2$ it was mentioned that the Dyson series can also be obtained by a power series expansion of the exponential form of the time development operator. ${ }^{1}$ Since series expansions of exponential functions are very familiar to even beginning physics students, it may be useful to repeat the derivation of some of the standard equations of time-dependent perturbation theory using the series expansion.

Consider a time-dependent Hamiltonian

$$
\hat{H}(t)=\hat{H}_{0}+\hat{V}(t)
$$

where $\left\|\hat{H}_{0}\right\| \gg\|\hat{V}\|$. The solution of $\hat{H}_{0}$ is presumed to be known:

$$
\hat{H}_{0}|n\rangle=E_{n}|n\rangle
$$

where the $\{|n\rangle\}$ are the eigenstates of $\hat{H}_{0}$ with corresponding eigenvalues $E_{n}=\hbar \omega_{n}=$ $\left\langle n\left|\hat{H}_{0}\right| n\right\rangle$. We wish to find the probability that the system starts out in some state $|i\rangle$ at time $t_{0}$ and ends up in some state $|f\rangle$ at time $t$. The transition probability is:

$$
P_{f \leftarrow i}=\left|A_{f \leftarrow i}\right|^{2}=\left|\left\langle f\left|\hat{U}\left(t ; t_{0}\right)\right| i\right\rangle\right|^{2}
$$

where $A_{f \leftarrow i}$ is the transition amplitude. We will assume that $|i\rangle$ and $|f\rangle$ are eigenstates of $\hat{H}_{0}$ with eigenvalues $E_{i}$ and $E_{f}$, respectively. If $\hat{H}(t)$ is homogeneous (i.e., $\left[\hat{H}(t), \hat{H}\left(t^{\prime}\right)\right] \neq$ 0 , usually due to $\left.\left[\hat{H}_{0}, \hat{V}(t)\right] \neq 0\right)$, then the time development operator $\hat{U}\left(t ; t_{0}\right)$ must be

\footnotetext{
${ }^{1}$ Obviously, this is the reverse process of the summation of the Dyson series to form an exponential operator $[506, \S 17.2]$.
} 
approximated. It is advantageous to make a transformation into the interaction frame of $\hat{H}_{0}$ such that the effective Hamiltonian is shrunk from the size of $\left\|\hat{H}_{0}\right\|$ to the size of $\|\hat{V}\|$ and any series approximation of the resulting time development operator will be valid for longer times. From $\S 2.3 .2$ we find that:

$$
\hat{U}\left(t ; t_{0}\right)=\hat{U}_{I}(t) \hat{\tilde{U}}\left(t ; t_{0}\right)=e^{-i \hat{H}_{0}\left(t-t_{0}\right) / \hbar} T\left\{\exp \left[-\frac{i}{\hbar} \int_{t_{0}}^{t} d t^{\prime} \hat{\tilde{V}}\left(t^{\prime}\right)\right]\right\}
$$

where $T$ is the Dyson time-ordering operator, the interaction frame transformation is defined by $\hat{U}_{I}(t)=e^{-i \hat{H}_{0}\left(t-t_{0}\right) / \hbar}$, and the interaction frame effective Hamiltonian is

$$
\hat{\tilde{V}}(t)=\hat{U}_{I}^{-1}(t) \hat{V}(t) \hat{U}_{I}(t)=e^{+i \hat{H}_{0}\left(t-t_{0}\right) / \hbar} \hat{V}(t) e^{-i \hat{H}_{0}\left(t-t_{0}\right) / \hbar}
$$

It can be seen from Eq. C.4 that the exponential operator to be approximated $\hat{\widetilde{U}}\left(t ; t_{0}\right)$ has an argument involving an integral over $\hat{\widetilde{V}}(t)$, which is of the order $\hat{V}$, so that the series expansion of the exponential will involve powers of $\hat{V}$. If a interaction frame transformation was not applied, the expansion would involve powers of $\hat{H}$, which is of the much larger order of $\hat{H}_{0}$. Eq. C.4 also cleanly separates the time evolution due to $\hat{H}_{0}$ and $\hat{\tilde{V}}(t)$, even if these operators do not commute with each other.

An exponential function may be expanded in a Maclaurin series as $e^{x} \simeq 1+x+$ $\frac{1}{2 !} x^{2}+\frac{1}{3 !} x^{3}+\ldots=\sum_{n=0}^{\infty} x^{n} / n !$. This expansion can be used in operator form on the 
interaction frame time development operator $\hat{\widetilde{U}}\left(t ; t_{0}\right)$ in Eq. C.4, such that:

$$
\begin{aligned}
& \hat{U}\left(t ; t_{0}\right) \simeq e^{-i \hat{H}_{0}\left(t-t_{0}\right) / \hbar} T\left\{\hat{1}+\left[\left(\frac{-i}{\hbar}\right) \int_{t_{0}}^{t} d t^{\prime} \hat{\tilde{V}}\left(t^{\prime}\right)\right]+\frac{1}{2 !}\left[\left(\frac{-i}{\hbar}\right) \int_{t_{0}}^{t} d t^{\prime} \hat{\tilde{V}}\left(t^{\prime}\right)\right]^{2}+\right. \\
&+\left.\frac{1}{3 !}\left[\left(\frac{-i}{\hbar}\right) \int_{t_{0}}^{t} d t^{\prime} \hat{\tilde{V}}\left(t^{\prime}\right)\right]^{3}+\ldots\right\} \\
&=e^{-i \hat{H}_{0}\left(t-t_{0}\right) / \hbar}\left\{\hat{1}+\left[\left(\frac{-i}{\hbar}\right) \int_{t_{0}}^{t} d t^{\prime} \hat{\tilde{V}}\left(t^{\prime}\right)\right]+\frac{1}{2 !}\left(\frac{-i}{\hbar}\right)^{2} T\left[\int_{t_{0}}^{t} d t^{\prime \prime} \int_{t_{0}}^{t} d t^{\prime} \hat{\tilde{V}}\left(t^{\prime \prime}\right) \hat{\tilde{V}}\left(t^{\prime}\right)\right]\right. \\
&+\left.\left.\frac{1}{3 !}\left(\frac{-i}{\hbar}\right)^{3} T\left[\int_{t_{0}}^{t} d t^{\prime \prime \prime} \int_{t_{0}}^{t} d t^{\prime \prime} \int_{t_{0}}^{t} d t^{\prime} \hat{\tilde{V}}\left(t^{\prime \prime \prime}\right) \hat{\tilde{V}}\left(t^{\prime \prime}\right) \hat{\tilde{V}}\left(t^{\prime}\right)\right]+\ldots\right\}, \quad \text { (C. } 6\right)
\end{aligned}
$$

where this series was also stated in Eq. 2.17. This equation can be cast into matrix element form by inserting factors of the identity $\hat{1}=\sum_{n}|n\rangle\langle n|$ appropriately, where the $\{|n\rangle\}$ are chosen to be eigenvectors of $\hat{H}_{0}$.

$$
\begin{aligned}
& A_{f \leftarrow i}=\left\langle f\left|\hat{U}\left(t ; t_{0}\right)\right| i\right\rangle \\
& \simeq\langle f| e^{-i \hat{H}_{0}\left(t-t_{0}\right) / \hbar}\left\{\hat{1}+\left[\left(\frac{-i}{\hbar}\right) \int_{t_{0}}^{t} d t^{\prime} \hat{\tilde{V}}\left(t^{\prime}\right)\right]\right. \\
&+ \frac{1}{2 !}\left(\frac{-i}{\hbar}\right)^{2} \sum_{n} T\left[\int_{t_{0}}^{t} d t^{\prime \prime} \int_{t_{0}}^{t} d t^{\prime} \hat{\tilde{V}}\left(t^{\prime \prime}\right)|n\rangle\langle n| \hat{\tilde{V}}\left(t^{\prime}\right)\right] \\
&+\left.\frac{1}{3 !}\left(\frac{-i}{\hbar}\right)^{3} \sum_{n, n^{\prime}} T\left[\int_{t_{0}}^{t} d t^{\prime \prime \prime} \int_{t_{0}}^{t} d t^{\prime \prime} \int_{t_{0}}^{t} d t^{\prime} \hat{\tilde{V}}\left(t^{\prime \prime \prime}\right)|n\rangle\left\langle n\left|\hat{\widetilde{V}}\left(t^{\prime \prime}\right)\right| n^{\prime}\right\rangle\left\langle n^{\prime}\right| \hat{\widetilde{V}}\left(t^{\prime}\right)\right]+\ldots\right\}|i\rangle \\
&=e^{-i \omega_{f}\left(t-t_{0}\right)}\left\{\delta_{f, i}+\left[\left(\frac{-i}{\hbar}\right) \int_{t_{0}}^{t} d t^{\prime} \widetilde{V}_{f i}\left(t^{\prime}\right)\right]+\right. \\
& \quad+\frac{1}{2 !}\left(\frac{-i}{\hbar}\right)^{2} \sum_{n} T\left[\int_{t_{0}}^{t} d t^{\prime \prime} \int_{t_{0}}^{t} d t^{\prime} \widetilde{V}_{f n}\left(t^{\prime \prime}\right) \widetilde{V}_{n i}\left(t^{\prime}\right)\right] \\
&\left.\left.+\frac{1}{3 !}\left(\frac{-i}{\hbar}\right)^{3} \sum_{n, n^{\prime}} T\left[\int_{t_{0}}^{t} d t^{\prime \prime \prime} \int_{t_{0}}^{t} d t^{\prime \prime} \int_{t_{0}}^{t} d t^{\prime} \widetilde{V}_{f n}\left(t^{\prime \prime \prime}\right) \widetilde{V}_{n n^{\prime}}\left(t^{\prime \prime}\right) \widetilde{V}_{n^{\prime} f}\left(t^{\prime}\right)\right]+\ldots\right\} \quad \text { (C. } 7\right)
\end{aligned}
$$


where $\delta_{f, i}=\langle f \mid i\rangle$ is a Kronecker delta function, and from the definition of $\hat{\tilde{V}}(t)$ in Eq. C.5:

$$
\widetilde{V}_{n n^{\prime}} \equiv\left\langle n|\hat{\tilde{V}}(t)| n^{\prime}\right\rangle=e^{i \omega_{n n^{\prime}}\left(t-t_{0}\right)} V_{n n^{\prime}}(t)
$$

where $\omega_{n n^{\prime}} \equiv \omega_{n}-\omega_{n^{\prime}}=\left\langle n\left|\hat{H}_{0} / \hbar\right| n\right\rangle-\left\langle n^{\prime}\left|\hat{H}_{0} / \hbar\right| n^{\prime}\right\rangle$ and $V_{n n^{\prime}}(t) \equiv\left\langle n|\hat{V}(t)| n^{\prime}\right\rangle$. Inserting Eq. C.8 into Eq. C.9 transforms back out of the interaction frame and gives (we take $t_{0}=0$ for more compact notation):

$$
\begin{aligned}
A_{f \leftarrow i} & =e^{-i \omega_{f} t}\left\{\delta_{f, i}+\left[\left(\frac{-i}{\hbar}\right) \int_{0}^{t} d t^{\prime} e^{i \omega_{f i} t^{\prime}} V_{f i}\left(t^{\prime}\right)\right]\right. \\
& +\frac{1}{2 !}\left(\frac{-i}{\hbar}\right)^{2} \sum_{n} T\left[\int_{0}^{t} d t^{\prime \prime} \int_{0}^{t} d t^{\prime} e^{i \omega_{f n} t^{\prime \prime}} V_{f n}\left(t^{\prime \prime}\right) e^{i \omega_{n i} t^{\prime}} V_{n i}\left(t^{\prime}\right)\right] \\
& +\frac{1}{3 !}\left(\frac{-i}{\hbar}\right)^{3} \sum_{n, n^{\prime}} T\left[\int_{0}^{t} d t^{\prime \prime \prime} \int_{0}^{t} d t^{\prime \prime} \int_{0}^{t} d t^{\prime} e^{i \omega_{f n} t^{\prime \prime \prime}} V_{f n}\left(t^{\prime \prime \prime}\right) e^{i \omega_{n n^{\prime}} t^{\prime \prime}} V_{n n^{\prime}}\left(t^{\prime \prime}\right) e^{i \omega_{n^{\prime} f} t^{\prime}} V_{n^{\prime} f}\left(t^{\prime}\right)\right] \\
& +\ldots\}
\end{aligned}
$$

In principle, this is the final result of the time-dependent perturbation theory. However, it may be more convenient for computational purposes to eliminate the formal Dyson timeordering operator $T$ by building the time ordering directly into the limits of integration. This approach also recovers the standard result of the Dyson series, which is developed by the iterative solution of the integral equation for $\hat{\widetilde{U}}\left(t ; t_{0}\right)$. As an example, consider the secondorder term in the expansion of $\tilde{\tilde{U}}\left(t ; t_{0}\right)$, which is $\frac{1}{2 !}\left(\frac{-i}{\hbar}\right)^{2}\left\langle f\left|T\left\{\int_{t_{0}}^{t} d t^{\prime \prime} \int_{t_{0}}^{t} d t^{\prime} \hat{\tilde{V}}\left(t^{\prime \prime}\right) \hat{\tilde{V}}\left(t^{\prime}\right)\right\}\right| i\right\rangle$. The integrand is symmetric around $t^{\prime}=t^{\prime \prime}$, such that equal contributions are given to the integral for $t^{\prime}>t^{\prime \prime}$ and $t^{\prime}<t^{\prime \prime}$ (where $t_{0} \leq t^{\prime}, t^{\prime \prime} \leq t$ ), and the integral is equal to just twice the contribution of either part. However, the restrictions $t^{\prime}>t^{\prime \prime}$ or $t^{\prime}<t^{\prime \prime}$ imply a time 
ordering. Therefore $\frac{1}{2 !} T\left\{\int_{t_{0}}^{t} d t^{\prime \prime} \int_{t_{0}}^{t} d t^{\prime}[\cdots]\right\}=\frac{1}{2 !} \times 2 \int_{t_{0}}^{t} d t^{\prime \prime} \int_{t_{0}}^{t^{\prime \prime}} d t^{\prime}[\cdots]$, where the limits of integration restrict $t_{0} \leq t^{\prime} \leq t^{\prime \prime} \leq t$. It can be demonstrated that for the $n^{t h}$-order term in the expansion, the time ordering operator can be removed by an appropriate time-ordered restriction of the integration domain, which happens to be smaller by a factor of $1 / n$ ! than the total integration volume (see e.g. Eq. B-19 of Ref. [123]). Therefore Eq. C.9 can be written in Dyson series form:

$$
\begin{aligned}
A_{f \leftarrow i} & =e^{-i \omega_{f} t}\left\{\delta_{f, i}+\left[\left(\frac{-i}{\hbar}\right) \int_{0}^{t} d t^{\prime} e^{i \omega_{f i} t^{\prime}} V_{f i}\left(t^{\prime}\right)\right]\right. \\
& +\left(\frac{-i}{\hbar}\right)^{2} \sum_{n}\left[\int_{0}^{t} d t^{\prime \prime} \int_{0}^{t^{\prime \prime}} d t^{\prime} e^{i \omega_{f n} t^{\prime \prime}} V_{f n}\left(t^{\prime \prime}\right) e^{i \omega_{n i} t^{\prime}} V_{n i}\left(t^{\prime}\right)\right] \\
& +\left(\frac{-i}{\hbar}\right)^{3} \sum_{n, n^{\prime}}\left[\int_{0}^{t} d t^{\prime \prime \prime} \int_{0}^{t^{\prime \prime \prime}} d t^{\prime \prime} \int_{0}^{t^{\prime \prime}} d t^{\prime} e^{i \omega_{f n} t^{\prime \prime \prime}} V_{f n}\left(t^{\prime \prime \prime}\right) e^{i \omega_{n n^{\prime}} t^{\prime \prime}} V_{n n^{\prime}}\left(t^{\prime \prime}\right) e^{i \omega_{n^{\prime} f} t^{\prime}} V_{n^{\prime} f}\left(t^{\prime}\right)\right] \\
& +\ldots\}
\end{aligned}
$$

where $0 \leq t^{\prime} \leq t^{\prime \prime} \leq t^{\prime \prime \prime} \leq \cdots \leq t$ for $t_{0}=0$. This is the textbook result for the transition amplitude via time-dependent perturbation theory, stated up to third order in the perturbation. Note that the time-dependent phase factor $e^{-i \omega_{f} t}$ is lost upon taking the square modulus to find the transition probability (Eq. C.3). A truncation of the perturbation series at any order gives an approximation to the transition amplitude. Unlike the Magnus expansion (§2.2), this approximation of the time evolution will not be unitary. Eq. C.10 can also be written with some of the time dependence explicitly divided up into intervals 
such that

$$
\begin{aligned}
A_{f \leftarrow i} & =\left\{e^{-i \omega_{f}\left(t-t_{0}\right)} \delta_{f, i}+\left[\left(\frac{-i}{\hbar}\right) \int_{t_{0}}^{t} d t^{\prime} e^{-i \omega_{f}\left(t-t^{\prime}\right)} V_{f i}\left(t^{\prime}\right) e^{-i \omega_{i}\left(t^{\prime}-t_{0}\right)}\right]\right. \\
& +\left(\frac{-i}{\hbar}\right)^{2} \sum_{n}\left[\int_{t_{0}}^{t} d t^{\prime \prime} \int_{t_{0}}^{t^{\prime \prime}} d t^{\prime} e^{-i \omega_{f}\left(t-t^{\prime \prime}\right)} V_{f n}\left(t^{\prime \prime}\right) e^{-i \omega_{n}\left(t^{\prime \prime}-t^{\prime}\right)} V_{n i}\left(t^{\prime}\right) e^{-i \omega_{i}\left(t^{\prime}-t_{0}\right)}\right] \\
& +\left(\frac{-i}{\hbar}\right)^{3} \sum_{n, n^{\prime}}\left[\int_{t_{0}}^{t} d t^{\prime \prime \prime} \int_{t_{0}}^{t^{\prime \prime \prime}} d t^{\prime \prime} \int_{t_{0}}^{t^{\prime \prime}} d t^{\prime}\right. \\
& \left.\times e^{-i \omega_{f}\left(t-t^{\prime \prime \prime}\right)} V_{f n}\left(t^{\prime \prime \prime}\right) e^{-i \omega_{n}\left(t^{\prime \prime \prime}-t^{\prime \prime}\right)} V_{n n^{\prime}}\left(t^{\prime \prime}\right) e^{-i \omega_{n^{\prime}}\left(t^{\prime \prime}-t^{\prime}\right)} V_{n^{\prime} f}\left(t^{\prime}\right) e^{-i \omega_{i}\left(t^{\prime}-t_{0}\right)}\right] \\
+ & \ldots\}, \quad \text { (C. } 11)
\end{aligned}
$$

where the initial condition is now $t=t_{0}$. Eq. C.11 may be compared to Eq. XVII.24 in $§$ XVII.I.2 of Messiah [272], which contains an intuitive graphical interpretation of this perturbation series. 


\section{Appendix D}

\section{Dynamics of two $J$-coupled}

\section{spin-1/2 nuclei}

This Appendix will present equations for the time evolution of some of the density operator components in a $J$-coupled system of two spin- $1 / 2$ nuclei in the presence of a magnetic field. These equations are exact and therefore valid over the full range between the strong and weak coupling limits. The Hamiltonian for the two-spin $I-S$ system is a sum of Zeeman and $J$-coupling contributions:

$$
\hat{H}=\hat{H}_{Z}+\hat{H}_{J}=\hbar\left(\omega_{I} \hat{I}_{z}+\omega_{S} \hat{S}_{z}+\omega_{J} \underline{\hat{I}} \cdot \underline{\hat{S}}\right),
$$

where $\omega_{J}=2 \pi J$ is the $J$-coupling constant in angular frequency units (it will be assumed that $\omega_{J}>0$ ), and $\omega_{I}$ and $\omega_{S}$ are the Larmor frequencies of spins $I$ and $S$, respectively. This Hamiltonian could represent a laboratory-frame spin system in a magnetic field $\underline{B}_{0}=B_{0} \underline{\tilde{z}}$ with $\omega_{I}=-\gamma B_{0}$ and $\omega_{I}=-\gamma B_{0}$, or a homonuclear rotating-frame spin system where $\omega_{I}$ 
and $\omega_{S}$ represent the resonance offset frequencies of the two spins. The quantization axis is along $z$, the direction of the magnetic field.

The Hamiltonian $\hat{H}$ can be diagonalized to give the following eigenfrequencies:

$$
\begin{aligned}
\omega_{1} & =\frac{1}{2}\left(\omega_{I}+\omega_{S}\right)+\frac{1}{4} \omega_{J} \\
\omega_{2} & =\frac{1}{2} \sqrt{\left(\omega_{I}-\omega_{S}\right)^{2}+\omega_{J}^{2}}-\frac{1}{4} \omega_{J}, \\
\omega_{3} & =-\frac{1}{2} \sqrt{\left(\omega_{I}-\omega_{S}\right)^{2}+\omega_{J}^{2}}-\frac{1}{4} \omega_{J}, \\
\omega_{4} & =-\frac{1}{2}\left(\omega_{I}+\omega_{S}\right)+\frac{1}{4} \omega_{J}
\end{aligned}
$$

If a mixing angle is defined as $\theta=\frac{1}{2} \tan ^{-1}\left[\omega_{J} /\left(\omega_{I}-\omega_{S}\right)\right][7, \S 2.1 .9]$, the corresponding eigenstates are $\left|\psi_{1}\right\rangle=|++\rangle,\left|\psi_{2}\right\rangle=\cos \theta|+-\rangle+\sin \theta|-+\rangle,\left|\psi_{3}\right\rangle=-\sin \theta|+-\rangle+\cos \theta|-+\rangle$, and $\left|\psi_{4}\right\rangle=|--\rangle$, where the Zeeman eigenstates are $| \pm \pm\rangle=\left|I=\frac{1}{2}, m_{I}= \pm \frac{1}{2} ; S=\frac{1}{2}, m_{S}= \pm \frac{1}{2}\right\rangle$. The addition of the $J$-coupling interaction to the Zeeman interaction mixes the $|+-\rangle$ and $|-+\rangle$ Zeeman eigenstates, or alternatively, the addition of the magnetic field to the $J$ coupled system breaks the isotropy of spin space and mixes the singlet and triplet total angular momentum eigenstates with zero projection on the quantization axis. Note that the individual $z$-components of angular momentum $\hat{I}_{z}$ and $\hat{S}_{z}$ are constants of the motion since $\left[\hat{I}_{z}, \hat{H}\right]=0$ and $\left[\hat{S}_{z}, \hat{H}\right]=0$. This implies that the system is invariant to rotations of either spin about the $z$-axis. A corollary of these facts is that the $z$-component of total angular momentum is also a constant of the motion since $\left[\left(\hat{I}_{z}+\hat{S}_{z}\right), \hat{H}\right]=0$. The total system is therefore axially symmetric about the $z$-axis and invariant to rotations in the combined space, i.e., in which both spins are rotated by the same angle about the $z$-axis. 
The dynamics of the density operator are given by:

$$
\hat{\rho}(t)=e^{-i \hat{H} t / \hbar} \hat{\rho}(0) e^{+i \hat{H} t / \hbar}
$$

This equation may be solved easily in the eigenbasis of the Hamiltonian. Some expressions are given for the evolution of the $\hat{I}_{x}, \hat{I}_{y}, \hat{I}_{z}, \hat{S}_{x}, \hat{S}_{y}$, and $\hat{S}_{z}$ single-spin magnetization components, e.g. $\hat{I}_{x}(t)=e^{-i \hat{H} t / \hbar} \hat{I}_{x} e^{+i \hat{H} t / \hbar}$.

\section{A. General case:}

$$
\begin{aligned}
\hat{I}_{x}(t)= & \frac{1}{2}\left[\sin ^{2} \theta\left(\cos \omega_{12} t+\cos \omega_{34} t\right)+\cos ^{2} \theta\left(\cos \omega_{24} t+\cos \omega_{13} t\right)\right] \hat{I}_{x} \\
& +\frac{1}{2}\left[\sin ^{2} \theta\left(\sin \omega_{12} t+\sin \omega_{34} t\right)+\cos ^{2} \theta\left(\sin \omega_{24} t+\sin \omega_{13} t\right)\right] \hat{I}_{y} \\
& +\frac{1}{2} \cos \theta \sin \theta\left[\cos \omega_{12} t-\cos \omega_{34} t+\cos \omega_{24} t-\cos \omega_{13} t\right] \hat{S}_{x} \\
& +\frac{1}{2} \cos \theta \sin \theta\left[\sin \omega_{12} t-\sin \omega_{34} t+\sin \omega_{24} t-\sin \omega_{13} t\right] \hat{S}_{y} \\
& +\frac{1}{2}\left[\sin ^{2} \theta\left(\cos \omega_{12} t-\cos \omega_{34} t\right)-\cos ^{2} \theta\left(\cos \omega_{24} t-\cos \omega_{13} t\right)\right] 2 \hat{I}_{x} \hat{S}_{z} \\
& +\frac{1}{2}\left[\sin { }^{2} \theta\left(\sin \omega_{12} t-\sin \omega_{34} t\right)-\cos ^{2} \theta\left(\sin \omega_{24} t-\sin \omega_{13} t\right)\right] 2 \hat{I}_{y} \hat{S}_{z} \\
& +\frac{1}{2} \cos \theta \sin \theta\left[\cos \omega_{12} t+\cos \omega_{34} t-\cos \omega_{24} t-\cos \omega_{13} t\right] 2 \hat{I}_{z} \hat{S}_{x} \\
& +\frac{1}{2} \cos \theta \sin \theta\left[\sin \omega_{12} t+\sin \omega_{34} t-\sin \omega_{24} t-\sin \omega_{13} t\right] 2 \hat{I}_{z} \hat{S}_{y}
\end{aligned}
$$




$$
\begin{aligned}
& \hat{I}_{y}(t)=\frac{1}{2}\left[\sin ^{2} \theta\left(\cos \omega_{12} t+\cos \omega_{34} t\right)+\cos ^{2} \theta\left(\cos \omega_{24} t+\cos \omega_{13} t\right)\right] \hat{I}_{y} \\
& -\frac{1}{2}\left[\sin ^{2} \theta\left(\sin \omega_{12} t+\sin \omega_{34} t\right)+\cos ^{2} \theta\left(\sin \omega_{24} t+\sin \omega_{13} t\right)\right] \hat{I}_{x} \\
& +\frac{1}{2} \cos \theta \sin \theta\left[\cos \omega_{12} t-\cos \omega_{34} t+\cos \omega_{24} t-\cos \omega_{13} t\right] \hat{S}_{y} \\
& -\frac{1}{2} \cos \theta \sin \theta\left[\sin \omega_{12} t-\sin \omega_{34} t+\sin \omega_{24} t-\sin \omega_{13} t\right] \hat{S}_{x} \\
& +\frac{1}{2}\left[\sin ^{2} \theta\left(\cos \omega_{12} t-\cos \omega_{34} t\right)-\cos ^{2} \theta\left(\cos \omega_{24} t-\cos \omega_{13} t\right)\right] 2 \hat{I}_{y} \hat{S}_{z} \\
& -\frac{1}{2}\left[\sin ^{2} \theta\left(\sin \omega_{12} t-\sin \omega_{34} t\right)-\cos ^{2} \theta\left(\sin \omega_{24} t-\sin \omega_{13} t\right)\right] 2 \hat{I}_{x} \hat{S}_{z} \\
& +\frac{1}{2} \cos \theta \sin \theta\left[\cos \omega_{12} t+\cos \omega_{34} t-\cos \omega_{24} t-\cos \omega_{13} t\right] 2 \hat{I}_{z} \hat{S}_{y} \\
& -\frac{1}{2} \cos \theta \sin \theta\left[\sin \omega_{12} t+\sin \omega_{34} t-\sin \omega_{24} t-\sin \omega_{13} t\right] 2 \hat{I}_{z} \hat{S}_{x} \\
& \hat{I}_{z}(t)=\frac{1}{4}\left[3+\left(\cos ^{2} 2 \theta-\sin ^{2} 2 \theta\right)+2 \sin ^{2} 2 \theta \cos \omega_{23} t\right] \hat{I}_{z} \\
& +\frac{1}{4}\left[1-\left(\cos ^{2} 2 \theta-\sin ^{2} 2 \theta\right)-2 \sin ^{2} 2 \theta \cos \omega_{23} t\right] \hat{S}_{z} \\
& +\sin \theta \cos \theta\left[\sin \omega_{23} t\right] 2\left(\hat{I}_{x} \hat{S}_{y}-\hat{I}_{y} \hat{S}_{x}\right) \\
& +\frac{1}{2} \sin 2 \theta \cos 2 \theta\left[1-\cos \omega_{23} t\right] 2\left(\hat{I}_{x} \hat{S}_{x}+\hat{I}_{y} \hat{S}_{y}\right)
\end{aligned}
$$




$$
\begin{aligned}
\hat{S}_{x}(t)= & \frac{1}{2}\left[\sin ^{2} \theta\left(\cos \omega_{12} t+\cos \omega_{34} t\right)+\cos ^{2} \theta\left(\cos \omega_{24} t+\cos \omega_{13} t\right)\right] \hat{S}_{x} \\
& +\frac{1}{2}\left[\sin ^{2} \theta\left(\sin \omega_{12} t+\sin \omega_{34} t\right)+\cos ^{2} \theta\left(\sin \omega_{24} t+\sin \omega_{13} t\right)\right] \hat{S}_{y} \\
& +\frac{1}{2} \cos \theta \sin \theta\left[\cos \omega_{12} t-\cos \omega_{34} t+\cos \omega_{24} t-\cos \omega_{13} t\right] \hat{I}_{x} \\
& +\frac{1}{2} \cos \theta \sin \theta\left[\sin \omega_{12} t-\sin \omega_{34} t+\sin \omega_{24} t-\sin \omega_{13} t\right] \hat{I}_{y} \\
& +\frac{1}{2}\left[\sin ^{2} \theta\left(\cos \omega_{12} t-\cos \omega_{34} t\right)-\cos ^{2} \theta\left(\cos \omega_{24} t-\cos \omega_{13} t\right)\right] 2 \hat{I}_{z} \hat{S}_{x} \\
& +\frac{1}{2}\left[\sin { }^{2} \theta\left(\sin \omega_{12} t-\sin \omega_{34} t\right)-\cos ^{2} \theta\left(\sin \omega_{24} t-\sin \omega_{13} t\right)\right] 2 \hat{I}_{z} \hat{S}_{y} \\
& +\frac{1}{2} \cos \theta \sin \theta\left[\cos \omega_{12} t+\cos \omega_{34} t-\cos \omega_{24} t-\cos \omega_{13} t\right] 2 \hat{I}_{x} \hat{S}_{z} \\
& +\frac{1}{2} \cos \theta \sin \theta\left[\sin \omega_{12} t+\sin \omega_{34} t-\sin \omega_{24} t-\sin \omega_{13} t\right] 2 \hat{I}_{y} \hat{S}_{z}
\end{aligned}
$$

$$
\begin{aligned}
\hat{S}_{y}(t)= & \frac{1}{2}\left[\sin ^{2} \theta\left(\cos \omega_{12} t+\cos \omega_{34} t\right)+\cos ^{2} \theta\left(\cos \omega_{24} t+\cos \omega_{13} t\right)\right] \hat{S}_{y} \\
& -\frac{1}{2}\left[\sin ^{2} \theta\left(\sin \omega_{12} t+\sin \omega_{34} t\right)+\cos ^{2} \theta\left(\sin \omega_{24} t+\sin \omega_{13} t\right)\right] \hat{S}_{x} \\
& +\frac{1}{2} \cos \theta \sin \theta\left[\cos \omega_{12} t-\cos \omega_{34} t+\cos \omega_{24} t-\cos \omega_{13} t\right] \hat{I}_{y} \\
& -\frac{1}{2} \cos \theta \sin \theta\left[\sin \omega_{12} t-\sin \omega_{34} t+\sin \omega_{24} t-\sin \omega_{13} t\right] \hat{I}_{x} \\
& +\frac{1}{2}\left[\sin ^{2} \theta\left(\cos \omega_{12} t-\cos \omega_{34} t\right)-\cos ^{2} \theta\left(\cos \omega_{24} t-\cos \omega_{13} t\right)\right] 2 \hat{I}_{z} \hat{S}_{y} \\
& -\frac{1}{2}\left[\sin { }^{2} \theta\left(\sin \omega_{12} t-\sin \omega_{34} t\right)-\cos ^{2} \theta\left(\sin \omega_{24} t-\sin \omega_{13} t\right)\right] 2 \hat{I}_{z} \hat{S}_{x} \\
& +\frac{1}{2} \cos \theta \sin \theta\left[\cos \omega_{12} t+\cos \omega_{34} t-\cos \omega_{24} t-\cos \omega_{13} t\right] 2 \hat{I}_{y} \hat{S}_{z} \\
& -\frac{1}{2} \cos \theta \sin \theta\left[\sin \omega_{12} t+\sin \omega_{34} t-\sin \omega_{24} t-\sin \omega_{13} t\right] 2 \hat{I}_{x} \hat{S}_{z}
\end{aligned}
$$




$$
\begin{aligned}
\hat{S}_{z}(t)= & \frac{1}{4}\left[3+\left(\cos ^{2} 2 \theta-\sin ^{2} 2 \theta\right)+2 \sin ^{2} 2 \theta \cos \omega_{23} t\right] \hat{S}_{z} \\
& +\frac{1}{4}\left[1-\left(\cos ^{2} 2 \theta-\sin ^{2} 2 \theta\right)-2 \sin ^{2} 2 \theta \cos \omega_{23} t\right] \hat{I}_{z} \\
& -\sin \theta \cos \theta\left[\sin \omega_{23} t\right] 2\left(\hat{I}_{x} \hat{S}_{y}-\hat{I}_{y} \hat{S}_{x}\right) \\
& -\frac{1}{2} \sin 2 \theta \cos 2 \theta\left[1-\cos \omega_{23} t\right] 2\left(\hat{I}_{x} \hat{S}_{x}+\hat{I}_{y} \hat{S}_{y}\right)
\end{aligned}
$$

B: Case $\omega_{I}=\omega_{S} \equiv \omega_{0}, \hat{H}=\hbar \omega_{0}\left(\hat{I}_{z}+\hat{S}_{z}\right)+\hat{H}_{J}$ :

This case could represent either a chemically-equivalent homonuclear spin pair in the rotating frame with a resonance offset, or any homonuclear spin pair in the laboratory frame at a low magnetic field where chemical shifts are negligible. In this case the Zeeman Hamiltonian is proportional to the $z$-component of total angular momentum $\hat{I}_{z}+\hat{S}_{z}$, which commutes with the $J$-coupling Hamiltonian (as do all components of total angular momentum, since $\hat{H}_{J}$ is isotropic in the combined spin space). Therefore $\left[\hat{H}_{Z}, \hat{H}_{J}\right]=0$, which is not the case when the spins are inequivalent. Of course, the $z$-component of total angular momentum is still conserved.

When the spins are identical the mixing angle reduces to: $\theta=\pi / 4 \Rightarrow \cos \theta=$ $\sqrt{1 / 2}, \sin \theta=\sqrt{1 / 2}, \cos 2 \theta=0, \sin 2 \theta=1$. The eigenfrequencies are: $\omega_{1}=\omega_{0}+\omega_{J} / 4$ $\omega_{2}=\omega_{J} / 4, \omega_{3}=-3 \omega_{J} / 4$, and $\omega_{4}=-\omega_{0}+\omega_{J} / 4$, such that $\omega_{12}=\omega_{0}, \omega_{34}=\omega_{0}-\omega_{J}$, $\omega_{24}=\omega_{0}, \omega_{13}=\omega_{0}+\omega_{J}$, and $\omega_{23}=\omega_{J}$. 


$$
\begin{aligned}
\hat{I}_{x}(t)= & \frac{1}{4}\left[2 \cos \omega_{0} t+\cos \left(\left(\omega_{0}+\omega_{J}\right) t\right)+\cos \left(\left(\omega_{0}-\omega_{J}\right) t\right)\right] \hat{I}_{x} \\
& +\frac{1}{4}\left[2 \sin \omega_{0} t+\sin \left(\left(\omega_{0}+\omega_{J}\right) t\right)+\sin \left(\left(\omega_{0}-\omega_{J}\right) t\right)\right] \hat{I}_{y} \\
& +\frac{1}{4}\left[2 \cos \omega_{0} t-\cos \left(\left(\omega_{0}+\omega_{J}\right) t\right)-\cos \left(\left(\omega_{0}-\omega_{J}\right) t\right)\right] \hat{S}_{x} \\
& +\frac{1}{4}\left[2 \sin \omega_{0} t-\sin \left(\left(\omega_{0}+\omega_{J}\right) t\right)-\sin \left(\left(\omega_{0}-\omega_{J}\right) t\right)\right] \hat{S}_{y} \\
& +\frac{1}{4}\left[\cos \left(\left(\omega_{0}+\omega_{J}\right) t\right)-\cos \left(\left(\omega_{0}-\omega_{J}\right) t\right)\right] 2 \hat{I}_{x} \hat{S}_{z} \\
& +\frac{1}{4}\left[\sin \left(\left(\omega_{0}+\omega_{J}\right) t\right)-\sin \left(\left(\omega_{0}-\omega_{J}\right) t\right)\right] 2 \hat{I}_{y} \hat{S}_{z} \\
& +\frac{1}{4}\left[-\cos \left(\left(\omega_{0}+\omega_{J}\right) t\right)+\cos \left(\left(\omega_{0}-\omega_{J}\right) t\right)\right] 2 \hat{I}_{z} \hat{S}_{x} \\
& +\frac{1}{4}\left[-\sin \left(\left(\omega_{0}+\omega_{J}\right) t\right)+\sin \left(\left(\omega_{0}-\omega_{J}\right) t\right)\right] 2 \hat{I}_{z} \hat{S}_{y}
\end{aligned}
$$

$$
\begin{aligned}
\hat{I}_{y}(t)= & \frac{1}{4}\left[2 \cos \omega_{0} t+\cos \left(\left(\omega_{0}+\omega_{J}\right) t\right)+\cos \left(\left(\omega_{0}-\omega_{J}\right) t\right)\right] \hat{I}_{y} \\
& -\frac{1}{4}\left[2 \sin \omega_{0} t+\sin \left(\left(\omega_{0}+\omega_{J}\right) t\right)+\sin \left(\left(\omega_{0}-\omega_{J}\right) t\right)\right] \hat{I}_{x} \\
& +\frac{1}{4}\left[2 \cos \omega_{0} t-\cos \left(\left(\omega_{0}+\omega_{J}\right) t\right)-\cos \left(\left(\omega_{0}-\omega_{J}\right) t\right)\right] \hat{S}_{y} \\
& -\frac{1}{4}\left[2 \sin \omega_{0} t-\sin \left(\left(\omega_{0}+\omega_{J}\right) t\right)-\sin \left(\left(\omega_{0}-\omega_{J}\right) t\right)\right] \hat{S}_{x} \\
& +\frac{1}{4}\left[\cos \left(\left(\omega_{0}+\omega_{J}\right) t\right)-\cos \left(\left(\omega_{0}-\omega_{J}\right) t\right)\right] 2 \hat{I}_{y} \hat{S}_{z} \\
& -\frac{1}{4}\left[\sin \left(\left(\omega_{0}+\omega_{J}\right) t\right)-\sin \left(\left(\omega_{0}-\omega_{J}\right) t\right)\right] 2 \hat{I}_{x} \hat{S}_{z} \\
& +\frac{1}{4}\left[-\cos \left(\left(\omega_{0}+\omega_{J}\right) t\right)+\cos \left(\left(\omega_{0}-\omega_{J}\right) t\right)\right] 2 \hat{I}_{z} \hat{S}_{y} \\
& -\frac{1}{4}\left[-\sin \left(\left(\omega_{0}+\omega_{J}\right) t\right)+\sin \left(\left(\omega_{0}-\omega_{J}\right) t\right)\right] 2 \hat{I}_{z} \hat{S}_{x}
\end{aligned}
$$




$$
\begin{aligned}
\hat{I}_{z}(t)=\frac{1}{2}[1+ & \left.\cos \omega_{J} t\right] \hat{I}_{z}+\frac{1}{2}\left[1-\cos \omega_{J} t\right] \hat{S}_{z}+\frac{1}{2}\left[\sin \omega_{J} t\right] 2\left(\hat{I}_{x} \hat{S}_{y}-\hat{I}_{y} \hat{S}_{x}\right) \\
\hat{S}_{x}(t)= & \frac{1}{4}\left[2 \cos \omega_{0} t+\cos \left(\left(\omega_{0}+\omega_{J}\right) t\right)+\cos \left(\left(\omega_{0}-\omega_{J}\right) t\right)\right] \hat{S}_{x} \\
& +\frac{1}{4}\left[2 \sin \omega_{0} t+\sin \left(\left(\omega_{0}+\omega_{J}\right) t\right)+\sin \left(\left(\omega_{0}-\omega_{J}\right) t\right)\right] \hat{S}_{y} \\
& +\frac{1}{4}\left[2 \cos \omega_{0} t-\cos \left(\left(\omega_{0}+\omega_{J}\right) t\right)-\cos \left(\left(\omega_{0}-\omega_{J}\right) t\right)\right] \hat{I}_{x} \\
& +\frac{1}{4}\left[2 \sin \omega_{0} t-\sin \left(\left(\omega_{0}+\omega_{J}\right) t\right)-\sin \left(\left(\omega_{0}-\omega_{J}\right) t\right)\right] \hat{I}_{y} \\
& +\frac{1}{4}\left[\cos \left(\left(\omega_{0}+\omega_{J}\right) t\right)-\cos \left(\left(\omega_{0}-\omega_{J}\right) t\right)\right] 2 \hat{I}_{z} \hat{S}_{x} \\
& +\frac{1}{4}\left[\sin \left(\left(\omega_{0}+\omega_{J}\right) t\right)-\sin \left(\left(\omega_{0}-\omega_{J}\right) t\right)\right] 2 \hat{I}_{z} \hat{S}_{y} \\
& +\frac{1}{4}\left[-\cos \left(\left(\omega_{0}+\omega_{J}\right) t\right)+\cos \left(\left(\omega_{0}-\omega_{J}\right) t\right)\right] 2 \hat{I}_{x} \hat{S}_{z} \\
& +\frac{1}{4}\left[-\cos \left(\left(\omega_{0}+\omega_{J}\right) t\right)+\sin \left(\left(\omega_{0}+\omega_{J}\right) t\right)+\sin \left(\left(\omega_{0}-\omega_{J}\right) t\right)\right] 2 \hat{I}_{x} \hat{S}_{z} \\
& +\frac{1}{4}\left[-\sin \left(\left(\omega_{0}+\omega_{J}\right) t\right)+\sin \left(\left(\omega_{0}-\omega_{J}\right) t\right)\right] 2 \hat{I}_{y} \hat{S}_{z} \\
& +\frac{1}{4}\left[\cos \left(\left(\omega_{0}+\omega_{J}\right) t\right)-\cos \left(\left(\omega_{0}-\omega_{J}\right) t\right)\right] 2 \hat{I}_{z} \hat{S}_{y} \\
& +\frac{1}{4}\left[2 \sin \left(\left(\omega_{0}+\omega_{J}\right) t\right)-\sin \left(\left(\omega_{0}-\omega_{J}\right) t\right)\right] 2 \hat{I}_{z} \hat{S}_{x} \\
\hat{S}_{y}(t)= & \frac{1}{4}\left[2 \cos \omega_{0} t-\cos \left(\left(\omega_{0}+\omega_{J}\right) t\right)-\cos \left(\left(\omega_{0}-\omega_{J}\right) t\right)\right] \hat{I}_{y} \\
& \\
& \\
& \\
& \\
& \\
&
\end{aligned}
$$




$$
\hat{S}_{z}(t)=\frac{1}{2}\left[1+\cos \omega_{J} t\right] \hat{S}_{z}+\frac{1}{2}\left[1-\cos \omega_{J} t\right] \hat{I}_{z}-\frac{1}{2}\left[\sin \omega_{J} t\right] 2\left(\hat{I}_{x} \hat{S}_{y}-\hat{I}_{y} \hat{S}_{x}\right)
$$

C: Case $\omega_{I}=\omega_{S} \equiv 0, \hat{H}=\hat{H}_{J}$

This is the case in which the two spins are indistinguishable by their chemical shifts, and only the $J$-coupling between them remains. It could represent the case of a chemically-equivalent homonuclear pair in the rotating frame, when the rf frequency is exactly on resonance. It could also represent any two-spin system in the laboratory frame at zero field, in which case even heteronuclei become "chemically equivalent" spins (of course, heteronuclei can be distinguished at zero field when a magnetic pulse is applied). It could also represent the case of a chemically-equivalent homonuclear pair in the rotating frame, when the rf is exactly on resonance. The $J$-coupling Hamiltonian represents a scalar quantity in the combined two-spin space, so it commutes with all components of the total $\operatorname{angular}$ momentum $\underline{\hat{I}}+\underline{\hat{S}}:\left[\left(\hat{I}_{x}+\hat{S}_{x}\right), \hat{H}_{J}\right]=0,\left[\left(\hat{I}_{y}+\hat{S}_{y}\right), \hat{H}_{J}\right]=0$, and $\left[\left(\hat{I}_{z}+\hat{S}_{z}\right), \hat{H}_{J}\right]=0$. The components of total angular momentum are therefore constants of the motion in the absence of a magnetic field, and the eigenstates of the system are the eigenstates of total angular momentum. It follows that the $J$-coupled spin system in zero field is isotropic and therefore invariant to all rotations in which both spins are rotated by the same angle about the same axis. Evolution under this Hamiltonian is called "isotropic mixing".

$$
\hat{I}_{x}(t)=\frac{1}{2}\left[1+\cos \omega_{J} t\right] \hat{I}_{x}+\frac{1}{2}\left[1-\cos \omega_{J} t\right] \hat{S}_{x}+\frac{1}{2}\left[\sin \omega_{J} t\right] 2\left(\hat{I}_{y} \hat{S}_{z}-\hat{I}_{z} \hat{S}_{y}\right)
$$




$$
\begin{aligned}
& \hat{I}_{y}(t)=\frac{1}{2}\left[1+\cos \omega_{J} t\right] \hat{I}_{y}+\frac{1}{2}\left[1-\cos \omega_{J} t\right] \hat{S}_{y}+\frac{1}{2}\left[\sin \omega_{J} t\right] 2\left(\hat{I}_{z} \hat{S}_{x}-\hat{I}_{x} \hat{S}_{z}\right) \\
& \hat{I}_{z}(t)=\frac{1}{2}\left[1+\cos \omega_{J} t\right] \hat{I}_{z}+\frac{1}{2}\left[1-\cos \omega_{J} t\right] \hat{S}_{z}+\frac{1}{2}\left[\sin \omega_{J} t\right] 2\left(\hat{I}_{x} \hat{S}_{y}-\hat{I}_{y} \hat{S}_{x}\right) \\
& \hat{S}_{x}(t)=\frac{1}{2}\left[1+\cos \omega_{J} t\right] \hat{S}_{x}+\frac{1}{2}\left[1-\cos \omega_{J} t\right] \hat{I}_{x}+\frac{1}{2}\left[\sin \omega_{J} t\right] 2\left(\hat{I}_{z} \hat{S}_{y}-\hat{I}_{y} \hat{S}_{z}\right) \\
& \hat{S}_{y}(t)=\frac{1}{2}\left[1+\cos \omega_{J} t\right] \hat{S}_{y}+\frac{1}{2}\left[1-\cos \omega_{J} t\right] \hat{I}_{y}+\frac{1}{2}\left[\sin \omega_{J} t\right] 2\left(\hat{I}_{x} \hat{S}_{z}-\hat{I}_{z} \hat{S}_{x}\right) \\
& \hat{S}_{z}(t)=\frac{1}{2}\left[1+\cos \omega_{J} t\right] \hat{S}_{z}+\frac{1}{2}\left[1-\cos \omega_{J} t\right] \hat{I}_{z}+\frac{1}{2}\left[\sin \omega_{J} t\right] 2\left(\hat{I}_{y} \hat{S}_{x}-\hat{I}_{x} \hat{S}_{y}\right)
\end{aligned}
$$




\section{Appendix E}

\section{Simulation of the distant dipolar}

\section{field for cylindrically-symmetric}

\section{geometries (Matlab code)}

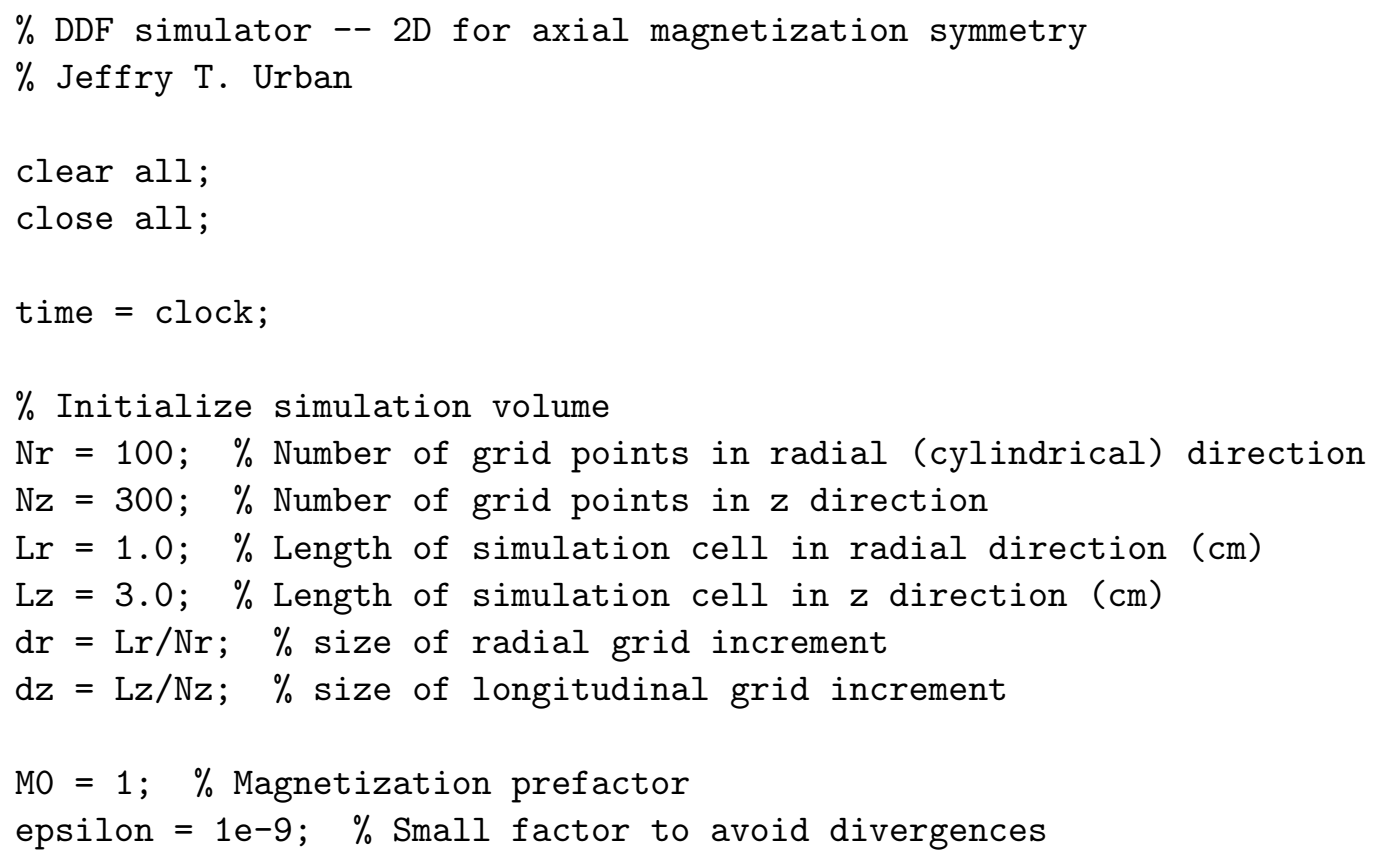


$\%$ Definition of coaxial cylinders (defined between $z=-d / 2$ and $z=+d / 2$ ) $\mathrm{R} 1=0.50 ; \%$ Radius of inner cylinder in $\mathrm{cm}$ R2 $=0.25 ; \%$ Radius of outer cylinder in $\mathrm{cm}$ $\mathrm{d}=1.5 ; \%$ Length of cylinder in $\mathrm{cm}$ $\mathrm{nt}=$ floor $(\mathrm{Nz} *(1+\mathrm{d} / \mathrm{Lz}) / 2+1 / 2) ; \% \mathrm{z}$-index for top of cylinder $\mathrm{nb}=\operatorname{ceil}(\mathrm{Nz} *(1-\mathrm{d} / \mathrm{Lz}) / 2+1 / 2) ; \% \mathrm{z}$-index for bottom of cylinder $\mathrm{kz}=1.0 / \mathrm{d} ; \% \mathrm{z}$-modulation wavenumber

$\% \mathrm{kz}=$ epsilon; $\%$ no modulation $(\mathrm{kz}=0)$

$\%$ Define $z$-directed magnetization density

$\%$ Define grid variables

$r=\mathrm{dr} / 2: \mathrm{dr}: \operatorname{Lr}-\mathrm{dr} / 2 ; \%$ Radial coordinate: center of grid squares

$\% r=$ epsilon:dr:Lr; \% Radial coordinate: edge of grid squares

$\mathrm{z}=-\mathrm{L} z / 2+\mathrm{dz} / 2: \mathrm{d} z: \mathrm{Lz} / 2-\mathrm{dz} / 2 ; \%$ Longitudinal coordinate

$\mathrm{hz}=(\mathrm{z}>=-\mathrm{d} / 2)+(\mathrm{z}<=\mathrm{d} / 2)-1 ; \%$ Defines cylinder volume in $\mathrm{z}$-direction $\mathrm{hr}=(\mathrm{r}>=\mathrm{R} 1)+(\mathrm{r}<=\mathrm{R} 2)-1 ; \%$ Defines cylinder volume in radial direction

\section{$\%$ Define z-magnetization}

psi $=0$;

$\% \mathrm{Mz}=(\mathrm{MO} * \cos (2 * \mathrm{pi} * \mathrm{kz} * \mathrm{z}+\mathrm{psi}) \cdot * \mathrm{hz}))^{\prime} *($ ones $(1, \mathrm{Nr}) \cdot * \mathrm{hr}) ;$

$\%$ Cosine-modulated $z$-magnetization $\mathrm{Mz}(z, r)$

$\% \mathrm{DM}=(-(2 * \mathrm{pi} * \mathrm{kz}) * \mathrm{M} 0 * \sin (2 * \mathrm{pi} * \mathrm{kz} * \mathrm{z}+\mathrm{psi}) . * \mathrm{hz})^{\prime} *($ ones $(1, \mathrm{Nr}) . * \mathrm{hr})$;

$\% \mathrm{dMz} / \mathrm{dz}$ for cosine-modulated $\mathrm{Mz}$

$\mathrm{Mz}=(\mathrm{MO} * \sin (2 * \mathrm{pi} * \mathrm{kz} *(\mathrm{z}+\mathrm{d} / 2)+\mathrm{psi}) \cdot * \mathrm{hz}){ }^{\prime} *($ ones $(1, \mathrm{Nr}) \cdot * \mathrm{hr}) ;$

$\%$ Sine-modulated $z$-magnetization $\mathrm{Mz}(z, r)$

$\mathrm{DM}=((2 * \mathrm{pi} * \mathrm{kz}) * \mathrm{M} 0 * \cos (2 * \mathrm{pi} * \mathrm{kz} *(\mathrm{z}+\mathrm{d} / 2)+\mathrm{psi}) . * \mathrm{hz})^{\prime} *($ ones $(1, \mathrm{Nr}) \cdot * \mathrm{hr})$;

$\% \mathrm{dMz} / \mathrm{dz}$ for sine-modulated $\mathrm{Mz}$

$\%$ Define magnetization projections at boundary surfaces

$\mathrm{t}=\mathrm{Mz}(\mathrm{nt}, \mathrm{:})$;

$\% \mathrm{nMt}=(\mathrm{MO} * \cos (2 * \mathrm{pi} * \mathrm{kz} *(\mathrm{~d} / 2)+\mathrm{psi}))^{\prime} *($ ones $(1, \mathrm{Nr}) \cdot * \mathrm{hr}) ;$

$\% \mathrm{n} . \mathrm{Mz}(\mathrm{zt}, \mathrm{r})$ for cosine-modulated $\mathrm{Mz}$

$\% \mathrm{nMb}=(-\mathrm{MO} * \cos (2 * \mathrm{pi} * \mathrm{kz} *(-\mathrm{d} / 2)+\mathrm{psi}))^{\prime} *($ ones $(1, \mathrm{Nr}) \cdot * \mathrm{hr}) ;$

$\% \mathrm{n} . \mathrm{Mz}(\mathrm{zb}, \mathrm{r})$ for cosine-modulated $\mathrm{Mz}$

$\mathrm{nMt}=(\mathrm{MO} * \sin (2 * \mathrm{pi} * \mathrm{kz} *(\mathrm{~d} / 2+\mathrm{d} / 2)+\mathrm{psi}))^{\prime} *($ ones $(1, \mathrm{Nr}) \cdot * \mathrm{hr})$;

$\% \mathrm{n} . \mathrm{Mz}(\mathrm{zt}, \mathrm{r})$ for sine-modulated $\mathrm{Mz}$

$\mathrm{nMb}=(-\mathrm{MO} * \sin (2 * \mathrm{pi} * \mathrm{kz} *(-\mathrm{d} / 2+\mathrm{d} / 2)+\mathrm{psi}))^{\prime} *($ ones $(1, \mathrm{Nr}) \cdot * \mathrm{hr}) ;$

$\% \mathrm{n} . \mathrm{Mz}(\mathrm{zb}, \mathrm{r})$ for sine-modulated $\mathrm{Mz}$

$\mathrm{z} 1=\mathrm{z}$;

$r 1=r ;$

$\mathrm{z} 2=\mathrm{z}^{\prime} *$ ones $(1, \mathrm{Nr})$;

$\mathrm{r} 2=\operatorname{ones}(\mathrm{Nz}, 1) * \mathrm{r}$; 
\% Calculate DDF, heteronuclear (z-component only)

$\%$ Point 2 = source, point 1 = field

$\%$ Calculate volume integral

for $(\mathrm{nr} 1=1: \mathrm{Nr})$;

for $(\mathrm{nz} 1=1: \mathrm{Nz})$;

$\mathrm{u}=\operatorname{sqrt}\left((4 * r 1(\mathrm{nr} 1) \cdot * r 2) \cdot /\left((\mathrm{r} 1(\mathrm{nr} 1)+\mathrm{r} 2) \cdot{ }^{\wedge} 2+\ldots\right.\right.$ $\left.\left.(z 1(n z 1)-z 2) .{ }^{-2}\right)\right)$ - epsilon; \% mu

$[\mathrm{K}, \mathrm{E}]=$ ellipke(u); \% Define complete elliptic integrals

$\mathrm{F}=(\mathrm{z} 1(\mathrm{nz} 1)-\mathrm{z} 2) \cdot *\left(\mathrm{u} \cdot{ }^{\wedge} 3\right) \cdot *(\mathrm{~K}+\mathrm{E} . /(1-\mathrm{u})) ; \%$ Integrand

$\operatorname{Iv}(\mathrm{nz} 1, \mathrm{nr} 1)=\operatorname{dz} * \operatorname{dr} *(-1 /(4 * \mathrm{pi})) *\left(1 /\left(4 * \mathrm{r} 1(\mathrm{nr} 1)^{\wedge}(3 / 2)\right)\right) * \ldots$ $\operatorname{sum}(\operatorname{sum}((1 . / \operatorname{sqrt}(r 2)) . * \mathrm{~F} . * \mathrm{DM})) ; \%$ Riemann sums end;

end;

$\%$ Calculate top and bottom surface integrals

for $(\mathrm{nr} 1=1: \mathrm{Nr})$;

for $(\mathrm{nz} 1=1: \mathrm{Nz})$;

ut $=\operatorname{sqrt}\left((4 * r 1(n r 1) * r) . /\left((r 1(n r 1)+r) \cdot{ }^{\wedge} 2+\ldots\right.\right.$ $\left.\left.(z 1(n z 1)-d / 2)^{\wedge} 2\right)\right)$ - epsilon; \% mu at top surface

$\mathrm{ub}=\operatorname{sqrt}\left((4 * \mathrm{r} 1(\mathrm{nr} 1) * r) \cdot /\left((\mathrm{r} 1(\mathrm{nr} 1)+\mathrm{r}) \cdot{ }^{\wedge} 2+\ldots\right.\right.$

$\left.\left.(z 1(n z 1)+d / 2)^{\wedge} 2\right)\right)-$ epsilon; $\%$ mu at bottom surface

[Kt,Et] = ellipke(ut); \% Complete elliptic integrals/top surface

$[\mathrm{Kb}, \mathrm{Eb}]=$ ellipke $(\mathrm{ub}) ; \% " \quad$ "/bottom surface

$\mathrm{Ft}=(\mathrm{z} 1(\mathrm{nz} 1)-\mathrm{d} / 2) \cdot *\left(\mathrm{ut} \cdot{ }^{\wedge} 3\right) \cdot *(\mathrm{Kt}+\mathrm{Et} . /(1-\mathrm{ut})) ;$

$\%$ Integrand for top surface

$\mathrm{Fb}=(\mathrm{z} 1(\mathrm{nz} 1)+\mathrm{d} / 2) \cdot *\left(\mathrm{ub} \cdot \wedge^{-3}\right) \cdot *(\mathrm{~Kb}+\mathrm{Eb} \cdot /(1-\mathrm{ub})) ;$

$\%$ Integrand for bottom surface

$\operatorname{Is}(\mathrm{nz} 1, \mathrm{nr} 1)=\operatorname{dr} *(1 /(4 * \mathrm{pi})) *\left(1 /\left(4 * \mathrm{r} 1(\mathrm{nr} 1)^{-}(3 / 2)\right)\right) * \ldots$

$\operatorname{sum}((1 . / \operatorname{sqrt}(r)) . *(\mathrm{Ft} . * \mathrm{nMt}+\mathrm{Fb} . * \mathrm{nMb})) ; \%$ Riemann sum end;

end;

$\mathrm{Hz}=\mathrm{Iv}+\mathrm{Is} ; \% \mathrm{H}-\mathrm{field}$ (volume + surface integral) $; \mathrm{H}=-\operatorname{grad}(\mathrm{Phi})$

$\mathrm{Bz}=\mathrm{Mz}+\mathrm{Hz} ; \quad \%$ B-field

$\%$ Reflect the magnetization/dipolar field across $\mathrm{x}=0$

Mz_symm = [fliplr(Mz), Mz $]$;

$\mathrm{Hz} \_$symm $=[\mathrm{fliplr}(\mathrm{Hz}), \mathrm{Hz}]$;

$\mathrm{Bz}_{-}$symm $=[\mathrm{fliplr}(\mathrm{Bz}), \mathrm{Bz}]$;

$r_{-}$symm $=[-f \operatorname{liplr}(r), r]$;

$\%$ Plot 


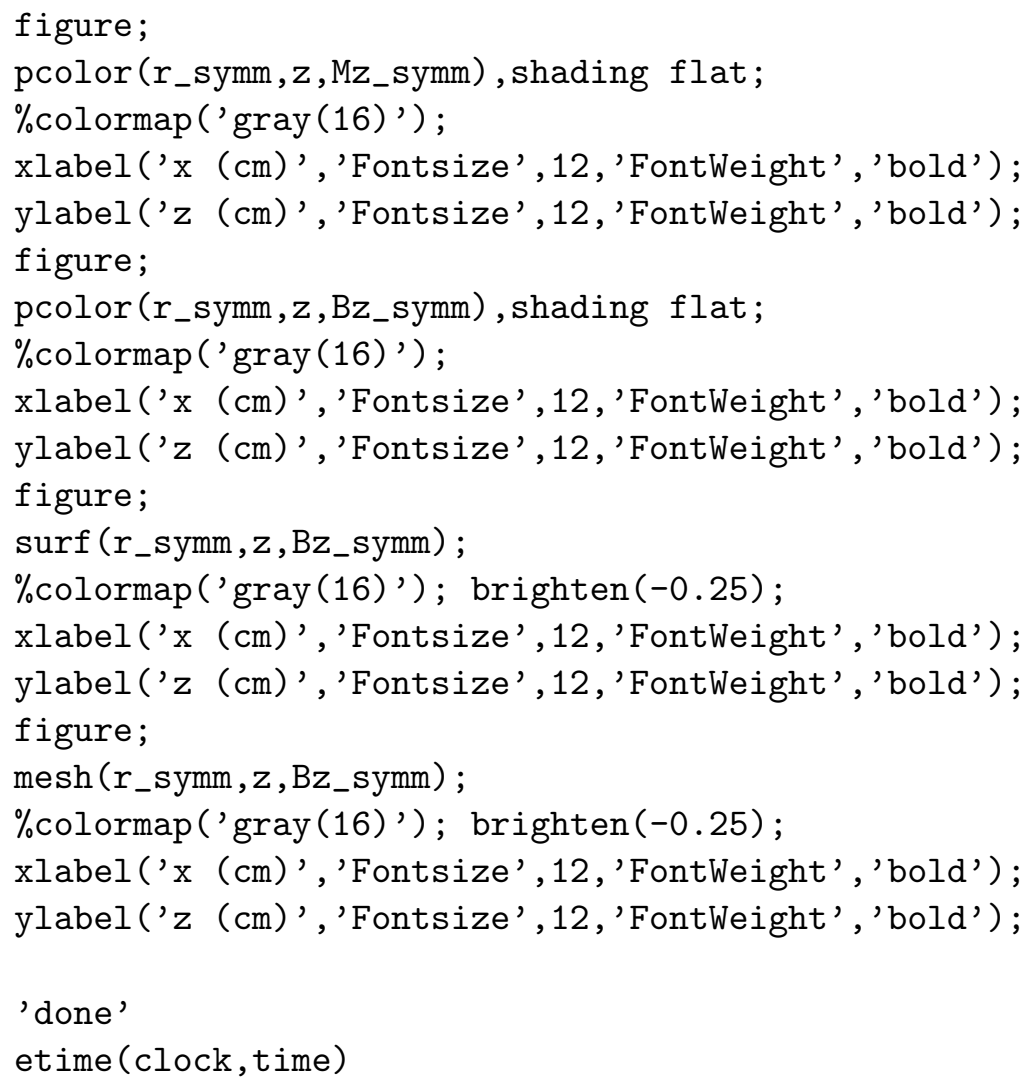

